\title{
A Search For Direct CP Violation in The Decay $K_{L, S} \rightarrow \pi^{+} \pi^{-} \gamma$
}

\author{
Michael Christopher Ronquest \\ Florissant, Missouri
}

B.S. Physics, University of Maryland, Baltimore County, 2000

A Dissertation presented to the Graduate Faculty of the University of Virginia in Candidacy for the Degree of

Doctor of Philosophy

Department of Physics

University of Virginia

December 2007 


\section{Abstract}

This thesis presents a search for direct CP violation in the decay $K_{L, S} \rightarrow \pi^{+} \pi^{-} \gamma$. Observation of direct $\mathrm{CP}$ violation is tantamount to observing the breaking of $\mathrm{CP}$ symmetry in the weak interaction, as it is this interaction which is responsible for this decay. If present, this phenomena is expected to manifest in direct photon emission from the $K_{L}$ decay. This direct CP violation is expected to be identified by the observation of the direct emission electric dipole (E1) photons. Since this process is small compared to the dominant M1 emission term, as well as the inner bremsstrahlung E1 photon emission process, observing the photon's energy and direction is not enough to isolate the process. Observing the time evolution of the photon emission from decays in a beam of coherent $K_{L}$ and $K_{S}$ affords a better method. However the signal is diluted by the kinematics of the decay, which are dominated by the inner bremsstrahlung process. Combining both methods - observing the momenta of the decay products and the time dependence of the decay, yields a powerful tool with which to search for this rare process.

Using data collected in 1997 and 1999 by the KTeV fixed-target experiment at Fermi National Accelerator Lab, we apply a theory describing the strength and characteristics of the various processes which are responsible for the decay $K_{L, S} \rightarrow \pi^{+} \pi^{-} \gamma$ : inner bremsstrahlung, M1 direct photon emission and finally E1 direct photon emission. The E1 direct photon emission process is split into two parts: a part that violates $\mathrm{CP}$ symmetry indirectly ( mixing type $\mathrm{CP}$ violation) and a part that violates $\mathrm{CP}$ directly ( via decay amplitudes ). The data consist of observed decays from KTeV's coherent $K_{L}$ and $K_{S}$ beam, as well as KTeV's pure $K_{L}$ beam. 


\section{Acknowledgments}

My foremost thanks go to my adviser, Brad Cox, with whom I have had the good fortune to have worked with for my entire graduate career. Not only did he prove to be an insightful and protective adviser, but he is also a good friend. He showed a great amount of understanding over the past year, which was certainly the hardest one of my life. I'd also like to thank Brad and his wife, Marguerite, for all the hospitality they've shown myself and others over the years - it was, and is, greatly appreciated. In addition to Brad, I also must thank Alexander Ledovskoy for a tremendous amount of help over the years both with this analysis and also with various other adventures. He was instrumental in dealing with a number of problems and bugs that cropped up over the years, especially in relation to the $\mathrm{KTeV}$ codebase. Finally, he was kind enough to put up with my naiveté and point out that more than a few of my "bright" ideas where in fact "crap"!

I must also thank John Shields for help in planting the seeds of understanding in my mind regarding the use and running of KTeVana and KTeVMC. He was also instrumental in dealing with the various $K_{L} \rightarrow \pi^{+} \pi^{-} \gamma$ theory papers that have been published over the years. Both Andrew Norman and Chad Materniak have over the past few years shown great patience and assistance in helping me deal with the computing resources here at UVa, in addition to teaching me a decent amount of physics and computer science along the way. Thanks also go to the rest of the UVa HEP crew, past and present, in no particular order, including Mike Balazs, Dan Andelin, Alexander Golossanov and of course the "Three Daves"; Williams, Smith and Phillips. I'd like to especially thank David Williams and his family for their friendship and support over the years and David Phillips for being a great officemate/building-jester during the past few years. 
My first two years at UVa were bearable due to my outstanding classmates including Scott Rohrbaugh, Charlie Molhoek, "Chairman" Mao Yi, Junzhong Yu, Jessica Reeves, Timour Kotchiev and Marianne Stefanini. Even though we seem to have all drifted our own ways over the years, those two years I'll never forget. Thanks to you, and what you helped me learn along the way, I didn't have to include a single hand-turkey in this dissertation.

Ofir Garcia and his family, including the wonderful Karina, have also been a great source of friendship and support over the years - I wish you all the best, no matter which continent each of you currently inhabit. And yes, I am willing to "speak into the microphone" regarding this.

The entire physics department at UVa has proved to be an excellent environment in which to work and study. Everyone from the graduate students, faculty, technical and office staff have in some way bailed me out of one jam or another over the years and for that I am grateful. Specific thanks go out to Chris Floyd, Pam Joseph, BH Kent, Theresa Marshal, and of course departmental über-moms Tammie Shifflett and Suzie Garrett for all their help.

This entire adventure started at UMBC, and I must thank all those at the physics department there who helped steer me in the right direction-Terrance Worchesky for introducing me to the joys of physics, Mark Henriksen for taking me on as a inexperienced research assistant and teaching me the basics of physics research and to Eric Titley, for teaching me much about the use of computers and Linux in a research environment. I'd also like to acknowledge the assistance and good times that came from my fellow undergrads Deandra Hughes, Reni Ayachitula, Colleen Jenkins and Jeremy Smith.

$\mathrm{KTeV}$ was the product of the collective efforts of hundreds of people. Their efforts in designing, building, running and calibrating the experiment resulted in the collection of an outstanding mass of data. It was this data that allowed this dissertation to be written and for that I thank the entire collaboration. Special thanks must also go to those whose special efforts contributed to the E832 dataset and its later analysis, including the "U. Chicago gang" - Ed Blucher, Rick Kessler, Alexander Glazov, Elizabeth Worchester and everyone else I forgot to mention and/or never had the fortune to meet.

My family has been a huge source of love, support and encouragement, and I 
wish to thank them as well, including David, Christine, Hannah and of course my mother. My thoughts also turn to my father, who laughed when he saw parts of this dissertation, but sadly he did not get to see me finish what I started.

Finally, my attention must turn to my wonderful wife, Naoko. Thanks for inviting yourself to my party three summers ago, and all the great things that followed (and will follow) afterward. Special thanks also go to Theodore Inu for taking care of Naoko while I finished my studies in Virginia.

My time spent on this dissertation was kindly supported by a number of sources. My final year was supported by the Faculty-Senate of the University of Virginia. Another year of my studies was supported by the Graduate School of Arts and Sciences of the University of Virginia. The UVa Department of Physics also contributed, in addition to the Department of Energy via my advisor's base grant. An additional year of my graduate studies was supported by the ARCS Foundation, for which I am quite grateful. KTeV was built and run using funds from the United States Department of Energy and the National Science Foundation in addition to the Japan Ministry of Education and Science. 


\section{Contents}

Abstract $\quad$ i

$\begin{array}{ll}\text { Acknowledgments } & \text { ii }\end{array}$

1 Introduction 1

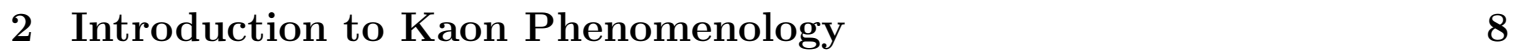

3 The Decay $K_{L, S} \rightarrow \pi^{+} \pi^{-} \gamma \quad 15$

3.1 Introduction . . . . . . . . . . . . . . . . 15

3.2 Decay rate as a function of invariant amplitude . . . . . . . . . . . . 19

3.3 Matrix Element for Kaon Decay . . . . . . . . . . . . . . . . . . . 20

3.4 Amplitudes for multipole transitions . . . . . . . . . . . . . 22

3.4.1 Inner Bremsstrahlung amplitudes . . . . . . . . . . . . . 22

3.4.2 Direct Emission Amplitudes . . . . . . . . . . . . . . . . . . . 23

3.4.3 Expected Values of Parameters . . . . . . . . . . . . . . 25

3.5 The Triple Differential Decay Rate of the Process $K_{L, S} \rightarrow \pi^{+} \pi^{-} \gamma$. . 27

4 The KTeV Experiment 33

$4.1 \mathrm{KTeV}$ Beamline . . . . . . . . . . . . . . . . . . 34

4.1.1 Kaon Production Target . . . . . . . . . . . . . . . 35

4.1.2 KTeV Coordinate System . . . . . . . . . . . . . . 35

4.1.3 Secondary Beamline Components . . . . . . . . . . . . . . . 38

4.1.4 Decay Volume . . . . . . . . . . . . . . . . . . . . . . . . . 39

4.1.5 Mask-Anti Veto and Ring Counters . . . . . . . . . . . . . . . 41

4.1.6 Regenerator .................. 41 
$4.2 \mathrm{KTeV} / \mathrm{E} 832$ Detector . . . . . . . . . . . . . . . . . . 46

4.2.1 Drift Chambers And Analysis Magnet _. . . . . . . . 48

4.2 .2 Spectrometer Antis . . . . . . . . . . . . . . 55

4.2 .3 VV' Counter . . . . . . . . . . . . . 56

4.2 .4 CsI Calorimeter . . . . . . . . . . . . . . . . 56

4.2 .5 Collar Anti . . . . . . . . . . . . . . . . . . . . 62

4.2 .6 Back Anti . . . . . . . . . . . . . . . . . . 63

4.2 .7 Hadron Anti . . . . . . . . . . . . . . . . . . . . . . 63

4.2 .8 Muon Filters and Vetoes . . . . . . . . . . . . . . . . 63

4.3 Trigger Electronics . . . . . . . . . . . . . . . . . . . . . . . 64

4.3 .1 Level 1 Trigger . . . . . . . . . . . . . . . . . . . . 65

4.3 .2 Level 2 Trigger . . . . . . . . . . . . . . . . . . 66

4.3 .3 Level 3 Trigger . . . . . . . . . . . . . . . . . . 67

5 Event Selection $\quad 69$

5.1 Data Used . . . . . . . . . . . . . . . . . . . . . . . . . . . . . . . . 69

5.2 Reconstruction of Data . . . . . . . . . . . . . . 70

5.3 Event Selection and Requirements . . . . . . . . . . . . . . 72

5.3 .1 Trigger Requirements . . . . . . . . . . . . . . . . . 72

5.3 .2 Crunch Requirements . . . . . . . . . . . . . . . . . 76

5.3.3 Final Analysis Requirements . . . . . . . . . . . . . . . . 82

5.3.4 Selection Efficiency and Event Yield . . . . . . . . . . 84

5.4 Useful Selection Criteria . . . . . . . . . . . . . . . . . . 87

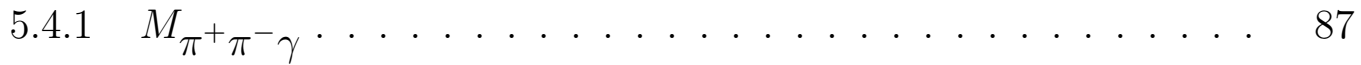

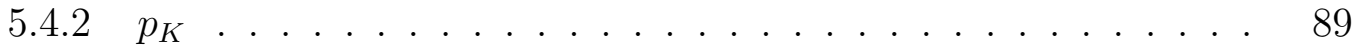

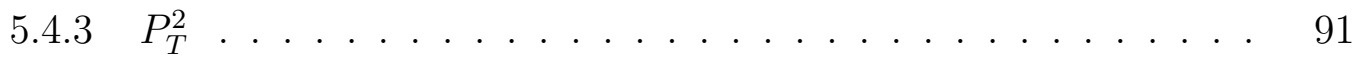

$5.4 .4 \mathrm{Z}$ vertex . . . . . . . . . . . . . . . . . 94

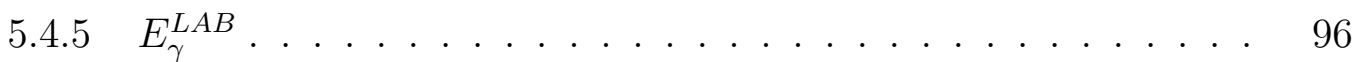

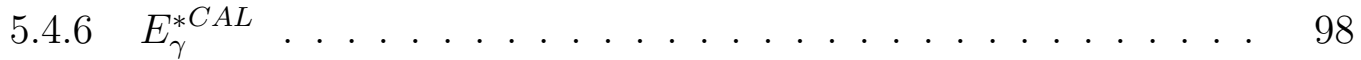

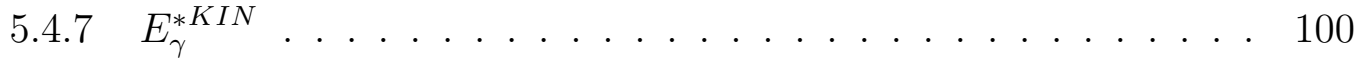

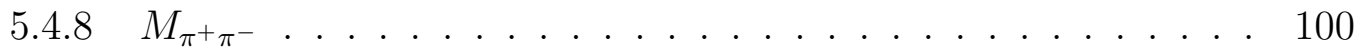

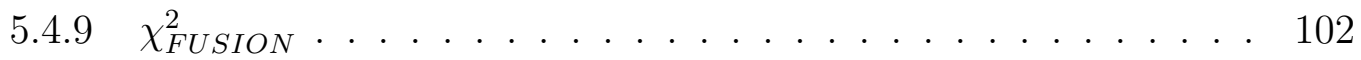

$5.4 .10 \pi-\gamma$ Separation at CsI . . . . . . . . . . . . . . 104 


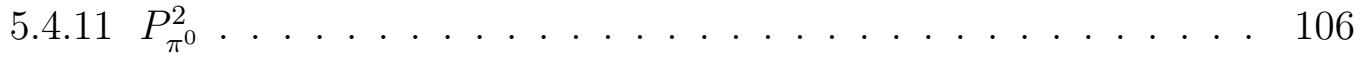

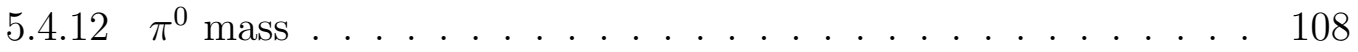

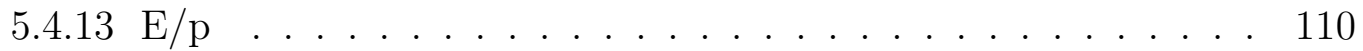

5.4.14 Upstream track projection / $\gamma$ separation . . . . . . . . . . 112

5.4 .15 Track Momentum . . . . . . . . . . . . . . . . . . . . . . 114

5.4 .16 Early and in-time cluster energy . . . . . . . . . . . . 116

$5.4 .17 p \pi$ invariant mass . . . . . . . . . . . . . . . . . . . . . 119

5.4 .18 Photon Cluster Location in the CsI . . . . . . . . . . . . . 121

5.4 .19 Vertex $\chi^{2} \ldots \ldots \ldots \ldots \ldots \ldots \ldots$

5.4 .20 Track Offset $\chi^{2} \ldots \ldots \ldots \ldots \ldots$

5.4 .21 Track Separation . . . . . . . . . . . . . . . . 128

5.4 .22 Veto Cuts . . . . . . . . . . . . . . . . . . 132

5.4 .23 Fiducial Cuts . . . . . . . . . . . . . . . . . . . . 132

6 Background Determination 134

6.1 Total Size of Background . . . . . . . . . . . . . . . . . . . . . 134

6.2 Background kaon decays . . . . . . . . . . . . . . . . . . . . . 139

$6.2 .1 \quad K_{L} \rightarrow \pi^{ \pm} e^{\mp} \nu \ldots \ldots \ldots \ldots \ldots \ldots \ldots \ldots \ldots$

6.2.2 $K_{L} \rightarrow \pi^{ \pm} \mu^{\mp} \nu$ with an accidental photon . . . . . . . . 140

6.2.3 $K_{L} \rightarrow \pi^{+} \pi^{-} \pi^{0}$ with one photon missed . . . . . . . . . 142

6.2.4 Comments about background due to kaon decays . . . . . . . 144

6.3 Non-kaon decays . . . . . . . . . . . . . . . . . . . . . . . . . . . 149

6.3 .1 Neutral Hyperons . . . . . . . . . . . . . . . . . . . . . . . . . 149

6.4 Scattered $K_{L, S} \rightarrow \pi^{+} \pi^{-} \gamma$ decays . . . . . . . . . . . . . . . . 149

6.4 .1 Regenerator Scattering . . . . . . . . . . . . . . . . . . 149

6.4 .2 Collimator Scattering . . . . . . . . . . . . . 150

6.5 Modified $K_{L, S} \rightarrow \pi^{+} \pi^{-} \gamma$ decays $\ldots \ldots \ldots \ldots \ldots \ldots$

6.5.1 $K_{L, S} \rightarrow \pi^{+} \pi^{-} \gamma \gamma \ldots \ldots \ldots \ldots \ldots \ldots \ldots \ldots$

7 Monte Carlo Simulation $\quad 152$

7.1 Generation and Propagation of Neutral Kaons . . . . . . . . . . . 153

7.1.1 Kaon wavefunction evolution and particle transport . . . . . 154

7.2 Generation of $K_{L, S} \rightarrow \pi^{+} \pi^{-} \gamma$ decays $\ldots \ldots \ldots \ldots \ldots$ 
7.2.1 Generation Using Matrix Element . . . . . . . . . . . . . . 155

$7.2 .2 \quad$ Kinematics of $K_{L, S} \rightarrow \pi^{+} \pi^{-} \gamma \ldots \ldots \ldots \ldots \ldots \ldots$

7.2.3 Radiative Corrections to $K_{L, S} \rightarrow \pi^{+} \pi^{-} \gamma \ldots \ldots \ldots \ldots$

7.3 Tracing of Decay Products Through KTeV Detector . . . . . . . . . 159

7.4 Simulation of Detector Response . . . . . . . . . . . . . 160

7.4.1 Drift Chambers . . . . . . . . . . . . . . . . . . 160

7.4 .2 CsI Calorimeter . . . . . . . . . . . . . . . . . . 161

7.5 Simulation of Accidental Activity . . . . . . . . . . . . . . 161

7.6 Simulation of Level 1 and 2 triggers . . . . . . . . . . . . . . . 162

8 Development of the Maximum Likelihood Fit 163

8.1 General Likelihood Function . . . . . . . . . . . . . . . . 163

8.2 Total Likelihood Function . . . . . . . . . . . . . . . . . 168

8.3 Execution of Maximum Likelihood Fit . . . . . . . . . . . . . . 173

8.3.1 Calculation of Likelihood using LCALC . . . . . . . . . . . 176

8.3 .2 Use of the program LMAX . . . . . . . . . . . . 180

9 Results of the Maximum Likelihood Fit 182

9.1 The Central Value . . . . . . . . . . . . . . . . . . . . . . . 182

9.2 Goodness of Fit . . . . . . . . . . . . . . . . . . . . 185

10 Systematic Errors $\quad 190$

$10.1 \Delta_{S}$ Method of Systematic Error Estimation _ . . . . . . . . . 192

10.2 Systematic Errors Due to Uncertainty in Input Parameters and Constants . . . . . . . . . . . . . . . . . . . . 193

10.3 Systematic Errors Due to Treatment of Data . . . . . . . . . . 196

10.3.1 Resolution of Phase Space variables . . . . . . . . . . . . 196

10.3 .2 Background Effects . . . . . . . . . . . . . . . . . 197

10.3.3 Systematic error due to scattering and incoherent regeneration 199

10.4 Systematic Errors Due to Treatment of Monte Carlo Samples . . . . . 200

10.4.1 Detector Acceptance and Simulation . . . . . . . . . . 200

10.5 Sum of Systematic Errors _ . . . . . . . . . . . . . . . 225 
11 Conclusions $\quad 227$

11.1 Final Result . . . . . . . . . . . . . . . . . . . . . 227

11.2 Comparison with previous results . . . . . . . . . . . . 229

11.3 Final Conclusions . . . . . . . . . . . . . . . . . . 230

A Kinematic Relations $\quad 232$

A.1 Invariant Quantities . . . . . . . . . . . . . . . . . . 232

A.2 Values in the kaon rest frame . . . . . . . . . . . . . . . . 232

A.3 Values in the $\pi \pi$ rest frame . . . . . . . . . . . . . . . . . 234

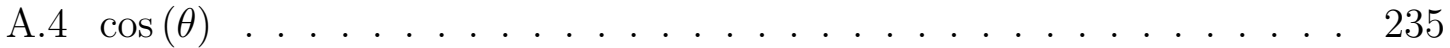

B Derivation of $P_{\pi^{0}}^{2} \quad 237$

C Manipulation of $K \rightarrow \pi^{+} \pi^{-} \gamma$ Matrix Element 239

C.1 Squaring the Matrix Element . . . . . . . . . . . . . . . . . . 239

C.2 Evaluation of the Momentum Terms in the Matrix Element . . . . . . 243

D Expansion of Decay Rate $\quad 247$

D.1 Numerical Estimates . . . . . . . . . . . . . . . . . . . . . 251

E The "average" decay rate for $K_{L, S} \rightarrow \pi^{+} \pi^{-} \gamma \quad 254$

$\begin{array}{lll}\text { F Average Kaon wavefunctions } & 260\end{array}$

G Simulation of $K_{L, S} \rightarrow \pi^{+} \pi^{-} \gamma \gamma$

H MC Generation of Kinematic Variables 268

I Plots for 1997 Vacuum Data 272

$\begin{array}{lll}\text { J Plots for } 1999 \text { Regenerator Data } & 298\end{array}$

K Plots for 1999 Vacuum Data 324 


\section{List of Figures}

1.1 The components of the decay rate as a function of the proper lifetime

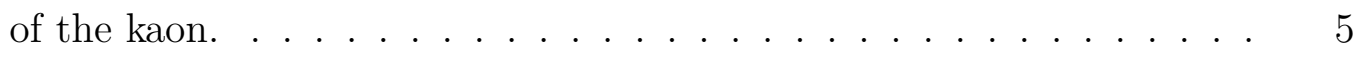

1.2 The components of the decay rate as a function of the photon energy in the kaon rest frame. . . . . . . . . . . . . 6

1.3 Effect of $\widehat{e}$ on decay rate . . . . . . . . . . . . . . 7

2.1 Feynman diagrams contributing to the process $\bar{K}^{0} \rightarrow K^{0} \ldots \ldots$.

2.2 Effect of $K_{L}-K_{S}$ interference $\ldots \ldots \ldots \ldots \ldots \ldots$

3.1 A Feynman diagram of the inner bremsstrahlung process . . . . . 16

3.2 A Feynman diagram of the direct emission process . . . . . . . . 17

4.1 A cartoon of the $\mathrm{KTeV}$ detector in the E832 configuration . . . . . 36

4.2 A more detailed diagram of the layout of the $\mathrm{KTeV}$ experiment in the E832 configuration. . . . . . . . . . . . . 37

4.3 A cartoon depicting parts of the $\mathrm{KTeV}$ beamline in its E832 configu-

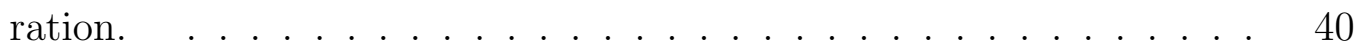

4.4 A cartoon of the shape of the $\mathrm{RC}$ and MA vetoes . . . . . . . 42

4.5 A cartoon depicting KTeV's active regenerator . . . . . . . . . 45

4.6 A candidate $K_{L, S} \rightarrow \pi^{+} \pi^{-} \gamma$ event $\ldots \ldots \ldots \ldots \ldots \ldots$

4.7 A cartoon depicting the design of the drift chambers . . . . . . 50

4.8 A cartoon depicting the sum of drift distance (SOD) measurement . . 51

4.9 A cartoon of the VV' trigger counter . . . . . . . . . . 57

4.10 A diagram of the CsI calorimeter . . . . . . . . . . 58

4.11 A cartoon depicting the CsI collar-anti . . . . . . . . . . . 62 
$5.1 \pi^{+} \pi^{-} \gamma$ invariant mass $(97 \mathrm{reg}) \ldots \ldots \ldots \ldots \ldots \ldots$

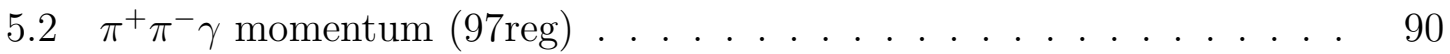

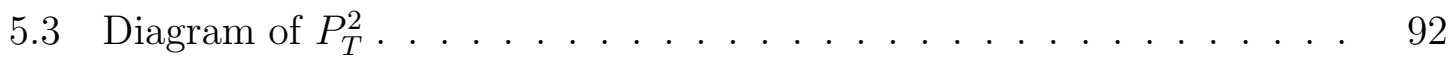

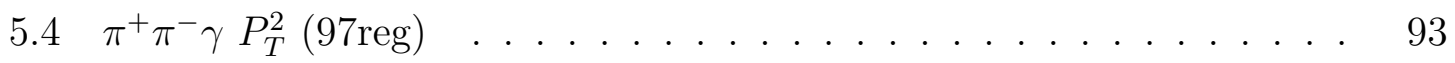

$5.5 \mathrm{Z}$ position of decay vertex $(97 \mathrm{reg}) \ldots \ldots \ldots \ldots \ldots$

$5.6 \quad E_{\gamma}^{*}$ in lab frame $(97 \mathrm{reg}) \ldots \ldots \ldots \ldots$

$5.7 E_{\gamma}^{*}$ in kaon rest frame $(97 \mathrm{reg}) \ldots \ldots \ldots \ldots$

$5.8 \pi \pi$ invariant mass $(97 \mathrm{reg}) \ldots \ldots \ldots \ldots$

5.9 Fusion $\chi^{2}(97 \mathrm{reg}) \ldots \ldots \ldots \ldots \ldots$

$5.10 \pi-\gamma$ separation $(97 \mathrm{reg}) \ldots \ldots \ldots \ldots \ldots$

$5.11 P_{\pi^{0}}^{2}$ with respect to the regenerator $(97 \mathrm{reg}) \ldots \ldots \ldots \ldots . \ldots 107$

$5.12 \pi^{0} \rightarrow \gamma \gamma$ invariant mass $(97 \mathrm{reg}) \ldots \ldots \ldots \ldots \ldots$

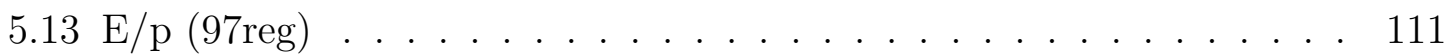

5.14 A cartoon of bremsstrahlung photon emission in a $K_{L} \rightarrow \pi^{ \pm} e^{\mp} \nu$ decay. 112

5.15 Upstream track/photon separation (97reg) . . . . . . . . . . 113

5.16 Track Momentum (97reg) _ . . . . . . . . . . . . . . 115

5.17 Early photon cluster energy $(97 \mathrm{reg}) \ldots \ldots \ldots \ldots$

5.18 In-time photon cluster energy $(97 \mathrm{reg}) \ldots \ldots \ldots \ldots \ldots$

$5.19 \Lambda \rightarrow p \pi$ invariant mass $(97 \mathrm{reg}) \ldots \ldots \ldots \ldots \ldots$

5.20 Outer CsI Ring $(97 \mathrm{reg}) \ldots \ldots \ldots \ldots \ldots$

5.21 Inner CsI Ring (97reg) . . . . . . . . . . . . . . . . 123

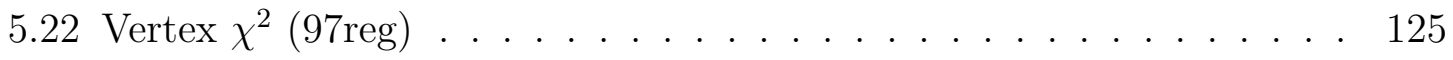

5.23 Offset $\chi^{2}$ (97reg) $\ldots \ldots \ldots \ldots \ldots \ldots \ldots \ldots$

5.24 Track X-separation $(97 \mathrm{reg}) \ldots \ldots \ldots \ldots$

5.25 Track Y-separation (97reg) … . . . . . . . . . . . . 130

5.26 Radial track separation $(97 \mathrm{reg}) \ldots \ldots \ldots \ldots \ldots$

$6.1 \quad M_{\pi^{+} \pi^{-} \gamma}$ background fit $\ldots \ldots \ldots \ldots \ldots \ldots \ldots \ldots$

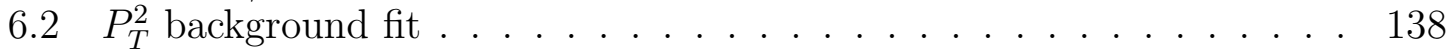

8.1 Schematic describing the reweighting method used to calculate the likelihood function . . . . . . . . . . . . . . . . . 172

9.1 Plots of $E_{\gamma}^{* C A L} \ldots \ldots \ldots \ldots \ldots \ldots \ldots \ldots \ldots \ldots$ 


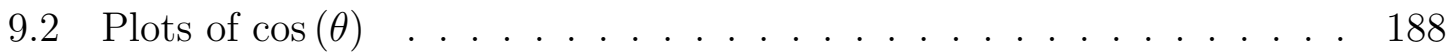

9.3 Plots of $\tau \ldots \ldots \ldots \ldots$

10.1 Parameter Shifts Due to Removal of Various Cuts . . . . . . . . . 203

10.2 Variation of the early photon cluster energy cut . . . . . . . . . . 204

10.3 Variation of the kinematic and CsI photon energy cuts in the kaon rest frame . . . . . . . . . . . . . . . . 205

10.4 Variation of the CsI photon energy cut in the lab frame . . . . . . 206

10.5 Variation of the fusion $\chi^{2}$ cut $\ldots \ldots \ldots \ldots 207$

10.6 Variation of the track offset $\chi^{2}$ cut $\ldots \ldots \ldots 20 . \ldots \ldots$

10.7 Variation of the vertex $\chi^{2}$ cut $\ldots \ldots \ldots . \ldots 209$

10.8 Variation of the $P_{T}^{2}$ cut $\ldots \ldots \ldots \ldots \ldots \ldots \ldots \ldots$

10.9 Variation of the SEEDRING cut . . . . . . . . . . . . . . 211

10.10 Variation of the ISMLRING cut . . . . . . . . . . . . . 212

10.11 Variation of the photon/pion separation cut . . . . . . . . . 213

10.12 Variation of the $P_{\pi^{0}}^{2}$ cut $\ldots \ldots \ldots \ldots \ldots \ldots \ldots$

10.13 Variation of the $\mathrm{E} / \mathrm{p}$ cut . . . . . . . . . . . . . 215

10.14 Variation of the track momentum cut . . . . . . . . . . 216

10.15 Variation of the track separation cut . . . . . . . . . . 217

10.16 Variation of the track X separation cut . . . . . . . . . . . . 218

10.17 Variation of the track Y separation cut . . . . . . . . . . . . . . 219

11.1 Magnitudes and phases of all measurements of $\eta_{+-\gamma} \ldots \ldots \ldots 231$

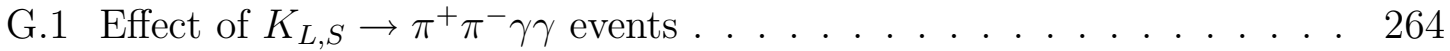

H.1 A picture of the traditional acceptance rejection method . . . . . 270

H.2 Preconditioning of $\tau$ in Monte Carlo . . . . . . . . . . 271

I.1 $\pi^{+} \pi^{-} \gamma$ invariant mass $(97 \mathrm{vac}) \ldots \ldots \ldots \ldots \ldots \ldots$

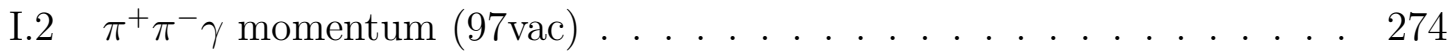

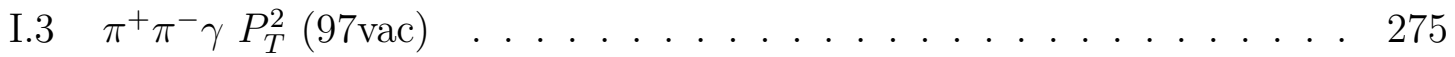

I.4 Z position of decay vertex $(97 \mathrm{vac}) \ldots \ldots \ldots \ldots \ldots \ldots$

I.5 $E_{\gamma}^{*}$ in lab frame $(97 \mathrm{vac}) \ldots \ldots \ldots \ldots \ldots \ldots . \ldots \ldots$

I.6 $E_{\gamma}^{*}$ in kaon rest frame $(97 \mathrm{vac}) \ldots \ldots \ldots \ldots$ 
I.7 $\pi \pi$ invariant mass $(97 \mathrm{vac}) \ldots \ldots \ldots \ldots \ldots \ldots$

I.8 Fusion $\chi^{2}(97 \mathrm{vac}) \ldots \ldots \ldots \ldots \ldots \ldots \ldots$

I.9 $\pi-\gamma$ seperation $(97 \mathrm{vac}) \ldots \ldots \ldots \ldots \ldots \ldots \ldots \ldots \ldots$

I.10 $P_{\pi^{0}}^{2}$ with respect to the regenerator $(97 \mathrm{vac}) \ldots \ldots \ldots \ldots \ldots$

I.11 $\pi^{0} \rightarrow \gamma \gamma$ invariant mass (97vac) . . . . . . . . . . . 283

$\mathrm{I} .12 \mathrm{E} / \mathrm{p}(97 \mathrm{vac}) \ldots \ldots \ldots \ldots \ldots \ldots \ldots \ldots \ldots \ldots$

I.13 Upstream track/photon seperation (97vac) . . . . . . . . . 285

I.14 Track Momentum (97vac) _ . . . . . . . . . . . . . . . 286

I.15 Early photon cluster energy $(97 \mathrm{vac}) \ldots \ldots \ldots \ldots . \ldots . \ldots 287$

I.16 In-time photon cluster energy $(97 \mathrm{vac}) \ldots \ldots \ldots \ldots \ldots$

I.17 $\Lambda \rightarrow p \pi$ invariant mass $(97 \mathrm{vac}) \ldots \ldots \ldots \ldots \ldots$

I.18 $\Lambda \rightarrow p \pi \gamma$ invariant mass $(97 \mathrm{vac}) \ldots \ldots \ldots \ldots \ldots \ldots$

I.19 Outer CsI Ring (97vac) . . . . . . . . . . . . . . . . . . 291

I.20 Inner CsI Ring (97vac) . . . . . . . . . . . . . . . . . 292

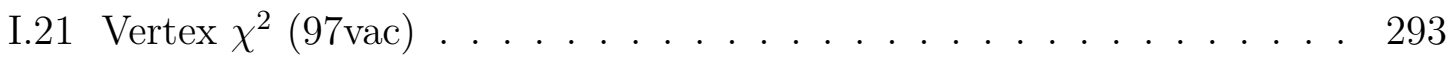

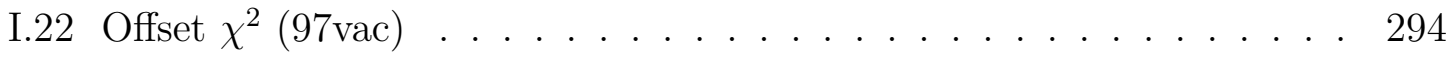

I.23 Track X-seperation (97vac) … . . . . . . . . . . . . . 295

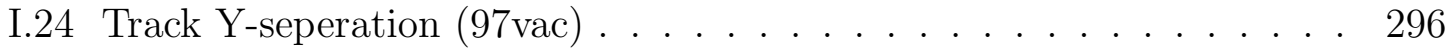

I.25 Radial track seperation $(97 \mathrm{vac}) \ldots \ldots \ldots \ldots$

J.1 $\pi^{+} \pi^{-} \gamma$ invariant mass (99reg) $\ldots \ldots \ldots \ldots \ldots$

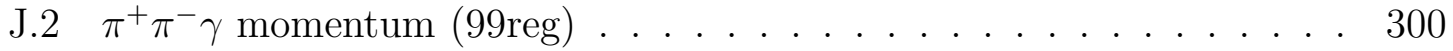

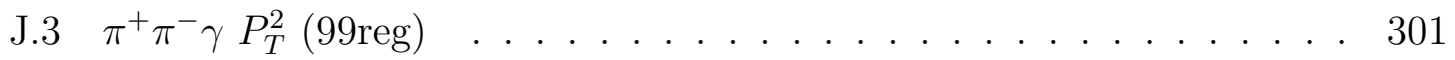

J.4 Z position of decay vertex (99reg) . . . . . . . . . . . 302

J.5 $E_{\gamma}^{*}$ in lab frame $(99 \mathrm{reg}) \ldots \ldots \ldots . \ldots \ldots$

J.6 $E_{\gamma}^{*}$ in kaon rest frame $(99 \mathrm{reg}) \ldots \ldots \ldots \ldots$

J.7 $\pi \pi$ invariant mass (99reg) $\ldots \ldots \ldots \ldots \ldots \ldots$

J.8 Fusion $\chi^{2}(99 \mathrm{reg}) \ldots \ldots \ldots \ldots \ldots \ldots$

J.9 $\quad \pi-\gamma$ seperation (99reg) $\ldots \ldots \ldots \ldots \ldots$

J.10 $P_{\pi^{0}}^{2}$ with respect to the regenerator $(99 \mathrm{reg}) \ldots \ldots \ldots \ldots \ldots$

J.11 $\pi^{0} \rightarrow \gamma \gamma$ invariant mass (99reg) $\ldots \ldots \ldots \ldots . \ldots \ldots$

$\mathrm{J} .12 \mathrm{E} / \mathrm{p}(99 \mathrm{reg}) \ldots \ldots \ldots \ldots$

J.13 Upstream track/photon seperation (99reg) . . . . . . . . . . 311 
J.14 Track Momentum (99reg) _ . . . . . . . . . . . . . . . . . . 312

J.15 Early photon cluster energy (99reg) … . . . . . . . . . . 313

J.16 In-time photon cluster energy (99reg) . . . . . . . . . . . . 314

J.17 $\Lambda \rightarrow p \pi$ invariant mass (99reg) . . . . . . . . . . . . 315

J.18 $\Lambda \rightarrow p \pi \gamma$ invariant mass $(99 \mathrm{reg}) \ldots \ldots \ldots \ldots \ldots$

J.19 Outer CsI Ring (99reg) . . . . . . . . . . . . . . . . 317

J.20 Inner CsI Ring (99reg) . . . . . . . . . . . . . . . . . 318

J.21 Vertex $\chi^{2}$ (99reg) . . . . . . . . . . . . . . . . . . . . . . 319

J.22 Offset $\chi^{2}$ (99reg) $\ldots \ldots \ldots \ldots \ldots \ldots$

J.23 Track X-seperation (99reg) … . . . . . . . . . . . . . . . 321

J.24 Track Y-seperation (99reg) … . . . . . . . . . . . . . . . 322

J.25 Radial track seperation (99reg) _ . . . . . . . . . . . 323

K.1 $\pi^{+} \pi^{-} \gamma$ invariant mass $(99 \mathrm{vac}) \ldots \ldots \ldots \ldots \ldots \ldots \ldots$

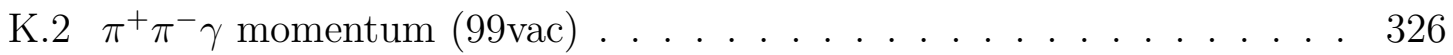

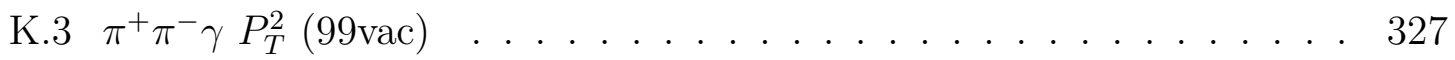

K.4 Z position of decay vertex $(99 \mathrm{vac}) \ldots \ldots \ldots \ldots \ldots$

K.5 $E_{\gamma}^{*}$ in lab frame $(99 \mathrm{vac}) \ldots \ldots \ldots . \ldots \ldots$

K.6 $E_{\gamma}^{*}$ in kaon rest frame $(99 \mathrm{vac}) \ldots \ldots \ldots \ldots$

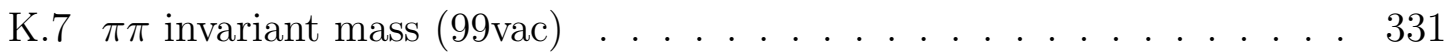

K.8 Fusion $\chi^{2}(99 \mathrm{vac}) \ldots \ldots \ldots \ldots \ldots \ldots$

K.9 $\pi-\gamma$ seperation $(99 \mathrm{vac}) \ldots \ldots \ldots \ldots$

K.10 $P_{\pi^{0}}^{2}$ with respect to the regenerator $(99 \mathrm{vac}) \ldots \ldots \ldots \ldots . \ldots 334$

K.11 $\pi^{0} \rightarrow \gamma \gamma$ invariant mass (99vac) . . . . . . . . . . . 335

K.12 E/p (99vac) . . . . . . . . . . . . . . . . 336

K.13 Upstream track/photon seperation (99vac) . . . . . . . . . . 337

K.14 Track Momentum (99vac) _. . . . . . . . . . . . . . 338

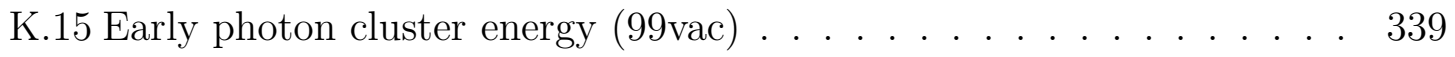

K.16 In-time photon cluster energy (99vac) … . . . . . . . . . 340

K.17 $\Lambda \rightarrow p \pi$ invariant mass (99vac) . . . . . . . . . . . . 341

K.18 $\Lambda \rightarrow p \pi \gamma$ invariant mass (99vac) . . . . . . . . . . . . . 342

K.19 Outer CsI Ring (99vac) . . . . . . . . . . . . . . . . . 343

K.20 Inner CsI Ring (99vac) . . . . . . . . . . . . . . . . . 344 
K.21 Vertex $\chi^{2}(99 \mathrm{vac}) \ldots \ldots \ldots \ldots \ldots \ldots \ldots \ldots \ldots \ldots$

K.22 Offset $\chi^{2}$ (99vac) $\ldots \ldots \ldots \ldots \ldots \ldots \ldots \ldots \ldots$

K.23 Track X-seperation (99vac) … . . . . . . . . . . . 347

K.24 Track Y-seperation (99vac) . . . . . . . . . . . . . . . 348

K.25 Radial track seperation $(99 \mathrm{vac}) \ldots \ldots \ldots$ 


\section{List of Tables}

3.1 The $\mathrm{CP}$ characteristics of various processes . . . . . . . . 23

5.1 Runs removed from 1997 and $1999 \mathrm{KTeV}$ datasets . . . . . . . . . 69

5.2 Analysis Cuts Applied To Data . . . . . . . . . . . . . . . 83

5.3 Number of events after all cuts . . . . . . . . . . . . 84

5.4 Number of Combinations in Data removed by each cut . . . . . . 85

5.5 Fraction of Combinations in Monte Carlo removed by each cut . . . . 86

$5.6 \quad K_{L, S} \rightarrow \pi^{+} \pi^{-} \gamma$ Acceptance . . . . . . . . . . . . 86

6.1 Background Estimates from Invariant Mass Plot . . . . . . . . . . 135

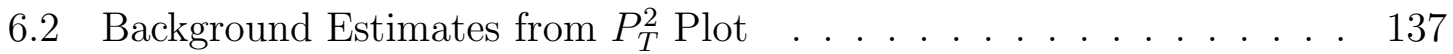

6.3 Fraction of Combinations in $K_{L} \rightarrow \pi^{ \pm} e^{\mp} \nu$ Monte Carlo removed by each cut . . . . . . . . . . . . . . . . . . . . 141

6.4 Fraction of Combinations in $K_{L} \rightarrow \pi^{ \pm} \mu^{\mp} \nu$ Monte Carlo removed by each cut . . . . . . . . . . . . . . . . . . . . 142

6.5 Fraction of Combinations in $K_{L} \rightarrow \pi^{+} \pi^{-} \pi^{0}$ Monte Carlo removed by each cut . . . . . . . . . . . . . . . . . 143

6.6 Distribution of events in the low invariant mass region . . . . . . 146

6.7 Distribution of events in the high invariant mass region . . . . . . 146

6.8 Distribution of events in the high invariant mass region . . . . . . 147

6.9 Distribution of events in the high invariant mass region . . . . . . 147

6.10 Estimated background composition in high invariant mass region . . . 148

9.1 Input parameters used in the maximum likelihood fit . . . . . . . 183

9.2 Nominal 4-parameter fit results . . . . . . . . . . . . . . . . 184 
9.3 Nominal 4-parameter fit correlation matrix . . . . . . . . . . . . . . . 184

9.4 Minimum $\chi^{2}$ values for various distributions . . . . . . . . . . . . . 186

10.1 Parameter shifts from nominal result due to variation of input parameters 194

10.2 Correlation matrix taken from the fit for $R e\left(\frac{\epsilon^{\prime}}{\epsilon}\right) \ldots \ldots$. . . . . . 195

10.3 Correlation matrix taken from the fit for $\phi_{+-} \ldots \ldots$. . . . . . . 195

10.4 Systematic errors due to input parameter uncertainty after proper treatment of correlations. . . . . . . . . . . . . . . . . 195

10.5 Systematic Errors Due to Event Reconstruction. . . . . . . . . . . . 197

10.6 Composition of background sample for the estimation of the background systematic error . . . . . . . . . . . . . . . . 199

10.7 Observed shifts and assigned systematic errors from the addition of the estimated background sample . . . . . . . . . . . . . . . . . . . 199

10.8 Observed shifts and assigned systematic errors from the effect of incoherent regeneration and regenerator scattering . . . . . . . . . . 200

10.9 Assigned systematic errors from the cut variation study . . . . . . . . 221

10.10Observed kaon momentum slopes relative to Monte Carlo . . . . . . . 222

10.11Observed parameter shifts due to flattening of the momentum spectrum. 222

10.12Observed z vertex slopes relative to Monte Carlo . . . . . . . . . . . . 223

10.13Observed parameter shifts due to flattening of the $\mathrm{z}$ vertex distribution. 223

10.14Systematic errors due to flattening of $Z_{\text {vertex }}$ and $p_{K}$ distributions . . 223

10.15 Observed shifts and assigned systematic errors from the uncertainty in the Monte Carlo accidental simulation . . . . . . . . . . . . . . . . . 224

10.16Assigned systematic errors from the uncertainty in PHOTOS normalization . . . . . . . . . . . . . . . . 225

10.17Total Systematic Errors . . . . . . . . . . . . . . . . 226

10.18Methods used to obtain systematic errors . . . . . . . . . . . 226

11.1 Results of measurements of $\eta_{+-\gamma} \quad \ldots \ldots$. . . . . . . . . . . . . . . . . 229

11.2 Results of measurements of the M1 direct emission parameters . . . . 230 


\section{Chapter 1}

\section{Introduction}

Symmetries are a common tool that physicists use to understand the fundamental laws that define the universe. In addition to the more well-known conserved quantities such as charge, energy and momentum ${ }^{1}$ there are other symmetries known as C,P and T, which stand for Charge conjugation, Parity reversal, and Time reversal respectively. Charge conjugation, when applied to a particle, transforms it to its corresponding antiparticle and reverses the electric charge. Parity reversal flips the sign of all dimensions in a coordinate system, which results in any linear momentum vectors being reversed. The final symmetry is Time reversal, which simply flips the flow of time. If these were all conserved at all times, physical law would be invariant between particles and antiparticles, the usual world and a matching "mirror-reversed world", and the usual world and the world in "reverse". It was once thought that each of these symmetries was in fact conserved, however it was observed [1] that not only does the weak interaction ${ }^{2}$ violate parity, it does so at the level of $100 \%$. It was very quickly assumed that the combined operation of $\mathrm{C}$ and $\mathrm{P}$, of course referred to as $\mathrm{CP}$, would then be conserved. However, once the decay $K_{L} \rightarrow \pi^{+} \pi^{-}$[2], which is a CP violating reaction, was observed, this hope was dashed. The final unvarnished symmetry is CPT, which has survived a number of very strong tests. An example of a violation of CPT would be the case where the mass of a given particle was not equal to the mass of it's corresponding anti-particle. Since CPT is conserved in the Standard

\footnotetext{
${ }^{1}$ Conservation of momentum and energy are actually linked by relativity. It is more accurate to say that 4-momentum is conserved. As a consequence, mass isn't conserved.

${ }^{2}$ The weak interaction is a short range force responsible for the $\beta$ decay of various unstable atoms.
} 
Model, any violation would be a major landscape changing event for physicists.

The violation of $\mathrm{CP}$ was fortuitous, as it is needed to explain the lack of naturally occurring anti-matter in the universe [3] ${ }^{3}$ The type of CP violation exposed in the decay $K_{L} \rightarrow \pi^{+} \pi^{-}$was later shown to be mostly due to mixing between $K^{0}$ and $\bar{K}^{04}$. This type of CP violation is referred to as "indirect" CP violation. However, direct $\mathrm{CP}$ violation, which is $\mathrm{CP}$ violation that occurs in weak decays, is shown to also contribute to $K_{L} \rightarrow \pi^{+} \pi^{-}$decays. This identifies the weak interaction as a source of $\mathrm{CP}$ violation. After much work with kaon and B-meson decays, the level of CP violation present in the weak interaction is well understood, and it is now obvious that the observed amount of $\mathrm{CP}$ violation is not sufficient to explain the absence of cosmological anti-matter. It is possible that the strong interaction ${ }^{5}$ also violates $\mathrm{CP}$, and after the discovery of neutrino-mixing, whatever process responsible is another possible source of $\mathrm{CP}$ violation. While $\mathrm{CP}$ violation in the quark sector may not explain the matter/anti-matter asymmetry in the universe, it can still serve as a sensitive probe for new physics.

The KTeV experiment had two primary goals: the first was the measurement of the value of $R e\left(\frac{\epsilon^{\prime}}{\epsilon}\right)$ which describes the amount of direct CP violation present in $K_{L} \rightarrow \pi^{+} \pi^{-}$while the second was the search for a number of very rare neutral kaon decays. It established a non-zero value for $R e\left(\frac{\epsilon^{\prime}}{\epsilon}\right)$ showing that direct $\mathrm{CP}$ violation exists, and hence the weak interaction violates CP symmetry. KTeV produced very intense, twin neutral kaon beams which then traveled through the $\mathrm{KTeV}$ detector. One beam was converted into a mixture of long lived and short lived neutral kaons. Some of these neutral kaons decay inside the $\mathrm{KTeV}$ detector, allowing various properties to be studied, mainly the physics responsible for determining how quickly and in what manner the kaons decay. Various models can be tested and refined by looking at how the kaon decay - how many daughter particles are produced by the decay, the energies and orientation of these decay products, and due to KTeV's unique design,

\footnotetext{
${ }^{3}$ Whenever energy is converted into matter, an equal amount of anti-matter is created. Correspondingly, matter and anti-matter will annihilate each other, the end result which will be pure energy.

${ }^{4} K_{L}$ is the longer lived neutral kaon. It is a real particle, with a well defined lifetime and mass. However, $K_{-}^{0}$ and $K^{0}$ are the particles that are produced by the weak interaction. $K_{L}$ is an admixture of $K^{0}$ and $\bar{K}^{0}$ particles.

${ }^{5}$ This is a short range force responsible for the binding of quarks together to form atomic nuclei
} 
the lifetime of each kaon before it decays.

This thesis presents a study of the neutral kaon decay $K_{L, S} \rightarrow \pi^{+} \pi^{-} \gamma$. It measures the amount of $\mathrm{CP}$ violation present in this particular decay by looking at how the probability of the decay varies with time. If we inspect Figure 1.1, which is a distribution of the time of flight of $K_{L, S} \rightarrow \pi^{+} \pi^{-} \gamma$ events ( the time it took them to pass a certain point in the detector and decay) we can see that it is composed of three components. Beginning at $\tau=0$ the histogram has a steep slope owing to the short lifetime of the $K_{S}$. Then the "wiggle" appears, indicating quantum mechanical interference between the $K_{L}$ and $K_{S}$. The fact that $K_{L}$ and $K_{S}$ interfere in this decay indicate that $\mathrm{CP}$ violation is present. Finally, the histogram smooths out into a region dominated by $K_{L}$ decays.

In the past, the amount of $\mathrm{CP}$ violation present in this decay ( as well as in $K_{L, S} \rightarrow \pi^{+} \pi^{-}$decays ) was measured by quantifying the size of this "wiggle" or "dip". The majority of the $\mathrm{CP}$ violation indicated by this bump will be "mixing" type $\mathrm{CP}$ violation, coming from the underlying $K_{L, S} \rightarrow \pi^{+} \pi^{-}$decay. A much smaller amount will be due to the direct $\mathrm{CP}$ violation present in the $K_{L, S} \rightarrow \pi^{+} \pi^{-}$decay. However, a small amount may also be due to direct CP violation present in $K_{L, S} \rightarrow \pi^{+} \pi^{-} \gamma$ only. This direct $\mathrm{CP}$ violation will also show up in a plot of the photon energy as measured in the rest frame of the decaying kaon. Figure 1.2 is such a plot. While the majority of $K_{L, S} \rightarrow \pi^{+} \pi^{-} \gamma$ decays occur via either the Inner Bremsstrahlung (IB) process, in which low energy photons are produced, or the direct emission (DE) process, in which higher energy photons are produced, a direct $\mathrm{CP}$ violating process would result in intermediate energy photons being produced. The strategy used in this analysis is then the search for a component in the $K_{L, S} \rightarrow \pi^{+} \pi^{-} \gamma$ decay which shows a "wiggle" which is both time and photon energy dependent. This "wiggle" can be clearly seen in Figure 1.3

After filtering the data in order to reject kaon decay which are not $K_{L, S} \rightarrow \pi^{+} \pi^{-} \gamma$ decays, the correlated distribution of photon energy, photon emission direction and proper kaon lifetime is used to estimate the amount of direct CP violation present in this decay. The method of maximum likelihood is used in order to perform the estimation. This process involves calculating the probability that a given $K_{L, S} \rightarrow \pi^{+} \pi^{-} \gamma$ decay with certain characteristics will be observed, and then computing the product, over the entire dataset, of all these probabilities. The parameters which describe the 
physics of the decay, including the amount of direct $\mathrm{CP}$ violation present, are varied until this product, called the likelihood, is maximized. Once this is done, the best estimation of the various parameters has been found. However, due to the limited number of $K_{L, S} \rightarrow \pi^{+} \pi^{-} \gamma$ events obtained, there will be a certain amount of uncertainty in the value of each of these parameters. This is referred to as "statistical" error, and describes the range in which there is a $68 \%$ chance that the actual value of the parameter is included. In addition to the statistical error, there is also a certain amount of uncertainity arising from the methodology or assumptions of the experiment and/or analysis. This is referred to as "systematic" error, and describes how sensitive the best estimates of the parameters are to the way the analysis was carried out.

The goal of this dissertation is then to obtain the best estimate of the amount of direct $\mathrm{CP}$ violation in the neutral kaon decay $K_{L, S} \rightarrow \pi^{+} \pi^{-} \gamma$. The statistical and systematic errors on this parameter will also be estimated in order to determine if the result is either consistent or inconsistent with no direct $\mathrm{CP}$ violation being present. In addition, various other characteristics describing the $K_{L, S} \rightarrow \pi^{+} \pi^{-} \gamma$ decay will also be studied. 


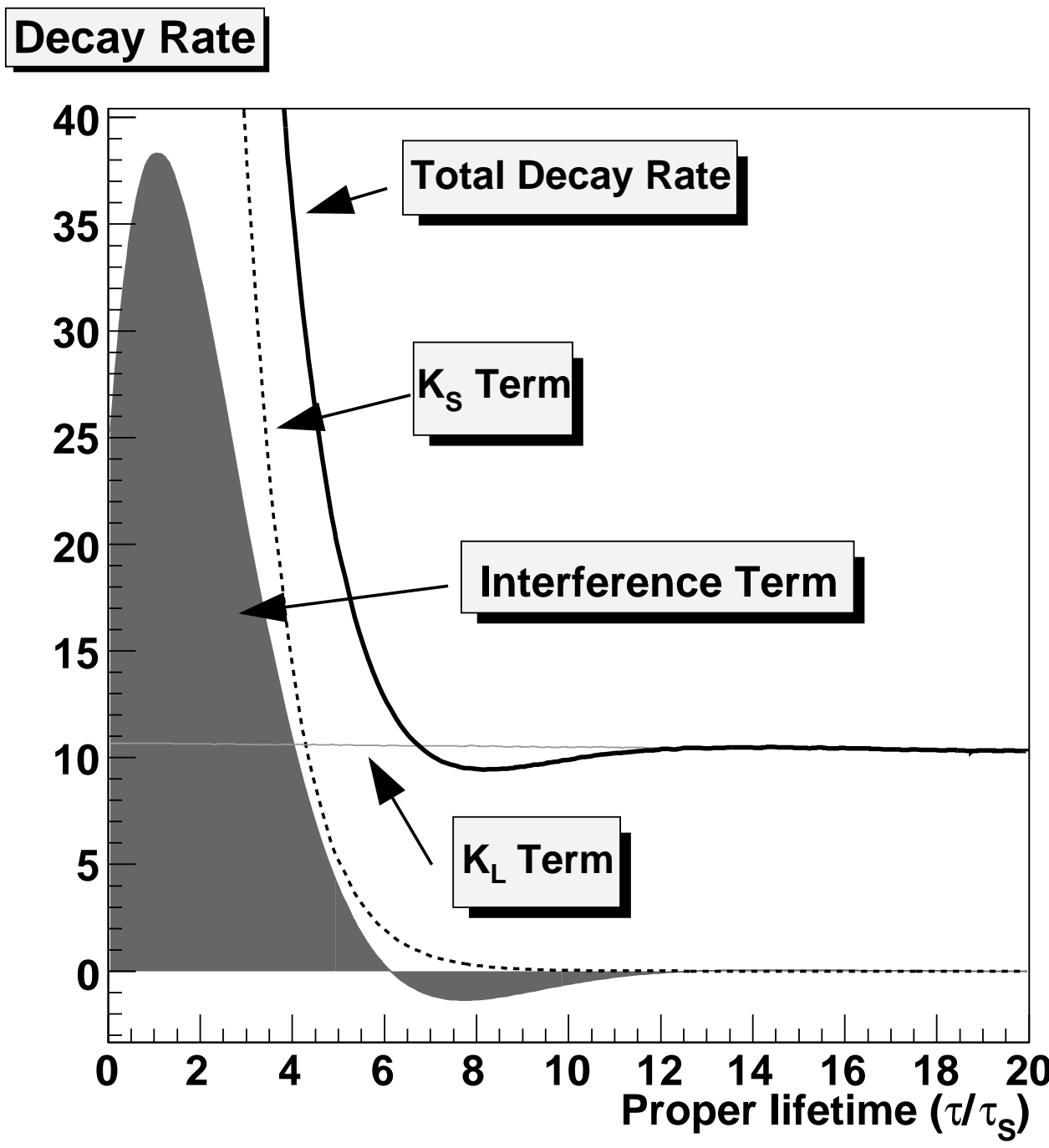

Figure 1.1: The components of the decay rate as a function of the proper lifetime of the kaon. 


\section{Decay Amplitudes}

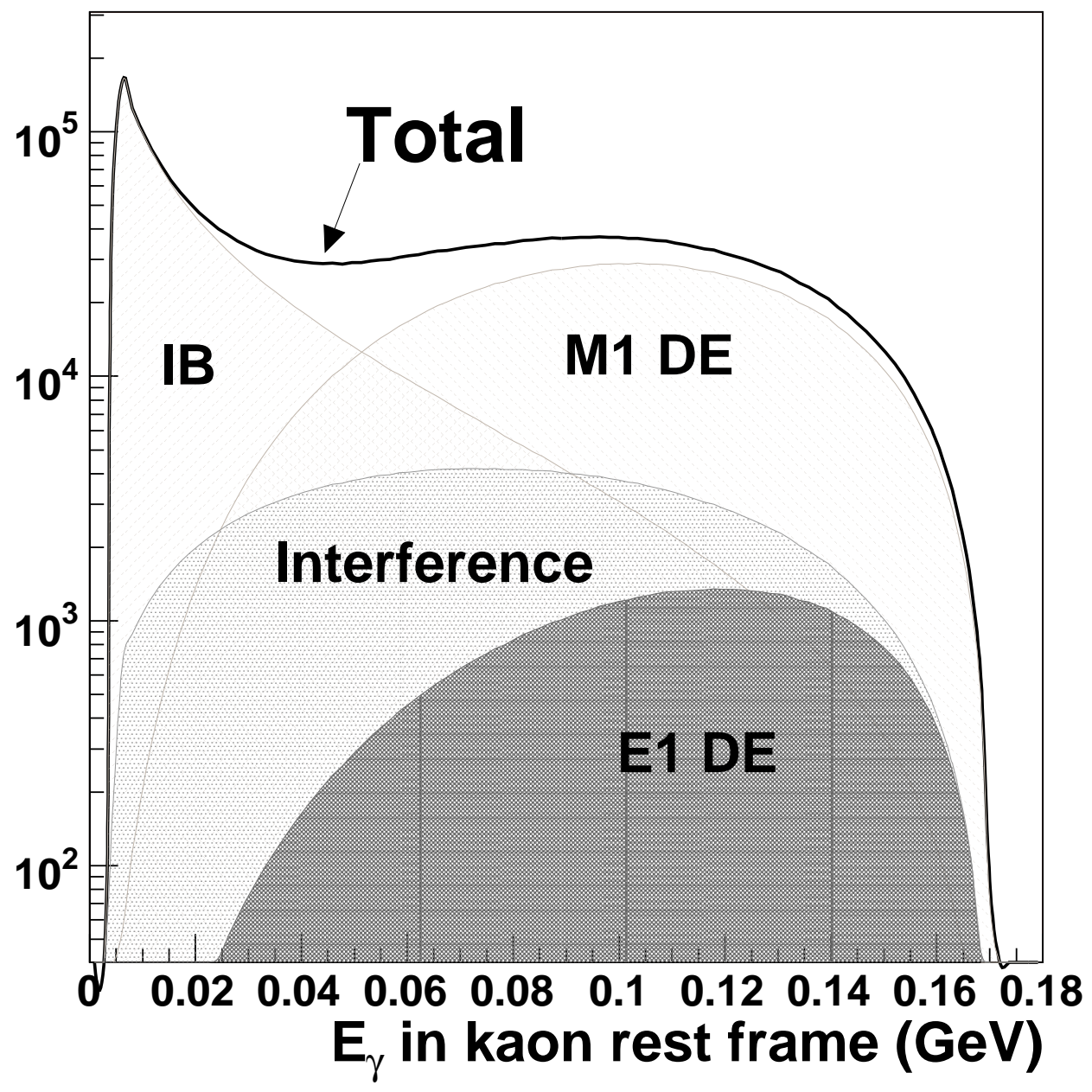

Figure 1.2: The components of the decay rate as a function of the photon energy in the kaon rest frame. 

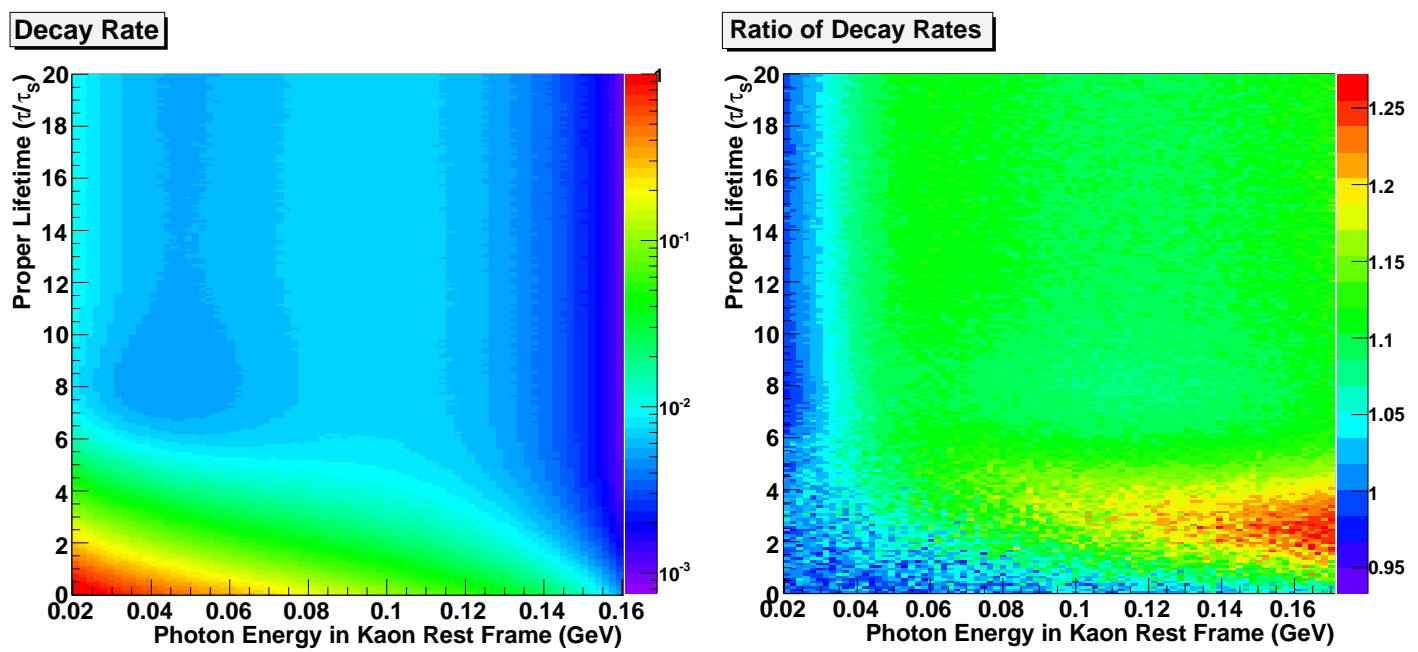

Figure 1.3: The decay rate as a function of photon energy and proper lifetime. a) shows the decay rate for events in the regenerator beam, and shows the various regions where $K_{S}$ decays dominate ( at small lifetimes ), where $K_{L}$ decays dominate ( at large lifetimes ) and where the interference term is dominant. b) shows the bin-by-bin ratio of the decay rate with $\widehat{e}=0.01$ to the decay rate with $\widehat{e}=0$. The ratio plot reveals that the direct $\mathrm{CP}$ violating component of this decay is most visible at high photon energies and at moderate proper time, where the constructive interference between $K_{L}$ and $K_{S}$ is greatest. Also note that there is a smaller effect in the destructive interference region around $\tau=8 \tau_{S}$. 


\section{Chapter 2}

\section{Introduction to Kaon Phenomenology}

Before going into the specifics of the $K_{L, S} \rightarrow \pi^{+} \pi^{-} \gamma$ decay, it is instructive to review a few facts about the kaon sector, in addition to the decay $K_{L, S} \rightarrow \pi^{+} \pi^{-}$which is responsible for a large part of the $K_{L, S} \rightarrow \pi^{+} \pi^{-} \gamma$ decay.

We first begin with the production of neutral kaons. Neutral kaons, which are mesons, are created via the strong interaction. The quark content of the two neutral kaons are:

$$
\begin{aligned}
& K^{0}=\bar{s} d \\
& \bar{K}^{0}=s \bar{d}
\end{aligned}
$$

They are the lightest mesons containing a strange quark. $K^{0}$ and $\bar{K}^{0}$ are each other's antiparticles. Recalling that the combined operation of CP transforms a particle to its corresponding antiparticle, we can also write:

$$
\begin{aligned}
& C P\left|K^{0}\right\rangle=\left|\bar{K}^{0}\right\rangle \\
& C P\left|\bar{K}^{0}\right\rangle=\left|K^{0}\right\rangle
\end{aligned}
$$


Note that since the CP operator does not return the same state, $\left|K^{0}\right\rangle$ and $\left|\bar{K}^{0}\right\rangle$ are not eigenstates of CP. They are simply strong interaction eigenstates. Instead, we can construct CP eigenstates out of these strong eigenstates by defining:

$$
\begin{aligned}
\left|K_{1}\right\rangle & =\frac{\left|K^{0}\right\rangle+\left|\bar{K}^{0}\right\rangle}{\sqrt{2}} \\
\left|K_{2}\right\rangle & =\frac{\left|K^{0}\right\rangle-\left|\bar{K}^{0}\right\rangle}{\sqrt{2}}
\end{aligned}
$$

note that both $K_{1}$ and $K_{2}$ are normalized. With this definition, we can then apply the $\mathrm{CP}$ operator to show:

$$
\begin{aligned}
& C P\left|K_{1}\right\rangle=+\left|K_{1}\right\rangle \\
& C P\left|K_{2}\right\rangle=-\left|K_{2}\right\rangle
\end{aligned}
$$

$K_{1}$ is then referred to as the $\mathrm{CP}$ even eigenstate, while $K_{2}$ is known as the CP-odd eigenstate.

In the early days of kaon physics, it was clear that there were two neutral kaons, both having approximately the same mass, but drastically different lifetimes. They also exhibited very different decay behavior. The longer lived kaon most commonly decayed into $\pi l \nu$, while the shorter lived kaon decayed into $\pi \pi$ (charged and neutral states) approximately $99 \%$ of the time. Since:

$$
\begin{aligned}
C P\left|\pi^{+} \pi^{-}\right\rangle & =+\left|\pi^{+} \pi^{-}\right\rangle \\
C P\left|\pi^{0} \pi^{0}\right\rangle & =+\left|\pi^{0} \pi^{0}\right\rangle
\end{aligned}
$$

the shorter lived kaon was assumed to be a pure CP eigenstate, and identified with the $\left|K_{1}\right\rangle$ At the time, the weak interaction, like all other interactions, was assumed to conserve CP symmetry. One then expected the CP eigenvalues of an initial and final state to always match. However, it was soon discovered [2] that the long lived neutral kaon does in fact decay into $\pi^{+} \pi^{-}$. This was the first observation of CP violation. After this point, it was clear that the long and short lived neutral kaons, now referred to as $K_{L}$ and $K_{S}$ respectively, were not pure CP eigenstates. Instead, 
they can be written as a superposition of both CP eigenstates:

$$
\begin{aligned}
\left|K_{L}\right\rangle & =\frac{\left|K_{2}\right\rangle+\epsilon\left|K_{1}\right\rangle}{\sqrt{1+|\epsilon|^{2}}} \\
\left|K_{S}\right\rangle & =\frac{\left|K_{1}\right\rangle+\epsilon\left|K_{2}\right\rangle}{\sqrt{1+|\epsilon|^{2}}}
\end{aligned}
$$

where $\epsilon$ is small ( $\approx 2.232 \times 10^{-3}$ ) and parameterizes the amount of the "wrong" CP eigenstate in the $K_{L}$ and $K_{S}$ states. Since the presence of this wrong CP eigenstate is responsible, at least partly, for the $\mathrm{CP}$ violating decay $K_{L} \rightarrow \pi^{+} \pi^{-} \epsilon$ parameterizes the size of $\mathrm{CP}$ violation in the neutral kaon system.

This type of $\mathrm{CP}$ violation is referred to as "indirect $\mathrm{CP}$ violation" as the physical eigenstate is not really a pure CP eigenstate. For example, the decay $K_{L} \rightarrow \pi^{+} \pi^{-}$ can instead be thought of as $K_{1} \rightarrow \pi^{+} \pi^{-}$. In this case, the initial and final states have equal $\mathrm{CP}$ eigenvalues, and hence $\mathrm{CP}$ is conserved.

This indirect $\mathrm{CP}$ violation arises due to asymmetric mixing of $K^{0}$ and $\bar{K}^{0}$ states, as can be seen when the $K_{L}$ and $K_{S}$ states are re-expressed as:

$$
\begin{aligned}
\left|K_{L}\right\rangle & =\frac{(1+\epsilon)\left|K^{0}\right\rangle-(1-\epsilon)\left|\bar{K}^{0}\right\rangle}{\sqrt{2\left(1+|\epsilon|^{2}\right)}} \\
\left|K_{S}\right\rangle & =\frac{(1+\epsilon)\left|K^{0}\right\rangle+(1-\epsilon)\left|\bar{K}^{0}\right\rangle}{\sqrt{2\left(1+|\epsilon|^{2}\right)}}
\end{aligned}
$$

where it can be seen that $\epsilon$ moderates the imbalance between $K^{0}$ and $\bar{K}^{0}$. The asymmetric mixing is in turn due to the transition rate of $K^{0} \rightarrow \bar{K}^{0}$ not being equal to the transition rate of $\bar{K}^{0} \rightarrow K^{0}$. This transition rate originates from the weak interaction, as shown in Figure 2.1. It is interesting to note that the CP eigenstate composition of the $K_{L}$ and $K_{S}$ states results in their very different lifetimes. The kinetically favored decay into $\pi \pi$ is $\mathrm{CP}$ conserving for $K_{S}$ however it is CP violating for the $K_{L}$. Without this contribution the $K_{L}$ has a lower decay rate, and hence a much longer lifetime.

Although there are other methods available with which to measure the value of 

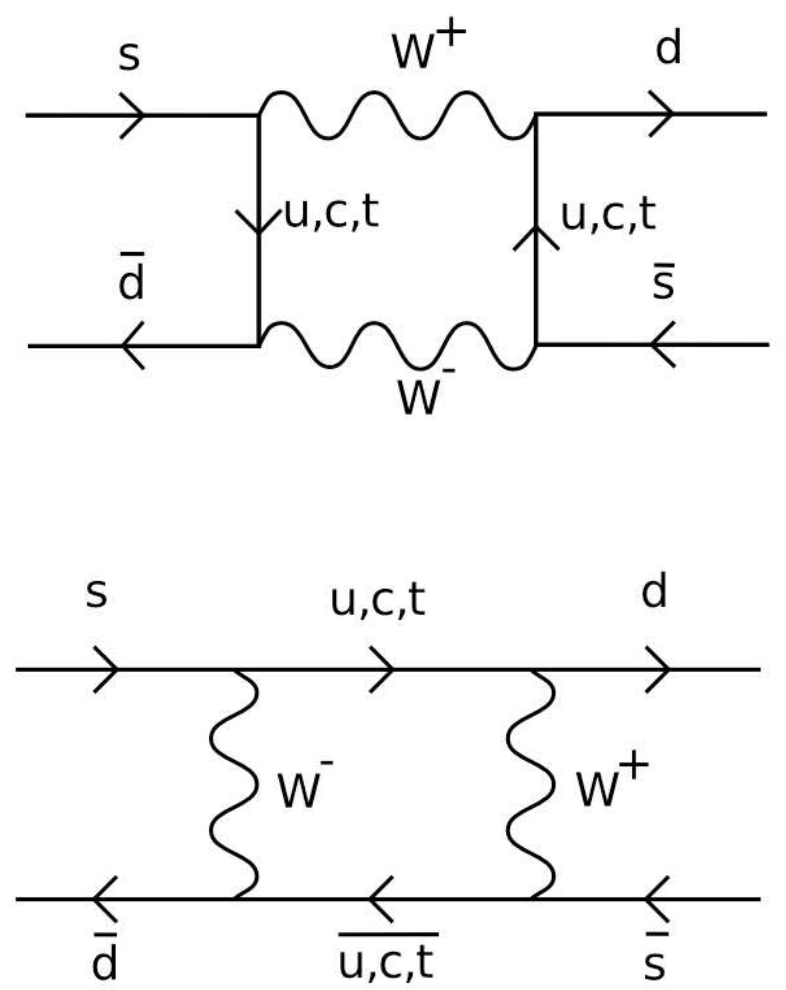

Figure 2.1: Feynman diagrams contributing to the process $\bar{K}^{0} \rightarrow K^{0}$

$\epsilon$, such as measuring the difference between the branching fractions for $K_{L} \rightarrow \pi^{+} e^{-} \nu$ and $K_{L} \rightarrow \pi^{-} e^{+} \nu$, another is to prepare a mixed neutral kaon state, and observe how the decay rate changes with time. It can be shown ${ }^{1}$ that the decay rate of a kaon state containing an admixture of both $K_{L}$ and $K_{S}$ in the form $\left|K_{L}\right\rangle+\rho\left|K_{S}\right\rangle$ will be, $\mathrm{E}$

${ }^{1}$ An analogous treatment of the $K_{L, S} \rightarrow \pi^{+} \pi^{-} \gamma$ decay rate is shown in Chapter 3 and Appendix 
for $K_{L, S} \rightarrow \pi^{+} \pi^{-}$decays:

$$
\begin{aligned}
\Gamma= & |A|^{2} \Gamma_{K_{S}}\left[|\rho|^{2} e^{-\left(\frac{1}{\tau_{S}}\right) \tau}+|\eta|^{2} e^{-\left(\frac{1}{\tau_{L}}\right) \tau}\right. \\
& \left.+2|\rho||\eta| \cos \left(\Delta M_{K} \tau+\phi_{\rho}-\phi_{\eta}\right) e^{-\left(\frac{1}{\tau_{S}}+\frac{1}{\tau_{L}}\right) \frac{\tau}{2}}\right]
\end{aligned}
$$

where $A$ is a normalization constant, $\Gamma_{K_{S}}$ is the decay rate of the $K_{S}, \tau$ is the proper lifetime of the kaon, and $\eta$ is a $\mathrm{CP}$ violation parameter defined by:

$$
\eta=\frac{A\left(K_{L} \rightarrow \pi \pi\right)}{A\left(K_{S} \rightarrow \pi \pi\right)}
$$

where $A\left(K_{L} \rightarrow \pi \pi\right)$ and $A\left(K_{S} \rightarrow \pi \pi\right)$ are the decay amplitudes, not rates, for the $K_{L}$ and $K_{S}$. KTeV measured [4] $\left|\eta_{+_{-}}\right|=(2.228 \pm 0.010) \times 10^{-3}$.

Detailed analysis $[5,6]$ of both the neutral and charged pion decays reveals that:

$$
\begin{aligned}
\eta_{+-} & =\epsilon+\epsilon^{\prime} \\
\eta_{00} & =\epsilon-2 \epsilon^{\prime}
\end{aligned}
$$

where $\epsilon^{\prime}$ is a parameter which describes the presence of direct CP violation, which occurs in the decay and not through mixing. The process which exhibits "direct" CP violation in this case is:

$$
\left|K_{2}\right\rangle(C P=-1) \rightarrow \pi \pi(C P=+1)
$$

Since $\epsilon^{\prime}$ is quite small compared to $\epsilon$, one can observe the shape of the decay distribution as written above, and measure $\epsilon$ by observing the amount of $K_{L^{-}} K_{S}$ interference present in addition to the tail due to $K_{L}$ decays. The shape of this decay distribution (as a function of the decay point instead of the proper lifetime of the kaon) is shown in Figure 2.2

However, the parameter $\epsilon^{\prime}$ can also be measured, if both the neutral and charge decays are observed. Squaring the amplitudes and then forming the ratio of ratios, 


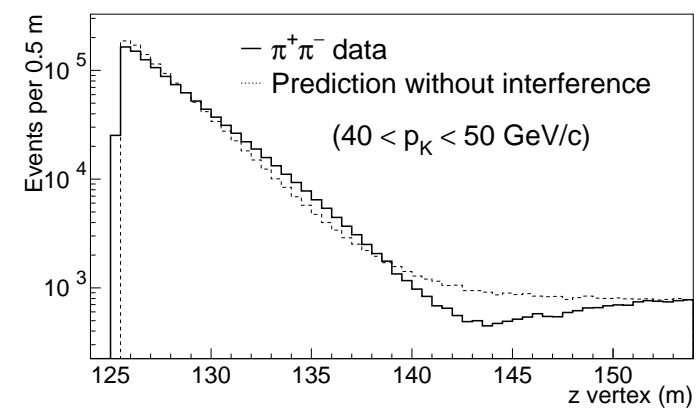

Figure 2.2: The location of the decay point of a beam of neutral kaons with and without $K_{L}-K_{S}$ interference. Plot taken from [7]

we can isolate the real part of $\epsilon^{\prime}$ :

$$
\frac{\left|\eta_{+-}\right|^{2}}{\left|\eta_{00}\right|^{2}} \approx 1+\operatorname{Re}\left(\epsilon^{\prime} / \epsilon\right)
$$

Forming the double ratio is useful from an experimental standpoint, as many systematic errors may cancel this way.

The measurement of $\operatorname{Re}\left(\frac{\epsilon^{\prime}}{\epsilon}\right)$ was long a major goal among high energy physics laboratories. After many years of effort, the $\mathrm{KTeV}$ experiment, along with the NA48 experiment at CERN, were able to measure the double ratio to a precision which allowed both to declare observation of direct CP violation. The current PDG world average is:

$$
\operatorname{Re}\left(\frac{\epsilon^{\prime}}{\epsilon}\right)=(1.67 \pm 0.23) \times 10^{-3}
$$

while the latest $\mathrm{KTeV}$ measurement is:

$$
\operatorname{Re}\left(\frac{\epsilon^{\prime}}{\epsilon}\right)=(2.07 \pm 0.148(\text { stat }) \pm 0.239(\text { syst })) \times 10^{-3}
$$

which establishes that direct $\mathrm{CP}$ violation exists - and that $\mathrm{CP}$ is not a symmetry of the weak interaction. However, due to hadronic interactions between the two pions, theoretical estimates of $R e\left(\frac{\epsilon^{\prime}}{\epsilon}\right)$ using the Standard Model can't easily be produced. By extension, the possibility of new physics contributing to this result can't be ruled out. Other probes of direct $\mathrm{CP}$ violation are then still interesting.

There are other possibilities for the observation of direct $\mathrm{CP}$ violation in the 
kaon sector. The decays $K_{L} \rightarrow \pi^{0} e^{+} e^{-}$and $K_{L} \rightarrow \pi^{0} \nu \bar{\nu}$ both have a dominant direct $\mathrm{CP}$ violating component in their decay amplitudes. However, their extremely low branching ratios put them out of experimental reach now and in the near to far future. However, $K_{L, S} \rightarrow \pi^{+} \pi^{-} \gamma$ can receive a contribution from a direct CP violating process, and its interference with a more common process might be large enough for the signature to be seen. One signature is completely analogous to $K_{L, S} \rightarrow \pi^{+} \pi^{-}$ namely interference between $K_{L}$ and $K_{S}$. If direct CP violation is present in this decay, then

$$
\eta_{+-\gamma} \neq \eta_{+-}
$$

where $\eta_{+-\gamma}$ is a CP violation parameter for $K_{L, S} \rightarrow \pi^{+} \pi^{-} \gamma$ and is extracted from the plot of the time dependent decay rate..

However, it is possible to use knowledge of the expected decay amplitudes for $K_{L, S} \rightarrow \pi^{+} \pi^{-} \gamma$ to extend and refine the search for CP violation as detailed in Chapter 3 . 


\section{Chapter 3}

\section{The Decay $K_{L, S} \rightarrow \pi^{+} \pi^{-} \gamma$}

\subsection{Introduction}

Shortly after the discovery of indirect CP violation in $K_{L} \rightarrow \pi^{+} \pi^{-}$it was realized $[8,9]$ that the associated radiative decay, $K_{L} \rightarrow \pi^{+} \pi^{-} \gamma$, could also provide a window into this phenomenon. Soon after the discovery [10] of $K_{L} \rightarrow \pi^{+} \pi^{-} \gamma$ it was observed [11] that this particular decay is composed of at least two different processes, inner bremsstrahlung (IB), where a photon is emitted, via bremsstrahlung, from one of the two charged pions in $K_{L} \rightarrow \pi^{+} \pi^{-}$, and direct emission (DE), where the photon originates from the decay vertex itself before the quarks hadronize into pions. This direct emission process is usually approximated as a pure magnetic dipole (M1) transition. In contrast, the decay $K_{S} \rightarrow \pi^{+} \pi^{-} \gamma$ occurs predominantly through the inner bremsstrahlung process. The reason for such asymmetry between $K_{L}$ and $K_{S}$ is the fact that the decay $K_{L} \rightarrow \pi^{+} \pi^{-}$is CP violating and thus suppressed, while the decay $K_{S} \rightarrow \pi^{+} \pi^{-}$is not. Since the IB process occurs after these decays, $K_{L} \rightarrow \pi^{+} \pi^{-} \gamma$ via IB is also suppressed, allowing the DE process to be seen. These two processes are illustrated in the Feynman diagrams in Figures 3.1 and 3.2.

For this study, the inner bremsstrahlung process is uninteresting, as it is just the radiative tail of the well studied process $K_{L, S} \rightarrow \pi^{+} \pi^{-}$. It is the direct emission processes which are most interesting here. The direct emission process has been observed and well measured in a number of analyses [11, 12, 13, 14, 15]. For the $K_{L}$ the direct emission process can be shown to be dominated by the magnetic transistion 


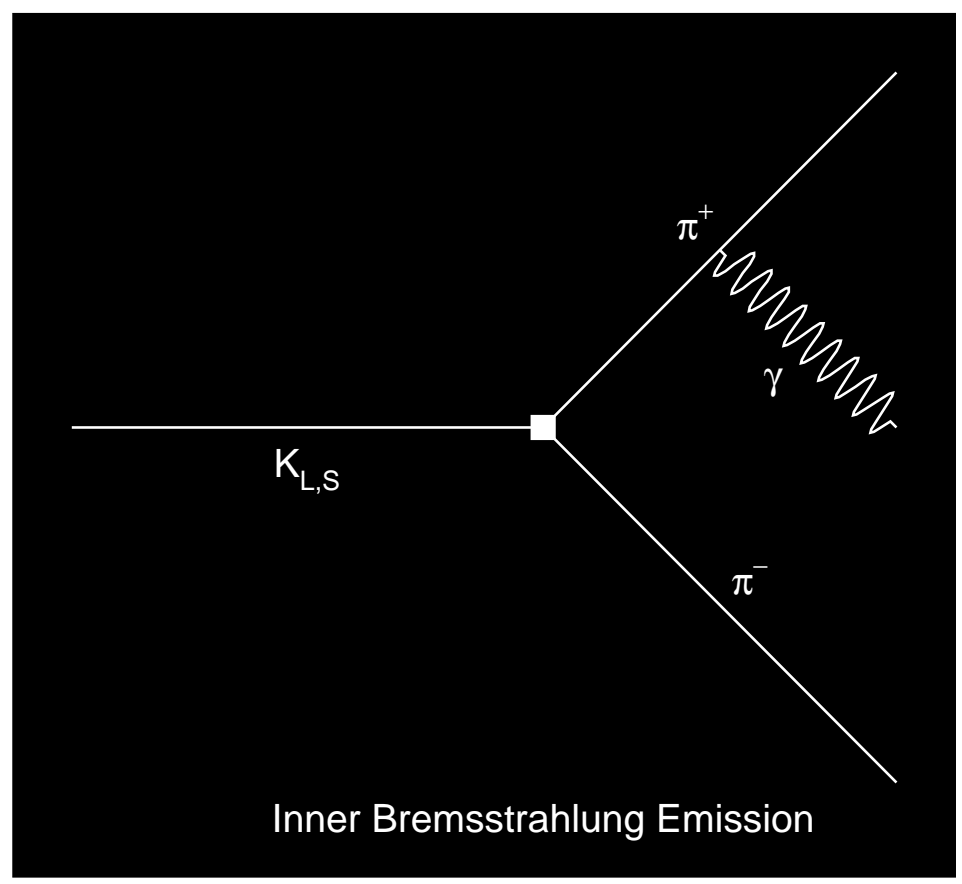

Figure 3.1: A Feynman diagram of the inner bremsstrahlung process. This process is an electric dipole (E1) process and is dominant in the decay of the $K_{S}$

due to the lack of a large amount of interference with the IB process, however the photon energy spectrum is not described as a pure M1 process owing to the presence of mediating vector mesons in the decay. Reference [11] was the first to observe this, and to attempt to fit a $\rho$ meson propagator to the photon energy spectrum. Reference [16] developed a model which agrees with the experimental study, while the models developed by $[17,18]$ do not. The M1 process will be present in the $K_{L}$ decay, where it is a $\mathrm{CP}$ conserving process. However, for the $K_{S}$ decay, this process is CP violating, so should be negligible. The M1 process is most interesting for practitioners of chiral perturbation theory, a method for performing calculations involving strong interaction processes at low energy, where perturbative QCD theory is by definition not valid. In chiral perturbation theory, $[19,20]$ the lowest order terms do not contribute to the M1 amplitude, which makes it sensitive to higher order terms which are not as well understood.

Finally, the most interesting process is the E1 direct emission process. Since it is an electric process, an interference term will be present between this process and the 


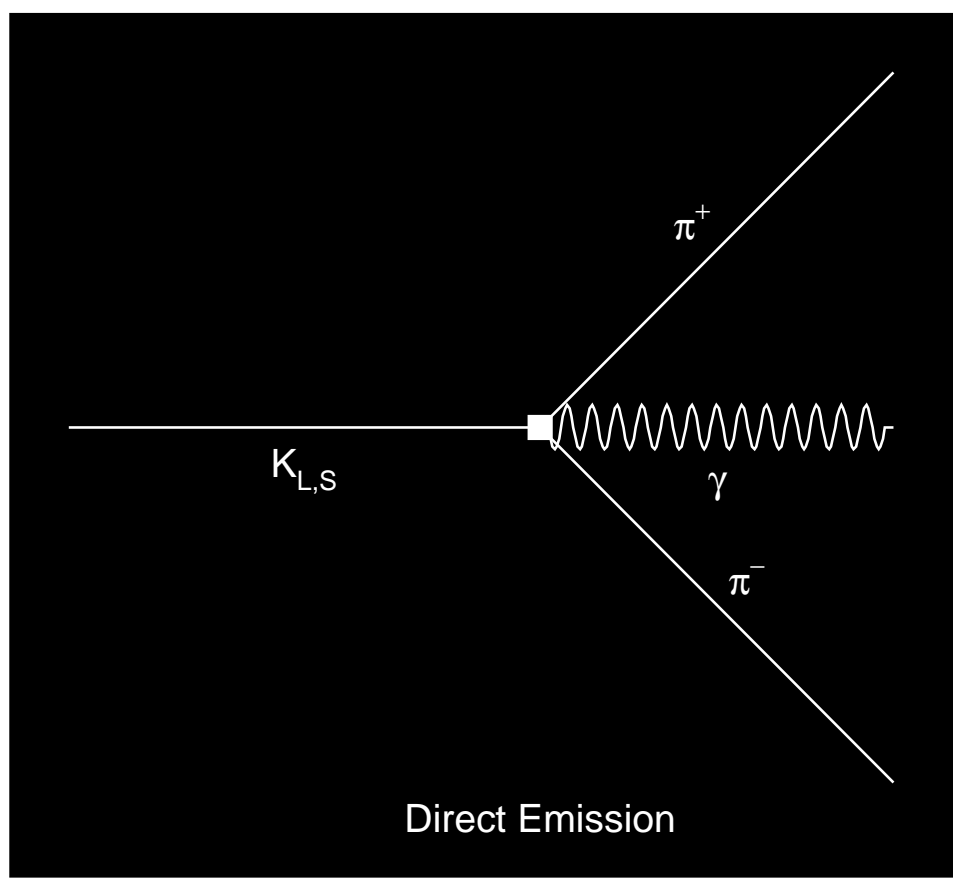

Figure 3.2: A Feynman diagram of the direct emission process. For the $K_{L}$, the multipole expansion for this process is dominated by the magnetic quadrupole (M1) term. The search for part of the electric dipole (E1) term is the purpose of this thesis.

IB process, boosting the contribution of this process, perhaps to an amount where observation is possible. Thus, even though the DE process is expected to be so much smaller than the IB process, it may still be observed by searching for interference with the dominant IB process. For the $K_{S}$ the E1 DE process will be CP conserving, implying this will be the largest term beyond the pure IB amplitude. However, for the $K_{L}$ this process is $\mathrm{CP}$ violating, meaning that it will be much smaller than the M1 process. An upper limit on the size of this term has been set using a study [15] of the Dalitz plot of the $K_{L}$ decay. Interestingly, a similar search using the decay $K_{L} \rightarrow \pi^{+} \pi^{-} e^{+} e^{-}$[14], with much lower statistics resulted in a tighter upper limit. In this particular decay, the dilepton pair act as an analyzer, allowing the polarization of the photon to be observed instead of being summed over. When the polarization is not summed over, the E1 and M1 DE amplitudes may interfere with each other, boosting the effect of any E1 DE term. More observables are also available in this mode which can be used in a Dalitz analysis. The conclusion to draw from this is 
that the Dalitz plot isn't particularly sensitive to interference between any E1 DE term and the IB term.

It should be noted that both of these studies only allowed for a E1 process which is due to mixing, which would then exhibit indirect $\mathrm{CP}$ violation. However, this process can also be due to a direct CP violating term in which the $K_{2} \mathrm{CP}$ eigenstate decays via the E1 DE process. This is the term of the most interest. This process would be present in the $K_{L}$ but not the $K_{S}$ where it would be doubly (indirect and direct) $\mathrm{CP}$ violating. Since it isn't present in the decay of the $K_{S}$, the direct CP violating term would be most visible in the interference between the $K_{L}$ and $K_{S}$. It should be mentioned that observation of this term would be an independent test of direct $\mathrm{CP}$ violation, different than the observation of non-zero $R e\left(\frac{\epsilon^{\prime}}{\epsilon}\right)$ and would constitute only the third sighting of direct $\mathrm{CP}$ violation ${ }^{1}$.

In order to search for this direct CP violating process, we must first develop a model of the decay, with dependence on the possible observables, which parameterizes the relative sizes of the different process that contribute to $K_{L, S} \rightarrow \pi^{+} \pi^{-} \gamma$. In order to allow the interference between the $K_{L}$ and $K_{S}$ states to be used, this model must also allow for the decay from both particles.

In the following sections, and throughout this thesis, the phenomenological model is parameterized according to [21], except for the form of the E1 DE term. Previous works $[14,15]$,which have used the notation of [21], have assumed that, if present, this decay is due solely to the indirect CP violating process. Doing so fixes the phase of the E1 DE amplitude to be given by:

$$
\arg \left[\frac{E_{D E}\left(K_{L}\right)}{M_{D E}\left(K_{L}\right)}\right]=\phi_{+-}
$$

However, a direct $\mathrm{CP}$ violating amplitude would have:

$$
\arg \left[\frac{E_{D E}\left(K_{L}\right)}{M_{D E}\left(K_{L}\right)}\right]=\frac{\pi}{2}
$$

In order to be consistent with the previous experimental formalism, the parameter

\footnotetext{
${ }^{1}$ The first definite proof of direct $\mathrm{CP}$ violation came from the measurement of $R e\left(\frac{\epsilon^{\prime}}{\epsilon}\right)$ while the second comes from neutral $\mathrm{B}$ meson decays
} 
$g_{E 1}$ is used to describe only the indirect $\mathrm{CP}$ violating part of the amplitude. The parameter $\widehat{e}$, identical to the parameter of the same name in [20], is used to denote the strength of the direct $\mathrm{CP}$ violating part of the amplitude. This parameter will be discussed more fully in Section 3.4.1.

\subsection{Decay rate as a function of invariant amplitude}

For an arbitrary initial particle state, the partial decay rate (i.e. the rate of the transition from the initial state into the final state, which in this case is into two charged pions and a photon) is given by [22, 23]:

$$
d \Gamma=\frac{(2 \pi)^{4}}{2 M}|\mathcal{M}|^{2} \delta^{4}\left(P-\sum_{i=1}^{n} p_{i}\right) \prod_{i=1}^{n} \frac{d^{3} p_{i}}{(2 \pi)^{3} 2 E_{i}}
$$

where

$\mathrm{M}$ is the invariant mass of the decaying particle, and is equal to $M_{K}$ in this case.

$\mathrm{P}$ is the momentum of the decaying particle.

$p_{i}$ is the 3 -momentum of the ith daughter particle.

$E_{i}$ is the energy of the ith daughter particle.

$\mathcal{M}$ is the invariant amplitude of the decay.

Then, for a three body decay:

$$
d \Gamma=\frac{1}{(2 \pi)^{5} 2 M}|\mathcal{M}|^{2} \delta^{4}\left(P-p_{1}-p_{2}-p_{3}\right) \frac{d^{3} p_{1}}{2 E_{1}} \frac{d^{3} p_{2}}{2 E_{2}} \frac{d^{3} p_{3}}{2 E_{3}}
$$

In the case of the $K_{L, S} \rightarrow \pi^{+} \pi^{-} \gamma$ decay, which is a three body process, two independent parameters are able to completely describe the orientation of the decay products. Since the purpose of this study is to measure the strengths of the different photon emission processes, the photon energy and direction will be the best choices for the independent observables. $E_{\gamma}^{*}$ is the energy of the emitted photon in the rest frame of the decaying kaon. The direction will be the angle between the $\gamma$ and $\pi^{+}$ 
momenta in the frame in which the pion 3-momenta are anti-parallel ${ }^{2}$. This frame is also referred to as the $\pi \pi$ rest frame.

According to section 3.7 of [23], the delta function in equation 3.4 can be removed by partial integration over particle momenta, which results in:

$$
d \Gamma=\frac{1}{2 M} \frac{1}{256 \pi^{5}}|\mathcal{M}|^{2} d E_{1} d E_{2} d_{\Omega} d \phi
$$

where $\Omega$ denotes two angles giving the orientation of $p_{1}, \phi$ is an angle of rotation and $E_{1}$ and $E_{2}$ are the energy of two of the daughter particles in the rest frame of the kaon. Since the matrix element is invariant over rotations in the coordinate system, we can integrate over $\Omega$ and $\phi$ yields:

$$
\begin{aligned}
d \Gamma & =\frac{1}{2 M} \frac{8 \pi^{2}}{256 \pi^{5}}|\mathcal{M}|^{2} d E_{1} d E_{2} \\
& =\frac{1}{2 M} \frac{1}{32 \pi^{3}}|\mathcal{M}|^{2} d E_{1} d E_{2}
\end{aligned}
$$

Since we desire that one of the kinematic variables be $E_{\gamma}^{*}$ let us choose $E_{1}=E_{\gamma}^{*}$ and $E_{2}$ to be the energy of the $\pi^{+}$. Using the result in section A.4 to make the change of variables $d E_{2}=\frac{\beta E_{\gamma}^{*}}{2} d \cos (\theta)$ we can then write:

$$
d \Gamma=\frac{1}{M_{K}} \frac{1}{128 \pi^{3}} \beta E_{\gamma}^{*}|\mathcal{M}|^{2} d E_{\gamma}^{*} d \cos (\theta)
$$

where $\beta$ is simply the pion velocity in the $\pi \pi$ rest frame.

As the decay rate depends on the square of the matrix element, the next step is introduce the matrix element for the decay $K \rightarrow \pi^{+} \pi^{-} \gamma$.

\subsection{Matrix Element for Kaon Decay}

The most general matrix element that describes a neutral kaon decaying into a $\pi^{+}$ with 4-momentum $p_{+}$, a $\pi^{-}$with 4-momentum $p_{-}$, and a $\gamma$ with 4-momentum $q$ and

\footnotetext{
${ }^{2}$ Using this frame greatly simplifies the evaluation of the matrix element.
} 
polarization vector $\varepsilon$ is $[24,25]$ :

$$
\begin{aligned}
\mathcal{M} & =\frac{e\left|f_{S}\right|}{M_{K}^{4}}\left[\left[E_{I B}(K)+E_{D E}(K)\right]\left[\left(\varepsilon \cdot p_{+}\right)\left(q \cdot p_{-}\right)-\left(\varepsilon \cdot p_{-}\right)\left(q \cdot p_{+}\right)\right]\right. \\
& \left.+M_{D E}(K)\left[\epsilon_{\lambda \mu \rho \sigma} \varepsilon^{\lambda} q^{\mu} p_{+}^{\rho} p_{-}^{\sigma}\right]\right]
\end{aligned}
$$

where

$E_{I B}$ is the amplitude for the inner bremsstrahlung transition.

$E_{D E}$ is the amplitude for the direct emission electric transition.

$M_{D E}$ is the amplitude for the direct emission magnetic transition.

$\epsilon_{\lambda \mu \rho \sigma}$ is the totally anti-symmetric Levi-Civita tensor.

e is the electron charge.

$f_{S}$ is a constant related to the decay rate of $K_{S} \rightarrow \pi^{+} \pi^{-}$.

$\mathcal{M}_{K \rightarrow \pi^{+} \pi^{-}}$is the matrix element for the dipion decay, and is simply a constant.

$M_{K}$ is the kaon mass.

Noting that that amplitude is squared in the decay rate, and that the polarization of the photon cannot be observed in this decay, we must square equation 3.8 and then sum over all photon polarizations. This is done in Appendix C.1.

Using the results as shown in equations C.12, C.11, and C.17 we can write:

$$
\begin{aligned}
|\mathcal{M}|^{2} & =\left(\frac{e\left|f_{S}\right|}{M_{K}^{4}}\right)^{2}\left[\left|E_{I B}(K)+E_{D E}(K)\right|^{2}+\left|M_{D E}(K)\right|^{2}\right] \times \\
& {\left[-M_{\pi}^{2}\left[\left(q \cdot p_{-}\right)^{2}+\left(q \cdot p_{+}\right)^{2}\right]+2\left(p_{+} \cdot p_{-}\right)\left(q \cdot p_{-}\right)\left(q \cdot p_{+}\right)\right] }
\end{aligned}
$$

where the interference term between the magnetic and electric amplitudes is zero due to the summation over photon polarizations.

In order to actually use the matrix element, we must evaluate the momenta. Since the matrix element describes the invariant amplitude, the actual value will be the same in all reference frames. For convenience, we shall chose to evaluate $|\mathcal{M}|^{2}$ in the 
$\pi \pi$ rest frame, as there are a number of useful kinematic relations in that frame which serve to simplify the mathematics involved. The result, which is derived in Appendix C.2, is:

$$
\begin{aligned}
|\mathcal{M}|^{2}= & \left(\frac{e^{2}\left|f_{S}\right|^{2}}{4 M_{K}^{4}}\right)\left(1-\frac{2 E_{\gamma}^{*}}{M_{K}}\right) E_{\gamma}^{* 2} \beta^{2} \sin ^{2}(\theta) \\
& {\left[\left|E_{I B}(K)+E_{D E}(K)\right|^{2}+\left|M_{D E}(K)\right|^{2}\right] }
\end{aligned}
$$

which is the final result for the squared matrix element after the summation over photon polarizations, and as before, $\theta$ is the angle between the photon and $\pi^{+}$in the $\pi \pi$ rest frame and $E_{\gamma}^{*}$ is the photon energy in the kaon rest frame.

We now have enough information to write down the decay rate of a pure kaon state. Plugging equation 3.10 into equation 3.7, we get:

$$
\begin{aligned}
\frac{d \Gamma}{d E_{\gamma}^{*} d \cos (\theta)}= & \frac{1}{128 \pi^{3}} \frac{1}{M_{K}} \beta E_{\gamma}^{*}\left(\frac{e^{2}\left|f_{S}\right|^{2}}{4 M_{K}^{4}}\right)\left(1-\frac{2 E_{\gamma}^{*}}{M_{K}}\right) E_{\gamma}^{* 2} \beta^{2} \sin ^{2}(\theta) \\
& {\left[\left|E_{I B}(K)+E_{D E}(K)\right|^{2}+\left|M_{D E}(K)\right|^{2}\right] } \\
= & \frac{1}{512 \pi^{3}}\left(\frac{\beta^{3} E_{\gamma}^{*} 3}{M_{K}^{3}}\right)\left(\frac{e^{2}\left|f_{S}\right|^{2}}{M_{K}^{2}}\right)\left(1-\frac{2 E_{\gamma}^{*}}{M_{K}}\right) \sin ^{2}(\theta) \\
& {\left[\left|E_{I B}(K)+E_{D E}(K)\right|^{2}+\left|M_{D E}(K)\right|^{2}\right] }
\end{aligned}
$$

\subsection{Amplitudes for multipole transitions}

\subsubsection{Inner Bremsstrahlung amplitudes}

Now that the form of the squared matrix element is manifest, we introduce the amplitude for each individual decay process.

The amplitude for the inner bremsstrahlung decay of the $K_{S}$ is given [24] by:

$$
E_{I B}\left(K_{S}\right)=\left(4 \frac{M_{K}^{2}}{E_{\gamma}^{* 2}}\right) \frac{e^{i \delta_{0}}}{1-\beta^{2} \cos ^{2}(\theta)}
$$

where

$E_{\gamma}^{*}$ is the photon energy in the kaon rest frame. 
$\theta$ is the angle between the photon and $\pi^{+}$momenta in the $\pi \pi$ rest frame.

$\delta_{0}$ is the strong interaction phase shift for $\mathrm{I}=0$ that is due to re-scattering amongst the two pions.

$$
\beta=\sqrt{1-\frac{4 M_{\pi}^{2}}{M_{K}^{2}-2 E_{\gamma}^{*} M_{K}}}
$$

The amplitude for the inner bremsstrahlung decay of the $K_{L}$ is given [24] by:

$$
E_{I B}\left(K_{L}\right)=\left(4 \frac{M_{K}^{2}}{E_{\gamma}^{* 2}}\right) \frac{\eta_{+-} e^{i \delta_{0}}}{1-\beta^{2} \cos ^{2}(\theta)}
$$

where $\eta_{+-}$is the CP violation parameter for the $K_{L} \rightarrow \pi^{+} \pi^{-}$decay.

\subsubsection{Direct Emission Amplitudes}

The terms $E_{D E}$ and $M_{D E}$ in equation 3.8 are the amplitudes for electric and magnetic dipole direct emission, respectively. Both can be expressed in a multipole expansion which conveniently produces terms with unique $\mathrm{CP}$ and $\mathrm{J}$ values.

Affecting the multipole expansion by expanding in powers of $\left(p_{+}-p_{-}\right) \cdot q / M_{K}{ }^{2}$, we have[9] :

$$
E_{D E}{ }^{\text {total }}=E_{D E}+\cdots
$$

as well as

$$
M_{D E}{ }^{\text {total }}=M_{D E}+\cdots
$$

where $E_{D E}$ and $M_{D E}$ are the electric and magnetic dipole terms respectively.

The CP characteristics of all the various amplitudes can be seen in Table 3.1

\begin{tabular}{||l|l|l|l|l|l||}
\hline & $E_{I B}$ & $E_{D E}$ & $M_{D E}$ & $E_{D E}^{(2)}$ & $M_{D E}^{(2)}$ \\
\hline$K_{L} \rightarrow \pi^{+} \pi^{-} \gamma$ & $\mathrm{CPV}$ & $\mathrm{CPV}$ & & & $\mathrm{CPV}$ \\
\hline$K_{S} \rightarrow \pi^{+} \pi^{-} \gamma$ & & & $\mathrm{CPV}$ & $\mathrm{CPV}$ & \\
\hline
\end{tabular}

Table 3.1: The $\mathrm{CP}$ characteristics of various processes

The amplitude for the decay of the $K_{L}$ via M1 direct photon emission is given [20] 
by:

$$
M_{D E}\left(K_{L}\right)=i \widetilde{g_{M 1}}\left(\frac{a_{1} / a_{2}}{M_{\rho}^{2}-M_{K}^{2}+2 E_{\gamma}^{*} M_{K}}+1\right) e^{i \delta_{1}}
$$

where

$\widetilde{g_{M 1}}$ is a parameter that describes the strength of this process. This is a parameter to be fit.

$a_{1} / a_{2}$ is a parameter that describes the effect of a possible vector meson influence is this decay. This is a parameter to be fit.

$M_{\rho}$ is the mass of the $\rho$ meson which is assumed to be the vector meson which influences this decay.

$\delta_{1}$ is the strong interaction phase shift for $\mathrm{I}=1$ that is due to rescattering amongst the two pions.

The term above involving $a_{1} / a_{2}$ is a form factor from the Vector Dominance Model. If $a_{1} / a_{2}$ were zero, effects arising from virtual vector mesons would be negligible here. This form factor will deform the photon energy spectrum due to this process, and will shift the mean photon energy to lower values. Measurements $[13,14,15]$ indicate that $a_{1} / a_{2}$ is non-zero, negative, and of order unity.

The amplitude for the decay of the $K_{S}$ via M1 direct photon emission is given [20] by:

$$
M_{D E}\left(K_{S}\right)=i \epsilon \widetilde{g_{M 1}}\left(\frac{a_{1} / a_{2}}{M_{\rho}{ }^{2}-M_{K}{ }^{2}+2 E_{\gamma}^{*} M_{K}}+1\right) e^{i \delta_{1}}
$$

where $\epsilon$ is the $\mathrm{CP}$ violation parameter which describes the amount of mixing type ( indirect ) $\mathrm{CP}$ violation in the neutral kaons. This indicates that decay of the $K_{S}$ via this process is $\mathrm{CP}$ violating. This process has not been observed, due to $\mathrm{CP}$ suppression, and it will most likely remain so.

The amplitude for the decay of the $K_{S}$ via E1 direct photon emission is given [20] by:

$$
E_{D E}\left(K_{S}\right)=\frac{g_{E 1}}{\epsilon} e^{i\left(\delta_{1}+\phi \epsilon\right)}
$$

where 
$g_{E 1}$ is a parameter that describes the strength of this process. This is a parameter to be fit.

$\phi_{+-}$is the complex phase of the CP violation parameter $\epsilon$.

This process conserves $\mathrm{CP}$, however the rate of the inner bremsstrahlung process for the $K_{S}$ is far larger, and hence this process has previously gone unobserved.

Finally the amplitude for the corresponding process for the $K_{L}$ is given as [20]:

$$
E_{D E}\left(K_{L}\right)=g_{E 1} e^{i\left(\delta_{1}+\phi_{\varepsilon}\right)}+i 16 \widehat{e} e^{i \delta_{1}}
$$

where

$\widehat{e}$ is a parameter that describes the amount of this process that is due to the decay of the $K_{2} \mathrm{CP}$ eigenstate. This is a parameter to be fit.

The form of the amplitudes for the E1 direct process imply that $g_{E 1}$ parameterizes the amount of indirect $\mathrm{CP}$ violation in the $K_{L}$ decay, while the $\widehat{e}$ term is a measure of direct $\mathrm{CP}$ violation in the $K_{L}$ decay. This amplitude was split into two pieces in order stay consistent with previous experimental limits on $g_{E 1}$ while also allowing direct CP violation, as previously mentioned in section 3.1. This is the first time this amplitude has been expressed in this way.

The measurement of $\widehat{e}$ is the central goal of this analysis. No part of this process has been previously observed. Also note that while $g_{E 1}$ and $\widehat{e}$ are real by definition, they can be positive or negative.

\subsubsection{Expected Values of Parameters}

Many of the parameters that appear in the decay amplitudes have already been measured, or enough experimental information exists so that estimates can be made. An analysis [15] of $K_{L} \rightarrow \pi^{+} \pi^{-} \gamma$ yielded

$$
\begin{aligned}
\widetilde{g_{M 1}} & =1.198 \pm 0.035(\text { stat }) \pm 0.086(\text { syst }) \\
a_{1} / a_{2} & =-0.738 \pm 0.007(\text { stat }) \pm 0.018(\text { syst }) G e V^{2} / c^{2}
\end{aligned}
$$


Two previous analyses have attempted to measure or constrain $g_{E 1}$. An analysis using $K_{L} \rightarrow \pi^{+} \pi^{-} e^{+} e^{-}[14]$ constrains

$$
g_{E 1}<0.03(90 \% \text { C.L. })
$$

The power of this constraint comes mainly from the fact that in this decay, the $E_{D E}$ component will interfere with the $M_{D E}$ component, further boosting the effect of the process. In addition, there are many more experimental observables in the analysis. In comparison, the previous analysis of $K_{L} \rightarrow \pi^{+} \pi^{-} \gamma$ [15] reveals that a limit only utilizing two observables is quite weak, yielding $g_{E 1}<0.21(90 \%$ C.L.). It is also possible to place a rough limit on $g_{E 1}$ using the branching ratio of the $K_{S}$ where the process would be CP conserving and would interfere with the bremsstrahlung process. One such limit [21] is $\left|\frac{g_{E 1}}{g_{M 1}}\right|<0.05$.Note that this limit incorporates $g_{M 1}$, the average amplitude of the process, not $\widetilde{g_{M 1}}$, which is a measure of the overall amplitude when the $\rho$ form factor is used.

The parameter $\widehat{e}$ was first introduced in [20] as a measure of direct CP violation in $K_{L, S} \rightarrow \pi^{+} \pi^{-} \gamma$. As such, the only estimate also comes from that paper. The estimate, using Chiral Perturbation arguments, is:

$$
\left|\frac{\widehat{e}}{\epsilon^{\prime}}\right| \sim 1
$$

to an order of magnitude.

Other authors instead look at the difference between the values of $\eta_{+-}$and $\eta_{+-\gamma}$ (see Section E) which is defined as $\epsilon_{+-\gamma}^{\prime}$. However, [20] was the first to treat $\eta_{+-\gamma}$ in the proper way, as a average over all photon energies, as opposed to just a ratio of amplitudes, which in this case is dependent on the photon energy cutoff.

This is unfortunate, since [20] points out that $\epsilon_{+-\gamma}^{\prime}$ is suppressed by phase space, however it can be related to $\widehat{e}$ by:

$$
\epsilon_{+-\gamma}^{\prime} \approx 0.041 \widehat{e}\left(E_{\gamma}>20 M e V\right)
$$


Previous experiments $[26,27]$ have measured $\eta_{+-\gamma}$, the PDG average of which is :

$$
\begin{aligned}
\left|\eta_{+-\gamma}\right| & =(2.35 \pm 0.07) \times 10^{-3} \\
\phi_{+-\gamma} & =(44 \pm 4)^{\circ}
\end{aligned}
$$

$\mathrm{KTeV}$ measured $\eta_{+-}$to be:

$$
\left|\eta_{+-}\right|=(2.228 \pm 0.010) \times 10^{-3}
$$

while the PDG average is:

$$
\begin{aligned}
& \left|\eta_{+-}\right|=(2.236 \pm 0.018) \times 10^{-3} \\
& \phi_{+-\gamma}=(43.4 \pm 0.7)^{\circ}
\end{aligned}
$$

If we use the PDG average of $\eta_{+-\gamma}$ and the $\mathrm{KTeV}$ value of $\eta_{+-}$, we estimate:

$$
\epsilon_{+-\gamma}^{\prime}=(1.2 \pm 0.7) \times 10^{-4}
$$

which then leads to

$$
\widehat{e} \approx \frac{\epsilon_{+-\gamma}^{\prime}}{0.041} \approx(3.0 \pm 1.7) \times 10^{-3}
$$

\subsection{The Triple Differential Decay Rate of the Pro- cess $K_{L, S} \rightarrow \pi^{+} \pi^{-} \gamma$}

For this particular analysis, the relative strength of the different photon emission amplitudes will be determined.

The $\mathrm{KTeV}$ experiment produces kaon beams of two different states. One beam, called the "vacuum" beam, consists mostly of pure $K_{L}$ particles. The other beam, called the "regenerator" beam, is composed of a mixture of $K_{S}$ and $K_{L}$ particles. Both beams are used in this analysis, so a time dependent particle state is defined as:

$$
|\Psi(t)\rangle=A\left[\left|K_{L}(t)\right\rangle+\rho\left|K_{S}(t)\right\rangle\right]
$$

This wavefunction represents an arbitrary superposition of the $K_{L}$ state and the $K_{S}$ 
state. Note that the $K_{S}$ part can arise from regeneration as well as production in the target. The factor of $A$ is a normalization constant. The factor of $\rho$ is the regenerator amplitude, as explained in detail in Section 4.1.6 and Chapter 7. It describes the phase and size of the $K_{S}$ component of the total wavefunction. Making the time dependence explicit, we get:

$$
|\Psi(\tau)\rangle=A\left[\left|K_{L}\right\rangle e^{-i\left(M_{L}-\frac{i}{2 \tau_{L}}\right) \tau}+\rho\left|K_{S}\right\rangle e^{-i\left(M_{S}-\frac{i}{2 \tau_{S}}\right) \tau}\right]
$$

in the rest frame of the decaying particle, where

$M_{L}$ is the mass of the $K_{L}$,

$M_{S}$ is the mass of the $K_{S}$,

$\tau_{L}$ is the mean lifetime of the $K_{L}$ and

$\tau_{S}$ is the mean lifetime of the $K_{S}$.

Given that we are observing the decay of a superposition of two particle states, $K_{L}$ and $K_{S}$ we must account for this in our definition of the decay rate. Instead of using the invariant amplitude for a pure $K_{L}$ or $K_{S}$ decay, we must determine the correct invariant amplitude for the state shown in equation 3.30. The invariant amplitude is given by [22] :

$$
\langle\alpha|i T| \Psi\rangle=i(2 \pi)^{4} \delta^{4}\left(P-\sum_{i=1}^{n} p_{i}\right) \frac{\mathcal{M}}{(2 E)^{1 / 2} \prod_{i=1}^{n}\left(2 E_{I}\right)^{1 / 2}}
$$

where

$\mathrm{T}$ is the operator which encodes the particle interactions, and is related to the $\mathrm{S}$ matrix, and thus the Hamiltonian, via $S=I+i T$,

$E$ is the energy of the initial particle, before decay,

$\alpha$ is the final state of the particles after decay and 
$\Psi$ is the initial particle state, in this case given in equation (3.30).

The total invariant amplitude for the state $|\Psi\rangle$ will then be given by:

$$
\begin{aligned}
\langle\alpha|i T| \Psi\rangle & \quad i(2 \pi)^{4} \delta^{4}\left(P-\sum_{i=1}^{n} p_{i}\right) \frac{\mathcal{M}_{T}}{(2 E)^{1 / 2} \prod_{i=1}^{n}\left(2 E_{i}\right)^{1 / 2}} \\
= & \left\langle\alpha|i T| A K_{L}\right\rangle e^{-i\left(M_{L}-\frac{i}{2 \tau_{L}}\right) \tau}+\left\langle\alpha|i T| A \rho K_{S}\right\rangle e^{-i\left(M_{S}-\frac{i}{2 \tau_{S}}\right) \tau} \\
= & i(2 \pi)^{4} \delta^{4}\left(P-\sum_{i=1}^{n} p_{i}\right) \times \\
& \frac{\left(A \mathcal{M}_{K_{L}} e^{-i\left(M_{L-} \frac{i}{2 \tau_{L}}\right) \tau}+A \rho \mathcal{M}_{K_{S}} e^{-i\left(M_{S}-\frac{i}{2 \tau_{S}}\right) \tau}\right)}{(2 E)^{1 / 2} \prod_{i=1}^{n}\left(2 E_{i}\right)^{1 / 2}}
\end{aligned}
$$

where the definitions of the wavefunction from Equations 3.29 and 3.30 in the second and third lines. This indicates that the total invariant amplitude for the state shown in equation (3.30) to decay into $\pi^{+} \pi^{-} \gamma$ can be expressed as the sum of the amplitudes for the $K_{L}$ decay and the $K_{S}$ decay:

$$
\mathcal{M}_{T}=A\left[\mathcal{M}_{K_{L}} e^{-i\left(M_{L-} \frac{i}{2 \tau_{L}}\right) \tau}+\rho \mathcal{M}_{K_{S}} e^{-i\left(M_{S}-\frac{i}{2 \tau_{S}}\right) \tau}\right]
$$

Since the square of the amplitude appears in the decay rate, we multiply the 
amplitude with its complex conjugate to yield:

$$
\begin{aligned}
\left|\mathcal{M}_{T}\right|^{2}= & |A|^{2}\left[\left|\mathcal{M}_{K_{L}}\right|^{2} e^{-\left(\frac{1}{\tau_{L}}\right) \tau}+|\rho|^{2}\left|\mathcal{M}_{K_{S}}\right|^{2} e^{-\left(\frac{1}{\tau_{S}}\right) \tau}\right. \\
& \left.+2 \operatorname{Re}\left[\rho \mathcal{M}_{K_{L}}^{\dagger} \mathcal{M}_{K_{S}} e^{i \Delta M_{K} \tau}\right] e^{-\left(\frac{1}{\tau_{S}}+\frac{1}{\tau_{L}}\right) \frac{1}{2} \tau}\right]
\end{aligned}
$$

Inserting this amplitude into the decay rate as shown in equation 3.7 produces:

$$
\begin{aligned}
\frac{d \Gamma}{d E_{\gamma}^{*} d \cos (\theta)}= & |A|^{2} \frac{1}{M_{K}} \frac{1}{128 \pi^{3}} \beta E_{\gamma}^{*}\left[\left|\mathcal{M}_{K_{L}}\right|^{2} e^{-\left(\frac{1}{\tau_{L}}\right) \tau}+|\rho|^{2}\left|\mathcal{M}_{K_{S}}\right|^{2} e^{-\left(\frac{1}{\tau_{S}}\right) \tau}\right. \\
& \left.+2 \operatorname{Re}\left[\rho \mathcal{M}_{K_{L}}^{\dagger} \mathcal{M}_{K_{S}} e^{i \Delta M_{K} \tau}\right] e^{-\left(\frac{1}{\tau_{S}}+\frac{1}{\tau_{L}}\right) \frac{1}{2} \tau}\right]
\end{aligned}
$$

Now two of the terms can be identified as the Dalitz plot densities for the $K_{L}$ and $K_{S}$ while the third term arises due to interference between the two particle states, which allows us to write:

$$
\begin{aligned}
\frac{d \Gamma}{d E_{\gamma}^{*} d \cos (\theta)}= & |A|^{2}\left[\frac{d \Gamma_{K_{L}}}{d E_{\gamma}^{*} d \cos (\theta)} e^{-\left(\frac{1}{\tau_{L}}\right) \tau}+|\rho|^{2} \frac{d \Gamma_{K_{S}}}{d E_{\gamma}^{*} d \cos (\theta)} e^{-\left(\frac{1}{\tau_{S}}\right) \tau}\right. \\
& \left.+2 \operatorname{Re}\left[\rho \frac{d \gamma_{L S}}{d E_{\gamma}^{*} d \cos (\theta)} e^{i \Delta M_{K} \tau}\right] e^{-\left(\frac{1}{\tau_{S}}+\frac{1}{\tau_{L}}\right) \frac{1}{2} \tau}\right]
\end{aligned}
$$


where

$$
\begin{aligned}
\frac{d \Gamma_{K_{L}}}{d E_{\gamma}^{*} d \cos (\theta)}= & \frac{1}{M_{K}} \frac{1}{128 \pi^{3}} \beta E_{\gamma}^{*}\left|\mathcal{M}_{K_{L}}\right|^{2} \\
= & \frac{e^{2}\left|f_{s}\right|^{2}}{M_{K}{ }^{2}}\left(\frac{\beta E_{\gamma}^{*}}{8 \pi M_{K}}\right)^{3}\left(1-\frac{2 E_{\gamma}^{*}}{M_{K}}\right) \sin ^{2} \theta \\
& \times\left(\left|E_{I B}\left(K_{L}\right)+E_{D E}\left(K_{L}\right)\right|^{2}+\left|M_{D E}\left(K_{L}\right)\right|^{2}\right)
\end{aligned}
$$

and

$$
\begin{aligned}
\frac{d \Gamma_{K_{S}}}{d E_{\gamma}^{*} d \cos (\theta)}= & \frac{1}{M_{K}} \frac{1}{128 \pi^{3}} \beta E_{\gamma}^{*}\left|\mathcal{M}_{K_{S}}\right|^{2} \\
= & \frac{e^{2}\left|f_{s}\right|^{2}}{M_{K}{ }^{2}}\left(\frac{\beta E_{\gamma}^{*}}{8 \pi M_{K}}\right)^{3}\left(1-\frac{2 E_{\gamma}^{*}}{M_{K}}\right) \sin ^{2} \theta \\
& \times\left(\left|E_{I B}\left(K_{S}\right)+E_{D E}\left(K_{S}\right)\right|^{2}+\left|M_{D E}\left(K_{S}\right)\right|^{2}\right)
\end{aligned}
$$

and finally

$$
\begin{aligned}
\frac{d \gamma_{L S}}{d E_{\gamma}^{*} d \cos (\theta)}= & \frac{1}{M_{K}} \frac{1}{128 \pi^{3}} \beta E_{\gamma}^{*} \mathcal{M}_{K_{L}}^{\dagger} \mathcal{M}_{K_{S}} \\
= & \frac{e^{2}\left|f_{s}\right|^{2}}{M_{K}{ }^{2}}\left(\frac{\beta E_{\gamma}^{*}}{8 \pi M_{K}}\right)^{3}\left(1-\frac{2 E_{\gamma}^{*}}{M_{K}}\right) \sin ^{2} \theta \\
& \times\left(\left[E_{I B}\left(K_{L}\right)+E_{D E}\left(K_{L}\right)\right]^{\dagger}\left[E_{I B}\left(K_{S}\right)+E_{D E}\left(K_{S}\right)\right]\right. \\
& \left.+M_{D E}{ }^{\dagger}\left(K_{L}\right) M_{D E}\left(K_{S}\right)\right)
\end{aligned}
$$

The factor of $\rho$ can now be identified as the regeneration amplitude due to the mostly pure $K_{S}$ particle beam transversing the active regenerator in the $\mathrm{KTeV}$ beamline. This parameter is momentum-dependent, so we express the rate in terms of the kaon 
momentum and the distance the kaon traveled before it decayed:

$$
\begin{aligned}
\frac{d \Gamma}{d E_{\gamma}^{*} d \cos (\theta)}= & |A|^{2}\left[\frac{d \Gamma_{K_{L}}}{d E_{\gamma}^{*} d \cos (\theta)} e^{-\left(\frac{1}{\tau_{L}}\right) \frac{M_{K} \Delta z}{p_{K}}}\right. \\
& +\left|\rho\left(p_{K}\right)\right|^{2} \frac{d \Gamma_{K}}{d E_{\gamma}^{*} d \cos (\theta)} e^{-\left(\frac{1}{\tau_{S}}\right) \frac{M_{K} \Delta z}{p_{K}}} \\
& +2 R e\left[\rho\left(p_{K}\right) \frac{d \gamma_{L S}}{d E_{\gamma}^{*} d \cos (\theta)} e^{\left.\left.i \Delta M_{K} \frac{M_{K} \Delta z}{p_{K}}\right] e^{-\left(\frac{1}{\tau_{S}}+\frac{1}{\tau_{L}}\right)} \frac{1}{2} \frac{M_{K} \Delta z}{p_{K}}\right]}\right.
\end{aligned}
$$

This equation describes an observable distribution of decays in the phase space $\left(p_{K}, \mathrm{z}\right.$ vertex, $\left.E_{\gamma}^{*}, \cos (\theta)\right)$, and explicitly depends on the amplitudes and form factors of the different photon emission processes. It is this Equation 3.40 which will be fit to the data in order to search for the direct CP-violating E1 direct emission process, whose presence would be indicated by a best fit value of $\widehat{e}$ (see Equation 3.19) that is non-zero and statistically significant.

Equation 3.40 can also be used in analyze the decays in the pure $K_{L}$ "vacuum" beam, for which we can set $\rho=0$. In doing this, equation 3.40 simply becomes the Dalitz density for the $K_{L}$.

Finally, it should be noted that Equation 3.40 can be integrated over $\cos (\theta)$ and $E_{\gamma}^{*}$, as shown in Appendix E. Doing so yields an "average" decay rate as shown in Equation E.18. 


\section{Chapter 4}

\section{The KTeV Experiment}

$\mathrm{KTeV}[28,7,29,6,30,31]$ was one of the last of a long line of fixed target flavor experiments at Fermi National Laboratory. Among the many topics of its experimental program were:

- The search for $K_{L} \rightarrow \pi^{0} e^{+} e^{-}$and $K_{L} \rightarrow \pi^{0} \mu^{+} \mu^{-}$both of which may exhibit a large degree of direct $\mathrm{CP}$ violation if observed. The observation of these decays would be useful probes for new physics beyond the Standard Model.

- The search for $K_{L} \rightarrow \pi^{0} \nu \bar{\nu}$, a ultra-rare decay which violates direct CP violation completely and has a branching ratio which can be easily computed in the Standard Model. The observation of this decay would be an excellent probe of new physics.

- The search for $K_{L} \rightarrow \pi^{0} \mu e$, a decay that if observed, would be the first example of lepton-flavor violation.

- The observation and study of $K_{L} \rightarrow \pi^{+} \pi^{-} e^{+} e^{-}$which in addition to containing a very large $\mathrm{CP}$ asymmetry, also in an example of explicit $\mathrm{T}$ violation.

- The study of a number of Hyperon decays.

- The high precision measurement of $\operatorname{Re}\left(\frac{\epsilon^{\prime}}{\epsilon}\right)$, neutral kaon parameters and a high sensitivity search for CPT violation. 
- The measurement of the major $K_{L}$ branching fractions in order to obtain a high precision measurement of $\left|V_{U S}\right|$ and $\left|\eta_{+-}\right|$

- The search for direct CP violation in $K_{L, S} \rightarrow \pi^{+} \pi^{-} \gamma$

$\mathrm{KTeV}$ ran in two different configurations-E799 which produced data for most of the rare decay studies and only produced $K_{L}$ particles, and E832, which produced data for the $\operatorname{Re}\left(\frac{\epsilon^{\prime}}{\epsilon}\right)$, CPT and kaon parameters analyses. E832 produced a beam of $K_{L}$ in addition to a second beam which produced kaons in a mixed $K_{L}-K_{S}$ state. Data for the $K_{L, S} \rightarrow \pi^{+} \pi^{-} \gamma$ analysis comes from the E832 configuration. The following description of the detector is for the E832 configuration.

\subsection{KTeV Beamline}

KTeV utilizes Fermilab's Tevatron particle accelerator in order to create beams of intense kaons. Fermilab's chain of accelerator consists of:

- Cockcroft-Walton $H^{-}$source-A small bottle of hydrogen is fed into this first stage accelerator, which ionizes the hydrogen to produce $H^{-}$ions, and then accelerates the ions up to an energy of $750 \mathrm{keV}$, which equates to $4 \%$ of the speed of light.

- Linear Accelerator - $H^{-}$ions are accelerated up to $400 \mathrm{MeV}$, or $71 \%$ of the speed of light. At the end of the 430 foot length of the linear accelerator, the $H^{-}$ions pass through a thin carbon foil, where they are stripped of electrons and become $H^{+}$ions, also known as bare protons.

- Booster - protons are accelerated to $8 \mathrm{GeV}$, or $99.5 \%$ of the speed of light in this synchotron. This is a circular accelerator, with a radius of 75 meters.

- Main Injector-Brought online in 1999, it accelerates protons up to $120 \mathrm{GeV}$, or $99.997 \%$ of the speed of light

- Tevatron - The final and most powerful accelerator, it accelerates protons to $99.99995 \%$ of the speed of light, or $800 \mathrm{GeV}$, in fixed target mode. 
The Tevatron directs a proton beam into the $\mathrm{KTeV}$ beamline for 20 seconds once every minute. This 20 second long burst of beam is referred to as a "spill". Within each spill, the protons form 1 nanosecond pulses with 19 nanoseconds between each pulse. Each 1 nanosecond pulse is also known as a "bucket".

\subsubsection{Kaon Production Target}

The beam is then directed into a small piece of metal which serves as the kaon production target. For $\mathrm{KTeV}$, a $3 \mathrm{~mm} \times 3 \mathrm{~mm} \times 30 \mathrm{~cm}$ Be0 target was selected. Note that the length of $30 \mathrm{~cm}$ corresponds to roughly one proton interaction length in $\mathrm{Be} 0$, ensuring a large portion of protons produce secondary particles in the target. The proton beam is focused on the end of the rod, with the beam and the long axis of the target forming an angle of 4.8 milliradians downward. The proton beam then interacts with the Be and 0 nuclei in the target and produces a secondary beam composed of neutral kaons. At this point, each neutral kaon exists in a state which can be described as either a pure $K^{0}$ or $\bar{K}^{0}$ state.

\subsubsection{KTeV Coordinate System}

At this point, it is useful to define a coordinate system in order to describe the layout of the remainder of the $\mathrm{KTeV}$ beamline and the $\mathrm{KTeV}$ detector. We fix the origin of the coordinate system at the center of the $\mathrm{Be} 0$ production target. We define the line segment between the center of the target to the center of the detector downstream to be the $\mathrm{z}$ axis of the $\mathrm{KTeV}$ coordinate system, with the beam direction to be the $+\mathrm{z}$ direction. The vertical direction is then taken as the y-axis, with up being $+y$. The horizontal direction is then taken as the $\mathrm{x}$-axis, with $+\mathrm{x}$ being taken as left of the beam direction downstream. The coordinate system is shown along with the layout of the detector in Figure 4.2. 


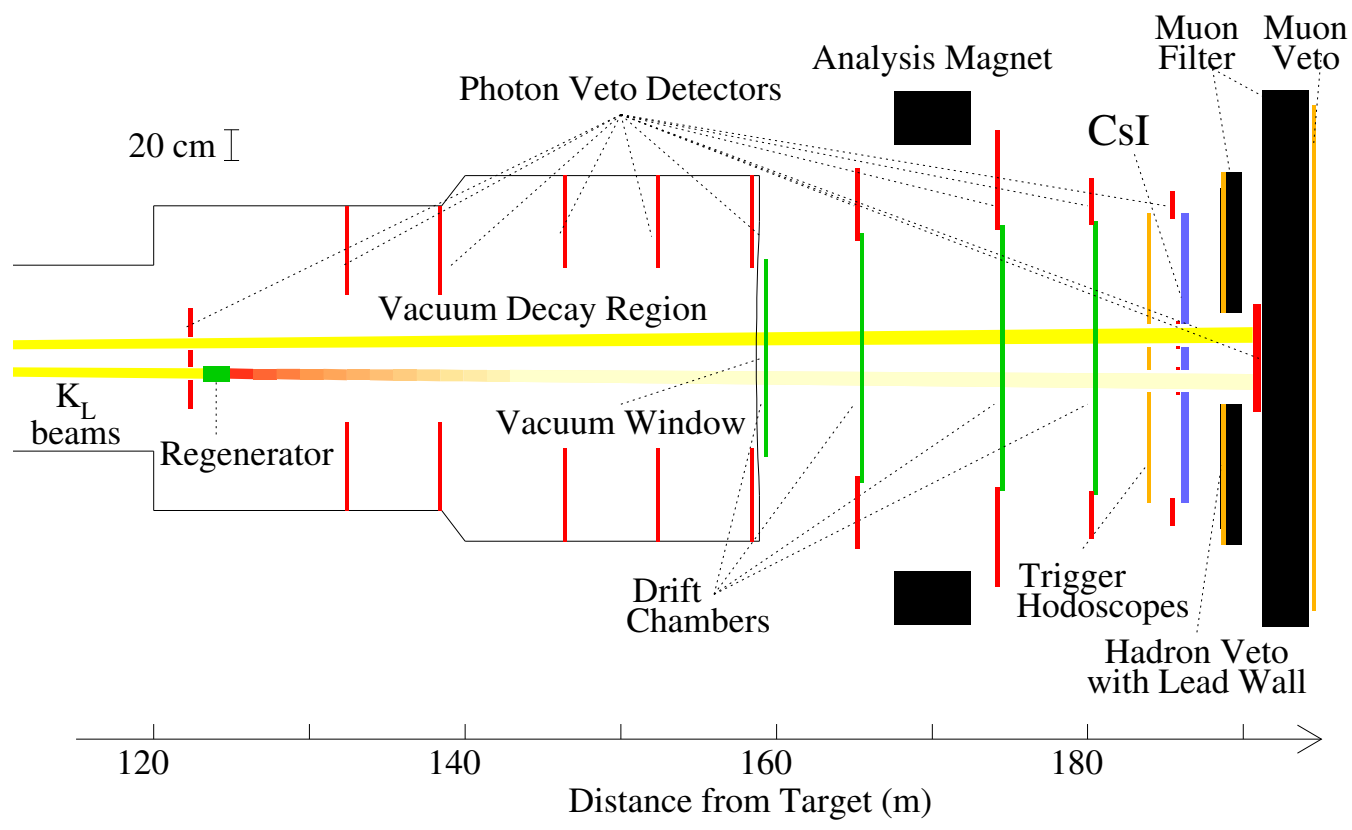

Figure 4.1: A cartoon of the $\mathrm{KTeV}$ detector in the E832 configuration. The relative positions of the major detector components are shown, including the drift chambers and CsI calorimeter. Notice the twin kaon beams entering the detector. 


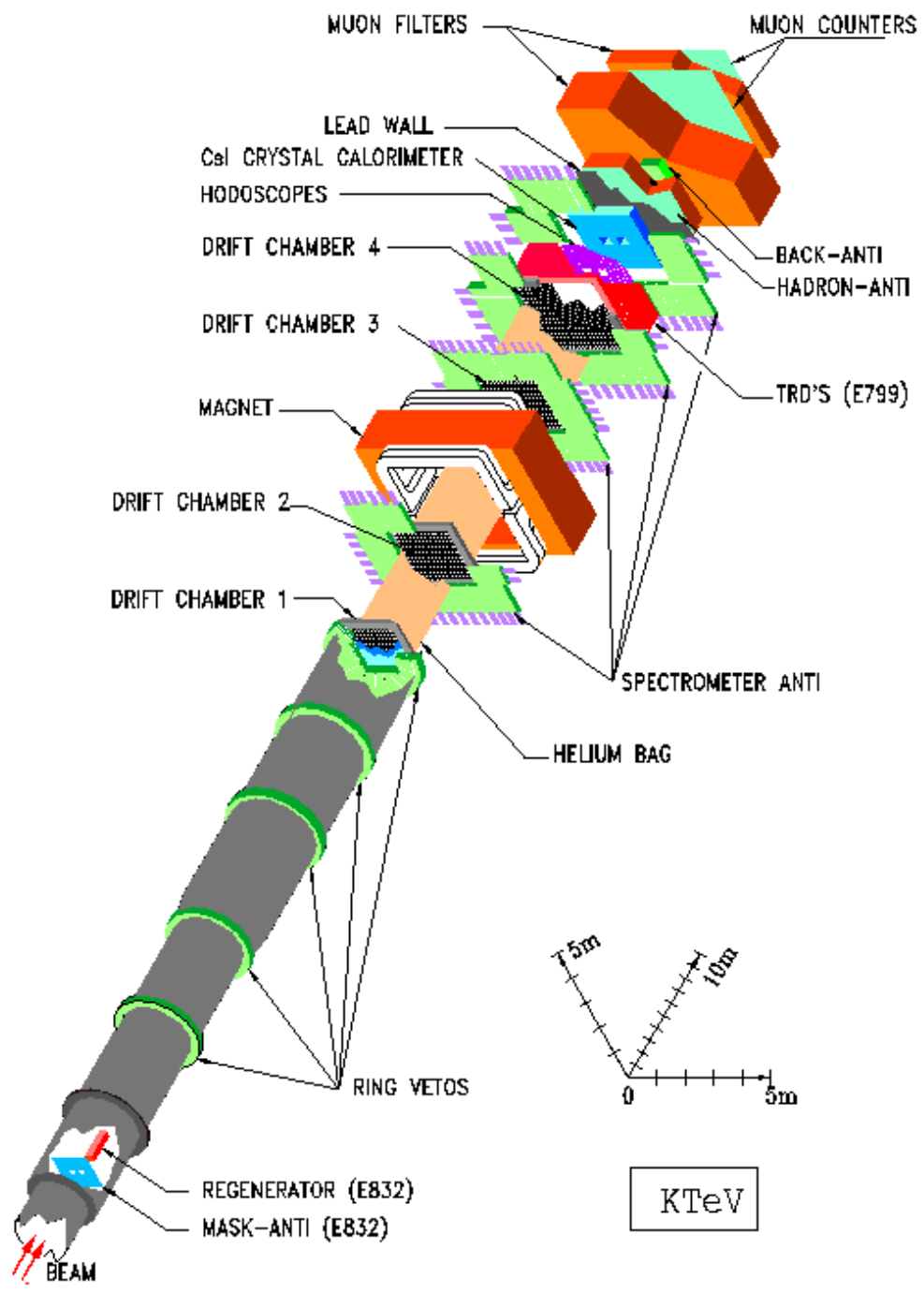

Figure 4.2: A more detailed diagram of the layout of the $\mathrm{KTeV}$ experiment in the E832 configuration. 


\subsubsection{Secondary Beamline Components}

The particle beam out of the target is known as the secondary beam, and travels the entire length of the detector. A number of sweeping magnets, collimators and filters are used to produce a high quality neutral kaon beam. The target is followed by a primary sweeping magnet which deflects the remaining proton beam into a water cooled $\mathrm{Cu}$ beam dump. Three more sweeping magnets deflect muons produced in the target, in various beam absorbers, and via particle decays close to the target.

As the neutral beam will pass through the $\mathrm{KTeV}$ detector, the neutral beam must be well defined in order to prevent radiation damage and accidental activity ${ }^{1}$ via neutrons interacting hadronically in the detector material. Additionally, two kaon beams are needed, as in the E832 configuration, a beam of pure $K_{L}$ and a mixed beam of $K_{L}$ and $K_{S}$ will be used. Various collimators are used in order to separate the secondary beam into two well defined, nearly parallel secondary beams. The first collimator is located at $\mathrm{z}=20 \mathrm{~m}$, and consists of two square holes bored through a $1.5 \mathrm{~m}$ block of brass and steel. As the secondary beam is expanding in solid angle away from the target, these holes are tapered in such a way as to represent an aperture of constant solid angle throughout its length. This helps prevent beam "scraping" at the end of the collimator. An absorber, known as the crossover absorber, is placed at $\mathrm{z}=40 \mathrm{~m}$, where it can intercept and eliminate particles which scatter from the first, or "primary" collimator, and may crossover to the other beam. Such crossover events are problematic, as each event must be assigned to a particular beam, and decays which produce all neutral daughter particles do not yield any directional informationmeaning that such crossover events can't be identified for neutral decays. The final, or defining, collimator is located at $\mathrm{z}=85 \mathrm{~m}$, and consists of a $3 \mathrm{~m}$ long block of tungsten, again with twin tapered holes to minimize scattering in either beam. Combined, the collimator system produces two twin neutral beams, which are $4.4 \times 4.4 \mathrm{~cm}^{2}$ in cross sectional area past the defining collimator, and diverge at an angle of $1.6 \mathrm{mrad}$. They are separated by $14.2 \mathrm{~cm}$ at this point as well.

\footnotetext{
${ }^{1}$ This term refers to extra particles produced in either the secondary beam, or in its interactions with various parts of the detector or beamline. These extra particles can combine with daughter particles of various kaon decays in order to form events which resemble other kaon decays. For example, a gamma ray coincident with a $K_{L, S} \rightarrow \pi^{+} \pi^{-}$decay which resemble the signal mode used in this analysis.
} 
After the sweeper magnets, the beam consists of neutral particles only- neutrons, gamma rays, neutral kaons and neutral hyperons ${ }^{2}$. Beam absorbers are used to enhance the relative kaon component of the beam. The first absorber is located at $\mathrm{z}=18 \mathrm{~m}$ and consists of $45.72 \mathrm{~cm}$ of Be. This absorber moves back and forth in the $\mathrm{x}$ direction, "shadowing" the movement of the regenerator (described in Section 4.1.6) much further downstream and as such only covers one of the two apertures of the primary collimator. Its purpose is to decrease the neutron flux in the secondary beam in order to reduce hadronic interactions in the regenerator, which can produce accidental particles which will pollute the data. This absorber is referred to as the shadow absorber due to its movement. It is closely followed by another Be absorber which intercepts the entire width of the beam, and is $50.8 \mathrm{~cm}$ thick. Both Be absorbers help decrease the relative neutron flux, as neutrons have a larger interaction cross section than kaons in beryllium. These absorbers have the secondary benefit of also preferentially removing neutral hyperons from the secondary beams. A $7.62 \mathrm{~cm}$ block of lead serves as the third beam absorber. Its purpose is to absorb gamma rays.

The sweepers, combined with the collimators and absorbers, produce two high quality kaon beams which then enter the main decay volume which begins near $\mathrm{z}=98 \mathrm{~m}$. The collimator and absorber arrangement is shown in Figure 4.3

\subsubsection{Decay Volume}

After passing through the beamline components, the twin neutral beams transverse an evacuated decay pipe held at $10^{-6}$ Torr. The vacuum eliminates the possibility that the neutral beam will interact with air molecules, which would produce accidental activity. It is in this region that acceptable, data-quality kaon decays occur, the daughter particles of which will continue downstream into the main portion of the $\mathrm{KTeV}$ detector, where their topologies may be studied. The entire evacuated volume ranges from $\mathrm{z}=28 \mathrm{~m}$ to $\mathrm{z} 159 \mathrm{~m}$, and is terminated at the downstream end by a sheet of mylar impregnated with Kevlar for strength - needed to resist the considerable vacuum pressure over this area. This mylar and Kevlar sandwich is known as the vacuum window, represents $0.14 \%$ of a radiation length and thus defines the end of

\footnotetext{
${ }^{2}$ Hyperons are particles containing three quarks, at least one of which is a strange quark. They may be roughly thought of as "strange" protons and neutrons
} 


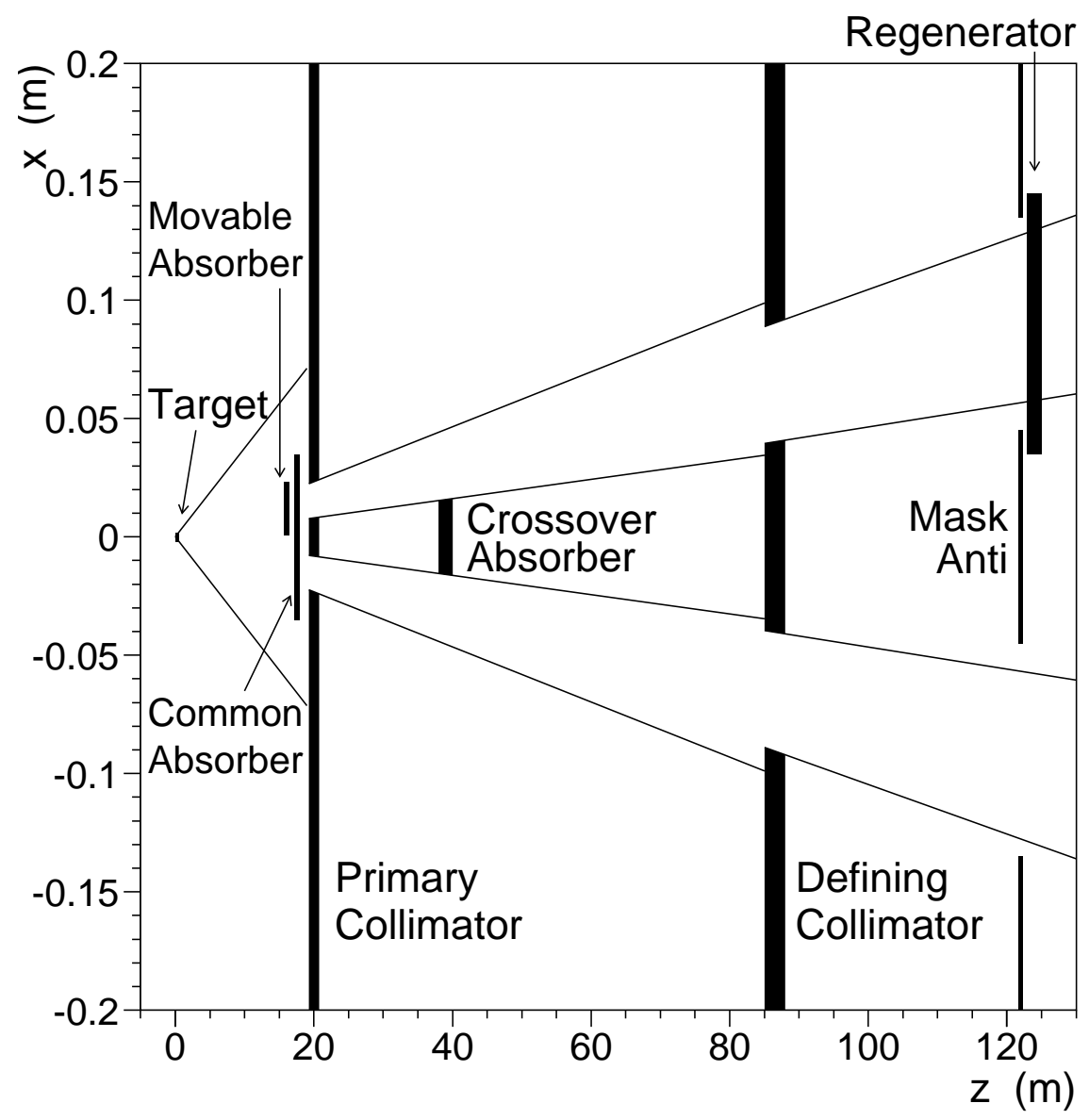

Figure 4.3: A cartoon depicting parts of the KTeV beamline in its E832 configuration.

the decay region. It should be noted that during times in which the detector was serviced, or otherwise not in use, the vacuum window was sealed with a steel cap, lest the vacuum window fail and cause a catastrophic implosion - a fate that KTeV's sister experiment at CERN, NA48/1 befell.

In addition to providing a region in which kaons may decay, the decay region contained a number of particle detectors and the active regenerator to be discussed later. 


\subsubsection{Mask-Anti Veto and Ring Counters}

The effective beginning of the decay volume is determined by the location of the Mask-Anti Veto Counter. It is composed of a sandwich of lead and scintillator, and is designed to detect photons and charged particles coming from the upstream area. When a photon or charged particle is detected, the readout can be used to reject, or "veto" the event, hence the name of the detector. It has two holes, measuring $9 \mathrm{~cm}^{2}$ in area, which allow the neutral beam to pass unmolested. The amount of lead and scintillator present 16 radiation lengths, ensuring that all photons, in addition to charged particles, are vetoed. The shape of the Mask-Anti (MA) can be seen in Figure 4.4. It is located at $\mathrm{z}=123 \mathrm{~m}$, just upstream of the active regenerator.

Serving a similar purpose, with a similar design, the Ring Counters (RC) are 5 lead-scintillator sandwiches, also 16 radiation lengths in thickness each. They are designed to detect any particles which may leave the fiducial volume of the detector. They contain a central square hole which progressively widens in successive downstream RCs. The size of this hole is selected so that any particles which go through the aperture will enter the $\mathrm{KTeV}$ spectrometer. The shape of the RCs may also be seen in Figure 4.4. The ring counters were stationed at $\mathrm{z}=132 \mathrm{~m}, 138 \mathrm{~m}, 146 \mathrm{~m}, 152 \mathrm{~m}$ and $158 \mathrm{~m}$.

The operations of the MA and $\mathrm{RC}$ detectors were identical. The lead is designed to intercept gamma rays, which will convert into a $e^{+} e^{-}$pair, which will then transverse and shower within the scintillator. The scintillator will fluoresce, and the light from this fluorescence is collected in light fibers which pipe the light into photo-multiplier tubes positioned outside the detector. The photo-multiplier tubes (PMTs) amplify the light signal, producing an analog electronic signal which is then feed into analogdigital converters (ADCs) where the signal pulse height and current can be measured out of each PMT. This information is then read out by KTeV's data acquisition system (DAQ) and/or trigger system.

\subsubsection{Regenerator}

The final component of the $\mathrm{KTeV}$ beamline is the Active Regenerator $[31,30,6]$.

The measurement of $\operatorname{Re}\left(\frac{\epsilon^{\prime}}{\epsilon}\right)$, as well as this analysis, require decays from a $K_{L}-K_{S}$ superposition in order to be successful. However, by the time the beam is well inside 


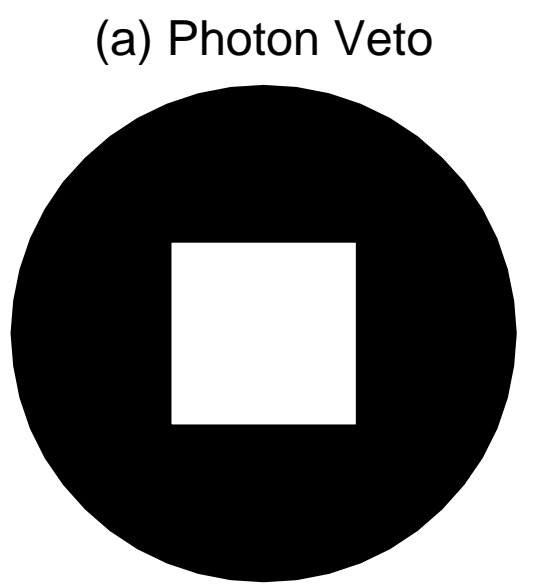

(b) Mask Anti (MA)

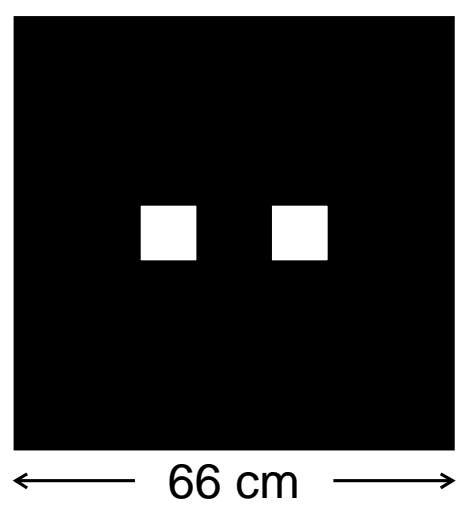

Figure 4.4: A cartoon depicting the shape of the a) Ring Counters and b) Mask-Anti veto.

the $\mathrm{KTeV}$ decay volume, most of the $K_{S}$ component from the target has decayed, and only the $K_{L}$ component remains. The solution to this problem is to exploit the properties of the $K^{0}-\bar{K}^{0}$ system in order to convert or "regenerate" $K_{S}$ from $K_{L}$ particles.

Recall from Chapter 2 that the $K_{L}$ may be written as the superposition of the strong eigenstates:

$$
\left|K_{L}\right\rangle=\frac{(1+\epsilon)\left|K^{0}\right\rangle-(1-\epsilon)\left|\bar{K}^{0}\right\rangle}{\sqrt{2\left(1+|\epsilon|^{2}\right)}}
$$

If a $K_{L}$ enters a block of material, its component $K^{0}$ and $\bar{K}^{0}$ states will see a different landscape via the strong interaction which acts between themselves and the nuclei in the material. Noting that the strong interaction conserves flavor ( i.e. it can't change one type of quark into another) any strong interaction must result in a strange particle also existing in the end. This requirement, combined with the lack of anti-quarks in regular matter, present many more possible interactions for the $\bar{K}^{0}$, for example the 
processes

$$
\begin{aligned}
& \bar{K}^{0}(\bar{d} s)+p(u u d) \rightarrow \Lambda(u d s)+\pi^{+}(u \bar{d}) \\
& \bar{K}^{0}(\bar{d} s)+n(u d d) \rightarrow \Lambda(u d s)+\pi^{0}(d \bar{d})
\end{aligned}
$$

are allowed and occur, however the analogous processes for the $K^{0}$

$$
\begin{aligned}
& K^{0}+\bar{p} \rightarrow \bar{\Lambda}+\pi^{-} \\
& K^{0}+\bar{n} \rightarrow \bar{\Lambda}+\pi^{0}
\end{aligned}
$$

are not possible except in the imaginary case in which the kaons encounter a block of antimatter. The result is that the $\bar{K}^{0}$ has a larger interaction cross-section in matter than the $K^{0}$.

Assuming that the initial kaon state was that of a pure $K_{L}$ before entering the material, and that the final amplitudes for the $K^{0}$ and $\bar{K}^{0}$ are $f$ and $\bar{f}$ respectively, we can then write the new state as

$$
\begin{aligned}
\frac{(1+\epsilon) f\left|K^{0}\right\rangle-(1-\epsilon) \bar{f}\left|\bar{K}^{0}\right\rangle}{\sqrt{2\left(1+|\epsilon|^{2}\right)}} & =\frac{(f+\bar{f})\left[(1+\epsilon)\left|K^{0}\right\rangle-(1-\epsilon)\left|\bar{K}^{0}\right\rangle\right]}{2 \sqrt{2\left(1+|\epsilon|^{2}\right)}} \\
& +\frac{(f-\bar{f})\left[(1+\epsilon)\left|K^{0}\right\rangle+(1-\epsilon)\left|\bar{K}^{0}\right\rangle\right]}{2 \sqrt{2\left(1+|\epsilon|^{2}\right)}} \\
& =\frac{f+\bar{f}}{2}\left|K_{L}\right\rangle+\frac{f-\bar{f}}{2}\left|K_{S}\right\rangle
\end{aligned}
$$

illustrating that unequal interaction cross sections result in regeneration of the $K_{S}$ state from a pure $K_{L}$ state, due to the effect of the strong interaction. Note that this process can be likened to the Stern-Gerlach experiment, where the strong interaction takes the place of a non-uniform magnetic field and the $K^{0} / \bar{K}^{0}$ states take the place of the spin-up / spin-down states.

The amplitudes $f$ and $\bar{f}$ can be computed in the case of coherent scattering, where the amount of momentum transfer between the kaons and nuclei is negligible. For this reason, we wish to produce only coherently scattered kaons for study. However, a large amount of unwanted scattering can also take place in material and is considered 
background. This unwanted scattering includes "diffractive scattering" in which a non-negligible amount of momentum transfer is imparted to the scattered kaon. The second type of unwanted scattering is inelastic scattering, where the kaon destroys an atomic nucleus. This often deposits a great amount of energy in the material, and is accompanied by secondary particle production, such as hyperons as indicated in the process shown in Equation 4.2

In order to remove the inelastic scatters from the data, $\mathrm{KTeV}$ used an object made mainly of scintillating plastic in order to regenerate $K_{S}$ from a beam of $K_{L}$. The scintillator will allow the energy deposited by inelastic scatters to be detected, and for this reason, the regnerator is an "active" one. This active regenerator is then both a part of the beamline and is also an important detector component.

The active regenerator is constructed of 84 blocks of scintillating plastic. Each block is $2 \mathrm{~cm}$ thick along the beam direction, and is $10 \mathrm{~cm}$ square transverse to the beam. Each block is instrumented with one PMT above and one PMT below in order to detect the scintillation light coming from charged decays within the regenerator as well as inelastic scattering between kaons or neutrons and the atomic nuclei in the scintillator. At the downstream end of the regenerator, two $5.6 \mathrm{~mm}$ thick lead sheets alternate with two more pieces of scintillator of $4 \mathrm{~mm}$ thickness. The purpose of this termination is to convert photons into electromagnetic showers which can then be detected in the remaining pieces of scintillator, allowing neutral decays which occur inside and upstream of the regenerator to be rejected. The total length of the regenerator was $1.7 \mathrm{~m}$, chosen in order to maximize the desirable coherent regeneration and minimize the unwanted diffractive regeneration. The downstream face of the regenerator was located at $\mathrm{z}=125.476 \mathrm{~m}$, meaning that the upstream face is just behind the MA. Note that the regenerator veto will also reject charged decays that occur within the regenerator as well. The end result is that there will be an effective edge upstream of which no events will be accepted, however this effective edge is slightly different for charged and neutral decays and is not given by the physical end of the regenerator. For charged decays, of which $K_{L, S} \rightarrow \pi^{+} \pi^{-} \gamma$ is an example, the effective edge is determined by how far two charged minimally ionizing particles can travel before they deposit an amount of energy in the last scintillator module equal to the threshold energy for veto, which is 0.7 times the energy a minimally ionizing particle would deposit into this last module. On the other hand, the effective 
edge for neutral decays will be given by the mean distance that photons can travel through the last lead sheet without converting to an $e^{+} e^{-}$pair. The effective edge also depends on the threshold of the regenerator veto, however for the 1997 data, the effective edges are $(6.2 \pm 0.1) \mathrm{mm}$ and $(1.65 \pm 0.45) \mathrm{mm}$ upstream of the downstream edge of the regenerator for the neutral and charged decays respectively. The relative positions of the effective edges, in addition to the general layout of the regenerator, can be seen in Figure 4.5. A given event which is reconstructed within the beam that

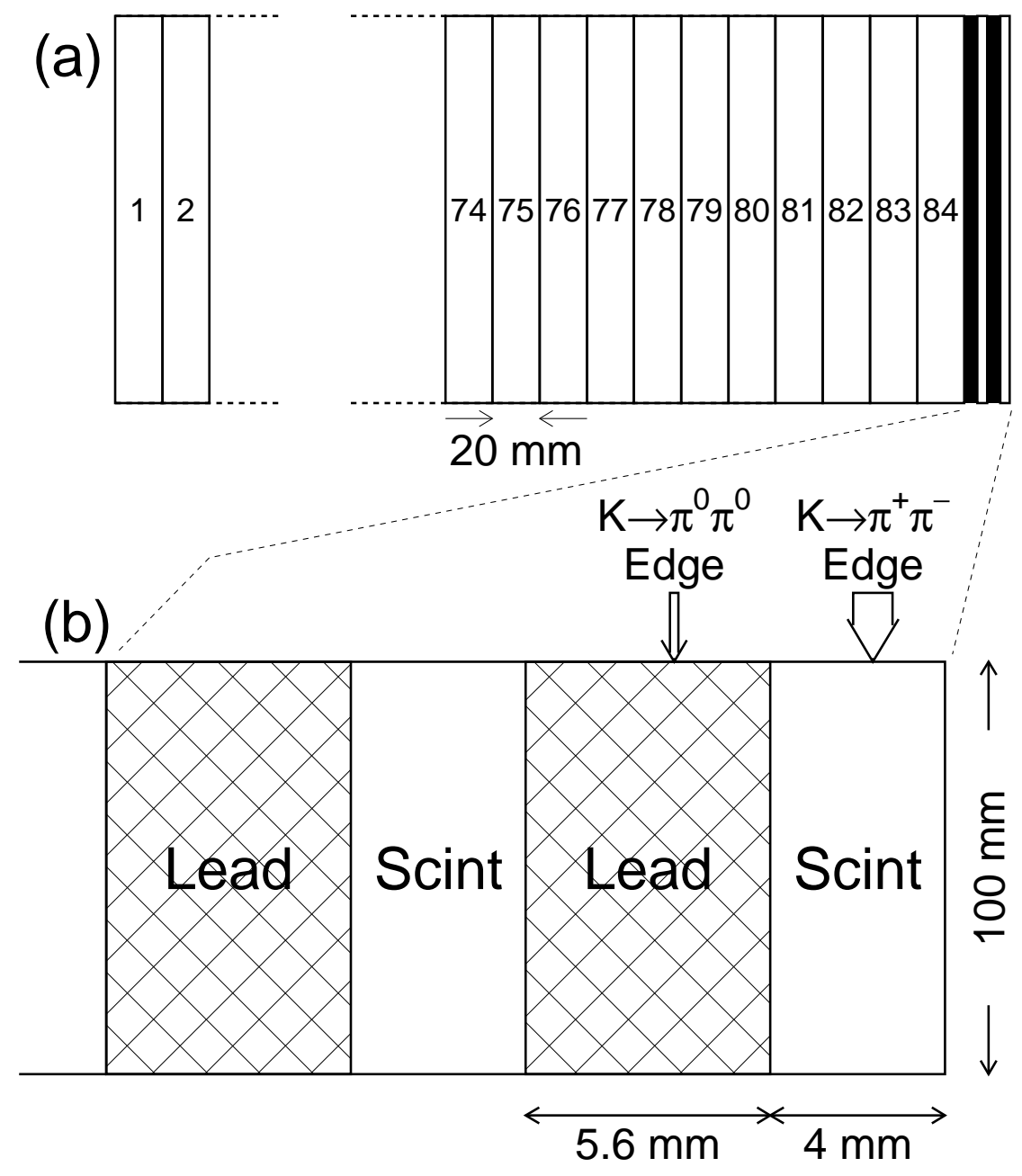

Figure 4.5: A cartoon depicting KTeV's active regenerator, with the effective edges for $\pi^{0} \pi^{0}$ and $\pi^{+} \pi^{-}$decays indicated. 
transected the regenerator is referred to as a "regenerator beam" event. Similarly, if a given event is reconstructed within the beam that did not transect the regenerator, it is known as a "vacuum" beam event. Recall that events in the regenerator beam will coming from the decay of a mixed beam of $K_{L}$ and $K_{S}$ in superposition. The form of this superposition is identical to that posited in Equation 3.29: $\left[\left|K_{L}(t)\right\rangle+\right.$ $\left.\rho\left|K_{S}(t)\right\rangle\right]$ where $\rho$ is known as the regenerator amplitude and comes completely from the characteristics of the Active Regenerator. Even though it has momentum dependence, the approximate magnitude is $|\rho| \approx 0.03$. Due to shorter lifetime of the $K_{S}$ and the correspondingly higher decay rate, this small admixture is enough for $K_{S}$ decays to dominate the regenerator beam for $\mathrm{CP}$ violating modes, like $K_{L, S} \rightarrow \pi^{+} \pi^{-} \gamma$

An additional feature of the active regenerator was the ability to alternate between the left and right beams. This ability was necessary to ensure that the any possible left-right asymmetry in the acceptance of the detector was averaged out for both the vacuum beam and regenerator beam data.

Downstream of the regenerator position, we then have twin neutral kaon beams, one which is nearly pure $K_{L}$ and another which is a coherent superposition of both $K_{L}$ and $K_{S}$. This allows us to use the interference in the regenerator beams to isolate the direct CP violating component of $K_{L, S} \rightarrow \pi^{+} \pi^{-} \gamma$, and at the same time to use the higher statistics of the vacuum beam to pin down the M1 direct emission parameters.

Downstream of the regenerator, the usable kaon decays take place. The daughter particles then travel through the vacuum window, out of the decay volume, and into the heart of the $\mathrm{KTeV}$ detector.

\section{2 $\mathrm{KTeV} / \mathrm{E} 832$ Detector}

Once a kaon has decayed, its daughter particles stream into the $\mathrm{KTeV}$ detector proper. It is here that the momenta and energies of the daughter particles are measured as well as where the identity of the particles can be ascertained. 


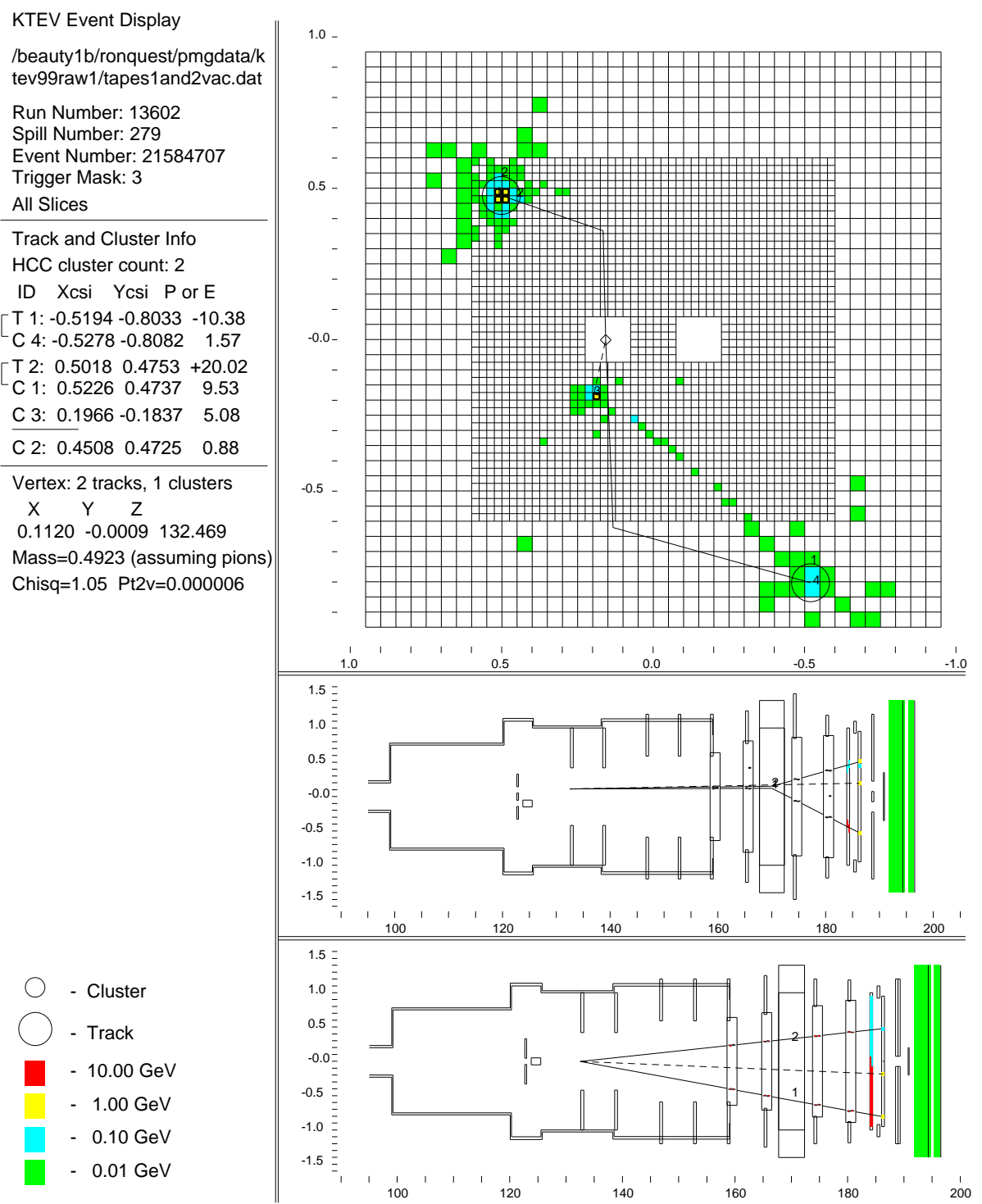

Figure 4.6: A snapshot of the $\mathrm{KTeV}$ event display, showing a candidate $K_{L, S} \rightarrow \pi^{+} \pi^{-} \gamma$ event. In this case, the event is from the 1999 run, and appears to be a decay in the vacuum beam. Note the "streamer" coming from the bottom cluster. Given the amount of energy deposited into the VV' counter - far above that for a minimum ionizing particle, it would appear that a pion has created a hadronic shower which has "splashed" onto the CsI calorimeter. There is a possibility that the candidate photon cluster in this event is not from a photon but is in fact from a particle from the pion shower. 


\subsubsection{Drift Chambers And Analysis Magnet}

For charged particles, the most important piece of the detector, as well as one of the first, is the magnetic spectrometer [6]- consisting of four drift chambers bracketing a high-current, high field electromagnet.

Each drift chamber consists of a large array of wires in a sealed volume of mixed argon/ethane gas (50\%/50\%). There are two types of wires in this array-gold-plated aluminum field wires held at ground and thus acting as cathodes, and tungsten sense wires, held at high voltage and acting as anodes. The sense wires are instrumented with readout electronics. The field wires are arrayed in a hexagonal pattern with as single sense wire at the center. This sets up a localized central electric field in each hexagon where the electric field points from the "corners" of the hexagon to the center sense wire. This arrangement can be seen in Figure 4.7, where it can be seen that the hexagonal cells are approximately $13 \mathrm{~mm}$ in width. As a charged particle traverses the gas volume, it will ionize the argon atoms along its path. The resulting ions are then accelerated away from each other by the strong electric field setup by the wires. The electron will be accelerated towards the sense wire until is acquires enough energy to ionize yet another argon atom, at which point the process begins again with two electrons, which then accelerate, etc... the result is a cascade of electrons attracted to the sense wire. This process results in an amplification of the weak primary ionization signal, to the point where the collected charge on the sense wire can be detected and measured by the readout electronics. The purpose of the ethane is to absorb photons from the cascade which would otherwise boost the effect and make the effective cascade region much larger. The average drift velocity in the gas mixture is approximately $50 \mu \mathrm{m} / \mathrm{ns}$ which means that the maximum drift time for an electron is about 150ns - in other words a maximum of $150 \mathrm{~ns}$ will pass between a charged particle barely grazing a cell and the last ion reaching the sense wire. In this case this "last ion" has traveled from the furthest edge of the cell to the center. This 150ns time window defines a smallest time slice in which drift chamber information must be collected in order to obtain all possible hits in the chamber.

In each chamber there are four layers or planes of "hexagons" - two rows in the $\mathrm{x}$ direction (horizontal) and two rows in the y-direction (vertical). The sense wires

have an offset of one half cell $(6.35 \mathrm{~mm})$ between each pair of parallel planes in order 
to remove geometric ambiguity in the track position. The two parallel planes in each chamber are referred to as "views".

Each drift chamber had a unique size, with the smallest $(1.26 m \times 12.6 m)$ being placed the furthest upstream, and the largest $(1.77 m \times 1.77 m)$ the furthest downstream in order to maintain constant solid angle in the detector sizes with respect to the target, in addition to account for the magnetic deflection of tracks in the analysis magnet. The first two are placed at $\mathrm{z}=159.4 \mathrm{~m}$ and $\mathrm{z}=165.6 \mathrm{~m}$ where they can used together to form straight tracks from the hits in both chambers. The analysis magnet, at $\mathrm{z}=170.0 \mathrm{~m}$, separates the first two DCs with the second pair at $\mathrm{z}=174.6 \mathrm{~m}$ and $\mathrm{z}=180.5 \mathrm{~m}$, which are used to form the straight tracks after the magnet. With upstream and downstream track segments, the deflection, and thus the momentum of each track can be measured.

The analysis magnet was a large dipole electro-magnet, centered at $\mathrm{z}=170 \mathrm{~m}$, which generated a 0.3 Tesla peak field, which equates to an average "pt-kick" ${ }^{3}$ of $0.4 \mathrm{GeV} / \mathrm{c}$. The magnetic field was parallel to the y-axis, meaning that the deflection was along the $\mathrm{x}$-axis. Just as the regenerator was switched between the twin beams to minimize the effect of asymmetries in the detector, the polarity of the magnet was inverted every few days to ensure that no positive/negative asymmetry could be introduced via differences in acceptance of the various parts of the detector.

To minimize the amount of multiple scattering of daughter particles, in addition to minimize the amount of interactions from the neutral beam, Helium filled bags were used to provide a "clean" path from the vacuum window to the last drift chamber.

\section{Track and Decay Vertex Reconstruction}

The magnetic spectrometer provides three important pieces of information about each event:the momentum vector of both charged particles and the location of the decay vertex, i.e. the $(\mathrm{x}, \mathrm{y}, \mathrm{z})$ coordinates of the point at which each kaon decayed.

First, the tracking software must reconstruct the individual track segments upstream and downstream of the magnet. Recall that the timing of each wire hit in the drift chambers is read out. This information gives the drift time for each hit- the

\footnotetext{
${ }^{3}$ pt-kick refers to the amount of transverse momentum the average particle acquires as a result of going through the magnet and having its momentum vector rotated due to the Lorenz force
} 


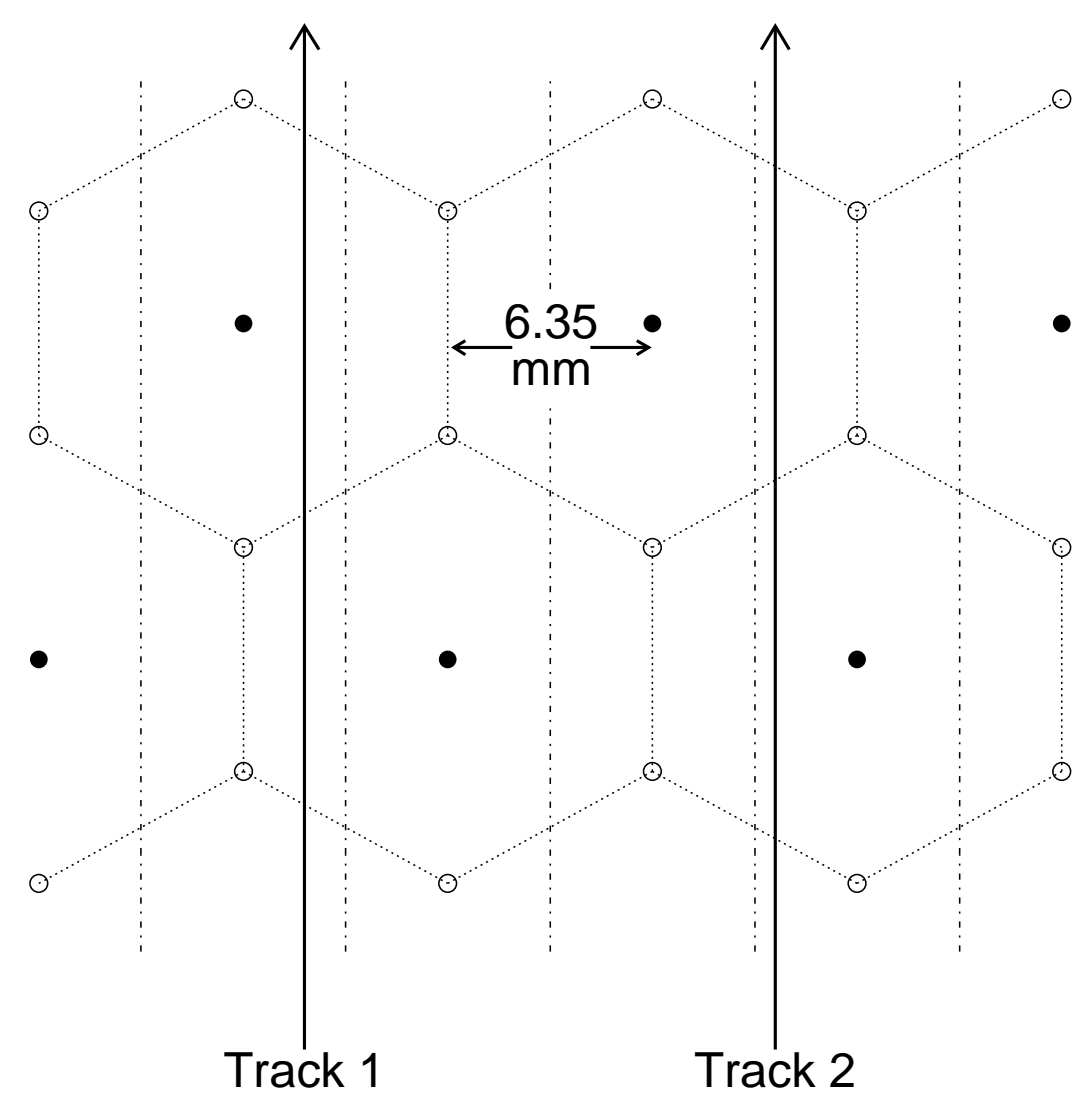

Figure 4.7: A cartoon depicting the design of the drift chambers. The solid dots denote sense wires while the open circles indicate the location of the field wires.

time elapsed between when the track crossed the drift chambers, as deduced by the RF signal sent by the Tevatron when producing beam to the experiment, and when there is an analog signal above a certain threshold for each wire. The result is a number of drift times for each active wire. A map of $x(t)$ for each chamber is then utilized which given the drift time, produces a drift distance from each wire.

"Hit pairs" are then formed between hits in adjoining, parallel planes in each chamber. This is done by computing the sum of drift (SOD) distances for each candidate hit pair, which is defined as simply the sum of the drift distances for each of the two wires. In the ideal case of tracks orthogonal to the plane of the drift 
chamber, a correct hit-pair is formed when the SOD of two hits is equal to the the cell width of $6.35 \mathrm{~mm}$, as the wires in the two planes are offset by half a cell. However, non-orthogonal tracks will result in a SOD measurement of greater than $6.35 \mathrm{~mm}$, which is illustrated in Figure 4.8. The reconstruction software accepts corrected SOD values within $1 \mathrm{~mm}$ of the ideal $6.35 \mathrm{~mm}$ in order to form hit pairs in each chamber view. The typical spatial resolution of the a hit-pair is $80 \mu m$ [7].

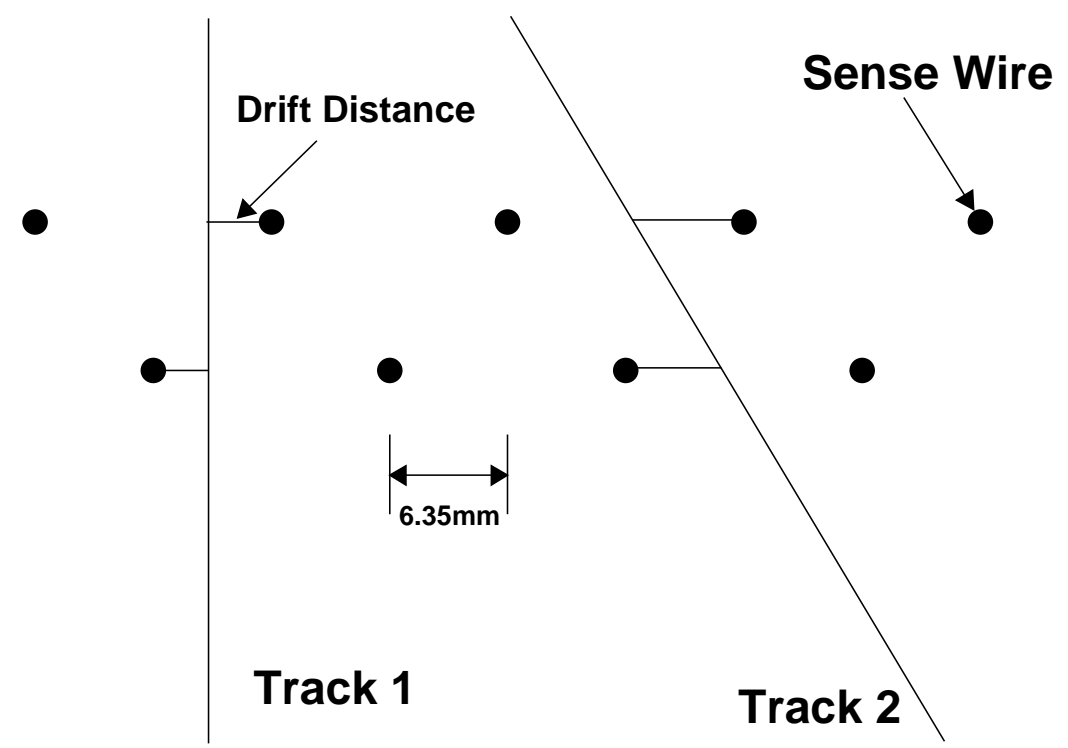

Figure 4.8: A cartoon depicting the sum of drift distance (SOD) measurement between two planes in a drift chamber. Only sense wires are shown. Note that the SOD measurement for track 2 will be greater than $6.35 \mathrm{~mm}$, illustrating that non-orthogonal tracks must be corrected.

Once $\mathrm{x}$ and $\mathrm{y}$ hit-pairs are found in each of the four drift chambers, candidate track projections in the YZ plane are formed by looking at possible y hit-pairs in DC1 and DC4. The y-tracks are found first because the tracks are not deflected in the y direction by the analysis magnet. Accordingly, the y-tracks should be straight. If hit-pairs in DC2 and DC3 fall within 5mm of the line formed by the hit-pairs in 
DC1 and DC4 and do not also fall within $5 \mathrm{~mm}$ of the other track candidate, the track trajectory is fit using all four hit-pairs. The result is two exclusive Y-track candidates if this part of the tracking is successful.

The next step is to form candidate track projections in the XZ plane, a task made more complicated by the bending of the tracks in the $\mathrm{x}$-direction by the analysis magnet. Here, track segment candidates are formed by matching hit-pairs between DC1 and DC2 and between DC3 and DC4, resulting in a number of possible upstream and downstream candidates. These candidates are then projected to the centerline of the analysis magnet at $\mathrm{z}=170 \mathrm{~m}$. If the separation between an upstream and downstream track projection is less than $6 \mathrm{~mm}$ at this point, this combination is considered as a possible $\mathrm{x}$-track candidate. If this $\mathrm{x}$-track candidate shares $\mathrm{x}$ hit-pairs with another candidate, both are rejected. The result is two exclusive $\mathrm{x}$-track candidates if this part of the tracking is successful.

The next step is to determine the decay vertex, which should appear as the common origin of both the $\mathrm{x}$ and $\mathrm{y}$ track candidates. The process is begun by projecting the $\mathrm{x}$-track candidates back to a common origin in order to obtain a $(\mathrm{x}, \mathrm{z})$ coordinate for the decay vertex. The process is repeated for the y-track candidates, obtaining a $(\mathrm{y}, \mathrm{z})$ coordinate for the decay vertex. The vertex candidate is considered acceptable if the $\mathrm{z}$ positions of the two vertex candidates are consistent within the expected vertex resolutions of both vertex candidates.

Once a decay vertex candidate is found, it must be corrected to obtain the best estimate of the actual $(\mathrm{x}, \mathrm{y}, \mathrm{z})$ position. For this to happen, the $\mathrm{X}$ and $\mathrm{Y}$ tracks must be paired together in order to form three dimensional track trajectories. As the drift chambers can only return separate $\mathrm{x}$ and $\mathrm{y}$ hits, there will be multiple track candidates, since each y hit-pair used in a y-track candidate can be paired with two other $\mathrm{x}$ hit-pairs used in the x-track candidates. Another way of thinking about this issue is to realize that each hit-pair constrains a track to have intercepted a drift chamber at a certain $\mathrm{x}$ or $\mathrm{y}$ position. Since the real track must meet two of these four requirements ( assuming 4 good hit-pairs in a chamber) the track will lie in one corner of a square given by the $\mathrm{x}$ and $\mathrm{y}$ hit-pair locations. In the ideal case, the two tracks will occupy two corners of the square. In order to remove the ambiguity, we require a correlated $\mathrm{x}-\mathrm{y}$ position of either track. This is conveniently supplied by the CsI calorimeter described later in this chapter. The CsI calorimeter is segmented, and 
a charged track impacting the calorimeter will result in a localized energy deposit, called a cluster, in the calorimeter which has a well defined $\mathrm{x}$ and $\mathrm{y}$ position.

We then project the $\mathrm{x}$ and $\mathrm{y}$ track candidates to the face of the CsI calorimeter. Any cluster lying within $7 \mathrm{~cm}$ of a track is associated to that track. If any $\mathrm{x}$ and $\mathrm{y}$ tracks match to the same cluster, they are in turn matched to each other. The result is three dimensional track candidates. The process of matching tracks to clusters also allows the CsI cluster to be used in the identification of the particle in question by looking at the reconstructed track momentum and the energy of its cluster. This process is described in more detail in Section 5.4.13.

The final step of the process is to use the three dimensional track information to obtain a number of corrections to the track information and to fit for the location of a combined three dimensional decay vertex. The full details of all these corrections can be found in [6], and include various corrections involving drift chamber orientation, the slight twisting of the chambers themselves, individual wire positions and signal propagation, angular corrections to SOD values and the fringe field from the magnet. The final result is a best estimate for the $(\mathrm{x}, \mathrm{y}, \mathrm{z})$ position of the decay vertex of an event, in addition to the momentum vectors of the charged particles.

\section{Drift Chamber Calibration and Alignment}

The first step in the calibration and alignment of the $\mathrm{KTeV}$ drift chambers is determine the $\mathrm{x}(\mathrm{t})$ maps. The operating assumption is that the track illumination of each individual cell will be effectively uniform. After a certain amount of data is collected, the TDC values of each wire are plotted, and the smallest value is assumed to represent tracks which nearly graze the wire, while the largest value is taken to be the drift time for tracks grazing the outer perimeter of the cell. The statistics of this procedure are greatly increased by summing the data for all cells within a chamber to produce a global $\mathrm{x}(\mathrm{t})$ map which is valid for every cell in a given chamber. Once this is done, a rough conversion between the drift times as measured by the TDC and the drift distance is obtained. This allows the reconstruction of tracks, and allows alignment to be done.

The alignment process begins by aligning the drift chambers to each other. Hits in DC1 and DC4 are used to align DC2 and DC3. Muons are produced in the beamline 
by reducing the current in the sweeper magnets near the target and placing a $2 \mathrm{~m}$ long steel beamstop within the beam. The beamstop absorbs kaons, neutrons and other neutral particles, but muons are able to tunnel through. The analysis magnet is also turned off, allowing straight tracks to be reconstructed in the $\mathrm{x}$ and y planes. Single tracks are selected using the trigger and muon hodoscopes described later. Hits in DC1 and DC4 are used to define a line through DC2 and DC3, then the location of hits in DC2 and DC3 relative to the intercept of the line are used to define the position and rotation of DC2 and DC3 relative to DC1 and DC4. However, rotations between DC1 and DC4 can cause problems with the process. In order to correct for this, two track events with a common decay vertex are used to define a common plane between all the drift chambers. The trajectory of the two tracks define an orthogonal plane, and the orientation of this plane can be measured in each chamber.

The next step is to align the drift chamber system with the $\mathrm{KTeV}$ coordinate system. This is done by aligning the drift chambers with the target and the CsI calorimeter. The target alignment is performed by reconstructing $K_{L, S} \rightarrow \pi^{+} \pi^{-}$decays. As this is a two-body decay, it is simple to fully reconstruct it and it is not necessary to use the calorimeter to reconstruct any particles. The reconstructed kaon momentum can then be traced back to the target. On the other hand, electrons from $K_{L} \rightarrow \pi^{ \pm} e^{\mp} \nu$ are traced to the face of the CsI and compared to the cluster associated with that track, locking the calorimeter's coordinate system to that of the drift chambers.

The final step in calibration is the treatment of the $p_{T}$ kick in the magnet. The momentum of each track is given by:

$$
p=\left|\frac{e \oint \vec{B} \cdot \overrightarrow{d l}}{\Delta \theta}\right|
$$

where $\oint \vec{B} \cdot \overrightarrow{d l}$ is the transverse momentum "kick" acquired by a particle transversing the analysis magnet, and $\Delta \theta$ was the angle between the track trajectory before and after the magnet. The value of the $p_{T}$ kick is adjusted until the resulting measured track momenta for $K_{L, S} \rightarrow \pi^{+} \pi^{-}$decays yields an average value of $M_{\pi^{+} \pi^{-}}$that is equal to the mass of the neutral kaon.

Note that the entire calibration and alignment process often uses information 
from later stages of the process. This requires an iterative procedure to be used which converges on stable calibration values. All stages of calibration were done continuously during data taking, with $K_{L} \rightarrow \pi^{ \pm} e^{\mp} \nu$ decays being collected by a dedicated trigger for CsI calibration and alignment during regular data taking, and muon runs being run after the magnet polarity was reversed which was normally every day or every other day.

After final alignment and calibration, which is described in much more detail in Reference [6], the average resolution of the magnetic spectrometer is:

$$
\frac{\sigma_{p}}{p(G e V / c))} \approx\left(1.7 \oplus \frac{p(G e V / c)}{14}\right) \times 10^{-3}
$$

while the decay vertex resolution is approximately $30 \mathrm{~cm}$ near the regenerator and $5 \mathrm{~cm}$ near the vacuum window.

\subsubsection{Spectrometer Antis}

Three veto detectors called Spectrometer Antis (SA2, SA3, SA4) together with a fourth called the CsI Anti (CIA) served to reject particles which would miss the drift chambers and/or the CsI calorimeter. Especially important are the last three elements, as particles could be swept out of the geometric acceptance of the detector by the analysis magnet. SA2 was placed just upstream of DC2, SA3 placed just upstream of RC3, SA4 was placed just upstream of DC4 and finally the CIA was placed just upstream of the face of the CsI (The last RC counter served a similar role for DC1). The construction of all four vetoes was similar to that of the RCs in the decay volume-they are lead-scintillator sandwiches read out by PMTs around their perimeter. The inner aperture of each of the SAs and the CsI subtend the same solid angle - which is slightly smaller than the face of the calorimeter and the drift chambers. This means that they will veto events grazing the edges of any of these detector elements, and thus provide the defining edge to the detector. 


\subsubsection{VV' Counter}

One of the most important scintillator based detector elements is the VV' counter, also known as the trigger hodoscope for its important part in both the charged and neutral triggers. Located at $\mathrm{z}=184 \mathrm{~m}$, it consists of two $5 \mathrm{~mm}$ layers of scintillator, each of which is separated into vertical strips as shown in Figure 4.9 The arrangement of the scintillator planks in the two planes is such that the cracks between elements never line up. This is done to prevent a particle from crossing the trigger counter without triggering it. The arrangement is also such that the beam region is left open - this is done in order to prevent hadronic interactions in the VV' counter which will then result in hadronic "spray" onto the CsI.

The VV' counter is used in analyses of charged particles to initiate the Level 1 trigger - signals in the DC are quite slow, while the PMT monitoring in the VV' is quite fast. The segmentation of the VV' is such that its readout can be used to roughly identify the topology of the decay - the analysis magnet will deflect the charged tracks along the $\mathrm{x}$ (horizontal) direction and thus each track will penetrate a separate plank, allowing the number of tracks to be counted. The vertical segmentation was also useful in the case in which in-bending tracks impacted the same plank.

For analyses of strictly neutral particles, the VV' was used to veto events with charged tracks.

\subsubsection{CsI Calorimeter}

The most advanced part of the $\mathrm{KTeV}$ detector is the Cesium Iodide (CsI) calorimeter [30]. It is a $1.9 m \times 1.9 m$ array of crystals arranged in a square pattern as shown in Figure 4.10, with larger $5 \mathrm{~cm} \times 5 \mathrm{~cm}$ crystals forming the outer perimeter of the calorimeter, and smaller $2.5 \mathrm{~cm} \times 2.5 \mathrm{~cm}$ crystals forming the center. The upstream face is located at $\mathrm{z}=186 \mathrm{~m}$. Two $15 \mathrm{~cm} \times 15 \mathrm{~cm}$ beam holes are left unfilled in order to allow the neutral beam to pass unmolested, lest the neutral hadrons interact with the edge of the crystals, thus producing radiation damage to the inner crystals and correspondingly high background rates in the calorimeter. Both the large and small crystals are $50 \mathrm{~cm}$, or 27 electromagnetic interaction lengths long. This length helped to ensure that the electromagnetic showers from electrons and photons were completely contained within the calorimeter. However, since one nuclear interaction 


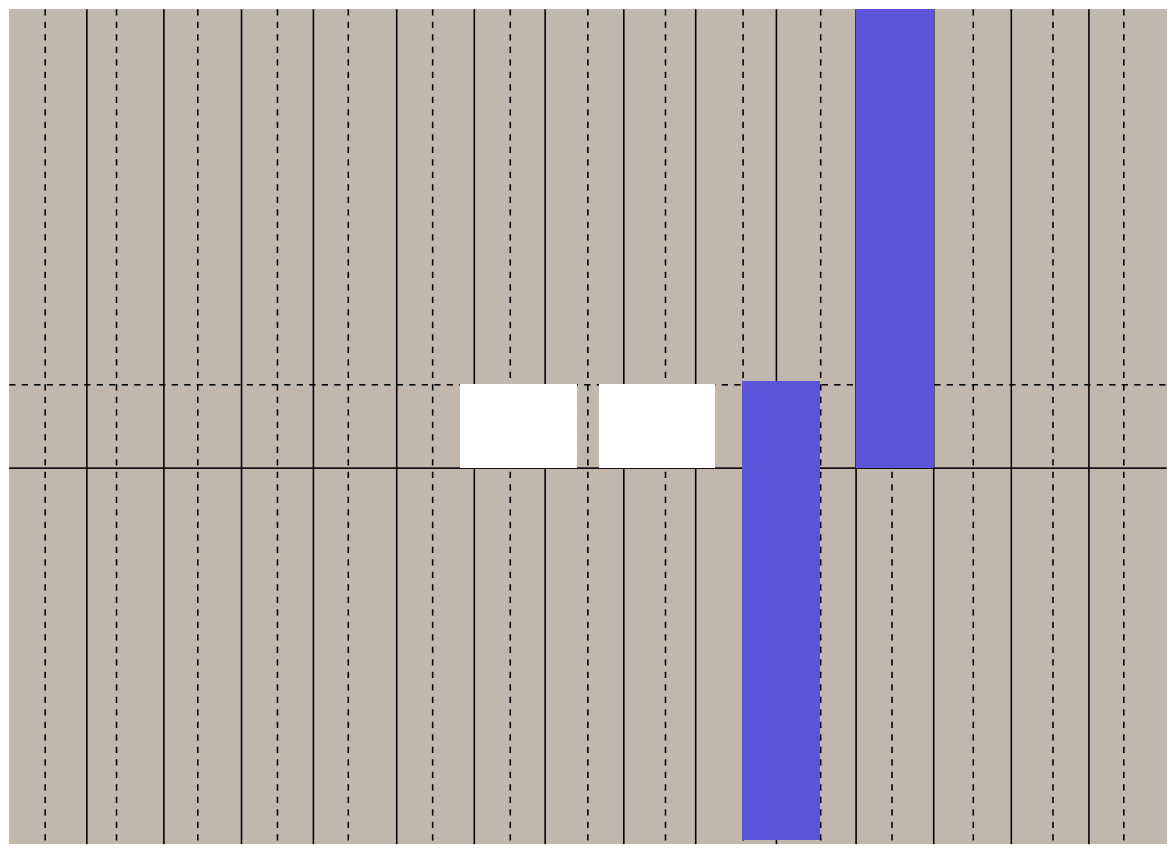

Figure 4.9: A cartoon of the VV' trigger counter. The joints between individual scintillator planks are shown - solid lines demarcate the orientation of the first plane, while dotted lines show the design of the second plane. Note that the cracks between the planes do not overlap. The beamholes are shown in the center, along with the area of two planks shown as the dark areas - one from each plane

length is approximately $37 \mathrm{~cm}$ for CsI, each crystal is only 1.35 nuclear interaction lengths long - many pions will slice through the calorimeter and only deposit a small amount of energy via ionization into the calorimeter. This, combined with efficient particle tracking will allow pions and electrons to be distinguished as detailed in Section 5.4.13.

In order to help improve the energy resolution of the CsI calorimeter, The longitudinal response of each crystal was tested using a radioactive source and reflective mylar wrapping was applied in specific places around each crystal in an effort to make the crystal response as uniform as possible as a function of the depth of the crystal, as viewed by an incoming particle in $\mathrm{KTeV}$. After wrapping, a silicon disk is then affixed to the back end of each crystal, unto which a Schott UV filter is placed. Finally, a custom built Hamamatsu PMT was mounted behind the Schott filter. The purpose 
of the silicon was to increase the amount of light which exited the CsI crystal - as CsI has a index of fraction of $n=1.80$, the critical angle for total internal reflection would be only 15 degrees if there was an air gap between the crystal and PMT lens. Using silicon, with an index of $n=3.95$, eliminates this problem. The Schott filter glass helps to remove the slow UV scintillation light which CsI produces. This is desired as the large $1 \mu s$ width of this signal would cause pile-up between activity in this part of the detector if it were not removed. The filter leaves the much faster (10ns and 36ns) components intact. The output of the PMT is fed into digitizer called a Digital PMT (DPMT) which integrated and digitized the PMT signal in 114ns windows. It also served as a data buffer for later readout into the $\mathrm{KTeV}$ datastream.

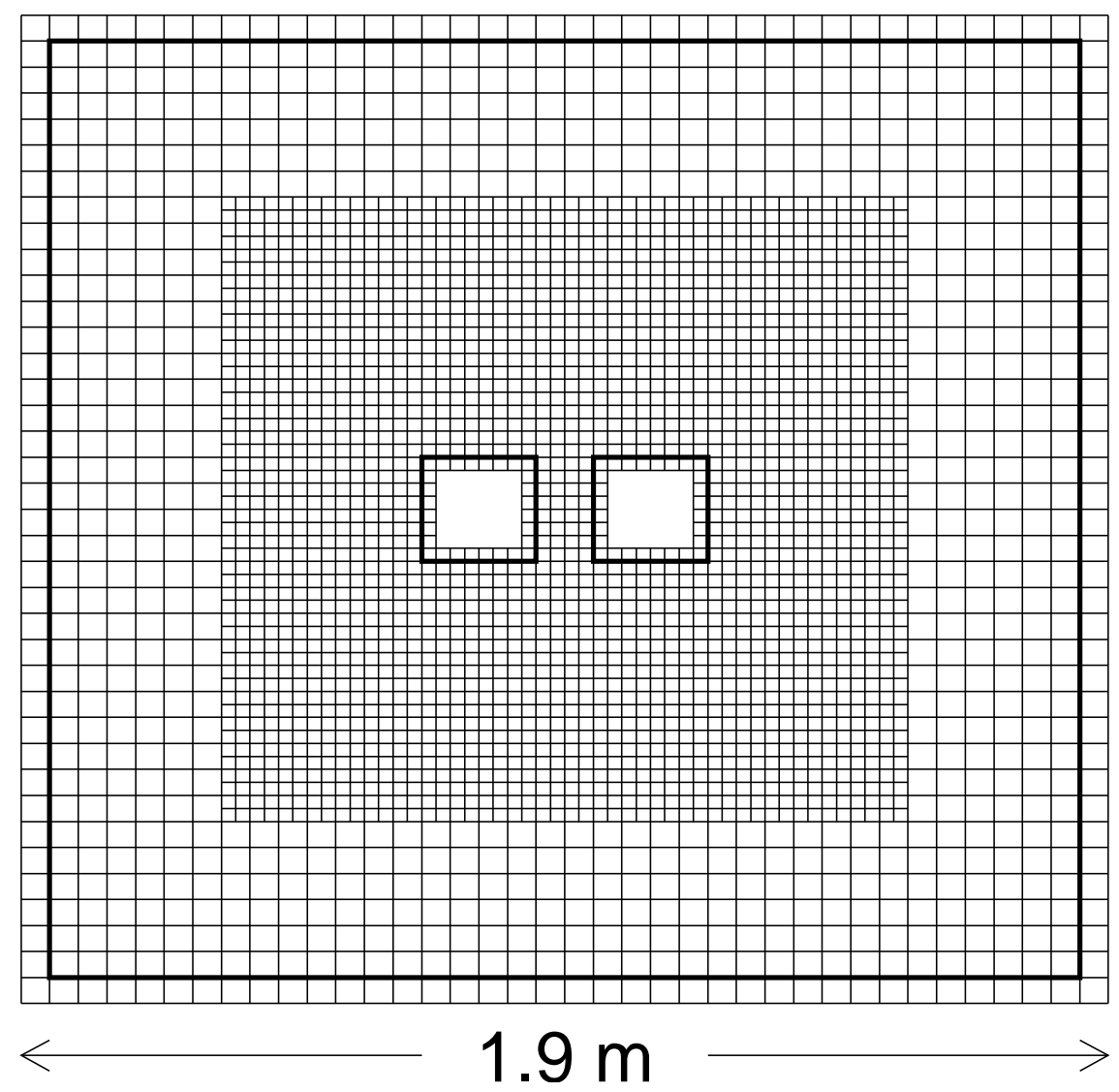

Figure 4.10: A diagram of the CsI calorimeter, showing the two beam holes and small crystals in the center, and the larger crystals in the perimeter. The crystals used in this analysis are bounded by the thick black lines. 


\section{Clustering}

Typically, when a particle interacts inside the calorimeter, the corresponding shower ${ }^{4}$ isn't contained within only a single crystal. In general electromagnetic showers are much tighter than hadronic ones. In all cases the $\mathrm{KTeV}$ reconstruction code must properly reconstruct the energy of the shower by properly finding and grouping all crystals in which the shower affects. This process is called "clustering" and a group of crystals which contains a single shower is called a CsI cluster.

KTeV's DAQ system was capable of finding clusters in the calorimeter using hardware only. These clusters are referred to as "hardware clusters" while KTeV's analysis routines, known as KTeVana, are capable of finding additional, lower energy clusters, which are referred to as "software clusters", which are used in this analysis.

The location of a cluster begins with the search for PMT channels ( each channel corresponds to a single crystal ) with a readout above the pedestal value. A "seed" crystal is defined as a crystal with a greater energy than all of its immediate neighbors. A seed crystal forms the center block of a cluster, hence the name. Once a seed crystal is found, either a $3 \times 3$ or $7 \times 7$ array of large or small crystals, respectively, is formed around the seed. This array is defined as a CsI cluster. The raw, uncorrected energy of the cluster is then taken as simply the sum of the energies in all the crystals making up the cluster.

The next step is to locate the "energy center" of the cluster, where the photon presumably interacted ${ }^{5}$ with the calorimeter. First, the crystals are grouped into rows and the energy summed in each row, producing an energy distribution as a function of the y position of the row. Then the ratios of energies in the rows are compared to those in a detailed lookup map in order to obtain the center of energy in the cluster, which the geographical center of the cluster is set equal to. The same process is used to obtain the $\mathrm{x}$ position of the cluster center, instead summing the energy in the

\footnotetext{
${ }^{4}$ The daughter particles that result from kaon decay are all relativistic, and have plenty of excess energy which can go into particle production in the event of a collision with an atom in the CsI. Pions will create quark debris within the calorimeter which will result in the production of more hadrons. Electrons and muons will create photons and electron/positron pairs. This particle production begins at the point of interaction in the calorimeter, and the particle multiplicities greatly increase from that point as newly created particles create more particles, etc. The resulting pattern looks vaguely reminiscent of a shower, hence the name.

${ }^{5}$ Photons and electrons will often penetrate some distance within the CsI calorimeter before actually initiating an electromagnetic shower.
} 
columns of the cluster. The lookup maps used in this process were obtained using a large sample of $K \rightarrow \pi^{0} \pi^{0}$ events which provided uniform photon illumination over the face of every individual crystal. The data was separated into events with different cluster energies and positions so that different maps were generated for clusters of certain energy ranges and in different parts of the calorimeter.

Once the position of the cluster has been obtained, the raw energy is corrected to obtain a best estimate of the true energy of the photon cluster. This correction takes place in many stages, and is fully described in [30]. For completeness, I outline some of these corrections here.

In the case of a photon cluster near the beam holes or the edge of the calorimeter, a geometric correction is applied where an average shower shape is utilized to estimate the amount of energy lost through the beam hole or the edge of the calorimeter, which is then added back to the total energy of the cluster. In addition, missing energy expected to be present in crystals that were not read-out due to the energy being below threshold or a malfunction in the PMT or DPMT was also estimated and added to the overall cluster energy, as was the approximate $5 \%$ of the energy expected to be deposited outside the nominal $3 \times 3$ or $7 \times 7$ cluster area.

In order to correct for differences in energy resolution as a function of position on a crystal face or along the crystal's depth, longitudinal and transverse correction were applied to the energy of each block. Due to the wrapping and testing of each individual crystal, the longitudinal corrections were small compared to the transverse corrections, which accounted for the drop in scintillation light output when the photon impacted near the edge of a crystal.

Other corrections due to long-time scale changes in the calorimeter due to temperature changes or radiation damage of the CsI crystals were also applied. Short term gain variations were corrected using the laser system described in the next section.

A linearity correction was computed separately for each seed block using electrons for $K_{L} \rightarrow \pi^{ \pm} e^{\mp} \nu$ decays, in which the momentum of the electron was known. A overlap correction was made that accounted for energy deposited in the cluster that actually originated from a neighboring cluster. A so-called "sneaky" energy correction was made to correct for "sneaky" energy which originated from electromagnetic shower products leaking across the beamhole and into blocks on the other side. This correction would come into play when two clusters existed on opposite 
sides of the beamhole. Finally, a correction was made in order to force the energy scale of $K \rightarrow \pi^{0} \pi^{0}$ to match between data and MC.

\section{Calibration}

Much like the calibration of the drift chambers, the calibration of the CsI calorimeter was a iterative process using many separate steps, and is more completely described in [30]. The process begins with the linear conversion of the DPMT readout to an energy in each block. This is done using a laser system developed and installed in order to calibrate the CsI. It consisted of a single Nb:YAG laser. Filters were installed to allow the output of the laser to be continuously adjusted in order to cover the entire range of the PMT/DPMT system. A system of beam splitters then divided the primary beam into four secondary beams, which then each traveled to a separate bowling ball ${ }^{6}$ containing dye that when illuminated, would fluoresce at a peak wavelength of $380 \mathrm{~nm}$. A series of optical fibers then carried the resulting light into the back (PMT side) of each CsI crystal. A photo-diode was also placed inside the bowling ball in order to monitor the light output to the crystals. Special "laser runs" were made in the beginning and throughout the data taking period, where the laser output was steadily increased as the output of the photo-diode was compared to the DPMT reading. This yields a measurement of the linearity of the PMT response. It should be noted that the laser system. in addition to being used to study the linearity of the PMTs, was also used to monitor the short-term changes in gain of the PMTs during data taking, by flashing the crystals regularly with a constant amplitude pulse.

The next step is to determine the absolute energy scale of calorimeter. This is done using electrons from $K_{L} \rightarrow \pi^{ \pm} e^{\mp} \nu$ decays. The momentum of the electrons can be measured using the magnetic spectrometer. Since the average kaon energy in the lab frame is approximately $50 \mathrm{GeV}$, the electrons from these decays are highly relativistic, meaning their rest mass may be safely neglected and then their energy is effectively equal to their momentum. This means that the momentum, as measured using magnetic deflection, should be equal to the energy of the electron cluster in the CsI. This allows the energy in the cluster to be related to the total charge collected by all the PMTs attached to the crystals in the blocks making up the cluster. This

\footnotetext{
${ }^{6}$ Yes, a bowling ball!
} 
yields the conversion factor between the charge collected by the DPMTs and the energy deposited into the crystals. After final calibration the average resolution of the CsI calorimeter is:

$$
\frac{\sigma_{E}}{E(G e V))} \approx\left(.004 \oplus \frac{.02}{\sqrt{E}}\right)
$$

while the average position resolution is $1.2 \mathrm{~mm}$ and $2.4 \mathrm{~mm}$ for small and large CsI blocks respectively, for electrons.

\subsubsection{Collar Anti}

There is a pair of veto detectors placed around the perimeter of the CsI calorimeter beam holes that help define the inner edge of the CsI, much as the CIA defines the outer edge of the CsI. It is placed immediately in front of the CsI, and consists of alternating layers of tungsten and scintillator, and is $1.5 \mathrm{~cm}$ wide and $8.7 \mathrm{EM}$ interaction lengths thick. Its shape and orientation relative to the CsI beamholes is shown in Figure 4.11. This veto counter was not used in this analysis.

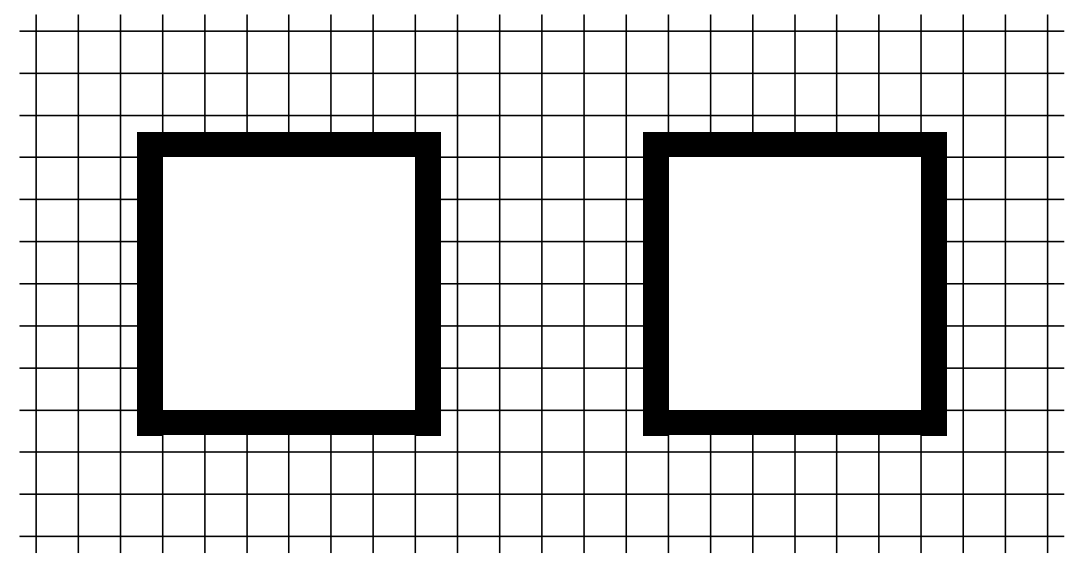

Figure 4.11: A cartoon depicting the CsI collar-anti. The veto counter's coverage area is indicated by the thick black line. 


\subsubsection{Back Anti}

Although unused in this analysis, there was a detector placed directly into the neutral beam at $\mathrm{z}=191 \mathrm{~m}$ that was designed to veto photons going through the beamholes of the CsI. This was a very demanding task, due to the hadronic showers coming from the beam neutrons and kaons. In order to succeed, it had to efficiently separate electromagnetic and hadronic showers and has to be well modeled by the Monte Carlo simulation of the detector. Sadly, this was not the case, and the BA is not used in many analyses.

The follow on to KTeV, KAMI ( Kaons at the Main Injector) needed to be completely hermetic to photons, meaning that it needed a high performance Beam Anti detector. After much hard work at UVa, the design was finalized around a segmented crystal calorimeter using $\mathrm{NaBi}\left(\mathrm{WO}_{4}\right)^{2}[32]$ crystals as a Cerenkov radiator. This design was to provide much better performance than the KTeV BA. Sadly, KAMI was canceled before the challenge of building this particular detector could be completed.

\subsubsection{Hadron Anti}

Yet another veto detector is placed behind the CsI calorimeter and a lead wall at $\mathrm{z}=189 \mathrm{~m}$ and is designed to detect and veto hadronic showers from pions. Its design is similar to the VV' counter and also includes beam holes in order to prevent hadronic beam interactions which may spill into other detector elements downstream. Although signals from this detector are not used in the trigger or analysis of this decay, the lead wall stops residual electromagnetic showers coming out of the CsI from triggering the downstream muon detectors. The lead also triggers hadronic showers which in turn helps prevent pion "punch-through" to the muon vetoes.

\subsubsection{Muon Filters and Vetoes}

The final part of the $\mathrm{KTeV}$ detector was the muon detector/veto system. While signals are required in this system in analyses involving muons, most often these large detectors were used to veto the presence of muons, and accordingly reduce the rate of the very common $K_{L} \rightarrow \pi^{ \pm} \mu^{\mp} \nu$ decays. The muon system consisted of three large, very thick steel walls designed to absorb all particles except for muons. 
The first element, MF1 (Muon Filter 1) was located at $\mathrm{z}=189 \mathrm{~m}$, right behind the HA, it simply consisted of 1 meter of solid steel, however it did contain beam holes through which the neutral beam could pass. The second element, located at $\mathrm{z}=192 \mathrm{~m}$, was another steel wall, this time $3 \mathrm{~m}$ thick, called MF2. MF2 did not contain beam holes. As such, it served as the neutral beam dump and absorbed the neutrons and kaons in the neutral beam. As could be expected, this has the potential to generate a large amount of hadronic showers, including "back-splash" which could in turn trigger the HA. The absorption of this "back-splash" is the primary role of MF1.

Behind MF2, the first muon hodoscope (MU2) is located at $\mathrm{z}=195 \mathrm{~m}$. Again, it was similar in design to the VV' counter, in that it consisted of planks of scintillator attached to PMTs for readout. The planks that made up MU2 were oriented vertically, with a joint running the width of the detector at $\mathrm{y}=0$, and with $1 \mathrm{~cm}$ of overlap between adjoining planks in order to prevent a muon from escaping detection through a crack in the scintillator. In order to account for possible wide angle multiple scattering in MF2, MU2 measured 4m wide and 3m high. MU2 was used in the Level 1 trigger to quickly veto muon events.

At $\mathrm{z}=195.5$, yet another steel wall is located, this time it is $1 \mathrm{~m}$ thick. Behind it, at $\mathrm{z}=196.5$, lies the final muon hodoscope MU3. It consists of two layers of scintillating planks. The first layer contains scintillator paddles lying in the vertical direction, while the second layer's planks lie in the horizontal direction. This, combined with division of the planks at $\mathrm{x}=0$ and $\mathrm{y}=0$ for the first and second planes respectively, allows the hit position of each muon to be located.

The combined $5 \mathrm{~m}$ of steel does an excellent job of filtering out the majority of the hadronic showers - a $20 \mathrm{GeV}$ pion only has a $0.5 \%$ probability of activating MU3 via a hadronic shower [30]. The filter steel will also absorb muons with momenta of less than $7 \mathrm{GeV} / \mathrm{c}$, which will force a cut on track momentum to be made in order to ensure that any tracks aren't in fact muons.

\subsection{Trigger Electronics}

$\mathrm{KTeV}$, like every other particle physics experiment, uses a combination of hardware and software based systems to decide whether to read out and/or record data from 
the detector. These systems are based upon many different layers of decisions logic of increasing complexity and exist in order to make the maximize the usability of the detector and the resulting data. These systems are collectively referred to as a "trigger" as they trigger the readout of the detector.

$\mathrm{KTeV}$ has a three layer trigger system. The first, most basic, layer is referred to as the Level 1 trigger, and it completely hardware based. The second layer is the Layer 2 trigger, and is also entirely hardware based. Finally, the Level 3 trigger begins to separate the data into groups which may contain certain decays, and actually writes data to tape. It is software based, and is based upon a version of the $\mathrm{KTeV}$ analysis code.

\subsubsection{Level 1 Trigger}

The Level 1 trigger used a variety of fast signals in order to determine if part of the detector should be read out. It used a variety of logic units in conjugation with signal discriminators in order to determine if certain detector components had been active or seen hits. This trigger was synchronized with the timing structure of the primary proton beam on target - as such, it ran whenever kaons could decay within the $\mathrm{KTeV}$ detector. This trigger was so fast, it was "dead-time less" - a Level 1 trigger decision was always made before the next RF bucket arrived.

There were are number of different Level 1 trigger definitions which used different logic associated with different detector components to determine if the current RF bucket had generated an interesting event. These definitions included one which summed the total amount of energy in the calorimeter, used for neutral decays such as $K \rightarrow \pi^{0} \pi^{0}$, one which looked for signals in the muon counters, used for decays resulting in muons, one which looked for six clusters in the CsI, used for $K \rightarrow \pi^{0} \pi^{0} \pi^{0}$, accidental triggers which were designed to record the random activity in the detector, and various alternative trigger definitions which ignored some trigger sources in order to collect data for systematic error studies. The trigger definition which is important for the analysis of $K_{L, S} \rightarrow \pi^{+} \pi^{-} \gamma$ is that of Trigger 1, which was also used to collect $K_{L, S} \rightarrow \pi^{+} \pi^{-}$decays for the measurement of $\operatorname{Re}\left(\frac{\epsilon^{\prime}}{\epsilon}\right)$.

Trigger 1 first looks at a subset of the veto counters to see if the event should be ignored due to the loss of particles or interactions in the regenerator. It used the 
signals from the PMTs attached to SA2,SA3,SA4, the CIA, the regenerator and the MU2 scintillator planks, to accomplish this, vetoing the event if the signals from any of these were above a certain threshold. It then looks at the PMTs connected to the VV' array. The trigger requires at least two hits in one plane of the VV' and at least one hit in the other. This is defined in this way to prevent an event from being vetoed if it simply goes through a crack between planks in the VV'. The trigger also looks at the distribution of hits on the face of the VV' counters, which were split into East/West and Up/Down regions, forming four quadrants in all. The trigger required that the hits in the $\mathrm{VV}^{\prime}$ counter satisfy a hit in two opposing quadrants of the four, i.e. that the hits be in the East/Up plus West/Down quadrants OR in the West/Up and East/Down quadrants. This is essentially a very rough cut on transverse momentum for two-body decays. The final Level 1 requirement counted the number of drift chamber hits in DC1 and DC2, and vetoed the event if more than one of the 4 views in DC1 and DC2 contains no hits. This helps to ensure that the decay occurred in the decay volume.

\subsubsection{Level 2 Trigger}

The Level 2 Trigger for Trigger 1 completed two tasks, both related to tracking. The first was the counting of hits in the drift chambers, while the second attempted to crudely reconstruct tracks in the Y-plane. The hit counting part of the Level 2 trigger, described in more detail in [33], used custom electronics and fast signals from each of the wires in each drift chamber to perform the complicated logic required at this level. The Level 2 trigger looks for neighboring hits, or hit-pairs, in the two wire layers that make up a view, which is what is needed to compute a SOD value later on. Only hits in the y-views were used in the trigger, as this view can not treat the regenerator or vacuum beams differently. A real track should have hit-pairs in each of the four views in the drift chambers, meaning that an ideal event should contain eight hit-pairs in total. In total, hit counting took about 800ns to run, introducing some dead-time into the trigger during which further activity in the detector was ignored.

The second part of the Level 2 trigger, called the y-track finder, simply looked at the topology of hits in the drift chambers in order to check to see if those hits were consistent with the behavior of real tracks - which for two-body decays such 
as $K_{L, S} \rightarrow \pi^{+} \pi^{-}$for which this part of the trigger was developed, should mean that one track reconstructs in the $+\mathrm{y}$ region(upward going track), and the other should reconstruct in the -y region (downward going track) of the drift chambers. Tracks in the central region of the drift chambers are counted as upward tracks and downward going tracks. The system, using off-the shelf logic modules and memory modules, did not use signals from individual wires, rather, it used signals from groups of 16 wires in order to operate more quickly. The result was that this component returned a decision regarding the event before the hit counter, even though this system relied upon the hit counter to sum the signals of the wires.

Upon an event passing all Level 2 requirements, the detector is read out into VME memory buffers, which can then be accessed by the the computers which run the Level 3 trigger. The memory buffers have enough space to save the data in a $20 \mathrm{~s}$ spill, leaving the Level 3 trigger a full minute to analyze the spill.

\subsubsection{Level 3 Trigger}

The Level 3 trigger was software based, and ran in parallel on 24 Silicon Graphics processors. The Level 3 software was a variant of the normal "off-line" reconstruction and analysis code known as KTeVana. It contained a number of different routines each used to perform a very rough analysis of a particular decay mode using only parts of the event information in order to process the data more quickly. Preliminary "on-line" calibrations where used, requiring loose cuts to be made. In general for the charged decay modes, only tracking information was used at first-only if the tracking appeared to indicate a desirable event was the CsI information unpacked and analyzed. This was done because the treatment of the CsI data required the greatest amount of CPU time.

For $K_{L, S} \rightarrow \pi^{+} \pi^{-} \gamma$ decays, the Level 3 trigger first required a Level 1 Trigger 1 tag. This indicates that the event was a " $K_{L, S} \rightarrow \pi^{+} \pi^{-}$like event" - no calorimeter information was used up to this point. The next step was to look for exactly two $\mathrm{x}$-tracks and two $\mathrm{y}$-tracks. Vertexing was then run in order to determine if acceptable $\mathrm{x}$ and $\mathrm{y}$ decay vertexes exist and if so, if the $\mathrm{z}$ vertexes they return are consistent with each other. At this point, the requirements used start to diverge depending if another Level 1 trigger was also satisfied by a particular event. If the Level 3 trigger 
was to read out the CsI information, which is the case for prescaled triggers which were designed to record $K_{L} \rightarrow \pi^{ \pm} e^{\mp} \nu$ decays, the CsI information was used to subject the event to further cuts, for example, to search for an extra cluster with an energy about $1 \mathrm{GeV}$. If the Level 3 trigger did not need to unpack the CsI for another decay, the tracking information is used to compute a special kinematic variable which is useful for rejecting $K_{L} \rightarrow \pi^{+} \pi^{-} \pi^{0}$ events. If an event passes the cut on this variable, then the CsI information is unpacked and the process above is carried out. Note that the cut is not applied when the CsI information is to be unpacked anyway. This fact will require special treatment later. If the Level 3 requirements are satisfied for any single decay mode defined in the trigger, the event is written out to Digital Linear Tape (DLT) and archived for future analysis.

More details regarding the cuts applied in Level 3 can be found in Section 5.3.1.

Considered together, KTeV's trigger did an excellent job of filtering the activity in the detector. The $100 \mathrm{kHz}$ rate of kaon decays, along with the $2 \mathrm{Mhz}$ rate of hadronic activity in the regenerator and $100 \mathrm{kHz}$ rate of hadronic activity in other parts of the detector, was reduced to $60 \mathrm{kHz}$ by the Level 1 trigger, was reduced further to $10 \mathrm{kHz}$ at Level 2 , and finally only $2 \mathrm{kHz}$ of events were written to disk ( for all decay modes) by the Level 3 trigger algorithm[7]. 


\section{Chapter 5}

\section{Event Selection}

The selection of $K_{L, S} \rightarrow \pi^{+} \pi^{-} \gamma$ events for analysis required multiple stages of analysis, beginning with a partial readout of the $\mathrm{KTeV}$ detector, and ending with final analysis cuts.

\subsection{Data Used}

This analysis utilizes the full 1997 and 1999 E832 data sets. For the 1997 sample, this corresponds to runs 9070-10300, for the 1999 sample, runs 13625 to 14530 were used. Data from some runs was not used however, as detailed in Table 5.1.

\begin{tabular}{|l|l|}
\hline Rejected Run & Reason \\
\hline 9884 & Analysis magnet current set to deliver $0.1 \mathrm{GeV} p_{T}$ kick \\
$9896-9909$ & Analysis magnet current set to deliver 0.1GeV $p_{T}$ kick \\
13860 & Cannot reproduce Level 3 filtering \\
14048 & Cannot reproduce Level 3 filtering \\
14308 & Missing accidental data \\
14374 & Cannot reproduce Level 3 filtering \\
14377 & Cannot reproduce Level 3 filtering \\
14383 & Cannot reproduce Level 3 filtering \\
14505 & Cannot reproduce Level 3 filtering \\
14518 & Cannot reproduce Level 3 filtering \\
\hline
\end{tabular}

Table 5.1: Runs removed from 1997 and $1999 \mathrm{KTeV}$ datasets 


\subsection{Reconstruction of Data}

In order to analyze the data, it is first necessary to unpack and reconstruct each event. This is done using a software package called KTeVana, which is a collection of software tools written in FORTRAN and used to read and analyze KTeV data. KTeVana provides the software foundation on which my own analysis code is written.

For each event, which represents an instance of reading out KTeV's various detector elements, KTeVana routines are used to do the following:

- Unpack drift chamber information from the data.

- Unpack trigger information from the data.

- Unpack ADC information from various detector elements in the data.

- Use drift chamber information to find X-projections and Y-projections of tracks.

- Use CsI calorimeter information in order to locate calorimeter clusters, and calculate various cluster parameters like energy, shape, etc.

- Use X-projection and Y-projections of tracks to locate the best decay vertex candidate - where the $\mathrm{X}$ and $\mathrm{Y}$ tracks should both intersect at the same point.

- Match tracks to clusters in the calorimeter.

- calculate other quantities like E/p for tracks, track offsets in the analysis magnet, and charged vertex $P_{T}^{2}$

- Check the Level 1 trigger information in order to verify the Level 1 trigger requirements are met by the event. If there are any inconsistencies, the event is tagged for later rejection.

- Check the readout of various veto detectors, and indicate any detectors for which the readout indicates that the amount of energy deposited is above a certain threshold. This information is used later in order to reject events.

After this process, the analysis program has access to the location of the decay vertex, the momentum and direction of each charged track, as well as other track 
information, and a list of clusters in the CsI, as well as the characteristics of the clusters.

From this information, an event can be completely reconstructed and analyzed. A "perfect" $K_{L, S} \rightarrow \pi^{+} \pi^{-} \gamma$ event would possess two charged tracks pointing to a decay vertex, three clusters in the CsI corresponding to one for each track plus an extra cluster from the photon. In reality, extra CsI clusters are quite common, often coming from hadronic interactions of the pions in the calorimeter, as well as various other sources. In this case, each cluster that is not associated with a track must be treated as a photon cluster so that all possibilities are considered.

For each combination of charged tracks plus one possible photon cluster ${ }^{1}$, the location of the CsI cluster relative to the decay vertex is used along with the cluster's energy to compute the momentum vector, in the lab frame, of the "candidate" photon. This momentum vector is then used in conjunction with the momenta of the charged tracks in order to compute:

$M_{\pi^{+} \pi^{-} \gamma}$ The invariant mass of the three particles, assuming that the tracks are pions and the cluster is due to a photon

$p_{K}$ The combined momentum of the $\pi^{+} \pi^{-} \gamma$ system in the lab frame. This should be equal to the momentum of the parent kaon

$P_{T}^{2}$ The transverse momentum of the $\pi^{+} \pi^{-} \gamma$ system in the lab frame.

among other quantities. Once the magnitude and direction of the kaon momentum are known, the momenta and energies of the three daughter particles can be computed in other reference frames, for example, in the kaon rest frame. Once all relevant quantities are calculated, the "combination" is subjected to a number of various requirements. If any of these requirements are not met, the "combination" is rejected. For this reason, the requirements are often referred to as "cuts". For each event in the data, if one and only one "combination" passes all cuts, information needed for the maximum likelihood fit is written out to a file. In the rare event that two different combinations in a single event pass all cuts, there is no way to determine which is the

\footnotetext{
${ }^{1}$ Henceforth referred to as just a "combination". Note that each event may have many possible "combinations"
} 
correct combination, and the event is rejected as a whole. This happens for roughly 1 out of every 10,000 events.

\subsection{Event Selection and Requirements}

In this selection, the complete event selection is traced from the Level 1 trigger to the final analysis stage, with exact requirements given.

Often, a requirement that was applied at a certain stage is tightened at a later stage of data filtering. All cuts which are tightened at a later stage are marked with

" $\dagger$ ". Cuts which are repeated at the same value at a later stage are marked with "^".

\subsubsection{Trigger Requirements}

The trigger for $K_{L, S} \rightarrow \pi^{+} \pi^{-} \gamma$ events was composed of three parts:

Level One (L1) uses information from a few specific detector elements, and was hardware based. If an event passes these requirements, the detector is read out and the event information is written to disk.

Level Two (L2) applied further tracking cuts, and was also hardware based

Level Three (L3) applied further cuts and applied decay mode specific tags that could be used later to split off the data that was useful for the mode in question. This was a software based trigger

\section{Level One Trigger Requirements}

Here are the Level One requirements:

VV' Hit Number There must be two or more hits in one of the two VV' scintillator banks, and one more hits in the other VV' scintillator bank

VV' Hit Pattern The hits in the VV' banks must be such that there is approximate symmetry in both the $\mathrm{X}$ and $\mathrm{Y}$ directions i.e. a hit in the top left must be accompanied by a hit in the lower right, etc. 
Veto Counters The Ring Counters (RC), CsI-Anti (CIA), Mask-Anti (MA), first Muon (MU2) and Regenerator vetoes must be free of activity

Drift Chamber Hits in $\mathbf{X}$ and $\mathrm{Y}$ There must be at least $3 \mathrm{X}$ or $\mathrm{Y}$ hits (out of 4 possible) in the first two drift chambers

The Level 1 Trigger requirements yield a sample in which there appears to be two nonmuon charged particles in each event, no extra particles escaping the detector, and the decay occurs before the first drift chamber. The requirement of quasi-symmetric hits in the VV' banks would nearly always be met by clean $K_{L, S} \rightarrow \pi^{+} \pi^{-}$events, since transverse momentum for these events must be zero. However, this requirement can remove some $K_{L, S} \rightarrow \pi^{+} \pi^{-} \gamma$ events, since the charged tracks will not have zero transverse momentum, due to the momentum carried by the photon.

The above definition is for KTeV's "Trigger 1". KTeV's "Trigger 2" was quite similar, except that it was prescaled ${ }^{2}$. As a consequence, all Trigger 2 events also pass Trigger 1, although the converse is of course not true.

\section{Level Two Trigger Requirements}

Here are the Level Two requirements:

2-Track Y-Hit Counter There must be at least two hits each in three of the four Y-view drift chamber planes, and at least one hit in the remaining Y-view drift chamber plane.

Y-Track Counter There must be one upward going and one downward going track found in the Y-view, with tracks in the central portion of the drift chambers being counted as both upward and downward going. One of tracks must be a good track which is composed of hits in each and every drift chamber and the other may be a marginal track in which a hit is missing in either DC1 or DC2. The upward going and downward going track definitions depend only on the $\mathrm{Y}$ coordinate- tracks with positive $\mathrm{Y}$ intercepts are considered to be upward going, and tracks with negative $\mathrm{Y}$ intercepts are considered as downward going.

\footnotetext{
${ }^{2} \mathrm{~A}$ prescale means that only a certain fraction of events are selected.
} 
The Level 2 Trigger requirements yield a sample in which there are two charged track candidates reconstructed in the drift chambers for every event. Only Y-view information is used so that only wires common to the regenerator and vacuum beams are used. This prevents any bias from being introduced between the two beams.

\section{Level Three Trigger Requirements}

The Level 3 Trigger, unlike the previous trigger levels, was a software trigger. The software in question was based on KTeVana. It imposed further requirements on each event, and tagged the event as a certain type if these requirements were met. In this analysis, data is selected which has the $K_{L, S} \rightarrow \pi^{+} \pi^{-} \gamma$ Level 3 tag. The requirements for an event to be labeled as $K_{L, S} \rightarrow \pi^{+} \pi^{-} \gamma$ are:

Tracks $\star$ There must be exactly two X-tracks and two Y-tracks found.

Decay Vertex $\star$ The range of permissible $\mathrm{z}$ locations of at least one decay vertex found using $\mathrm{X}$-tracks must overlap with the $\mathrm{z}$ range of at least one decay vertex found using Y-tracks. This ensures that there is an acceptable 3-dimensional vertex candidate.

Level $3 P_{\pi^{0}}^{2}$ cut The value of a kinematic variable called $P_{\pi^{0}}^{2}$ was used to distinguish $K_{L, S} \rightarrow \pi^{+} \pi^{-} \gamma$ events from $K_{L} \rightarrow \pi^{+} \pi^{-} \pi^{0}$ events. $P_{\pi^{0}}^{2}$ took a positive value on average for the latter, and a negative value for the former. The Level 3 requirement was $-0.03<P_{\pi^{0}}^{2}<-0.002$. The tracking information used in this calculation is different than that used in the final analysis stage, so this cut must be treated carefully - it is effectively a different cut than the analysis level $P_{\pi^{0}}^{2}$ cut, so this exact cut is not repeated later in the analysis.

Track Cluster Match $\star$ Each downstream portion of a track must point to within $7 \mathrm{~cm}$ of a cluster in the calorimeter.

Track $\mathrm{E} / \mathrm{p} \dagger$ The ratio of $\mathrm{E} / \mathrm{p}$ for each track, where $\mathrm{E}$ is measured using the energy of each track's cluster, and $\mathrm{p}$ is measured using the deflection of each charged track in the magnetic spectrometer, is required to be $0.00<E / p<0.90$. This rejects electron tracks. 
Extra Cluster Energy $\star$ There must be at least one extra cluster, beyond those associated with the charged tracks, with an energy greater than $1.0 \mathrm{GeV}$.

Invariant Mass The reconstructed value of the invariant mass of the $\pi^{+} \pi^{-} \gamma$ system must be greater than $0.450 \mathrm{GeV} / \mathrm{c}^{2}$. The tracking information used in this calculation is different than that used in the final analysis stage, so this cut must be treated carefully - it is essentially a different cut that the analysis level $M_{\pi^{+} \pi^{-} \gamma}$ cut, so this exact cut is not repeated later in the analysis.

It should be noted that at the beginning of the 1997 run ( before run 9070 ) only $1 / 8$ of the $K_{L, S} \rightarrow \pi^{+} \pi^{-} \gamma$ tags were allowed to actually pass. This was done in order to reduce the large amount of events from this particular tag, which were due mostly to $K_{L} \rightarrow \pi^{+} \pi^{-} \pi^{0}$. However, after run 9070 the Level $3 P_{\pi^{0}}^{2}$ cut was applied. This decreased the $K_{L} \rightarrow \pi^{+} \pi^{-} \pi^{0}$ background, decreased the overall event rate for this trigger, and allowed the "pre-scale" to be removed. The net effect of this trigger change was that more $K_{L} \rightarrow \pi^{+} \pi^{-} \gamma$ events were collected. Additionally, during the 1999 run, tracking requirements were modified after run 13704.

\section{Rerun of Level Three Trigger}

The Level $3 P_{\pi^{0}}^{2}$ cut was added to the filter code shortly after the beginning of KTeV's 1997 E832 data taking period. It's purpose was to select candidate $K_{L, S} \rightarrow \pi^{+} \pi^{-} \gamma$ events without undertaking the CPU intensive task of reading the calorimeter information [34]. If a particular event did in fact pass the $P_{\pi^{0}}^{2}$ cut, only then would the calorimeter information be referenced in order to search for a third cluster. Since the $P_{\pi^{0}}^{2}$ cut was used to avoid referencing calorimeter information, when the CsI calorimeter information was actually read in, as was the case for the events which also passed Trigger $2,{ }^{3}$ the $P_{\pi^{0}}^{2}$ cut was not applied. The CsI information was also used for all regenerator events. Thus the $P_{\pi^{0}}^{2}$ cut was not applied to regenerator events. The lack of the cut in a subset of the data is advantageous as the effect of the Level 3 $P_{\pi^{0}}^{2}$ can be studied using data without the cut. The disadvantage is that the entire $K_{L, S} \rightarrow \pi^{+} \pi^{-} \gamma$ sample does not have consistent cuts. The proportion of the events

\footnotetext{
${ }^{3}$ Trigger 2 was a prescaled version of Trigger 1 with the same Level 1 and Level 2 trigger requirements. The difference is that the CsI information is always unpacked at Level 3, which isn't always true for Trigger 1 .
} 
without the $P_{\pi^{0}}^{2}$ cut ranged from $1 / 9$ of the total for the 1999 vacuum beam sample, to $1 / 7$ for the 1997 vacuum beam sample, to all events for the regenerator beam. The proportions originate from the prescale of Trigger 2 .

The solution to this cut inconsistency was to rerun the Level 3 trigger on all the $K_{L, S} \rightarrow \pi^{+} \pi^{-} \gamma$ candidate events, and tag all events which pass the Level $3 P_{\pi^{0}}^{2}$ cut. The presence of this additional tag will then be required in order for a particular event to contribute to the final data sample.

The Level 3 trigger was rerun as part of the data crunch. This trigger rerun utilized a specialized version of the Level 3 trigger code which subjected every event to the $P_{\pi^{0}}^{2}$ cut, and issued a special tag to every event which passed this cut.

\subsubsection{Crunch Requirements}

For both data sets, a "crunch" was run on the data where additional, loose requirements were used to select a purer sample of $K_{L, S} \rightarrow \pi^{+} \pi^{-} \gamma$ events, while trying to remove background events. Data was read from tapes at Fermilab which were the output of the Level 3 trigger during data taking. A preliminary set of cuts were made on the data, then the Level 3 trigger was rerun was detailed above. Finally, an additional set of requirements was made to further reduce the background, the data was output into a compressed data format where clustering and tracking information was recorded, and the data files were then transferred to UVa. for final analysis.

\section{Crunch of 1997 Data}

The preliminary crunch cuts for the 1997 data were:

Level 1 Trigger Tag The Level 1 trigger tag for Trigger $1\left(\eta_{+-}\right.$trigger) was required to be present.

Level 3 Trigger Tag The Level 3 tag for $K_{L, S} \rightarrow \pi^{+} \pi^{-} \gamma$ was required to be present.

Data periods * "Good" spills were required, as well as pt-kicks above $0.1 \mathrm{GeV}^{4}$

\footnotetext{
${ }^{4}$ The nominal setting of the analysis magnet was to set a transverse momentum kick of $0.4 \mathrm{GeV}$
} 
General Event Reconstruction $\star$ No errors in reading the event information, finding charged tracks in the drift chambers,finding a charged track decay vertex, or reading the veto detectors were allowed.

Clusters $\star 3$ CsI calorimeter clusters were required to be present.

Regenerator Veto $\star$ The amount of energy deposited in the regenerator was required to be less than that of 2 MIPS ${ }^{5}$

Downstream regenerator veto $\star$ The amount of energy deposited in the downstream end of the regenerator - that part downstream of the last lead section, was required to be less than 0.7 MIPS.

Ring Counter $\star$ The Ring counter Level 1 trigger readout was required to be quiet.

Mask-Anti veto $\star$ The amount of energy deposited in the Mask-Anti veto counter was required to be less than $0.1 \mathrm{GeV}$.

$P_{\pi^{0}}^{2}$ Cut If the event is not an overlap with trigger 2 nor a regenerator event (i.e. if the event received the Level $3 P_{\pi^{0}}^{2}$ cut ) the event must have a $P_{\pi^{0}}^{2}$ value less than $-0.002 \mathrm{GeV}^{2} / \mathrm{c}^{2}$. where $P_{\pi^{0}}^{2}$ is computed using the $P_{T}^{2}$ value of the charged tracks only as measured from the target.

Decay vertex $\dagger$ The $\mathrm{z}$ position of the decay vertex was required to be $100.0 \mathrm{~m}<$ $z<159.99 m$.

$\mathbf{E} / \mathbf{p}$ value $\dagger$ The $\mathrm{E} / \mathrm{p}$ value of each of the two charged tracks was required to be less than 0.90 .

$\pi$ - Collar Anti Separation The distance between each track's intercept point on the face of the CsI calorimeter and the Collar Anti Veto counter was required to be greater than 0.0

\footnotetext{
${ }^{5}$ The definition of MIPS is Minimum Ionizing Particle, and refers to the minimum amount of energy that can be deposited in material by a charged particle. The actual energy deposited by a charged particle is dependent on the particle's energy, and receives many contributions from various physical processes. However, there is a energy at which a particle deposits a minimum amount of energy, and the amount of energy deposited is one MIP. In this case, it is only important to ensure that two charged particles have not passed through the regenerator, as would happen from a decay upstream of the regenerator.
} 
Track momentum $\dagger$ The momentum of each track, determined from magnetic deflection, was required to be greater than $7.0 \mathrm{GeV} / \mathrm{c}$

Photon Cluster Energy $\left(E_{\gamma}^{L A B}\right) \dagger$ The energy of the photon cluster was required to be greater than $0.900 \mathrm{GeV}$.

Photon Cluster Shape $\nmid$ The value of $\chi_{\text {FUSION }}^{2}$ for the candidate photon cluster must be less than 1000 .

$\pi-\gamma$ separation $\dagger$ The separation between each track's intercept point at the face of the CsI calorimeter and the candidate photon cluster must be greater than $0.18 \mathrm{~m}$.

$\pi^{+} \pi^{-} \gamma$ energy $\dagger$ The energy of the $\pi^{+} \pi^{-} \gamma$ system in the lab frame must be $10.0<$ $E_{\pi^{+} \pi^{-} \gamma}<180.0 \mathrm{GeV}$.

$\pi^{+} \pi^{-} \gamma$ invariant mass $\dagger$ The invariant mass of the $\pi^{+} \pi^{-} \gamma$ system must be $0.460 \mathrm{GeV} / \mathrm{c}^{2}<M_{\pi^{+} \pi^{-} \gamma}<0.540 \mathrm{GeV} / \mathrm{c}^{2}$.

$\pi^{+} \pi^{-} \gamma P_{T}^{2} \dagger$ The transverse momentum squared of the $\pi^{+} \pi^{-} \gamma$ system relative to the target must be $P_{T}^{2}<0.005 \mathrm{GeV}^{2} / \mathrm{c}^{2}$.

The additional set of crunch cuts, used immediately prior to the output of the data in compressed format, were:

Regenerator present $\star$ Runs in which the regenerator was swung out of the beam were discarded

General Event Reconstruction $\star$ No errors in reading the event information, finding charged tracks in the drift chambers,finding a charged track decay vertex, or reading the veto detectors were allowed.

Level 1 trigger $\star$ The event must pass a verification of the Level 1 trigger

Mask Anti Fiducial Cut $\star$ The charged tracks must pass $>0.003 m$ from the edge of the holes in the Mask Anti. This requires the tracks to pass within the beam holes in the MA. 
$V V^{\prime}$ Fiducial Cut $\star$ The charged tracks must pass $>0.002 m$ from the edge of the holes in the $V V^{\prime}$. This requires the tracks to pass outside the beam holes in the $V V^{\prime}$ counter. counters.

Collar Anti Fiducial Cut $\star$ The charged tracks must pass $>0.002 m$ from the edge of the Collar Anti counters. This requires that the tracks pass outside the outer edge of the CA.

CsI Calorimeter Fiducial Cut $\star$ The charged tracks must pass $>0.029 m$ from the outside edge of the CsI calorimeter. This requires the tracks to pass inside the outer edge of the CsI.

Cell Separation Cut $\star$ The charged tracks must have a minimum separation greater than 3 wire-centered cells in all drift chambers.

Number of Candidate photon clusters $\dagger$ At least one candidate photon cluster must pass all other cuts.

$\pi-\gamma$ separation $\dagger$ The separation between each track's intercept point at the face of the CsI calorimeter and the candidate photon cluster must be greater than $0.20 \mathrm{~m}$.

Photon Cluster Shape $\nmid$ The value of $\chi_{F U S I O N}^{2}$ for the candidate photon cluster must be less than 100 .

Dipion invariant mass The invariant mass of the two pions must be $M_{\pi^{+} \pi^{-}}<$ 0.492 .

Photon Cluster Energy $\left(E_{\gamma}^{L A B}\right) \star$ The energy of the photon cluster was required to be $1.0 \mathrm{GeV}<E_{\gamma}^{L A B}<180 \mathrm{GeV}$.

\section{Crunch of 1999 Data}

Compared to the 1997 crunch, the requirements applied to the 1999 data were quite loose. This was done in order to retain $K_{L, S} \rightarrow \pi^{+} \pi^{-}$decays, which are useful for possible normalization studies. The preliminary set of crunch cuts for the $1999 \mathrm{crunch}$ were: 
Level 1 Trigger Tag The Level 1 trigger tag for Trigger 1 ( $\eta_{+-}$trigger) was required to be present.

Level 3 Trigger Tag The Level 3 tag for either $K_{L, S} \rightarrow \pi^{+} \pi^{-} \gamma$ or $K_{L, S} \rightarrow \pi^{+} \pi^{-}$ was required to be present.

Data periods * "Good" spills were required, as well as pt-kicks above $0.1 \mathrm{GeV}$.

General Event Reconstruction $\star$ No errors in reading the event information, finding charged tracks in the drift chambers, finding a charged track decay vertex, or reading the veto detectors were allowed.

Regenerator Veto $\star$ The amount of energy deposited in the regenerator was required to be less than that of 1.75 MIPS.

Downstream regenerator veto $\star$ The amount of energy deposited in the downstream end of the regenerator - that part downstream of the last lead section, was required to be less than 0.7 MIPS.

Ring Counter $\star$ The Ring counter Level 1 trigger readout was required to be quiet.

Mask-Anti veto $\star$ The MA Level 1 trigger readout was required to be quiet.

$P_{\pi^{0}}^{2}$ Cut If the event is not an overlap with trigger 2 nor a regenerator event (i.e. if the event received the Level $3 P_{\pi^{0}}^{2}$ cut ) the event must have a $P_{\pi^{0}}^{2}$ value less than $-0.002 G e V^{2} / c^{2}$. where $P_{\pi^{0}}^{2}$ is computed using the $P_{T}^{2}$ value of the charged tracks only as measured from the target.

Decay vertex $\dagger$ The $\mathrm{z}$ position of the decay vertex was required to be $100.0 \mathrm{~m}<$ $z<159.99 m$.

$\mathrm{E} / \mathrm{p}$ value $\dagger$ The $\mathrm{E} / \mathrm{p}$ value of each of the two charged tracks was required to be less than 0.90 .

$\pi$ - Collar Anti Separation The distance between each track's intercept point on the face of the CsI calorimeter and the Collar Anti Veto counter was required to be greater than 0.0 
Track momentum $\dagger$ The momentum of each track, determined from magnetic deflection, was required to be greater than $7.0 \mathrm{GeV} / \mathrm{c}$

Note that the $P_{\pi^{0}}^{2}$ cut will not remove any $K_{L, S} \rightarrow \pi^{+} \pi^{-}$events.

The additional set of crunch cuts, used prior the output of the data in compressed format were:

Regenerator present $\star$ Runs in which the regenerator was swung out of the beam were discarded

General Event Reconstruction $\star$ No errors in reading the event information, finding charged tracks in the drift chambers,finding a charged track decay vertex, or reading the veto detectors were allowed.

Level 1 trigger $\star$ The event must pass a verification of the Level 1 trigger

Mask Anti Fiducial Cut $\star$ The charged tracks must pass $>0.003 m$ from the edge of the holes in the Mask Anti. This requires the tracks to pass within the beam holes in the MA.

$V V^{\prime}$ Fiducial Cut $\star$ The charged tracks must pass $>0.002 m$ from the edge of the holes in the $V V^{\prime}$. This requires the tracks to pass outside the beam holes in the $V V^{\prime}$ counter. counters.

Collar Anti Fiducial Cut $\star$ The charged tracks must pass $>0.002 m$ from the edge of the Collar Anti counters. This requires that the tracks pass outside the outer edge of the CA.

CsI Calorimeter Fiducial Cut $\star$ The charged tracks must pass $>0.029 m$ from the outside edge of the CsI calorimeter. This requires the tracks to pass inside the outer edge of the CsI.

Cell Separation Cut $\star$ The charged tracks must have a minimum separation greater than 3 wire-centered cells in all drift chambers. 


\subsubsection{Final Analysis Requirements}

After the crunch, one more set of requirements were applied to the data. Unlike the crunch cuts, these requirements can be quite tight, and can be set so that we obtain maximum background rejection while retaining maximum sensitivity to the fit parameters. The final analysis requirements can all be found in Table 5.2. For each of the cuts, we have attempted to retain regions in which the Monte Carlo, which is described in Chapter 7, matches data, ensuring that we can accurately calculate the acceptance. 


\begin{tabular}{|l|l|}
\hline Cut Variable & Keep Event If... \\
\hline Kaon Mass & $0.48967 \mathrm{GeV} / \mathrm{c}^{2}<M_{\pi^{+}} \pi^{-} \gamma<0.50567 \mathrm{GeV} / \mathrm{c}^{2}$ \\
$P_{T}^{2}$ w.r.t Regenerator & $P_{T}^{2}<2.5 \times 10^{-4} \mathrm{GeV}^{2} / \mathrm{c}^{2}$ \\
Kaon Momentum & $40.0 \mathrm{GeV} / \mathrm{c}<P_{\pi^{+}} \pi^{-} \gamma<160.0 \mathrm{GeV} / \mathrm{c}$ \\
Photon Energy in Lab Frame & $E_{\gamma}^{L A B}>1.5 \mathrm{GeV}$ \\
Photon Energy in Kaon Rest Frame, From & $20.0 \mathrm{MeV}<E_{\gamma}^{*}<175.0 \mathrm{MeV}$ \\
Calorimeter & \\
Photon Energy in Kaon Rest Frame, From & $20.0 \mathrm{MeV}<E_{\gamma}^{*}<175.0 \mathrm{MeV}$ \\
Kinematics & \\
$\pi \pi$ Invariant Mass, Implied From Above Cut & $0.2711 \mathrm{GeV} / \mathrm{c}^{2}<M_{\pi \pi}<0.4772 \mathrm{GeV} / \mathrm{c}^{2}$ \\
Fusion $\chi^{2}$ For Photon Cluster & $\chi_{F U S I O N}^{2}<48$ \\
Outer Fiducial Cut For Photon Cluster & ISEEDRING $<18.1$ \\
Inner Fiducial Cut For Photon Cluster & ISMLRING2 $>4.5$ \\
Photon/Track Separation at CsI & d $>30 \mathrm{~cm}$ \\
Number of CsI clusters & NCLUS $\geq 3$ \\
$P_{\pi^{0}}^{2}$ w.r.t. Target & $-0.10 \mathrm{GeV} / \mathrm{c}^{2}<P_{\pi^{0}}^{2}<-0.0055 \mathrm{GeV} / \mathrm{c}^{2}$ \\
L3 $P_{\pi^{0}}^{2}$ & passes \\
Z vertex & $125.5 \mathrm{~m}<\mathrm{VTXZ}<158.0 \mathrm{~m}$ \\
E/p & E/p $<0.85$ \\
Track Momentum & TRKP $>8.0 \mathrm{GeV}$ \\
Vertex $\chi^{2}$ & VTXCHI $<50.0$ \\
Magnet Offset $\chi^{2}$ & TRKOCHI $<50.0$ \\
Track x separation at CsI & $\Delta x>3.0 \mathrm{~cm}$ \\
Track y separation at CsI & $\Delta y>3.0 \mathrm{~cm}$ \\
Total track separation at CsI & $\Delta r>20.0 \mathrm{~cm}$ \\
Number of Tracks & NTRK $=2$ \\
$\Lambda \rightarrow p \pi$ invariant mass & $M_{p \pi}<1.112 \mathrm{GeV} / \mathrm{c}^{2}$ or $M_{p \pi}>1.119 \mathrm{GeV} / \mathrm{c}^{2}$ \\
Early energy in photon cluster & ADCSI_EARLY $<150 \mathrm{counts}$ \\
In-time energy in photon cluster & ADCSI_INTIM $>115 \mathrm{counts}$ \\
Photon/Upstream Track Projection at CsI & d $>2.0 \mathrm{~cm} \mathrm{distance}$ \\
Reconstruction Routines & Return no errors \\
Veto Cuts & All pass \\
Level 1 Trigger Verification & Event passes \\
Fiducial Cuts & All pass \\
Number of Photon Candidates That Pass ALL & $N_{C O M B I N A T I O N S}=1 \mathrm{ONLY}$ \\
Cuts & \\
\hline & \\
\hline
\end{tabular}

Table 5.2: Analysis Cuts Applied To Data 
In addition, it was necessary to identify if events were associated with the regenerator beam or the vacuum beam. This was done in two stages. In the first stage, the location, in $\mathrm{Y}$, of the decay vertex was compared to the $\mathrm{Y}$ location of the vacuum and regenerator beams. During the second stage, the kaon momentum vector is used for identification - the kaon's trajectory should point away from the regenerator for a regenerator beam event. Ideally, the identification from both stages should be consistent with each other. If not, this indicates the event is a crossover event ${ }^{6}$, and the event is rejected.

\subsubsection{Selection Efficiency and Event Yield}

After all cuts, the number of events remaining in the data are shown in Table 5.3.

\begin{tabular}{|l|l|r|}
\hline Beam & Year & Number of Events After All Cuts \\
\hline Regenerator & 1997 & 58,755 \\
Regenerator & 1999 & 75,666 \\
Vacuum & 1997 & 75,506 \\
Vacuum & 1999 & 97,276 \\
\hline \hline Total & Total & 307,203 \\
\hline
\end{tabular}

Table 5.3: Number of events after all cuts

Each of the final analysis cuts detailed previously may remove $K_{L, S} \rightarrow \pi^{+} \pi^{-} \gamma$ events in addition to background. The effect of each cut on the number of combinations passing all cuts in the data is shown in Table 5.4 while the same is shown for Monte Carlo simulated events in Table 5.5.

Using Monte Carlo simulated events, we can calculate the total acceptance for these cuts - in other words, we can see find the fraction of events that are left after all cuts. This information is in Table 5.6.

\footnotetext{
${ }^{6} \mathrm{~A}$ cross over event refers to an event in which the kaon scatters from one beam over to another, and then decays within the other beam.
} 


\begin{tabular}{|c|c|c|c|c|}
\hline \multirow[t]{2}{*}{ Cut Variable } & \multicolumn{4}{|c|}{$\begin{array}{c}\text { Number of combinations removed only by this cut } \\
\text { and relative loss (\%) }\end{array}$} \\
\hline & 97REG & 99REG & 97VAC & 99VAC \\
\hline$\pi^{0}$ mass & $\begin{array}{c}246 \\
0.42 \pm 0.03\end{array}$ & $\begin{array}{c}273 \\
0.36 \pm 0.02\end{array}$ & $\begin{array}{c}628 \\
0.82 \pm 0.03\end{array}$ & $\begin{array}{c}675 \\
0.68 \pm 0.03\end{array}$ \\
\hline $\mathrm{Z}$ vertex & $\begin{array}{c}1204 \\
2.01 \pm 0.06\end{array}$ & $\begin{array}{c}1453 \\
1.86 \pm 0.05\end{array}$ & $\begin{array}{c}21961 \\
22.53 \pm 0.13\end{array}$ & $\begin{array}{c}28444 \\
22.44 \pm 0.12\end{array}$ \\
\hline$M_{\pi^{+} \pi^{-} \gamma}$ & $\begin{array}{c}469 \\
0.79 \pm 0.04\end{array}$ & $\begin{array}{c}623 \\
0.81 \pm 0.03\end{array}$ & $\begin{array}{c}866 \\
1.13 \pm 0.04\end{array}$ & $\begin{array}{c}1074 \\
1.08 \pm 0.03\end{array}$ \\
\hline$P_{T}^{2}$ & $\begin{array}{c}1376 \\
2.29 \pm 0.06\end{array}$ & $\begin{array}{c}1950 \\
2.49 \pm 0.06\end{array}$ & $\begin{array}{c}1287 \\
1.68 \pm 0.05\end{array}$ & $\begin{array}{c}1949 \\
1.94 \pm 0.04\end{array}$ \\
\hline$p_{K}$ & $\begin{array}{c}8781 \\
13.00 \pm 0.13\end{array}$ & $\begin{array}{c}11963 \\
13.53 \pm 0.12\end{array}$ & $\begin{array}{c}18856 \\
19.98 \pm 0.13\end{array}$ & $\begin{array}{c}25054 \\
20.31 \pm 0.11\end{array}$ \\
\hline$E_{\gamma}^{*}$ & $\begin{array}{c}1037 \\
1.73 \pm 0.05\end{array}$ & $\begin{array}{c}1299 \\
1.67 \pm 0.05\end{array}$ & $\begin{array}{c}427 \\
0.56 \pm 0.03\end{array}$ & $\begin{array}{c}542 \\
0.55 \pm 0.02\end{array}$ \\
\hline$E_{\gamma}^{L A B}$ & $\begin{array}{c}2444 \\
3.99 \pm 0.08\end{array}$ & $\begin{array}{c}3224 \\
4.05+0.07\end{array}$ & $\begin{array}{c}2184 \\
2.81 \pm 0.06\end{array}$ & $\begin{array}{c}2842 \\
2.81+0.05\end{array}$ \\
\hline$M_{\pi+\pi}-$ & $\begin{array}{c}1057 \\
1.77 \pm 0.05\end{array}$ & $\begin{array}{c}1448 \\
1.86 \pm 0.05\end{array}$ & $\begin{array}{c}430 \\
0.57 \pm 0.03\end{array}$ & $\begin{array}{c}509 \\
0.52 \pm 0.02\end{array}$ \\
\hline $\mathrm{E} / \mathrm{p}$ & $\begin{array}{c}564 \\
0.95 \pm 0.04\end{array}$ & $\begin{array}{c}803 \\
1.04 \pm 0.04\end{array}$ & $\begin{array}{c}775 \\
1.02 \pm 0.04\end{array}$ & $\begin{array}{c}951 \\
0.96 \pm 0.03\end{array}$ \\
\hline Track Momentum & $\begin{array}{c}985 \\
1.65 \pm 0.05\end{array}$ & $\begin{array}{c}1385 \\
1.78 \pm 0.05\end{array}$ & $\begin{array}{c}1736 \\
2.25 \pm 0.05\end{array}$ & $\begin{array}{c}2256 \\
2.24 \pm 0.05\end{array}$ \\
\hline$\chi_{V E R T E X}^{2}$ & $\begin{array}{c}92 \\
0.16 \pm 0.02\end{array}$ & $\begin{array}{c}79 \\
0.10 \pm 0.01\end{array}$ & $\begin{array}{c}160 \\
0.21 \pm 0.02\end{array}$ & $\begin{array}{c}198 \\
0.20 \pm 0.01\end{array}$ \\
\hline$\chi_{F U S I O N}^{2}$ & $\begin{array}{c}31 \\
0.05 \pm 0.01\end{array}$ & $\begin{array}{c}76 \\
0.10 \pm 0.01\end{array}$ & $\begin{array}{c}23 \\
0.03 \pm 0.01\end{array}$ & $\begin{array}{c}52 \\
0.05 \pm 0.01\end{array}$ \\
\hline$M_{p \pi}$ & $\begin{array}{c}1045 \\
1.75 \pm 0.05\end{array}$ & $\begin{array}{c}1397 \\
1.79 \pm 0.05\end{array}$ & $\begin{array}{c}1611 \\
2.09 \pm 0.05\end{array}$ & $\begin{array}{c}2054 \\
2.05 \pm 0.04\end{array}$ \\
\hline$\chi_{O F F S E T}^{2}$ & $\begin{array}{c}528 \\
0.89 \pm 0.04\end{array}$ & $\begin{array}{c}661 \\
0.86 \pm 0.03\end{array}$ & $\begin{array}{c}714 \\
0.94^{ \pm} 0.03\end{array}$ & $\begin{array}{c}1057 \\
1.06 \pm 0.03\end{array}$ \\
\hline Track Separation & $\begin{array}{c}173 \\
0.29 \pm 0.02\end{array}$ & $\begin{array}{c}240 \\
0.31 \pm 0.02\end{array}$ & $\begin{array}{c}272 \\
0.36 \pm 0.02\end{array}$ & $\begin{array}{c}378 \\
0.38 \pm 0.02\end{array}$ \\
\hline$\pi-\gamma$ separation & $\begin{array}{c}15142 \\
20.49 \pm 0.15\end{array}$ & $\begin{array}{c}19403 \\
20.24 \pm 0.13\end{array}$ & $\begin{array}{c}19740 \\
20.73 \pm 0.13\end{array}$ & $\begin{array}{c}25329 \\
20.48 \pm 0.11\end{array}$ \\
\hline Seedring & $\begin{array}{c}803 \\
1.35 \pm 0.05\end{array}$ & $\begin{array}{c}1069 \\
1.38 \pm 0.04\end{array}$ & $\begin{array}{c}1008 \\
1.32 \pm 0.04\end{array}$ & $\begin{array}{c}1254 \\
1.26 \pm 0.04\end{array}$ \\
\hline Smlring & $\begin{array}{c}1216 \\
2.03 \pm 0.06\end{array}$ & $\begin{array}{c}1483 \\
1.90 \pm 0.05\end{array}$ & $\begin{array}{c}1755 \\
2.27 \pm 0.05\end{array}$ & $\begin{array}{c}2238 \\
2.23 \pm 0.05\end{array}$ \\
\hline Track X-separation & $\begin{array}{c}1506 \\
2.50 \pm 0.06\end{array}$ & $\begin{array}{c}1854 \\
2.37 \pm 0.05\end{array}$ & $\begin{array}{c}649 \\
0.85 \pm 0.03\end{array}$ & $\begin{array}{c}937 \\
0.94 \pm 0.03\end{array}$ \\
\hline Track Y-separation & $\begin{array}{c}51 \\
0.09 \pm 0.01\end{array}$ & $\begin{array}{c}54 \\
0.07 \stackrel{ \pm}{ \pm} 0.01\end{array}$ & $\begin{array}{c}24 \\
0.03 \pm 0.01\end{array}$ & $\begin{array}{c}30 \\
0.03 \pm 0.01\end{array}$ \\
\hline$P_{\pi^{0}}^{2}(\mathrm{VAC})$ & $\begin{array}{c}125 \\
0.21 \pm 0.02\end{array}$ & $\begin{array}{c}163 \\
0.21 \pm 0.02\end{array}$ & $\begin{array}{c}486 \\
0.64 \pm 0.03\end{array}$ & $\begin{array}{c}615 \\
0.62 \pm 0.02\end{array}$ \\
\hline Early Cluster Energy & $\begin{array}{c}416 \\
0.70 \pm 0.03\end{array}$ & $\begin{array}{c}2582 \\
3.27 \pm 0.06\end{array}$ & $\begin{array}{c}707 \\
0.93^{ \pm} 0.03\end{array}$ & $\begin{array}{c}4769 \\
4.63 \pm 0.07\end{array}$ \\
\hline Intime Cluster Energy & $\begin{array}{c}44 \\
0.07 \pm 0.01\end{array}$ & $\begin{array}{c}59 \\
0.08 \pm 0.01\end{array}$ & $\begin{array}{c}53 \\
0.07 \pm 0.01\end{array}$ & $\begin{array}{c}63 \\
0.06 \pm 0.01\end{array}$ \\
\hline Upstream Track - $\gamma$ separation & $\begin{array}{c}31 \\
0.05 \pm 0.01\end{array}$ & $\begin{array}{c}37 \\
0.05 \pm 0.01\end{array}$ & $\begin{array}{c}63 \\
0.08 \pm 0.01\end{array}$ & $\begin{array}{c}69 \\
0.07 \pm 0.01\end{array}$ \\
\hline Veto Cuts & $\begin{array}{c}35 \\
0.06 \pm 0.01\end{array}$ & $\begin{array}{c}43 \\
0.06 \pm 0.01\end{array}$ & $\begin{array}{c}59 \\
0.08 \pm 0.01\end{array}$ & $\begin{array}{c}49 \\
0.05 \pm 0.01\end{array}$ \\
\hline L3 $P_{\pi^{0}}^{2}$ & $\begin{array}{c}0 \\
0.00 \pm 0.00\end{array}$ & $\begin{array}{c}0 \\
0.00 \pm 0.00\end{array}$ & $\begin{array}{c}189 \\
0.25 \pm 0.02\end{array}$ & $\begin{array}{c}197 \\
0.20 \pm 0.01\end{array}$ \\
\hline Bad Spills and Runs & $\begin{array}{c}0 \\
0.00 \pm 0.00\end{array}$ & $\begin{array}{c}1606 \\
2.06 \pm 0.05\end{array}$ & $\begin{array}{c}0 \\
0.00 \pm 0.00\end{array}$ & $\begin{array}{c}2125 \\
2.12 \pm 0.05\end{array}$ \\
\hline Number of Good combinations & $\begin{array}{c}7 \\
0.01 \pm 0.00\end{array}$ & $\begin{array}{c}13 \\
0.02 \pm 0.00\end{array}$ & $\begin{array}{c}8 \\
0.01 \pm 0.00\end{array}$ & $\begin{array}{c}6 \\
0.01 \pm 0.00\end{array}$ \\
\hline
\end{tabular}

Table 5.4: Number of Combinations in Data removed by each cut. Each value gives the difference between the total number of events before and after each particular cut after all other cuts have already been applied 


\begin{tabular}{|l|c|c|c|c|}
\hline \multicolumn{1}{|c|}{ Cut Variable } & \multicolumn{3}{|c|}{ Relative loss due to only this cut (\%) } \\
\hline & 97REG & 99REG & 97VAC & 99VAC \\
\hline$\pi^{0}$ mass & $0.33 \pm 0.02$ & $0.33 \pm 0.02$ & $0.62 \pm 0.03$ & $0.55 \pm 0.02$ \\
\hline$Z_{\text {vertex }}$ & $1.97 \pm 0.06$ & $1.74 \pm 0.04$ & $22.40 \pm 0.14$ & $22.24 \pm 0.12$ \\
\hline$M_{\pi^{+} \pi^{-} \gamma}$ & $0.92 \pm 0.04$ & $0.98 \pm 0.03$ & $0.73 \pm 0.03$ & $0.70 \pm 0.03$ \\
\hline$P_{T}^{2}$ & $1.68 \pm 0.05$ & $8.08 \pm 0.08$ & $0.20 \pm 0.02$ & $0.17 \pm 0.01$ \\
\hline$p_{K}$ & $13.22 \pm 0.13$ & $13.17 \pm 0.09$ & $19.99 \pm 0.14$ & $20.42 \pm 0.12$ \\
\hline$E_{\gamma}^{*}$ & $1.93 \pm 0.06$ & $1.80 \pm 0.04$ & $0.59 \pm 0.03$ & $0.60 \pm 0.03$ \\
\hline$E_{\gamma}^{L A B}$ & $4.05 \pm 0.08$ & $4.07 \pm 0.06$ & $2.96 \pm 0.06$ & $2.96 \pm 0.06$ \\
\hline$M_{\pi^{+} \pi^{-}}$ & $2.04 \pm 0.06$ & $1.84 \pm 0.04$ & $0.57 \pm 0.03$ & $0.63 \pm 0.03$ \\
\hline$E_{\text {p }}$ & $1.54 \pm 0.05$ & $1.61 \pm 0.04$ & $1.58 \pm 0.05$ & $1.62 \pm 0.04$ \\
\hline Track Momentum & $1.75 \pm 0.05$ & $1.67 \pm 0.04$ & $2.16 \pm 0.05$ & $2.16 \pm 0.05$ \\
\hline$\chi_{V E R T E X}^{2}$ & $0.10 \pm 0.01$ & $0.10 \pm 0.01$ & $0.10 \pm 0.01$ & $0.12 \pm 0.01$ \\
\hline$\chi_{F U S I O N}^{2}$ & $0.03 \pm 0.01$ & $0.08 \pm 0.01$ & $0.02 \pm 0.01$ & $0.04 \pm 0.01$ \\
\hline$M_{p \pi}$ & $1.88 \pm 0.06$ & $1.82 \pm 0.04$ & $2.01 \pm 0.05$ & $2.08 \pm 0.05$ \\
\hline$\chi_{O F F S E T}^{2}$ & $0.82 \pm 0.04$ & $0.85 \pm 0.03$ & $0.81 \pm 0.03$ & $0.93 \pm 0.03$ \\
\hline Track Separation & $0.29 \pm 0.02$ & $0.28 \pm 0.02$ & $0.38 \pm 0.02$ & $0.39 \pm 0.02$ \\
\hline$\pi-\gamma$ separation & $20.51 \pm 0.15$ & $20.73 \pm 0.11$ & $20.60 \pm 0.14$ & $20.90 \pm 0.12$ \\
\hline Seedring & $1.44 \pm 0.05$ & $1.40 \pm 0.03$ & $1.48 \pm 0.05$ & $1.39 \pm 0.04$ \\
\hline Smlring & $1.89 \pm 0.06$ & $1.80 \pm 0.04$ & $2.37 \pm 0.06$ & $2.15 \pm 0.05$ \\
\hline Track X-separation & $2.30 \pm 0.06$ & $2.31 \pm 0.04$ & $0.80 \pm 0.03$ & $0.88 \pm 0.03$ \\
\hline Track Y-separation & $0.08 \pm 0.01$ & $0.07 \pm 0.01$ & $0.02 \pm 0.01$ & $0.03 \pm 0.01$ \\
\hline$P_{\pi^{0}}^{2}$ (VAC) & $0.19 \pm 0.02$ & $0.16 \pm 0.01$ & $0.62 \pm 0.03$ & $0.60 \pm 0.03$ \\
\hline Early Cluster Energy & $3.91 \pm 0.08$ & $2.41 \pm 0.05$ & $4.92 \pm 0.08$ & $3.98 \pm 0.06$ \\
\hline Intime Cluster Energy & $0.07 \pm 0.01$ & $0.05 \pm 0.01$ & $0.03 \pm 0.01$ & $0.02 \pm 0.00$ \\
\hline Upstream Track - $\gamma$ separation & $0.04 \pm 0.01$ & $0.04 \pm 0.01$ & $0.08 \pm 0.01$ & $0.06 \pm 0.01$ \\
\hline Veto Cuts & $0.47 \pm 0.03$ & $0.43 \pm 0.02$ & $0.47 \pm 0.03$ & $0.43 \pm 0.02$ \\
\hline Number of Good combinations & $0.01 \pm 0.00$ & $0.01 \pm 0.00$ & $0.00 \pm 0.00$ & $0.00 \pm 0.00$ \\
\hline & & & & \\
\hline & \multicolumn{4}{|c|}{}
\end{tabular}

Table 5.5: Fraction of Combinations in Monte Carlo removed by each cut. Each value gives the relative difference between the total number of events before and after each particular cut after all other cuts have already been applied. Note that the Monte Carlo does not generate events over the full range of $Z_{v e r t e x}, p_{K}$ or $E_{\gamma}^{*}$, as such these efficiencies will depend on how the particular Monte Carlo sample was generated.

\begin{tabular}{|l|l|c|}
\hline Beam & Year & Total Acceptance (\%) \\
\hline Regenerator & 1997 & 3.55 \\
Regenerator & 1999 & 3.51 \\
Vacuum & 1997 & 2.27 \\
Vacuum & 1999 & 2.22 \\
\hline
\end{tabular}

Table 5.6: Total Monte Carlo Acceptance for events with $10 \mathrm{GeV} / \mathrm{c}<p_{K}<200 \mathrm{GeV} / \mathrm{c}$, $105.5 m<Z_{\text {vertex }}<159.9 \mathrm{~m}$ and $E_{\gamma}^{*}>4 \mathrm{MeV}$ after the stated analysis cuts. 


\subsection{Useful Selection Criteria}

The selection requirements used to extract $K_{L, S} \rightarrow \pi^{+} \pi^{-} \gamma$ events from the data has been presented in the previous section. However, it is instructive to go over each requirement or cut in detail. For each cut, we present a plot of the cut variable from data, with a similar plot of Monte Carlo events overlaid as a histogram. We also present the bin by bin ratio of data to Monte Carlo events, which is useful in studying the quality of the Monte Carlo simulation. In this chapter, we present plots for the 1997 regenerator beam data sample only. For the most part, the 1999 data will resemble the 1997 data, while many regenerator beam distributions will be quite distinct in relation to the vacuum beam data. The regenerator beam data is expected to provide the best contraint on the value of $\widehat{e}$. For that reason, plots of the 1997 regenerator beam data are shown here. The plots for the 1997 vacuum beam, 1999 regenerator beam and 1999 vacuum beam data samples can be found in Appendices I, $J$, and $K$ respectively.

\subsection{1 $M_{\pi^{+}} \pi^{-} \gamma$}

The plot of the invariant mass of the $\pi^{+} \pi^{-} \gamma$ system provides an excellent way of observing the background present in this decay. The signal can be clearly seen as a sharp peak centered on the mass of the neutral kaon. The wings of this peak are caused by various resolution effects inherent in the event reconstruction, and can come from minor problems with tracking, clustering, as well as the choice of the "wrong" cluster as the photon cluster. Additionally, the area beyond the wings is populated by events coming from background events. If the shape of this background is understood, one can extrapolate the amount of background under the kaon mass peak, and thus estimate the number of background events present in the final data sample after all cuts. The value of the $\pi^{+} \pi^{-} \gamma$ invariant mass for each event is shown in Figure 5.1. 

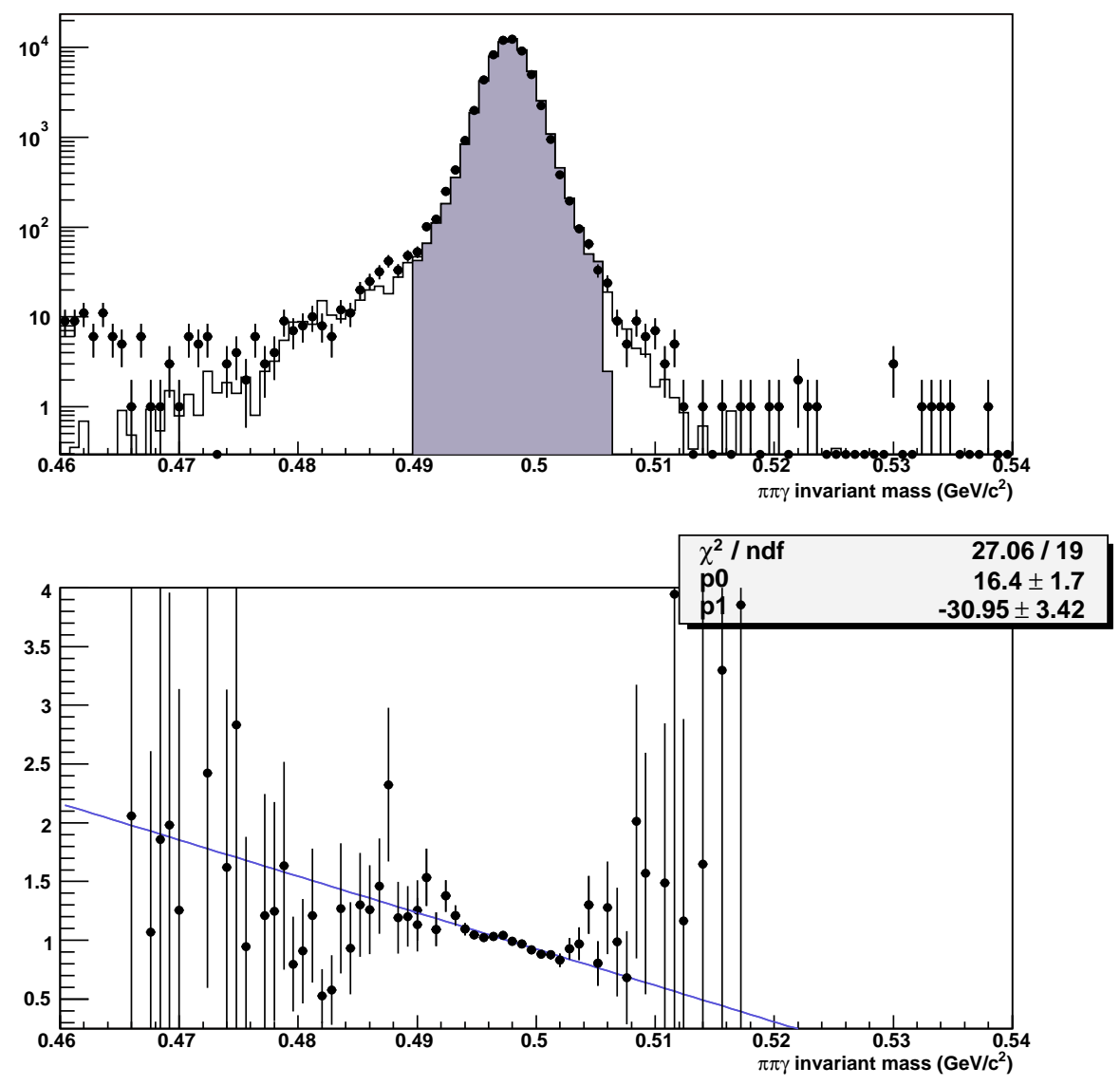

Figure 5.1: A plot of the $\pi^{+} \pi^{-} \gamma$ invariant mass before the cut on $M_{\pi^{+} \pi^{-}}$but after all other cuts. Data are points, while the histogram is of Monte Carlo simulated events. The solid part of the histogram denotes the areas that pass after this particular cut. A plot of the ratio of the number of data events to Monte Carlo events, before this cut, is shown on the bottom. The number of Monte Carlo events is normalized to be equal to the total number of data events in this plot. A fit of the ratio using a linear function is also shown.

It should be noted that this is the only observable which provides clear evidence of the presence of $K_{L, S} \rightarrow \pi^{+} \pi^{-} \gamma \gamma$ decays. Due to the missing photon, these events will reconstruct at values of $M_{\pi^{+}} \pi^{-} \gamma$ lower than the kaon mass, however they are most visible from $0.49 \mathrm{GeV} / \mathrm{c}^{2}$ to $0.48 \mathrm{GeV} / \mathrm{c}^{2}$. The Monte Carlo sample presented here includes the corrected PHOTOS simulation, which explains the good agreement in this area. 


\section{$5.4 .2 \quad p_{K}$}

It is necessary to cut on the value of the momentum of the $\pi^{+} \pi^{-} \gamma$ system for two reasons. The first is that the effect of the regenerator on the kaon's wavefunction is only well understood between 40 and $160 \mathrm{GeV}$. Second, higher momentum events, due to time dilation and time of flight, have a higher probability to be $K_{S}$ from the target, which are still considered signal, or hyperons like the $\Lambda$, which are background. The $p_{K}$ spectrum is shown in Figure 5.2. 

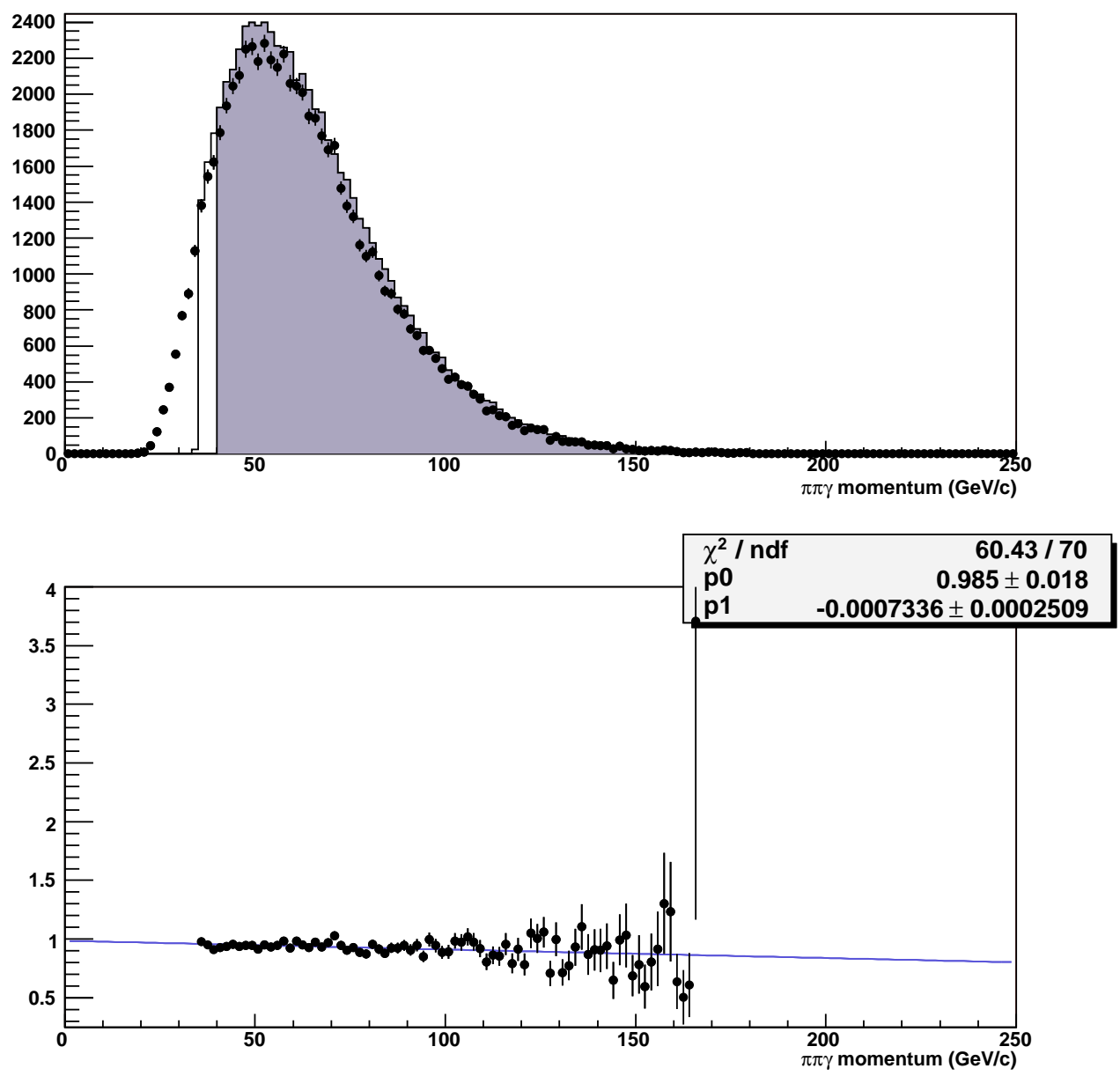

Figure 5.2: The reconstructed kaon momentum before the cut. Data are points, while the histogram is of Monte Carlo simulated events. The solid part of the histogram denotes the areas that pass after this particular cut. A plot of the ratio of the number of data events to Monte Carlo events, before this cut, is shown on the bottom. The number of Monte Carlo events is normalized to be equal to the total number of data events in this plot. A fit of the ratio using a linear function is also shown.

The deficiency in Monte Carlo events at the extreme low end of the spectrum is due to the momentum range used in Monte Carlo generation.

There is a sizable slope in this plot, which can cause problems with the energy scale of the analysis. The Monte Carlo sample was reweighted in order to correct for this momentum slope, and a systematic error was assigned due to the slope. 


\subsection{3 $P_{T}^{2}$}

An ideal $K_{L, S} \rightarrow \pi^{+} \pi^{-} \gamma$ event should have $P_{T}^{2}$ equal to zero, where $P_{T}^{2}$ denotes the component of the $\pi^{+} \pi^{-} \gamma$ combined momentum that is orthogonal to the direction of the neutral kaon beam. A non-zero value indicates that either the parent kaon scattered in a part of the $\mathrm{KTeV}$ beamline, or there was a problem with the reconstruction (i.e. the wrong cluster used as the photon cluster) or the event is in fact another kind of decay, for which particles have been misidentified, or lost, or extra particles have been gained.

The calculation of $P_{T}^{2}$ requires a reference point. It is common to use the target as this reference point, since this is the point at which the kaon was produced an unscattered kaon should point back to its production point. However, analyses utilizing data involving KTeV's regenerator - such as this one- use the center of the downstream face of the regenerator as the reference point for the calculation of $P_{T}^{2}$. The reason behind this is that the dominant scattering source in the regenerator beam is the regenerator itself. The definition used in subsequent references will state whether the target (VAC) or regenerator (REG) definitions were used. The value of $P_{T}^{2}$ with respect to the regenerator is shown in Figure 5.4 


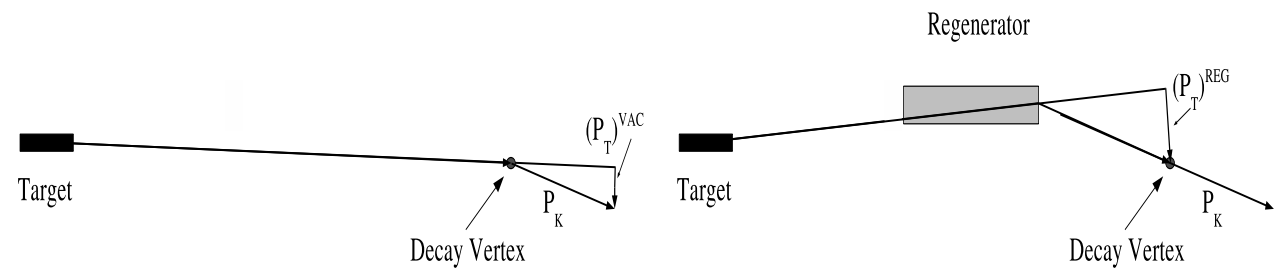

Figure 5.3: Two cartoons showing a scattered kaon decay in order to illustrate the difference between the "vacuum" and "regenerator" values of $P_{T}^{2}$. On the top, a kaon in the vacuum beam has scattered and the $P_{T}^{2}$ value is computed using the component of the kaon momentum that is perpendicular to a line connecting the decay vertex and the target. On the bottom, a kaon in the regenerator beam has scattered and the $P_{T}^{2}$ value is computed using the component of the kaon momentum that is perpendicular to a line connecting the center of the downstream face of the regenerator and the target. The two diagrams illustrate that the "regenerator" definition yields a larger value of $P_{T}^{2}$ than the "vacuum" definition, as the decay vertex and kaon momentum vectors are identical in this example. 

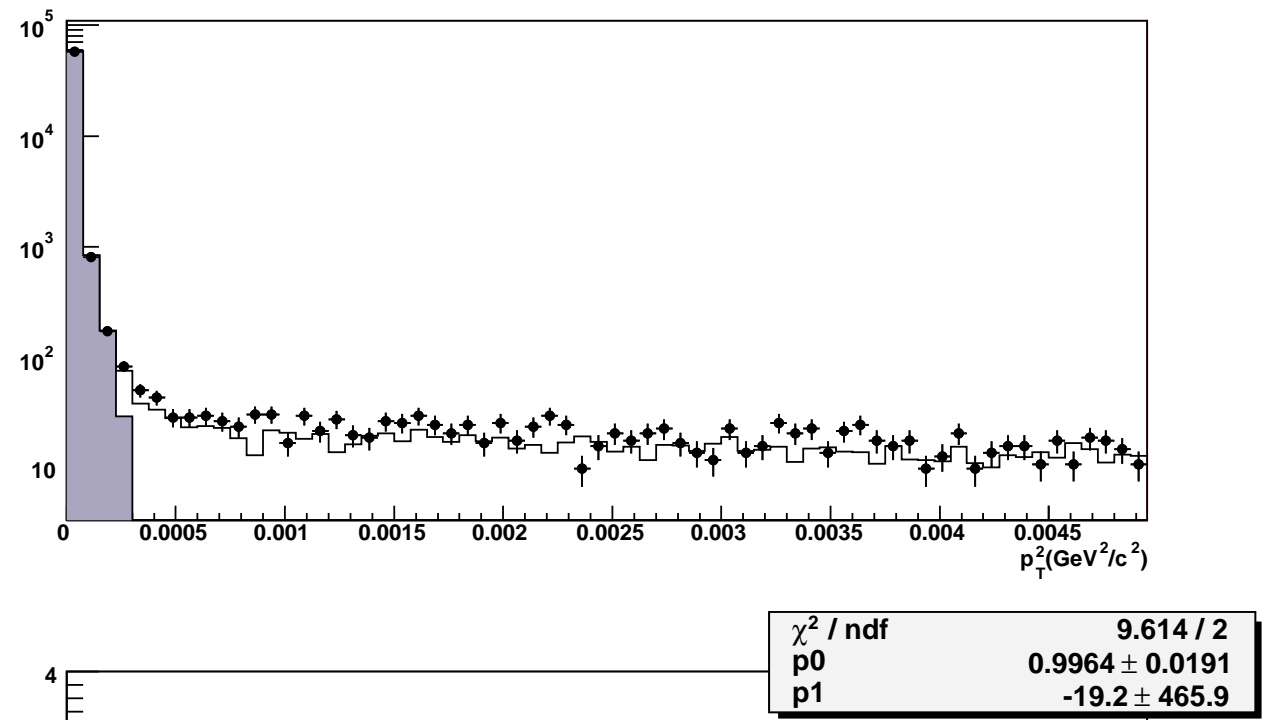

Figure 5.4: A plot of $P_{T}^{2}$ with respect to the downstream face of the regenerator for the $\pi^{+} \pi^{-} \gamma$ system before the cut. Data are points, while the histogram is of Monte Carlo simulated events. The solid part of the histogram denotes the areas that pass after this particular cut. A plot of the ratio of the number of data events to Monte Carlo events, before this cut, is shown on the bottom. The number of Monte Carlo events is normalized to be equal to the total number of data events in this plot. A fit of the ratio using a linear function is also shown. Note that the fit is done to points in the accepted region only. 


\subsubsection{Z vertex}

A cut is made on $Z_{\text {vertex }}$ which is the distance between the kaon production target and the decay point of the kaon. Events with smaller values of $Z_{\text {vertex }}$ are removed in order to decrease the amount of "target" $K_{S}$ in the data, as well as to reject short lived hyperons like $\Lambda$ and $\Sigma$. Events with higher values of $Z_{\text {vertex }}$ are removed in order to minimize beam interactions from the vacuum window, from which some hadronic and electromagnetic interactions come. The distribution of $Z_{\text {vertex }}$ is shown in Figure 5.5 . 

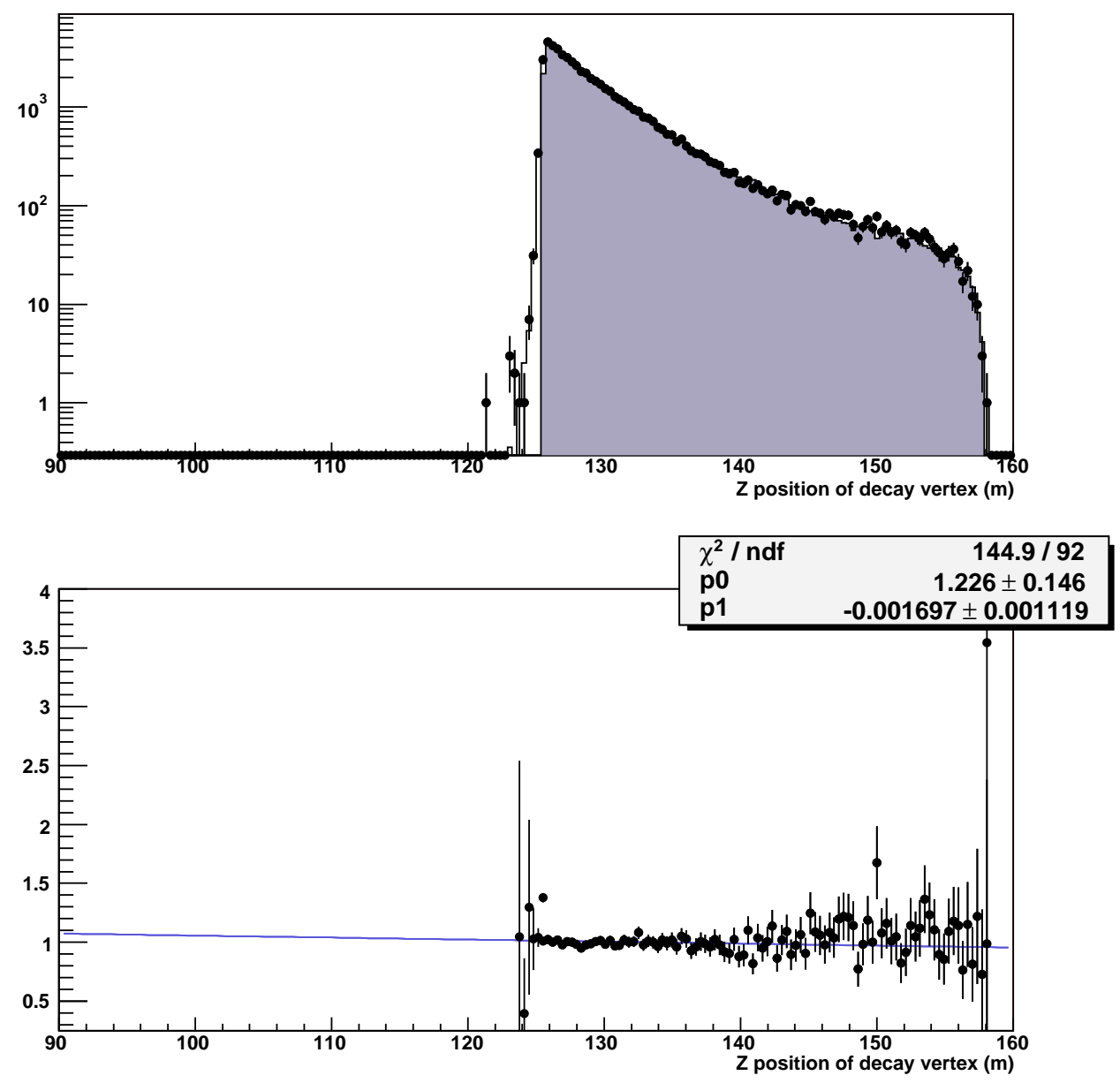

Figure 5.5: A plot of the $\mathrm{z}$ location of the decay vertex, before the cut is applied. Data are points, while the histogram is of Monte Carlo simulated events. The solid part of the histogram denotes the areas that pass after this particular cut. A plot of the ratio of the number of data events to Monte Carlo events, before this cut, is shown on the bottom. The number of Monte Carlo events is normalized to be equal to the total number of data events in this plot. A fit of the ratio using a linear function is also shown. 


\subsection{5 $E_{\gamma}^{L A B}$}

The value of $E_{\gamma}^{L A B}$ is simply the corrected energy of the cluster identified as having come from the photon. Accidental activity ( in this case photons from the neutral beam ) will produce many fake clusters in the CsI, however these fake clusters often have low energy, so a cut on $E_{\gamma}^{L A B}$ is useful for eliminating background. Care should be taken that this cut doesn't result in a tighter effective cut on $E_{\gamma}^{* C A L}$, which is the photon energy in the kaon rest frame. The $E_{\gamma}^{L A B}$ spectrum is shown in Figure 5.6. 

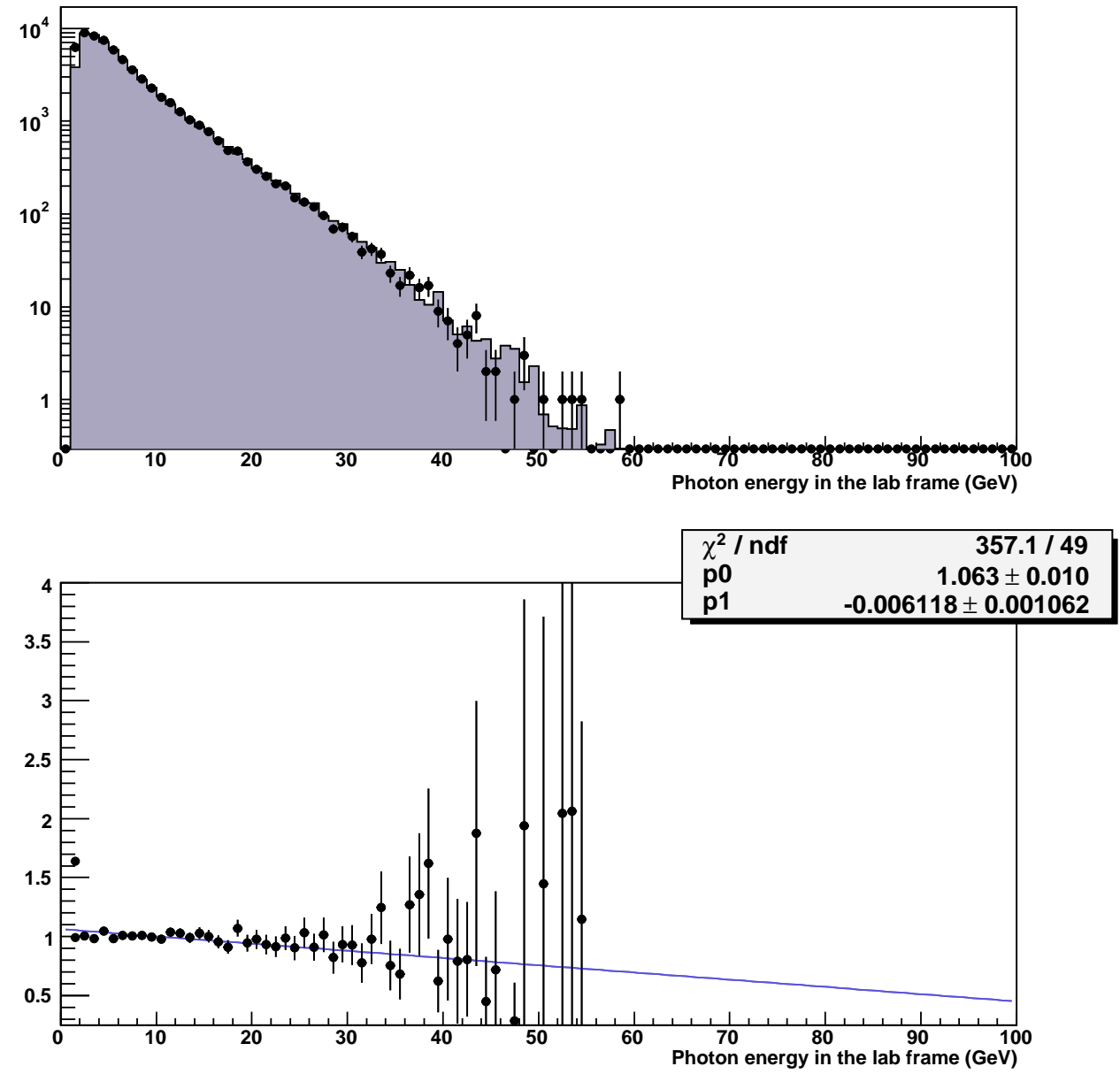

Figure 5.6: The photon cluster energy in the lab frame, before the final analysis cut. Data are points, while the histogram is of Monte Carlo simulated events. The solid part of the histogram denotes the areas that pass after this particular cut. A plot of the ratio of the number of data events to Monte Carlo events, before this cut, is shown on the bottom. The number of Monte Carlo events is normalized to be equal to the total number of data events in this plot. A fit of the ratio using a linear function is also shown. 


\subsection{6 $E_{\gamma}^{* C A L}$}

This is simply the value of the photon energy boosted into the kaon's rest frame. The cut was made at $20 \mathrm{MeV}$ in order to stay consistent with previous analyses. In addition, using the $20 \mathrm{MeV}$ cut also allows the most recent published branching ratios to be used for both $K_{L}$ and $K_{S}$. Radiative corrections are also expected to be reduced by using this cut value. However, this is a variable that directly enters into the likelihood fit, so this cut may alter the resolution of the fit. In fact, when all four data samples are used in the fit, the statistical error of $\widehat{e}$ is seen to decrease by a factor

of approximately $8 \%$ when the cut on $E_{\gamma}^{*}$ is lowered to $6 \mathrm{MeV}$. The $E_{\gamma}^{* C A L}$ spectrum is shown in Figure 5.7. 

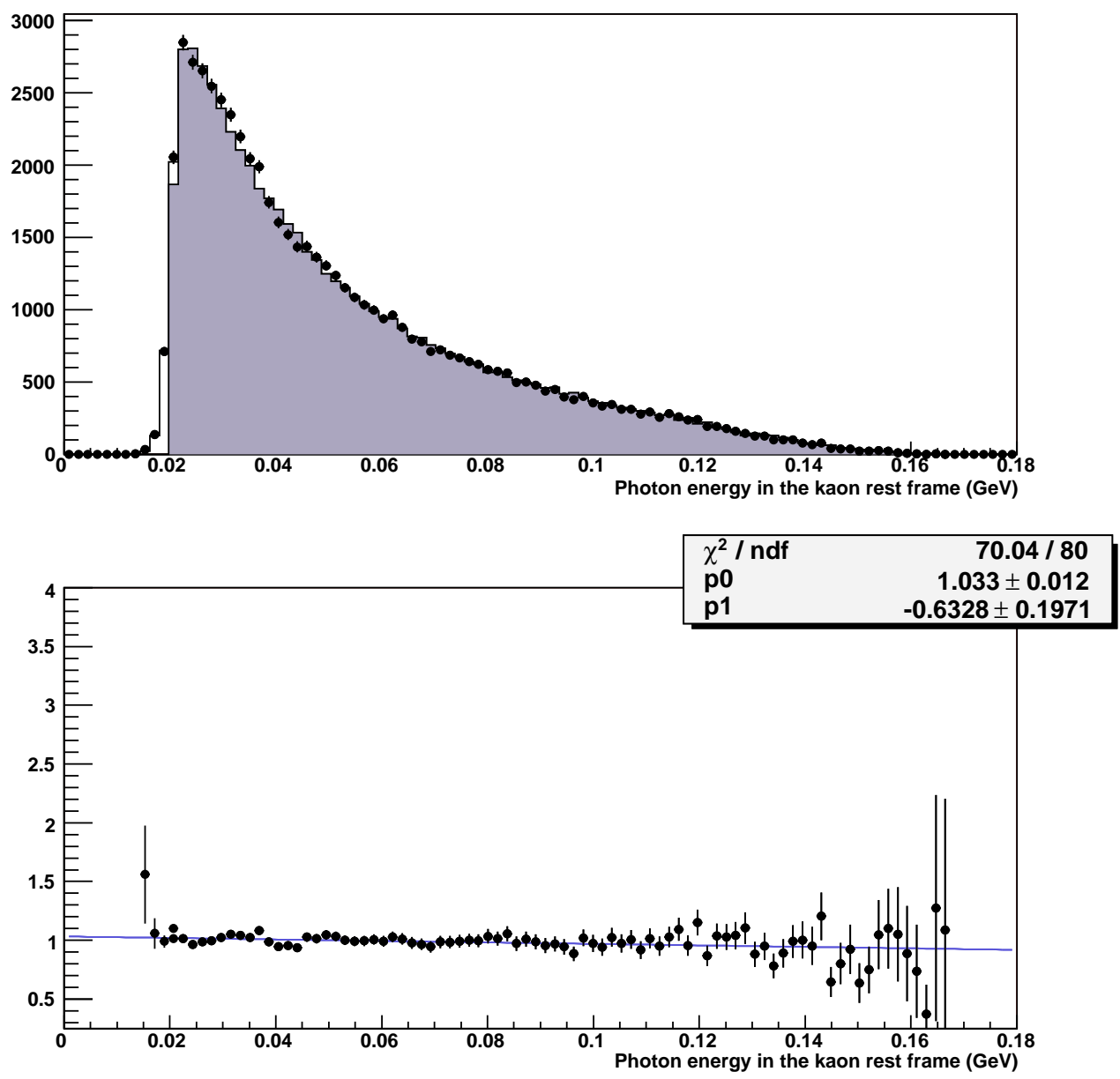

Figure 5.7: A plot of the photon energy in the kaon rest frame before the cut. Data are points, while the histogram is of Monte Carlo simulated events. The solid part of the histogram denotes the areas that pass after this particular cut. A plot of the ratio of the number of data events to Monte Carlo events, before this cut, is shown on the bottom. The number of Monte Carlo events is normalized to be equal to the total number of data events in this plot. A fit of the ratio using a linear function is also shown. 


\subsection{7 $E_{\gamma}^{* K I N}$}

One advantage to the $K_{L, S} \rightarrow \pi^{+} \pi^{-} \gamma$ decay is that in principle it can be fulled reconstructed. It is also a three body decay. These two facts lead to the ability to predict what the value of $E_{\gamma}^{*}$ should be in the kaon rest frame, given information about the pions, in this case, the invariant mass of the pion pair.

In the kaon rest frame, the value of $E_{\gamma}^{* K I N}$ can be expressed as:

$$
E_{\gamma}^{* K I N}=\frac{M_{K}^{2}-M_{\pi^{+} \pi^{-}}^{2}}{2 M_{K}}
$$

The value of $E_{\gamma}^{*}$ derived using the kinematics of the dipion pair, $E_{\gamma}^{* K I N}$, can be compared to the value, $E_{\gamma}^{* C A L}$ measured from the calorimeter and used to determine the energy resolution of the calorimeter. This value isn't sensitive to extra clusters in the calorimeter like $E_{\gamma}^{* C A L}$ is, however it is sensitive to second order radiative effects, as well as tracking resolution.

A cut is made on this variable at a value equal to the cut on $E_{\gamma}^{* C A L}$. Doing so helps to improve the rejection of the already small $K_{L, S} \rightarrow \pi^{+} \pi^{-}$background.

\section{$5.4 .8 M_{\pi^{+} \pi^{-}}$}

In order to suppress $K_{L, S} \rightarrow \pi^{+} \pi^{-}$decays, it is necessary to cut on the invariant mass of the dipion system. For $K_{L, S} \rightarrow \pi^{+} \pi^{-} \gamma$ decays, the value should be less than the mass of the neutral kaon. Careful attention needs to be paid to this cut, since, as seen above, the value of $M_{\pi^{+} \pi^{-}}$is directly correlated to the value of the photon energy in the kaon rest frame. A cut on $M_{\pi^{+} \pi^{-}}$is the same as a cut on $E_{\gamma}^{* K I N}$ and both are strongly correlated to the value of $E_{\gamma}^{* C A L}$. The value of $M_{\pi^{+} \pi^{-}}$is plotted in Figure 5.8 . 

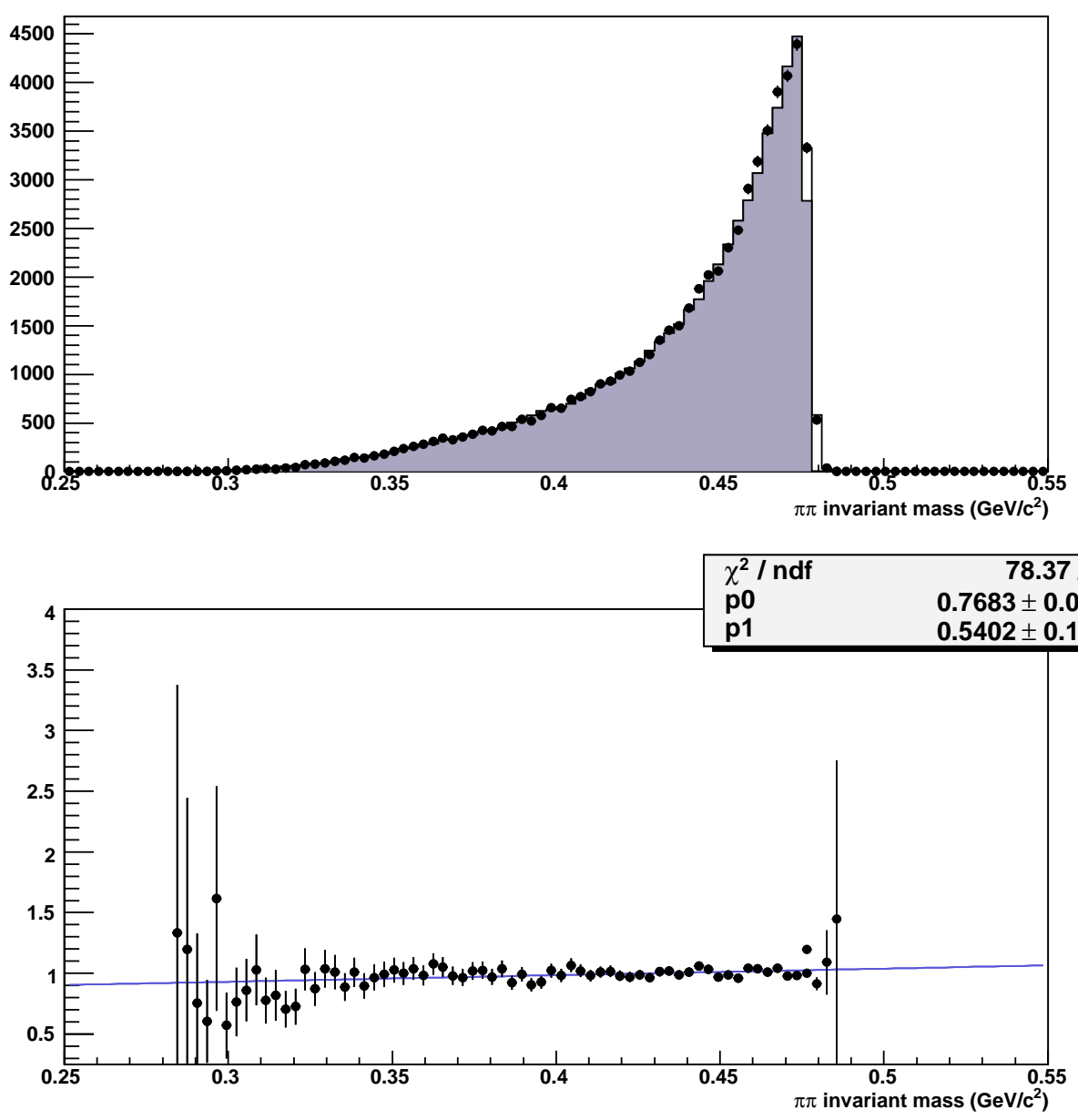

Figure 5.8: A plot of the invariant mass of the pion pair before the $M_{\pi^{+} \pi^{-}}$cut. Note that any cut on $E_{\gamma}^{* K I N}$ will also be reflected here. Data are points, while the histogram is of Monte Carlo simulated events. The solid part of the histogram denotes the areas that pass after this particular cut. A plot of the ratio of the number of data events to Monte Carlo events, before this cut, is shown on the bottom. The number of Monte Carlo events is normalized to be equal to the total number of data events in this plot. A fit of the ratio using a linear function is also shown. 


\section{$\mathbf{5 . 4 . 9} \chi_{F U S I O N}^{2}$}

While the CsI provides excellent identification of electrons and pions through the use of the variable $\mathrm{E} / \mathrm{p}$, it is more difficult to distinguish between a satellite hadronic cluster, which tend to be very broad,asymmetric and often deposit energy far from the center of the shower, and a photon. The main characteristic is the transverse energy shape, which for electromagnetic clusters tends to be well focused and asymmetric. Using the large sample of electron induced electromagnetic showers in the CsI collected by the experiment, it is possible to define an "ideal" transverse shower shape which describes how the energy of a cluster is transversely distributed from its center to its edges. The $\chi^{2}$ between this ideal shower shape and the actual transverse energy deposition of a cluster is then computed and known as $\chi_{F U S I O N}^{2}$, which serves as a rough measure of how close to an ideal EM cluster a given real cluster is. In addition to distinguishing between hadronic and electromagnetic clusters, $\chi_{\text {FUSION }}^{2}$ can also expose overlapped clusters in which two photons enter the CsI and shower in roughly the same location, hence the name.

For the candidate photon cluster, we require the value of $\chi_{F U S I O N}^{2}$ to be smaller than a certain value in order to maximize the chance that it is in fact a photon cluster. As the Monte Carlo in general does not simulate this variable well, we do not apply a tight cut on the value of the quantity. The value of $\chi_{F U S I O N}^{2}$ is plotted in Figure 5.9 . 

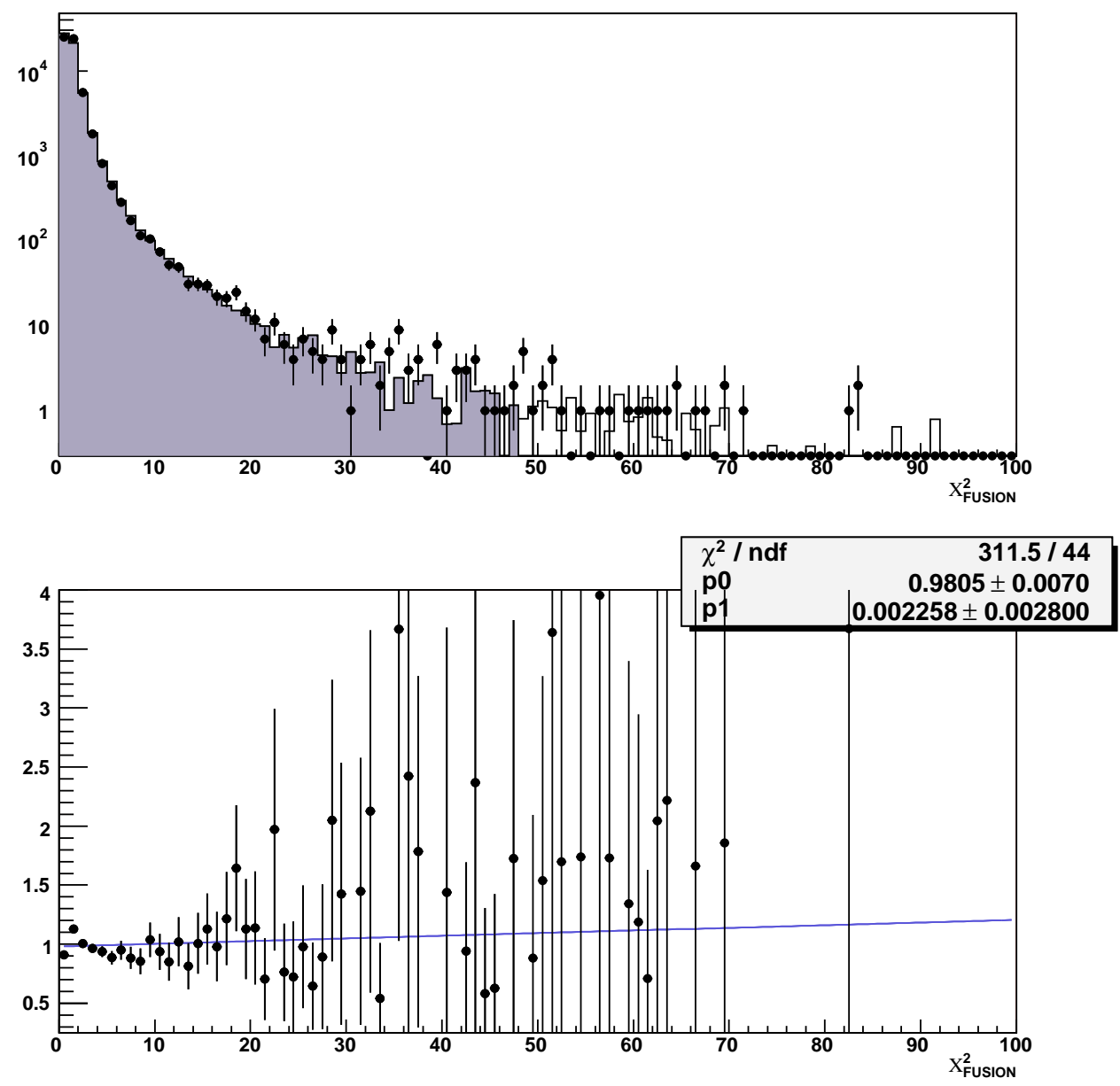

Figure 5.9: A plot of fusion $\chi^{2}$ before the cut on this variable. Data are points, while the histogram is of Monte Carlo simulated events. The solid part of the histogram denotes the areas that pass after this particular cut. A plot of the ratio of the number of data events to Monte Carlo events, before this cut, is shown on the bottom. The number of Monte Carlo events is normalized to be equal to the total number of data events in this plot. A fit of the ratio using a linear function is also shown. 


\subsubsection{0 $\pi-\gamma$ Separation at CsI}

As noted above, CsI clusters arising due to hadronic interactions - from pions for example, are often very spread out and non-symmetric. As an example, see the schematic of the $\mathrm{KTeV}$ event display in Figure 4.6. It is quite possible that an energy leak from a pion cluster may be treated as a second cluster, and may be used incorrectly as a candidate photon cluster. Pion interactions upstream of the CsI have an even larger effect here. In order to control the amount of contamination coming from "satellite" pion clusters, we cut on the distance between the candidate photon and pion clusters, with the assumption that satellite pion clusters increase in likelihood at smaller distances from the pion cluster.

It should also be noted that the required tightness of this cut is correlated with the $\chi_{F U S I O N}^{2}$ cut. If a tight cut on $\chi_{F U S I O N}^{2}$ is made, we may accept candidate photon clusters closer to the pion clusters, and vice versa. The minimum value of the $\pi-\gamma$ seperation for each event is plotted in Figure 5.10. 

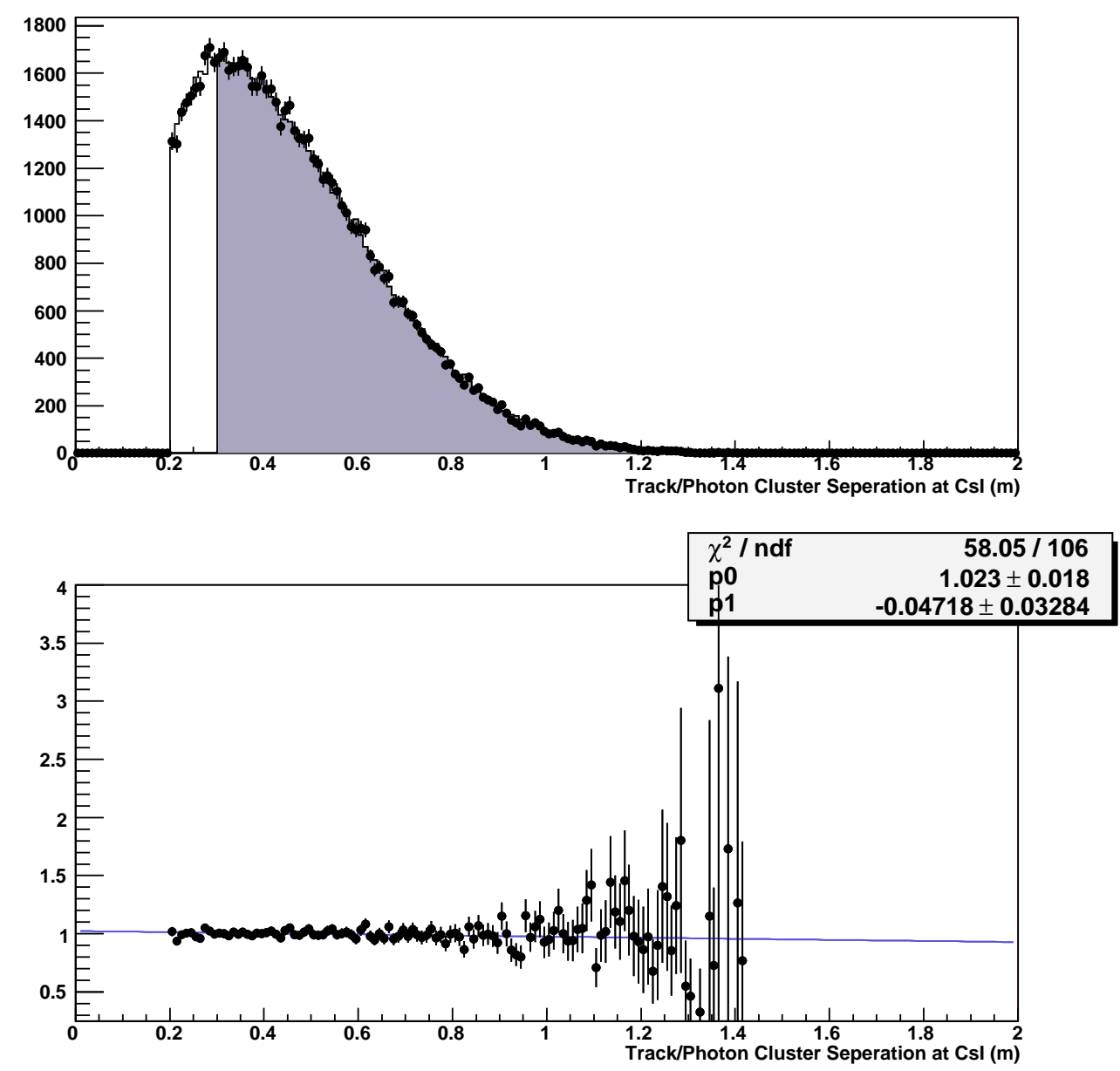

Figure 5.10: Minimum of the two pion/gamma separations at the CsI for each event, before the cut. Data are points, while the histogram is of Monte Carlo simulated events. The solid part of the histogram denotes the areas that pass after this particular cut. A plot of the ratio of the number of data events to Monte Carlo events, before this cut, is shown on the bottom. The number of Monte Carlo events is normalized to be equal to the total number of data events in this plot. A fit of the ratio using a linear function is also shown. 


\subsubsection{1 $P_{\pi^{0}}^{2}$}

$P_{\pi^{0}}^{2}$ is a kinematic quantity defined as the longitudinal momentum squared that a $\pi^{0}$ would have in a frame in which the combined momenta of the $\pi^{+}$and $\pi^{-}$are perpendicular to the kaon momentum, if the decay was a $K_{L} \rightarrow \pi^{+} \pi^{-} \pi^{0}$ decay. For an actual $K_{L} \rightarrow \pi^{+} \pi^{-} \pi^{0}$ decay, this quantity is always positive when resolution effects are ignored, however for a $K_{L, S} \rightarrow \pi^{+} \pi^{-} \gamma$ decay, this quantity is often negative - the only way in which 4 -momentum can be conserved when the decay is misidentified is if the hypothetical $\pi^{0}$ particle has an imaginary momentum. If we make the additional assumption that the kaon has zero transverse momentum in the lab frame, the expression for $P_{\pi^{0}}^{2}$ is:

$$
P_{\pi^{0}}^{2}=\frac{\left(M_{K}^{2}-M_{\pi^{0}}^{2}-M_{\pi^{+} \pi^{-}}\right)^{2}-4 M_{\pi^{0}}^{2} M_{\pi^{+} \pi^{-}}^{2}-4 M_{K}^{2}\left(P_{T}^{2}\right)_{\pi^{+} \pi^{-}}}{4\left(M_{\pi^{+} \pi^{-}}{ }^{2}+\left(P_{T^{+}}^{2}\right)_{\pi^{+} \pi^{-}}\right)}
$$

where $\left(P_{T}^{2}\right)_{\pi^{+} \pi^{-}}$is the combined transverse momentum of the dipion pair only. It should be noted that $\left(P_{T}^{2}\right)_{\pi^{+} \pi^{-}}$can either be calculated with respect to the target or the face of the regenerator. This impacts the value of $P_{\pi^{0}}^{2}$ so one should pay mind to which definition is used. During the crunch and final analysis stage, the value of $\left(P_{T}^{2}\right)_{\pi^{+} \pi^{-}}$with respect to the target was used. It should also be noted that since the tracking information used by the Level 3 trigger is different than that used by KTeVana, the Level 3 values of $M_{\pi^{+} \pi^{-}}$and $\left(P_{T}^{2}\right)_{\pi^{+} \pi^{-}}$will be different than those of KTeVana, and thus the Level $3 P_{\pi^{0}}^{2}$ cut is effectively a different cut than the $P_{\pi^{0}}^{2}$ cut used in later stages of data analysis. $P_{\pi^{0}}^{2}$ is plotted in Figure 5.11.

Note that the definition of $P_{\pi^{0}}^{2}$ involves both $M_{\pi^{+} \pi^{-}}$and the transverse momentum of the dipion pair. This means that the $P_{\pi^{0}}^{2}$ cut will bias the $E_{\gamma}^{*}$ spectrum, and this cut will also be correlated with the cut on $P_{T}^{2}$ through the transverse momentum of the pions. 

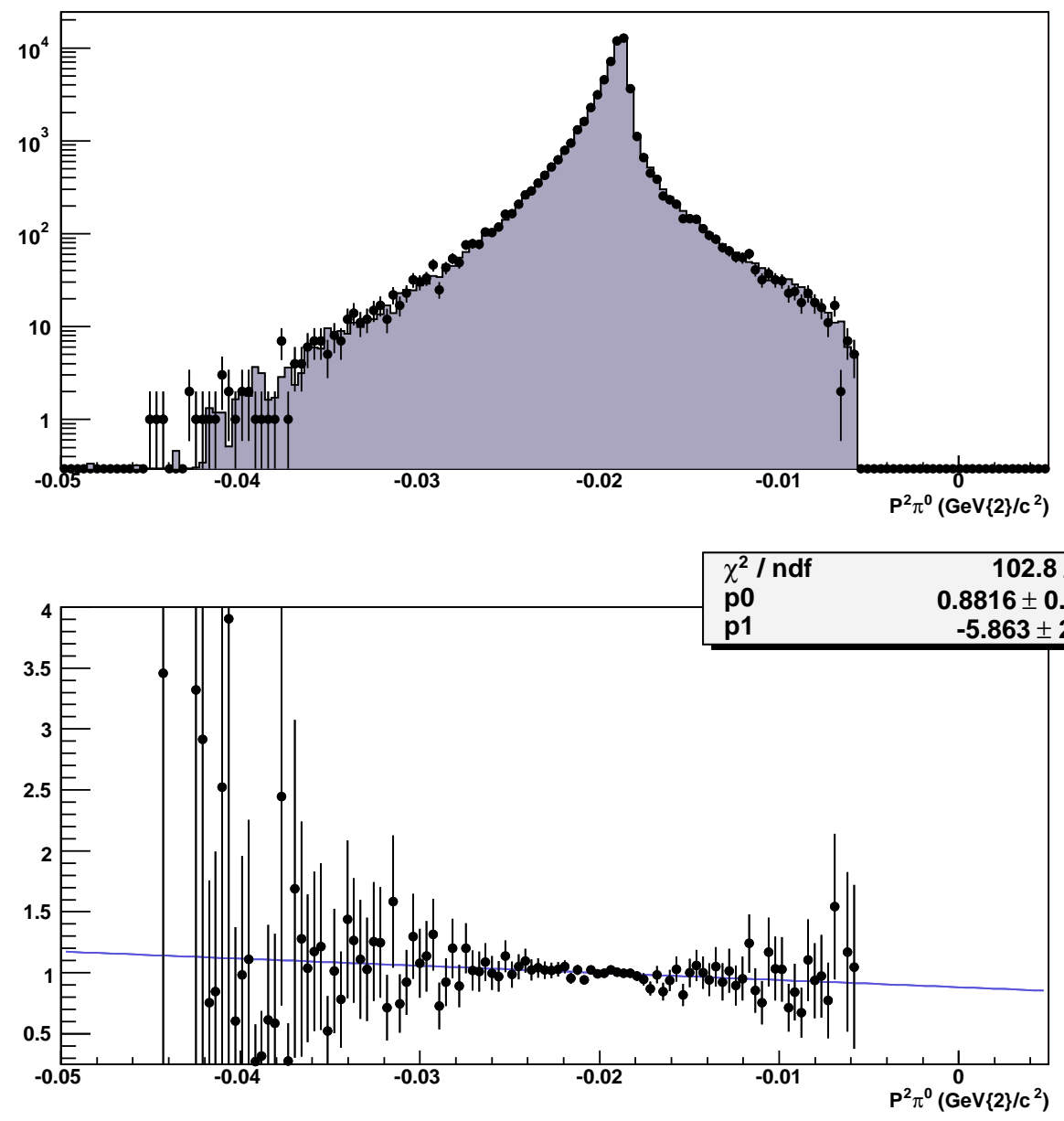

Figure 5.11: A plot of $P_{\pi^{0}}^{2}$ with respect to the downstream face of the regenerator, before the final analysis cut. Data are points, while the histogram is of Monte Carlo simulated events. The solid part of the histogram denotes the areas that pass after this particular cut. A plot of the ratio of the number of data events to Monte Carlo events, before this cut, is shown on the bottom. The number of Monte Carlo events is normalized to be equal to the total number of data events in this plot. A fit of the ratio using a linear function is also shown. 


\subsubsection{2 $\pi^{0}$ mass}

While the cut on $P_{\pi^{0}}^{2}$ serves as an excellent method with which to reject $K_{L} \rightarrow \pi^{+} \pi^{-} \pi^{0}$ decays, it is not perfectly efficient at removing these events. To further suppress $K_{L} \rightarrow \pi^{+} \pi^{-} \pi^{0}$ decays, it is possible to look for evidence of the $\pi^{0}$ in the event as well by computing the invariant mass of all pairs of non-pion CsI clusters, assuming that the photons originated at the charged decay vertex. Events which have a $\gamma \gamma$ combination with an invariant mass near that of the $\pi^{0}$ can then be removed. This cut has the small added benefit of suppressing other decay modes containing $\pi^{0} \mathrm{~s}$, for example, $K_{L} \rightarrow \pi^{0} \pi^{ \pm} e^{\mp} \nu$ and $K_{L} \rightarrow \pi^{0} \pi^{ \pm} \mu^{\mp} \nu$. A plot of the $\gamma \gamma$ invariant mass value which is closest to the $\pi^{0}$ mass for each event is shown in Figure 5.12. 

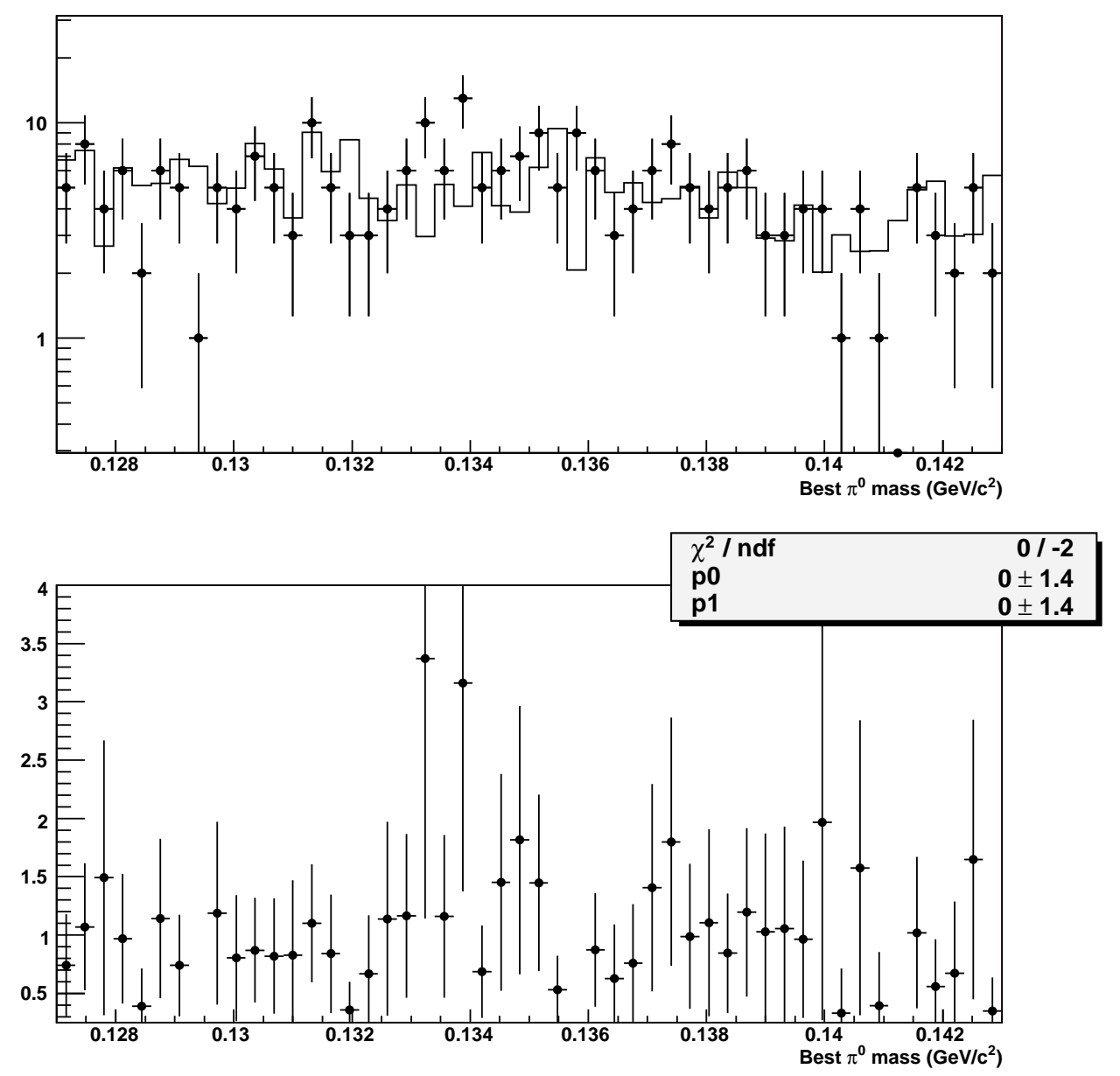

Figure 5.12: A plot of the best $\pi^{0}$ mass found in each event using two clusters in the CsI calorimeter, before the cut on this variable. Data are points, while the histogram is of Monte Carlo simulated events. The solid part of the histogram denotes the areas that pass after this particular cut. A plot of the ratio of the number of data events to Monte Carlo events, before this cut, is shown on the bottom. The number of Monte Carlo events is normalized to be equal to the total number of data events in this plot. Note that all events in this plot are removed by the $\pi^{0}$ mass cut. 


\section{$5.4 .13 \mathrm{E} / \mathrm{p}$}

The ratio of a track's cluster energy in the CsI to its momentum as measured in the magnetic spectrometer, known as "E/p", provides an excellent tool with which to discriminate different types of particles from one another. Electrons interact electromagnetically in the CsI, producing clusters that are tightly focused, allowing the cluster to collect the majority of the energy of the electron. Electrons then will have an $\mathrm{E} / \mathrm{p}$ spectrum that is sharply peaked at $E / p=1$. Muons on the other hand, have a much longer interaction length. The vast majority of these particles will minimally interact, and will only deposit approximately $0.4 \mathrm{GeV}[35]$ of energy into the CsI on average. Muons will then have an $\mathrm{E} / \mathrm{p}$ spectrum peaked slightly above $E / p=0$. Finally, some pions, with a mass roughly comparable to muons, will also pass through the CsI and leave little energy behind. These pions will also produce a peak near $E / p=0$. However, pions can also interact hadronically in the CsI. These hadronic clusters will be larger and more poorly defined compared to electromagnetic clusters, meaning the CsI will sample varying fractions of each pion's energy. These pions will produce a broad spectrum of $\mathrm{E} / \mathrm{p}$ values, from 0 up to 1, with a broad peak roughly near 0.5. The total pion $\mathrm{E} / \mathrm{p}$ spectrum will then have a sharp peak near 0 , with a broad shoulder peaking near 0.5 and stretching to $E / p=1$.

Rejecting events with large values of $\mathrm{E} / \mathrm{p}$ will quite effectively remove events with electrons. However, muons can't be removed with a cut on events with low E/p values without also losing a large portion of $K_{L, S} \rightarrow \pi^{+} \pi^{-} \gamma$. In general, the Monte Carlo cannot not perfectly reproduce the distribution of $\mathrm{E} / \mathrm{p}$ for pions, due to the extreme difficulty of simulating hadronic interactions in the CsI calorimeter, and hence, their energy deposition. The larger of the two E/p values for each event is plotted in Figure 5.13 . 

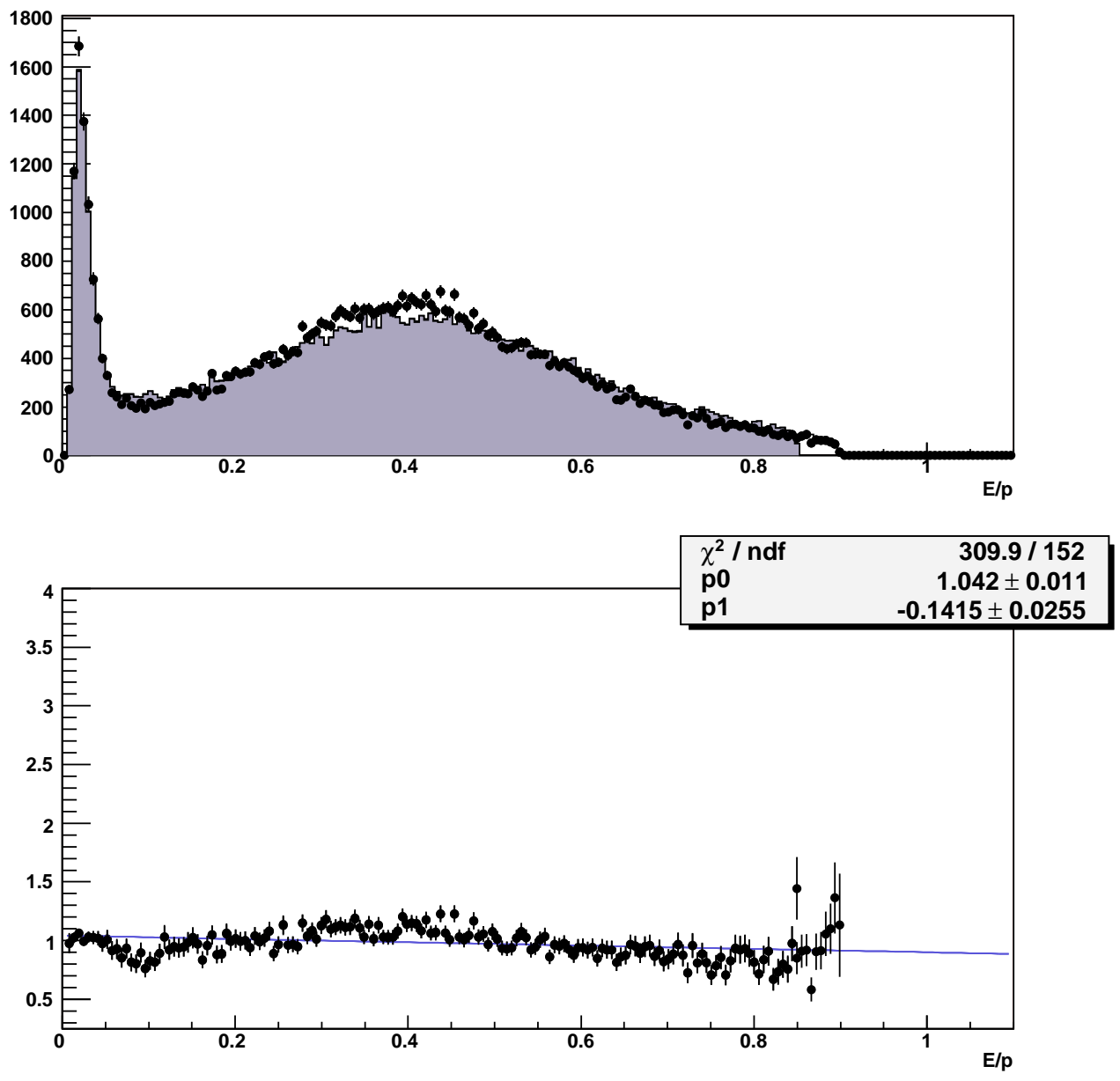

Figure 5.13: A plot of the greater of the two values of $\mathrm{E} / \mathrm{p}$ for each event, before the final E/p cut. Data are points, while the histogram is of Monte Carlo simulated events. The solid part of the histogram denotes the areas that pass after this particular cut. A plot of the ratio of the number of data events to Monte Carlo events, before this cut, is shown on the bottom. The number of Monte Carlo events is normalized to be equal to the total number of data events in this plot. A fit of the ratio using a linear function is also shown.

This distribution is not well reproduced by Monte Carlo, owing to difficulties in simulating hadronic interactions in the calorimeter, 


\subsubsection{Upstream track projection / $\gamma$ separation}

Another characteristic of electrons, other than $\mathrm{E} / \mathrm{p}$ values near unity, is the likelihood that they will emit bremsstrahlung radiation. If this radiation occurs when the electrons are upstream of the analysis magnet, the magnet will deflect the electron to a different trajectory, and the emitted photon will then produce an isolated CsI cluster as illustrated in Figure 5.14. If the radiation occurs downstream of the analysis magnet, the photon will overlap with the electron cluster in the CsI, and will not produce an isolated photon cluster.

For this reason, some additional $K_{L} \rightarrow \pi^{ \pm} e^{\mp} \nu$ events can be removed by rejecting events in which one of the upstream tracks, projected to the CsI, is near a photon cluster. This quantity is plotted in Figure 5.15.

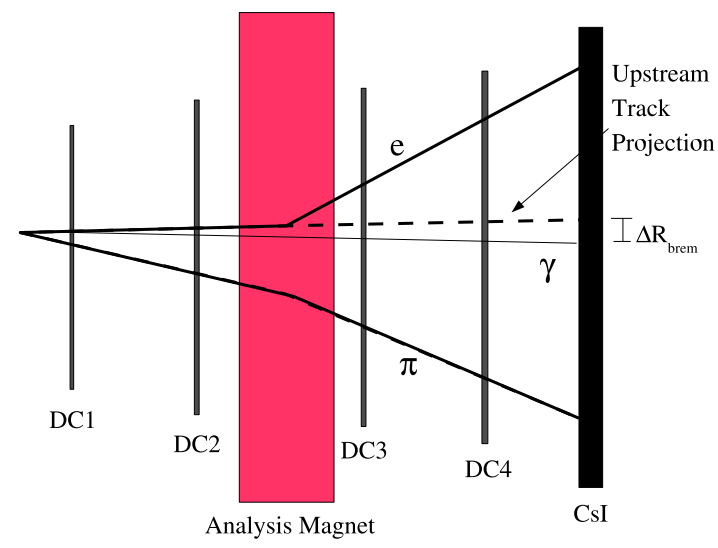

Figure 5.14: A cartoon of bremsstrahlung photon emission in a $K_{L} \rightarrow \pi^{ \pm} e^{\mp} \nu$ decay. 

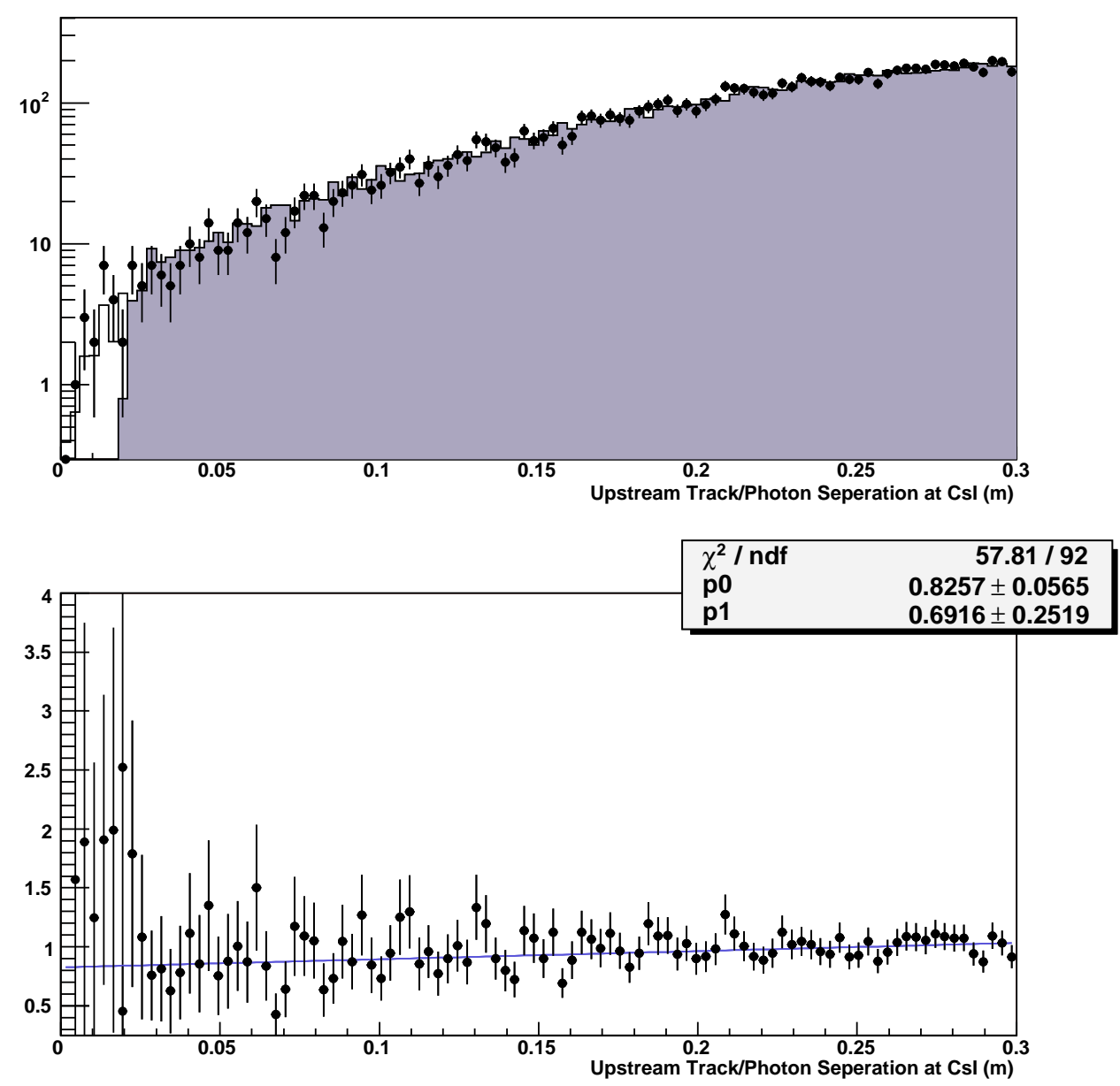

Figure 5.15: A plot of the upstream track/photon separation distance at the CsI before the cut. Data are points, while the histogram is of Monte Carlo simulated events. The solid part of the histogram denotes the areas that pass after this particular cut. A plot of the ratio of the number of data events to Monte Carlo events, before this cut, is shown on the bottom. The number of Monte Carlo events is normalized to be equal to the total number of data events in this plot. A fit of the ratio using a linear function is also shown. 


\subsubsection{Track Momentum}

While $\mathrm{E} / \mathrm{p}$ is an excellent method with which to remove electrons, we rely on the

muon veto in order to reject events with muons. Low energy muons( those with momenta less than $7 \mathrm{GeV} / \mathrm{c}$ ) will be absorbed by the steel filter, without triggering the muon veto, so we must remove these events by cutting on events with low track momentum. The minimum track momentum of both tracks in each event is plotted in Figure 5.16. 

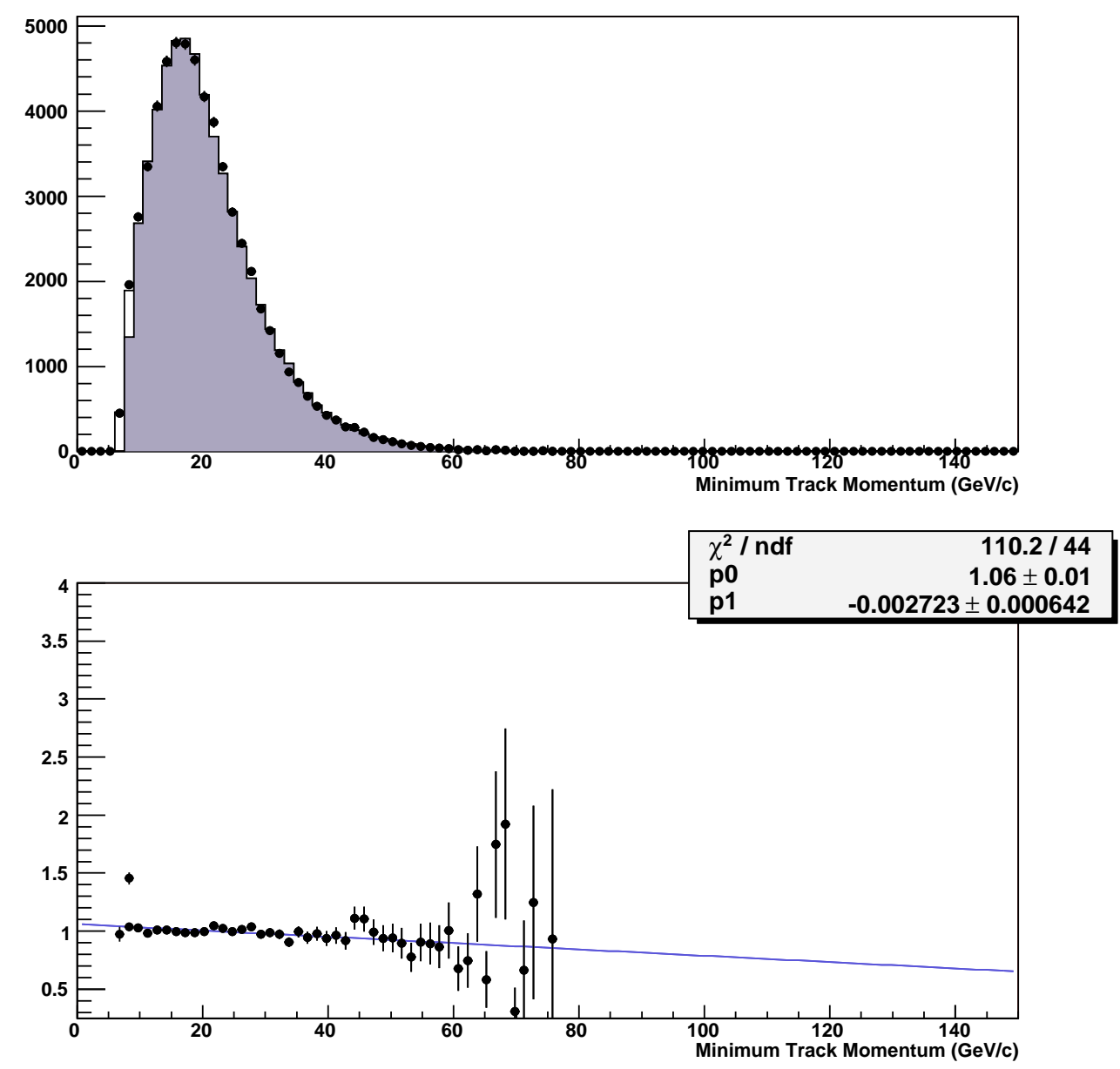

Figure 5.16: A plot of the lesser of two track momenta per event before the cut. Data are points, while the histogram is of Monte Carlo simulated events. The solid part of the histogram denotes the areas that pass after this particular cut. A plot of the ratio of the number of data events to Monte Carlo events, before this cut, is shown on the bottom. The number of Monte Carlo events is normalized to be equal to the total number of data events in this plot. A fit of the ratio using a linear function is also shown. 


\subsubsection{Early and in-time cluster energy}

In order for a photon candidate cluster to be a good candidate, it should coincide in time with the charged tracks. If there is a large amount of energy occurring before, or conversely, an insignificant amount of energy during during the "in-time" period, the cluster should be rejected. The energy in a CsI cluster is taken from an 120ns long integration time, and the in-time cluster energy which is cut on here is taken from the first 20ns of the integration. The early energy cut is applied to the time window 20ns prior to the in-time energy slice and will remove activity in the CsI which may extend into the in-time window. The early and in-time cluster energies are plotted in Figures 5.17 and 5.18.

This cut will help eliminate events that overlap with accidental photons, which will in turn help to reject non-radiative $K_{L} \rightarrow \pi^{ \pm} e^{\mp} \nu$ and $K_{L} \rightarrow \pi^{ \pm} \mu^{\mp} \nu$ events which depend on an accidental photon in order to pass the selection requirements. 

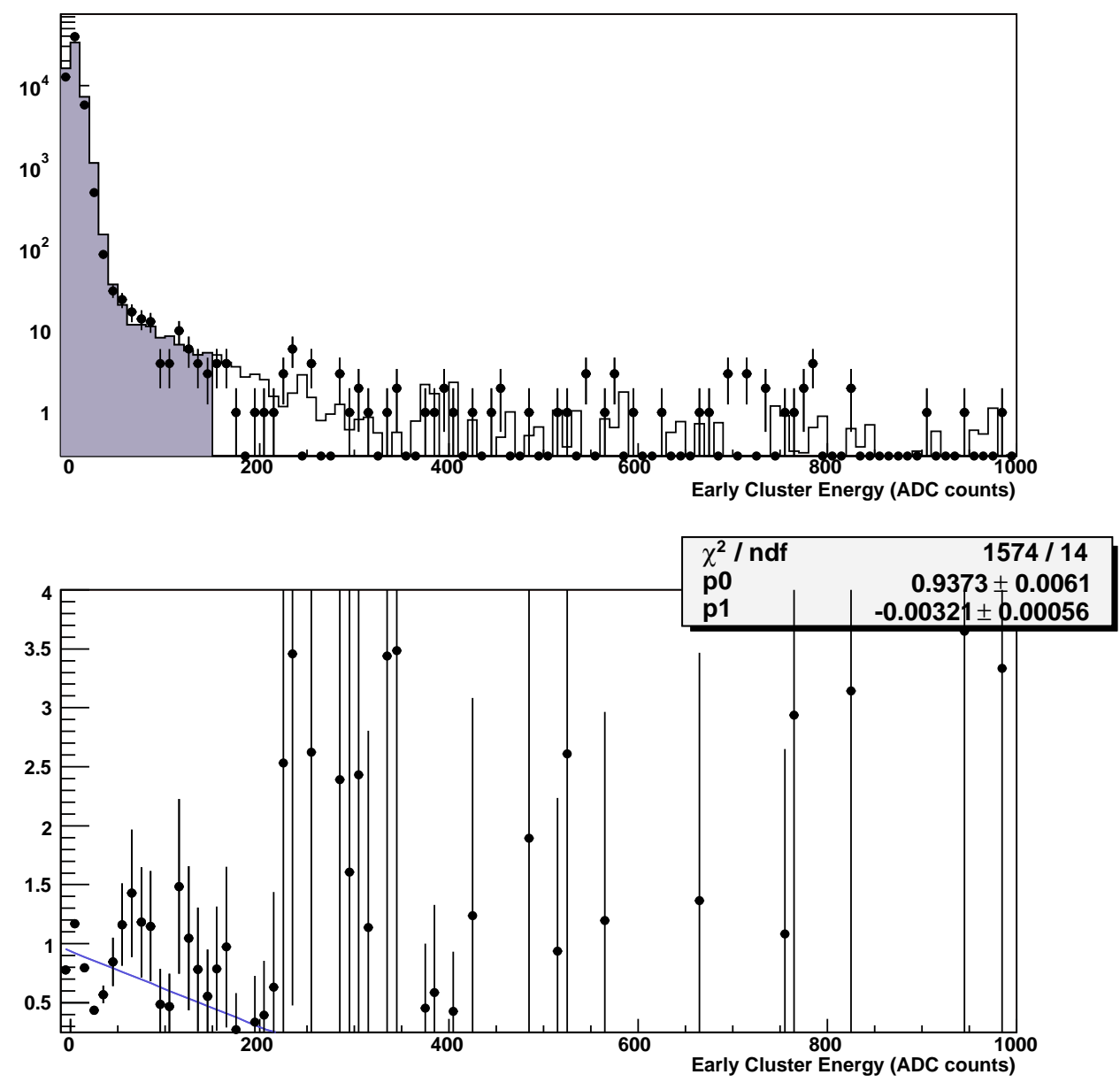

Figure 5.17: A plot of the "early" energy of the photon candidate cluster before the cut. Data are points, while the histogram is of Monte Carlo simulated events. The solid part of the histogram denotes the areas that pass after this particular cut. A plot of the ratio of the number of data events to Monte Carlo events, before this cut, is shown on the bottom. The number of Monte Carlo events is normalized to be equal to the total number of data events in this plot. A fit of the ratio using a linear function is also shown. 

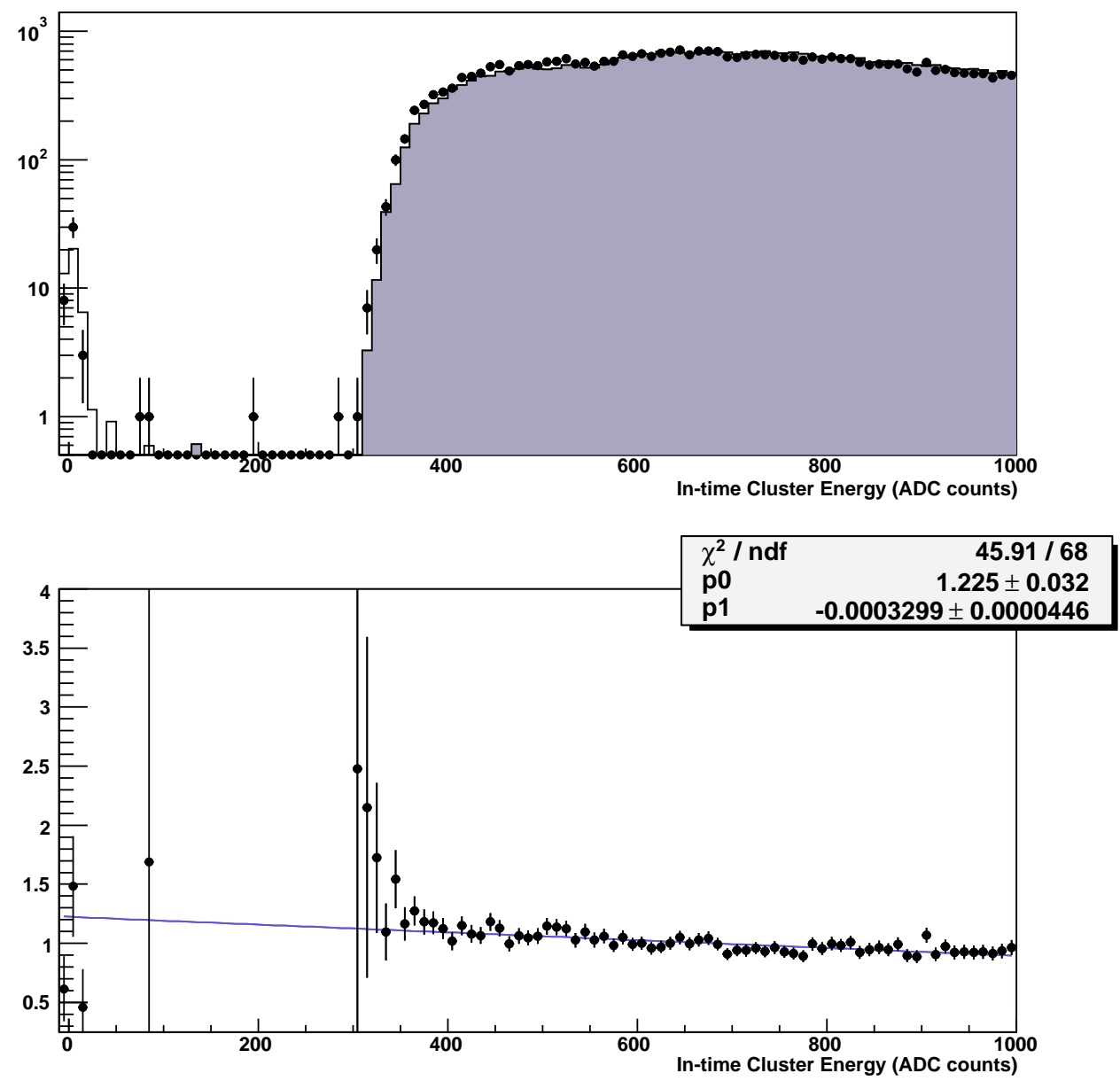

Figure 5.18: A plot of the "in-time" energy of the photon candidate cluster before the cut. Data are points, while the histogram is of Monte Carlo simulated events. The solid part of the histogram denotes the areas that pass after this particular cut. A plot of the ratio of the number of data events to Monte Carlo events, before this cut, is shown on the bottom. The number of Monte Carlo events is normalized to be equal to the total number of data events in this plot. A fit of the ratio using a linear function is also shown. 


\subsubsection{7 $p \pi$ invariant mass}

In addition to neutral kaons, a variety of neutral hyperons will also be created in the $\mathrm{KTeV}$ target, creating another possible source of background. $\Lambda$ particles are created in the target, the regenerator and as daughters of other hyperon decay such as $\Xi \rightarrow \Lambda \pi^{0}$ and $\Sigma \rightarrow \Lambda \gamma$. Thus, controlling the possible hyperon background involves suppressing $\Lambda$ decays, the most common of which is $\Lambda \rightarrow p \pi$. Noting that this is a two body decay involving hadronic daughter particles, this decay will not be considerably suppressed by other cuts used to suppress other background modes. However, the $p \pi$ invariant mass can be computed by assuming that the "stiffer" track is the one with the higher mass, and thus the most attractive proton candidate. A cut can then be made in the region of the $\Lambda$ mass. The $p \pi$ invariant mass is plotted in Figure 5.19. 

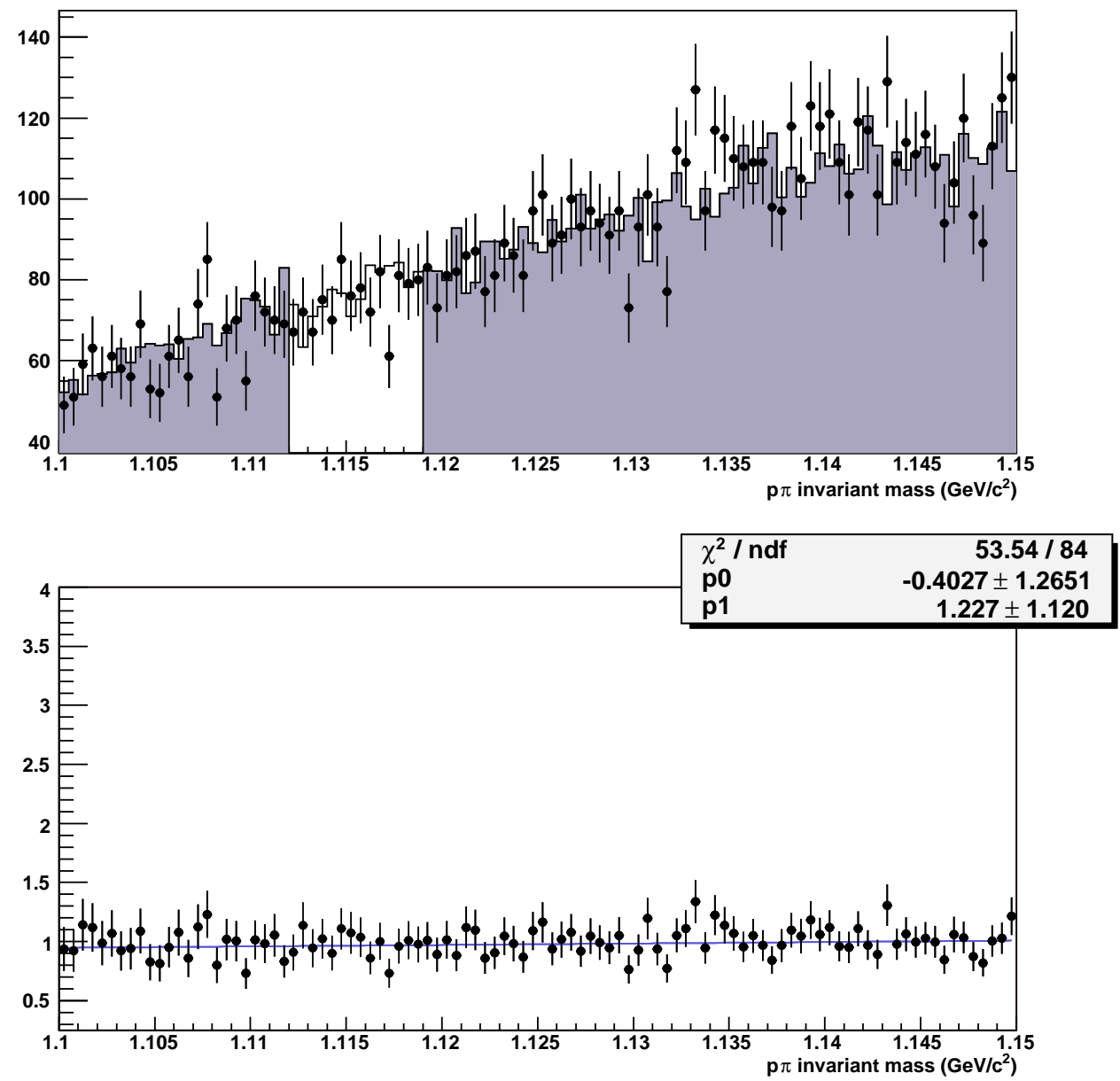

Figure 5.19: A plot of the proton/pion invariant mass before the cut. Data are points, while the histogram is of Monte Carlo simulated events. The solid part of the histogram denotes the areas that pass after this particular cut. A plot of the ratio of the number of data events to Monte Carlo events, before this cut, is shown on the bottom. The number of Monte Carlo events is normalized to be equal to the total number of data events in this plot. A fit of the ratio using a linear function is also shown. 


\subsubsection{Photon Cluster Location in the CsI}

In order to prevent energy leakage out of the edges of the calorimeter, we remove combinations in which the candidate photon cluster is near the outer edge of the CsI calorimeter. The units of this cut variable, called ISEEDRING, are large CsI blocks, where a cluster with a seed in the outermost layer of CsI blocks has ISEEDRING= 19. A cut at $I S E E D R I N G<18.1$ would then require that the cluster's seed is at least one large block away from the edge of the calorimeter.

In order to prevent energy leakage out of the inner edges of the calorimeter ( the beam holes ) we also remove combinations in which the candidate cluster is near the CsI's beam holes. Here, the units of distance used are the number of small CsI blocks between the seed of the candidate photon cluster and the nearest beam hole. The smallest value any cluster can have is 4 , which indicates that the seed is in a block adjacent to the beam hole. The name of this cut variable is ISMLRING. Both ring variables are plotted in Figures 5.20 and 5.21. 

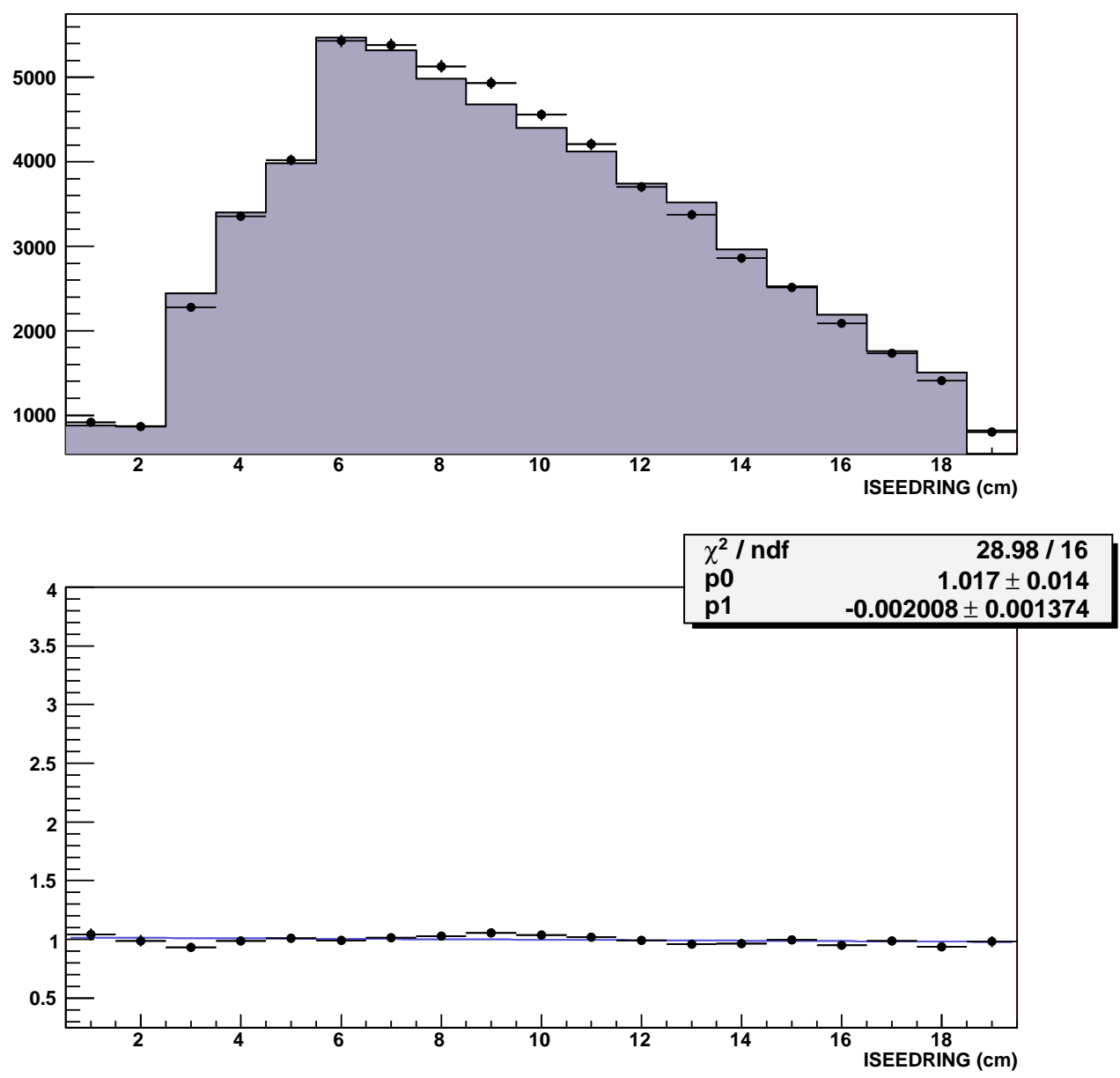

Figure 5.20: A plot of the outer photon fiducial cut variable ISEEDRING, before the cut. Data are points, while the histogram is of Monte Carlo simulated events. The solid part of the histogram denotes the areas that pass after this particular cut. A plot of the ratio of the number of data events to Monte Carlo events, before this cut, is shown on the bottom. The number of Monte Carlo events is normalized to be equal to the total number of data events in this plot. A fit of the ratio using a linear function is also shown. 

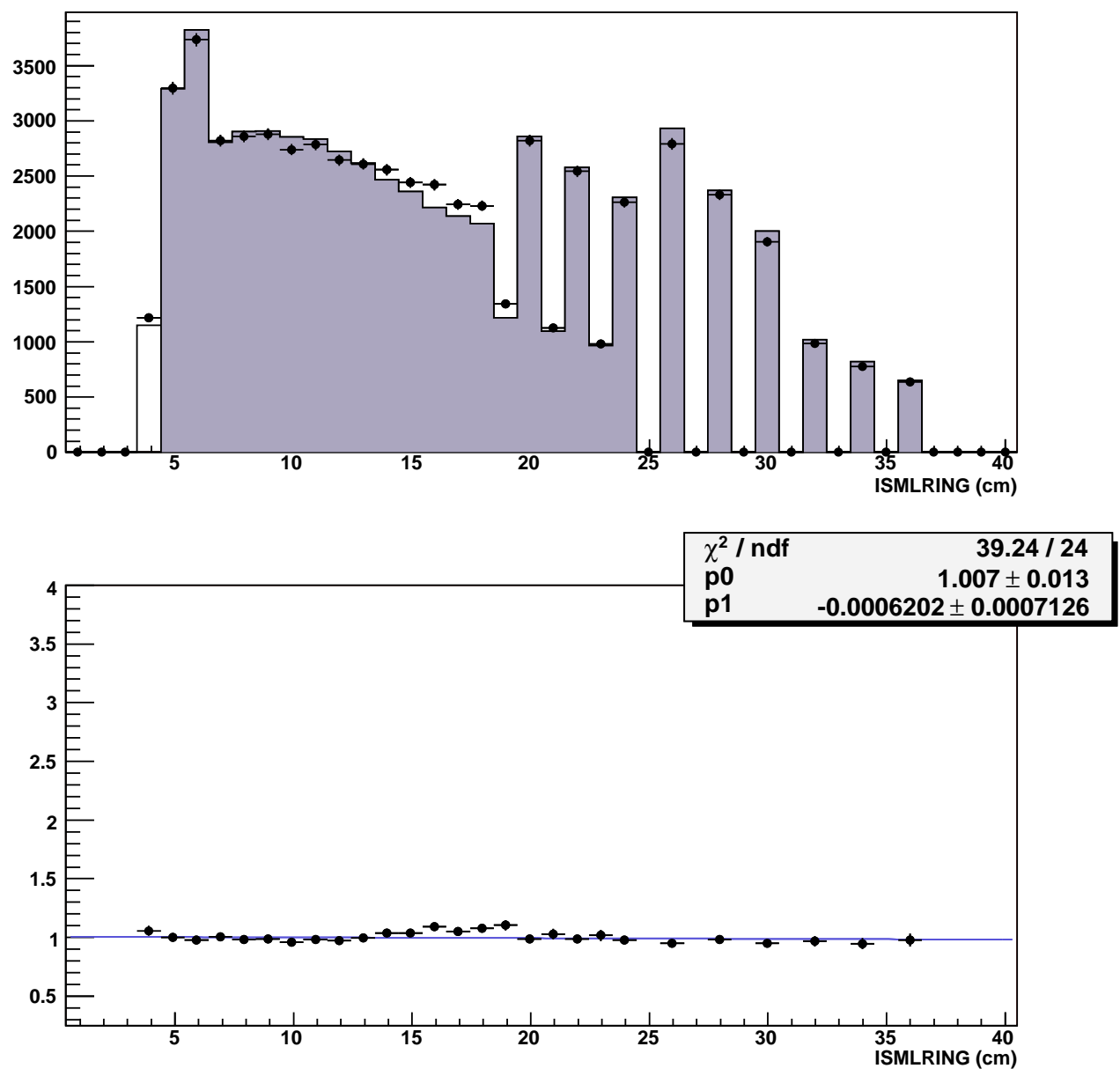

Figure 5.21: A plot of the inner photon fiducial cut variable ISMLRNG before the cut. Data are points, while the histogram is of Monte Carlo simulated events. The solid part of the histogram denotes the areas that pass after this particular cut. A plot of the ratio of the number of data events to Monte Carlo events, before this cut, is shown on the bottom. The number of Monte Carlo events is normalized to be equal to the total number of data events in this plot. A fit of the ratio using a linear function is also shown. 


\subsubsection{Vertex $\chi^{2}$}

The value of $\chi_{V E R T E X}^{2}$ describes how well the two tracks matched to form a common decay vertex in the $\mathrm{X}$ and $\mathrm{Y}$ views. Smaller values indicate a more precise vertex determination. Removing events with large values of $\chi_{V E R T E X}^{2}$ eliminates events for which the decay vertex is not well localized. Like most variables which are defined as $\chi^{2}$, the $\mathrm{KTeV}$ Monte Carlo does not reliably simulate the distribution of values for $\chi_{V E R T E X}^{2}$ owing to the difficulty in estimating the size of the errors which appear in the denominator of the definition of the $\chi^{2}$ characteristic. The value of $\chi_{V E R T E X}^{2}$ for each event is plotted in Figure 5.22. 

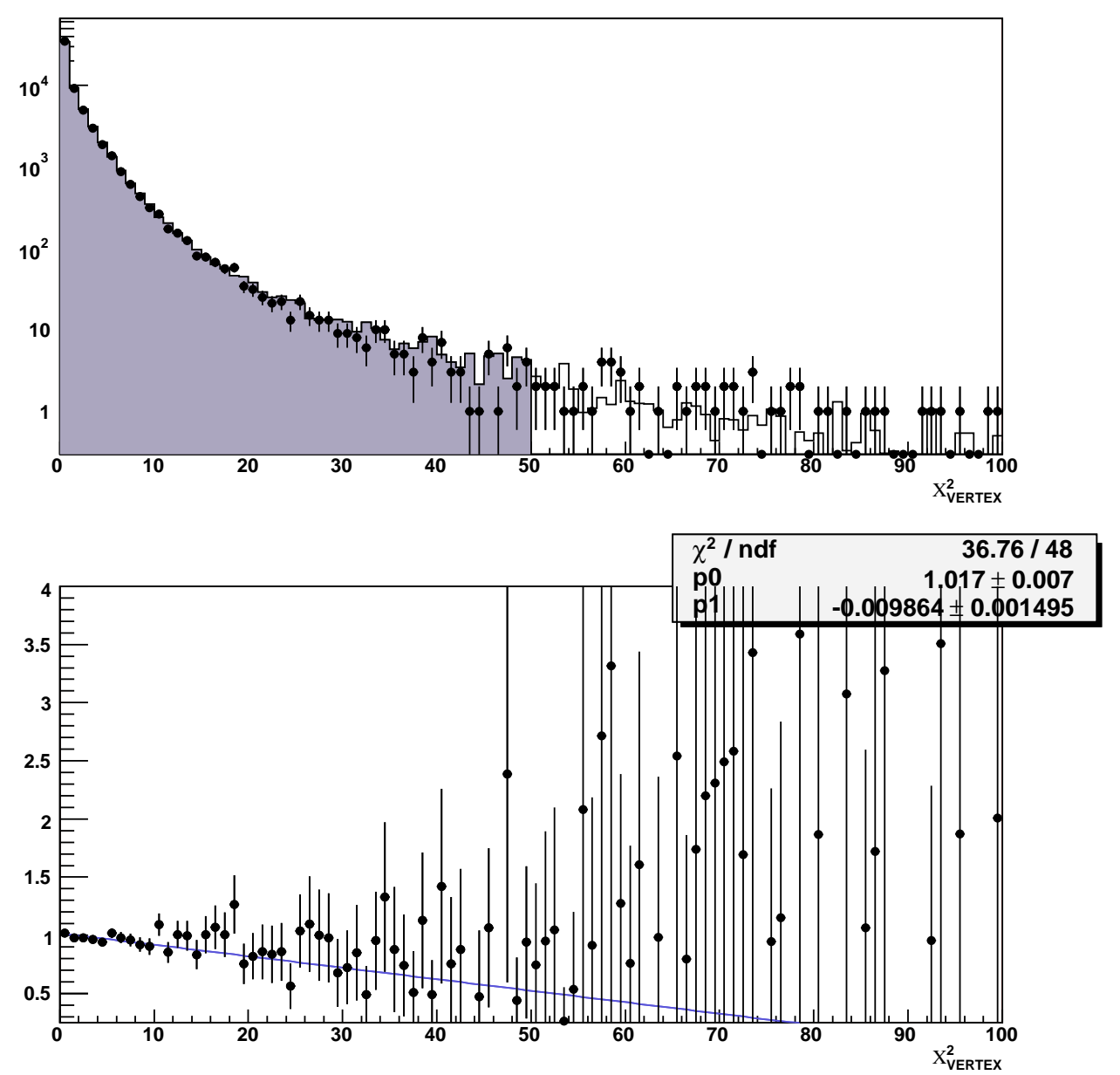

Figure 5.22: A plot of vertex $\chi^{2}$ before the cut. Data are points, while the histogram is of Monte Carlo simulated events. The solid part of the histogram denotes the areas that pass after this particular cut. A plot of the ratio of the number of data events to Monte Carlo events, before this cut, is shown on the bottom. The number of Monte Carlo events is normalized to be equal to the total number of data events in this plot. A fit of the ratio using a linear function is also shown. 


\subsubsection{Track Offset $\chi^{2}$}

The value of $\chi_{O F F S E T}^{2}$ for each track describes how closely the upstream and downstream track segments match in the center of the analysis magnet, and is given by:

$$
\chi_{O F F S E T}^{2}=\left(\frac{\Delta x}{\sigma_{x}}\right)^{2}+\left(\frac{\Delta y}{\sigma_{y}}\right)^{2}
$$

where $\Delta x$ and $\Delta y$ are the offsets between the upstream and downstream track segments, when projected to the magnet, and $\sigma_{x}$ and $\sigma_{y}$ are the estimated uncertainties due to track reconstruction in the offset values, taking into account the number of hits used and multiple scattering effects.

Removing events with larger values of $\chi_{O F F S E T}^{2}$ eliminates events for which the magnetic deflection and hence the momentum of a track is poorly reconstructed. The Track Offset $\chi^{2}$ is plotted in Figure 5.23. 

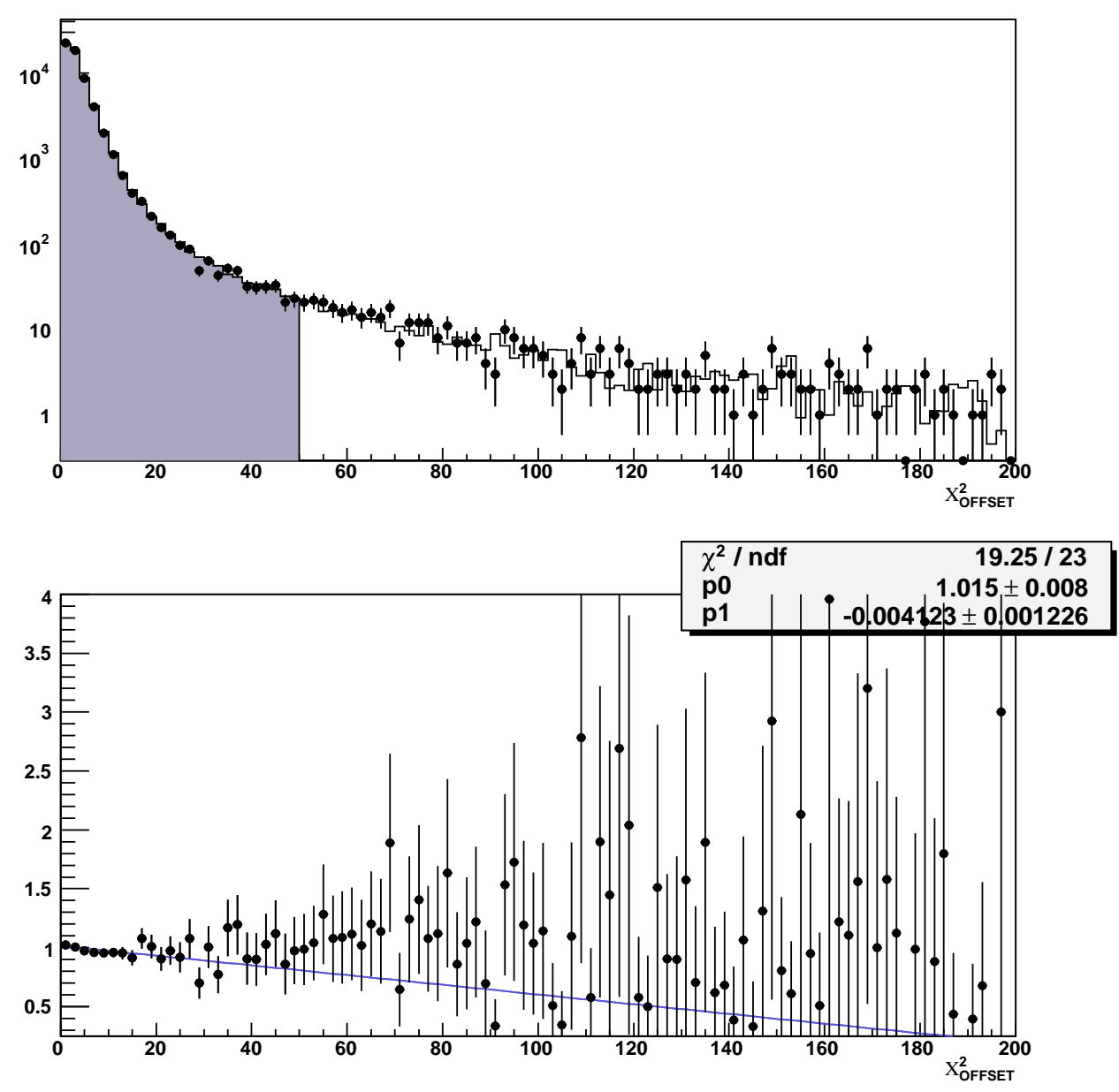

Figure 5.23: A plot of the greater of the two track offset $\chi^{2}$ values in each event before the cut. Data are points, while the histogram is of Monte Carlo simulated events. The solid part of the histogram denotes the areas that pass after this particular cut. A plot of the ratio of the number of data events to Monte Carlo events, before this cut, is shown on the bottom. The number of Monte Carlo events is normalized to be equal to the total number of data events in this plot. A fit of the ratio using a linear function is also shown. 


\subsubsection{Track Separation}

It is necessary to examine the track separation at the CsI in both directions. If either of the track's projections to the CsI fall within a certain distance to each other in either the $\mathrm{X}$ or $\mathrm{Y}$ direction, the $\mathrm{X}$ or $\mathrm{Y}$ track candidates may be interchanged, preventing the tracking from being done correctly. Additionally, if the radial separation between the track projections is too small, the tracks' clusters will overlap, and an accurate value of $\mathrm{E} / \mathrm{p}$, needed for particle ID, will be impossible to obtain. For these reasons, we cut on the X,Y and radial separation of the tracks at the CsI. Plots of the X,Y and radial track separations can be found in Figures 5.24, 5.25 and 5.26. 

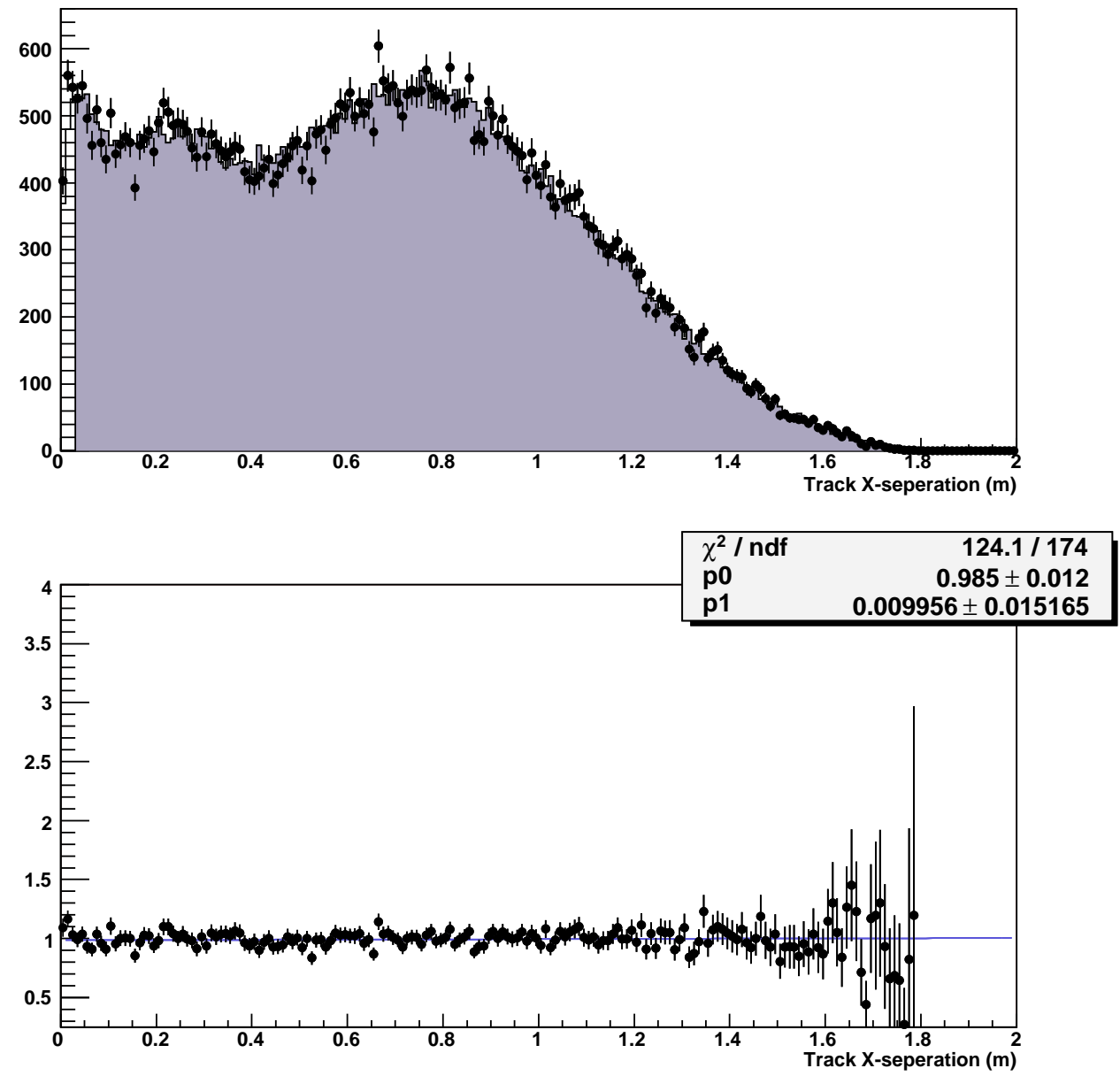

Figure 5.24: A plot of the separation of the tracks in the $\mathrm{x}$ direction at the CsI, before the cut. Data are points, while the histogram is of Monte Carlo simulated events. The solid part of the histogram denotes the areas that pass after this particular cut. A plot of the ratio of the number of data events to Monte Carlo events, before this cut, is shown on the bottom. The number of Monte Carlo events is normalized to be equal to the total number of data events in this plot. A fit of the ratio using a linear function is also shown. 

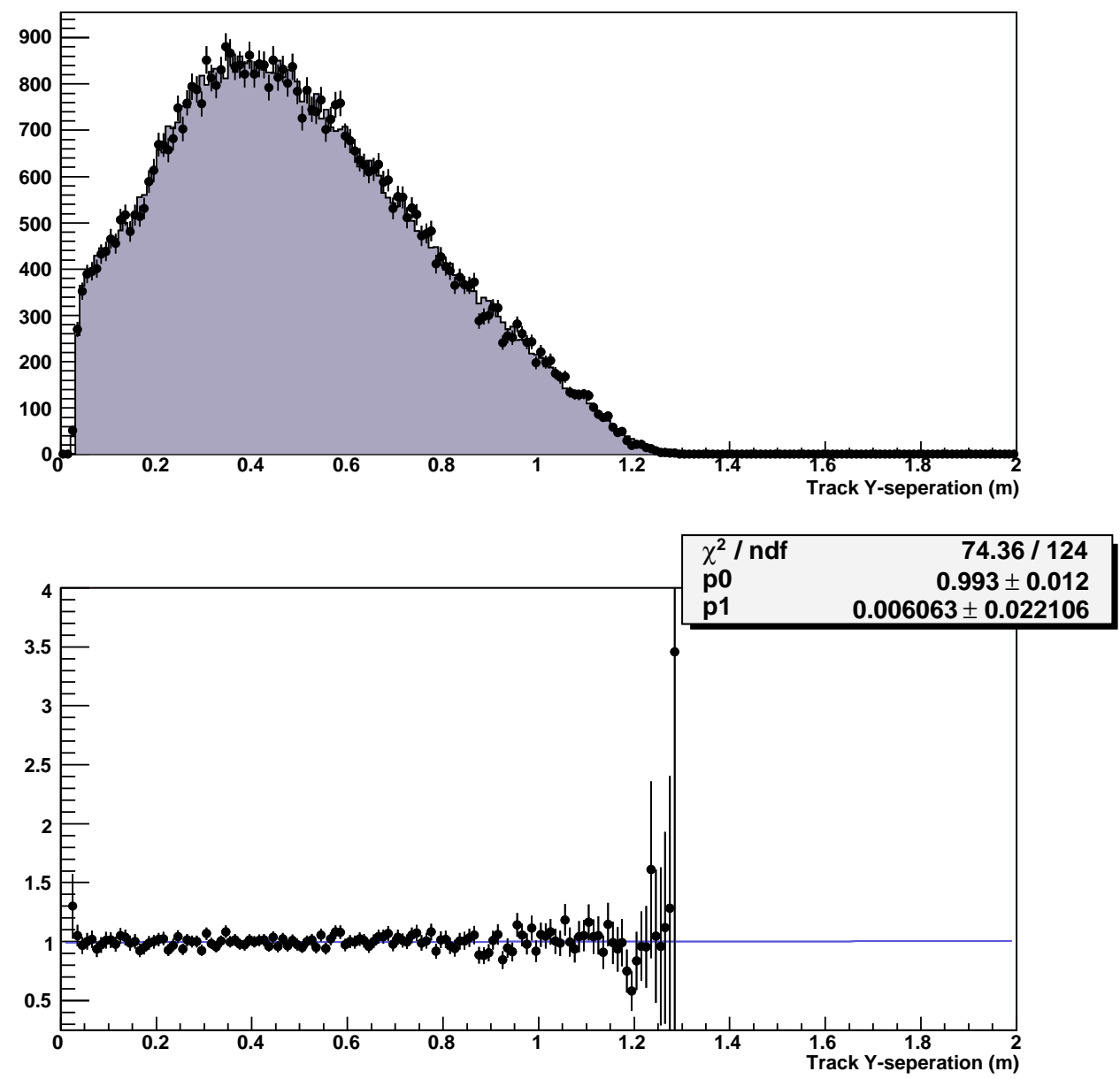

Figure 5.25: A plot of the separation of the tracks in the y direction at the CsI, before the cut. Data are points, while the histogram is of Monte Carlo simulated events. The solid part of the histogram denotes the areas that pass after this particular cut. A plot of the ratio of the number of data events to Monte Carlo events, before this cut, is shown on the bottom. The number of Monte Carlo events is normalized to be equal to the total number of data events in this plot. A fit of the ratio using a linear function is also shown. 

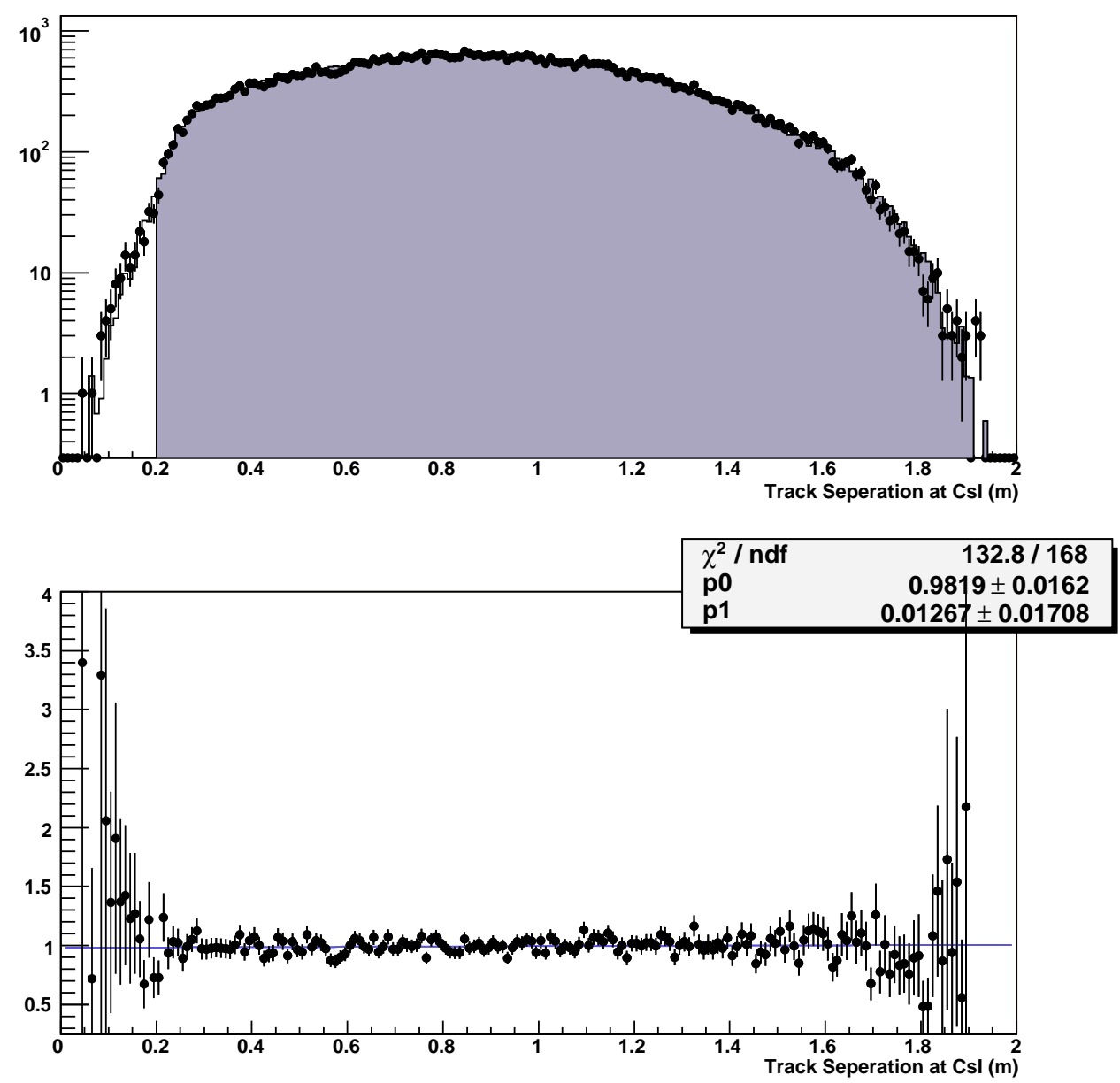

Figure 5.26: A plot of the track separation at the CsI before the cut. Data are points, while the histogram is of Monte Carlo simulated events. The solid part of the histogram denotes the areas that pass after this particular cut. A plot of the ratio of the number of data events to Monte Carlo events, before this cut, is shown on the bottom. The number of Monte Carlo events is normalized to be equal to the total number of data events in this plot. A fit of the ratio using a linear function is also shown. 


\subsubsection{Veto Cuts}

The numerous veto counters in the experiment are designed to reject background decays. While some veto detectors are not used, most are useful and their behavior is well understood. The veto detectors which are used in this analysis are:

RC The Ring-Counters are used to also remove events with extra charged particles or extra photons that escape the fiducial volume of the detector.

MA The Mask-Anti was used to reject decays upstream of the regenerator in both beams.

SA/CIA vetoes The Spectrometer-Antis and CsI-Anti are both used to veto events with extra charged particles and extra photons that escape the fiducial volume of the detector.

Regenerator The downstream and upstream parts of the regenerator veto are both used to reject decays upstream and inside of the regenerator, as well as to veto events in which the kaon regeneration is not coherent (i.e. inelastic scatters in the regenerator). The final part of the regenerator veto, that downstream of the final $\mathrm{Pb}$ section, is crucial in vetoing neutral decays upstream and inside of the regenerator.

\subsubsection{Fiducial Cuts}

The numerous fiducial cuts are meant to limit activity in the detector to regions in which it is well understood, and to thus allow an accurate acceptance simulation. The trajectory of the charged tracks are constrained by the following cuts:

VV' clearance Both tracks must pass no closer than $2.0 \mathrm{~mm}$ within the beam holes in the VV' counter, which ensures they are detected by the VV' array.

CsI outside edge clearance Both tracks must pass no closer than $29.0 \mathrm{~mm}$ from the outside edge of the calorimeter. This ensures that the tracks pass within the CsI, and can be matched to clusters for particle ID. 
CA clearance Both tracks must pass outside the CA veto by at least $2.0 \mathrm{~mm}$. This ensures that the tracks pass within the CsI, and can be matched to clusters for particle ID.

Track Separation in DC In order to keep the track efficiency high, the tracks are required to be separated by at least 3 wire cells in each drift chamber.

DC edge clearance In order to keep the tracks within the central region of each drift chamber in which the position resolution is acceptable, both charged tracks are required to pass a certain distance from the outside of each drift chamber. This distance is different for each drift chamber. 


\section{Chapter 6}

\section{Background Determination}

\subsection{Total Size of Background}

The number of background events in the four different samples can be estimated using the plots of $M_{\pi^{+} \pi^{-} \gamma}$ and $P_{T}^{2}$. Events which reconstruct with high or low invariant mass will contribute to the side bands of the kaon mass peak in the $M_{\pi^{+} \pi^{-} \gamma}$ plot as can be seen in Figure 6.1, and will be composed of events with missing energy and/or particles. Events which reconstruct with higher values of $P_{T}^{2}$ will contribute to the sideband of the $P_{T}^{2}$ plot, and will be composed of events with missing energy and/or particles, and will also include otherwise good $K_{L, S} \rightarrow \pi^{+} \pi^{-} \gamma$ events in which the kaons have undergone scattering.

In order to obtain a background estimate from data, the $M_{\pi^{+} \pi^{-} \gamma}$ plot is fit with a combination of the $M_{\pi^{+}} \pi^{-} \gamma$ shape taken from Monte Carlo events plus the sum of a decaying exponential distribution and a linear distribution. All components are varied until a best fit is obtained, and then the background estimate is integrated over the range of the kaon mass peak. The estimated number of events using this method are shown in Table 6.1 while the plots themselves are shown in Figure 6.1 


\begin{tabular}{|l|l|l|l|}
\hline Beam & Year & $\begin{array}{l}\text { Estimated Number } \\
\text { Of Background Events }\end{array}$ & $\begin{array}{l}\text { Total Number } \\
\text { Of Events }\end{array}$ \\
\hline Regenerator & 1997 & $20 \pm 5$ & 58755 \\
Regenerator & 1999 & $25 \pm 5$ & 75666 \\
Vacuum & 1997 & $44_{-14}^{+24}$ & 75506 \\
Vacuum & 1999 & $98 \pm 36$ & 97276 \\
\hline \hline Total & Total & $187 \pm 44$ & 307203 \\
\hline
\end{tabular}

Table 6.1: Background Estimates from Invariant Mass Plot 


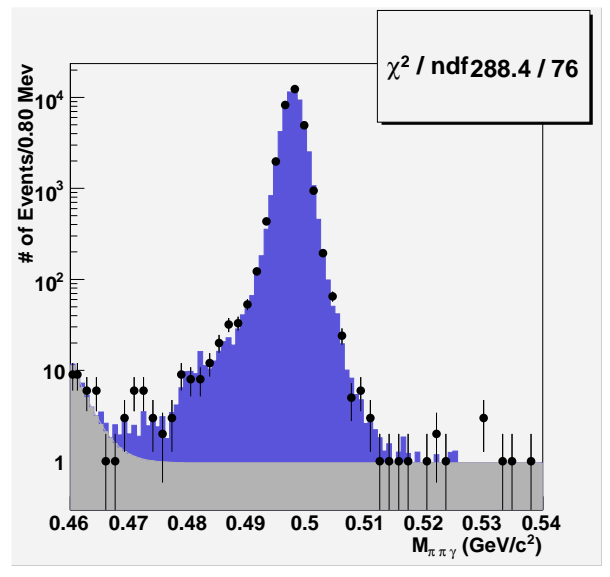

(a) 1997 Regenerator Beam

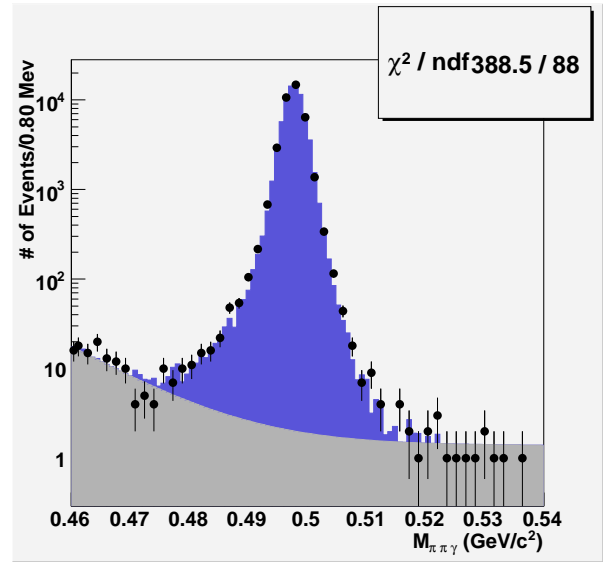

(c) 1997 Vacuum Beam

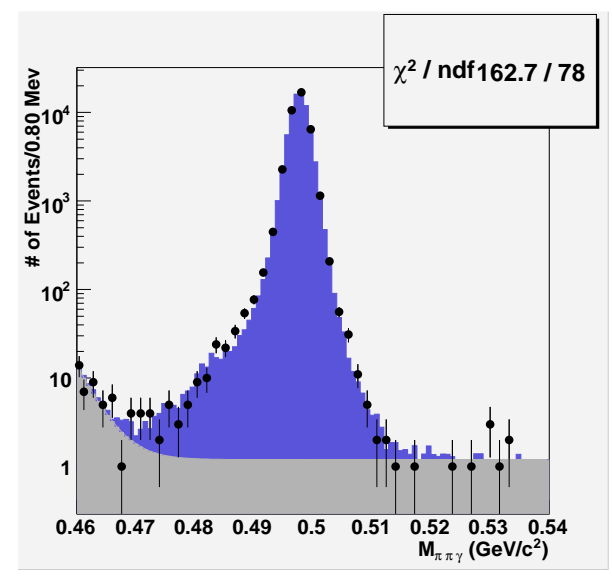

(b) 1999 Regenerator Beam

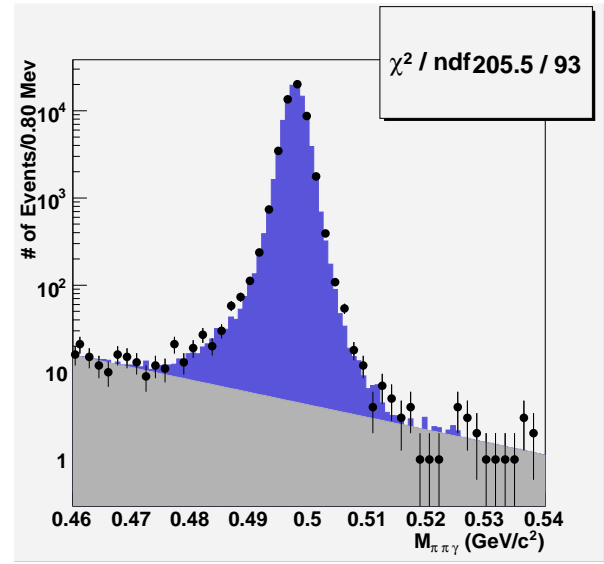

(d) 1999 Vacuum Beam

Figure 6.1: Invariant mass plots of the 4 data samples shown with the fitted background component. Points are data, the light gray area represents the estimated background, and the dark histogram is the sum of the background and Monte Carlo $K_{L, S} \rightarrow \pi^{+} \pi^{-} \gamma$ events. 
We can also perform a cross check by examining the plot of $P_{T}^{2}$. As with the $M_{\pi^{+} \pi^{-} \gamma}$ plot, the data is fit with a combination of Monte Carlo events plus the sum of a decaying exponential and a linear function. All components are varied until a best fit is obtained, and then the background term is integrated under the $P_{T}^{2}$ peak in order to obtain a second background estimate which in this case also includes scattered events. The estimated background found using this technique can be found in Table 6.2 while the plots, including the background estimation can be found in Figure 6.2

\begin{tabular}{|l|l|l|l|}
\hline Beam & Year & $\begin{array}{l}\text { Estimated Number } \\
\text { Of Background Events }\end{array}$ & $\begin{array}{l}\text { Total Number } \\
\text { Of Events }\end{array}$ \\
\hline Regenerator & 1997 & $18 \pm 7$ & 58755 \\
Regenerator & 1999 & $35 \pm 8$ & 75666 \\
Vacuum & 1997 & $82 \pm 11$ & 75506 \\
Vacuum & 1999 & $88 \pm 7$ & 97276 \\
\hline Total & Total & $223 \pm 17$ & 307203 \\
\hline
\end{tabular}

Table 6.2: Background Estimates from $P_{T}^{2}$ Plot 


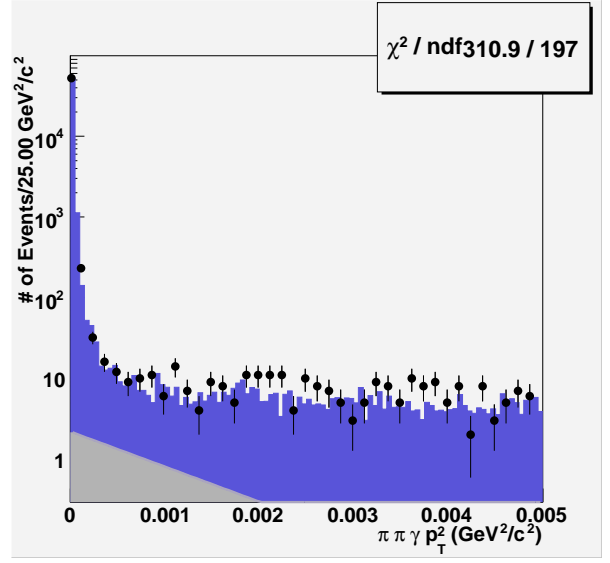

(a) 1997 Regenerator Beam

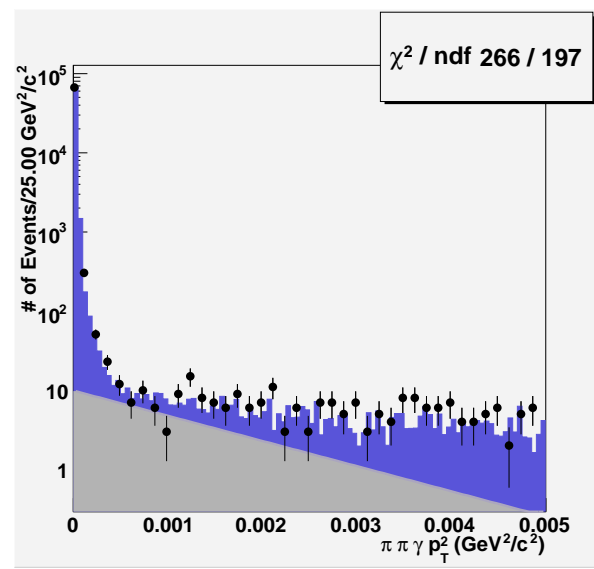

(c) 1997 Vacuum Beam

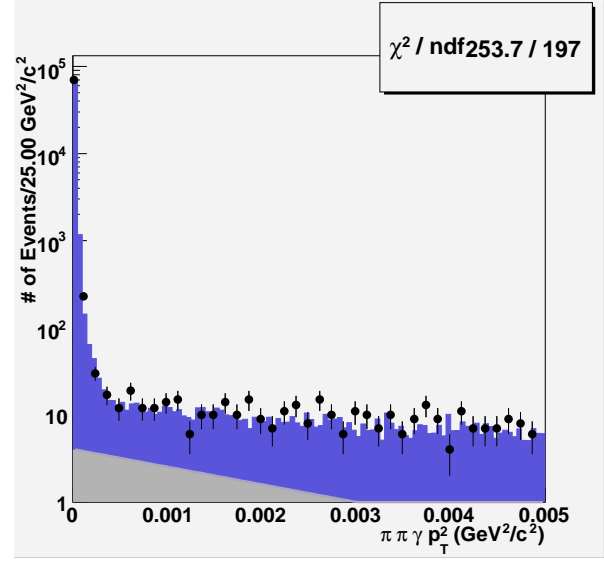

(b) 1999 Regenerator Beam

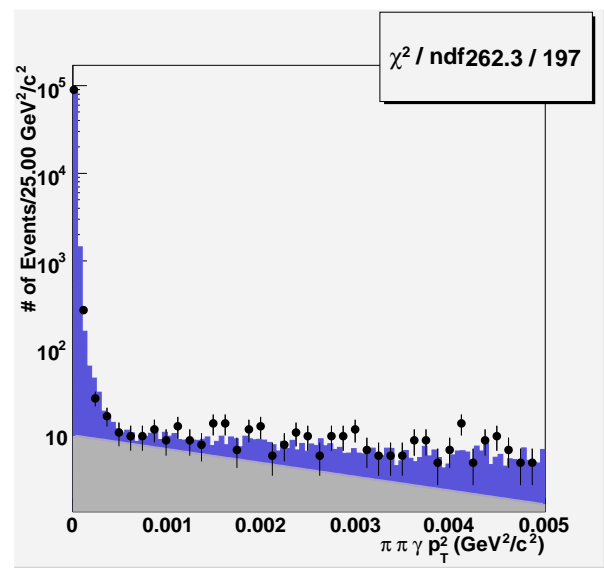

(d) 1999 Vacuum Beam

Figure 6.2: $P_{T}^{2}$ plots of the 4 data samples shown with the fitted background component. Points are data, the light gray area represents the estimated background, and the dark histogram is the sum of the background and Monte Carlo $K_{L, S} \rightarrow \pi^{+} \pi^{-} \gamma$ events. The $K_{L, S} \rightarrow \pi^{+} \pi^{-} \gamma$ Monte Carlo used as the signal component in this fit includes clean signal events plus full scattering simulations, which includes collimator scattering as well as regenerator scattering. The rate at which these scattering processes occur is determined by the Monte Carlo simulation and is not adjusted in the fit. The level at which these processes occur is explored in Section 6.4 
While the requirements placed on the data sample yield a relatively clean sample of $K_{L, S} \rightarrow \pi^{+} \pi^{-} \gamma$ events, there still remains a background at the $0.073 \%$ level in the entire data sample that may contaminate the signal. There are four classes of background:

Background Kaon decays These are kaon decays other than $K_{L, S} \rightarrow \pi^{+} \pi^{-} \gamma$. Often these must coincide with accidental activity in order to pass the selection criteria

Non-kaon decays These include hyperon decays.

Scattered $K_{L, S} \rightarrow \pi^{+} \pi^{-} \gamma$ decays These are $K_{L, S} \rightarrow \pi^{+} \pi^{-} \gamma$ decays in which the the kaon is scattered-resulting in a modification of the kaon wavefunction. These may include inelastic regenerator scattering, diffractive regenerator scattering and collimator scattering.

Modified $K_{L, S} \rightarrow \pi^{+} \pi^{-} \gamma$ decays These are decays in which the dynamics of the $K_{L, S} \rightarrow \pi^{+} \pi^{-} \gamma$ are poorly reconstructed, either by mis-identifying the photon calorimeter cluster, or by missing a second radiative photon in a $K_{L, S} \rightarrow \pi^{+} \pi^{-} \gamma \gamma$ decay.

\subsection{Background kaon decays}

\subsection{1 $\quad K_{L} \rightarrow \pi^{ \pm} e^{\mp} \nu$}

The semileptonic decay $K_{L} \rightarrow \pi^{ \pm} e^{\mp} \nu$ can contribute to the background of $K_{L, S} \rightarrow \pi^{+} \pi^{-} \gamma$ when the electron is misidentified. The decay must also be accompanied by a photon, which can come from accidental activity, hadronic interactions in the detector, or bremsstrahlung radiation from the electron. Note that the bremsstrahlung can occur during the kaon decay (inner bremsstrahlung) or when the electron's trajectory is bent by the analysis magnet.

In an attempt to estimate the contribution of this mode to the background, large samples of Monte Carlo events were generated for both years and beams. During generation, PHOTOS was used to simulate radiative corrections. Hadronic interactions in the detector where also simulated, as was accidental activity in the detector. 
36,440,142,309 events (2.6 times the expected number) were generated for the 1997 vacuum beam, 36,096,869,497 events (2.4 times the expected number) were generated for the 1999 vacuum beam sample, 3,552,275,845 events (3.2 times the expected number) were generated for the 1997 regenerator beam sample, and 3,907,431,749 events (3.2 times the expected number) were generated for the 1999 regenerator beam sample. These decays were generated in the ranges $30 \mathrm{GeV} / \mathrm{c}^{2}<p_{K}<162 \mathrm{GeV} / \mathrm{c}^{2}$ and $115 m<Z_{\text {vertex }}<160 m$.

After the generated samples are subjected to all final analysis cuts, 1 event remains in the 1997 regenerator beam sample, 5 events remain in the 1999 regenerator beam sample, 15 events remain in the 1997 vacuum beam sample, and 16 events remain in the 1999 vacuum beam sample.

Among the events remaining after all cuts, some are accepted due to hadronic interactions in the VV' trigger counter. When pions hadronically interact in the VV' counters, the shower may extend downstream into the CsI and result in one or more clusters being formed from the debris. Any of these clusters may be used as a photon cluster candidate. In the 1997 sample, 3 events in the vacuum sample are of this type. For the 1999 sample, 1 event in the regenerator beam and 4 in the vacuum beam were due to interactions in the $\mathrm{VV}^{\prime}$ counter. The remainder were events in which an accidental photon allowed the event to be accepted.

\subsection{2 $K_{L} \rightarrow \pi^{ \pm} \mu^{\mp} \nu$ with an accidental photon}

The semileptonic decay $K_{L} \rightarrow \pi^{ \pm} \mu^{\mp} \nu$ can also contribute to the background. While $\mathrm{KTeV}$ includes muon detectors which can be used to veto this decay, they are not $100 \%$ efficient in detecting muons, thus they are not $100 \%$ efficient in rejecting them. Another cut which is particularly effective in rejecting muons is to remove events for which a particular track's associated CsI cluster has a energy of less than $2 \mathrm{GeV}-$ which indicates that the particle was minimum ionizing. However, a large percentage of charged pions are also minimum ionizing, preventing this cut from being used due to the loss in statistics which would result. Thus, only the muon detectors are used to identify and reject muons. Except in the case of the radiative decay, the photon must be supplied by an accidental photon, or by a hadronic interaction in the spectrometer. A large sample of Monte Carlo generated $K_{L} \rightarrow \pi^{ \pm} \mu^{\mp} \nu$ event was generated in order 


\begin{tabular}{|l|c|c|c|c|}
\hline \multicolumn{1}{|c|}{ Cut Variable } & \multicolumn{3}{|c|}{ Relative loss due to only this cut (\%) } \\
\hline & 97REG & 99REG & 97VAC & 99VAC \\
\hline$\pi^{0}$ mass & $50.00 \pm 35.36$ & $0.00 \pm 0.00$ & $6.25 \pm 6.05$ & $5.88 \pm 5.71$ \\
\hline$Z_{\text {vertex }}$ & $0.00 \pm 0.00$ & $0.00 \pm 0.00$ & $25.00 \pm 9.68$ & $20.00 \pm 8.94$ \\
\hline$M_{\pi^{+} \pi^{-} \gamma}$ & $85.71 \pm 13.23$ & $50.00 \pm 15.81$ & $72.22 \pm 6.10$ & $83.33 \pm 3.80$ \\
\hline$P_{T}^{2}(\mathrm{VAC})$ & $96.43 \pm 3.51$ & $96.21 \pm 1.66$ & $95.15 \pm 1.22$ & $98.25 \pm 0.43$ \\
\hline$p_{K}$ & $0.00 \pm 0.00$ & $28.57 \pm 17.07$ & $21.05 \pm 9.35$ & $33.33 \pm 9.62$ \\
\hline$E_{\gamma}^{*}$ & $50.00 \pm 35.36$ & $16.67 \pm 15.21$ & $0.00 \pm 0.00$ & $5.88 \pm 5.71$ \\
\hline$E_{\gamma}^{L A B}$ & $0.00 \pm 0.00$ & $0.00 \pm 0.00$ & $0.00 \pm 0.00$ & $15.79 \pm 8.37$ \\
\hline$M_{\pi^{+} \pi^{-}}$ & $0.00 \pm 0.00$ & $0.00 \pm 0.00$ & $0.00 \pm 0.00$ & $15.79 \pm 8.37$ \\
\hline E/p & $0.00 \pm 0.00$ & $44.44 \pm 16.56$ & $37.50 \pm 9.88$ & $5.88 \pm 5.71$ \\
\hline$\chi_{F U S I O N}^{2}$ & $0.00 \pm 0.00$ & $0.00 \pm 0.00$ & $0.00 \pm 0.00$ & $15.79 \pm 8.37$ \\
\hline Track Separation & $0.00 \pm 0.00$ & $0.00 \pm 0.00$ & $6.25 \pm 6.05$ & $0.00 \pm 0.00$ \\
\hline$\pi-\gamma$ separation & $0.00 \pm 0.00$ & $28.57 \pm 17.07$ & $11.76 \pm 7.81$ & $54.29 \pm 8.42$ \\
\hline Smlring & $50.00 \pm 35.36$ & $0.00 \pm 0.00$ & $0.00 \pm 0.00$ & $0.00 \pm 0.00$ \\
\hline Track X-separation & $0.00 \pm 0.00$ & $37.50 \pm 17.12$ & $51.61 \pm 8.98$ & $52.94 \pm 8.56$ \\
\hline Early Cluster Energy & $0.00 \pm 0.00$ & $16.67 \pm 15.21$ & $28.57 \pm 9.86$ & $23.81 \pm 9.29$ \\
\hline Intime Cluster Energy & $66.67 \pm 27.22$ & $37.50 \pm 17.12$ & $55.88 \pm 8.52$ & $40.74 \pm 9.46$ \\
\hline Upstream Track - $\gamma$ separation & $50.00 \pm 35.36$ & $16.67 \pm 15.21$ & $40.00 \pm 9.80$ & $33.33 \pm 9.62$ \\
\hline Veto Cuts & $0.00 \pm 0.00$ & $0.00 \pm 0.00$ & $0.00 \pm 0.00$ & $15.79 \pm 8.37$ \\
\hline
\end{tabular}

Table 6.3: Fraction of Combinations in $K_{L} \rightarrow \pi^{ \pm} e^{\mp} \nu$ Monte Carlo removed by each cut. Each value gives the relative difference between the total number of events before and after each particular cut after all other cuts have already been applied

to try to estimate the significance of this decay as a background. This simulation, much like $K_{L} \rightarrow \pi^{ \pm} e^{\mp} \nu$ included radiative corrections using PHOTOS, as well as accidental event overlays and hadronic interaction simulations.

24,331,280,362 events (2.6 times the expected number) were generated in the 1997 vacuum beam, 25,269,646,220 events (2.5 times the expected number) were generated for the 1999 vacuum beam, while 1,764,850,286 (2.4 times the expected number) events were generated in the 1997 regenerator beam, and 2,709,962,060 event (3.3 times the expected number) were generated in the 1999 regenerator beam. These decays were generated in the ranges $30 \mathrm{GeV} / \mathrm{c}^{2}<p_{K}<162 \mathrm{GeV} / \mathrm{c}^{2}$ and $115 \mathrm{~m}<$ $Z_{\text {vertex }}<160 m$.

After the generated samples are subjected to all final analysis cuts, 1 event remains in the 1997 regenerator beam sample, no events remain in the 1999 regenerator beam sample, 2 events remain in the 1997 vacuum beam sample, and 1 event remains in the 1999 vacuum beam sample. 
Of the events remaining after all cuts, an accidental photon allowed each event to be accepted.

\begin{tabular}{|l|c|c|c|c|}
\hline \multicolumn{1}{|c|}{ Cut Variable } & \multicolumn{4}{c|}{ Relative loss due to only this cut (\%) } \\
\hline & 97REG & 99REG & 97VAC & 99VAC \\
\hline$\pi^{0}$ mass & $50.00 \pm 35.36$ & $0.00 \pm 0.00$ & $0.00 \pm 0.00$ & $0.00 \pm 0.00$ \\
\hline$Z_{\text {vertex }}$ & $0.00 \pm 0.00$ & $0.00 \pm 0.00$ & $33.33 \pm 27.22$ & $50.00 \pm 35.36$ \\
\hline$M_{\pi^{+} \pi^{-} \gamma}$ & $85.71 \pm 13.23$ & $0.00 \pm 0.00$ & $77.78 \pm 13.86$ & $83.33 \pm 15.21$ \\
\hline$P_{T}^{2}$ & $96.43 \pm 3.51$ & $100.00 \pm 0.00$ & $91.30 \pm 5.88$ & $97.14 \pm 2.82$ \\
\hline$p_{K}$ & $0.00 \pm 0.00$ & $0.00 \pm 0.00$ & $50.00 \pm 25.00$ & $50.00 \pm 35.36$ \\
\hline$E_{\gamma}^{*}$ & $50.00 \pm 35.36$ & $0.00 \pm 0.00$ & $0.00 \pm 0.00$ & $0.00 \pm 0.00$ \\
\hline$E_{\gamma}^{L A B}$ & $0.00 \pm 0.00$ & $0.00 \pm 0.00$ & $33.33 \pm 27.22$ & $0.00 \pm 0.00$ \\
\hline $\mathrm{E} / \mathrm{p}$ & $0.00 \pm 0.00$ & $0.00 \pm 0.00$ & $60.00 \pm 21.91$ & $0.00 \pm 0.00$ \\
\hline Track Momentum & $0.00 \pm 0.00$ & $0.00 \pm 0.00$ & $66.67 \pm 19.25$ & $66.67 \pm 27.22$ \\
\hline$\pi-\gamma$ separation & $0.00 \pm 0.00$ & $0.00 \pm 0.00$ & $33.33 \pm 27.22$ & $0.00 \pm 0.00$ \\
\hline Smlring & $50.00 \pm 35.36$ & $0.00 \pm 0.00$ & $0.00 \pm 0.00$ & $0.00 \pm 0.00$ \\
\hline Intime Cluster Energy & $66.67 \pm 27.22$ & $0.00 \pm 0.00$ & $66.67 \pm 19.25$ & $66.67 \pm 27.22$ \\
\hline Upstream Track - $\gamma$ separation & $50.00 \pm 35.36$ & $0.00 \pm 0.00$ & $0.00 \pm 0.00$ & $0.00 \pm 0.00$ \\
\hline
\end{tabular}

Table 6.4: Fraction of Combinations in $K_{L} \rightarrow \pi^{ \pm} \mu^{\mp} \nu$ Monte Carlo removed by each cut. Each value gives the relative difference between the total number of events before and after each particular cut after all other cuts have already been applied Note that the 97REG sample contains no events for which one cut is failed.

\subsection{3 $\quad K_{L} \rightarrow \pi^{+} \pi^{-} \pi^{0}$ with one photon missed}

The final kaon decay which is likely to contribute to the background owing to its large branching ratio is $K_{L} \rightarrow \pi^{+} \pi^{-} \pi^{0}$. When the $\pi^{0}$ undergoes the most common two photon decay, a $K_{L} \rightarrow \pi^{+} \pi^{-} \pi^{0}$ event can easily fake a $K_{L, S} \rightarrow \pi^{+} \pi^{-} \gamma$ event without the need for accidental photons, bremsstrahlung radiation or hadronic interactions in the detector. In order to study this possible source of background, large Monte Carlo samples were generated, with radiative corrections, hadronic interactions and accidental activity all included in the simulations.

6,618,147,560 events (1.5 times the expected number) were generated in the 1997 vacuum beam sample, 7,297,958,376 events (1.5 times the expected number) for 1999 vacuum beam, 473,214,696 events (1.4 times the expected number) were generated in the 1997 regenerator beam sample, and 562,490,175 events (1.5 times the expected 
number) in the 1999 regenerator beam sample. These decays were generated in the ranges $30 \mathrm{GeV} / \mathrm{c}^{2}<p_{K}<162 \mathrm{GeV} / \mathrm{c}^{2}$ and $115 \mathrm{~m}<Z_{\text {vertex }}<160 \mathrm{~m}$.

After the generated samples are subjected to all analysis cuts, 7 events remain in the 1997 regenerator beam sample and 4 events remain in the 1999 regenerator beam sample, while there are 12 events in the 1997 vacuum beam and 17 events in the 1999 vacuum beam. samples.

\begin{tabular}{|l|c|c|c|c|}
\hline \multicolumn{1}{|c|}{ Cut Variable } & \multicolumn{4}{|c|}{ Relative loss due to only this cut (\%) } \\
\hline & 97REG & 99REG & 97VAC & 99VAC \\
\hline$\pi^{0}$ mass & $30.00 \pm 14.49$ & $50.00 \pm 17.68$ & $40.00 \pm 10.95$ & $34.62 \pm 9.33$ \\
\hline $\mathrm{Z}$ vertex & $0.00 \pm 0.00$ & $0.00 \pm 0.00$ & $25.00 \pm 10.83$ & $34.62 \pm 9.33$ \\
\hline$M_{\pi^{+} \pi^{-} \gamma}$ & $92.86 \pm 2.60$ & $99.52 \pm 0.24$ & $94.47 \pm 1.55$ & $96.86 \pm 0.75$ \\
\hline$P_{T}^{2}$ & $92.47 \pm 2.74$ & $99.48 \pm 0.26$ & $94.34 \pm 1.59$ & $97.63 \pm 0.57$ \\
\hline$p_{K}$ & $0.00 \pm 0.00$ & $20.00 \pm 17.89$ & $0.00 \pm 0.00$ & $0.00 \pm 0.00$ \\
\hline Track Momentum & $0.00 \pm 0.00$ & $0.00 \pm 0.00$ & $7.69 \pm 7.39$ & $0.00 \pm 0.00$ \\
\hline$\chi_{V E R T E X}^{2}$ & $0.00 \pm 0.00$ & $20.00 \pm 17.89$ & $45.45 \pm 10.62$ & $29.17 \pm 9.28$ \\
\hline$\chi_{O F F S E T}^{2}$ & $56.25 \pm 12.40$ & $73.33 \pm 11.42$ & $86.96 \pm 3.51$ & $87.59 \pm 2.82$ \\
\hline$\pi-\gamma$ separation & $12.50 \pm 11.69$ & $50.00 \pm 17.68$ & $33.33 \pm 11.11$ & $22.73 \pm 8.93$ \\
\hline Seedring & $30.00 \pm 14.49$ & $55.56 \pm 16.56$ & $0.00 \pm 0.00$ & $0.00 \pm 0.00$ \\
\hline$P_{\pi^{0}}^{2}$ (VAC) & $0.00 \pm 0.00$ & $20.00 \pm 17.89$ & $14.29 \pm 9.35$ & $10.53 \pm 7.04$ \\
\hline Early Cluster Energy & $30.00 \pm 14.49$ & $20.00 \pm 17.89$ & $14.29 \pm 9.35$ & $5.56 \pm 5.40$ \\
\hline Intime Cluster Energy & $56.25 \pm 12.40$ & $42.86 \pm 18.70$ & $7.69 \pm 7.39$ & $0.00 \pm 0.00$ \\
\hline Veto Cuts & $0.00 \pm 0.00$ & $0.00 \pm 0.00$ & $0.00 \pm 0.00$ & $10.53 \pm 7.04$ \\
\hline
\end{tabular}

Table 6.5: Fraction of Combinations in $K_{L} \rightarrow \pi^{+} \pi^{-} \pi^{0}$ Monte Carlo removed by each cut. Each value gives the relative difference between the total number of events before and after each particular cut after all other cuts have already been applied

This decay has some very striking characteristics which distinguish it from the signal mode. For $K_{L} \rightarrow \pi^{+} \pi^{-} \pi^{0}$ events, the average value of $P_{\pi^{0}}^{2}$ is positive due to the mass of the emitted neutral pion, while for the $K_{L, S} \rightarrow \pi^{+} \pi^{-} \gamma$ signal mode this is not the case. When $K_{L} \rightarrow \pi^{+} \pi^{-} \pi^{0}$ events are reconstructed, one photon is not used - causing the $\pi^{+} \pi^{-} \gamma$ invariant mass to reconstruct well below the value of the mass of the neutral kaon. The most probable value of the vertex $P_{T}^{2}$ is near 0.006 $\mathrm{GeV}^{2} / \mathrm{c}^{2}$ unlike $K_{L, S} \rightarrow \pi^{+} \pi^{-} \gamma$ for which the most probable value is zero. Finally, these events often have a value of $M_{\pi^{+} \pi^{-}}$near $0.35 \mathrm{GeV} / \mathrm{c}^{2}$. While $P_{\pi^{0}}^{2}$ and vertex $P_{T}^{2}$ are correlated, combinations of the other observables can be plotted and serve as an excellent way to identify this mode. 


\subsubsection{Comments about background due to kaon decays}

As can be seen in the results above, the $\mathrm{KTeV}$ Monte Carlo predicts that the three most common kaon decays do not appreciably contribute to the background. However, the suppression due to the analysis cuts is so great that it is not realistic to expect that the KTeV Monte Carlo would be able to model the acceptance of the detector to one part in a billion. In reality, the actual contribution from these modes is most likely much higher than predicted.

$K_{L} \rightarrow \pi^{+} \pi^{-} \pi^{0}$ will be very sensitive to the level of accidental activity in the detector, which can supply a photon with enough energy to cause the $\pi^{+} \pi^{-} \gamma$ invariant mass to reconstruct within the mass cut window. In addition, it will be very sensitive to track resolution, as the $P_{\pi^{0}}^{2}$ cut removes a great deal of these events.

The rejection of $K_{L} \rightarrow \pi^{ \pm} e^{\mp} \nu$ depends mainly on the modeling and reconstruction of electromagnetic showers in the CsI calorimeter. However, there are a number of rare and/or exotic phenomena which could cause the energy of the electron to not be properly measured and thus skew E/p towards a value which may be accepted.

Finally, properly modeling the effectiveness of the muon vetoes which are used to reject $K_{L} \rightarrow \pi^{ \pm} \mu^{\mp} \nu$ proves especially challenging due the their size and complexity.

In a previous analysis [6] of $K_{L, S} \rightarrow \pi^{+} \pi^{-}, K_{L} \rightarrow \pi^{ \pm} e^{\mp} \nu$ and $K_{L} \rightarrow \pi^{ \pm} \mu^{\mp} \nu$ were found to contribute to the background to that particular decay. By applying additional cuts to the data, it was possible to select $K_{L} \rightarrow \pi^{ \pm} e^{\mp} \nu$ or $K_{L} \rightarrow \pi^{ \pm} \mu^{\mp} \nu$ events. To enhance $K_{L} \rightarrow \pi^{ \pm} e^{\mp} \nu$ and further suppress $K_{L} \rightarrow \pi^{ \pm} \mu^{\mp} \nu$ the cuts applied where to keep events in which one track had $E / p>0.75$ and the other track deposited at least $1 \mathrm{GeV}$ of energy into the CsI. This selected events in which an electron appeared to be present, in addition to a particle which was not minimum ionizing. On the other hand, requiring one particle to be minimum ionizing (a muon) and the other particle to have $E / p<0.5$ (not an electron) enhances $K_{L} \rightarrow \pi^{ \pm} \mu^{\mp} \nu$ over $K_{L} \rightarrow \pi^{ \pm} e^{\mp} \nu$ D்oing so resulted in an estimate of the backgrounds due to $K_{L} \rightarrow \pi^{ \pm} e^{\mp} \nu$ and $K_{L} \rightarrow \pi^{ \pm} \mu^{\mp} \nu$ to be $0.036 \%$ and $0.054 \%$ respecitively for the vacuum beam and $0.001 \%$ and $0.002 \%$ for the regenerator beam. This indicates that contrary to the Monte Carlo prediction, the analysis cuts have a harder time rejecting $K_{L} \rightarrow \pi^{ \pm} \mu^{\mp} \nu$ decays.

However, in the $K_{L, S} \rightarrow \pi^{+} \pi^{-}$analysis [6], the absolute amount of background in that analysis is much higher, allowing studies of the background regions in the 
invariant mass and $P_{T}^{2}$ plots to reveal the identity and size of the various background components. In the $K_{L, S} \rightarrow \pi^{+} \pi^{-} \gamma$ samples, the background level is much lower ( the sum of the sidebands in the invariant mass plot of all four datasets is 5000 events) frustrating attempts to make an accurate estimate of the exact composition of the background.

It is possible, however, to make a rough estimate of the background composition. First, we confirm that the majority of the low mass region background, in the region of $0.46 \mathrm{GeV} / \mathrm{c}^{2}$ is in fact due to $K_{L} \rightarrow \pi^{+} \pi^{-} \pi^{0}$ decays.

\section{Investigation of events with low invariant mass}

The shape of the $\pi^{+} \pi^{-} \gamma$ invariant mass plot for $K_{L} \rightarrow \pi^{+} \pi^{-} \pi^{0}$ is strongly peaked in this region. For this reason, $K_{L} \rightarrow \pi^{+} \pi^{-} \pi^{0}$ is most likely to be seen in this region.

First, events with $0.46 \mathrm{GeV} / \mathrm{c}^{2}<M_{\pi^{+} \pi^{-} \gamma}<0.475 \mathrm{GeV} / \mathrm{c}^{2}$ are selected in order to minimize the presence of $K_{L, S} \rightarrow \pi^{+} \pi^{-} \gamma$ events. Second, the number of events for which $0.30 \mathrm{GeV} / \mathrm{c}^{2}<M_{\pi^{+} \pi^{-}}<0.35 \mathrm{GeV} / \mathrm{c}^{2}$ and $0.01 \mathrm{GeV}^{2} / \mathrm{c}^{2}<$ Vertex $P_{T}^{2}<$ $0.02 \mathrm{GeV}^{2} / \mathrm{c}^{2}$ are counted. This region is chosen as one for which $K_{L} \rightarrow \pi^{+} \pi^{-} \pi^{0}$ decays are concentrated, but other background decays do not. The number of events in the total low invariant mass sample, and those in the restricted sample, are shown in Table 6.6. The fraction of events residing in the restricted area compared to the total low mass invariant mass region is approximately $50 \%$ in the regenerator beam and $30 \%$ in the vacuum beam. Looking at the same ratios for various background Monte Carlo samples, we notice that $K_{L} \rightarrow \pi^{+} \pi^{-} \pi^{0}$ is most likely the source, with ratios of $45 \%$ and $30 \%$ at the crunch level. The other background decays have fractions of less than $2 \%$, while $K_{L, S} \rightarrow \pi^{+} \pi^{-} \gamma$ decays have fractions of less than $7 \%$.

\section{Investigation of events with high invariant mass}

The higher invariant mass region, which is taken to be $0.5150 \mathrm{GeV} / \mathrm{c}^{2}<M_{\pi^{+} \pi^{-} \gamma}<$ $0.5400 \mathrm{GeV} / \mathrm{c}^{2}$ is not as easy to study. Here, the larger background modes, $K_{L} \rightarrow \pi^{+} \pi^{-} \pi^{0}, K_{L} \rightarrow \pi^{ \pm} e^{\mp} \nu$ and $K_{L} \rightarrow \pi^{ \pm} \mu^{\mp} \nu$ are all expected to contribute.

We first repeat the above process in order to check for the presence of $K_{L} \rightarrow \pi^{+} \pi^{-} \pi^{0}$ events. Counting the total number of events in the reduced sample, and those with 


\begin{tabular}{|l|c|c|}
\hline Data Sample & $\begin{array}{c}\text { Number of Events } \\
\text { in low } M_{\pi^{+} \pi^{-} \gamma} \text { region }\end{array}$ & $\begin{array}{c}\text { Number of Events } \\
\text { in low } M_{\pi^{+} \pi^{-} \gamma} \text { region } \\
\text { with additional } M_{\pi^{+} \pi^{-}} \\
\text {and Vertex } P_{T}^{2} \text { cuts }\end{array}$ \\
\hline 1997 Regenerator & 91 & 46 \\
\hline 1999 Regenerator & 112 & 56 \\
\hline 1997 Vacuum & 224 & 79 \\
\hline 1999 Vacuum & 247 & 72 \\
\hline
\end{tabular}

Table 6.6: Distribution of events in the region defined by $0.46 \mathrm{GeV} / \mathrm{c}^{2}<M_{\pi^{+}} \pi^{-} \gamma$ $0.475 \mathrm{GeV} / \mathrm{c}^{2}$ The fraction of these events which reside in the more restricted sample matches the fraction found in $K_{L} \rightarrow \pi^{+} \pi^{-} \pi^{0}$ Monte Carlo events, indicating that the majority of background events in the low invariant mass region are in fact $K_{L} \rightarrow \pi^{+} \pi^{-} \pi^{0}$ events.

even tighter cuts on Vertex $P_{T}^{2}$ and $M_{\pi^{+} \pi^{-}}$it appears as though $K_{L} \rightarrow \pi^{+} \pi^{-} \pi^{0}$ candidates only make up a small part of the background in this region.

\begin{tabular}{|l|c|c|}
\hline Data Sample & $\begin{array}{c}\text { Number of Events } \\
\text { in high } M_{\pi^{+} \pi^{-} \gamma} \text { region }\end{array}$ & $\begin{array}{c}\text { Number of Events } \\
\text { in high } M_{\pi^{+} \pi^{-} \gamma}^{\text {region }} \\
\text { with additional } M_{\pi^{+} \pi^{-}} \\
\text {and Vertex } P_{T}^{2} \text { cuts }\end{array}$ \\
\hline 1997 Regenerator & 17 & 2 \\
\hline 1999 Regenerator & 22 & 4 \\
\hline 1997 Vacuum & 41 & 4 \\
\hline 1999 Vacuum & 69 & 6 \\
\hline
\end{tabular}

Table 6.7: Distribution of events in the region defined by $0.515 \mathrm{GeV} / \mathrm{c}^{2}<M_{\pi^{+}} \pi^{-} \gamma<$ $0.540 \mathrm{GeV} / \mathrm{c}^{2}$ The fraction of these events which reside in the more restricted sample indicate that the majority of background events in this region are not $K_{L} \rightarrow \pi^{+} \pi^{-} \pi^{0}$ events, unlike the case at low invariant masses.

The next step is to determine if any part of the background may be due to $K_{L} \rightarrow \pi^{ \pm} \mu^{\mp} \nu$. We follow Reference [6] and apply further cuts to the higher mass background region in order to try to identify the background composition.

First, we require that one track in the event has $E / p<0.5$ and require that the other track deposited less than $1.0 \mathrm{GeV}$ of energy into the CsI. This will remove the vast majority of remaining $K_{L} \rightarrow \pi^{ \pm} e^{\mp} \nu$ events, while keeping most $K_{L} \rightarrow \pi^{ \pm} \mu^{\mp} \nu$ events. The number of events before and after this cut are shown in Table 6.8. 


\begin{tabular}{|l|c|c|}
\hline Data Sample & $\begin{array}{c}\text { \# of Events } \\
\text { in high } M_{\pi^{+} \pi^{-} \gamma} \text { region }\end{array}$ & $\begin{array}{c}\text { \# of Events } \\
\text { in high } M_{\pi^{+}} \pi^{-} \gamma \text { region } \\
\text { with } K_{L} \rightarrow \pi^{ \pm} \mu^{\mp} \nu \text { enhancement cuts }\end{array}$ \\
\hline 1997 Regenerator & 17 & 11 \\
\hline 1999 Regenerator & 22 & 5 \\
\hline 1997 Vacuum & 41 & 10 \\
\hline 1999 Vacuum & 69 & 18 \\
\hline
\end{tabular}

Table 6.8: Distribution of events in the region defined by $0.515 \mathrm{GeV} / \mathrm{c}^{2}<M_{\pi^{+}} \pi^{-} \gamma<$ $0.540 \mathrm{GeV} / \mathrm{c}^{2}$ The fraction of these events which reside in the more restricted sample indicate that $K_{L} \rightarrow \pi^{ \pm} \mu^{\mp} \nu$ events are present in the background.

Instead removing $K_{L} \rightarrow \pi^{ \pm} \mu^{\mp} \nu$ events and keeping most $K_{L} \rightarrow \pi^{ \pm} e^{\mp} \nu$ events results in the number of events indicated in Table 6.9

\begin{tabular}{|l|c|c|}
\hline Data Sample & $\begin{array}{c}\text { Number of Events } \\
\text { in high } M_{\pi^{+} \pi^{-} \gamma} \text { region }\end{array}$ & $\begin{array}{c}\text { Number of Events } \\
\text { in high } M_{\pi^{+} \pi^{-} \gamma}^{\text {region }} \\
\text { with } K_{L} \rightarrow \pi^{ \pm} e^{\mp} \nu \text { enhancement cuts }\end{array}$ \\
\hline 1997 Regenerator & 17 & 3 \\
\hline 1999 Regenerator & 22 & 3 \\
\hline 1997 Vacuum & 41 & 7 \\
\hline 1999 Vacuum & 69 & 13 \\
\hline
\end{tabular}

Table 6.9: Distribution of events in the region defined by $0.515 \mathrm{GeV} / \mathrm{c}^{2}<M_{\pi^{+}} \pi^{-} \gamma<$ $0.540 \mathrm{GeV} / \mathrm{c}^{2}$ The fraction of these events which reside in the more restricted sample indicate that $K_{L} \rightarrow \pi^{ \pm} e^{\mp} \nu$ events are present in the background.

However, not all $K_{L} \rightarrow \pi^{ \pm} \mu^{\mp} \nu$ events will pass the $K_{L} \rightarrow \pi^{ \pm} \mu^{\mp} \nu$ enhancement cuts, nor will all $K_{L} \rightarrow \pi^{ \pm} e^{\mp} \nu$ events pass the $K_{L} \rightarrow \pi^{ \pm} e^{\mp} \nu$ enhancement cuts. Given the limited statistics of the $K_{L} \rightarrow \pi^{ \pm} \mu^{\mp} \nu$ and $K_{L} \rightarrow \pi^{ \pm} e^{\mp} \nu$ Monte Carlo samples after all cuts, we are forced to compute the efficiency of the enhancement cuts using the MC samples after crunch cuts but before the analysis cuts. As the enhancement cuts depend only on E/p, track momentum and and track cluster energy, the efficiency at the crunch level and after all analysis cuts should be approximately the same. Applying crunch cuts to the $K_{L} \rightarrow \pi^{ \pm} \mu^{\mp} \nu \mathrm{MC}$ samples, and then applying the $K_{L} \rightarrow \pi^{ \pm} \mu^{\mp} \nu$ enhancement cuts to the result, we find that the efficiency for this extra 
cut is approximately $72 \%$. Applying crunch cuts to the $K_{L} \rightarrow \pi^{ \pm} e^{\mp} \nu$ MC samples, and then applying the $K_{L} \rightarrow \pi^{ \pm} e^{\mp} \nu$ enhancement cuts to the result, we find that the efficiency for this extra cut to be very approximately $57 \%$. Recalling that the efficiency for the $K_{L} \rightarrow \pi^{+} \pi^{-} \pi^{0}$ enahancement cut on $K_{L} \rightarrow \pi^{+} \pi^{-} \pi^{0}$ events is approximately $40 \%$ we can attempt to compute the total number of background events before the enhancement cuts. Assuming that events which pass the $K_{L} \rightarrow \pi^{ \pm} e^{\mp} \nu$ enhancement cut are only $K_{L} \rightarrow \pi^{ \pm} e^{\mp} \nu$ events, and likewise for the $K_{L} \rightarrow \pi^{ \pm} \mu^{\mp} \nu$ and $K_{L} \rightarrow \pi^{+} \pi^{-} \pi^{0}$ enhancement cuts, we arrive at a very rough estimate for the composition of the background as shown in Table 6.10

\begin{tabular}{|l|c|c|c|}
\hline Data Sample & $\begin{array}{c}\text { \# of Estimated } \\
K_{L} \rightarrow \pi^{+} \pi^{-} \pi^{0} \text { Events }\end{array}$ & $\begin{array}{c}\text { \# of Estimated } \\
K_{L} \rightarrow \pi^{ \pm} \mu^{\mp} \nu \text { events }\end{array}$ & $\begin{array}{c}\text { \# of Estimated } \\
K_{L} \rightarrow \pi^{ \pm} e^{\mp} \nu \text { events }\end{array}$ \\
\hline 1997 Regenerator & 5 & 15 & 5 \\
\hline 1999 Regenerator & 10 & 7 & 5 \\
\hline 1997 Vacuum & 10 & 14 & 12 \\
\hline 1999 Vacuum & 15 & 25 & 23 \\
\hline
\end{tabular}

Table 6.10: Estimated background composition of events in the region defined by $0.515 \mathrm{GeV} / \mathrm{c}^{2}<M_{\pi^{+} \pi^{-} \gamma}<0.540 \mathrm{GeV} / \mathrm{c}^{2}$

The high mass background appears to be a mixture of different decay modes as expected with a composition of $40 \% K_{L} \rightarrow \pi^{ \pm} \mu^{\mp} \nu, 30 \% K_{L} \rightarrow \pi^{ \pm} e^{\mp} \nu$ and $30 \%$ $K_{L} \rightarrow \pi^{+} \pi^{-} \pi^{0}$. It should be restated that this is only a very approximate estimation, meant mainly as a qualitative rather than quantitative study. Additionally, the results of this study in no way impact the results of the maximum likelihood fit, as we do not use knowledge of the background composition in that result.

Further, since the $K_{L} \rightarrow \pi^{+} \pi^{-} \pi^{0}$ peak at lower invariant mass falls off sharply with increasing invariant mass, the actual background in the signal region can be approximated as having the same composition as that at invariant masses larger than the kaon mass. The consequence is that our best estimate for the total (for both years and both beams) composition of the background would be $40 \% K_{L} \rightarrow \pi^{ \pm} \mu^{\mp} \nu$, $30 \% K_{L} \rightarrow \pi^{ \pm} e^{\mp} \nu$ and $30 \% K_{L} \rightarrow \pi^{+} \pi^{-} \pi^{0}$. 


\subsection{Non-kaon decays}

\subsubsection{Neutral Hyperons}

In addition to neutral kaons, neutral hyperons are produced in the target and regenerator, and thus are possible sources of background.

The lambda decay $\Lambda \rightarrow p \pi$ provides a possible source of background when the decay is accompanied by a photon and the proton is misidentified as a pion. In addition to $\Lambda$ particles produced in the target and in the regenerator, $\Lambda$ s are also produced by other hyperon decays, such as $\Sigma^{0} \rightarrow \Lambda \gamma \Xi \rightarrow \Lambda \pi^{0}$ and $\Xi \rightarrow \Lambda \gamma$. A study of the hyperon background [36] for the 1997 analysis on $K_{L} \rightarrow \pi^{+} \pi^{-} \gamma$ [15] revealed that the background due to $\Lambda \rightarrow p \pi$ is approximately $(2.17 \pm .27)$ events when the conservative value of $730: 10: 1^{1}$ is used from the ratios of $K_{L}$ to $\Lambda$ s to $\Xi$ produced in the target. The estimate for $\Xi \rightarrow \Lambda \pi^{0}$ is even lower. In this analysis, an even smaller amount of these decays are expected due to a tighter cut on $Z_{\text {vertex }}$. However, in order to erase uncertainty on these estimates due to the kaon to hyperon flux ratios, we have chosen to apply a cut on the $p \pi$ invariant mass in the region of the $\Lambda$ mass. Doing so will effectively eliminate the hyperon background, as the most common modes involve $\Lambda$ decays. Only the radiative $\Lambda$ decay will escape this cut, however due to its low branching ratio and the presence of the $p \pi$ invariant mass cut, this mode is not expected to contribute to the background. Inspection of the $p \pi \gamma$ invariant mass plot reveals that no peak exists. In summary, the background due to hyperon decays is expected to be negligible.

\subsection{Scattered $K_{L, S} \rightarrow \pi^{+} \pi^{-} \gamma$ decays}

\subsubsection{Regenerator Scattering}

These are unwanted regenerated kaon decays which do not acquire a coherent phase during regeneration. There are two types of these events. The first class of events are due to the inelastic process which deposits energy into the regenerator, so most of this process can be removed using the regenerator veto. These events will also

\footnotetext{
${ }^{1}$ This ratio is valid for the range $20 \mathrm{GeV}<E_{K}<230 \mathrm{GeV}$ and $95 \mathrm{~m}<Z_{\text {vertex }}<158 \mathrm{~m}$ only
} 
have non-zero $P_{T}^{2}$ values. These events will have a modified wavefunction, and their presence may affect the likelihood fit. As the $\mathrm{KTeV}$ Monte Carlo which simulates these events also properly included their frequency, the size of this background is extracted using the Monte Carlo prediction.

Events which have undergone diffractive regenerator scattering do not leave energy in the regenerator, however the length of the regenerator has been designed to minimize these events. These events will have a modified wavefunction, and as such can affect the likelihood fit. Again, the Monte Carlo does simulate and predict the frequency of these events, which is used to estimate the number of these events in the final data sample.

The fraction of events which do not coherently regenerate, as predicted by the Monte Carlo simulation, are $0.09 \%$ and $0.10 \%$ for the 1997 and 1999 regenerator beam samples respectively. Applying these percentages to the total number of events in each sample, we estimate that incoherent regeneration contributes approximately 129 events to the background.

\subsubsection{Collimator Scattering}

These are events in which the kaon either grazes one of the collimators, or scatters within it. Most often these events will acquire some transverse momentum during the scattering process which can then be used to reject this background. As the $\mathrm{KTeV}$ Monte Carlo simulates these events along with their frequency, the number of events in the sample of this type is estimated using the Monte Carlo simulation. The Monte Carlo predicts roughly $0.0084 \%, 0.0057 \%, 0.0062 \%$, and $0.0112 \%$ of events will have undergone some kind of scattering in the collimators from the 1997 regenerator, 1997 vacuum, 1999 regenerator and 1999 vacuum beams samples respectively. Applying these percentages to the total number of events in each sample, we estimate that collimator scattering contributes approximately 25 events in total to the background. We assume that this background is negligible. 


\subsection{Modified $K_{L, S} \rightarrow \pi^{+} \pi^{-} \gamma$ decays}

\subsection{1 $\quad K_{L, S} \rightarrow \pi^{+} \pi^{-} \gamma \gamma$}

This process can affect the reconstruction of $K_{L, S} \rightarrow \pi^{+} \pi^{-} \gamma$ events a number of different ways which can then lead to a bias in the likelihood fit. However, we choose to treat this process as signal, as its effect will be taken into account during the treatment of other systematic errors. As this process is simulated in the Monte Carlo by PHOTOS, we can estimate how many events of this type are present in the sample. The estimate is approximately $1.4 \%$ in the 1997 samples, $2.8 \%$ in the 1999 regenerator sample and $2.2 \%$ in the 1999 vacuum beam sample. For all samples, the number of these events for which the 2 nd photon has an energy above $20 \mathrm{MeV}$ in the kaon rest frame is completely negligible. 


\section{Chapter 7}

\section{Monte Carlo Simulation}

Any analysis of experimental data requires an understanding of how the apparatus used in the collection of the data may effect the data sample, and an analysis of $\mathrm{KTeV}$ data is no different. The $\mathrm{KTeV}$ collaboration has written a detailed Monte Carlo simulation of the detector and beam-line [7, 6, 30].

The Monte Carlo simulation integrates the physics of particle production, relativistic kinematics, the interaction of particles with matter, and the physical layout of the experiment. The Monte Carlo simulation is used primarily in the calculation of the "acceptance" for each event, which is a quantity that describes the probability that a event with a particular set of characteristics may be accepted into a dataset. The Monte Carlo is also useful for simulating the ability of the detector to measure the characteristics of each event. In addition to acting as a model of the detector, the $\mathrm{KTeV}$ Monte Carlo, also known as KTeVMC, also contains the physics of a number of neutral kaon and hyperon decays, which can be used to model each particular decay.

The result is the ability to produce "fake" data samples of particular decays which approximate the actual data, allowing the effects of various cuts and backgrounds to be understood in relation to a desired signal, which is typically a single kaon decay channel. 


\subsection{Generation and Propagation of Neutral Kaons}

Each Monte Carlo event begins with the selection of the beam in which the parent kaon will decay, in addition to the choice of which beam the regenerator occupies. $\mathrm{KTeVMC}$ has the ability to generate the proper relative number of events in the vacuum and regenerator beams at the same time, however this analysis produces Monte Carlo data samples which consist of only vacuum beam or regenerator beam decays, and the two samples are always kept separate. Once the location and type of beam are selected, the timing of the event is determined, i.e. where in the 1-ns long $\mathrm{RF}$ bucket the kaon is produced.

The next step is to choose the identity $\left(K^{0}\right.$ or $\left.\bar{K}^{0}\right)$ of the parent kaon, choose its momentum and finally its direction of propagation. For all three tasks, the Malensik kaon production functions [37] are utilized. These production cross sections, which are functions of the resulting kaon momentum and production angle, are first integrated to obtain the total cross-section for $K^{0}$ and $\bar{K}^{0}$ production. These probabilities are then used to select either a $K^{0}$ or $\bar{K}^{0}$ state. Once that is done, the selected state is then re-expressed as a $K_{L}-K_{S}$ basis state. This is then the initial kaon wavefunction. The kaon momentum and production angle are then selected using the production cross-section which is appropriate for the type of particle in question. The consequence is that $K^{0}$ and $\bar{K}^{0}$ states will have slightly different momentum distributions. It should be noted that a small correction term is added to both the $K^{0}$ and $\bar{K}^{0}$ production cross-sections which forces the kaon momentum distribution to match that of the data. This correction is based on $K_{L, S} \rightarrow \pi^{+} \pi^{-}$data, as this is the only decay which can be completely reconstructed and contains no adjustable parameters which describe the kinematics of the decay, as it is a simple two-body decay. Finally, the point along the target at which the kaon was produced is selected using a simple decaying exponential distribution of the form

$$
P(z) \propto \exp -\frac{z}{L_{a b s}}
$$

where $z$ is the position along the target, and $L_{a b s}$ is the effective absorption length of the $\mathrm{KTeV}$ target.

After this point, the initial kaon wavefunction must be evolved up to the the 
beginning of the decay volume.

\subsubsection{Kaon wavefunction evolution and particle transport}

Once the kaon is produced, it will travel through the remainder of the target, the beam absorbers, and the rest of the KTeV beam-line. Every time the kaon passes through matter, it may scatter. Additionally, the kaon's wavefunction will evolve from a pure $K^{0}$ or $\bar{K}^{0}$ state to a mixed state. In the $K_{L}-K_{S}$ basis, the $K_{S}$ component will be reduced except in cases in which the kaon passes through matter, then the $K_{S}$ state may be regenerated, as in the active regenerator. Without regeneration, except in cases of extreme scattering or extremely high kaon momentum, towards the end of the decay volume the kaon state will be predominantly that of a $K_{L}$.

KTeVMC transports the kaon throughout the beam-line and detector element by element, including the space between elements. The evolution of the kaon wavefunction is done using a matrix transformation. This matrix transformation takes the form of:

$$
\begin{aligned}
\left(\begin{array}{c}
K_{S^{\prime}}{ }^{\prime} \\
K_{L^{\prime}}{ }^{\prime}
\end{array}\right) & =\left(\begin{array}{cc}
\cosh (i \delta \tau)-\frac{H}{\delta} \sinh (i \delta \tau) & \frac{G}{\delta} \sinh (i \delta \tau) \\
\frac{G}{\delta} \sinh (i \delta \tau) & \cosh (i \delta \tau)+\frac{H}{\delta} \sinh (i \delta \tau)
\end{array}\right) \\
& \times e^{-\left(\frac{1}{4}\left(\Gamma_{S}+\Gamma_{L}\right)+\Im\left(\left(\frac{p_{K}}{M_{K}}\right)\left(\frac{N_{A}}{Z}\right) \pi c d f_{+}\right)\right) \tau}\left(\begin{array}{c}
K_{S} \\
K_{L}
\end{array}\right)
\end{aligned}
$$

where

$$
\begin{aligned}
\delta & =\sqrt{H^{2}+G^{2}} \\
H & =\frac{\Delta_{M}}{2}-\frac{i}{4}\left(\Gamma_{S}-\Gamma_{L}\right) \\
G & =\left(\frac{N_{A}}{Z}\right)\left(\frac{p_{K}}{M_{K}}\right)\left(\frac{p_{K}}{70 G e V / c}\right)^{\alpha} \pi c d f_{-}
\end{aligned}
$$

and $\tau$ is the proper time of flight of the kaon during the current round of propagation, $c$ is the speed of light, $d$ is the density of the material that the kaon is transecting, $Z$ denotes the atomic number of the matter, $N_{A}$ is Avogadro's number, $\alpha$ is a power law parameter intrinsic to the material, and both $f_{+}$and $f_{-}$are kaon-nucleon scattering amplitudes for the material in question. Note that $d, Z, \alpha, f_{+}, f_{-}$are all needed to 
describe the transformation matrix for a given part of the beamline. Also note that in the case of vacuum, $d=f_{+}=f_{-}=0$ and the transformation matrix becomes:

$$
\begin{aligned}
\left(\begin{array}{c}
K_{S}{ }^{\prime} \\
K_{L}{ }^{\prime}
\end{array}\right) & =\left(\begin{array}{cc}
\exp (-i \delta \tau) & 0 \\
0 & \exp (+i \delta \tau)
\end{array}\right) \\
& \times e^{-\left(\frac{1}{4}\left(\Gamma_{S}+\Gamma_{L}\right)\right) \tau}\left(\begin{array}{c}
K_{S} \\
K_{L}
\end{array}\right) \\
& =\left(\begin{array}{cc}
\exp -\frac{1}{2}\left(i \Delta_{M} \tau-\Gamma_{S} \tau\right) & 0 \\
0 & \exp +\frac{1}{2}\left(i \Delta_{M} \tau-\Gamma_{L} \tau\right)
\end{array}\right)\left(\begin{array}{c}
K_{S} \\
K_{L}
\end{array}\right)
\end{aligned}
$$

which indicates that there is no oscillation between $K_{L}$ and $K_{S}$ in vacuum. Using the above transformation matrix, the wavefunction is propagated through each beam-line element, using the proper material specifications for each element until the beginning of the decay volume is reached, at which point the Monte Carlo chooses the point at which the kaon will decay.

\subsection{Generation of $K_{L, S} \rightarrow \pi^{+} \pi^{-} \gamma$ decays}

The purpose of the Monte Carlo simulation is two fold. First, it allows us to correct our results for the effect of the acceptance of the $\mathrm{KTeV}$ detector without having to determine a closed-form solution for the acceptance. Secondly, it allows us to generate "fake" data which can then be compared to real data in order to expose any problem in the understanding of the detector. In order to achieve the second goal, it is imperative that the Monte Carlo also include a detailed simulation of the decay in question itself. $\mathrm{KTeVMC}$ is able to generate a wide variety of kaon decays, as well as hyperon decays. However, due to the complexity of this analysis, it was necessary to completely rewrite the decay routine that handles $K_{L, S} \rightarrow \pi^{+} \pi^{-} \gamma$ events.

\subsubsection{Generation Using Matrix Element}

The new feature introduced in the routine which generates $K_{L, S} \rightarrow \pi^{+} \pi^{-} \gamma$ decays is the use of the triple differential decay rate (Equation 3.40) which allows values of $E_{\gamma}^{*}$, $\cos (\theta)$ and $Z_{\text {vertex }}$ to be generated at the same time. This triple differential decay 
rate contains all the physics of the $K_{L, S} \rightarrow \pi^{+} \pi^{-} \gamma$ decay, and also serves as our model which will be fit to the data.

The first step in the procedure is the choice of a trial value of the kaon proper lifetime $\tau$ relative to the beginning of the decay volume. While one could in principle generate this from a uniform distribution, we instead pick $^{1}$ a value of $\tau$ using the following function:

$$
\tau=\frac{-0.005}{c}+\frac{1.010}{c} t_{\text {rand }}^{3}
$$

where $t_{\text {rand }}$ is a uniform random number between 0 and 1 and $c$ is the speed of light. This function maps $t_{\text {rand }}$ in such a way that $\tau$ will tend to take on smaller values - in other words we take a unbiased random number and produce a biased variable. This is done because we expect to see many more $K_{L, S} \rightarrow \pi^{+} \pi^{-} \gamma$ events with small values of $\tau$ than large values. However, we will need to correct for this bias when we make the acceptance-rejection decision, so we also assign a statistical weight of

$$
w_{\tau}=3\left(\frac{1.010}{c}\right) t_{\text {rand }}^{2}
$$

to this choice of $\tau$, which will be used later. If $\tau$ takes on a value between 0 and the value that corresponds to the kaon escaping the decay volume, we continue on. If not, we repeat the process again.

Next, a value of $E_{\gamma}^{*}$ is selected using

$$
E_{\gamma}^{*}=\left(E_{\gamma}^{* \min }-0.005\right)+\left(E_{\gamma}^{* \max }-E_{\gamma}^{* \min }+0.01\right) * E_{\text {rand }}^{3}
$$

where $E_{\gamma}^{* \min }$ is the minimum allowable value of $E_{\gamma}^{*}$, which is used as a infrared cutoff here, $E_{\gamma}^{* \max }$ is the maximum possible value of $E_{\gamma}^{*}$ as limited by conservation of four momentum, and $E_{\text {rand }}$ is a uniform random number from 0 to 1 . Note that $E_{\gamma}^{* \max }=$ $\frac{M_{K}{ }^{2}-4 M_{\pi}{ }^{2}}{2 M_{K}}$. As before, the use of this function will tend to produce lower values of $E_{\gamma}^{*}$, just was we expect to see more low $E_{\gamma}^{*}$ events in the data. Again, we must correct

\footnotetext{
${ }^{1}$ Note that we will sometimes choose negative values using this function. As noted in the text, these negative values are rejected, as are values which are too large. This is done in order to avoid floating point issues near the boundaries of $\tau$. While this method introduces a small amount of inefficiency, the result is a sharp cut at low and high values. This method is used with each kinematic variable to be selected.
} 
for this bias later, so we assign a statistical weight of

$$
w_{E_{\gamma}^{*}}=3\left(E_{\gamma}^{* \max }-E_{\gamma}^{* \min }+0.01\right) * E_{\text {rand }}^{2}
$$

to this choice of $E_{\gamma}^{*}$. If $E_{\gamma}^{*}$ takes on a value between $E_{\gamma}^{* \min }$ and $E_{\gamma}^{* \max }$ we continue on. If not, we choose another value of $E_{\gamma}^{*}$.

Finally, we choose $\cos (\theta)$ according to

$$
\cos (\theta)=-1.05+2.10 \times \text { rand }
$$

where rand is a random number from 0 to 1 . We simply assign a statistical weight of 1 here, and continue on if $\cos (\theta)$ takes on a value between -1 and 1 .

The result after this process is a set of values for $E_{\gamma}^{*}, \cos (\theta)$ and $\tau$ which cover the entire range of allowable values, while at the same time are free from "edge effects"

The location of the decay vertex ( $Z_{\text {vertex }}$ ) is then computed from the kaon lifetime using

$$
Z_{\text {vertex }}=z_{b e g}+\frac{\tau c p_{K}}{M_{K}}
$$

where $z_{\text {beg }}$ is the location of the beginning of the decay volume. The kaon wavefunction is then propagated up to this point as described in Section 7.1. Finally, using the values of $E_{\gamma}^{*}, \cos (\theta)$ and the computed kaon wavefunction, the probability density $w_{\text {decay }}$ of this particular choice of kinematic variables is computed using Equation 3.40. Note that the kaon wavefunction handles the time dependence in Equation 3.40 .

We now utilize von Neumann's acceptance rejection method to generate events distributed according to Equation 3.40. First, we must correct for the biased values of $E_{\gamma}^{*}$ and $\tau$ which we have selected. We define an overall statistical weight according to

$$
w_{\text {total }}=w_{\text {decay }} w_{\tau} w_{E_{\gamma}^{*}}
$$

and then ask if

$$
w_{\text {total }} \geq w_{\max } \times \text { rand }
$$

where rand is a uniform random number between 0 and 1 and $w_{\max }$ is a constant chosen such that it is greater than all possible values of $w_{\text {total }}$. If Equation 7.12 is 
satisfied, then the choice of $\tau, E_{\gamma}^{*}$ and $\cos (\theta)$ is accepted. If not, another set of values of $\tau, E_{\gamma}^{*}$ and $\cos (\theta)$ are selected in the same fashion, until a set is accepted.

To review, we have used importance sampling in order to try to improve the efficiency of the acceptance-rejection procedure. This was accomplished by preconditioning the values of $E_{\gamma}^{*}$ and $\tau$ so that selected values were more often accepted by the von Neumann method. This technique is explained further in Appendix H.

One consequence to the method which we have employed is that the generation of $K_{L, S} \rightarrow \pi^{+} \pi^{-} \gamma$ decays is quite inefficient, leading to very long times needed to generate a substantial number of decays. The root cause of this is the very sharp probability density we are using to generate events - for regenerator beam events the density is sharply peaked in $E_{\gamma}^{*}$ and $\tau$. The preconditioning of these two variables helps this issue, but it can still take up to a full second to generate a single $K_{L, S} \rightarrow \pi^{+} \pi^{-} \gamma$ decay in the regenerator beam. The biggest demand for large Monte Carlo samples comes from the maximum likelihood fit, detailed later, which requires samples which are approximately 20 larger in size than the data. However, since a reweighting method is utilized in the fit, we can simply use a matrix element equal to 1 for these samples, meaning that all trials pass. This allows the samples to be quickly produced.

\subsubsection{Kinematics of $K_{L, S} \rightarrow \pi^{+} \pi^{-} \gamma$}

Once values of $E_{\gamma}^{*}$ and $\cos (\theta)$ have been generated for a given event, their values can be used, along with 4-momentum conservation, to compute the momentum vectors of both pions in the kaon rest frame. Initially, the decay is produced without any specific orientation, so the momentum of the $\pi^{+}$is assumed to point along the $\mathrm{z}$ axis ( directly downstream ). Then the entire system is rotated about the $\mathrm{x}$-axis by a uniformly random angle between 0 and $2 \pi$. The vectors are then also rotated about the y-axis and finally the z-axis. This procedure ensures that the generated decays have a random orientation in solid angle.

Once the magnitude and direction of all three momentum vectors are known in the kaon rest frame, we use the kaon momentum vector to boost the entire system into the lab frame, at which point the daughter particles can be propagated through the $\mathrm{KTeV}$ detector. At this point, the generated values of all the interesting quantities 
describing the decay, such as $E_{\gamma}^{*}, \cos (\theta), \tau, p_{K}$ and $Z_{\text {vertex }}$ are saved for future use.

\subsubsection{Radiative Corrections to $K_{L, S} \rightarrow \pi^{+} \pi^{-} \gamma$}

Once the decay itself has been produced, there is another physical process which must be taken into account - that of additional radiation, via bremsstrahlung, from one of the pions. In this case, the decay is actually an example of $K_{L, S} \rightarrow \pi^{+} \pi^{-} \gamma \gamma$. While this particular decay has not been directly observed, evidence of it exists in the left hand side of the $\pi^{+} \pi^{-} \gamma$ invariant mass plot, which contains a larger than expected tail which is reminiscent of the analogous tail seen in $K_{L, S} \rightarrow \pi^{+} \pi^{-}$decays (which comes from $K_{L, S} \rightarrow \pi^{+} \pi^{-} \gamma$ ).

In order to account for this additional process, we enlist the PHOTOS [38] software package in order to produce radiative corrections for, and thus additional photons out of the $K_{L, S} \rightarrow \pi^{+} \pi^{-} \gamma$ decays. PHOTOS calculates the probability with which it should produce a second photon, and when appropriate, the energy of the second photon. PHOTOS is a general tool used for a wide variety of decays, so it comes as no surprise that using it "out of the box" produces less than satisfactory results while its use results in a closer match between data and Monte Carlo in the region just to the left of the kaon mass peak in the plot of $\pi^{+} \pi^{-} \gamma$ invariant mass, compared to the case without PHOTOS, the match isn't great. Appendix G details corrections made to PHOTOS specifically for the $K_{L, S} \rightarrow \pi^{+} \pi^{-} \gamma$ decay. These corrections are used throughout this analysis.

\subsection{Tracing of Decay Products Through KTeV De- tector}

Once the kinematics of the daughter particles are determined in the lab frame, each particle is traced through the detector. Multiple scattering through the various detector elements is handled using GEANT [39] a powerful and detailed software package used to simulate the passage of radiation ( charged and neutral) through matter. GEANT is used to determine the scattering characteristics for the various detector elements including the vacuum window, the helium bags between the drift chambers, 
the drift chambers themselves, the VV' counter and the muon steel. Electrons and pions are traced to the back-anti veto only, while muons are propagated through the muon steel as well. For each detector element, GEANT was used to produce a distribution of scattering angles which could then be applied within $\mathrm{KTeVMC}$ when tracing each particle. Note that GEANT is not directly used within the detector simulation as is the case with many experiments, instead GEANT-produced scattering distributions are used.

In addition to propagating each daughter particle through the $\mathrm{KTeV}$ detector, the Monte Carlo also allows daughter particles to decay ( in the case of pions ) emit secondary radiation ( in the case of electrons ) and to convert (in the case of pair production from photons ). Muons are assumed stable, and are not allowed to decay. In all cases, the secondary particles are also traced through the detector.

\subsection{Simulation of Detector Response}

Once the daughter particles have been traced through the detector, the response of the various detector components is simulated.

\subsubsection{Drift Chambers}

Given the importance of the drift chambers, they were treated with a very detailed treatment in the Monte Carlo which is well described elsewhere [6]. The primary process which the simulation was required to reproduce was the basic resolution of the drift chamber, which was built into the model by smearing the hit position in a given location in each chamber using resolution maps taken from data. The hit position was then translated into a drift time using the inverse of the drift maps also obtained from data. The simulation of the chambers also includes a certain amount of inefficiency coming from different sources. The first is a position dependent inefficiency which is measured for each wire, and has an increasing effect with radial distance from the wire. The second is a "late" hit inefficiency where the primary ions produced by the track which initially lie closest to the wire do not initiate a large enough cascade to create a hit. In this case, ions further from the wire first trigger a hit which comes later than the ideal case. This effect is largest in the region where the neutral beam 
transects the drift chambers. The final source of inefficiency is due to delta rays, which are electrons knocked out of the atoms comprising the chamber gas. Since delta rays often have a large amount of kinetic energy, they may reach a neighboring wire before the ionization cascade and thus cause an early hit which may be rejected by the tracking algorithm.

\subsubsection{CsI Calorimeter}

Modeling the CsI calorimeter was also a major effort [30]. Once again GEANT was used to simulate this detector element, this time to model the interaction of a daughter particle with the CsI crystals. GEANT was used to generate a number of electromagnetic (in the case of electrons and photons ) and hadronic (in the case of pions ) showers in a simulated model of the CsI calorimeter. In this case, a sample of showers were generated at various incident particle energies and impact locations around a central crystal in an array. However, the The result is a "shower library" consisting of showers appropriate for photons, electrons and pions at various energies which can then be utilized to yield a simulated shower within the calorimeter.It should be noted that all showers were generated for particles normal to the face of the CsI. Finally, it should be mentioned that hadronic interactions in the VV' trigger counter are also included in the simulation of hadronic showers in the calorimeter, as these interactions result in a substantially different energy deposition pattern in the calorimeter.

Once the Monte Carlo selects an appropriate shower to create a cluster, the energy contained within that shower is spread over six RF buckets as the scintillation light and its collection by the phototubes is not instantaneous. The energy is smeared and then converted to a digital value using the inverse of the calorimeter energy calibration map.

\subsection{Simulation of Accidental Activity}

Accidental activity - additional particles propagating in the detector, is also included in the Monte Carlo simulation. Accidental activity can veto otherwise good events by activating veto counters, causing out of time hits in drift chamber wires, and 
can create isolated, photon-like clusters in the CsI, among other effects. Accidental activity can also cause unwanted events to pass for $K_{L, S} \rightarrow \pi^{+} \pi^{-} \gamma$ decays. Accidental activity was observed and recorded during the experimental run by means of a special trigger which read out the entire detector and whose trigger rate was correlated with the overall beam intensity. This accidental data is then read during Monte Carlo generation and the energy present in each detector in a single accidental event is then added to the detectors for a given Monte Carlo event.

\subsection{Simulation of Level 1 and 2 triggers}

The final stage in the simulation of a Monte Carlo event is the simulation of the Level 1 and Level 2 triggers. This allows the effect of the various veto detectors and trigger counters to be folded into the simulation, and is quite straightforward, as the Level 1 and Level 2 triggers used simple digital logic to make their decisions. Various triggers can be selected such that events are only written to disk if certain triggers are satisfied. This ensures that the data written to disk by the Monte Carlo is a close approximation to the information actually read out from the $\mathrm{KTeV}$ experiment during the experimental run. This allows data and Monte Carlo files to be treated in much the same way during further, offline processing and analysis.

After the Level 1 and Level 2 triggers are run, the Monte Carlo data can then be feed into the software based Level 3 trigger. After this stage, the Monte Carlo data can be crunched in the same fashion as the data. The result is Monte Carlo data which has been subjected to the same requirements as the data, and should closely approximate the data as well. The quality of the simulation can be seen in the data/MC plots appearing in Chapter 5 and Appendices I,J, and K 


\section{Chapter 8}

\section{Development of the Maximum Likelihood Fit}

A method must be selected which may be used to estimate the model parameters which result in the best agreement between data and the model used. One of the most commonly used methods of estimation is the method of maximum likelihood $[40,22]$.

\subsection{General Likelihood Function}

Given $N_{D}$ sets (events) of measured quantities $\overrightarrow{x_{i}}$ and a model that predicts the probability of observing the set $\overrightarrow{x_{i}}$ as being $f\left(\overrightarrow{x_{i}} ; \vec{\alpha}\right)$ where $\vec{\alpha}$ is a vector of parameters to be estimated, the likelihood function, given by:

$$
L(\vec{\alpha})=\prod_{i=1}^{N_{D}} f\left(\overrightarrow{x_{i}} ; \vec{\alpha}\right)
$$

is maximized when $\vec{\alpha}=\hat{\vec{\alpha}}$ where $\hat{\vec{\alpha}}$ is the "best estimate" of the vector $\vec{\alpha}$ which results in the best agreement between the observed distribution of $\overrightarrow{x_{i}}$ and $f\left(\overrightarrow{x_{i}} ; \vec{\alpha}\right)$.

Equation 8.1 is intuitive, in that the total probability of a sequence of observations is equal to the product of the probability of each observation.

Since it is a probability, $f\left(\overrightarrow{x_{i}} ; \vec{\alpha}\right)$ must satisfy this equality over the entire phase 
space observed:

$$
\int_{V\left(\overrightarrow{x_{i}}\right)} d \overrightarrow{x_{i}} f\left(\overrightarrow{x_{i}} ; \vec{\alpha}\right)=1
$$

for any choice of parameters $\vec{\alpha}$. However, in this analysis we cannot obtain a closed form expression for $f\left(\overrightarrow{x_{i}} ; \vec{\alpha}\right)$, since we must take into account not only the physics of the decay to be studied, but also the detector response and effects of cuts made against the data, the latter two being handled using Monte Carlo simulation techniques. We can instead define $f\left(\overrightarrow{x_{i}} ; \vec{\alpha}\right)$ in such a way that it is explicitly normalized. Denoting the quantity $\mathcal{O}\left(\overrightarrow{x_{i}} ; \vec{\alpha}\right)$ as the relative probability for an event to be observed ${ }^{1}$, we can then define $f\left(\overrightarrow{x_{i}} ; \vec{\alpha}\right)$ as:

$$
f\left(\overrightarrow{x_{i}} ; \vec{\alpha}\right)=\frac{\mathcal{O}\left(\overrightarrow{x_{i}} ; \vec{\alpha}\right)}{\int_{V\left(\vec{x}_{i}\right)} d \overrightarrow{x_{i}} \mathcal{O}\left(\overrightarrow{x_{i}} ; \vec{\alpha}\right)}
$$

and then evaluate the integral using the theorem of Monte Carlo integration [41]:

$$
\int \mathcal{O}\left(\overrightarrow{x_{i}} ; \vec{\alpha}\right) d V \approx V\langle\mathcal{O}\rangle \pm V \sqrt{\frac{\left\langle\mathcal{O}^{2}\right\rangle-\langle\mathcal{O}\rangle^{2}}{N_{0}}}
$$

where $V$ is the volume of phase space $\overrightarrow{x_{i}}$ in which the accepted data events reside, and is bounded by cuts and detector acceptance, and

$$
\langle\mathcal{O}\rangle \equiv \frac{1}{N_{0}} \sum_{i=1}^{N_{0}} \mathcal{O}\left(\overrightarrow{x_{i}} ; \vec{\alpha}\right)
$$

and

$$
\left\langle\mathcal{O}^{2}\right\rangle \equiv \frac{1}{N_{0}} \sum_{i=1}^{N_{0}}\left[\mathcal{O}\left(\overrightarrow{x_{i}} ; \vec{\alpha}\right)\right]^{2}
$$

and $N_{0}$ is the number of points used in the Monte Carlo integration. The error term of equation 8.4 indicates that the error of the value of the integral decreases as $\frac{1}{\sqrt{N_{0}}}$ which implies that a large number of points must be utilized to get acceptable precision in the calculation.

The traditional method of Monte Carlo integration must be modified in order to be usable in this analysis. The Monte Carlo simulation of the $\mathrm{KTeV}$ detector uses a

\footnotetext{
${ }^{1}$ As will be shown later, this is the product of the probability that the observed decay will occur and the probability that the event will be accepted into the final data sample after all cuts
} 
series of steps that each uses the acceptance-rejection (Von Neumann) method [22] to obtain a distribution of events with characteristics that should be consistent with reality. Instead of a series of uniformly distributed points over the phase space $V$ each with a closed form expression for the weight $\mathcal{O}\left(\overrightarrow{x_{i}} ; \vec{\alpha}\right)$, the $\mathrm{KTeV}$ Monte Carlo will produce a number of events which are distributed according to $\mathcal{O}\left(\overrightarrow{x_{i}} ; \vec{\alpha}\right)$, each with a weight of 1 . With a method such as this, instead of the traditional method of Monte Carlo integration, we instead must use:

$$
\langle\mathcal{O}\rangle=\frac{1}{N_{0}} \sum_{i=1}^{N_{M C}} 1=\frac{N_{M C}}{N_{0}}
$$

where

$N_{0}$ is now the number of acceptance-rejection trials ${ }^{2}$ used to generate the Monte Carlo sample

$N_{M C}$ is the number of Monte Carlo events that pass all analysis cuts, including the geometric acceptance of the detector, trigger simulation and crunch cuts.

The disadvantage of this technique is that if $\mathcal{O}\left(\overrightarrow{x_{i}} ; \vec{\alpha}\right)$ is dependent on the parameters $\vec{\alpha}$, then a new Monte Carlo sample must be generated whenever a new set of parameters is to be used in a likelihood calculation. Instead, we will produce a single set of Monte Carlo data, including the detector simulation and all cuts, and re-weight these Monte Carlo events in order to obtain a distribution of events which is consistent with any set of parameters $\vec{\alpha}$. First, we must remove by division the weight $\mathcal{O}\left(\overrightarrow{x_{i}} ; \overrightarrow{\alpha_{0}}\right)$ which was used to produce the Monte Carlo sample, so that we have a "flat" distribution. Then, the current weight, i.e. that using the current choice of parameters $\vec{\alpha}$, is applied. The use of this reweighting technique will modify equation 8.7 to read:

$$
\langle\mathcal{O}\rangle \equiv \frac{1}{N_{0}} \sum_{i=1}^{N_{M C}} \frac{\mathcal{O}\left(\overrightarrow{x_{i}} ; \vec{\alpha}\right)}{\mathcal{O}\left(\overrightarrow{x_{i}} ; \overrightarrow{\alpha_{0}}\right)}
$$

This equation can be interpreted as the number of events which would have passed

\footnotetext{
${ }^{2}$ this would be the number of times a set of $\overrightarrow{x_{i}}$ is generated. This number is decreased as events are accepted or rejected to make up the decay distribution defined by the matrix element for the decay, and as events are lost due to detector acceptance, the triggers and the analysis cuts.
} 
all cuts if the Monte Carlo sample had been generated using $\vec{\alpha}$ instead of $\overrightarrow{\alpha_{0}}$.

Inserting this result into equation 8.4 leads to:

$$
\int \mathcal{O}\left(\overrightarrow{x_{i}} ; \vec{\alpha}\right) d V \approx V \frac{1}{N_{0}} \sum_{i=1}^{N_{M C}} \frac{\mathcal{O}\left(\overrightarrow{x_{i}} ; \vec{\alpha}\right)}{\mathcal{O}\left(\overrightarrow{x_{i}} ; \overrightarrow{\alpha_{0}}\right)}
$$

This is the final result for the integral when the reweighting method is to be used.

Now using equation 8.9 along with equations 8.3 and 8.1 we can write:

$$
L(\vec{\alpha})=\prod_{i=1}^{N_{D}} \frac{\mathcal{O}\left(\overrightarrow{x_{i}} ; \vec{\alpha}\right)}{\frac{V}{N_{0}} \sum_{i=1}^{N_{M C}} \frac{\mathcal{O}\left(\overrightarrow{x_{i}} ; \vec{\alpha}\right)}{\mathcal{O}\left(\overrightarrow{x_{i}} ; \overrightarrow{\alpha_{0}}\right)}}
$$

When evaluated over a large number of events, equation 8.10 will become asymptotically approach zero, since $f\left(\overrightarrow{x_{i}} ; \vec{\alpha}\right)$ is by definition less than 1 . Instead, it is more appropriate to maximize the logarithm of the likelihood function, and not the likelihood function itself. Doing so will still yield the same final estimates for $\vec{\alpha}$. The "log likelihood function" is given by:

$$
\log L(\vec{\alpha})=\sum_{i=1}^{N_{D}}\left[\log \mathcal{O}\left(\overrightarrow{x_{i}} ; \vec{\alpha}\right)-\log V-\log \sum_{i=1}^{N_{M C}} \frac{\mathcal{O}\left(\overrightarrow{x_{i}} ; \vec{\alpha}\right)}{\mathcal{O}\left(\overrightarrow{x_{i}} ; \overrightarrow{x_{0}}\right)}+\log N_{0}\right]
$$

However, as the sum runs over all data points, the sum of all the terms, except the first, may be evaluated to get:

$$
\begin{aligned}
\log L(\vec{\alpha}) & =\sum_{i=1}^{N_{D}}\left[\log \mathcal{O}\left(\overrightarrow{x_{i}} ; \vec{\alpha}\right)\right]-N_{D} \log V-N_{D} \log \sum_{i=1}^{N_{M C}} \frac{\mathcal{O}\left(\overrightarrow{x_{i}} ; \vec{\alpha}\right)}{\mathcal{O}\left(\overrightarrow{x_{i}} ; \overrightarrow{\alpha_{0}}\right)} \\
& +N_{D} \log N_{0}
\end{aligned}
$$

We can now split $\mathcal{O}$ into two pieces:

$$
\mathcal{O}\left(\overrightarrow{x_{i}} ; \vec{\alpha}\right)=\mathcal{D}\left(\overrightarrow{x_{i}} ; \vec{\alpha}\right) \times \mathcal{A}\left(\overrightarrow{x_{i}}\right)
$$

where

$\mathcal{D}\left(\overrightarrow{x_{i}} ; \vec{\alpha}\right)$ describes the relative probability for the decay to occur. The decay rate as 
given in equation 3.40 will be used in this analysis.

$\mathcal{A}\left(\overrightarrow{x_{i}}\right)$ is the acceptance of the $\mathrm{KTeV}$ detector for an event with characteristics $\overrightarrow{x_{i}}$. This can be thought of as the probability for a decay with characteristics $\overrightarrow{x_{i}}$ to result in a positive trigger at Levels 1, 2, and 3, pass all crunch and analysis cuts, and thus be represented in the data.

Inserting this into equation 8.11 yields:

$$
\begin{aligned}
\log L(\vec{\alpha}) & =\sum_{i=1}^{N_{D}}\left[\log \mathcal{D}\left(\overrightarrow{x_{i}} ; \vec{\alpha}\right) \mathcal{A}\left(\overrightarrow{x_{i}}\right)\right]-N_{D} \log V-N_{D} \log \sum_{i=1}^{N_{M C}} \frac{\mathcal{D}\left(\overrightarrow{x_{i}} ; \vec{\alpha}\right) \mathcal{A}\left(\overrightarrow{x_{i}}\right)}{\mathcal{D}\left(\overrightarrow{x_{i}} ; \overrightarrow{\alpha_{0}}\right) \mathcal{A}\left(\overrightarrow{x_{i}}\right)} \\
& +N_{D} \log N_{0} \\
& =\sum_{i=1}^{N_{D}} \log \mathcal{D}\left(\overrightarrow{x_{i}} ; \vec{\alpha}\right)+\sum_{i=1}^{N_{D}} \log \mathcal{A}\left(\overrightarrow{x_{i}}\right)-N_{D} \log V \\
& -N_{D} \log \sum_{i=1}^{N_{M C}} \frac{\mathcal{D}\left(\overrightarrow{x_{i}} ; \vec{\alpha}\right)}{\mathcal{D}\left(\overrightarrow{x_{i}} ; \overrightarrow{\alpha_{0}}\right)}+N_{D} \log N_{0}
\end{aligned}
$$

Finally, making the definitions:

$$
\begin{aligned}
& \log \mathcal{L}(\vec{\alpha})=\sum_{i=1}^{N_{D}} \log \mathcal{D}\left(\overrightarrow{x_{i}} ; \vec{\alpha}\right)-N_{D} \log \sum_{i=1}^{N_{M C}} \frac{\mathcal{D}\left(\overrightarrow{x_{i}} ; \vec{\alpha}\right)}{\mathcal{D}\left(\overrightarrow{x_{i}} ; \overrightarrow{\alpha_{0}}\right)} \\
& \log \mathcal{C}=\sum_{i=1}^{N_{D}} \log \mathcal{A}\left(\overrightarrow{x_{i}}\right)+N_{D} \log N_{0}-N_{D} \log V
\end{aligned}
$$

We can write

$$
\log L(\vec{\alpha})=\log \mathcal{L}(\vec{\alpha})+\log \mathcal{C}
$$

taking the derivative of this expression yields:

$$
\frac{\partial \log L(\vec{\alpha})}{\partial \alpha_{j}}=\frac{\partial \log \mathcal{L}(\vec{\alpha})}{\partial \alpha_{j}}
$$

which indicates that we can drop the constant term $\mathcal{C}$ and maximize instead

$$
\log \mathcal{L}(\vec{\alpha})=\sum_{i=1}^{N_{D}} \log \mathcal{D}\left(\overrightarrow{x_{i}} ; \vec{\alpha}\right)-N_{D} \log \sum_{i=1}^{N_{M C}} \frac{\mathcal{D}\left(\overrightarrow{x_{i}} ; \overrightarrow{\mathcal{\alpha}}\right)}{\mathcal{D}\left(\overrightarrow{x_{i}} ; \overrightarrow{\alpha_{0}}\right)}
$$


We can now explicitly identify the fit parameters:

$$
\vec{\alpha}=\left\{\widehat{e}, g_{E 1}, \widetilde{g_{M 1}}, a_{1} / a_{2}\right\}
$$

as well as the observables for each event

$$
\overrightarrow{x_{i}}=\left\{E_{\gamma}^{*}, \cos (\theta), p_{K}, Z_{\text {vertex }}\right\}
$$

where $E_{\gamma}^{*}$ is the photon energy in the kaon rest frame, $\cos (\theta)$ is the angle between the photon and $\pi^{+}$momenta in the $\pi \pi$ rest frame, $p_{K}$ is the momentum of the parent kaon, and $Z_{\text {vertex }}$ is the distance between the decay vertex and the target.

Equation 8.18 will be evaluated with $\mathcal{D}\left(\overrightarrow{x_{i}} ; \vec{\alpha}\right)$ set equal to the decay rate as shown in equation 3.40. The first part of the modified log likelihood function depends only on the distribution of data and the value of $\mathcal{D}\left(\overrightarrow{x_{i}} ; \vec{\alpha}\right)$. The second part depends only on the Monte Carlo sample used and the values of $\mathcal{D}\left(\overrightarrow{x_{i}} ; \vec{\alpha}\right)$ and $\mathcal{D}\left(\overrightarrow{x_{i}} ; \overrightarrow{\alpha_{0}}\right)$ for each Monte Carlo event. Note that the detector acceptance $\mathcal{A}\left(\overrightarrow{x_{i}}\right)$ doesn't appear explicitly in this equation, nor does the phase space volume $V$ nor does $N_{0}$. Figure 8.1 illustrates the use of this method.

\subsection{Total Likelihood Function}

The modified $\log$ likelihood function as shown in Equation 8.18 is applicable to any general data set, but careful attention must be paid to what is summed over. The part of the modified log likelihood function that arises due to the normalization Monte Carlo sample must sum over a Monte Carlo sample which approximates, as closely as possible, the data sample used. For example, if we perform the log likelihood fit on the 1997 regenerator data only, the normalization Monte Carlo must consist of Monte Carlo simulated 1997 regenerator events only. The same would hold if we fit the 1997 vacuum data only. However, when different data samples are used, the normalization Monte Carlo must consist of events corresponding to each different data sample. In addition, the proportion of each Monte Carlo subsample that makes up the total must mirror the relative size of each data sample. If this is not the case, the likelihood function is not properly normalized, and will give unreliable results. After noting 
this, we may break up the sums in equation 8.18 to explicitly show the contribution from each sample:

$$
\begin{aligned}
& \sum_{i=1}^{N_{D}} \log \mathcal{D}\left(\overrightarrow{x_{i}} ; \vec{\alpha}\right)=\sum_{i=1}^{N_{D}^{97 V A C}} \log \mathcal{D}\left(\overrightarrow{x_{i}} ; \vec{\alpha}\right)+\sum_{i=1}^{N_{D}^{97 R E G}} \log \mathcal{D}\left(\overrightarrow{x_{i}} ; \vec{\alpha}\right) \\
& +\sum_{i=1}^{N_{D}^{99 V A C}} \log \mathcal{D}\left(\overrightarrow{x_{i}} ; \vec{\alpha}\right)+\sum_{i=1}^{N_{D}^{99 R E G}} \log \mathcal{D}\left(\overrightarrow{x_{i}} ; \vec{\alpha}\right)
\end{aligned}
$$

for the data contribution, and for the normalization Monte Carlo:

$$
\begin{aligned}
N_{D} \log \sum_{i=1}^{N_{M C}} \frac{\mathcal{D}\left(\overrightarrow{x_{i}} ; \vec{\alpha}\right)}{\mathcal{D}\left(\overrightarrow{x_{i}} ; \overrightarrow{\alpha_{0}}\right)} & =N_{D} \log \left[\sum_{i=1}^{N_{M C}^{97 V A C}} \frac{\mathcal{D}\left(\overrightarrow{x_{i}} ; \vec{\alpha}\right)}{\mathcal{D}\left(\overrightarrow{x_{i}} ; \overrightarrow{\alpha_{0}}\right)}+\sum_{i=1}^{N_{M C}^{97 R E G}} \frac{\mathcal{D}\left(\overrightarrow{x_{i}} ; \overrightarrow{\alpha^{\prime}}\right)}{\mathcal{D}\left(\overrightarrow{x_{i}} ; \overrightarrow{\alpha_{0}}\right)}\right. \\
& \left.+\sum_{i=1}^{N_{M C}^{99 V A C}} \frac{\mathcal{D}\left(\overrightarrow{x_{i}} ; \vec{\alpha}\right)}{\mathcal{D}\left(\overrightarrow{x_{i}} ; \overrightarrow{\alpha_{0}}\right)}+\sum_{i=1}^{N_{M C E G}^{99 R E G}} \frac{\mathcal{D}\left(\overrightarrow{x_{i}} ; \vec{\alpha}\right)}{\mathcal{D}\left(\overrightarrow{x_{i}} ; \overrightarrow{\alpha_{0}}\right)}\right]
\end{aligned}
$$

where $N_{D}$ is the number of data events in the entire data sample, and $N_{M C}^{97 V A C}$, $N_{M C}^{97 R E G}, N_{M C}^{99 V A C}, N_{M C}^{99 R E G}$ must be in exact proportion to $N_{D}^{97 V A C}, N_{D}^{97 R E G}, N_{D}^{99 V A C}$, $N_{D}^{99 R E G}$.

However, there are two complications. The first is the fact that it is very difficult to generate large Monte Carlo samples of an exact size, as hundreds of separate jobs are used to generate the normalization Monte Carlo sample, and some of these jobs may fail, resulting in the loss of some events. The second problem is that the relative size of the Monte Carlo samples should only match that of the data at the true value of $\vec{\alpha}$. The solution is this:

- Choose values of $\vec{\alpha}$ close to the expected best fit values, denote these choices by $\vec{\alpha}_{g}$

- Using the reweighting method as shown in (8.8), compute the number of generated events which would have passed all cuts, if the Monte Carlo had been generated with $\vec{\alpha}=\vec{\alpha}_{g}$

- For each of the Monte Carlo samples, scale the sum by the factor $N_{\text {wanted }} / N_{\text {generated }}$ where $N_{\text {wanted }}$ is the number of data events in each sample, 
and $N_{\text {generated }}$ is the number expected to have passed all cuts at $\vec{\alpha}_{g}$

Using the above procedure, the Monte Carlo contribution to the likelihood becomes:

$$
\begin{aligned}
& N_{D} \log \sum_{i=1}^{N_{M C}} \frac{\mathcal{D}\left(\overrightarrow{x_{i}} ; \vec{\alpha}\right)}{\mathcal{D}\left(\overrightarrow{x_{i}} ; \overrightarrow{\alpha_{0}}\right)} \rightarrow \\
& N_{D} \log \left[N_{D}^{97 V A C} \frac{\sum_{i=1}^{N_{M C}^{97 V A C}} \frac{\mathcal{D}\left(\overrightarrow{x_{i}} ; \vec{\alpha}\right)}{\mathcal{D}\left(\overrightarrow{x_{i}} ; \overrightarrow{\alpha_{0}}\right)}}{\sum_{i=1}^{N_{M C}^{97 V A C}} \frac{\mathcal{D}\left(\overrightarrow{x_{i}} ; \overrightarrow{\alpha_{g}}\right)}{\mathcal{D}\left(\overrightarrow{x_{i}} ; \overrightarrow{\alpha_{0}}\right)}}+N_{D}^{97 R E G} \frac{\sum_{i=1}^{N_{M C}^{97 R E G}} \frac{\mathcal{D}\left(\overrightarrow{x_{i}} ; \vec{\alpha}\right)}{\mathcal{D}\left(\overrightarrow{x_{i}} ; \overrightarrow{\alpha_{0}}\right)}}{\sum_{i=1}^{N_{M C}^{97 R E G}} \frac{\mathcal{D}\left(\overrightarrow{x_{i}} ; \overrightarrow{\alpha_{g}}\right)}{\mathcal{D}\left(\overrightarrow{x_{i}} ; \overrightarrow{\alpha_{0}}\right)}}\right. \\
& \left.+N_{D}^{99 V A C} \frac{\sum_{i=1}^{N_{M C}^{99 V A C}} \frac{\mathcal{D}\left(\overrightarrow{x_{i}} ; \vec{\alpha}\right)}{\mathcal{D}\left(\overrightarrow{x_{i}} ; \overrightarrow{\alpha_{0}}\right)}}{\sum_{i=1}^{N_{M C}^{99 V} A C} \frac{\mathcal{D}\left(\overrightarrow{x_{i}} ; \overrightarrow{\alpha_{g}}\right)}{\mathcal{D}\left(\overrightarrow{x_{i}} ; \overrightarrow{\alpha_{0}}\right)}}+N_{D}^{99 R E G} \frac{\sum_{i=1}^{N_{M C}^{99 R E G}} \frac{\mathcal{D}\left(\overrightarrow{x_{i}} ; \vec{\alpha}\right)}{\mathcal{D}\left(\overrightarrow{x_{i}} ; \overrightarrow{\alpha_{0}}\right)}}{\sum_{i=1}^{N_{M C}^{99 R E G}} \frac{\mathcal{D}\left(\overrightarrow{x_{i}} ; \overrightarrow{\alpha_{g}}\right)}{\mathcal{D}\left(\overrightarrow{x_{i}} ; \overrightarrow{\alpha_{0}}\right)}}\right]
\end{aligned}
$$

We can now write the final form of the total modified log likelihood function, which can be used to fit the entire $\mathrm{KTeV} K_{L, S} \rightarrow \pi^{+} \pi^{-} \gamma$ dataset in both beams and years when using arbitrary amounts of normalization Monte Carlo events. The equation is: 


$$
\begin{aligned}
& \log \mathcal{L}(\vec{\alpha})=\sum_{i=1}^{N_{D}^{97 V A C}} \log \mathcal{D}\left(\overrightarrow{x_{i}} ; \vec{\alpha}\right)+\sum_{i=1}^{N_{D}^{97 R E G}} \log \mathcal{D}\left(\overrightarrow{x_{i}} ; \vec{\alpha}\right) \\
& +\sum_{i=1}^{N_{D}^{99 V A C}} \log \mathcal{D}\left(\overrightarrow{x_{i}} ; \vec{\alpha}\right)+\sum_{i=1}^{N_{D}^{99 R E G}} \log \mathcal{D}\left(\overrightarrow{x_{i}} ; \vec{\alpha}\right) \\
& -\left(N_{D}^{97 V A C}+N_{D}^{97 R E G}+N_{D}^{99 V A C}+N_{D}^{99 R E G}\right) \\
& \times \log \left[N_{D}^{97 V A C} \frac{\sum_{i=1}^{N_{M C}^{97 V A C}} \frac{\mathcal{D}\left(\overrightarrow{x_{i}} ; \vec{\alpha}\right)}{\mathcal{D}\left(\overrightarrow{x_{i}} ; \overrightarrow{\alpha_{0}}\right)}}{\sum_{i=1}^{N_{M C}^{97 V A C}} \frac{\mathcal{D}\left(\overrightarrow{x_{i}} ; \vec{\alpha}_{g}\right)}{\mathcal{D}\left(\overrightarrow{x_{i}} ; \overrightarrow{\alpha_{0}}\right)}}+N_{D}^{97 R E G} \frac{\sum_{i=1}^{N_{M C}^{97 R E G}} \frac{\mathcal{D}\left(\overrightarrow{x_{i}} ; \vec{\alpha}\right)}{\mathcal{D}\left(\overrightarrow{x_{i}} ; \overrightarrow{\alpha_{0}}\right)}}{\sum_{i=1}^{N_{M C}^{97 R E G}} \frac{\mathcal{D}\left(\overrightarrow{x_{i}} ; \vec{\alpha}_{g}\right)}{\mathcal{D}\left(\overrightarrow{x_{i}} ; \overrightarrow{\alpha_{0}}\right)}}\right. \\
& \left.+N_{D}^{99 V A C} \frac{\sum_{i=1}^{N_{M C}^{99 V A C}} \frac{\mathcal{D}\left(\overrightarrow{x_{i}} ; \vec{\alpha}\right)}{\mathcal{D}\left(\overrightarrow{x_{i}} ; \overrightarrow{\alpha_{0}}\right)}}{\sum_{i=1}^{N_{M C}^{99 V A C}} \frac{\mathcal{D}\left(\overrightarrow{x_{i}} ; \overrightarrow{\alpha_{g}}\right)}{\mathcal{D}\left(\overrightarrow{x_{i}} ; \overrightarrow{\alpha_{0}}\right)}}+N_{D}^{99 R E G} \frac{\sum_{i=1}^{N_{M C}^{99 R E G}} \frac{\mathcal{D}\left(\overrightarrow{x_{i}} ; \vec{\alpha}\right)}{\mathcal{D}\left(\overrightarrow{x_{i}} ; \overrightarrow{\alpha_{0}}\right)}}{\sum_{i=1}^{N_{M C}^{99 R E G}} \frac{\mathcal{D}\left(\overrightarrow{x_{i}} ; \overrightarrow{\alpha_{g}}\right)}{\mathcal{D}\left(\overrightarrow{x_{i}} ; \overrightarrow{\alpha_{0}}\right)}}\right]
\end{aligned}
$$




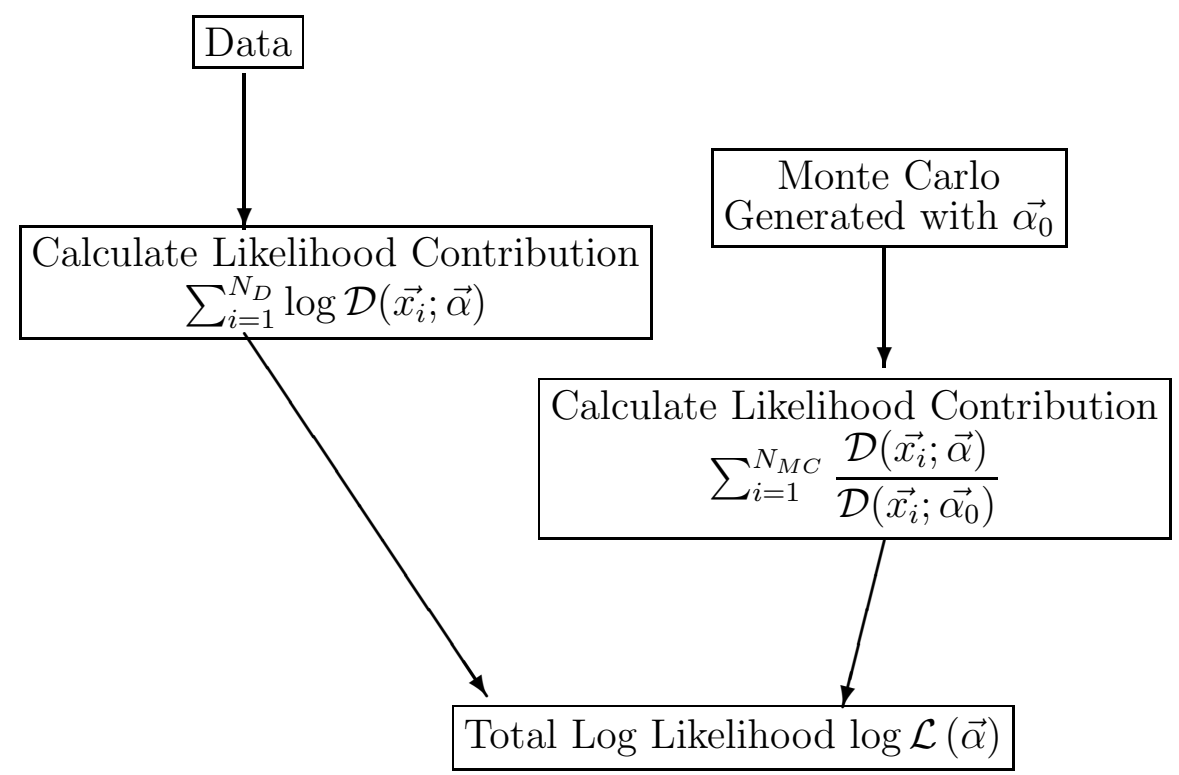

Figure 8.1: Schematic describing the reweighting method used to calculate the likelihood function 


\subsection{Execution of Maximum Likelihood Fit}

In order to perform a maximum likelihood fit on the data, a computer program must be written that evaluates the modified log likelihood function (Eq. 8.24). This program consists of two general parts:

- A routine that maximizes a general multivariate function, in this case, the log likelihood function.

- A routine that calculates the log likelihood function for the entire data set given a set of parameters $\vec{\alpha}$.

Noting that these two parts are completely independent gives us freedom in choosing which maximization algorithm to use. Given that the log likelihood function is a sum over all data and Monte Carlo events, we can also choose to break up the likelihood calculation into a number of smaller parts. This is quite advantageous, as the number of Monte Carlo events must exceed the number of data events by a sizable margin in order to minimize uncertainty due to the statistical error in the Monte Carlo integration. This would normally cause the calculation time for the likelihood to become unacceptably long. However, by breaking up the likelihood calculation into many smaller pieces, we can spread the pieces among many different CPUs, reducing the time required for a likelihood fit by a factor roughly equal to the number of CPUs used.

The complete set of tools developed in order to realize this scheme is as follows:

- RUNPMGFIT is the highest level program, and spawns all others. It:

- creates the initial files that control the function of LMAX and specifies the initial guess of the fit parameters.

- spawns a single instance of LMAX

- spawns an instance of LCALC for every data file used

- combines likelihood values from various LCALC instances into the total likelihood, which is then fed back into LMAX

- LMAX is the program that maximizes the log likelihood function as shown in (Eq. 8.24) It: 
- writes the current choice of parameters to a file that can be accessed by RUNLCALC

- reads in the total likelihood value from a file written by RUNPMGFIT

- determines the set of parameters $\vec{\alpha}$ which maximizes the likelihood function.

It employs two different techniques to maximize the likelihood:

- Powell's Method as implemented in Numerical Recipes in Fortran [41]

- A modified version [42] of the Davidson-Fletcher-Powell Method, implemented in CERN's MINUIT package [43]

It also is able to estimate parameter errors using the MINOS routine, which is also part of MINUIT.

- RUNLCALC fetches the value of the current fit parameters, passes these to LCALC, and then passes the likelihood contribution to RUNPMGFIT. It also spawns one instance of LCALC when the likelihood fitter is first started.

- LCALC reads in the values of the current parameters, calculates the likelihood contribution from a subset of the data and/or Monte Carlo samples, and then writes out the likelihood contribution of this subset.

As MINUIT and Powell's Method are existing packages, or program listings, in FORTRAN 77, LMAX is written in FORTRAN 77. LCALC must make use of the same matrix element as that in the Monte Carlo of $K_{L, S} \rightarrow \pi^{+} \pi^{-} \gamma$, as well as handling the same physics of the evolution of kaon states and $K_{S}$ regeneration so LCALC was also written in FORTRAN 77, so that as many routines could be reused as possible. Since RUNPMGFIT and RUNLCALC must spawn separate, parallel processes, as well as transfer files to and from remote computers, these were both written on Python, an object oriented programming language which is able to make system calls.

These programs and scripts together constitute the fitting package in its entirety. The sequence of execution is:

1. Execute RUNPMGFIT 
2. RUNPMGFIT spawns LMAX

3. RUNPMGFIT spawns RUNLCALC on a remote computer

4. RUNLCALC spawns LCALC.

5. Step 3 is repeated for each separate data or Monte Carlo sample

6. LMAX chooses a trial set of parameters, and writes them to a file

7. RUNLCALC retrieves this file, checks to see if the parameters are new, and then copies it to another file

8. which is then read by LCALC. LCALC uses this set of parameters to calculate the likelihood, which is then written to another file.

9. RUNLCALC then transfers this file to a location readable by RUNPMGFIT.

10. RUNPMGFIT scans through these files and calculates the total likelihood.

11. LMAX reads in the total likelihood, and then chooses a new set of parameters.

12. Step 6 is repeated until the maximum log likelihood value is obtained.

One important feature of the code above is the treatment of the parameter $\widehat{e}$. This parameter has not been previously measured and it the most important parameter of the analysis. In order to prevent the measurement of this parameter from being biased, the true value of $\widehat{e}$ is hidden by the addition of a unknown offset of a random size and sign. The fitter only prints out the shifted value of $\widehat{e}$, and all parameter files used by the fitter also use the shifted value of $\widehat{e}$. Only in LCALC - immediately before the calculation of the decay amplitudes, is the true value of $\widehat{e}$ calculated and used.

Once the analysis is finished the true value of $\widehat{e}$ is revealed by running a standalone program that removes the offset. 


\subsubsection{Calculation of Likelihood using LCALC}

LCALC is the program that forms the core of the likelihood fitter, as it is the piece which actually calculates the likelihood itself. In order to calculate the likelihood, it must first calculate:

- A best guess of the kaon wavefunction for a particular event

- The decay amplitudes for each photon emission process

In order to estimate the kaon wavefunction, LCALC uses the kaon momentum and z position of the decay vertex from each event. In order to calculate the decay amplitudes, LCALC must use the values of $E_{\gamma}^{*}$ and $\cos (\theta)$ found for each event.

\section{Treatment of Kaon Wavefunction for Data}

The first step in the likelihood calculation is the determination of the kaon wavefunction for each event. The importance of the kaon wavefunctions can be seen by expressing Equation 3.40 as:

$$
\begin{aligned}
\mathcal{D}\left(\overrightarrow{x_{i}} ; \vec{\alpha}\right)= & \frac{d \Gamma}{d E_{\gamma}^{*} d \cos (\theta)} \\
= & \frac{d \Gamma_{K_{L}}}{d E_{\gamma}^{*} d \cos (\theta)}\left|a_{L}(t)\right|^{2}+\frac{d \Gamma_{K_{S}}}{d E_{\gamma}^{*} d \cos (\theta)}\left|a_{S}(t)\right|^{2} \\
& +2 \operatorname{Re}\left[\frac{d \gamma_{L S}}{d E_{\gamma}^{*} d \cos (\theta)} a_{L}(t)^{\dagger} a_{S}(t)\right]
\end{aligned}
$$

where $a_{S}(t)$ and $a_{L}(t)$ are complex quantities encoding the relative amplitudes for

the $K_{S}$ and $K_{L}$ in the overall kaon wavefunction. Recall that a $K^{0}$ produced in the target would have:

$$
\begin{aligned}
& a_{S}(t=0)=1 \\
& a_{L}(t=0)=1
\end{aligned}
$$

While a $\bar{K}^{0}$ would have:

$$
\begin{aligned}
& a_{S}(t=0)=1 \\
& a_{L}(t=0)=-1
\end{aligned}
$$


Some fit parameters may change the composition of the kaon wavefunction, so this step may be repeated many times during a fit. Conversely, if these parameters are not changed the wavefunction is not recalculated in order to speed up the execution of LCALC.

In the beginning of each event, one of the strong interaction eigenstates $K^{0}$ and $\bar{K}^{0}$ are produced in the target. However it is not possible to identify which particle was produced. The solution is to calculate the value of $\mathcal{D}\left(\overrightarrow{x_{i}} ; \vec{\alpha}\right)$ using both possibilities and then to take the average between the two values of $\mathcal{D}\left(\vec{x}_{i} ; \vec{\alpha}\right)$. As detailed in Appendix F the average value of $\mathcal{D}\left(\vec{x}_{i} ; \vec{\alpha}\right)$ can be expressed instead as the value of $\mathcal{D}\left(\vec{x}_{i} ; \vec{\alpha}\right)$ that arises from using the average squared moduli of all possible kaon wavefunctions.

For data, each set of moduli are computed by first generating either a $K^{0}$ or $\bar{K}^{0}$ somewhere in the target, and expressing the initial wavefunction in the $K_{L^{-}} K_{S}$ basis shown above. However, the relative probability of a $K^{0}$ or $\bar{K}^{0}$ to be produced varies over the range of kaon momentum. To account for this, the production cross sections (see section 7.1) for $K^{0}$ and $\bar{K}^{0}$ are calculated using the reconstructed kaon momentum for each event, treated as dimensionless probabilities, and then the relative probability of either possibility is computed by dividing each cross section by the sum of both cross sections. The initial wavefunction is then propagated (see Section 7.1) from the production point in the target through all material in the beamline up to the measured $\mathrm{z}$ position of the decay vertex. The moduli of the kaon wavefunction in the $K_{L^{-}} K_{S}$ basis is then computed and the three moduli are then weighted by the relative production probability mentioned earlier.

Once the moduli using the $K^{0}$ and $\bar{K}^{0}$ initial states are obtained, the average of each modulus is computed and store for future iterations of the fit and used in the calculation of $\mathcal{D}\left(\overrightarrow{x_{i}} ; \vec{\alpha}\right)$. Doing this accounts for all the physics of particle production and propagation.

It should be reinforced that the production cross sections for $K^{0}$ and $\bar{K}^{0}$ are taken from the KTeVMC. The treatment of the wavefunction evolution mirrors that used by the fitting program for $R e\left(\frac{\epsilon^{\prime}}{\epsilon}\right)$ namely KFIT. KFIT's kaon evolution includes a more detailed model of the regenerator which includes parameters for differences in the flux between the vacuum and regenerator beams, a momentum dependent regenerator transmission factor, and of course the regenerator. The regenerator's amplitude is assumed to have a power law dependence on momentum, and the modeling of the 
phase of the regenerator amplitude is included as well. More information can be found in [6] and [7]. For the purposes of cross-checks and the estimation of systematic errors, many of the parameters that effect production and propagation of the wavefunction can be changed in order to observe the effect on the value of $\mathcal{D}\left(\overrightarrow{x_{i}} ; \vec{\alpha}\right)$ and thus the total value of the log likelihood function.

\section{Treatment of Wavefunctions For Monte Carlo Events}

In the course of Monte Carlo generation, the kaon wavefunctions are produced, propagated, and saved for each event. In principle, these saved wavefunctions could be used to calculate the value of $\mathcal{D}\left(\overrightarrow{x_{i}} ; \vec{\alpha}\right)$ however LCALC uses the more detailed treatment of the regenerator that is found in KFIT. This requires that the generated wavefunction be discarded and recomputed by LCALC. The production position in the target of each simulated kaon, the kaon momentum, along with the kaon's identity $\left(K^{0}\right.$ or $\left.\bar{K}^{0}\right)$ as generated by the Monte Carlo are used to generate an initial state wavefunction. Since the kaon's identity is already determined, the value of $\mathcal{D}\left(\overrightarrow{x_{i}} ; \vec{\alpha}\right)$ is not multiplied by the production cross-sections. ${ }^{3}$ The initial kaon state is then evolved using the KFIT model in order for the Monte Carlo to receive the same treatment as the data, and the moduli are then produced in order to calculate $\mathcal{D}\left(\overrightarrow{x_{i}} ; \vec{\alpha}\right)$.

\section{Treatment of Decay Amplitudes}

The next step in each likelihood calculation is the evaluation of the decay amplitudes. The current values of the parameters $\vec{\alpha}$ are used in each iteration. For data events, measured values of $\overrightarrow{x_{i}}\left(p_{K}, Z_{\text {vertex }}, \cos (\theta)\right.$ and $\left.E_{\gamma}^{*}\right)$ are used. The value of $E_{\gamma}^{*}$ was determined using the CsI cluster energy $E_{\gamma}^{* C A L}$ in order to minimize systematic errors and maximize the precision of the fit. It is possible to use $E_{\gamma}^{* K I N}$ instead, however the candidate photon cluster must still be used to obtain the photon momentum vector which is needed to calculate the value of $\cos (\theta)$. Accordingly, some use of the candidate photon cluster is unavoidable. Additionally, each source of $E_{\gamma}^{*}$ has different

\footnotetext{
${ }^{3}$ The reason for this is that the cross-sections have already been used during the generation of the Monte Carlo events in order to determine if a $K^{0}$ or $\bar{K}^{0}$ should be produced, so the effect is already built into each event, although implicity.

This is not the case for data, since the identity of each kaon is unknown. The solution in this case is to allow for both possiblities and to weight each using the production cross-sections
} 
resolution effects which are a function of $E_{\gamma}^{*}$ itself - using $E_{\gamma}^{* K I N}$ results in a better

resolution at higher values of $E_{\gamma}^{*}$ however at lower photon energies $E_{\gamma}^{* C A L}$ is more precise.

For Monte Carlo events, the true, generated values of $\overrightarrow{x_{i}}$ are used, not the reconstructed values - the purpose of the normalization Monte Carlo is to properly normalize the likelihood function, NOT to account for various resolution effects in the fit.

For both data and Monte Carlo events, the calculation of the the decay amplitudes is done using the same subroutines used in the Monte Carlo.

\section{Treatment of Reweighting Factors}

Once $\mathcal{D}\left(\overrightarrow{x_{i}} ; \vec{\alpha}\right)$ is calculated, the likelihood contribution from data is fully known. However, for Monte Carlo, it is necessary to apply reweighting factors to each event. The first reweighting factor is calculated in the final analysis stage and is used to fine-tune the Monte Carlo so that the best agreement between data and Monte Carlo is obtained. An example would be the application of a reweighting factor to remove a slope in the data/MC plot of CsI cluster energy. This first reweighting factor is multiplied with the $\mathcal{D}\left(\overrightarrow{x_{i}} ; \vec{\alpha}\right)$. The second reweighting factor applied to each Monte Carlo event is the original generated value of $\mathcal{D}\left(\overrightarrow{x_{i}} ; \vec{\alpha}\right), \mathcal{D}\left(\overrightarrow{x_{i}} ; \overrightarrow{\alpha_{0}}\right)$ which is saved during Monte Carlo generation. The value of $\mathcal{D}\left(\overrightarrow{x_{i}} ; \vec{\alpha}\right)$ is divided by this factor in order to remove the effect of the original generation parameters. 


\subsubsection{Use of the program LMAX}

Two different fitting algorithms are available in LMAX. The first, based on Powell's method, is quite robust. However the second, MIGRAD, which is a part of the fitting program MINUIT, often obtains a maximum in a fewer number of iterations and is thus faster. Both take an initial set of starting parameters and then compute the gradient of the function in order to seek out the minimum. Since MIGRAD is a minimizer, the $\log$ likelihood function is multiplied by -1 in order to allow MIGRAD to find the set of parameters that maximizes the log likelihood function.

As discussed above, LCALC does the computation of the parts of the log likelihood function, and RUNPMGFIT combines the results from the various instances of LCALC. LMAX takes the summed results and actually computes the log likelihood function as shown in (8.24) . However, as mentioned previously, a "best guess" of the parameters $\vec{\alpha}_{g}$ must be made in order to accurately estimate the normalization part of the log likelihood function. A bootstrap method is used by LMAX in order to do this. Upon execution, LMAX uses the initial guess of the parameters $\vec{\alpha}$ input by the user as the value of $\vec{\alpha}_{g}$. During the first iteration of LCALC, the various likelihood contributions are computed at this value of $\vec{\alpha}$ and the Monte Carlo's likelihood sums are used to compute the values of $\sum_{i=1}^{N_{M C}} \frac{\mathcal{D}\left(\overrightarrow{x_{i}} ; \overrightarrow{\alpha_{g}}\right)}{\mathcal{D}\left(\overrightarrow{x_{i}} ; \vec{\alpha}_{0}\right)}$ for each Monte Carlo sample, and is saved to be used during the rest of the fit. The values are then used to compute the value of the log likelihood function. Note that for the first iteration of the fit, the sums are corrected by themselves, and the likelihood function becomes:

$$
\begin{aligned}
& \log \mathcal{L}\left(\vec{\alpha}=\vec{\alpha}_{g}\right)=\sum_{i=1}^{N_{D}^{97 V A C}} \log \mathcal{D}\left(\overrightarrow{x_{i}} ; \vec{\alpha}\right)+\sum_{i=1}^{N_{D}^{97 R E G}} \log \mathcal{D}\left(\overrightarrow{x_{i}} ; \vec{\alpha}\right)
\end{aligned}
$$

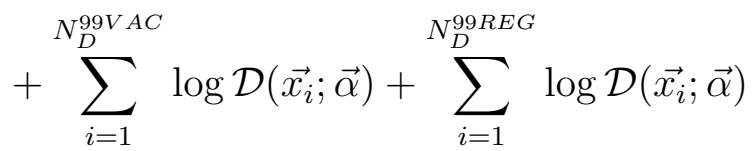

$$
\begin{aligned}
& -\left(N_{D}^{97 V A C}+N_{D}^{97 R E G}+N_{D}^{99 V A C}+N_{D}^{99 R E G}\right) \\
& \times \log \left[N_{D}^{97 V A C}+N_{D}^{97 R E G}+N_{D}^{99 V A C}+N_{D}^{99 R E G}\right]
\end{aligned}
$$

Once MIGRAD has found a maximum, $\vec{\alpha}_{g}$ is set equal to the current set of the best fit parameters, the fit is restarted, the improved correction factors are computed, 
and then used in the second execution of MIGRAD. This is continued until the fit results converge. 


\section{Chapter 9}

\section{Results of the Maximum Likelihood Fit}

Now that a estimation method has been developed, we can apply it in order to obtain the most likely estimates for the $K_{L, S} \rightarrow \pi^{+} \pi^{-} \gamma$ amplitude parameters, as well as the confidence intervals for said parameters. This is a multistage process. First, we run the maximum likelihood fit program on the nominal data set and nominal normalization Monte Carlo sample. This fit gives us the most probable parameter values, also known as the central values, and also gives us $1 \sigma$ confidence intervals which are due to limited statistics in the data. This uncertainty is also known as the "statistical error". The second step is to locate the sources of systematic error and estimate the size of each error. The third is to perform a series of cross checks to ensure our result is valid, and that we do not need to assign any further systematic errors.

\subsection{The Central Value}

As indicated in Chapter 8, we have instituted a re-weighting scheme that allows us to use in the likelihood fit Monte Carlo samples of $K_{L, S} \rightarrow \pi^{+} \pi^{-} \gamma$ that have been generated using any set of matrix element parameters. The logical extension is that we can also choose to use events for which the matrix element is always set equal to 1 , which can be generated by the Monte Carlo much more quickly than events 
using the normal $K_{L, S} \rightarrow \pi^{+} \pi^{-} \gamma$ matrix element - the reweighting scheme can also properly handle Monte Carlo samples of this type. This type of sample is referred to as a "flat" Monte Carlo sample.

The size of this and any other Monte Carlo sample must be reasonably larger than the data sample to ensure that statistical fluctuations from the Monte Carlo sample do not greatly bias the parameter estimates of the fit. After generating a large sample of flat $K_{L, S} \rightarrow \pi^{+} \pi^{-} \gamma$ Monte Carlo events, after all cuts, we have:

3,297,079 flat Monte Carlo events for the 1997 regenerator beam sample

3,734,735 flat Monte Carlo events for the 1997 vacuum beam sample

3,959,905 flat Monte Carlo events for the 1999 regenerator vacuum beam sample

4,868,692 flat Monte Carlo events for the 1999 vacuum beam sample

for use in the normalization of the likelihood function.

As was mentioned in Section 8.3.1, we follow the lead of the $\operatorname{Re}\left(\frac{\epsilon^{\prime}}{\epsilon}\right)$ analysis in the treatment of kaon evolution through the regenerator. In order to maintain consistency between this analysis and that one, we use the parameters taken from the $\operatorname{Re}\left(\frac{\epsilon^{\prime}}{\epsilon}\right)$ and $\phi_{+-}$result. These parameters and their values are shown in Table 9.1. Using the

\begin{tabular}{|c|c|}
\hline Input Parameter & Value Used with Error \\
\hline$K_{L} \rightarrow \pi^{+} \pi^{-}$CP violation parameter & $\begin{array}{c}\eta_{+-}=(2.2280 \pm 0.010) \times 10^{-3} \\
\phi_{+-}=(0.76201 \pm 0.02443) \text { radians }\end{array}$ \\
\hline Kaon Mass & $M_{K}=0.497648 \pm 0.000022 \mathrm{GeV}^{2} \mathrm{c}^{2}$ \\
\hline Neutral Kaon Mass Difference & $\Delta_{M}=-(0.52620 \pm 0.0043) \times 10^{10} \hbar \mathrm{s}^{-1}$ \\
\hline Decay Width of $K_{S}$ & $\Gamma_{S}=(1.1155 \pm .00646) \times 10^{10} \mathrm{~s}^{-1}$ \\
\hline Decay Width of $K_{L}$ & $\Gamma_{L}=(1.9420 \pm 0.015) \times 10^{7} \mathrm{~s}^{-1}$ \\
\hline Regenerator Amplitude & $|\rho|=1.2087 \pm 0.0003$ \\
\hline$\rho$ Momentum Scaling Factor & $\alpha_{R E G}=-0.53831 \pm 0.0008$ \\
\hline Regenerator/Vacuum Normalization & $\mathcal{N}_{R E G}=1.0413 \pm 0.0006$ \\
\hline Reg Attenuation Slope & $\mathcal{T}_{R E G}=-0.000685072 \pm 0.000029 \mathrm{GeV}^{-1}$ \\
\hline
\end{tabular}

Table 9.1: Input parameters used in the maximum likelihood fit

above input parameters, we obtain the results shown in Table 9.1. The parabolic 


\begin{tabular}{||c|c|c|c||}
\hline \hline Parameter & $\begin{array}{c}\text { Value and } \\
\text { Parabolic Error }\end{array}$ & $\begin{array}{c}\text { MINOS } \\
\text { Lower Error }\end{array}$ & $\begin{array}{c}\text { MINOS } \\
\text { Upper Error }\end{array}$ \\
\hline Offset $\widehat{e}$ & $0.43919 \pm 0.0010$ & -0.00065 & 0.00065 \\
\hline$g_{E 1}$ & $-0.0061 \pm 0.0016$ & -0.0015 & 0.0015 \\
\hline $\bar{g}_{M 1}$ & $1.133 \pm 0.076$ & -0.030 & 0.030 \\
\hline$a_{1} / a_{2}$ & $-0.7503 \pm 0.018$ & -0.0072 & 0.0068 \\
\hline \hline
\end{tabular}

Table 9.2: Nominal 4-parameter fit results

error is estimated using the second derivative of the modified log likelihood function. MINOS[43] however will explicitly trace out the error boundary given by:

$$
\log \mathcal{L}\left(\vec{\alpha}^{\prime}\right)=\log \mathcal{L}\left(\vec{\alpha}_{B E S T}\right)-1 / 2
$$

where $\vec{\alpha}^{\prime}$ is the set of all possible parameter values that lie within one standard deviation of the best fit values. Given the explicit nature of the MINOS errors, they are more reliable than parabolic estimates, as such, we will use MINOS whenever possible to estimate statistical errors. The price of such precision is of course the added computation time. The correlation matrix of the fit is shown in Table 9.1, and was obtained from the MINOS result. As explained in Chapter 8, the relative normalization of the Monte Carlo samples is unknown ahead of time - which necessitates a bootstrapping method when fitting where the likelihood fit must be run a number of times in order to update the Monte Carlo sample normalization each time. This fit used 22 cycles, however between the 4th and 22nd cycle, none of the parameter estimates varied outside of their respective statistical error.

\begin{tabular}{|c|cccc|}
\hline & $\widehat{e}$ & $g_{E 1}$ & $\widetilde{g_{M 1}}$ & $a_{1} / a_{2}$ \\
\hline$\widehat{e}$ & 1 & -0.367 & -0.651 & -0.527 \\
$g_{E 1}$ & -0.367 & 1 & 0.327 & 0.267 \\
$\widehat{g_{M 1}}$ & -0.651 & 0.327 & 1 & 0.983 \\
$a_{1} / a_{2}$ & -0.527 & 0.267 & 0.983 & 1 \\
\hline
\end{tabular}

Table 9.3: Nominal 4-parameter fit correlation matrix

A quick review of Table 9.1 reveals that the goal of this analysis has been fulfilled. The use of the regenerator beam data has removed most of the correlation between the 
parameters $\widehat{e}$ and $g_{E 1}$ which would be present in a likelihood fit using only vacuum beam data. Additionally, the rather large correlation between êand the M1 direct emission parameters indicates that fixing $\widetilde{g_{M 1}}$ or $a_{1} / a_{2}$ at some values may bias the estimate for $\widehat{e}$, indicating that the use of the vacuum beam data in the fit is also desirable.

\subsection{Goodness of Fit}

One disadvantage to using the unbinned maximum likelihood method is that it does not present the opportunity for a reliable goodness of fit test[44], only binned fits allow this [22]. We shall compute the $\chi^{2}$ per degrees of freedom for $E_{\gamma}^{*}, \cos (\theta)$ and $\tau$ distributions, between the data and a sample of Monte Carlo events. We ignore the very strong possiblity that testing the goodness of a likelihood fit with a $\chi^{2}$ computation is wholly inappropriate, and may produce misleading results. Samples of Monte Carlo events were generated using the best fit parameters shown in Table 9.1 and then crunched and subjected to all analysis cuts. Once this is done, the $\chi^{2}$ between the data and Monte Carlo distributions can be calculated, where the $\chi^{2}$ was calculated using:

$$
\chi^{2}=\sum_{i=1}^{N} \frac{\left(N_{D A T A}(i)-A_{M C} N_{M C}(i)\right)^{2}}{\sigma_{D A T A}^{2}(i)+\left(A_{M C}\right) \sigma_{M C}^{2}(i)}
$$

where $N_{X}(i)$ refers to the number of events in bin $i$ in either the data or Monte Carlo samples, and $\sigma_{X}(i)$ is the standard deviation in the number of events in bin $i$. Since we are dealing with random processes, we must use Poisson statistics which indicates that the error for a bin containing $N$ events is $\sqrt{N}$. $A_{M C}$ is a normalization factor for the Monte Carlo sample. Notice that the standard deviation of both the data and Monte Carlo samples are used. This is done in order to account for statistical fluctuations in the Monte Carlo sample. We then re-write the equation to obtain:

$$
\chi^{2}=\sum_{i=1}^{N} \frac{\left(N_{D A T A}(i)-A_{M C} N_{M C}(i)\right)^{2}}{N_{D A T A}(i)+A_{M C}^{2} N_{M C}(i)}
$$

The overall normalization $A_{M C}$ of the Monte Carlo distributions was then scaled until the $\chi^{2}$ is minimized, since the method of maximum likelihood, unlike the $\chi^{2}$ method, 
is not sensitive to the overall normalization of the distributions. This procedure produces a minimum $\chi^{2}$ value for each distribution. For purposes of this calculation, the 1997 and 1999 data samples were added together to approximate the global likelihood fit, however the samples were kept separated according to beam ( regenerator or vacuum ) owing to the difference in the shape of the distributions. The minimum $\chi^{2}$ values are shown in Table 9.2, while the results are shown graphically in Figures $9.1,9.2$, and 9.3 .

\begin{tabular}{|c|c|c|c|}
\hline Sample & Distribution & $\chi^{2}$ & Number of Degrees of Freedom \\
\hline Regenerator & $E_{\gamma}^{* C A L}$ & 109 & 85 \\
Vacuum & $E_{\gamma}^{* C A L}$ & 93 & 86 \\
\hline Regenerator & $\cos (\theta)$ & 88 & 100.0 \\
Vacuum & $\cos (\theta)$ & 98 & 100.0 \\
\hline Regenerator & $\tau$ & 150 & 151.0 \\
Vacuum & $\tau$ & 120 & 118.0 \\
\hline
\end{tabular}

Table 9.4: Minimum $\chi^{2}$ values for various distributions. Degrees of freedom refer to the number of bins in each histogram that are populated by either data or Monte Carlo.

Now that the best fit to the data has been obtained along with the statistical error of the parameter estimates, the systematic errors of the fit results must now be determined. 


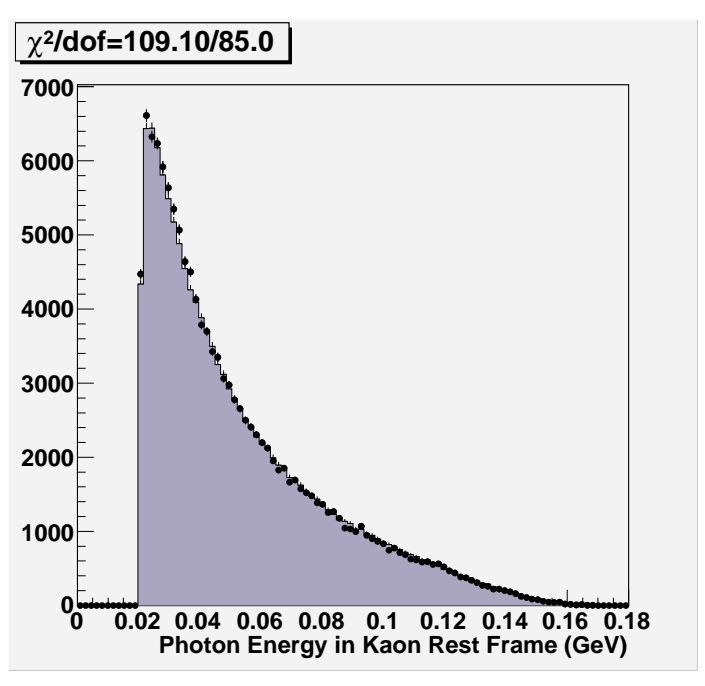

(a) Regenerator Beam

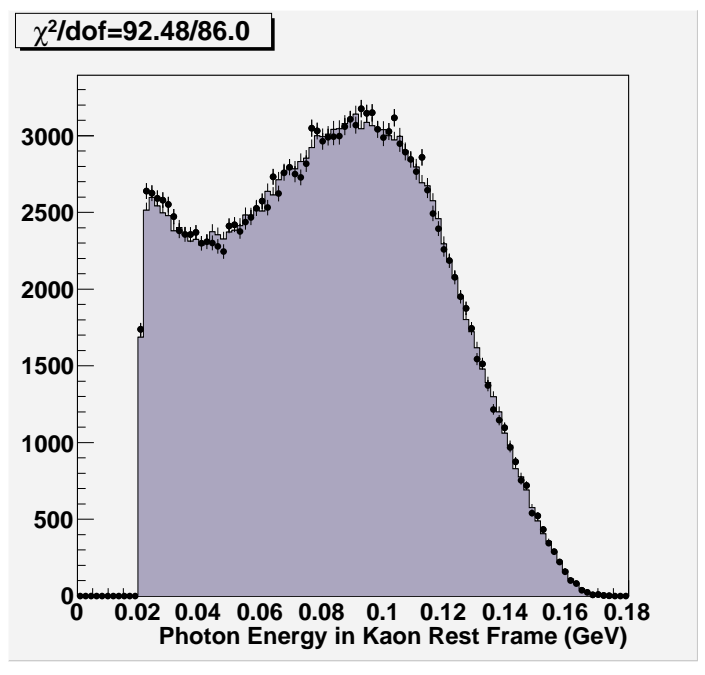

(b) Vacuum Beam

Figure 9.1: Plots of $E_{\gamma}^{* C A L}$ for a) the regenerator beam and b) vacuum beam events. Points are data and the histogram is Monte Carlo generated with best fit parameters. 


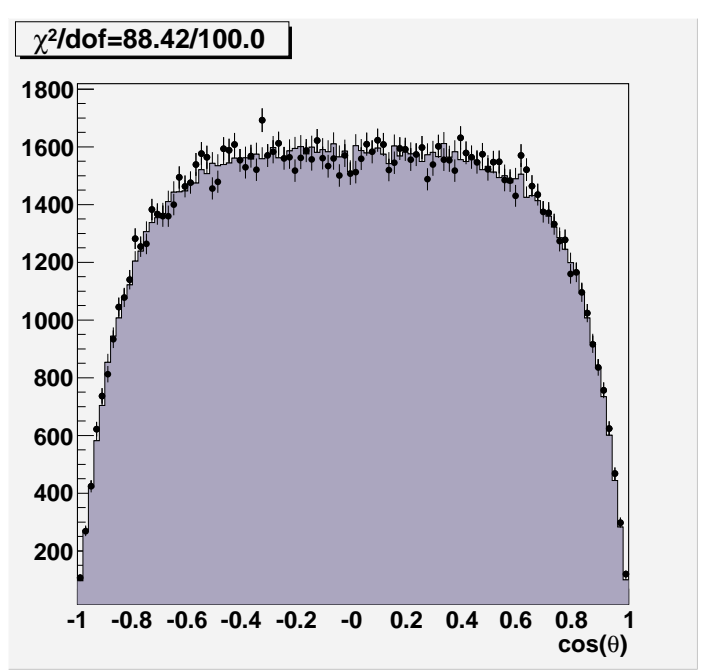

(a) Regenerator Beam

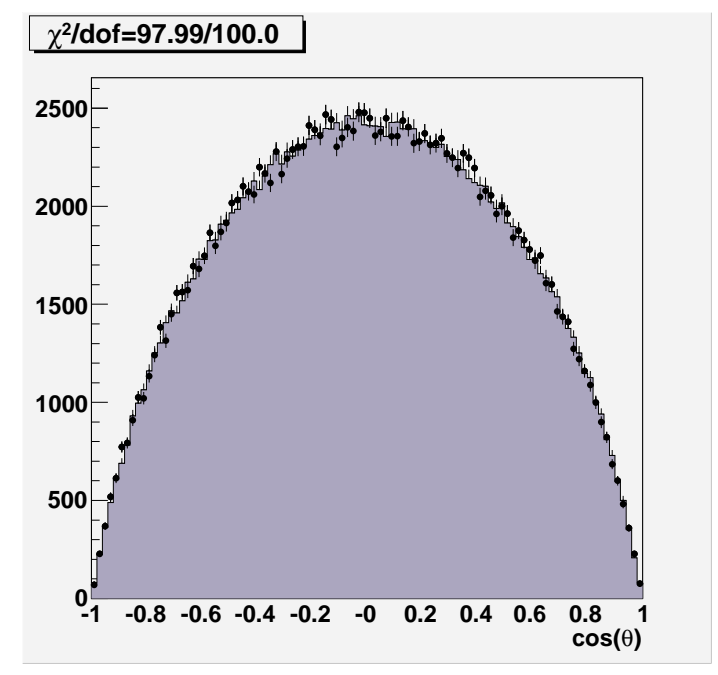

(b) Vacuum Beam

Figure 9.2: Plots of $\cos (\theta)$ for a) the regenerator beam and $b$ ) vacuum beam events. Points are data and the histogram is Monte Carlo generated with best fit parameters. 


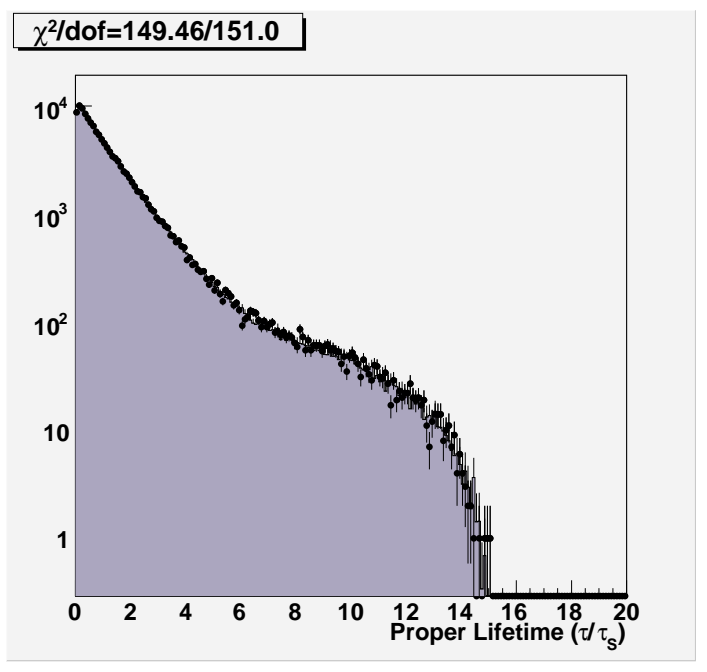

(a) Regenerator Beam

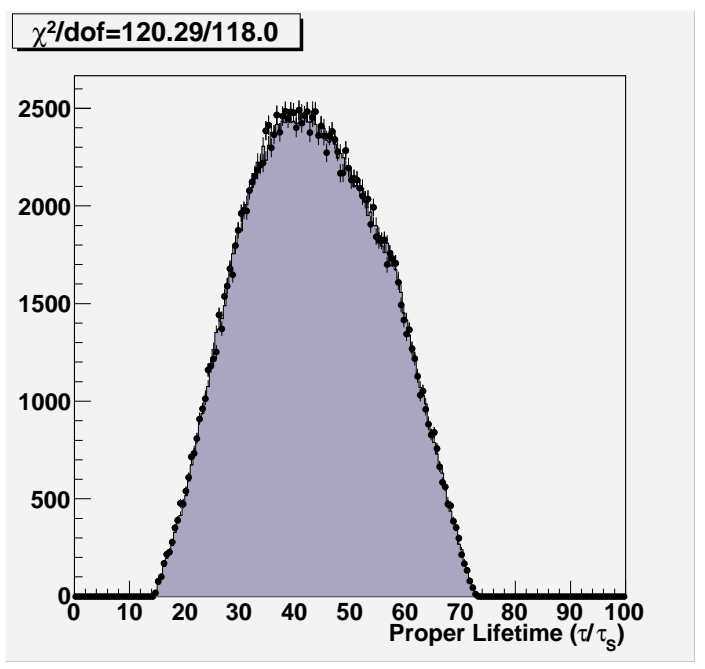

(b) Vacuum Beam

Figure 9.3: Plots of $\tau$ for a) the regenerator beam and b) vacuum beam events. Points are data and the histogram is Monte Carlo generated with best fit parameters. In this case, the regenerator beam plot is the proper time of flight from the downstream face of the regenerator, while for the vacuum beam plot is of the proper time of flight from the target. 


\section{Chapter 10}

\section{Systematic Errors}

Now that the statistical error of each parameter estimate has been determined, we must now estimate the uncertainty, or systematic error, introduced in the parameter estimates by the methodology used in this analysis. We will make these estimates by changing many of our initial assumptions, and observing how these new assumptions change the parameter estimates.

In order to formulate a complete study of the possible sources of systematic bias and uncertainty, we must look for changes in the likelihood function, as that is how the systematic biases and uncertainties can effect the answer. First, recall that the likelihood function contains a part that involves only the data:

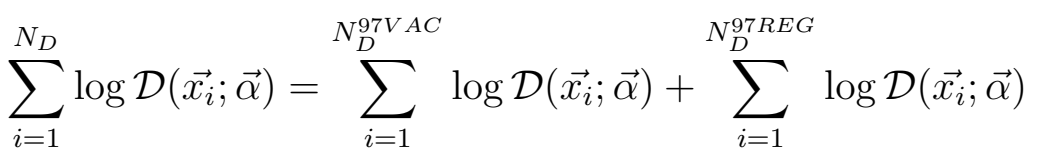

$$
\begin{aligned}
& +\sum_{i=1}^{N_{D}^{99 V A C}} \log \mathcal{D}\left(\overrightarrow{x_{i}} ; \vec{\alpha}\right)+\sum_{i=1}^{N_{D}^{99 R E G}} \log \mathcal{D}\left(\overrightarrow{x_{i}} ; \vec{\alpha}\right)
\end{aligned}
$$

and the normalization factor which is computed from the Monte Carlo sample only:

$$
\log \sum_{i=1}^{N_{M C}} \frac{\mathcal{D}\left(\overrightarrow{x_{i}} ; \vec{\alpha}\right)}{\mathcal{D}\left(\overrightarrow{x_{i}} ; \overrightarrow{\alpha_{0}}\right)}
$$

Each part can contribute bias to the parameter estimates. For clarity, the contribution from each can be separated. 
The systematic error due to the data term can only arise due to problems in calculating $\mathcal{D}\left(\overrightarrow{x_{i}} ; \vec{\alpha}\right)$ for each event. Such problems could be due incomplete reconstruction of a photon cluster, and thus an error in the measurement of $E_{\gamma}^{*}$ and $\cos (\theta)$ using the wrong cluster altogether, and problems with pion tracking which will effect the reconstructed kaon momentum and z vertex position. Other potential problems include the estimated kaon wavefunction not matching with reality, for example, if the kaon underwent scattering somewhere in the beamline or detector. Finally, background events will also result in an error in the calculation of the data portion of the likelihood function. Thus, we must evaluate the systematic errors due to the following issues when treating data:

- problems with photon energy reconstruction ( not using true value of $E_{\gamma}^{* C A L}$ )

- problems with photon direction reconstruction ( not using true value $\cos (\theta)$ )

- problems with z vertex reconstruction ( not using true value $Z_{\text {vertex }}$ )

- problems with pion momentum reconstruction ( and this kaon momentum reconstruction ) (not using true value of $p_{K}$ )

- effect of backgrounds, including scattered $K_{L, S} \rightarrow \pi^{+} \pi^{-} \gamma$ events

- other problems in computing $\mathcal{D}\left(\overrightarrow{x_{i}} ; \vec{\alpha}\right)$ including uncertainty in physical constants used

On the other hand, systematic error can contribute in two different ways in the likelihood normalization term. The first way is similar to data: any problems in computing $\mathcal{D}\left(\overrightarrow{x_{i}} ; \vec{\alpha}\right)$ for each Monte Carlo event will result in a systematic error. This would occur if the reconstructed value of generated quantities were to be used in the computation of $\mathcal{D}\left(\overrightarrow{x_{i}} ; \vec{\alpha}\right)$, if scattered events were to be used, etc. However, the true, generated values of the phase space variables are used in the computation, so this isn't an issue. Additionally, unlike the data, the Monte Carlo's event distribution in phase space can also introduce systematic error. Ideally, the Monte Carlo would reproduce the data exactly in every way, for every distribution. In reality, the accuracy of the Monte Carlo's simulation of the $\mathrm{KTeV}$ beamline and detector determines how well the acceptance is modeled, which in turn determines how closely the data and 
Monte Carlo match. Additionally, as this term is actually an example of Monte Carlo integration, the finite statistics of the Monte Carlo sample may also affect the accuracy of the Monte Carlo's event distribution in phase space. We then need to evaluate systematic errors due to the treatment of MC, including:

- the error due to finite normalization Monte Carlo statistics

- the error due to improper modeling of the detector acceptance

- the error due to uncertainty in physical constants used to compute $\mathcal{D}\left(\overrightarrow{x_{i}} ; \vec{\alpha}\right)$

Note that other than the physical constants and inputs used to compute $\mathcal{D}\left(\overrightarrow{x_{i}} ; \vec{\alpha}\right)$, the systematic errors can be cleanly split into those coming from data and those coming from the Monte Carlo.

\section{1 $\Delta_{S}$ Method of Systematic Error Estimation}

In the course of many of the studies to be done in order to estimate various systematic errors, the number of events in the data may increase or decrease. In this case, the statistical sample changes, which means that the pure shift in parameter estimates between the nominal and new fit should not be used, as a component of such as shift may be due to statistical fluctuations only. Instead, if the shift in the parameter estimates is larger than the statistical error of the shift, only then is the shift considered - otherwise the systematic error is considered to be negligible.

The statistical error of the shift $\sigma_{s}$ is the error due to the difference in statistics between two samples, and is given by

$$
\sigma_{s}=\sqrt{\left|\sigma_{\text {nominal }}^{2}-\sigma_{\text {new }}^{2}\right|}
$$

where $\sigma_{\text {nominal }}$ is the statistical error for each parameter estimate from the nominal fit, and $\sigma_{n e w}$ is the statistical error from the new fit. The statistical error of the shift should be near zero when the varied cut does not add or remove many events, while the said error will be large when many events are added or removed. 
If the shift is larger than its statistical error, we assign a symmetric systematic error $\Delta_{s}$ found using the relation [7]:

$$
\frac{1}{\sigma_{s} \sqrt{2 \pi}} \int_{-\Delta_{s}}^{+\Delta_{s}} d x \exp \left[\frac{(x-s)^{2}}{2 \sigma_{s}}\right]=0.683
$$

which simply finds the interval along a shifted Gaussian distribution which accounts for $68.3 \%$ of the area under the curve. This technique shall be referred to as the " $\Delta_{S}$ " method.

\subsection{Systematic Errors Due to Uncertainty in In- put Parameters and Constants}

There is a certain amount of uncertainty in many of the input parameters used in the fit as indicated in Table 9.1. This will effect the computation of $\mathcal{D}\left(\overrightarrow{x_{i}} ; \vec{\alpha}\right)$ for both data and Monte Carlo. The strategy is to increase and decrease the value of each parameter by an amount of one standard deviation, as given by Table 9.1 and run the maximum likelihood fit with the changed parameter each time. The new parameter is used in both the data and Monte Carlo likelihood calculations. This will yield two sets of new parameter estimates for each input parameter or constant, which will define the range of the possible shift to $68 \%$ confidence. We take the larger of the two shifts to be the systematic error in order to be conservative. As this procedure does not affect the statistics of the data nor the Monte Carlo ( due to the reweighting method used) the systematic error due to each parameter is taken as the shift, regardless of the size of the shift - the statistical error is ignored here. The resulting parameter shifts are given in Table 10.2

Caution should be used when combining these results however. Many of the input parameters are taken from other measurements by $\mathrm{KTeV}$, specifically from the $R e\left(\frac{\epsilon^{\prime}}{\epsilon}\right)$ analysis, and as such will exhibit varying degrees of correlation. When dealing with correlated errors, the total error cannot be obtained by adding individual contributions in quadrature. Instead, we must use the covariance matrices from the $R e\left(\frac{\epsilon^{\prime}}{\epsilon}\right)$ fits to integrate the correlation between input parameters into the error propagation here. The propagation of errors in this case can be determined using 


\begin{tabular}{|c|c|c|c|c|}
\hline Parameter Variation & Shift in $\widehat{e}$ & Shift in $g_{E 1}$ & Shift in $\widetilde{g_{M 1}}$ & Shift in $a_{1} / a_{2}$ \\
\hline$\eta_{+-}=2.218 \times 10^{-3}$ & $\overline{0.00032}$ & -0.00033 & -0.0072 & -0.00047 \\
\hline$\eta_{+-}=2.238 \times 10^{-3}$ & -0.0003 & 0.000313 & 0.0073 & 0.00052 \\
\hline$\phi_{+-}=0.73758$ radians & 0.00037 & -0.00055 & -0.0034 & -0.00059 \\
\hline$\phi_{+-}=0.78644$ radians & -0.00037 & 0.00057 & 0.0033 & 0.00062 \\
\hline$M_{K}=0.497626 \mathrm{GeV} / \mathrm{c}^{2}$ & $1 \times 10^{-5}$ & $2.15 \times 10^{-5}$ & -0.0014 & -0.0004 \\
\hline$M_{K}=0.49767 \mathrm{GeV} / \mathrm{c}^{2}$ & 0.0 & $-4.02 \times 10^{-5}$ & 0.0015 & 0.00041 \\
\hline$\Delta_{M}=-0.5219 \times 10^{10} \hbar s^{-1}$ & -0.00012 & 0.00026 & 0.0004 & $3 \times 10^{-5}$ \\
\hline$\Delta_{M}=-0.5304 \times 10^{10} \hbar s^{-1}$ & 0.00013 & -0.00028 & -0.0003 & $-3 \times 10^{-5}$ \\
\hline$\Gamma_{S}=1.1139 \times 10^{10} s^{-1}$ & $-8 \times 10^{-5}$ & 0.00016 & 0.0021 & 0.00039 \\
\hline$\Gamma_{S}=1.11713 \times 10^{10} s^{-1}$ & $1 \times 10^{-4}$ & -0.000175 & -0.002 & -0.00037 \\
\hline$\Gamma_{L}=1.92678 \times 10^{7} s^{-1}$ & 0.0 & $9.0 \times 10^{-7}$ & $1 \times 10^{-4}$ & $2 \times 10^{-5}$ \\
\hline$\Gamma_{L}=1.95695 \times 10^{7} s^{-1}$ & $1 \times 10^{-5}$ & $-1.93 \times 10^{-5}$ & 0.0 & $-1.0 \times 10^{-5}$ \\
\hline$|\rho|=1.2084$ & $-1 \times 10^{-5}$ & $2.7 \times 10^{-6}$ & 0.0002 & $3 \times 10^{-5}$ \\
\hline$|\rho|=1.209$ & $2 \times 10^{-5}$ & $-2.17 \times 10^{-5}$ & $-1 \times 10^{-4}$ & $-2 \times 10^{-5}$ \\
\hline$\alpha_{R E G}=-0.53911$ & $2 \times 10^{-5}$ & $-3.17 \times 10^{-5}$ & $-1 \times 10^{-4}$ & $-2 \times 10^{-5}$ \\
\hline$\alpha_{R E G}=-0.53751$ & $-1 \times 10^{-5}$ & $1.26 \times 10^{-5}$ & $1 \times 10^{-4}$ & $3 \times 10^{-5}$ \\
\hline $\mathcal{N}_{R E G}=1.04068$ & $1 \times 10^{-5}$ & $-9.3 \times 10^{-6}$ & $\overline{0.0}$ & $1.0 \times 10^{-5}$ \\
\hline $\mathcal{N}_{R E G}=1.04188$ & $1 \times 10^{-5}$ & $-9.2 \times 10^{-6}$ & 0.0 & 0.0 \\
\hline $\mathcal{T}_{R E G}=-0.000714 \mathrm{GeV}^{-1}$ & $1 \times 10^{-5}$ & $9 \times 10^{-6}$ & 0.0 & $1.0 \times 10^{-5}$ \\
\hline $\mathcal{T}_{R E G}=-0.000656 G e V^{-1}$ & $1 \times 10^{-5}$ & $-3.17 \times 10^{-5}$ & $1 \times 10^{-4}$ & $1.0 \times 10^{-5}$ \\
\hline
\end{tabular}

Table 10.1: Parameter shifts from nominal result due to variation of input parameters $[22]:$

$$
U_{i j}=\left.\sum_{k, l} \frac{\partial \Phi_{i}}{\partial \theta_{k}} \frac{\partial \Phi_{j}}{\partial \theta_{l}}\right|_{\hat{\theta}} V_{k l}
$$

where $U_{i j}$ gives the covariance between the fit parameters $\Phi_{i}$ and $\Phi_{j}, \frac{\partial \Phi_{i}}{\partial \theta_{k}}$ captures the dependence of the estimated parameter $\Phi_{i}$ on the input parameter $\theta_{k}$ and $V_{k l}$ is the covariance between the input parameters $\theta_{k}$ and $\theta_{l}$. In this case, $V_{k l}$ comes from the $\operatorname{Re}\left(\frac{\epsilon^{\prime}}{\epsilon}\right)$ analysis fits from which we take the values of the input parameters. The diagonal elements of $U_{i j}$ give the total variance of the parameter estimates due to the uncertainty in a group of input parameters.

First, we note that variation of the regeneration parameters $\alpha_{R E G}, \mathcal{N}_{R E G}$ and $\mathcal{T}_{R E G}$ result in negligible shifts compared to the other parameters - they shall be treated as independent. Second, we note that $M_{K}$ and $\Gamma_{L}$ are not among the parameters measured by the $\operatorname{Re}\left(\frac{\epsilon^{\prime}}{\epsilon}\right)$ analysis - they shall also be treated as independent. Next, we note that the value of $\rho$ and $\alpha_{R E G}$ are taken from the actual fit for $\operatorname{Re}\left(\frac{\epsilon^{\prime}}{\epsilon}\right)$ so they will be correlated with the magnitude of $\eta_{+-}$. Finally, we note that the values 
of $\phi_{+-}, \Delta_{M}$ and $\tau_{S}$ are all taken from the fit for $\phi_{+-}$, which is also a part of the $\operatorname{Re}\left(\frac{\epsilon^{\prime}}{\epsilon}\right)$ analysis. From these fits, we can read off the correlations which can be found in Tables 10.2 and 10.2. Using the correlation matrices, the parameter shifts in

\begin{tabular}{|c|ccc|}
\hline & $\rho$ & $\alpha_{R E G}$ & $\eta_{+-}$ \\
\hline$\rho$ & 1 & 0.231 & 0.601 \\
$\alpha_{R E G}$ & 0.231 & 1 & -0.082 \\
$\eta_{+-}$ & 0.601 & -0.082 & 1 \\
\hline
\end{tabular}

Table 10.2: Correlation matrix taken from the fit for $\operatorname{Re}\left(\frac{\epsilon^{\prime}}{\epsilon}\right)$ The correlation for $\eta_{+-}$ is taken to be the same as that for $R e\left(\frac{\epsilon^{\prime}}{\epsilon}\right)$.

\begin{tabular}{|c|ccc|}
\hline & $\phi_{+-}$ & $\Delta_{M}$ & $\tau_{S}$ \\
\hline$\phi_{+-}$ & 1 & 0.975 & -0.908 \\
$\Delta_{M}$ & 0.975 & 1 & -0.857 \\
$\tau_{S}$ & -0.908 & -0.857 & 1 \\
\hline
\end{tabular}

Table 10.3: Correlation matrix taken from the fit for $\phi_{+-}$[7]. Note that the $\phi_{+-}$fit uses $\tau_{S}$, while we varied the value of $\Gamma_{S}$. This will introduce a sign change.

Table 10.2 and Equation 10.2 to add the parameters, we get the following combined errors as shown in Table 10.2. We assume that the only parameter correlations are contained within the two groups defined. It should be noted that this method of combining errors results in errors that are smaller than one would obtain by adding in quadrature.

\begin{tabular}{|c|c|c|c|c|}
\hline Combination of Inputs & Error in $\widehat{e}$ & Error in $g_{E 1}$ & Error in $\widehat{g_{M 1}}$ & Error in $a_{1} / a_{2}$ \\
\hline$\rho, \alpha_{R E G}, \eta_{+-}$ & 0.000307 & 0.000316 & 0.00717 & 0.000501 \\
\hline$\phi_{+--}, \Delta_{M}, \Gamma_{S}$ & 0.000158 & 0.000160 & 0.00140 & 0.000287 \\
\hline$M_{K}, \Gamma_{L}, \mathcal{N}_{R E G}, \mathcal{I}_{R E G}$ & $2.00 \times 10^{-5}$ & $5.55 \times 10^{-5}$ & 0.00151 & 0.000411 \\
\hline \hline Total Error & 0.000346 & 0.000358 & 0.00746 & 0.000708 \\
\hline
\end{tabular}

Table 10.4: Systematic errors due to input parameter uncertainty after proper treatment of correlations.

One final possible source of systematic uncertainty comes from the uncertainty in the evaluation of the strong interaction phase shift for the inner bremsstrahlung 
decay amplitude. We choose to follow previous $\mathrm{KTeV}$ analyses and evaluate this at the mass of the neutral kaon. References [9] and [21] also follow this procedure. However, Reference [20] implicitly assumes that the phase shift should instead be evaluated at $M_{\pi^{+} \pi^{-}}$which is often close to the value of $M_{K}$ for Inner bremsstrahlung events, but not always so. In order to discover the size of this effect, we use this competing prescription to compute $\mathcal{D}\left(\overrightarrow{x_{i}} ; \vec{\alpha}\right)$ for data and Monte Carlo events, and rerun the maximum likelihood fit. Doing this, we find the parameters shift by :

$$
\begin{aligned}
& \Delta \widehat{e}=0.00028 \\
& \Delta g_{E 1}=0.0035 \\
& \Delta \widetilde{g_{M 1}}=-0.0156 \\
& \Delta a_{1} / a_{2}=-0.00419
\end{aligned}
$$

We do not treat this as a systematic error, as it is an external theoretical uncertainty.

\subsection{Systematic Errors Due to Treatment of Data}

As mentioned in the beginning of the chapter, the treatment of the data may introduce some systematic uncertainty which must be estimated.

\subsubsection{Resolution of Phase Space variables}

The likelihood contribution of each event is a function of the phase space variables $E_{\gamma}^{*}, \cos (\theta), Z_{\text {vertex }}$, and $p_{K}$. While the $\mathrm{KTeV}$ detector in general has excellent performance, there will be some resolution effects that come into play with the measurement of all of these variables. This also means that there will be a certain amount of error introduced into the likelihood contribution for each event.

Our primary tool with which to attack this issue is the $\mathrm{KTeV}$ Monte Carlo, which in general does an excellent job of simulating the resolution effects of the detector. The method employed here is the generation of a Monte Carlo sample which will be used as "fake" data. The option of whether or not to use generated ( Monte Carlo truth ) or reconstructed variables is available, and used here. First, we run the maximum likelihood fit on a sample of fake data with similar statistics as the 
real data. For this first fit, all phase space variables used by the fitter take their generated values. Then, the fit is rerun on the same fake data sample, however this time all phase space variables $\left(E_{\gamma}^{* C A L}, \cos (\theta), Z_{\text {vertex }}, p_{K}\right)$ take on their reconstructed values. The pure shifts in parameter estimates is then taken as the systematic errors due to resolution effects of all phase variables in question. The pure shift is used, as the statistical sample remains identical between the two fits. The systematic error estimate is as reliable as the resolution simulation of the $\mathrm{KTeV}$ detector. For this study all phase space variables are switched from their generated to reconstructed values at the same time, rather than one at a time, due to correlations between variables in which resolution effects in one variable will create resolution effects in another. For example, the resolution in the value of $E_{\gamma}^{* C A L}$ will result in smearing of the value of the kaon momentum $p_{K}$. Table 10.5 gives the shift in parameter estimates from the result using generated variables to the result using reconstructed variables. Note that the effects of fake photon clusters and $K_{L, S} \rightarrow \pi^{+} \pi^{-} \gamma \gamma$ are also both included in this study.

\begin{tabular}{|c|c|c|c|c|}
\hline & $\widehat{e}$ Shift & $g_{E 1}$ Shift & $\widetilde{g_{M 1}}$ Shift & $a_{1} / a_{2}$ Shift \\
\hline Observed Shifts & 0.00052 & -0.0003254 & -0.0033 & -0.00009 \\
\hline
\end{tabular}

Table 10.5: Systematic Errors Due to Event Reconstruction.

\subsubsection{Background Effects}

In Chapter 6 we estimated the amount of background present in the data sample after all analysis cuts have been applied. Now we wish to estimate the effect of this background on the parameter estimates from the likelihood fit. As we are using an unbinned likelihood fit, it is not possible for us to subtract the background from the signal, as could be done in a binned likelihood or $\chi^{2}$ fit. Instead, we shall add background into the data sample, and observe how the parameter estimates shift. Since we will be changing the statistics of the data sample when we introduce the background, we shall use the $\Delta_{S}$ method to estimate the systematic error due to the background.

We shall add a background sample to the nominal data sample in order to perform 
this study. Additionally, we shall extract a background sample directly from the data, as Chapter 6 also revealed the difficulty in simulating the kaon decays which make up the largest contribution to the background - the proper statistics are extremely hard to obtain.

The background sample can either be extracted from the tail in the $P_{T}^{2}$ distribution, or from the tails on either side of the $M_{\pi^{+}} \pi^{-} \gamma$ mass peak. If the tail of the $P_{T}^{2}$ plot is used, the background will consist of both background kaon decays, but also scattered $K_{L, S} \rightarrow \pi^{+} \pi^{-} \gamma$ events. However, the tails of the $M_{\pi^{+} \pi^{-} \gamma}$ distribution will contain mostly background kaon decay events. Because of this, we shall extract the background sample from the $M_{\pi^{+}} \pi^{-} \gamma$ wings.

Finally, we must choose a region in the invariant mass plot from which to extract the background sample. We select events in the region of $0.46 \mathrm{GeV} / \mathrm{c}^{2}$ to $0.475 \mathrm{GeV} / \mathrm{c}^{2}$ as the low invariant mass sideband in an effort to keep the number of $K_{L, S} \rightarrow \pi^{+} \pi^{-} \gamma$ decays to a minimum, while also selecting events from $0.515 \mathrm{GeV} / \mathrm{c}^{2}$ to $0.54 \mathrm{GeV} / \mathrm{c}^{2}$ as the high invariant mass sideband. ${ }^{1}$ We must be careful when dealing with these two background samples as the low invariant mass sample, according to the analysis presented in Section 6.2.4 is dominated by $K_{L} \rightarrow \pi^{+} \pi^{-} \pi^{0}$ decays, which are associated with large values of $E_{\gamma}^{*}$ and thus may fake direct emission events. On the other hand, the high invariant mass sample, and presumably the background under the mass peak, is a mixture of the three largest kaon decay modes, $K_{L} \rightarrow \pi^{ \pm} e^{\mp} \nu, K_{L} \rightarrow \pi^{ \pm} \mu^{\mp} \nu$ and $K_{L} \rightarrow \pi^{+} \pi^{-} \pi^{0}$ and is not dominated by the $K_{L} \rightarrow \pi^{+} \pi^{-} \pi^{0}$ decay. This implies that if we wish to extract additional events which approximate the background under the mass peak, we should do so from the high invariant mass region. However, the number of events present in the data between $0.515 \mathrm{GeV} / \mathrm{c}^{2}$ to $0.54 \mathrm{GeV} / \mathrm{c}^{2}$ is less than the total estimated background. In order to acquire enough events, we first collect all events between $0.515 \mathrm{GeV} / \mathrm{c}^{2}$ to $0.54 \mathrm{GeV} / \mathrm{c}^{2}$ and then add to this sample enough events between $0.46 \mathrm{GeV} / \mathrm{c}^{2}$ to $0.475 \mathrm{GeV} / \mathrm{c}^{2}$ to yield a sample which is approximately equal in statistics to the estimated background under the mass peak. See Table 10.6 for the relative contribution to the sample from the two regions. Note that in determining the total number of additional events to inject into the sample,

\footnotetext{
${ }^{1}$ Plotting the $\pi^{+} \pi^{-} \gamma$ invariant mass for both data and Monte Carlo reveals that the two plots start to greatly diverge below $0.475 \mathrm{GeV} / \mathrm{c}^{2}$ and above $0.515 \mathrm{GeV} / \mathrm{c}^{2}$. This can be seen clearly in Figures 5.1, I.1, J.1, and K.1.
} 
we have chosen between the invariant mass and $P_{T}^{2}$ estimates by selecting the larger of the two for each sample.

\begin{tabular}{|c|c|c|c|c|}
\hline & 1997 Reg & 1997 Vac & 1999 Reg & 1999 Vac \\
\hline \# of Background Events & 20 & 82 & 44 & 98 \\
\hline \# with Low Invariant Mass & 3 & 41 & 22 & 30 \\
\# with High Invariant Mass & 17 & 41 & 22 & 68 \\
\hline \# Total Used & 20 & 82 & 44 & 98 \\
\hline
\end{tabular}

Table 10.6: Composition of background sample for the estimation of the background systematic error

This background sample is added to the nominal data sample and fit. The resulting parameter shifts from the nominal value are shown, in addition to the resulting systematic errors if any, are shown in Table 10.7 .

\begin{tabular}{|c|c|c|c|c|}
\hline & $\widehat{e}$ & $g_{E 1}$ & $\widehat{g}_{M 1}$ & $a_{1} / a_{2}$ \\
\hline Parameter Shift & $(1.7 \pm 0.3) \times 10^{-4}$ & $(0.82 \pm 6.93) \times 10^{-5}$ & $(-5.5 \pm 1.1) \times 10^{-3}$ & $(-1.34 \pm 0.90) \times 10^{-3}$ \\
Assigned Error & 0.000185 & 0.0 & 0.00603 & 0.00177 \\
\hline
\end{tabular}

Table 10.7: Observed shifts and assigned systematic errors from the addition of the estimated background sample

\subsubsection{Systematic error due to scattering and incoherent re- generation}

Another source of background comes from kaon scattering, a process which modifies the wavefunction of the kaon. This possibility is not taken into account by the fitter, so a systematic error must be estimated. The primary scattering processes are collimator and regenerator scattering. Section 6.4.2 determined that the expected amount of collimator scattering in the data sample is quite low, so we neglect it here. On the other hand the amount of regenerator scattering, folded in with other incoherent regeneration, is non-negligible as pointed out in Section 6.4.1.

The method employed here is to generate Monte Carlo with incoherent regeneration and regenerator scattering turned on. Then, the scattered and incoherently regenerated events are removed from the sample, and the Monte Carlo sample is used 
as fake data and fit. The Monte Carlo sample is then used as fake data once again, but this time the incoherently regenerated events, in addition to events in which the kaon scattered within the regenerator, are kept and fit. The shift in parameters then determines the systematic error, where the error is estimated using the $\Delta_{S}$ method. Note that the Monte Carlo handles the normalization of the various terms here. Table 10.8 presents the parameter shifts between the sample with incoherent regeneration and the sample without.

\begin{tabular}{|c|c|c|c|c|}
\hline & $\widehat{e}$ & $g_{E 1}$ & $\widetilde{g_{M 1}}$ & $a_{1} / a_{2}$ \\
\hline $\begin{array}{c}\text { Parameter } \\
\text { Shift }\end{array}$ & $(11.0 \pm 2.7) \times 10^{-5}$ & $(-8.62 \pm 4.78) \times 10^{5}$ & $(4.0 \pm 7.1) \times 10^{-4}$ & $(7 \pm 29) \times 10^{-5}$ \\
$\begin{array}{c}\text { Assigned } \\
\text { Error }\end{array}$ & 0.000123 & 0.000109 & 0.0 & 0.0 \\
\hline
\end{tabular}

Table 10.8: Observed shifts and assigned systematic errors from the effect of incoherent regeneration and regenerator scattering

\subsection{Systematic Errors Due to Treatment of Monte Carlo Samples}

The systematic error due to Monte Carlo mainly involves problems with simulating the $\mathrm{KTeV}$ detector response, however the frequency of specific processes may also introduce some systematic uncertainty into the result.

\subsubsection{Detector Acceptance and Simulation \\ Data/MC agreement}

Getting a correct value for the Monte Carlo integration depends on the phase space being accurately modeled. This means that any problem with acceptance could lead to a systematic error. Problems in acceptance may be worsened when a cut is made on the variable in question. In general, there are two ways to address the possible impact of selection cuts on the parameter estimates. The first is to vary each cut and rerun the fit multiple times in order to see if there is a systematic shift as a cut is loosened or 
tightened. However, doing this changes the statistical sample used in the fit, which introduces a certain measure of statistical error into the result. Additionally, it is possible to pick up background events in this way. Finally, it is often not clear where to stop the cut variations.

The second method to quantify the effect of poor detector Monte Carlo modeling is to choose a cut distribution and reweight it in order to force the Monte Carlo to reproduce the data. This correction comes from a plot of the ratio of the data distribution to the Monte Carlo distribution. This plot can then be applied as a bin by bin correction, or in the case of a linear slope, a linear correction function can be used. The main disadvantage to this technique is that the slope of a number of data/MC plots may be correlated with each other. In this case, if one distribution is flattened, others may also be effected, rendering the determination of a pure shift due to each distribution very difficult, if not impossible.

The flattening method proceeds as follows: first, obtain the nominal fit results before the correction is applied. Then, the necessary correction to the distribution in question is computed for each data subsample. Each correction is applied to its corresponding Monte Carlo sample - we must correct Monte Carlo to match data, not the other way around, and the corrected Monte Carlo samples are used in another fit. The shift in each parameter estimate is then taken as the systematic error.

The cut variation method is as follows: we evaluate the systematic error due to the choice of each cut by varying each cut and seeing if the shift in parameter values is larger than would be expected given the change in statistics that results from the change in each cut. The assumption is any shift reveals problems with the way the MC models the data.

We vary each cut independently, keeping all others at their nominal values. We fit data and Monte Carlo using a number of different cut values, being careful not to remove too much of the sample each time.

Each of the analysis cuts is varied, and the resulting shifts can be seen graphically in Figures 10.1 through 10.17, where the size of the error bar for the shift is given by Equation 10.3. If a given variation of a cut results in a shift larger than its error bar, the $\Delta_{S}$ method is used to compute a symmetric error interval. For a given cut, the cut value with the largest symmetric error interval is chosen as the systematic error for that parameter due to that particular cut. If no shifts are statistically significant, 
no systematic error is assigned. Additionally, if other reasons exist for the shift, for example, an increase in background resulted from the variation of the cut, we neglect to assign a systematic error.

Since the flattening method presents serious problems involving correlations between different observables, we shall use the cut variation method to address most of the systematic errors here. 

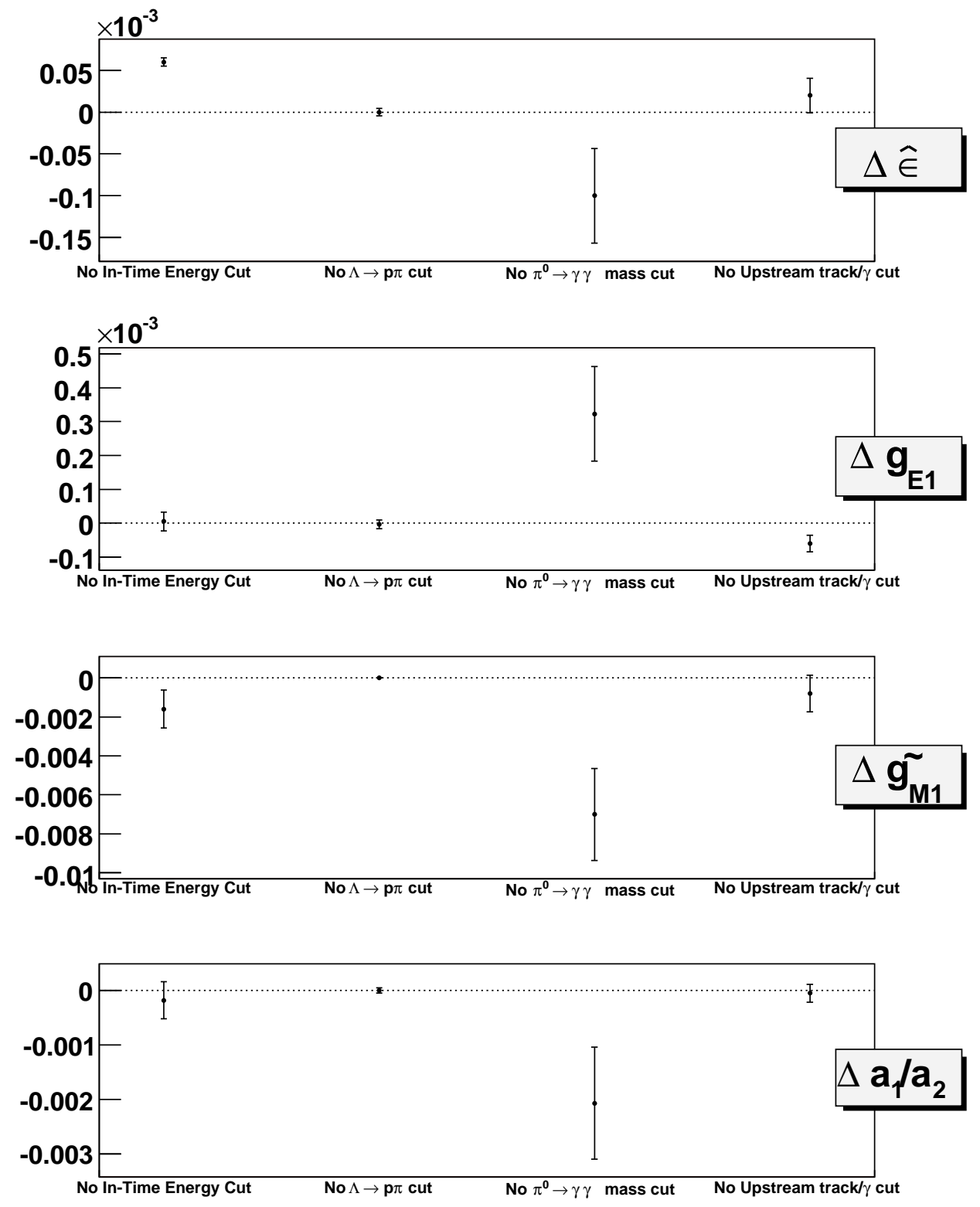

Figure 10.1: Resulting parameter shifts due to the removal of a number of different cuts. Although its removal results in a sizable shift in parameters, we choose not to assign a systematic error due to the In-time energy cut, as the distribution is very clearly separated into desirable and undesirable areas(see Figures 5.18, I.16, J.16, and K.16 ), and variation of the cut would result in no change in events. Simply removing this cut by definition lets in additional background, which has already been dealt with. The other three points are included in the study. 


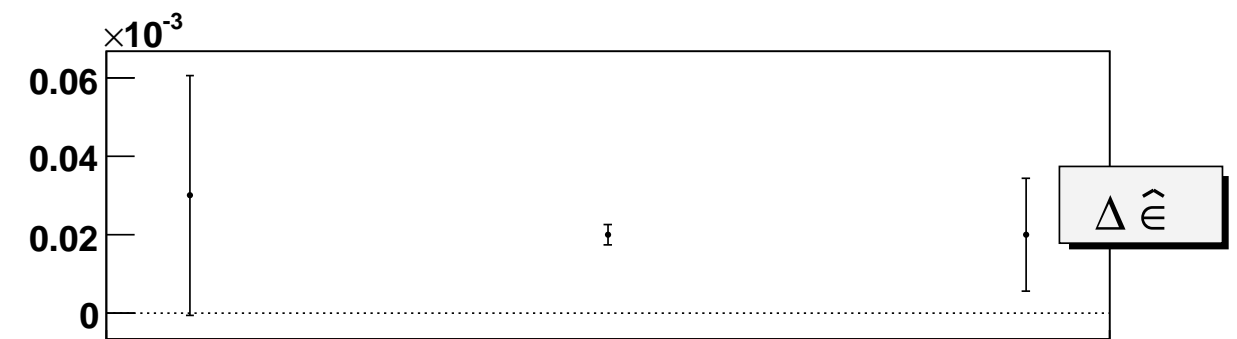

Early Energy $<50$ counts Early Energy $<250$ counts Early Energy $<500$ counts
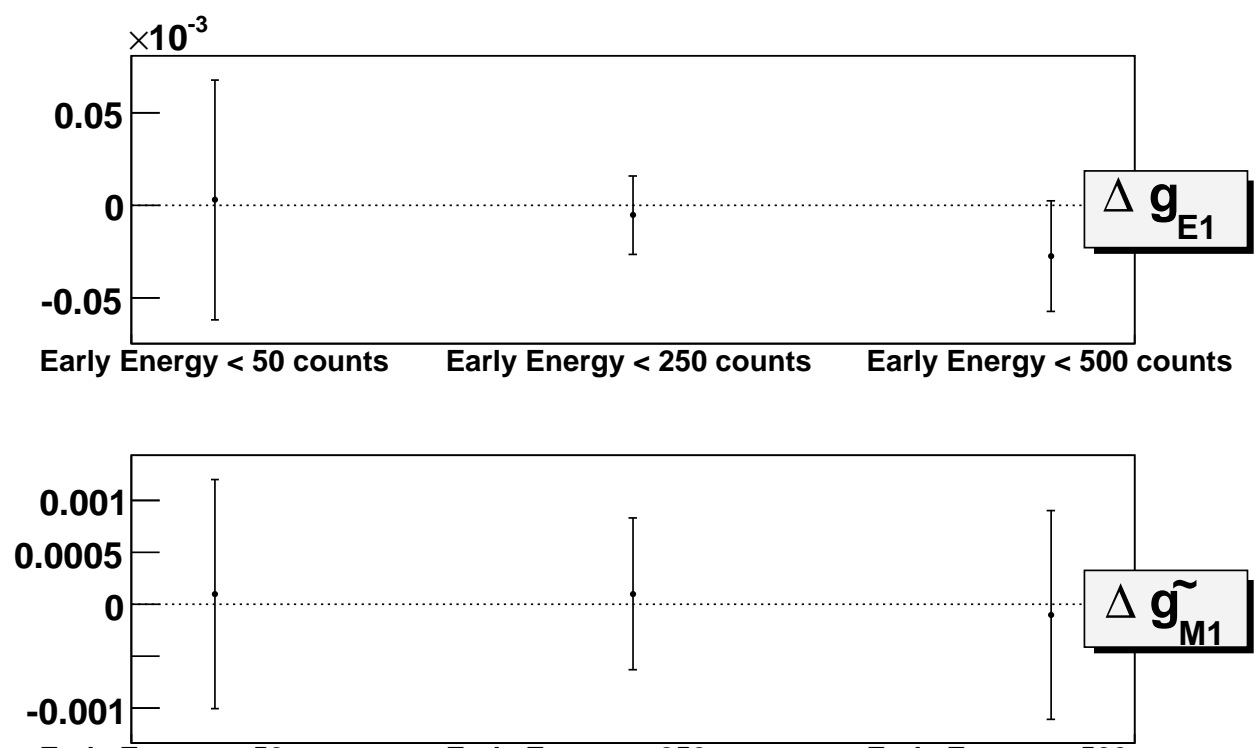

Early Energy $<50$ counts Early Energy $<250$ counts Early Energy $<500$ counts

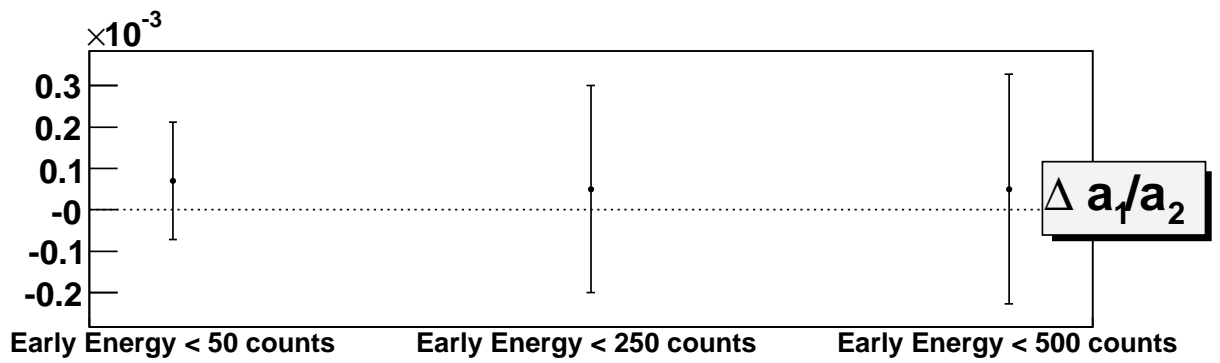

Figure 10.2: Resulting parameter shifts due to the variation of the early photon cluster energy cut. The default cut requires a early photon cluster energy of $<150$ counts. 

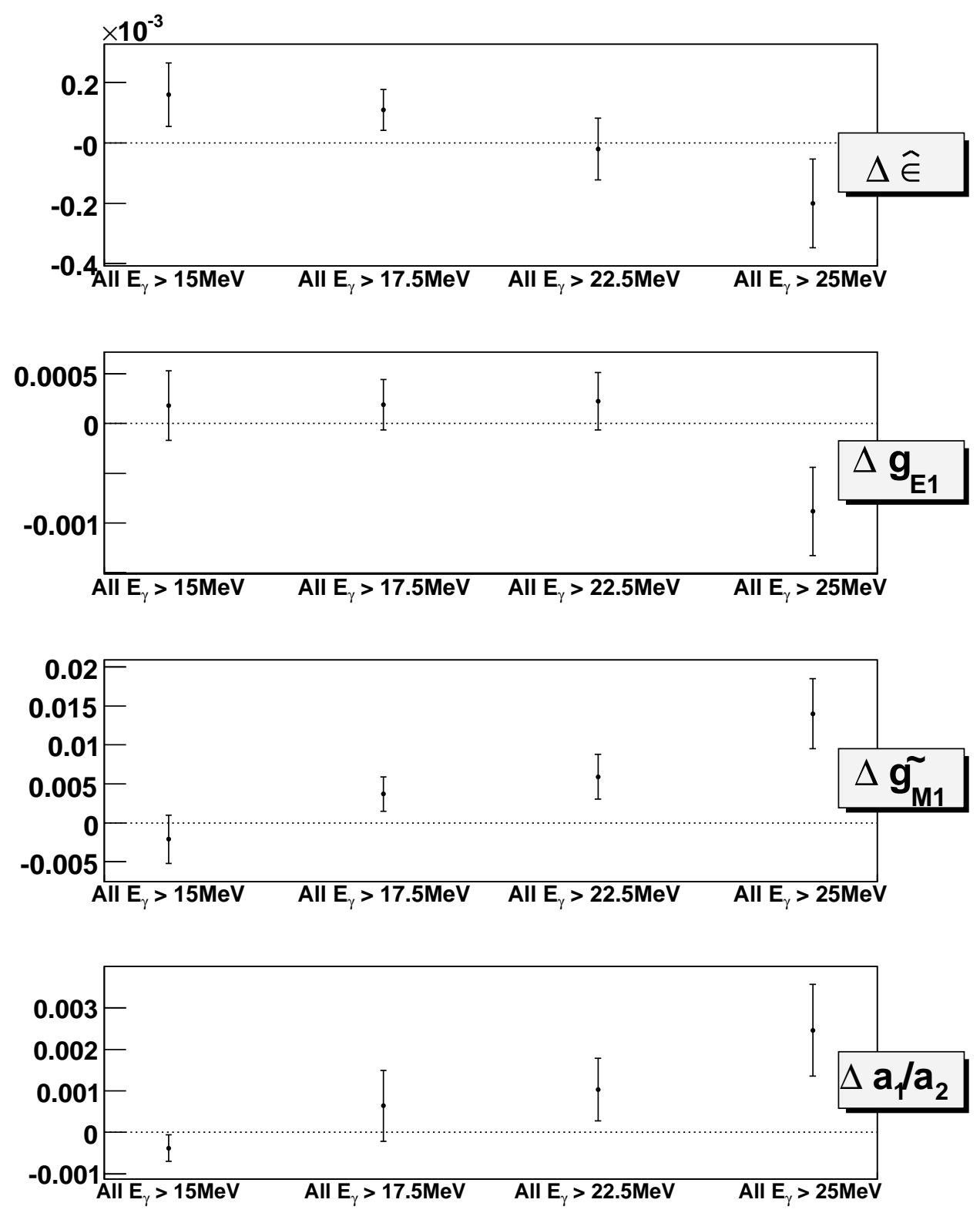

Figure 10.3: Resulting parameter shifts due to the variation of the kinematic and CsI photon energy cuts in the kaon rest frame. For each point, as well as in the nominal set of cuts, the two values are subjected to the same cut. The nominal cut requires that $E_{\gamma}^{*}>20 \mathrm{MeV}$. 

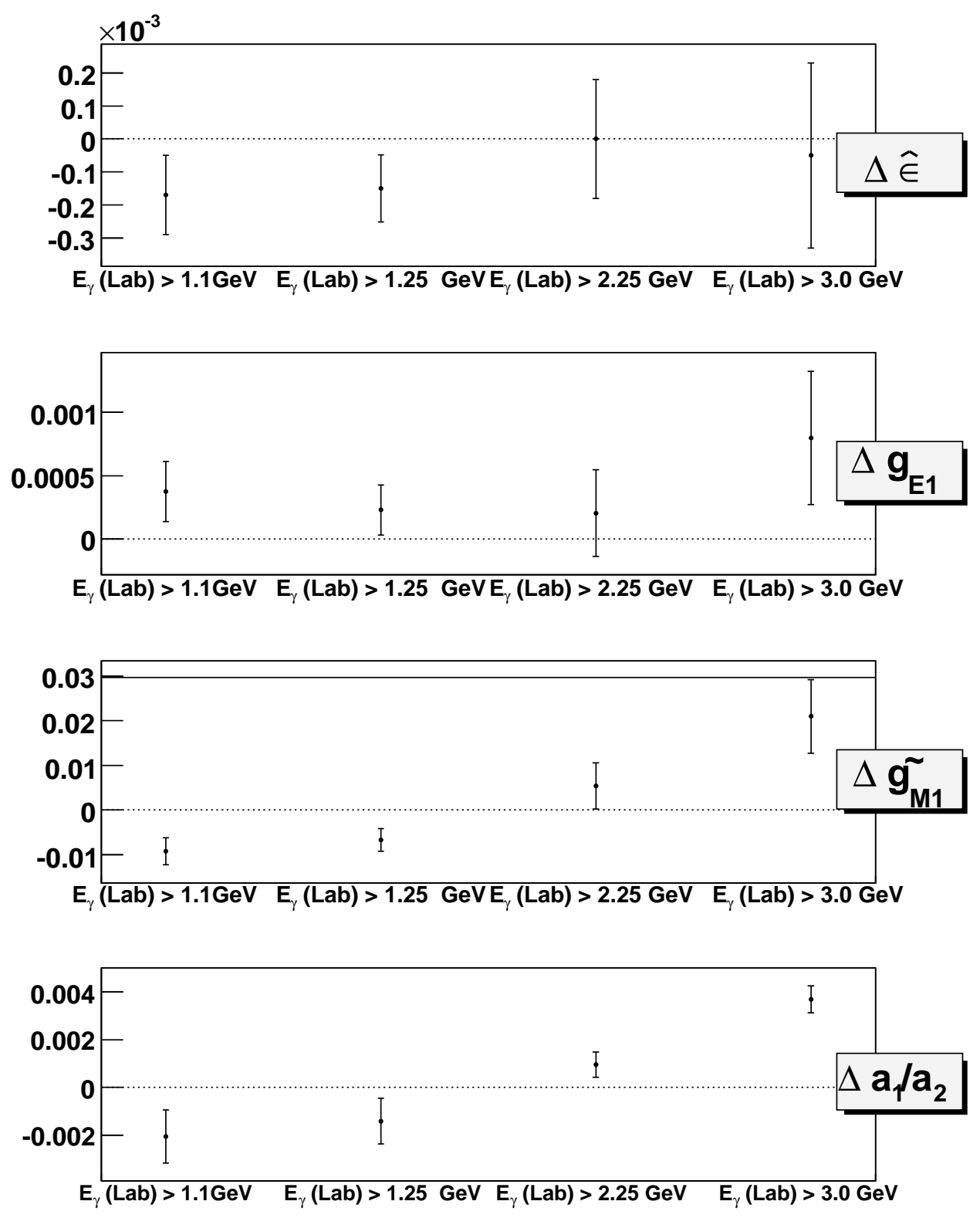

Figure 10.4: Resulting parameter shifts due to the variation of the CsI photon energy cut in the lab frame. The nominal cut requires that $E_{\gamma}^{L A B}>1.5 \mathrm{GeV}$. The crunch cut was made at $1.0 \mathrm{GeV}$ while cutting at $3.0 \mathrm{GeV}$ removes approximately $15 \%$ of events compared to the nominal cut. This also represents the agressive cut used in Reference [35]. The solid line in the $\widetilde{g_{M 1}}$ plot indicates the upper statistical error bound for the nominal result. 

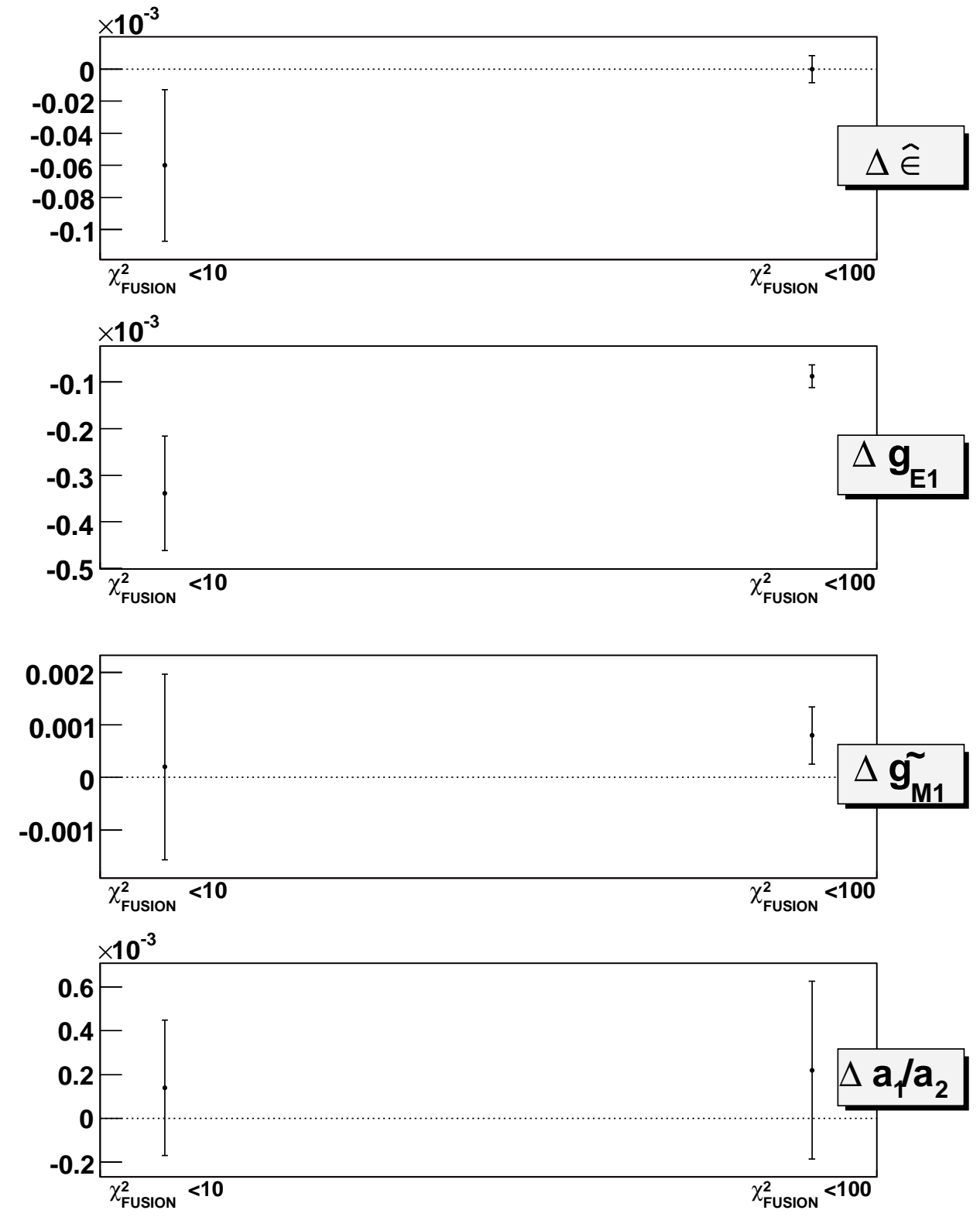

Figure 10.5: Resulting parameter shifts due to the variation of the fusion $\chi^{2}$ cut. The nominal cut requires fusion $\chi^{2}<48$ 

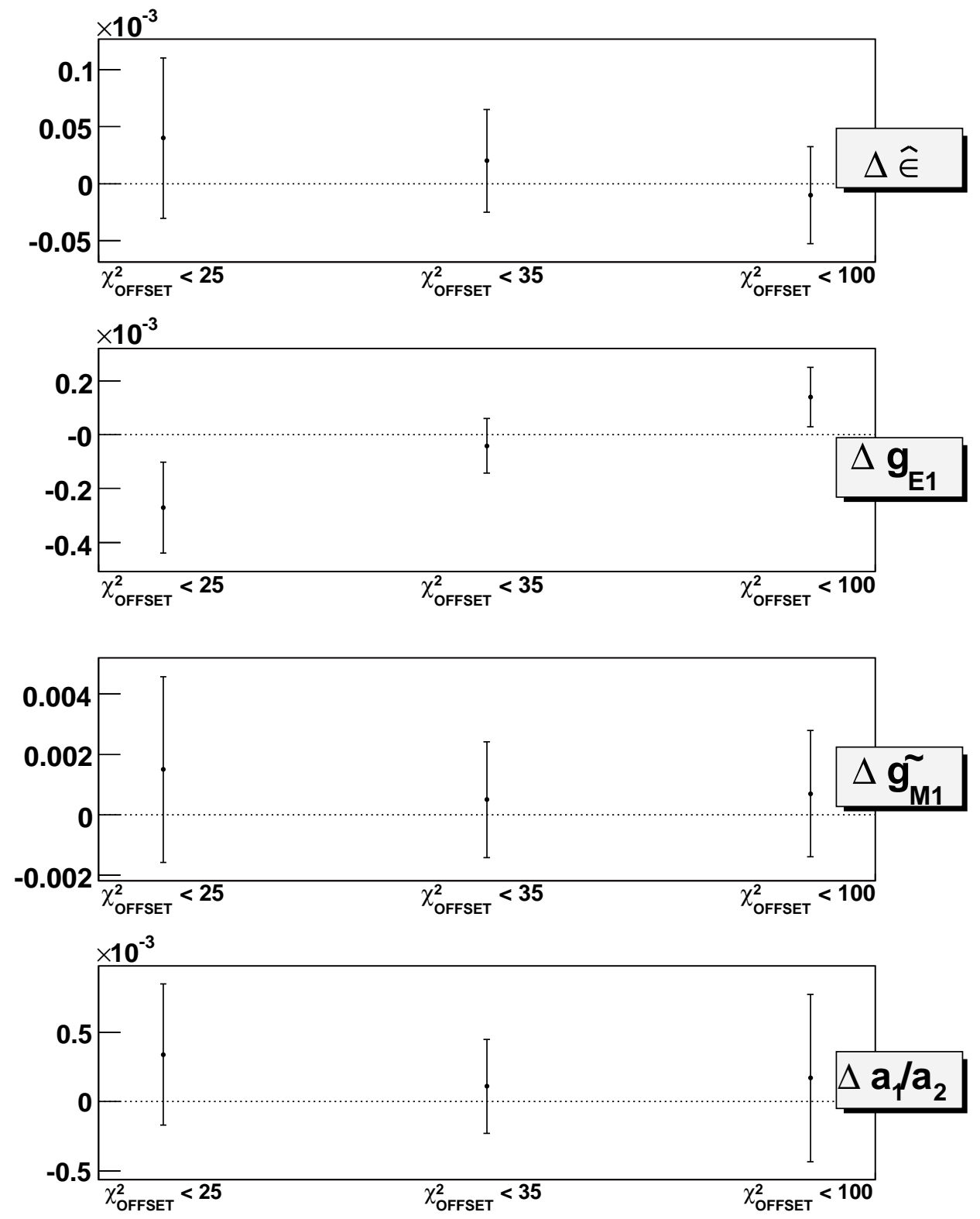

Figure 10.6: Resulting parameter shifts due to the variation of the track offset $\chi^{2}$ cut. The nominal cut requires $\chi^{2}<50$. 

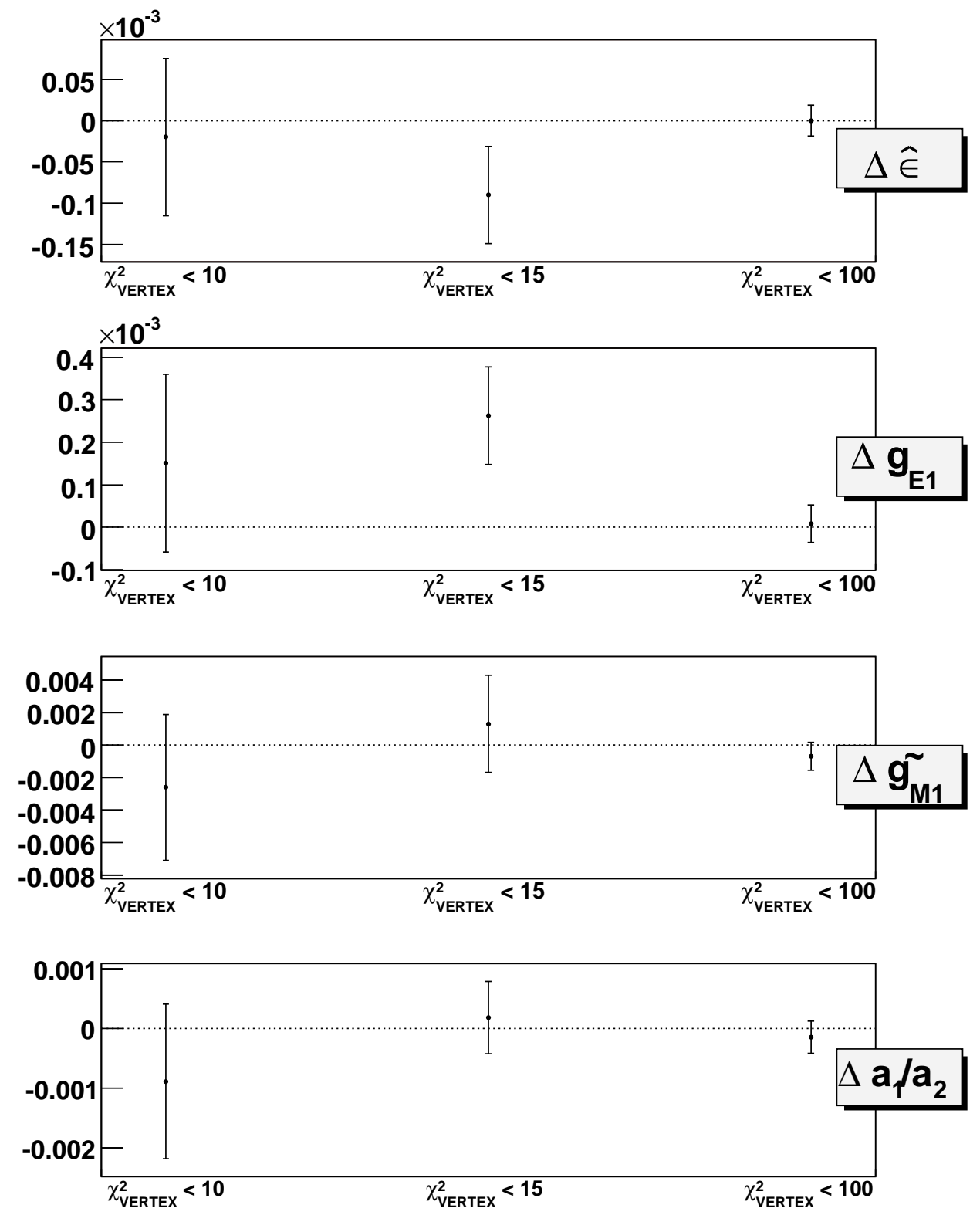

Figure 10.7: Resulting parameter shifts due to the variation of the vertex $\chi^{2}$ cut. The nominal cut requires $\chi^{2}<50$. 

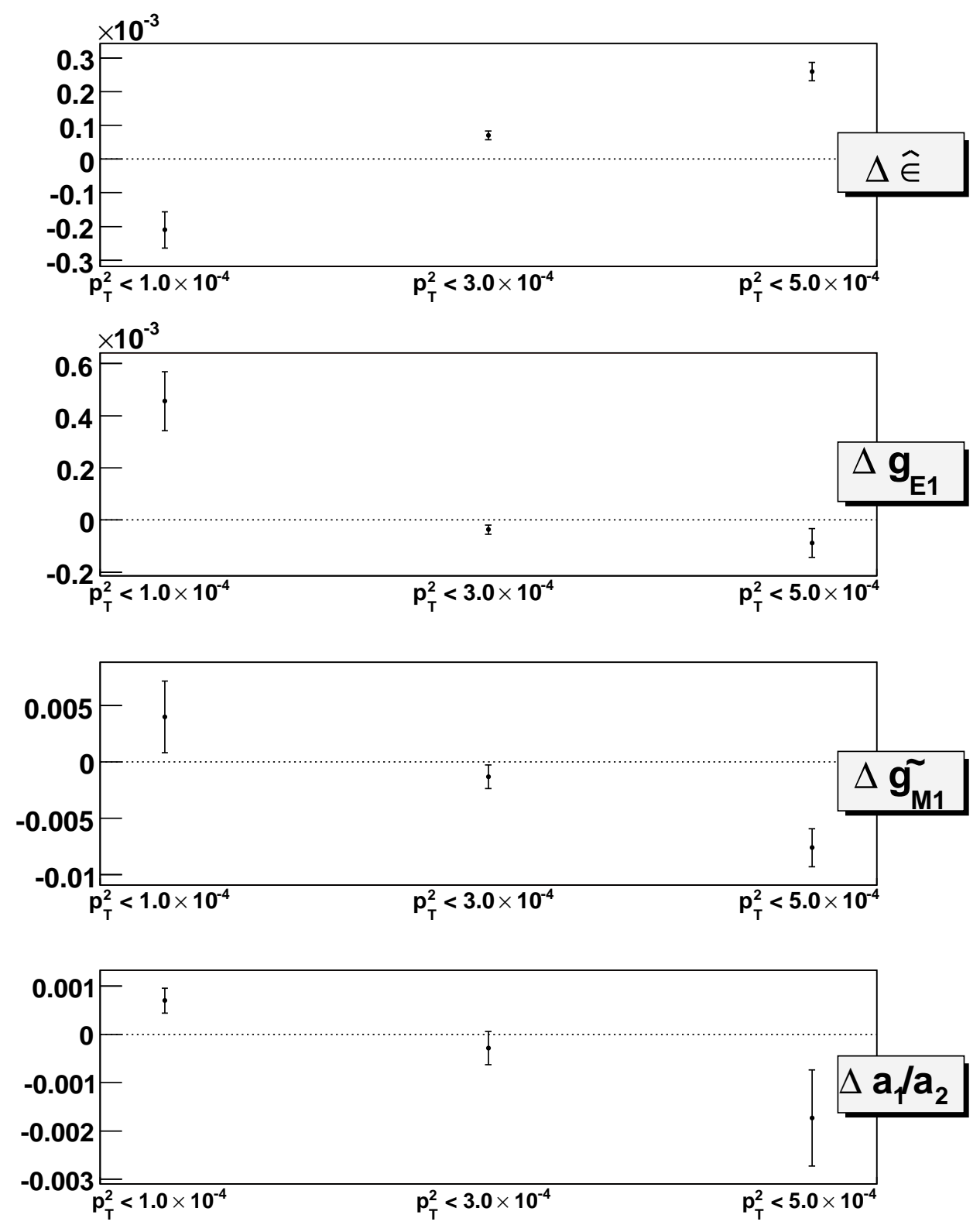

Figure 10.8: Resulting parameter shifts due to the variation of the $P_{T}^{2}$ cut. The nominal cut requires $P_{T}^{2}<2.5 \times 10^{-4} \mathrm{GeV}^{2} / \mathrm{c}^{2}$. 

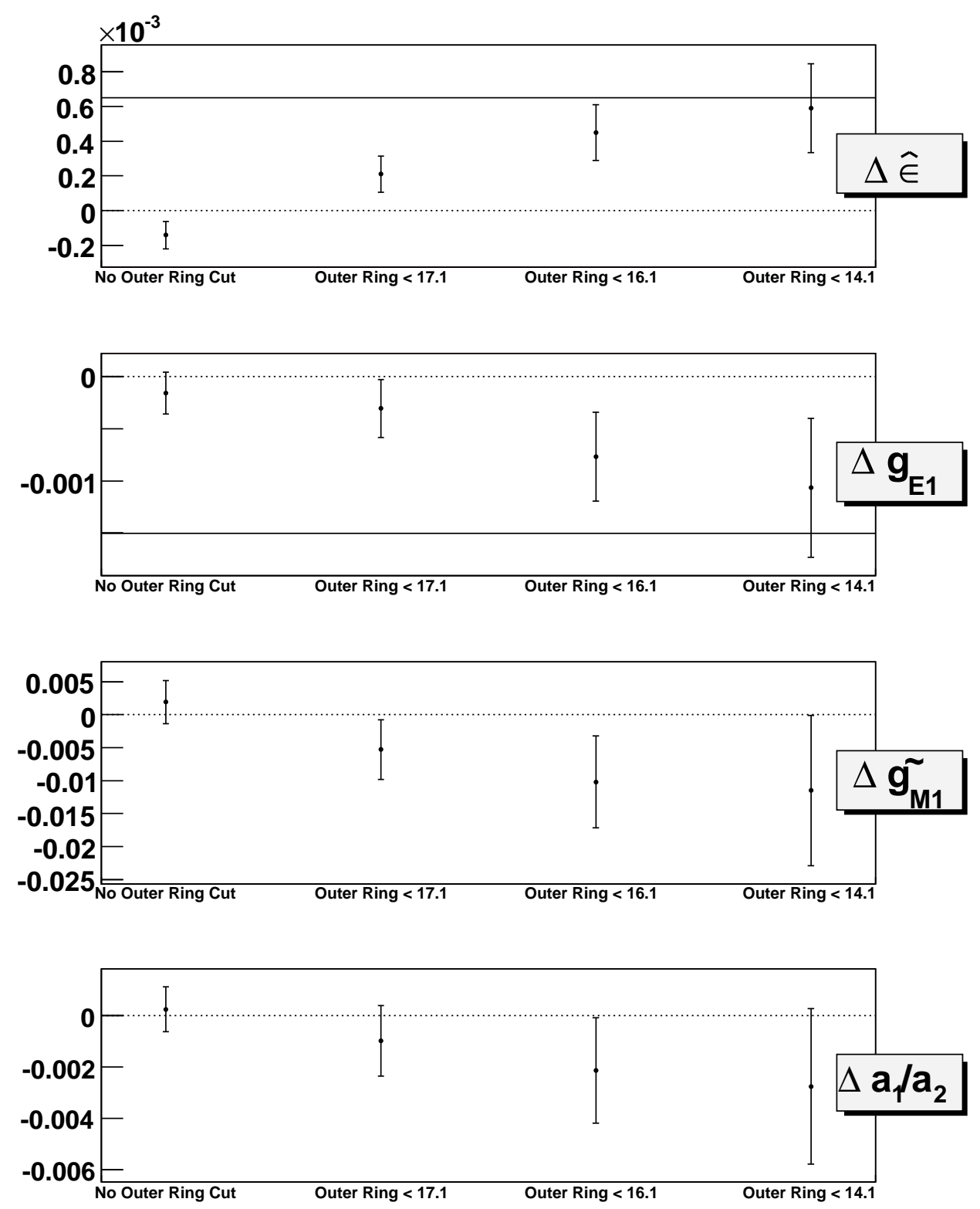

Figure 10.9: Resulting parameter shifts due to the variation of the SEEDRING cut, which is the outer fiducial cut for CsI cluster seeds. The nominal cut is SEEDRING $<$ 18.1. This cut variation reveals a large shift as the cut is tightened, however we assign a systematic error based on the parameter shift due to the removal of the cut. The solid line indicates the statistical error bound for the nominal results. 

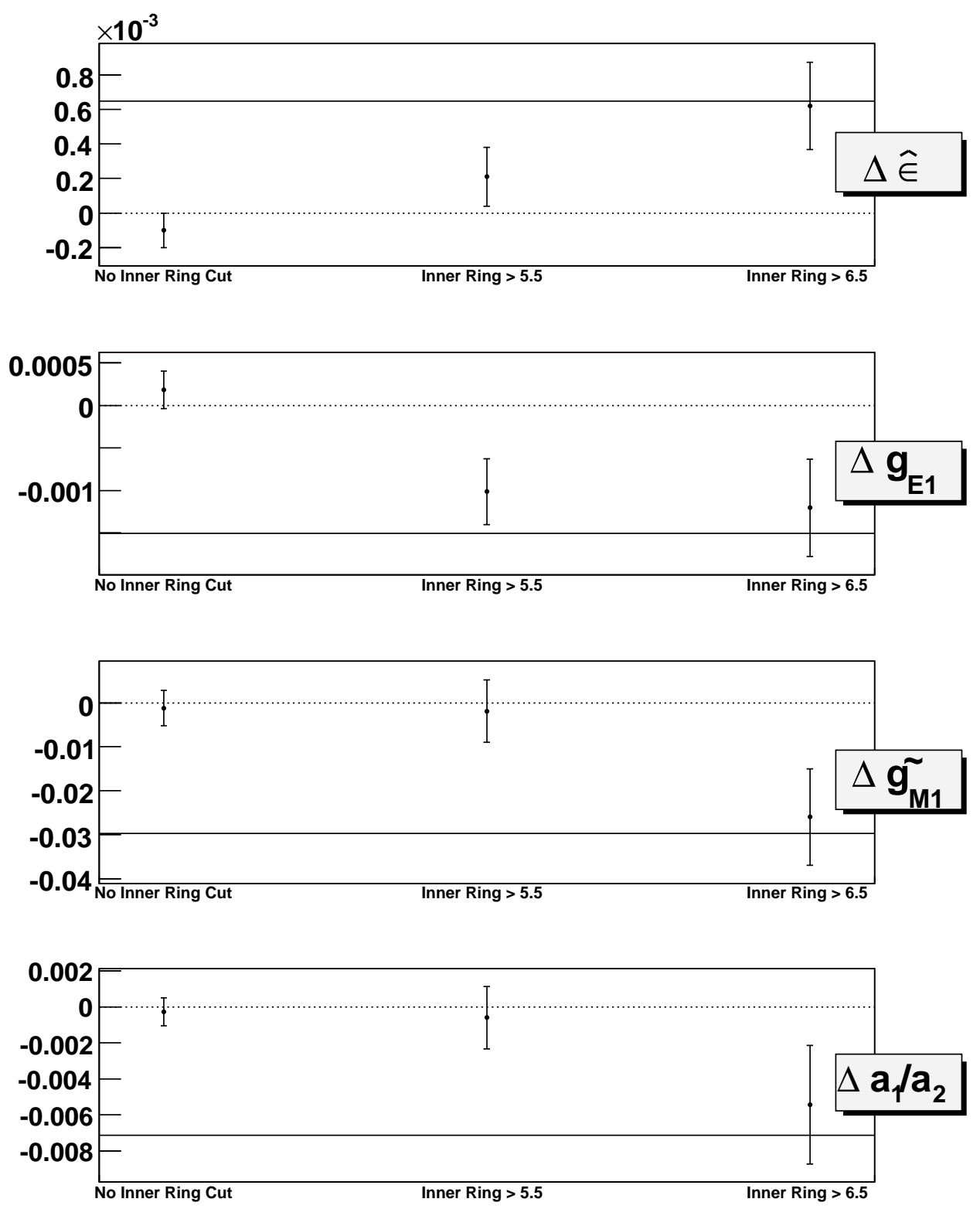

Figure 10.10: Resulting parameter shifts due to the variation of the ISMLRING cut, which is the inner fiducial cut for CsI cluster seeds. The nominal cut is ISMLRING $>$ 4.5. This cut variation reveals a large shift as the cut is tightened, however we assign a systematic error based on the parameter shift due to the removal of the cut. The solid line indicates the statistical error bound for the nominal results. 

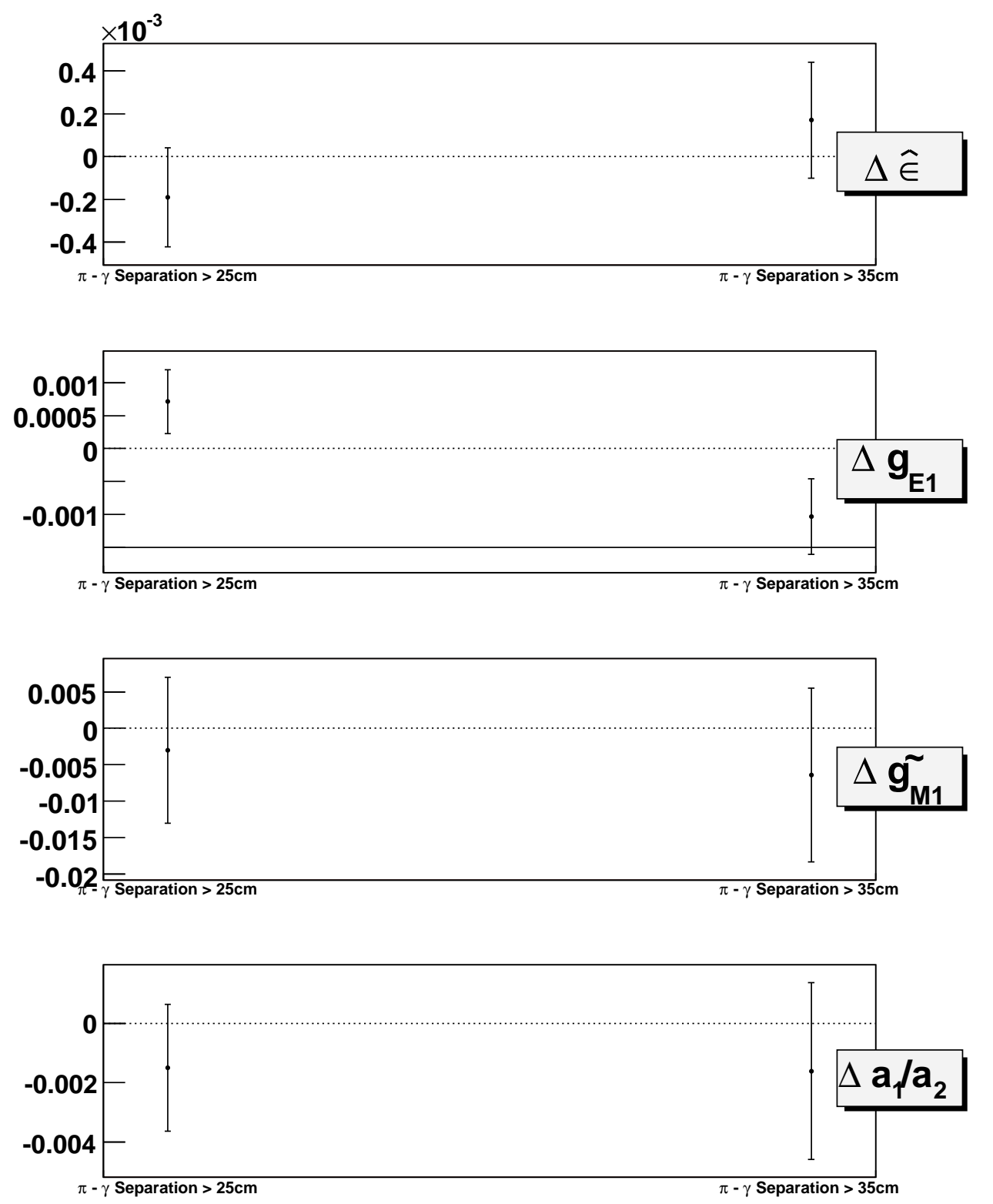

Figure 10.11: Resulting parameter shifts due to the variation of the photon/pion separation cut. The nominal cut is $\pi-\gamma$ separation $>30 \mathrm{~cm}$. Each cut value represents the point where approximately $10 \%$ of the data are removed or added to the sample. 

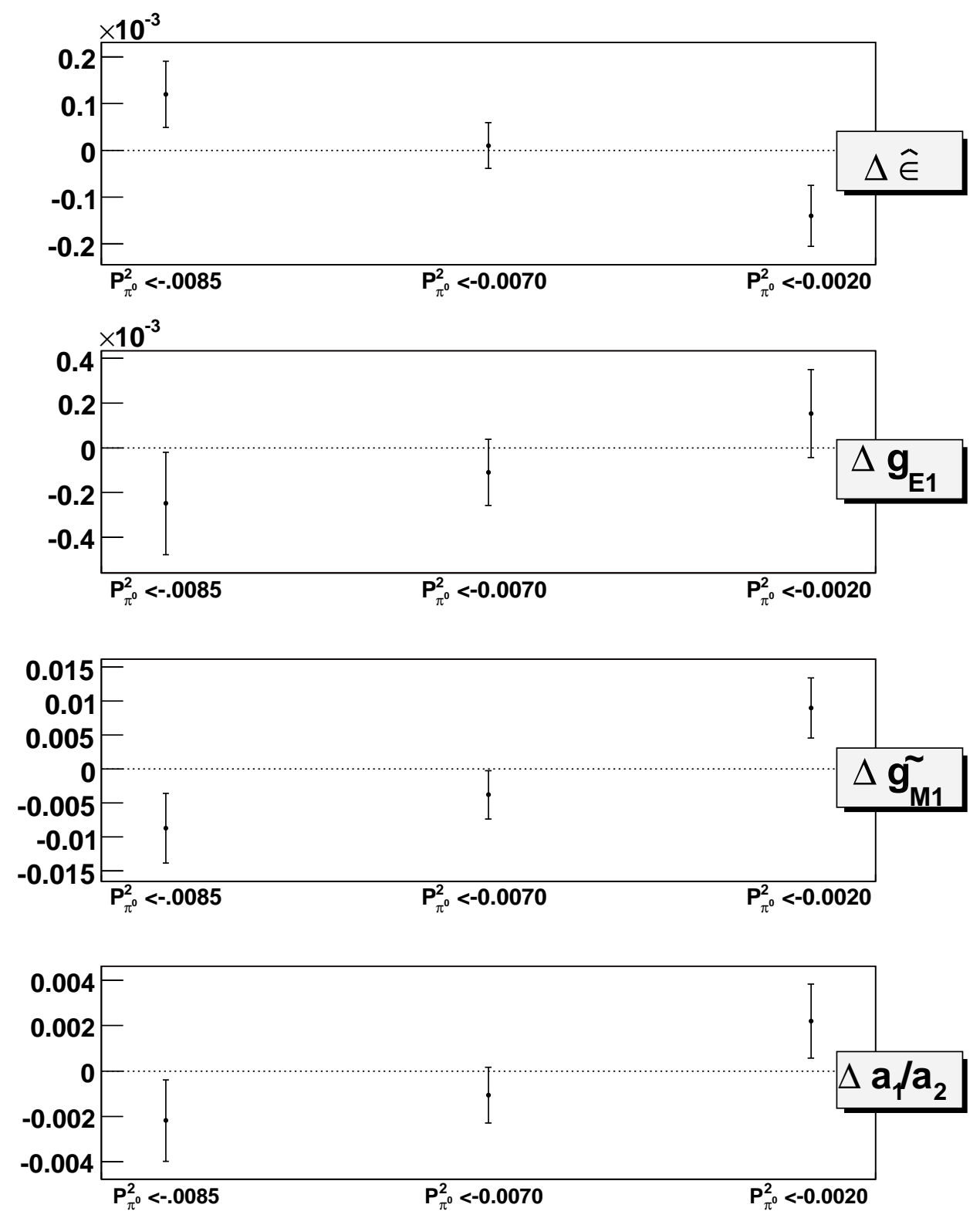

Figure 10.12: Resulting parameter shifts due to the variation of the $P_{\pi^{0}}^{2}$ cut. The nominal cut is $-0.10 \mathrm{GeV}^{2} / \mathrm{c}^{2}<P_{\pi^{0}}^{2}<-0.0055 \mathrm{GeV}^{2} / \mathrm{c}^{2}$. 

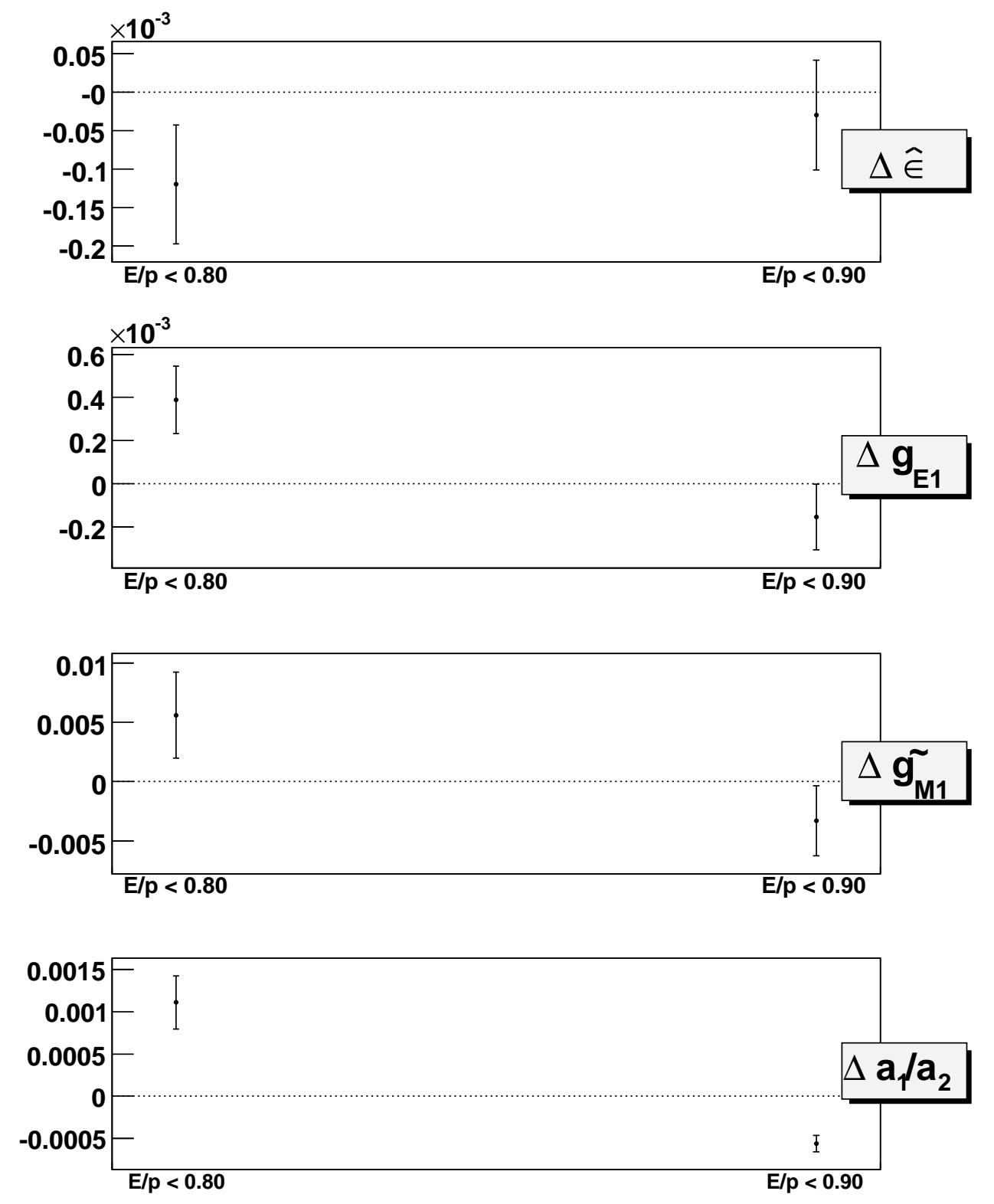

Figure 10.13: Resulting parameter shifts due to the variation of the $\mathrm{E} / \mathrm{p}$ cut. The nominal cut requires $\mathrm{E} / \mathrm{p}<0.85$. 

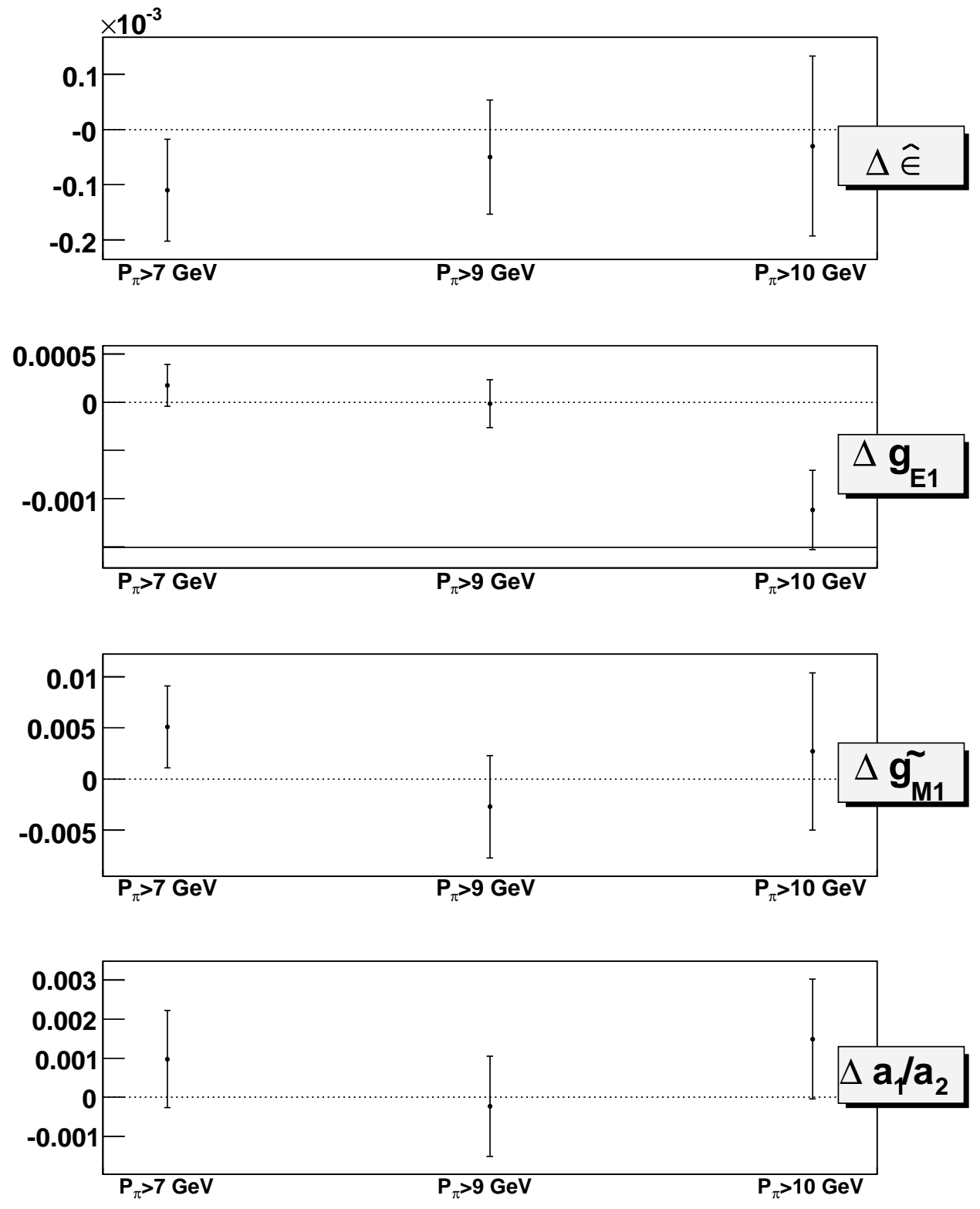

Figure 10.14: Resulting parameter shifts due to the variation of the track momentum cut. The nominal cut requires $P_{\pi}>8.0 \mathrm{GeV}$. The solid line indicates the statistical error bound for the nominal results. 

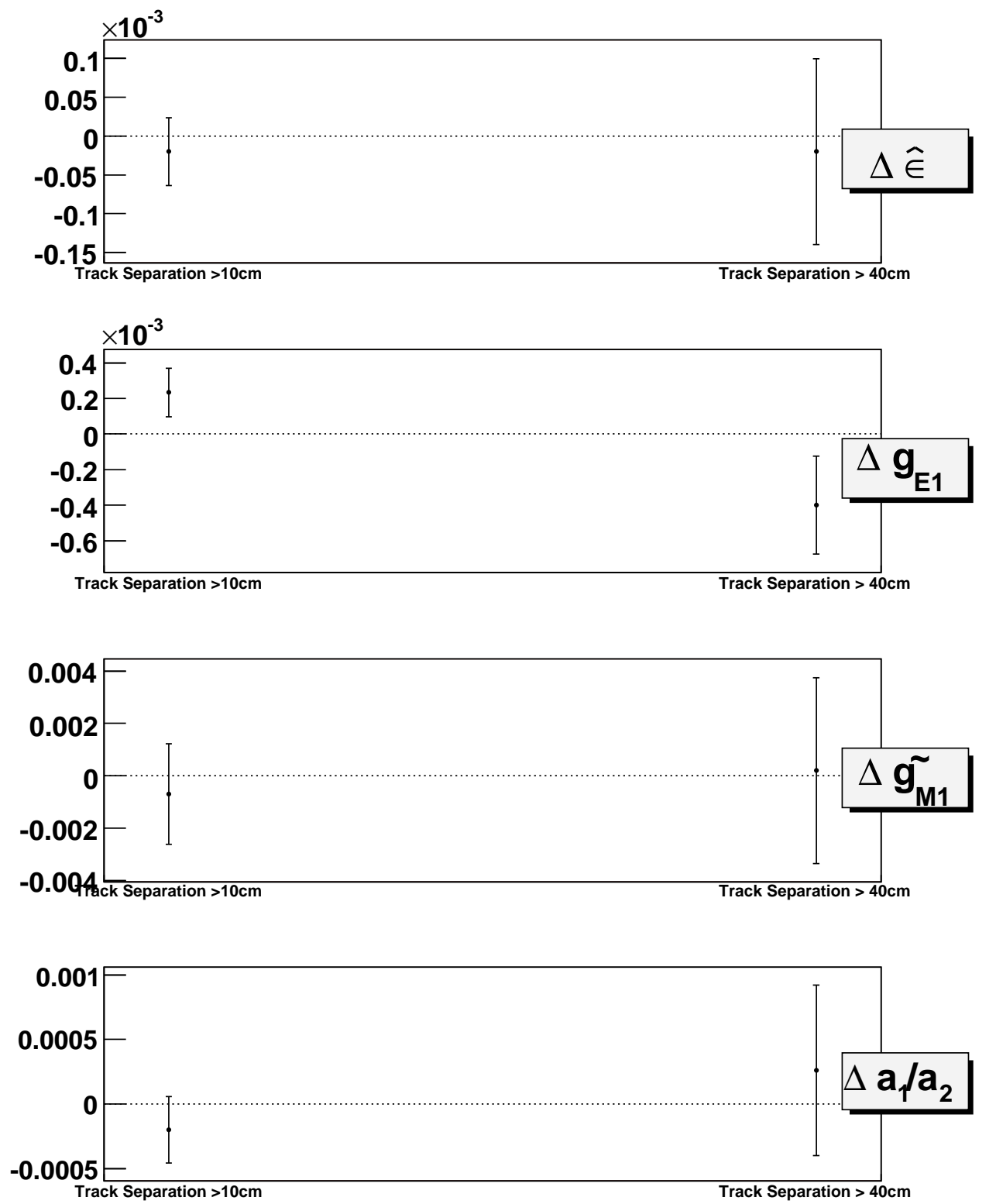

Figure 10.15: Resulting parameter shifts due to the variation of the track separation cut. The nominal cut requires the total track separation to be $>20 \mathrm{~cm}$ at the CsI. 

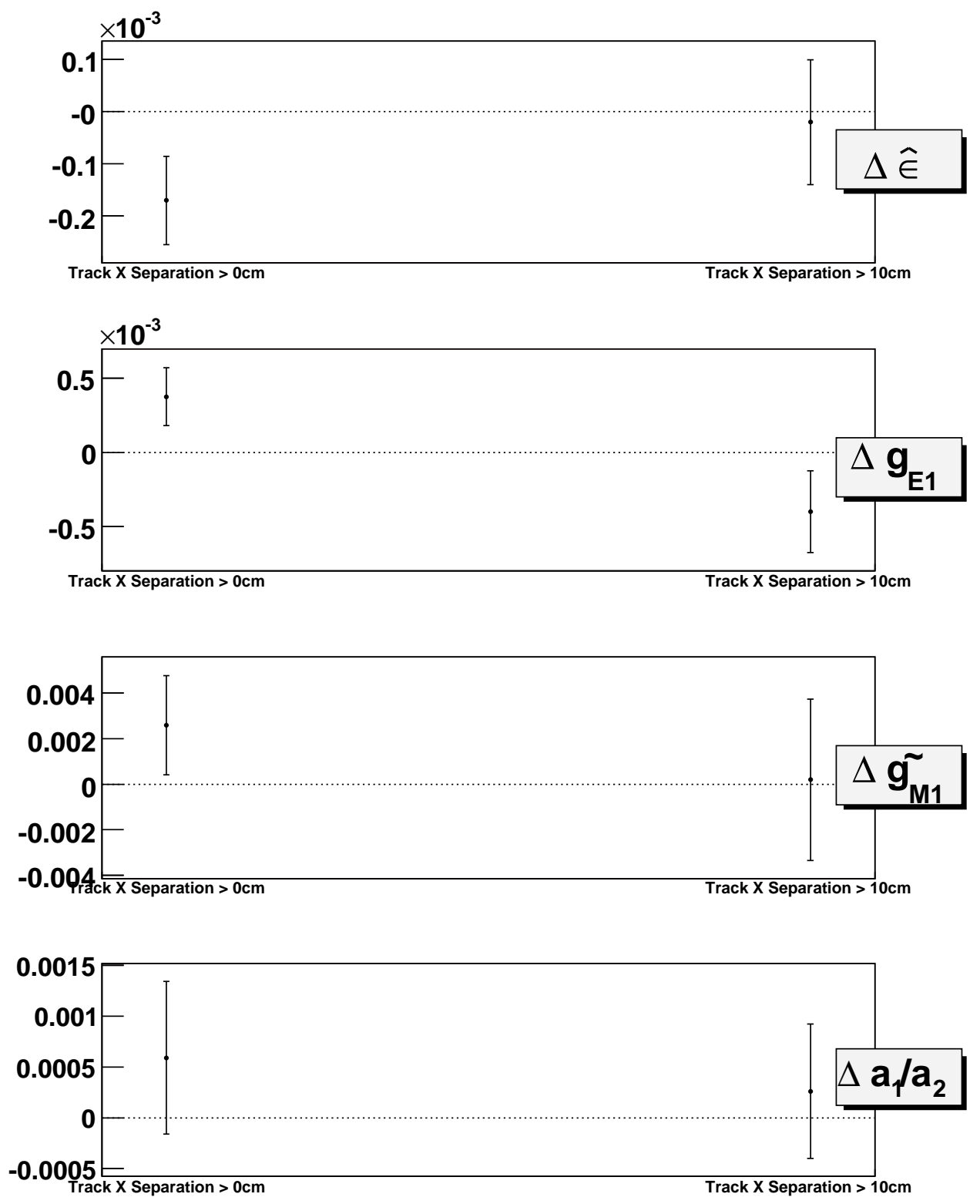

Figure 10.16: Resulting parameter shifts due to the variation of the track X separation cut. The nominal cut requires the $\mathrm{X}$ track separation to be $>3.0 \mathrm{~cm}$ at the CsI. The cut at $10 \mathrm{~cm}$ shows signs that the cut is too extreme, for this reason we only consider the situation where the cut is removed. 

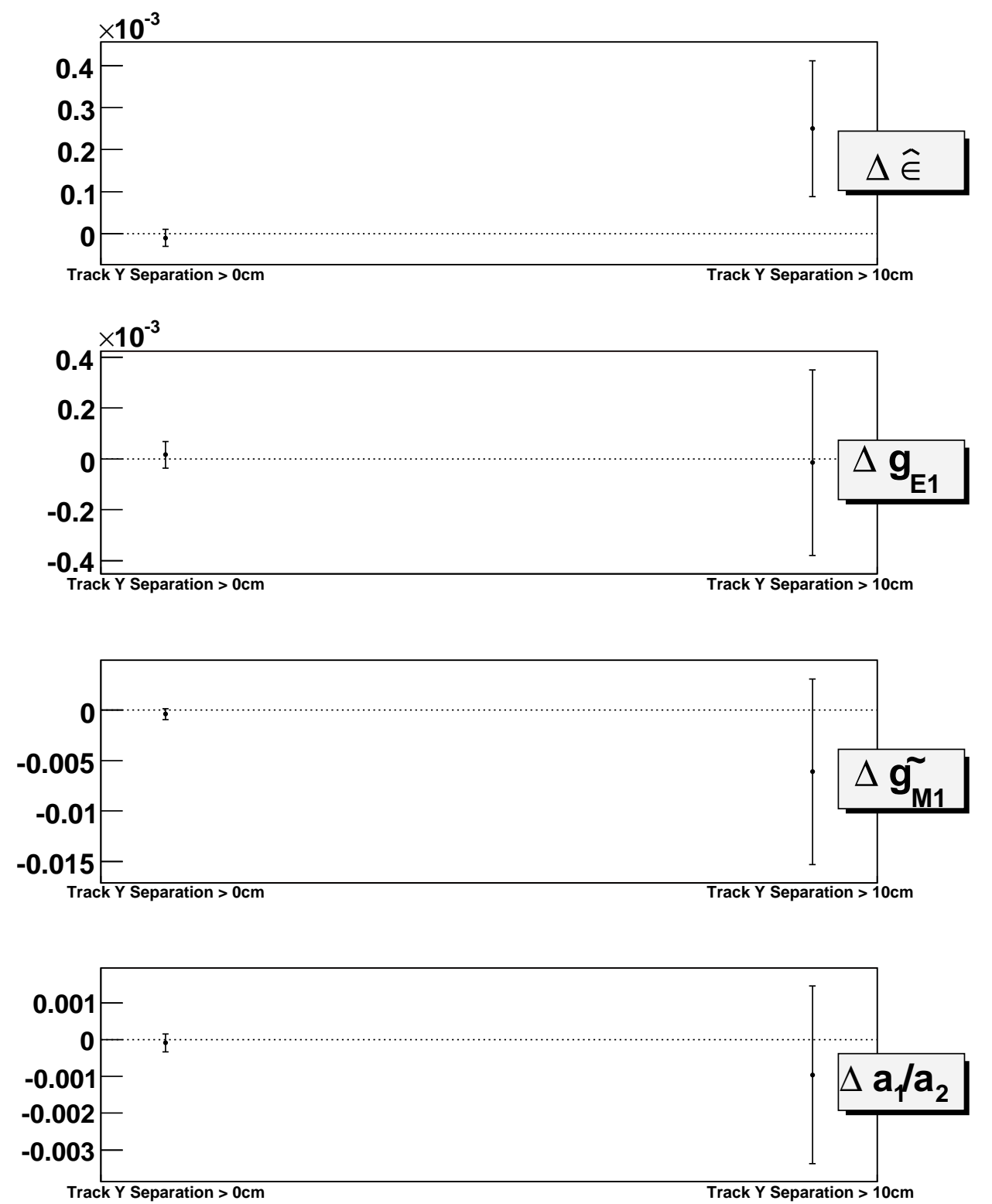

Figure 10.17: Resulting parameter shifts due to the variation of the track Y separation cut. The nominal cut requires the $Y$ track separation to be $>3.0 \mathrm{~cm}$ at the CsI. The cut at $10 \mathrm{~cm}$ shows signs that the cut is too extreme, for this reason we only consider the situation where the cut is removed. 
The cut variation plots indicate how the parameter estimates vary with different cut values. There are some cuts which are not included in this study, or the largest symmetric error is not used. The first cut which is not included in the fit variation study is the In-time Photon Cluster Energy cut. Small variations in the cut will not result in any parameter shift, as the cut is made in a region with a deficiency of both data and MC events. Also, it suppresses background events, so we would expect a shift if we were to remove the cut entirely.

Two other cuts which are treated in a different fashion are the inner and outer CsI ring cuts. Tightening each of these cuts results in a large shift in many of the fit parameters. Further inspection reveals that tightening these cuts warps other distributions, for example the kaon momentum spectrum. Since these cuts are designed to prevent energy leakage out of the sides and/or center of the calorimeter, the largest true systematic error possible would be that of not making these cuts at all, thus letting the energy leakage become an issue. We thus only include the situation where the inner and outer ring cuts are not made. Much in the same fashion, we also see a shift in parameters when we tighten the $\mathrm{X}$ and $\mathrm{Y}$ track separation cuts. Again, we see other distributions become warped, including the kaon momentum spectrum. These cuts are made in order to avoid problems in tracking that result from the tracks not matching to the correct cluster, and the largest possible systematic error would be due to these problems actually occurring. For that reason, we instead assign systematic errors using the parameter shifts that result from removing the $\mathrm{X}$ and $\mathrm{Y}$ track separation cuts.

The next cut that is treated differently is the $\mathrm{Z}$ vertex cut. Again, tightening this cut reveals large parameter shifts. Further inspection reveals alteration of the kaon momentum, indicating that we cannot tighten this cut so much. The purpose behind making this cut is to cut away from the regenerator edge, and thus remove kaons which decayed within the regenerator. These events would have a different wavefunction than we had assumed in the fitter, and the daughter pions will also lose energy as they transverse the regenerator. Given our purpose for making this cut, the largest systematic error should occur when we loosen this cut and begin to accept events coming from decays in the regenerator. As can be seen from the cut variation plot, when this is done, the resulting parameter shifts are far smaller than those obtained by tightening the cut. Because of this, we do not assign systematic 
errors based on the result obtained by tightening the cuts. However, in order to avoid this issue, we shall instead flatten the $\mathrm{z}$ vertex distribution in order to obtain the systematic error for this variable.

The results of the cut variation studies, including the modified treatment of some parameters as discussed above, are summarized in Table 10.9.

\begin{tabular}{|l|l|l|l|l|}
\hline Varied Cut & $\widehat{e}$ Error & $g_{E 1}$ Error & $\widetilde{g_{M 1}}$ Error & $a_{1} / a_{2}$ Error \\
\hline No $\Lambda \rightarrow p \pi$ cut & 0.0 & 0.0 & 0.0 & 0.0 \\
No $\pi^{0} \rightarrow \gamma \gamma$ mass cut & 0.000127 & 0.00039 & 0.0081 & 0.00256 \\
No Upstream track $/ \gamma$ cut & 0.0 & 0.000071 & 0.0 & 0.0 \\
No Inner Ring Cut & 0.000149 & 0.0 & 0.0 & 0.0 \\
No Outer Ring Cut & 0.000178 & 0.0 & 0.0 & 0.0 \\
Track Y Separation & 0.0 & 0.0 & 0.0 & 0.0 \\
Track X Separation & 0.00021 & 0.000468 & 0.00365 & 0.0 \\
Track Separation & 0.0 & 0.00053 & 0.0 & 0.0 \\
$P_{\pi}$ & 0.000154 & 0.00131 & 0.007 & 0.0 \\
E/p & 0.000157 & 0.000463 & 0.0073 & 0.00126 \\
$P_{\pi^{0}}^{2}$ & 0.000171 & 0.00036 & 0.0111 & 0.00306 \\
$\pi-\gamma$ Separation & 0.0 & 0.00131 & 0.0 & 0.0 \\
$p_{T}^{2}$ & 0.000273 & 0.00051 & 0.0084 & 0.00221 \\
All $E_{\gamma}$ & 0.00027 & 0.00109 & 0.0161 & 0.00299 \\
$E_{\gamma}$ (Lab) & 0.000228 & 0.00105 & 0.0249 & 0.00396 \\
$\chi_{O F F S E T}^{2}$ & 0.0 & 0.000351 & 0.0 & 0.0 \\
$\chi_{V E R T E X}^{2}$ & 0.000118 & 0.000317 & 0.0 & 0.0 \\
$\chi_{F U S I O N}^{2}$ & 0.000083 & 0.000397 & 0.00106 & 0.0 \\
Early Energy & 0.0000269 & 0.0 & 0.0 & 0.0 \\
\hline Total Error & 0.00064 & 0.00271 & 0.0355 & 0.0069 \\
\hline
\end{tabular}

Table 10.9: Assigned systematic errors from the cut variation study. All systematic errors were obtained using the $\Delta_{S}$ method. Errors which read " 0.0 " indicate that the observed shift in the parameter was not larger than the statistical error of the shift, and hence no systematic error was assigned.

There is one more distribution that we have not yet addressed - the kaon momentum spectrum. Recall that the $\mathrm{KTeV}$ Monte Carlo generates its kaon momentum spectrum using the observed spectrum from $K_{L, S} \rightarrow \pi^{+} \pi^{-}$decays. This strongly implies that the observed kaon momentum spectrum for $K_{L, S} \rightarrow \pi^{+} \pi^{-} \gamma$ should match that from the Monte Carlo - if everything is correctly modeled. Differences in the two spectra can come from a multitude of different sources, however it is still useful to see how the estimated parameter values depend on the kaon momentum. We first plot the ratio of the kaon momentum from data and Monte Carlo on a bin by bin 
basis. If there was perfect agreement, the result would be a linear distribution with a slope of zero. A slope indicates problems with the simulation. We fit this distribution for each of the 4 data subsamples to obtain a slope for each. The observed slopes are indicated in Table 10.4.1, and indicate that while the vacuum beam samples have a slope which is consistent with being equal to zero, the regenerator beam samples do have statistically significant slopes. In order to evaluate this possible systematic

\begin{tabular}{|l|l|}
\hline Subsample & Kaon Momentum Slope \\
\hline 1997 Reg & $(-2.01 \pm 0.59) \times 10^{-4}$ \\
\hline 1999 Reg & $(-1.33 \pm 0.31) \times 10^{-4}$ \\
\hline 1997 Vac & $(4.1 \pm 9.4) \times 10^{-5}$ \\
\hline 1999 Vac & $(0.23 \pm 4.7) \times 10^{-5}$ \\
\hline
\end{tabular}

Table 10.10: Observed kaon momentum slopes relative to Monte Carlo

error, we take the momentum slopes from the regenerator beam and apply them as corrections to the normalization Monte Carlo sample, which will flatten the momentum spectrum. Since the vacuum beam samples do not have a statistically significant slope, we do not correct those two samples. The shift in parameters that results from flattening the momentum spectrum is shown in Table 10.11. Since we only adjusted the weights of the events in the normalization Monte Carlo sample, we simply take the observed shift as the systematic error.

\begin{tabular}{|c|c|c|c|c|}
\hline & $\widehat{e}$ Shift & $g_{E 1}$ Shift & $\widetilde{g_{M 1}}$ Shift & $a_{1} / a_{2}$ Shift \\
\hline Observed Shift & -0.00007 & 0.0006 & -0.0009 & -0.00013 \\
\hline
\end{tabular}

Table 10.11: Observed parameter shifts due to flattening of the momentum spectrum.

As outlined above, we have also chosen to flatten the $\mathrm{z}$ vertex distribution due to difficulty in performing a cut variation on that variable. The procedure is the same as that used in handling the kaon momentum. The observed slopes are indicated in Table 10.12. The shift in parameters from flattening the $\mathrm{z}$ vertex distributions can be found in Table 10.13.

There is one final complication to the flattening procedure as performed - the kaon momentum and z vertex are highly correlated with one another. The consequence 


\begin{tabular}{|l|l|}
\hline Subsample & Z Vertex Slope \\
\hline 1997 Reg & $(-0.20 \pm 2.41) \times 10^{-4}$ \\
\hline 1999 Reg & $(-1.68 \pm 1.23) \times 10^{-4}$ \\
\hline 1997 Vac & $(-6.22 \pm 2.17) \times 10^{-4}$ \\
\hline 1999 Vac & $(-1.87 \pm 1.10) \times 10^{-4}$ \\
\hline
\end{tabular}

Table 10.12: Observed z vertex slopes relative to Monte Carlo

\begin{tabular}{|c|c|c|c|c|}
\hline & $\widehat{e}$ Shift & $g_{E 1}$ Shift & $\widetilde{g_{M 1}}$ Shift & $a_{1} / a_{2}$ Shift \\
\hline Observed Shift & 0.00045 & -0.000759 & -0.0023 & -0.00049 \\
\hline
\end{tabular}

Table 10.13: Observed parameter shifts due to flattening of the z vertex distribution.

is that these errors ( actually their magnitudes ) must be added linearly and not in quadrature. The combined result can be found in Table 10.14

\begin{tabular}{|c|c|c|c|c|}
\hline & $\widehat{e}$ Error & $g_{E 1}$ Error & $\widetilde{g_{M 1}}$ Error & $a_{1} / a_{2}$ Error \\
\hline Total Error & 0.00052 & 0.001359 & 0.0032 & 0.00062 \\
\hline
\end{tabular}

Table 10.14: Systematic errors due to flattening of $Z_{\text {vertex }}$ and $p_{K}$ distributions

\section{Accidental Simulation}

While the Monte Carlo does include a simulation of accidental particles, there is some uncertainty in the level of this. The nominal result was obtained with the accidental simulation turned on. Since the simulation is known to be accurate to roughly $10 \%$ [4] we generate a sample of normalization Monte Carlo with the accidental simulation turned off, compute the resulting shift in parameters, and the multiply the shift by $10 \%$ in order to obtain the corresponding systematic error. The results of this study are shown in Table 10.15 .

\section{Radiative Corrections}

The KTeV Monte Carlo utilizes the radiative correction package PHOTOS in order to simulate the emission of a second photon, i.e. $K_{L, S} \rightarrow \pi^{+} \pi^{-} \gamma \gamma$. However, we must allow for the possibility that the probability of this occurring is not well repro- 


\begin{tabular}{|c|c|c|c|c|}
\hline & $\widehat{e}$ Shift & $g_{E 1}$ Shift & $\widetilde{g_{M 1}}$ Shift & $a_{1} / a_{2}$ Shift \\
\hline No Accidental Simulation & -0.00027 & -0.0013061 & -0.0053 & -0.00157 \\
Assigned Error & 0.000027 & 0.00013061 & 0.00053 & 0.000157 \\
\hline
\end{tabular}

Table 10.15: Observed shifts and assigned systematic errors from the uncertainty in the Monte Carlo accidental simulation

duced. In order to account for this, we introduce a correction factor which scales the probability of second photon emission, and use this correction factor to match the data in the region just to the left of the nominal kaon mass in the plot of $M_{\pi^{+}} \pi^{-} \gamma$ - which is where these events reconstruct. This procedure is outlined in Appendix G. The observed PHOTOS normalization is different between the vacuum and regenerator beams, and each measured value also has an associated error. We use the average value of the two to actually generate the normalization Monte Carlo sample for the nominal fit, and determine the systematic error due to the uncertainty in the PHOTOS normalization. The shifts from the average value to the values specific to each beam are:

$$
\begin{aligned}
& \Delta w_{\pi \pi \gamma \gamma}=0.232 \pm 0.056 \quad(V A C) \\
& \Delta w_{\pi \pi \gamma \gamma}=-0.123 \pm 0.034 \quad(R E G)
\end{aligned}
$$

where the errors are taken from the error on the specific value for each beam. As can be seen, the two values are quite different. In order to better cover the possible range of values of $w_{\pi \pi \gamma \gamma}$ that these represent, we will generate two samples of normalization Monte Carlo, one using a lower value of $w_{\pi \pi \gamma \gamma}$ than nominal, and another using a higher value of $w_{\pi \pi \gamma \gamma}$ than nominal. In order to avoid underestimating the systematic error here, we will not merely use the values of $w_{\pi \pi \gamma \gamma}$ found from the vac and reg beams, but will utilize the $\Delta_{S}$ method to determine maximum and minimum values of $w_{\pi \pi \gamma \gamma}$.

We first use the $\Delta_{S}$ method to determine a symmetric error interval for each shift. The $\Delta_{S}$ method yields:

$$
\begin{gathered}
\sigma_{w_{\pi \pi \gamma \gamma}^{V A C}}^{V A}=0.259 \\
\sigma_{w_{\pi \pi \gamma \gamma}^{R E G}}^{R E}=0.139
\end{gathered}
$$


Each value of $\sigma_{w_{\pi \gamma \gamma}}$ gives a symmetric error interval around the average (nominal) value of $w_{\pi \pi \gamma \gamma}$. This error interval can be interpreted as the interval in which there is a expectation, at $68.3 \%$ confidence, that the true value value of $w_{\pi \pi \gamma \gamma}$ lies within. The expectation comes from the measurement of $w_{\pi \pi \gamma \gamma}$ in either beam.

The next step is to use the intervals to determine the maximum and minimum bounds for $w_{\pi \pi \gamma \gamma}$. We use the interval corresponding to the regenerator beam for the lower bound, as it yielded the lower of the two values, and use the interval corresponding to the vacuum beam for the higher bound. The result is:

$$
\begin{aligned}
w_{\pi \pi \gamma \gamma}^{A V G}-\sigma w_{\pi \pi \gamma \gamma}^{R E G} & \leq w_{\pi \pi \gamma \gamma} \leq w_{\pi \pi \gamma \gamma}^{A V G}+\sigma w_{\pi \pi \gamma \gamma}{ }^{A A C} \\
0.499 & \leq w_{\pi \pi \gamma \gamma} \leq 0.897
\end{aligned}
$$

The result is two bounds which give an area slightly past the two measured values of $w_{\pi \pi \gamma \gamma}$ and partially incorporate the errors measured. We generate two samples of normalization Monte Carlo, setting $w_{\pi \pi \gamma \gamma}=0.499$ for one and $w_{\pi \pi \gamma \gamma}=0.897$ for another, and fit the nominal data sample using these MC samples. The shifts in parameter estimates from the nominal results are shown in Table 10.16, which also indicates that we take the maximum shift in each parameter as the systematic error.

\begin{tabular}{|c|c|c|c|c|}
\hline & $\widehat{e}$ Shift & $g_{E 1}$ Shift & $\widetilde{g_{M 1}}$ Shift & $a_{1} / a_{2}$ Shift \\
\hline$w_{\pi \pi \gamma \gamma}=0.499$ & -0.00016 & -0.0010051 & -0.0073 & -0.00141 \\
$w_{\pi \pi \gamma \gamma}=0.897$ & -0.00003 & 0.0007523 & -0.0165 & -0.00537 \\
\hline Assigned Error & 0.00016 & 0.0010051 & 0.0165 & 0.00537 \\
\hline
\end{tabular}

Table 10.16: Assigned systematic errors from the uncertainty in PHOTOS normalization

\subsection{Sum of Systematic Errors}

The final results of the systematic error studies, along with the total systematic errors, are shown in Table 10.17. The total systematic errors were obtained by adding the totals within each group in quadrature. Table 10.18 reviews how each systematic error was obtained. 


\begin{tabular}{|l|l|l|l|l|}
\hline Systematic Error & $\widehat{e}$ Error & $g_{E 1}$ Error & $\widetilde{g_{M 1}}$ Error & $a_{1} / a_{2}$ Error \\
\hline Input Error & 0.000346 & 0.000358 & 0.00746 & 0.000708 \\
Cut Variations & 0.000642 & 0.00271 & 0.0355 & 0.00685 \\
PHOTOS Error & 0.00016 & 0.00101 & 0.0165 & 0.00537 \\
Accidental Simulation Error & 0.000027 & 0.000131 & 0.00053 & 0.000157 \\
Reconstruction Error & 0.00052 & 0.000325 & 0.0033 & 0.00009 \\
Background & 0.000185 & - & 0.00603 & 0.00177 \\
Incoherent Regeneration & 0.000123 & 0.000109 & - & - \\
Flattened Distributions & 0.00052 & 0.00136 & 0.0032 & 0.00062 \\
\hline \hline Total Error & 0.00107 & 0.00324 & 0.0406 & 0.00893 \\
\hline
\end{tabular}

Table 10.17: Total Systematic Errors

\begin{tabular}{|l|c|}
\hline Systematic Error & Method Used to Estimate Error \\
\hline Input Error & Addition of Correlated Parameter Shifts \\
Cut Variations & $\Delta_{S}$ Method Using Correlated Shifts \\
PHOTOS Error & Pure Shift \\
Accidental Simulation Error & Pure Shift $\times$ Error on Accidental Rate \\
Reconstruction Error & Pure Shift \\
Background & $\Delta_{S}$ Method Using Correlated Shifts \\
Incoherent Regeneration & $\Delta_{S}$ Method Using Correlated Shifts \\
Flattened Distributions & Pure Shift \\
\hline \hline Total Error & \\
\hline
\end{tabular}

Table 10.18: Methods used to obtain systematic errors 


\section{Chapter 11}

\section{Conclusions}

Now that the systematic errors for all parameters have been estimated, we can now remove the unknown offset of $\widehat{e}$ and present the final result.

\subsection{Final Result}

The final result of this analysis, including systematic errors, is:

$$
\begin{aligned}
\widehat{e}+o f f \text { set } & =0.43919 \pm 0.00065(\text { stat }) \pm 0.00107(\text { syst }) \\
g_{E 1} & =-0.0061 \pm 0.0015(\text { stat }) \pm 0.00324(\text { syst }) \\
\widetilde{g_{M 1}} & =1.133 \pm 0.030(\text { stat }) \pm 0.0406(\text { syst }) \\
a_{1} / a_{2} & =-0.7503_{-0.0072}^{+0.0068}(\text { stat }) \pm 0.00893(\text { syst })
\end{aligned}
$$

Removing the offset on $\widehat{e}$, which is equal to -0.43532 , and combining errors yields:

$$
\begin{aligned}
\widehat{e} & =0.00387 \pm 0.00125 \\
g_{E 1} & =-0.0061 \pm 0.00357 \\
\widetilde{g_{M 1}} & =1.133 \pm 0.051 \\
a_{1} / a_{2} & =-0.7503 \pm 0.0113
\end{aligned}
$$

which reveals that we have measured a non-zero value for $\widehat{e}$ at $3.1 \sigma$ confidence and a non-zero value for $g_{E 1}$ at $1.7 \sigma$ confidence. Looking at the form of the entire E1 
direct emission amplitude for the $K_{L}$ as shown in Equation 3.19, we can compute the fraction of this amplitude which violates CP directly:

$$
\begin{aligned}
\frac{|16 \widehat{e}|}{\left|g_{E 1}\right|+|16 \widehat{e}|} & =\frac{16(0.00387 \pm 0.00125)}{0.0061 \pm 0.00357+16(0.00387 \pm 0.00125)} \\
& =91 \pm 4.5 \%
\end{aligned}
$$

where the correlation between $\widehat{e}$ and $g_{E 1}$ has been taken into account, assuming that the systematic errors introduce no new correlation.

These parameter values can be used to compute the value of $\epsilon_{+-\gamma}^{\prime}$ as defined in Equation E.14. The magnitude is:

$$
\begin{aligned}
\left|\epsilon_{+-\gamma}^{\prime}\right| & =(0.164 \pm 0.052) \times 10^{-3} \quad\left(E_{\gamma}^{*}>20 \mathrm{MeV}\right) \\
\arg \left(\epsilon_{+-\gamma}^{\prime}\right) & =(53.09 \pm 0.02)^{\circ}
\end{aligned}
$$

where the errors are due to the uncertainity in $\widehat{e}$ and $g_{E 1}$ only. ${ }^{1}$ Adding to this value $\eta_{+-}$yields:

$$
\begin{aligned}
\eta_{+-\gamma} & =\eta_{+-}+\epsilon_{+-\gamma}^{\prime} \\
\left|\eta_{+-\gamma}\right| & =(2.390 \pm 0.053) \times 10^{-3} \quad\left(E_{\gamma}^{*}>20 \mathrm{MeV}\right) \\
\phi_{+-} & =(44.30 \pm 1.37)^{\circ}
\end{aligned}
$$

where the $\mathrm{KTeV}$ measurement $\left|\eta_{+-}\right|=(2.228 \pm 0.010) \times 10^{-3}$ was used. For this calculation, the correlation between $\widehat{e}$ and $g_{E 1}$ has been taken into account, and is assumed to be not effected by systematic errors. The computation of $\eta_{+-\gamma}$ allows this result to be compared to older results produced by previous analyses $[26,27]$ of $K_{L, S} \rightarrow \pi^{+} \pi^{-} \gamma$.

\footnotetext{
assumption are used, the result is instead:

$$
\begin{aligned}
\left|\epsilon_{+-\gamma}^{\prime}\right| & =0.176 \times 10^{-3} \\
\arg \left(\epsilon_{+-\gamma}^{\prime}\right) & =69.31^{\circ}
\end{aligned}
$$
}

${ }^{1}$ Note that this calculation suffers from the same uncertainity regarding the evaluation of the strong interaction phase shifts as that mentioned in Section 10.2. If these two quantites are computed by evaluating $\delta_{0}$ at $M_{\pi^{+} \pi^{-}}$, and the corresponding parameter estimates coming from the same 


\subsection{Comparison with previous results}

This is the first measurement of $\widehat{e}$. However, previous experiments at Fermilab utilizing the $K_{S}$ regeneration technique $[26,27]$ have measured the related parameter $\eta_{+-\gamma}$ and its phase $\phi_{+-\gamma}$. These results, along with the computed result using our values for $\widehat{e}$ and $g_{E 1}$, can be seen in Table 11.1 Two previous KTeV analyses have attempted

\begin{tabular}{|c|c|c|}
\hline Experiment & $\eta_{+-\gamma} \times 10^{-3}$ & $\phi_{+-\gamma}$ (degrees) \\
\hline This Result & $2.390 \pm 0.053$ & $44.30 \pm 1.37$ \\
\hline E773 [27] & $2.359 \pm 0.062($ stat $) \pm 0.040($ syst $)$ & $43.8 \pm 3.5($ stat $) \pm 1.9($ syst $)$ \\
\hline E731 [26] & $2.15 \pm 0.26($ stat $) \pm 0.20($ syst $)$ & $72 \pm 23($ stat $) \pm 17($ syst $)$ \\
\hline
\end{tabular}

Table 11.1: Results of measurements of $\eta_{+-\gamma}$. Note that the E773 and E731 experiments directly measured the magnitude and phase of $\eta_{+-\gamma}$, while for this result it is computed from the estimated decay amplitudes.

to measure $g_{E 1}$. The best limit comes from $K_{L} \rightarrow \pi^{+} \pi^{-} e^{+} e^{-}$, which placed the upper limit [14, 45] of:

$$
\left\langle\left|g_{E 1}\right|\right\rangle_{E_{\gamma}^{*}}<0.03 \quad(90 \% \text { C.L. })
$$

which is compatible with this result. The other result came from the analysis of the 1997 vacuum beam sample [46] of $K_{L} \rightarrow \pi^{+} \pi^{-} \gamma$ which produced a surprisingly weak result of $\left|g_{E 1}\right|<0.21 \quad(90 \% \quad C . L$.$) Both results are consistent with the result of this$ analysis.

The NA48 experiment has recently reported [47] evidence of interference from the E1 direct emission amplitude in $K^{ \pm} \rightarrow \pi^{ \pm} \pi^{0} \gamma$. While beyond the scope of this thesis, the size of this effect can be related [48] to the similar amplitude in $K_{L, S} \rightarrow \pi^{+} \pi^{-} \gamma$.

$\mathrm{KTeV}$ has also measured $[14,46]$ the M1 direct emission parameters as shown in Table 11.2. These results also include the measurement from the NA48 experiment [49]. The results from this analysis are consistent with those from previous analyses. 


\begin{tabular}{|c|c|c|}
\hline Experiment/Data Sample & $\widetilde{g_{M 1}}$ & $a_{1} / a_{2}\left(\mathrm{GeV}^{2} / \mathrm{c}^{2}\right)$ \\
\hline This Result & $1.133 \pm 0.030($ stat $) \pm 0.0406($ syst $)$ & $-0.7503_{-0.0072}^{+0.0068}($ stat $) \pm 0.00893($ syst $)$ \\
\hline $\mathrm{KTeV}$ & $1.198 \pm 0.035($ stat $) \pm 0.086($ syst $)$ & $-0.738 \pm 0.007($ stat $) \pm 0.018($ syst $)$ \\
\hline$K_{L} \rightarrow \pi^{+} \pi^{-} \gamma[46]$ & & \\
\hline $\mathrm{KTeV}$ & $1.11 \pm 0.12($ stat $) \pm 0.08($ syst $)$ & $-0.744 \pm 0.027($ stat $) \pm 0.032($ syst $)$ \\
\hline$K_{L} \rightarrow \pi^{+} \pi^{-} e^{+} e^{-}[14]$ & $0.99_{-0.27}^{+0.28}($ stat $) \pm 0.07($ syst $)$ & $-0.81_{-0.13}^{+0.07}($ stat $) \pm 0.02($ syst $)$ \\
\hline NA48 $[49]$ & \multicolumn{2}{|c|}{} \\
\hline
\end{tabular}

Table 11.2: Results of measurements of the M1 direct emission parameters

\subsection{Final Conclusions}

This dissertation has presented a detailed study of the $K_{L, S} \rightarrow \pi^{+} \pi^{-} \gamma$ decay using data from the $\mathrm{KTeV}$ experiment. In addition to measuring the parameters describing the M1 direct emission process, this analysis has also determined the amplitude for E1 direct emission. The parameter describing the part of this process that violates $\mathrm{CP}$ indirectly was determined to be $1.7 \sigma$ away from zero, while the direct CP violating part was measured to be $3.1 \sigma$ away from zero. This result implies that $91 \pm 4.5 \%$ of the E1 direct emission process violates CP directly.

This analysis provides further proof that a well designed yet flexible experiment such as $\mathrm{KTeV}$ that provides for high precision studies and high sensitivity searches can provide unexpected and intriguing results. 


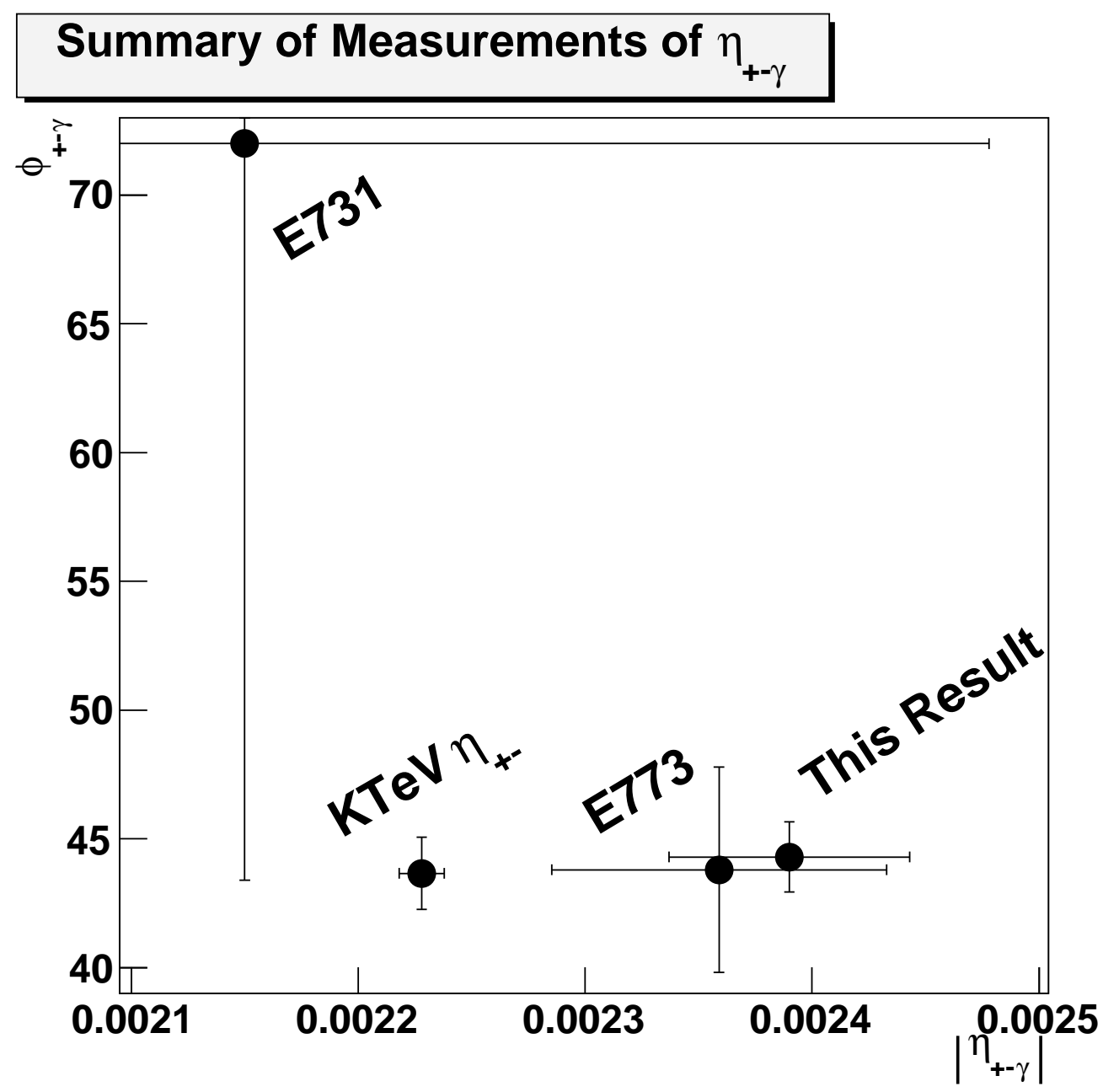

Figure 11.1: Magnitudes and phases of all measurements of $\eta_{+-\gamma}$ in addition to the $\mathrm{KTeV}$ measurement of $\eta_{+-}$ 


\section{Appendix A}

\section{Kinematic Relations}

This appendix derives a number of useful kinematic relations among the kinematic variables that are involved in the decay $K_{L, S} \rightarrow \pi^{+} \pi^{-} \gamma$.

\section{A.1 Invariant Quantities}

Some quantities give results useful in all frames. One is:

$$
\begin{aligned}
{M_{\pi^{+} \pi^{-}}}^{2} & =\left(p_{+}+p_{-}\right) \cdot\left(p_{+}+p_{-}\right) \\
& =\left(p_{+} \cdot p_{+}\right)+\left(p_{-} \cdot p_{-}\right)+2\left(p_{+} \cdot p_{-}\right) \\
& =2 M_{\pi}^{2}+2\left(p_{+} \cdot p_{-}\right)
\end{aligned}
$$

\section{A.2 Values in the kaon rest frame}

It is useful to compute some quantities in the kaon rest frame, in which the pion and gamma momenta cancel. First we shall derive a relation between $E_{\gamma}^{*}$ and $M_{\pi^{+} \pi^{-}}$

$$
\begin{aligned}
M_{K}^{2} & =p_{K} \cdot p_{K} \\
& =\left(p_{\pi \pi}+p_{\gamma}\right) \cdot\left(p_{\pi \pi}+p_{\gamma}\right) \\
& =\left(p \pi \pi \cdot p_{\pi \pi}\right)+2\left(p_{\pi \pi} \cdot p_{\gamma}\right)+\left(p_{\gamma} \cdot p_{\gamma}\right) \\
& =M_{\pi^{+} \pi^{-}}{ }^{2}+2\left(p_{\pi \pi} \cdot p_{\gamma}\right)+0
\end{aligned}
$$


In the kaon rest frame, $\overrightarrow{p_{\pi \pi}^{*}}=-\overrightarrow{p_{\gamma}^{*}}$ and $E_{K}^{*}=M_{K}=E_{\pi \pi}^{*}+E_{\gamma}^{*}$ which leads to:

$$
\begin{aligned}
M_{K}{ }^{2} & =M_{\pi^{+} \pi^{-}}{ }^{2}+2\left(\left(M_{K}-E_{\gamma}^{*}\right) E_{\gamma}^{*}\right)+\left|\overrightarrow{p_{\gamma}^{*}}\right|^{2} \\
& =M_{\pi^{+} \pi^{-}}{ }^{2}+2 E_{\gamma}^{*} M_{K}
\end{aligned}
$$

which leads to the final result:

$$
E_{\gamma}^{*}=\frac{M_{K}^{2}-M_{\pi^{+} \pi^{-}}{ }^{2}}{2 M_{K}}
$$

Alternatively, we can note that

$$
\begin{aligned}
\left(p_{K} \cdot p_{\gamma}\right) & =\left(p_{\pi \pi} \cdot p_{\gamma}\right)+\left(p_{\gamma} \cdot p_{\gamma}\right) \\
& =\left(p_{\pi \pi} \cdot p_{\gamma}\right)
\end{aligned}
$$

and then note that in the kaon rest frame, $\left(p_{K} \cdot p_{\gamma}\right)=E_{K} E_{\gamma}^{*}$, which then leads to the same result.

We can also derive the relation between the photon energy in the lab frame and in the kaon rest frame:

In the lab frame, we have:

$$
\begin{aligned}
\left(p_{K} \cdot p_{\gamma}\right) & =E_{K} E_{\gamma}^{L A B}-\left|p_{K}\right| E_{\gamma}^{L A B} \cos \psi \\
& =E_{\gamma}^{L A B}\left|p_{K}\right|\left[\sqrt{\frac{M_{K}^{2}}{\left|p_{K}\right|^{2}}+1}-\cos \psi\right]
\end{aligned}
$$

where $\psi$ is the angle between the kaon and photon momenta in the lab frame. We can then use Equations A.2 and A.4 to write

$$
\begin{aligned}
E_{\gamma}^{*} M_{K} & =E_{\gamma}^{L A B}\left|p_{K}\right|\left[\sqrt{\frac{M_{K}^{2}}{\left|p_{K}\right|^{2}}+1}-\cos \psi\right] \\
& \approx E_{\gamma}^{L A B}\left|p_{K}\right|[1+\cos \psi]
\end{aligned}
$$

leading to

$$
E_{\gamma}^{*} \approx E_{\gamma}^{L A B}\left|p_{K}\right| \frac{1-\cos \psi}{M_{K}}
$$




\section{A.3 Values in the $\pi \pi$ rest frame}

It is useful to compute some quantities in the $\pi \pi$ rest frame, where the pion momenta cancel. The first quantity is the photon energy in this frame. First, in the kaon rest frame:

$$
\left(p_{K} \cdot p_{\gamma}\right)=M_{K} E_{\gamma}^{*}
$$

while in the $\pi \pi$ rest frame:

$$
\left(p_{K} \cdot p_{\gamma}\right)=M_{\pi^{+} \pi^{-}} E_{\gamma}
$$

leading to:

$$
E_{\gamma}=\frac{M_{K}}{M_{\pi^{+} \pi^{-}}} E_{\gamma}^{*}
$$

The pion momenta are easy to determine in this frame as well:

$$
\begin{aligned}
M_{\pi^{+} \pi^{-}}{ }^{2} & =\left(p_{+}+p_{-}\right) \cdot\left(p_{+}+p_{-}\right) \\
& =\left(E_{+}+E_{-}\right)^{2}
\end{aligned}
$$

which, together with equal pion momenta ( and thus energies ) leads to:

$$
E_{+}=E_{-}=\frac{M_{\pi^{+} \pi^{-}}}{2}
$$

Now the magnitude of the pion momenta can be calculated:

$$
\left|p_{+}\right|=\left|p_{-}\right|=\sqrt{E^{2}-M^{2}}=\sqrt{\frac{M_{\pi^{+} \pi^{-}}^{2}}{4}-M_{\pi}^{2}}
$$

We can also calculate the invariant product of the pion and photon momentum:

$$
\begin{aligned}
p_{+} \cdot p_{\gamma} & =E_{+} E_{\gamma}-\overrightarrow{p_{+}} \cdot \overrightarrow{p_{\gamma}} \\
& =E_{+} E_{\gamma}-\left|p_{+}\right|\left|p_{\gamma}\right| \cos (\theta)
\end{aligned}
$$

where all quantities are in the $\pi \pi$ frame. Using the fact that in this frame $\overrightarrow{p_{+}}=-\overrightarrow{p_{-}}$:

$$
\begin{aligned}
p_{-} \cdot p_{\gamma} & =E_{-} E_{\gamma}-\overrightarrow{p_{-}} \cdot \overrightarrow{p_{\gamma}} \\
& =E_{-} E_{\gamma}+\left|p_{+}\right|\left|p_{\gamma}\right| \cos (\theta)
\end{aligned}
$$


If we use equations A.13, A.11, A.14 and the fact that $E_{\gamma}=\left|p_{\gamma}\right|$ then we get:

$$
\begin{aligned}
p_{ \pm} \cdot p_{\gamma} & =\frac{M_{K}}{2} E_{\gamma}^{*} \mp \sqrt{\frac{M_{\pi^{+} \pi^{-}}^{2}}{4}-M_{\pi}^{2}}\left(\frac{M_{K}}{M_{\pi^{+} \pi^{-}}} E_{\gamma}^{*}\right) \cos (\theta) \\
& =M_{K} E_{\gamma}^{*}\left[\frac{1}{2} \mp \sqrt{\frac{M_{\pi^{+} \pi^{-}}^{2}}{4}-M_{\pi}^{2}}\left(\frac{1}{M_{\pi^{+} \pi^{-}}}\right) \cos (\theta)\right] \\
& =M_{K} E_{\gamma}^{*}\left[\frac{1}{2} \mp \sqrt{\frac{1}{4}-\frac{M_{\pi}^{2}}{M_{\pi^{+} \pi^{-}}^{2}}} \cos (\theta)\right] \\
& =\frac{M_{K} E_{\gamma}^{*}}{2}\left[1 \mp \sqrt{1-\frac{4 M_{\pi}^{2}}{M_{\pi^{+} \pi^{-}}^{2}}} \cos (\theta)\right]
\end{aligned}
$$

\section{A.4 $\cos (\theta)$}

The kinematic variable $\cos (\theta)$, which is defined as the angle between the $\pi^{+}$and the $\gamma$ in the $\pi \pi$ rest frame, can also be expressed in terms of variables in the kaon rest frame.

Subtracting equation A.16 from A.15 results in:

$$
\left(p_{+}-p_{-}\right) \cdot p_{\gamma}=-2\left|p_{+}\right|\left|p_{\gamma}\right| \cos (\theta)
$$

However, the kaon 4-momentum $P_{K}=p_{+}+p_{-}+p_{\gamma}$ which, when evaluated in the kaon rest frame, leads to:

$$
\begin{aligned}
\left(p_{+}-p_{-}\right) \cdot p_{\gamma} & =\left(p_{+}-p_{-}\right) \cdot\left(P_{K}-\left(p_{+}+p_{-}\right)\right) \\
& =\left(E_{+}^{*}-E_{-}^{*}\right) M_{K}
\end{aligned}
$$

Equations A.18 and A.19 can then be used to obtain:

$$
\left(E_{+}^{*}-E_{-}^{*}\right) M_{K}=-2\left|p_{+}\right|\left|p_{\gamma}\right| \cos (\theta)
$$


Using the values of $p_{+}$and $E_{\gamma}$ in equations A.14 and A.11 in the above yields:

$$
\begin{aligned}
\cos (\theta) & =-\frac{\left(E_{+}^{*}-E_{-}^{*}\right) M_{K}}{2\left(\sqrt{\frac{M_{\pi^{+} \pi^{-}}^{2}}{4}-M_{\pi}^{2}}\right)\left(\frac{M_{K}}{M_{\pi^{+} \pi^{-}}} E_{\gamma}^{*}\right)} \\
& =-\frac{\left(E_{+}^{*}-E_{-}^{*}\right)}{E_{\gamma}^{*} \sqrt{1-\frac{4 M_{\pi}^{2}}{M_{\pi^{+} \pi^{-}}^{2}}}}
\end{aligned}
$$

The energy of the $\pi^{-}$can be removed by noting that $M_{K}=E_{+}^{*}+E_{-}^{*}+E_{\gamma}^{*}$ which then leads to:

$$
\begin{aligned}
\cos (\theta) & =-\frac{\left(E_{+}^{*}-M_{K}+E_{+}^{*}+E_{\gamma}^{*}\right)}{E_{\gamma}^{*} \sqrt{1-\frac{4 M_{\pi}^{2}}{M_{\pi^{+} \pi^{-}}{ }^{2}}}} \\
& =-\frac{\left(2 E_{+}^{*}-M_{K}+E_{\gamma}^{*}\right)}{E_{\gamma}^{*} \sqrt{1-\frac{4 M_{\pi}^{2}}{M_{\pi^{+} \pi^{-}}{ }^{2}}}} \\
& =-\frac{\left(2 E_{+}^{*}-M_{K}+E_{\gamma}^{*}\right)}{\beta E_{\gamma}^{*}}
\end{aligned}
$$

Solving for $E_{+}^{*}$ then yields:

$$
E_{+}^{*}=-\frac{\beta E_{\gamma}^{*} \cos (\theta)+M_{K}-E_{\gamma}^{*}}{2}
$$




\section{Appendix B}

\section{Derivation of $P_{\pi^{0}}^{2}$}

The longitudinal momentum of a $\pi^{0}$ in a $K_{L} \rightarrow \pi^{+} \pi^{-} \pi^{0}$ decay can be found by first treating $K_{L} \rightarrow \pi^{+} \pi^{-} \pi^{0}$ as a two body decay, where the $\pi^{+} \pi^{-}$pair are treated as a single particle referred to by " $\pi \pi$ ". Then, the covariant product of the kaon momentum with itself will read:

$$
\begin{aligned}
p_{K}{ }^{\mu} p_{K \mu}=M_{K}^{2} & =\left(P_{\pi \pi}+P_{\pi^{0}}\right)^{\mu}\left(P_{\pi \pi}+P_{\pi^{0}}\right)_{\mu} \\
& ={M_{\pi^{+} \pi^{-}}}^{2}+M_{\pi^{0}}^{2}+2\left(P_{\pi \pi}\right)^{\mu}\left(P_{\pi^{0}}\right)_{\mu}
\end{aligned}
$$

Now evaluate this in the frame in which the kaon momentum and $\pi \pi$ momentum are orthogonal and let

$$
\begin{aligned}
\overrightarrow{P_{\pi^{0}}} & =\overrightarrow{P_{\pi^{0}}^{L}}+\overrightarrow{P_{\pi^{0}}^{T}} \\
\overrightarrow{P_{\pi \pi}} & =\underbrace{\overrightarrow{P_{\pi \pi}^{L}}}_{=0}+\overrightarrow{P_{\pi \pi}^{T}}
\end{aligned}
$$

where $\overrightarrow{P_{\pi^{0}}^{L}}$ is parallel to the kaon momentum vector, and $\overrightarrow{P_{\pi^{0}}^{T}}$ as well as $\overrightarrow{P_{\pi \pi}^{T}}$ are orthogonal to the kaon momentum vector.

$$
\begin{aligned}
M_{K}^{2} & =M_{\pi^{+} \pi^{-}}{ }^{2}+M_{\pi^{0}}^{2}+2 E_{\pi \pi} E_{\pi^{0}}-2 \overrightarrow{P_{\pi^{0}}} \cdot \overrightarrow{P_{\pi \pi}} \\
& =M_{\pi^{+} \pi^{-}}{ }^{2}+M_{\pi^{0}}^{2}+2 E_{\pi \pi} E_{\pi^{0}}-2 \overrightarrow{P_{\pi^{0}}^{T}} \cdot \overrightarrow{P_{\pi \pi}^{T}}
\end{aligned}
$$


Nothing that $\overrightarrow{P_{\pi^{0}}^{T}}=-\overrightarrow{P_{\pi \pi}^{T}}$ leads to

$$
M_{K}{ }^{2}=M_{\pi^{+} \pi^{-}}{ }^{2}+M_{\pi^{0}}^{2}+2 E_{\pi \pi} E_{\pi^{0}}+2\left|\overrightarrow{P_{\pi \pi}^{T}}\right|^{2}
$$

now solve for the square of $E_{\pi^{0}}$

$$
\begin{aligned}
E_{\pi^{0}}^{2} & =\left(\frac{M_{K}{ }^{2}-M_{\pi^{+} \pi^{-}}{ }^{2}-M_{\pi^{0}}^{2}-2\left|\overrightarrow{P_{\pi \pi}^{T}}\right|^{2}}{2 E_{\pi \pi}}\right)^{2} \\
& =\frac{\left(M_{K}{ }^{2}-M_{\pi^{+} \pi^{-}}{ }^{2}-M_{\pi^{0}}^{2}\right)^{2}+4\left|\overrightarrow{P_{\pi \pi}^{T}}\right|^{4}-4\left|\overrightarrow{P_{\pi \pi}^{T}}\right|^{2}\left(M_{K}{ }^{2}-M_{\pi^{+} \pi^{-}}{ }^{2}-M_{\pi^{0}}^{2}\right)}{4\left(M_{\pi^{+} \pi^{-}}{ }^{2}+\left|\overrightarrow{P_{\pi \pi}^{T}}\right|^{2}\right)}
\end{aligned}
$$

however

$$
\begin{aligned}
E_{\pi^{0}}^{2} & =\sqrt{M_{\pi^{0}}^{2}+\left|\overrightarrow{P_{\pi^{0}}^{L}}\right|^{2}+\left|\overrightarrow{P_{\pi^{0}}^{T}}\right|^{2}} \\
& =\sqrt{M_{\pi^{0}}^{2}+\left|\overrightarrow{P_{\pi^{0}}^{L}}\right|^{2}+\left|\overrightarrow{P_{\pi \pi}^{T}}\right|^{2}}
\end{aligned}
$$

Solving for $\left|\overrightarrow{P_{\pi^{0}}^{L}}\right|^{2}$ then leads to:

$$
\left|\overrightarrow{P_{\pi^{0}}^{L}}\right|^{2}=\frac{\left(M_{K}^{2}-M_{\pi^{+} \pi^{-}}{ }^{2}-M_{\pi^{0}}^{2}\right)^{2}-4\left|\overrightarrow{P_{\pi \pi}^{T}}\right|^{2} M_{K}^{2}-4 M_{\pi^{+} \pi^{-}}{ }^{2} M_{\pi^{0}}^{2}}{4\left(M_{\pi^{+} \pi^{-}}{ }^{2}+\left|\overrightarrow{P_{\pi \pi}^{T}}\right|^{2}\right)}
$$




\section{Appendix C}

\section{Manipulation of $K \rightarrow \pi^{+} \pi^{-} \gamma$ Matrix Element}

\section{C.1 Squaring the Matrix Element}

In order to be used in the decay rate, the matrix element for the general decay $K \rightarrow \pi^{+} \pi^{-} \gamma$ must be squared and summed over all photon polarizations. Beginning with the matrix element as defined in equation 3.8, we separate the momentum dependent parts of the matrix element into the following:

$$
\begin{aligned}
& \Upsilon=\left(\varepsilon \cdot p_{+}\right)\left(q \cdot p_{-}\right)-\left(\varepsilon \cdot p_{-}\right)\left(q \cdot p_{+}\right) \\
& \Omega=\epsilon_{\lambda \mu \rho \sigma} \varepsilon^{\lambda} q^{\mu} p_{+}^{\rho} p_{-}^{\sigma}
\end{aligned}
$$

Then the total matrix element will have the form:

$$
\begin{aligned}
\mathcal{M} & =\sum_{\varepsilon}[A \Upsilon+B \Omega]^{\dagger}[A \Upsilon+B \Omega] \\
& =\sum_{\varepsilon}[A \Upsilon]^{\dagger}[A \Upsilon]+[B \Omega]^{\dagger}[B \Omega]+[A \Upsilon]^{\dagger}[B \Omega]+[B \Omega]^{\dagger}[A \Upsilon]
\end{aligned}
$$


notice that the last two terms are interference terms between the electric and magnetic amplitudes. Let us expand these first:

$$
\begin{aligned}
\sum_{\varepsilon} \Omega^{\dagger} \Upsilon= & \sum_{\varepsilon} \epsilon_{\lambda \mu \rho \sigma} \varepsilon^{* \lambda} q^{\mu} p_{+}^{\rho} p_{-}^{\sigma}\left[\left(\varepsilon_{\alpha} p_{+}^{\alpha}\right)\left(q \cdot p_{-}\right)-\left(\varepsilon_{\gamma} p_{-}^{\gamma}\right)\left(q \cdot p_{+}\right)\right] \\
= & \sum_{\varepsilon} \varepsilon^{* \lambda} \varepsilon_{\alpha} \epsilon_{\lambda \mu \rho \sigma} q^{\mu} p_{+}^{\rho} p_{-}^{\sigma} p_{+}^{\alpha}\left(q \cdot p_{-}\right) \\
& -\sum_{\varepsilon} \varepsilon^{* \lambda} \varepsilon_{\gamma} \epsilon_{\lambda \mu \rho \sigma} q^{\mu} p_{+}^{\rho} p_{-}^{\sigma} p_{-}^{\gamma}\left(q \cdot p_{+}\right) \\
= & -g_{\alpha}^{\lambda} \epsilon_{\lambda \mu \rho \sigma} q^{\mu} p_{+}^{\rho} p_{-}^{\sigma} p_{+}^{\alpha}\left(q \cdot p_{-}\right) \\
& +g_{\gamma}^{\lambda} \epsilon_{\lambda \mu \rho \sigma} q^{\mu} p_{+}^{\rho} p_{-}^{\sigma} p_{-}^{\gamma}\left(q \cdot p_{+}\right) \\
= & -\epsilon_{\lambda \mu \rho \sigma} q^{\mu} p_{+}^{\rho} p_{-}^{\sigma} p_{+}^{\lambda}\left(q \cdot p_{-}\right) \\
& +\epsilon_{\lambda \mu \rho \sigma} q^{\mu} p_{+}^{\rho} p_{-}^{\sigma} p_{-}^{\lambda}\left(q \cdot p_{+}\right)
\end{aligned}
$$

where the Ward Identity: [50]:

$$
\sum_{\varepsilon} \varepsilon_{\mu}^{*} \varepsilon_{\nu} \mathcal{M}^{\mu} \mathcal{M}^{\nu *}=-g_{\mu \nu} \mathcal{M}^{\mu} \mathcal{M}^{\nu *}
$$

was used to evaluate the sum. In the same fashion,

$$
\begin{aligned}
\sum_{\varepsilon} \Upsilon^{\dagger} \Omega= & \sum_{\varepsilon}\left[\left(\varepsilon_{\alpha} p_{+}^{\alpha}\right)\left(q \cdot p_{-}\right)-\left(\varepsilon_{\gamma} p_{-}^{\gamma}\right)\left(q \cdot p_{+}\right)\right]^{\dagger} \epsilon_{\lambda \mu \rho \sigma} \varepsilon^{\lambda} q^{\mu} p_{+}^{\rho} p_{-}^{\sigma} \\
= & \sum_{\varepsilon} \varepsilon_{\alpha}^{*} \varepsilon^{\lambda} \epsilon_{\lambda \mu \rho \sigma} q^{\mu} p_{+}^{\rho} p_{-}^{\sigma} p_{+}^{\alpha}\left(q \cdot p_{-}\right) \\
- & \sum_{\varepsilon} \varepsilon_{\gamma}^{*} \varepsilon^{\lambda} \epsilon_{\lambda \mu \rho \sigma} q^{\mu} p_{+}^{\rho} p_{-}^{\sigma} p_{-}^{\gamma}\left(q \cdot p_{+}\right) \\
= & -g_{\alpha}^{\lambda} \epsilon_{\lambda \mu \rho \sigma} q^{\mu} p_{+}^{\rho} p_{-}^{\sigma} p_{+}^{\alpha}\left(q \cdot p_{-}\right) \\
& +g_{\gamma}^{\lambda} \epsilon_{\lambda \mu \rho \sigma} q^{\mu} p_{+}^{\rho} p_{-}^{\sigma} p_{-}^{\gamma}\left(q \cdot p_{+}\right) \\
= & -\epsilon_{\lambda \mu \rho \sigma} q^{\mu} p_{+}^{\rho} p_{-}^{\sigma} p_{+}^{\lambda}\left(q \cdot p_{-}\right) \\
& +\epsilon_{\lambda \mu \rho \sigma} q^{\mu} p_{+}^{\rho} p_{-}^{\sigma} p_{-}^{\lambda}\left(q \cdot p_{+}\right)
\end{aligned}
$$


Which finally leads to:

$$
\begin{aligned}
\sum_{\varepsilon}\left[\Omega^{\dagger} \Upsilon+\Upsilon^{\dagger} \Omega\right] & =-2 \epsilon_{\lambda \mu \rho \sigma} q^{\mu} p_{+}^{\rho} p_{-}^{\sigma} p_{+}^{\lambda}\left(q \cdot p_{-}\right) \\
& +2 \epsilon_{\lambda \mu \rho \sigma} q^{\mu} p_{+}^{\rho} p_{-}^{\sigma} p_{-}^{\lambda}\left(q \cdot p_{+}\right)
\end{aligned}
$$

However, since the Levi-Civita tensor is anti-symmetric:

$$
\epsilon_{\lambda \mu \rho \sigma}=-\epsilon_{\rho \mu \lambda \sigma}
$$

which then implies:

$$
\epsilon_{\lambda \mu \rho \sigma} q^{\mu} p_{+}^{\rho} p_{-}^{\sigma} p_{+}^{\lambda}=-\epsilon_{\rho \mu \lambda \sigma} q^{\mu} p_{+}^{\rho} p_{-}^{\sigma} p_{+}^{\lambda}
$$

however, we could have just swapped indices : $\lambda \rightarrow \rho$ and $\rho \rightarrow \lambda$ to yield:

$$
\epsilon_{\lambda \mu \rho \sigma} q^{\mu} p_{+}^{\rho} p_{-}^{\sigma} p_{+}^{\lambda}=\epsilon_{\rho \mu \lambda \sigma} q^{\mu} p_{+}^{\lambda} p_{-}^{\sigma} p_{+}^{\rho}
$$

Equations C.8 and C.9, when used together, imply:

$$
\epsilon_{\rho \mu \lambda \sigma} q^{\mu} p_{+}^{\lambda} p_{-}^{\sigma} p_{+}^{\rho}=-\epsilon_{\rho \mu \lambda \sigma} q^{\mu} p_{+}^{\rho} p_{-}^{\sigma} p_{+}^{\lambda}
$$

which can only be true if this quantity is in fact zero. The other term has the same form, so

$$
\begin{aligned}
\sum_{\varepsilon}\left[\Omega^{\dagger} \Upsilon+\Upsilon^{\dagger} \Omega\right] & =-2 \epsilon_{\lambda \mu \rho \sigma} q^{\mu} p_{+}^{\rho} p_{-}^{\sigma} p_{+}^{\lambda}\left(q \cdot p_{-}\right) \\
& +2 \epsilon_{\lambda \mu \rho \sigma} q^{\mu} p_{+}^{\rho} p_{-}^{\sigma} p_{-}^{\lambda}\left(q \cdot p_{+}\right) \\
& =0
\end{aligned}
$$

So the interference term between the magnetic and electric amplitudes is zero, because the same vector is contracted into the Levi Civita tensor twice. The same vector appears twice because it is contracted with the metric tensor, which appears when the photon polarization is summed over. It is necessary to sum over all polarizations because this quantity is not observed in the data. This leads to the observation that the electric and magnetic terms do not interfere when the photon's polarization is not observed. This is true for all reference frames. 
Now moving on to the pure electric term:

$$
\begin{aligned}
\sum_{\varepsilon} \Upsilon^{\dagger} \Upsilon= & \sum_{\varepsilon}\left[\left(\varepsilon_{\alpha} p_{+}^{\alpha}\right)\left(q \cdot p_{-}\right)-\left(\varepsilon_{\gamma} p_{-}^{\gamma}\right)\left(q \cdot p_{+}\right)\right]^{\dagger} \\
& \times\left[\left(\varepsilon_{\beta} p_{+}^{\beta}\right)\left(q \cdot p_{-}\right)-\left(\varepsilon_{\delta} p_{-}^{\delta}\right)\left(q \cdot p_{+}\right)\right] \\
= & \sum_{\varepsilon}\left[\left(\varepsilon_{\alpha}^{*} p_{+}^{\alpha}\right)\left(q \cdot p_{-}\right)-\left(\varepsilon_{\gamma}^{*} p_{-}^{\gamma}\right)\left(q \cdot p_{+}\right)\right] \\
& \times\left[\left(\varepsilon_{\beta} p_{+}^{\beta}\right)\left(q \cdot p_{-}\right)-\left(\varepsilon_{\delta} p_{-}^{\delta}\right)\left(q \cdot p_{+}\right)\right] \\
= & \sum_{\varepsilon} \varepsilon_{\alpha}^{*} \varepsilon_{\beta} p_{+}^{\alpha} p_{+}^{\beta}\left(q \cdot p_{-}\right)^{2}+\sum_{\varepsilon} \varepsilon_{\gamma}^{*} \varepsilon_{\delta} p_{-}^{\gamma} p_{-}^{\delta}\left(q \cdot p_{+}\right)^{2} \\
& -\sum_{\varepsilon} \varepsilon_{\alpha}^{*} \varepsilon_{\delta} p_{+}^{\alpha} p_{-}^{\delta}\left(q \cdot p_{-}\right)\left(q \cdot p_{+}\right) \\
& -\sum_{\varepsilon} \varepsilon_{\gamma}^{*} \varepsilon_{\beta} p_{+}^{\beta} p_{-}^{\gamma}\left(q \cdot p_{+}\right)\left(q \cdot p_{-}\right) \\
= & -g_{\alpha \beta} p_{+}^{\alpha} p_{+}^{\beta}\left(q \cdot p_{-}\right)^{2}+-g_{\gamma \delta} p_{-}^{\gamma} p_{-}^{\delta}\left(q \cdot p_{+}\right)^{2} \\
& +g_{\alpha \delta} p_{+}^{\alpha} p_{-}^{\delta}\left(q \cdot p_{-}\right)\left(q \cdot p_{+}\right) \\
& +g_{\gamma \beta} p_{+}^{\beta} p_{-}^{\gamma}\left(q \cdot p_{+}\right)\left(q \cdot p_{-}\right) \\
= & -\left(p_{+} \cdot p_{+}\right)\left(q \cdot p_{-}\right)^{2}-\left(p_{-} \cdot p_{-}\right)\left(q \cdot p_{+}\right)^{2} \\
& +2\left(p_{+} \cdot p_{-}\right)\left(q \cdot p_{-}\right)\left(q \cdot p_{+}\right) \\
= & -M_{\pi}^{2}\left[\left(q \cdot p_{-}\right)^{2}+\left(q \cdot p_{+}\right)^{2}\right] \\
& +2\left(p_{+} \cdot p_{-}\right)\left(q \cdot p_{-}\right)\left(q \cdot p_{+}\right)
\end{aligned}
$$

Finally, moving onto the pure magnetic term:

$$
\begin{aligned}
\sum_{\varepsilon} \Omega^{\dagger} \Omega & =\sum_{\varepsilon} \epsilon_{\lambda \mu \rho \sigma} \varepsilon^{* \lambda} q^{\mu} p_{+}^{\rho} p_{-}^{\sigma} \epsilon_{\alpha \beta \gamma \delta} \varepsilon^{\alpha} q^{\beta} p_{+}^{\gamma} p_{-}^{\delta} \\
& =-g^{\lambda \alpha} \epsilon_{\lambda \mu \rho \sigma} \epsilon_{\alpha \beta \gamma \delta} q^{\mu} p_{+}^{\rho} p_{-}^{\sigma} q^{\beta} p_{+}^{\gamma} p_{-}^{\delta} \\
& =-\epsilon_{\mu \rho \sigma}^{\alpha} \epsilon_{\alpha \beta \gamma \delta} q^{\mu} p_{+}^{\rho} p_{-}^{\sigma} q^{\beta} p_{+}^{\gamma} p_{-}^{\delta}
\end{aligned}
$$

but since $q^{\mu}=g^{\mu \theta} q_{\theta}$ :

$$
\sum_{\varepsilon} \Omega^{\dagger} \Omega=-\epsilon_{\rho \sigma}^{\alpha \theta} \epsilon_{\alpha \beta \gamma \delta} q_{\theta} p_{+}^{\rho} p_{-}^{\sigma} q^{\beta} p_{+}^{\gamma} p_{-}^{\delta}
$$


and $\epsilon_{\rho \sigma}^{\alpha \theta}=g_{\rho \eta} g_{\sigma \kappa} \epsilon^{\alpha \theta \eta \kappa}$ :

$$
\sum_{\varepsilon} \Omega^{\dagger} \Omega=-g_{\rho \eta} g_{\sigma \kappa} \epsilon^{\alpha \theta \eta \kappa} \epsilon_{\alpha \beta \gamma \delta} q_{\theta} p_{+}^{\rho} p_{-}^{\sigma} q^{\beta} p_{+}^{\gamma} p_{-}^{\delta}
$$

The Levi-Civita tensors can be removed with the following identity [51]:

$$
\begin{aligned}
\epsilon^{\alpha \theta \eta \kappa} \epsilon_{\alpha \beta \gamma \delta} & =-\delta_{\beta \gamma \delta}^{\theta \eta \kappa} \\
& =-\delta_{\beta}^{\theta} \delta_{\gamma}^{\eta} \delta_{\delta}^{\kappa}+\delta_{\gamma}^{\theta} \delta_{\beta}^{\eta} \delta_{\delta}^{\kappa}+\delta_{\beta}^{\theta} \delta_{\delta}^{\eta} \delta_{\gamma}^{\kappa}+\delta_{\delta}^{\theta} \delta_{\gamma}^{\eta} \delta_{\beta}^{\kappa}-\delta_{\gamma}^{\theta} \delta_{\delta}^{\eta} \delta_{\beta}^{\kappa}-\delta_{\delta}^{\theta} \delta_{\beta}^{\eta} \delta_{\gamma}^{\kappa}
\end{aligned}
$$

which together with the fact that, for photons, $q_{\theta} q^{\theta}=0 \rightarrow \theta \neq \beta$ yields:

$$
\begin{aligned}
\sum_{\varepsilon} \Omega^{\dagger} \Omega= & g_{\rho \eta} g_{\sigma \kappa}\left[-\delta_{\gamma}^{\theta} \delta_{\beta}^{\eta} \delta_{\delta}^{\kappa}-\delta_{\delta}^{\theta} \delta_{\gamma}^{\eta} \delta_{\beta}^{\kappa}+\delta_{\gamma}^{\theta} \delta_{\delta}^{\eta} \delta_{\beta}^{\kappa}+\delta_{\delta}^{\theta} \delta_{\beta}^{\eta} \delta_{\gamma}^{\kappa}\right] q_{\theta} p_{+}^{\rho} p_{-}^{\sigma} q^{\beta} p_{+}^{\gamma} p_{-}^{\delta} \\
= & {\left[-\delta_{\gamma}^{\theta} \delta_{\beta}^{\eta} \delta_{\delta}^{\kappa}-\delta_{\delta}^{\theta} \delta_{\gamma}^{\eta} \delta_{\beta}^{\kappa}+\delta_{\gamma}^{\theta} \delta_{\delta}^{\eta} \delta_{\beta}^{\kappa}+\delta_{\delta}^{\theta} \delta_{\beta}^{\eta} \delta_{\gamma}^{\kappa}\right] q_{\theta} p_{+\eta} p_{-\kappa} q^{\beta} p_{+}^{\gamma} p_{-}^{\delta} } \\
= & {\left[-q_{\gamma} p_{+\beta} p_{-\delta} q^{\beta} p_{+}^{\gamma} p_{-}^{\delta}-q_{\delta} p_{+\gamma} p_{-\beta} q^{\beta} p_{+}^{\gamma} p_{-}^{\delta}\right.} \\
& \left.+q_{\gamma} p_{+\delta} p_{-\beta} q^{\beta} p_{+}^{\gamma} p_{-}^{\delta}+q_{\delta} p_{+\beta} p_{-\gamma} q^{\beta} p_{+}^{\gamma} p_{-}^{\delta}\right] \\
= & {\left[-\left(q \cdot p_{+}\right)\left(p_{+} \cdot q\right)\left(p_{-} \cdot p_{-}\right)-\left(q \cdot p_{-}\right)\left(p_{+} \cdot p_{+}\right)\left(p_{-} \cdot q\right)\right.} \\
& \left.+\left(q \cdot p_{+}\right)\left(p_{+} \cdot p_{-}\right)\left(p_{-} \cdot q\right)+\left(q \cdot p_{-}\right)\left(p_{+} \cdot q\right)\left(p_{-} \cdot p_{+}\right)\right] \\
= & {\left[-M_{\pi}^{2}\left(q \cdot p_{+}\right)^{2}-M_{\pi}^{2}\left(q \cdot p_{-}\right)^{2}+2\left(p_{+} \cdot p_{-}\right)\left(q \cdot p_{+}\right)\left(q \cdot p_{-}\right)\right] }
\end{aligned}
$$

Which is identical to the pure electric term! With this completed, the form of the squared matrix element, after summing over photon polarizations, is now known.

\section{C.2 Evaluation of the Momentum Terms in the Matrix Element}

The matrix element is only useful once the momentum terms have been evaluated, however since the value of the matrix element yields an invariant amplitude once it is squared and summed over spins, any reference frame may be used For convenience, we shall chose to evaluate $|\mathcal{M}|^{2}$ in the $\pi \pi$ rest frame. for which a number of useful relations are derived in Appendix A. 
First, define:

$$
\beta=\sqrt{1-\frac{4 M_{\pi}^{2}}{M_{\pi^{+} \pi^{-}}{ }^{2}}}
$$

then, according to equation A.17:

$$
\begin{aligned}
& p_{+} \cdot q=\frac{M_{K} E_{\gamma}^{*}}{2}[1-\beta \cos (\theta)] \\
& p_{-} \cdot q=\frac{M_{K} E_{\gamma}^{*}}{2}[1+\beta \cos (\theta)]
\end{aligned}
$$

and in any frame, using equation A.1:

$$
\left(p_{+} \cdot p_{-}\right)=\frac{M_{\pi^{+} \pi^{-}}{ }^{2}}{2}-M_{\pi}^{2}
$$


which leads to:

$$
\begin{aligned}
|\mathcal{M}|^{2}= & \left(\frac{e\left|f_{S}\right|}{M_{K}^{4}}\right)^{2}\left[\left|E_{I B}(K)+E_{D E}(K)\right|^{2}+\left|M_{D E}(K)\right|^{2}\right] \\
& {\left[-M_{\pi}^{2}\left(\frac{M_{K} E_{\gamma}^{*}}{2}\right)^{2}\left[2+2 \beta^{2} \cos ^{2}(\theta)\right]\right.} \\
& \left.+2\left(\frac{M_{\pi^{+} \pi^{-}}{ }^{2}}{2}-M_{\pi}{ }^{2}\right)\left(\frac{M_{K} E_{\gamma}^{*}}{2}\right)^{2}\left[1-\beta^{2} \cos ^{2}(\theta)\right]\right] \\
= & \left(\frac{e\left|f_{S}\right|}{M_{K}^{4}}\right)^{2}\left[\left|E_{I B}(K)+E_{D E}(K)\right|^{2}+\left|M_{D E}(K)\right|^{2}\right] \\
& 2\left(\frac{M_{K} E_{\gamma}^{*}}{2}\right)^{2}\left[-M_{\pi}{ }^{2}\left[1+\beta^{2} \cos ^{2}(\theta)\right]\right. \\
& \left.+\left(\frac{M_{\pi^{+} \pi^{-}}{ }^{2}}{2}-M_{\pi}{ }^{2}\right)\left[1-\beta^{2} \cos ^{2}(\theta)\right]\right] \\
= & \left(\frac{e\left|f_{S}\right|}{M_{K}^{4}}\right)^{2}\left[\left|E_{I B}(K)+E_{D E}(K)\right|^{2}+\left|M_{D E}(K)\right|^{2}\right] \\
& 2\left(\frac{M_{K} E_{\gamma}^{*}}{2}\right)^{2}\left[-2 M_{\pi}^{2}+\left(\frac{M_{\pi^{+} \pi^{-}}{ }^{2}}{2}\right)\left[1-\beta^{2} \cos ^{2}(\theta)\right]\right] \\
= & \left(\frac{e\left|f_{S}\right|}{M_{K}^{4}}\right)^{2}\left[\left|E_{I B}(K)+E_{D E}(K)\right|^{2}+\left|M_{D E}(K)\right|^{2}\right] \\
& 2\left(\frac{M_{K} E_{\gamma}^{*}}{2}\right)^{2}\left(\frac{M_{\pi^{+} \pi^{-}}{ }^{2}}{2}\right)\left[-\frac{4 M_{\pi}{ }^{2}}{M_{\pi^{+} \pi^{-}}{ }^{2}}+\left[1-\beta^{2} \cos ^{2}(\theta)\right]\right] \\
= & \left(\frac{e\left|f_{S}\right|}{M_{K}^{4}}\right)^{2}\left[\left|E_{I B}(K)+E_{D E}(K)\right|^{2}+\left|M_{D E}(K)\right|^{2}\right] \\
& \left(\frac{M_{K} E_{\gamma}^{*}}{2}\right)^{2} M_{\pi^{+} \pi^{-}}{ }^{2} \beta^{2}\left[1-\cos ^{2}(\theta)\right] \\
= & \left(\frac{e\left|f_{S}\right|}{M_{K}^{4}}\right)^{2}\left[\left|E_{I B}(K)+E_{D E}(K)\right|^{2}+\left|M_{D E}(K)\right|^{2}\right] \\
& \left(M_{\gamma}^{*}\right)^{2} M_{K}{ }^{2}\left(1-\frac{2 E_{\gamma}^{*}}{M_{K}}\right) \beta^{2} \sin ^{2}(\theta) \\
& \\
2 &
\end{aligned}
$$

Rearranging, we finally arrive at: 


$$
\begin{aligned}
|\mathcal{M}|^{2}= & \left(\frac{e^{2}\left|f_{S}\right|^{2}}{4 M_{K}^{4}}\right)\left(1-\frac{2 E_{\gamma}^{*}}{M_{K}}\right) E_{\gamma}^{* 2} \beta^{2} \sin ^{2}(\theta) \\
& {\left[\left|E_{I B}(K)+E_{D E}(K)\right|^{2}+\left|M_{D E}(K)\right|^{2}\right] }
\end{aligned}
$$




\section{Appendix D}

\section{Expansion of Decay Rate}

While equation 3.40 gives the triple differential decay rate in compact form, it can be informative to expand out the amplitudes.

Let us begin with the $K_{L}$ :

$$
\begin{aligned}
& \left|E_{I B}\left(K_{L}\right)+E_{D E}\left(K_{L}\right)\right|^{2}+\left|M_{D E}\left(K_{L}\right)\right|^{2}=\left|E_{I B}\left(K_{L}\right)\right|^{2}+\left|E_{D E}\left(K_{L}\right)\right|^{2} \\
& +E_{I B}{ }^{\dagger}\left(K_{L}\right) E_{D E}\left(K_{L}\right)+E_{I B}\left(K_{L}\right) E_{D E}^{\dagger}\left(K_{L}\right) \\
& +\left|M_{D E}\left(K_{L}\right)\right|^{2}
\end{aligned}
$$


Plugging in the amplitudes:

$$
\begin{aligned}
& \left|E_{I B}\left(K_{L}\right)+E_{D E}\left(K_{L}\right)\right|^{2}+\left|M_{D E}\left(K_{L}\right)\right|^{2}= \\
& \left(16 \frac{M_{K}^{4}}{E_{\gamma}^{* 4}}\right) \frac{\left|\eta_{+-}\right|^{2}}{\left(1-\beta^{2} \cos ^{2}(\theta)\right)^{2}}+g_{E 1}^{2}+256 \widehat{e}^{2}+16 \widehat{e} g_{E 1}\left(i e^{-i \phi_{\varepsilon}}-i e^{i \phi_{\varepsilon}}\right) \\
& +\left(4 \frac{M_{K}^{2}}{E_{\gamma}^{* 2}}\right) \frac{\left|\eta_{+-}\right|}{1-\beta^{2} \cos ^{2}(\theta)} \\
& \times\left[\left(g_{E 1} e^{i\left(\delta_{1}+\phi_{\varepsilon}-\phi_{+-}-\delta_{0}\right)}+i 16 \widehat{e} e^{i\left(\delta_{1}-\phi_{+-}-\delta_{0}\right)}\right)\right. \\
& \left.+\left(g_{E 1} e^{-i\left(\delta_{1}+\phi_{\varepsilon}-\phi_{+-}-\delta_{0}\right)}-i 16 \widehat{e} e^{-i\left(\delta_{1}-\phi_{+-}-\delta_{0}\right)}\right)\right] \\
& +{\widetilde{g_{M 1}}}^{2}\left(\frac{a_{1} / a_{2}}{M_{\rho}^{2}-M_{K}{ }^{2}+2 E_{\gamma}^{*} M_{K}}+1\right)^{2} \\
& =\left(16 \frac{M_{K}^{4}}{E_{\gamma}^{* 4}}\right) \frac{\left|\eta_{+-}\right|^{2}}{\left(1-\beta^{2} \cos ^{2}(\theta)\right)^{2}}+g_{E 1}^{2}+256 \widehat{e}^{2}+32 \widehat{e} g_{E 1} \sin \phi_{\varepsilon} \\
& +\left(4 \frac{M_{K}^{2}}{E_{\gamma}^{* 2}}\right) \frac{\left|\eta_{+-}\right|}{1-\beta^{2} \cos ^{2}(\theta)} \\
& \times\left[2 g_{E 1} \cos \left(\delta_{1}+\phi_{\varepsilon}-\phi_{+-}-\delta_{0}\right)-32 \widehat{e} \sin \left(\delta_{1}-\phi_{+-}-\delta_{0}\right)\right] \\
& +{\widetilde{g_{M 1}}}^{2}\left(\frac{a_{1} / a_{2}}{M_{\rho}{ }^{2}-M_{K}{ }^{2}+2 E_{\gamma}^{*} M_{K}}+1\right)^{2}
\end{aligned}
$$

Rearranging the terms into order of importance and dropping pure E1 DE terms finally gives:

$$
\begin{aligned}
& \left|E_{I B}\left(K_{L}\right)+E_{D E}\left(K_{L}\right)\right|^{2}+\left|M_{D E}\left(K_{L}\right)\right|^{2} \\
& =\left(16 \frac{M_{K}^{4}}{E_{\gamma}^{* 4}}\right) \frac{\left|\eta_{+-}\right|^{2}}{\left(1-\beta^{2} \cos ^{2}(\theta)\right)^{2}}+{\widetilde{g_{M 1}}}^{2}\left(\frac{a_{1} / a_{2}}{M_{\rho}{ }^{2}-M_{K}{ }^{2}+2 E_{\gamma}^{*} M_{K}}+1\right)^{2} \\
& +\left(4 \frac{M_{K}^{2}}{E_{\gamma}^{* 2}}\right) \frac{\left|\eta_{+-}\right|}{1-\beta^{2} \cos ^{2}(\theta)} \\
& \times\left[2 g_{E 1} \cos \left(\delta_{1}+\phi_{\varepsilon}-\phi_{+-}-\delta_{0}\right)-32 \widehat{e} \sin \left(\delta_{1}-\phi_{+-}-\delta_{0}\right)\right]
\end{aligned}
$$


Moving on to the $K_{S}$ :

$$
\begin{aligned}
& \left|E_{I B}\left(K_{S}\right)+E_{D E}\left(K_{S}\right)\right|^{2}+\left|M_{D E}\left(K_{S}\right)\right|^{2} \\
& =\left(16 \frac{M_{K}^{4}}{E_{\gamma}^{* 4}}\right) \frac{1}{\left(1-\beta^{2} \cos ^{2}(\theta)\right)^{2}}+\frac{g_{E 1}{ }^{2}}{|\epsilon|^{2}} \\
& +\left(4 \frac{M_{K}^{2}}{E_{\gamma}^{* 2}}\right) \frac{g_{E 1} /|\epsilon|}{1-\beta^{2} \cos ^{2}(\theta)}\left[e^{i\left(\delta_{1}-\delta_{0}\right)}+e^{\left.-i\left(\delta_{1}-\delta_{0}\right)\right]}\right. \\
& +\epsilon^{2}{\widetilde{g_{M 1}}}^{2}\left(\frac{a_{1} / a_{2}}{M_{\rho}{ }^{2}-M_{K}{ }^{2}+2 E_{\gamma}^{*} M_{K}}+1\right)^{2} \\
& =\left(16 \frac{M_{K}^{4}}{E_{\gamma}^{* 4}}\right) \frac{1}{\left(1-\beta^{2} \cos ^{2}(\theta)\right)^{2}}+\frac{g_{E 1}{ }^{2}}{|\epsilon|^{2}} \\
& +\left(4 \frac{M_{K}^{2}}{E_{\gamma}^{* 2}}\right) \frac{g_{E 1} /|\epsilon|}{1-\beta^{2} \cos ^{2}(\theta)} 2 \cos \left(\delta_{1}-\delta_{0}\right) \\
& +\epsilon^{2}{\widetilde{g_{M 1}}}^{2}\left(\frac{a_{1} / a_{2}}{M_{\rho}^{2}-M_{K}{ }^{2}+2 E_{\gamma}^{*} M_{K}}+1\right)^{2}
\end{aligned}
$$

Dropping pure DE terms results in:

$$
\begin{aligned}
& \left|E_{I B}\left(K_{S}\right)+E_{D E}\left(K_{S}\right)\right|^{2}+\left|M_{D E}\left(K_{S}\right)\right|^{2} \\
& =\left(16 \frac{M_{K}^{4}}{E_{\gamma}^{* 4}}\right) \frac{1}{\left(1-\beta^{2} \cos ^{2}(\theta)\right)^{2}}+\left(4 \frac{M_{K}^{2}}{E_{\gamma}^{* 2}}\right) \frac{g_{E 1} /|\epsilon|}{1-\beta^{2} \cos ^{2}(\theta)} 2 \cos \left(\delta_{1}-\delta_{0}\right)
\end{aligned}
$$

Finally moving onto the $K_{L, S}$ interference term:

$$
\begin{aligned}
& {\left[E_{I B}\left(K_{L}\right)+E_{D E}\left(K_{L}\right)\right]^{\dagger}\left[E_{I B}\left(K_{S}\right)+E_{D E}\left(K_{S}\right)\right]+\left[M_{D E}\left(K_{L}\right)\right]^{\dagger}\left[M_{D E}\left(K_{S}\right)\right]} \\
& =\left[E_{I B}\left(K_{L}\right)\right]^{\dagger}\left[E_{I B}\left(K_{S}\right)\right]+\left[E_{D E}\left(K_{L}\right)\right]^{\dagger}\left[E_{D E}\left(K_{S}\right)\right] \\
& +\left[E_{I B}\left(K_{L}\right)\right]^{\dagger}\left[E_{D E}\left(K_{S}\right)\right]+\left[E_{D E}\left(K_{L}\right)\right]^{\dagger}\left[E_{I B}\left(K_{S}\right)\right]+\epsilon\left|M_{D E}\left(K_{L}\right)\right|^{2} \\
& =\eta_{+-}^{\dagger}\left|E_{I B}\left(K_{S}\right)\right|^{2}+\left[E_{D E}\left(K_{L}\right)\right]^{\dagger}\left[E_{D E}\left(K_{S}\right)\right] \\
& +\left[E_{I B}\left(K_{L}\right)\right]^{\dagger}\left[E_{D E}\left(K_{S}\right)\right]+\left[E_{D E}\left(K_{L}\right)\right]^{\dagger}\left[E_{I B}\left(K_{S}\right)\right]+\epsilon\left|M_{D E}\left(K_{L}\right)\right|^{2}
\end{aligned}
$$


Plugging in the amplitudes results in:

$$
\begin{gathered}
\eta_{+-}{ }^{\dagger}\left|E_{I B}\left(K_{S}\right)\right|^{2}=\left(16 \frac{M_{K}{ }^{4}}{E_{\gamma}^{* 4}}\right) \frac{\left|\eta_{+-}\right| e^{-i \phi_{+-}}}{\left(1-\beta^{2} \cos ^{2}(\theta)\right)^{2}} \\
\epsilon\left|M_{D E}\left(K_{L}\right)\right|^{2}=|\epsilon| e^{i \phi_{\varepsilon}}{\widetilde{g_{M 1}}}^{2}\left(\frac{a_{1} / a_{2}}{M_{\rho}{ }^{2}-M_{K}{ }^{2}+2 E_{\gamma}^{*} M_{K}}+1\right)^{2} \\
{\left[E_{I B}\left(K_{L}\right)\right]^{\dagger}\left[E_{D E}\left(K_{S}\right)\right]=\left(4 \frac{M_{K}{ }^{2}}{E_{\gamma}^{* 2}}\right) \frac{\left|\eta_{+-}\right| e^{-i\left(\delta_{0}+\phi_{+-}\right)}}{1-\beta^{2} \cos ^{2}(\theta)} \frac{g_{E 1}}{\epsilon} e^{i\left(\delta_{1}+\phi \epsilon\right)}} \\
{\left[E_{D E}\left(K_{L}\right)\right]^{\dagger}\left[E_{I B}\left(K_{S}\right)\right]=\left(4 \frac{M_{K}{ }^{2}}{E_{\gamma}^{* 2}}\right) \frac{g_{E 1} e^{i\left(\delta_{0}-\delta_{1}-\phi_{\varepsilon}\right)}-i 16 \widehat{e} e^{i\left(\delta_{0}-\delta_{1}\right)}}{1-\beta^{2} \cos ^{2}(\theta)}} \\
{\left[E_{D E}\left(K_{L}\right)\right]^{\dagger}\left[E_{D E}\left(K_{S}\right)\right]} \\
=\left(g_{E 1} e^{-i\left(\delta_{1}+\phi_{\varepsilon}\right)}-i 16 \widehat{e} e^{-i \delta_{1}}\right) \frac{g_{E 1}}{\epsilon} e^{i\left(\delta_{1}+\phi_{\epsilon}\right)} \\
=\left(\frac{g_{E 1}{ }^{2}}{\epsilon}-i \frac{16 \widehat{e} g_{E 1}}{\epsilon} e^{i \phi_{\varepsilon}}\right)
\end{gathered}
$$

putting everything together:

$$
\begin{aligned}
& {\left[E_{I B}\left(K_{L}\right)+E_{D E}\left(K_{L}\right)\right]^{\dagger}\left[E_{I B}\left(K_{S}\right)+E_{D E}\left(K_{S}\right)\right]+\left[M_{D E}\left(K_{L}\right)\right]^{\dagger}\left[M_{D E}\left(K_{S}\right)\right]} \\
& =\left(16 \frac{M_{K}^{4}}{E_{\gamma}^{* 4}}\right) \frac{\left|\eta_{+-}\right| e^{-i \phi_{+-}}}{\left(1-\beta^{2} \cos ^{2}(\theta)\right)^{2}} \\
& +\left(4 \frac{M_{K}^{2}}{E_{\gamma}^{* 2}}\right) \frac{g_{E 1} e^{i\left(\delta_{0}-\delta_{1}-\phi_{\varepsilon}\right)}-i 16 \widehat{e} e^{i\left(\delta_{0}-\delta_{1}\right)}+\frac{\left|\eta_{+-}\right|}{\epsilon} g_{E 1} e^{-i\left(\delta_{0}-\delta_{1}+\phi_{+--\phi_{\varepsilon}}\right)}}{1-\beta^{2} \cos ^{2}(\theta)} \\
& +\left(\frac{g_{E 1}{ }^{2}}{\epsilon}-i \frac{16 \widehat{e} g_{E 1}}{\epsilon} e^{i \phi_{\varepsilon}}\right)+|\epsilon| e^{i \phi_{\varepsilon}}{\widetilde{g_{M 1}}}^{2}\left(\frac{a_{1} / a_{2}}{M_{\rho}{ }^{2}-M_{K}{ }^{2}+2 E_{\gamma}^{*} M_{K}}+1\right)^{2} \\
& \approx\left(16 \frac{M_{K}{ }^{4}}{E_{\gamma}^{* 4}}\right) \frac{\left|\eta_{+-}\right| e^{-i \phi_{+-}}}{\left(1-\beta^{2} \cos ^{2}(\theta)\right)^{2}} \\
& +\left(4 \frac{M_{K}^{2}}{E_{\gamma}^{* 2}}\right) \frac{g_{E 1} 2 \cos \left(\delta_{0}-\delta_{1}-\phi_{\varepsilon}\right)-i 16 \widehat{e} e^{i\left(\delta_{0}-\delta_{1}\right)}+\frac{\left|\epsilon^{\prime}\right|}{|\epsilon|} g_{E 1} e^{-i\left(\delta_{0}-\delta_{1}-\phi_{\varepsilon}\right)}}{1-\beta^{2} \cos ^{2}(\theta)} \\
& +\left(\frac{g_{E 1}{ }^{2}}{\epsilon}-i \frac{16 \widehat{e} g_{E 1}}{\epsilon} e^{i \phi_{\varepsilon}}\right)+|\epsilon| e^{i \phi_{\varepsilon}}{\widetilde{g_{M 1}}}^{2}\left(\frac{a_{1} / a_{2}}{M_{\rho}^{2}-M_{K}{ }^{2}+2 E_{\gamma}^{*} M_{K}}+1\right)^{2}
\end{aligned}
$$


dropping pure DE terms, which are small, results in:

$$
\begin{aligned}
& {\left[E_{I B}\left(K_{L}\right)+E_{D E}\left(K_{L}\right)\right]^{\dagger}\left[E_{I B}\left(K_{S}\right)+E_{D E}\left(K_{S}\right)\right]+\left[M_{D E}\left(K_{L}\right)\right]^{\dagger}\left[M_{D E}\left(K_{S}\right)\right]} \\
& \approx\left(16 \frac{M_{K}^{4}}{E_{\gamma}^{* 4}}\right) \frac{\left|\eta_{+-}\right| e^{-i \phi_{+-}}}{\left(1-\beta^{2} \cos ^{2}(\theta)\right)^{2}} \\
& +\left(4 \frac{M_{K}^{2}}{E_{\gamma}^{* 2}}\right) \frac{g_{E 1} 2 \cos \left(\delta_{0}-\delta_{1}-\phi_{\varepsilon}\right)-i 16 \widehat{e} e^{i\left(\delta_{0}-\delta_{1}\right)}+\frac{\left|\epsilon^{\prime}\right|}{|\epsilon|} g_{E 1} e^{-i\left(\delta_{0}-\delta_{1}-\phi_{\varepsilon}\right)}}{1-\beta^{2} \cos ^{2}(\theta)}
\end{aligned}
$$

Repeating the other results:

$$
\begin{aligned}
& \left|E_{I B}\left(K_{S}\right)+E_{D E}\left(K_{S}\right)\right|^{2}+\left|M_{D E}\left(K_{S}\right)\right|^{2} \\
& =\left(16 \frac{M_{K}^{4}}{E_{\gamma}^{* 4}}\right) \frac{1}{\left(1-\beta^{2} \cos ^{2}(\theta)\right)^{2}}+\left(4 \frac{M_{K}^{2}}{E_{\gamma}^{* 2}}\right) \frac{g_{E 1} /|\epsilon|}{1-\beta^{2} \cos ^{2}(\theta)} 2 \cos \left(\delta_{1}-\delta_{0}\right)
\end{aligned}
$$

and

$$
\begin{aligned}
& \left|E_{I B}\left(K_{L}\right)+E_{D E}\left(K_{L}\right)\right|^{2}+\left|M_{D E}\left(K_{L}\right)\right|^{2} \\
& =\left(16 \frac{M_{K}^{4}}{E_{\gamma}^{* 4}}\right) \frac{\left|\eta_{+-}\right|^{2}}{\left(1-\beta^{2} \cos ^{2}(\theta)\right)^{2}}+{\widetilde{g_{M 1}}}^{2}\left(\frac{a_{1} / a_{2}}{M_{\rho}^{2}-M_{K}{ }^{2}+2 E_{\gamma}^{*} M_{K}}+1\right)^{2} \\
& +\left(4 \frac{M_{K}^{2}}{E_{\gamma}^{* 2}}\right) \frac{\left|\eta_{+-}\right|}{1-\beta^{2} \cos ^{2}(\theta)} \\
& \times\left[2 g_{E 1} \cos \left(\delta_{1}+\phi_{\varepsilon}-\phi_{+-}-\delta_{0}\right)-32 \widehat{e} \sin \left(\delta_{1}-\phi_{+-}-\delta_{0}\right)\right]
\end{aligned}
$$

\section{D.1 Numerical Estimates}

By computing the phases of the terms in the above expressions, some information can be gleaned about the size of the angular terms, and their sign which will indicate if constructive or destructive interference occurs.

While the strong interaction phase shifts are energy dependent, their averages can be computed. Usually $\delta_{0}$ is evaluated at the kaon mass, which will result in energy independent angle of $39^{\circ}$. If it is evaluated at $M_{\pi^{+} \pi^{-}}$, the average value will be $23^{\circ}$. The average value of $\delta_{1}$ is $2.3^{\circ}$. The current PDG [22] value of $\phi_{+-}$is $\approx 43.5^{\circ}$ which is consistent with the value of $\phi_{\varepsilon}$. 
Using the above information, and assuming $\delta_{0}$ is energy independent we can compute:

$$
\begin{gathered}
\cos \left(\delta_{1}-\delta_{0}\right) \approx \cos \left(\delta_{1}+\phi_{\varepsilon}-\phi_{+-}-\delta_{0}\right) \approx 0.8 \\
\sin \left(\delta_{1}-\phi_{+-}-\delta_{0}\right) \approx-1.0 \\
\cos \left(\delta_{0}-\delta_{1}-\phi_{\varepsilon}\right) \approx 1.0
\end{gathered}
$$

also

$$
\begin{gathered}
\delta_{0}-\delta_{1} \approx 36.7^{\circ} \\
\delta_{0}-\delta_{1}-\frac{\pi}{2} \approx-53.3^{\circ} \\
\delta_{0}-\delta_{1}-\phi_{\varepsilon} \approx-6.8^{\circ}
\end{gathered}
$$

plugging these all into equations D.13, D.14 and D.15 results in:

$$
\begin{aligned}
& {\left[E_{I B}\left(K_{L}\right)+E_{D E}\left(K_{L}\right)\right]^{\dagger}\left[E_{I B}\left(K_{S}\right)+E_{D E}\left(K_{S}\right)\right]+\left[M_{D E}\left(K_{L}\right)\right]^{\dagger}\left[M_{D E}\left(K_{S}\right)\right]} \\
& \approx\left(16 \frac{M_{K}^{4}}{E_{\gamma}^{* 4}}\right) \frac{\left|\eta_{+-}\right| e^{-i\left(43.5^{\circ}\right)}}{\left(1-\beta^{2} \cos ^{2}(\theta)\right)^{2}} \\
& +\left(4 \frac{M_{K}^{2}}{E_{\gamma}^{* 2}}\right) \frac{2 g_{E 1}+16 \widehat{e} e^{i\left(-53.3^{\circ}\right)}+\frac{\left|\epsilon^{\prime}\right|}{|\epsilon|} g_{E 1} e^{-i\left(-6.8^{\circ}\right)}}{1-\beta^{2} \cos ^{2}(\theta)} \\
& \left|E_{I B}\left(K_{S}\right)+E_{D E}\left(K_{S}\right)\right|^{2}+\left|M_{D E}\left(K_{S}\right)\right|^{2} \\
& =\left(16 \frac{M_{K}^{4}}{E_{\gamma}^{* 4}}\right) \frac{1}{\left(1-\beta^{2} \cos ^{2}(\theta)\right)^{2}}+\left(4 \frac{M_{K}^{2}}{E_{\gamma}^{* 2}}\right) \frac{1.6 g_{E 1} /|\epsilon|}{1-\beta^{2} \cos ^{2}(\theta)}
\end{aligned}
$$


and

$$
\begin{aligned}
& \left|E_{I B}\left(K_{L}\right)+E_{D E}\left(K_{L}\right)\right|^{2}+\left|M_{D E}\left(K_{L}\right)\right|^{2} \\
& =\left(16 \frac{M_{K}^{4}}{E_{\gamma}^{* 4}}\right) \frac{\left|\eta_{+-}\right|^{2}}{\left(1-\beta^{2} \cos ^{2}(\theta)\right)^{2}}+{\widetilde{g_{M 1}}}^{2}\left(\frac{a_{1} / a_{2}}{M_{\rho}^{2}-M_{K}{ }^{2}+2 E_{\gamma}^{*} M_{K}}+1\right)^{2} \\
& +\left(4 \frac{M_{K}^{2}}{E_{\gamma}^{* 2}}\right) \frac{\left|\eta_{+-}\right|}{1-\beta^{2} \cos ^{2}(\theta)} \\
& \times\left[1.6 g_{E 1}+32 \widehat{e}\right]
\end{aligned}
$$

Equation D.22 indicates that the presence of the direct CP violating E1 term will not shift the phase of the interference term in the decay distribution, while the presence of the indirect $\mathrm{CP}$ violating $\mathrm{E} 1$ term will. Additionally, the indirect and direct $\mathrm{CP}$ violating processes both interfere constructively with the inner bremsstrahlung process, as shown in equations D.23 and D.24 . 


\section{Appendix E}

\section{The "average" decay rate for $K_{L, S} \rightarrow \pi^{+} \pi^{-} \gamma$}

While equation 3.40 serves as excellent prediction function with which to compare to data, in the past $[12,27]$ only the decay rate as a function of proper time was used. This can be thought of as the decay rate as given in equation 3.40 averaged over $E_{\gamma}^{*}$ and $\cos (\theta)$. Doing so results in the measurement of a set of parameters different than that of Section 3.5. In order to compare the results of the present study to past studies, we derive the average decay rate. For this derivation, I follow the procedure of [20]. We begin by integrating Equation 3.40 with respect $E_{\gamma}^{*}$ and $\cos (\theta)$. Doing so yields:

$$
\begin{aligned}
\Gamma= & |A|^{2}\left[\Gamma_{K_{L}} e^{-\left(\frac{1}{\tau_{L}}\right) \frac{M_{K} \Delta z}{p_{K}}}\right. \\
& +\left|\rho\left(p_{K}\right)\right|^{2} \Gamma_{K_{S}} e^{-\left(\frac{1}{\tau_{S}}\right) \frac{M_{K} \Delta z}{p_{K}}} \\
& \left.+2 \operatorname{Re}\left[\rho\left(p_{K}\right) \gamma_{L S} e^{i \Delta M_{K} \frac{M_{K} \Delta z}{p_{K}}}\right] e^{-\left(\frac{1}{\tau_{S}}+\frac{1}{\tau_{L}}\right) \frac{1}{2} \frac{M_{K} \Delta z}{p_{K}}}\right]
\end{aligned}
$$

where $\Gamma_{K_{S}} \rightarrow \pi^{+} \pi^{-} \gamma$ and $\Gamma_{K_{L}} \rightarrow \pi^{+} \pi^{-} \gamma$ are the partial widths of this decay for the $K_{S}$ and $K_{L}$ respectively and 


$$
\begin{aligned}
\gamma_{L S}= & \iint d E_{\gamma}^{*} d \cos (\theta)\left[\frac{e^{2}\left|f_{s}\right|^{2}}{M_{K}{ }^{2}}\left(\frac{\beta E_{\gamma}^{*}}{8 \pi M_{K}}\right)^{3}\left(1-\frac{2 E_{\gamma}^{*}}{M_{K}}\right) \sin ^{2} \theta\right. \\
& \times\left(\left[E_{I B}\left(K_{L}\right)+E_{D E}\left(K_{L}\right)\right]^{\dagger}\left[E_{I B}\left(K_{S}\right)+E_{D E}\left(K_{S}\right)\right]\right. \\
& \left.\left.+M_{D E}^{\dagger}\left(K_{L}\right) M_{D E}\left(K_{S}\right)\right)\right]
\end{aligned}
$$

is the interference term. However, we can manipulate this equation by breaking up the partial width of the $K_{L}$ in this fashion:

$$
\Gamma_{K_{L} \rightarrow \pi^{+} \pi^{-} \gamma}=\Gamma_{K_{L}}^{E 1} \rightarrow \pi^{+} \pi^{-} \gamma+\Gamma_{K_{L}}^{M 1} \rightarrow \pi^{+} \pi^{-} \gamma
$$

where

$\Gamma^{E 1}$ is the part of the partial width due to inner bremsstrahlung and E1 direct emission.

$\Gamma^{M 1}$ is the part of the partial width due to M1 direct emission only.

Now make the following definition:

$$
r=\frac{\Gamma_{K_{L}}^{M 1} \rightarrow \pi^{+} \pi^{-} \gamma}{\Gamma_{K_{L}}^{E 1} \rightarrow \pi^{+} \pi^{-} \gamma}
$$

in order to yield:

$$
\begin{aligned}
\Gamma_{K_{L} \rightarrow \pi^{+} \pi^{-} \gamma} & =\Gamma_{K_{L} \rightarrow \pi^{+} \pi^{-} \gamma}^{E 1}+r \Gamma_{K_{L}}^{E 1} \rightarrow \pi^{+} \pi^{-} \gamma \\
& =(1+r) \Gamma_{K_{L}}^{E 1} \rightarrow \pi^{+} \pi^{-} \gamma
\end{aligned}
$$

Now we can make the following definition:

$$
\widetilde{\eta_{+-\gamma}}=\frac{E_{I B}\left(K_{L}\right)+E_{D E}\left(K_{L}\right)}{E_{I B}\left(K_{S}\right)+E_{D E}\left(K_{S}\right)}
$$


however $E_{D E}$ is very small compared to $E_{I B}$, so making use of a power series expansion:

$$
\begin{aligned}
\frac{1}{E_{I B}\left(K_{S}\right)+E_{D E}\left(K_{S}\right)} & =\frac{1}{E_{I B}\left(K_{S}\right)}\left[\frac{1}{1+\frac{E_{D E}\left(K_{S}\right)}{E_{I B}\left(K_{S}\right)}}\right] \\
& \approx \frac{1}{E_{I B}\left(K_{S}\right)}\left[1-\frac{E_{D E}\left(K_{S}\right)}{E_{I B}\left(K_{S}\right)}+\left(\frac{E_{D E}\left(K_{S}\right)}{E_{I B}\left(K_{S}\right)}\right)^{2}+\ldots\right]
\end{aligned}
$$

which then leads to:

$$
\begin{aligned}
\widetilde{\eta_{+-\gamma}} & \approx \frac{E_{I B}\left(K_{L}\right)+E_{D E}\left(K_{L}\right)}{E_{I B}\left(K_{S}\right)}\left[1-\frac{E_{D E}\left(K_{S}\right)}{E_{I B}\left(K_{S}\right)}+\left(\frac{E_{D E}\left(K_{S}\right)}{E_{I B}\left(K_{S}\right)}\right)^{2}+\ldots\right] \\
& \approx\left[\frac{E_{I B}\left(K_{L}\right)}{E_{I B}\left(K_{S}\right)}+\frac{E_{D E}\left(K_{L}\right)}{E_{I B}\left(K_{S}\right)}\right]\left[1-\frac{E_{D E}\left(K_{S}\right)}{E_{I B}\left(K_{S}\right)}+\left(\frac{E_{D E}\left(K_{S}\right)}{E_{I B}\left(K_{S}\right)}\right)^{2}+\ldots\right] \\
& \approx \frac{E_{I B}\left(K_{L}\right)}{E_{I B}\left(K_{S}\right)}+\frac{E_{D E}\left(K_{L}\right)}{E_{I B}\left(K_{S}\right)}-\frac{E_{I B}\left(K_{L}\right)}{E_{I B}\left(K_{S}\right)} \frac{E_{D E}\left(K_{S}\right)}{E_{I B}\left(K_{S}\right)}
\end{aligned}
$$

Then using the definition of the $\mathrm{CP}$ violating parameter for $K_{L} \rightarrow \pi^{+} \pi^{-}$decays:

$$
\eta_{+-}=\frac{\left.A\left(K_{L} \rightarrow \pi^{+} \pi^{-}\right)\right)}{\left.A\left(K_{S} \rightarrow \pi^{+} \pi^{-}\right)\right)}=\frac{E_{I B}\left(K_{L} \rightarrow \pi^{+} \pi^{-} \gamma\right)}{E_{I B}\left(K_{S} \rightarrow \pi^{+} \pi^{-} \gamma\right)}=\epsilon+\epsilon^{\prime}
$$

and using the amplitudes for $K_{L, S} \rightarrow \pi^{+} \pi^{-} \gamma$ as written in section 3.4.1 we then yield:

$$
\begin{aligned}
\widetilde{\eta_{+-\gamma}} & \approx \eta_{+-}+\frac{E_{D E}\left(K_{L}\right)}{E_{I B}\left(K_{S}\right)}-\eta_{+-} \frac{E_{D E}\left(K_{S}\right)}{E_{I B}\left(K_{S}\right)} \\
& \approx \eta_{+-}+\frac{\left.g_{E 1} e^{i\left(\delta_{1}+\phi_{\varepsilon}\right.}\right)+i 16 \widehat{e} e^{i \delta_{1}}-\eta_{+-} \frac{g_{E 1}}{\epsilon} e^{i\left(\delta_{1}+\phi \epsilon\right)}}{\left(4 \frac{M_{K}^{2}}{E_{\gamma}^{* 2}}\right) \frac{e^{i \delta_{0}}}{1-\beta^{2} \cos ^{2}(\theta)}} \\
& \approx \eta_{+-}+\left[\widehat{e}+\frac{i}{16} \frac{\epsilon^{\prime}}{\epsilon} g_{E 1} e^{i \phi_{\epsilon}}\right] e^{i\left(\delta_{1}-\delta_{0+\frac{\pi}{2}}\right)}\left(\frac{2 E_{\gamma}^{*}}{M_{K}}\right)^{2}\left(1-\beta^{2} \cos ^{2}(\theta)\right)
\end{aligned}
$$


Neglecting the term $\propto g_{E 1}{ }^{1}$ we can define:

$$
\widetilde{\epsilon_{+-\gamma}^{\prime}}=\widehat{e} e^{i\left(\delta_{1}-\delta_{0}+\frac{\pi}{2}\right)}\left(\frac{2 E_{\gamma}^{*}}{M_{K}}\right)^{2}\left(1-\beta^{2} \cos ^{2}(\theta)\right)
$$

which then leads to:

$$
\widetilde{\eta_{+-\gamma}}=\eta_{+-}+\widetilde{\epsilon_{+-\gamma}^{\prime}}
$$

Finally, we can express $\Gamma_{K_{L}}^{E 1} \rightarrow \pi^{+} \pi^{-} \gamma$ as:

$$
\begin{aligned}
\Gamma_{K_{L}}^{E 1} \rightarrow \pi^{+} \pi^{-} \gamma= & \iint d E_{\gamma}^{*} d \cos (\theta) \frac{e^{2}\left|f_{s}\right|^{2}}{M_{K}^{2}}\left(\frac{\beta E_{\gamma}^{*}}{8 \pi M_{K}}\right)^{3}\left(1-\frac{2 E_{\gamma}^{*}}{M_{K}}\right) \sin ^{2} \theta \\
& \times\left|E_{I B}\left(K_{L}\right)+E_{D E}\left(K_{L}\right)\right|^{2} \\
= & \iint d E_{\gamma}^{*} d \cos (\theta) \frac{e^{2}\left|f_{s}\right|^{2}}{M_{K}{ }^{2}}\left(\frac{\beta E_{\gamma}^{*}}{8 \pi M_{K}}\right)^{3}\left(1-\frac{2 E_{\gamma}^{*}}{M_{K}}\right) \sin ^{2} \theta \\
& \times\left|\widetilde{\eta_{+-\gamma}}\right|^{2}\left|E_{I B}\left(K_{S}\right)+E_{D E}\left(K_{S}\right)\right|^{2} \\
= & \iint d E_{\gamma}^{*} d \cos (\theta) \frac{e^{2}\left|f_{s}\right|^{2}}{M_{K}{ }^{2}}\left(\frac{\beta E_{\gamma}^{*}}{8 \pi M_{K}}\right)^{3}\left(1-\frac{2 E_{\gamma}^{*}}{M_{K}}\right) \sin ^{2} \theta \\
& \times\left|\eta_{+-}+\widetilde{\epsilon_{+-\gamma}^{\prime}}\right|^{2}\left|E_{I B}\left(K_{S}\right)+E_{D E}\left(K_{S}\right)\right|^{2} \\
\approx & \iint d E_{\gamma}^{*} d \cos (\theta) \frac{e^{2}\left|f_{s}\right|^{2}}{M_{K}{ }^{2}}\left(\frac{\beta E_{\gamma}^{*}}{8 \pi M_{K}}\right)^{3}\left(1-\frac{2 E_{\gamma}^{*}}{M_{K}}\right) \sin ^{2} \theta \\
& \times\left[\left|\eta_{+-}\right|^{2}+2 R e\left(\eta_{+-}^{\dagger} \widetilde{\epsilon_{+-\gamma}^{\prime}}\right)\right]\left|E_{I B}\left(K_{S}\right)+E_{D E}\left(K_{S}\right)\right|^{2} \\
\approx & {\left[\left|\eta_{+-}\right|^{2}+2 R e\left(\eta_{+-}^{\dagger} \epsilon_{+-\gamma}^{\prime}\right)\right] \Gamma_{K_{S}}^{E 1} \rightarrow \pi^{+} \pi^{-} \gamma } \\
\approx & \left|\eta_{+-}+\epsilon_{+-\gamma}^{\prime}\right|^{2} \Gamma^{E 1} K_{S} \rightarrow \pi^{+} \pi^{-} \gamma
\end{aligned}
$$

where the following definition was used:

$$
\begin{aligned}
\epsilon_{+-\gamma}^{\prime}= & \frac{1}{\Gamma_{K_{S} \rightarrow \pi^{+} \pi^{-} \gamma}} \iint d E_{\gamma}^{*} d \cos (\theta) \frac{e^{2}\left|f_{s}\right|^{2}}{M_{K}^{2}}\left(\frac{\beta E_{\gamma}^{*}}{8 \pi M_{K}}\right)^{3}\left(1-\frac{2 E_{\gamma}^{*}}{M_{K}}\right) \sin ^{2} \theta \\
& \times \widetilde{\epsilon_{+-\gamma}^{\prime}}\left|E_{I B}\left(K_{S}\right)+E_{D E}\left(K_{S}\right)\right|^{2}
\end{aligned}
$$

\footnotetext{
${ }^{1} \operatorname{Ref}[14]$ constrains $g_{E 1}<0.04$ which leads to an upper limit $\left|\frac{i}{16} \frac{\epsilon^{\prime}}{\epsilon} g_{E 1}\right| \lesssim\left|\epsilon^{\prime}\right|$ This is likely far beyond the sensitivity of this analysis.
} 
A similar procedure can also be done with $\gamma_{L S}$ :

$$
\begin{aligned}
\gamma_{L S}= & \iint d E_{\gamma}^{*} d \cos (\theta)\left[\frac{e^{2}\left|f_{s}\right|^{2}}{M_{K}^{2}}\left(\frac{\beta E_{\gamma}^{*}}{8 \pi M_{K}}\right)^{3}\left(1-\frac{2 E_{\gamma}^{*}}{M_{K}}\right) \sin ^{2} \theta\right. \\
& \times\left(\left[E_{I B}\left(K_{L}\right)+E_{D E}\left(K_{L}\right)\right]^{\dagger}\left[E_{I B}\left(K_{S}\right)+E_{D E}\left(K_{S}\right)\right]\right. \\
& \left.\left.+M_{D E}^{\dagger}\left(K_{L}\right) M_{D E}\left(K_{S}\right)\right)\right] \\
= & \iint d E_{\gamma}^{*} d \cos (\theta)\left[\frac{e^{2}\left|f_{s}\right|^{2}}{M_{K}^{2}}\left(\frac{\beta E_{\gamma}^{*}}{8 \pi M_{K}}\right)^{3}\left(1-\frac{2 E_{\gamma}^{*}}{M_{K}}\right) \sin ^{2} \theta\right. \\
& \times\left({\widetilde{\eta_{+-\gamma}}}^{\dagger}\left[E_{I B}\left(K_{S}\right)+E_{D E}\left(K_{S}\right)\right]^{\dagger}\left[E_{I B}\left(K_{S}\right)+E_{D E}\left(K_{S}\right)\right]\right. \\
& \left.\left.+M_{D E}^{\dagger}\left(K_{L}\right) M_{D E}\left(K_{S}\right)\right)\right] \\
= & {\left[\eta_{+-}+\epsilon_{+-\gamma}^{\prime}\right]^{\dagger} \Gamma K_{S} \rightarrow \pi^{+} \pi^{-} \gamma }
\end{aligned}
$$

All results in this section can now be reviewed:

$$
\begin{aligned}
\Gamma_{K_{L}} & =(1+r) \Gamma_{K_{L}}^{E 1} \rightarrow \pi^{+} \pi^{-} \gamma=(1+r)\left|\eta_{+-}+\epsilon_{+-\gamma}^{\prime}\right|^{2} \Gamma_{K_{S}}^{E 1} \rightarrow \pi^{+} \pi^{-} \gamma \\
\gamma_{L S} & =\left[\eta_{+-}+\epsilon_{+-\gamma}^{\prime}\right]^{\dagger} \Gamma_{K_{S}} \rightarrow \pi^{+} \pi^{-} \gamma
\end{aligned}
$$

and when plugged into equation E.1 yield:

$$
\begin{aligned}
& \Gamma=|A|^{2}\left[(1+r)\left|\eta_{+-}+\epsilon_{+-\gamma}^{\prime}\right|^{2} \Gamma_{K_{S}} e^{-\left(\frac{1}{\tau_{L}}\right) \frac{M_{K} \Delta z}{p_{K}}}\right. \\
& +\left|\rho\left(p_{K}\right)\right|^{2} \Gamma_{K_{S}} e^{-\left(\frac{1}{\tau_{S}}\right) \frac{M_{K} \Delta z}{p_{K}}} \\
& \left.+2 R e\left[\rho\left(p_{K}\right)\left[\eta_{+-}+\epsilon_{+-\gamma}^{\prime}\right]^{\dagger} \Gamma_{K} K_{S} e^{i \Delta M_{K} \frac{M_{K} \Delta z}{p_{K}}}\right] e^{-\left(\frac{1}{\tau_{S}}+\frac{1}{\tau_{L}}\right) \frac{1}{2} \frac{M_{K} \Delta z}{p_{K}}}\right]
\end{aligned}
$$


Factoring out the common factor of $\Gamma_{K_{S}}$, employing the identity and making the definition $\eta_{+-\gamma}=\eta+\epsilon_{+-\gamma}^{\prime}$ we finally arrive at:

$$
\begin{aligned}
\Gamma= & |A|^{2} \Gamma_{K_{S}}\left[\left|\rho\left(p_{K}\right)\right|^{2} e^{-\left(\frac{1}{\tau_{S}}\right) \frac{M_{K} \Delta z}{p_{K}}}+(1+r)\left|\eta_{+-\gamma}\right|^{2} e^{-\left(\frac{1}{\tau_{L}}\right) \frac{M_{K} \Delta z}{p_{K}}}\right. \\
& \left.+2\left|\rho\left(p_{K}\right)\right|\left|\eta_{+-\gamma}\right| \cos \left(\Delta M_{K} \frac{M_{K} \Delta z}{p_{K}}+\phi_{\rho}-\phi_{+-\gamma}\right) e^{-\left(\frac{1}{\tau_{S}}+\frac{1}{\tau_{L}}\right) \frac{1}{2} \frac{M_{K} \Delta z}{p_{K}}}\right]
\end{aligned}
$$

which is the final result for the average decay rate of $K_{L, S} \rightarrow \pi^{+} \pi^{-} \gamma$. Note that this decay rate has the same form as that for $K_{L, S} \rightarrow \pi^{+} \pi^{-}$, except for the extra factor of $(1+r)$ and presence of the $\mathrm{CP}$ violating parameter $\eta_{+-\gamma}$, which is the equivalent of $\eta_{+-}$for this decay. It should be noted that in the absence of the direct $\mathrm{CP}$ violating part of the E1 DE process, $\eta_{+-\gamma}$ should be identical to $\eta_{+-}$. Equation E.14 shows this, in that if $\widehat{e}=0$ then $\epsilon_{+-\gamma}^{\prime}=0$ and $\eta_{+-\gamma}=\eta_{+-}$. So by fitting the average decay rate, it is also possible to search for direct $\mathrm{CP}$ violation in the DE process.

However, as is pointed out in [20], $\epsilon_{+-\gamma}^{\prime}$ is heavily suppressed due to kinematics. 


\section{Appendix F}

\section{Average Kaon wavefunctions}

As mentioned in Section 8.3.1, it is often necessary to calculate the probability $\mathcal{D}\left(\overrightarrow{x_{i}} ; \vec{\alpha}\right)$ averaged over a number of different possible values of the kaon wavefunctions in order to account for various possible ways a kaon can be produced and propagated. Recalling the form of Equation 8.25 suppose that we wish to calculate the value of $\mathcal{D}\left(\overrightarrow{x_{i}} ; \vec{\alpha}\right)$ averaged over a number of different kaon wavefunctions. We would then 
calculate:

$$
\begin{aligned}
\mathcal{D}\left(\overrightarrow{x_{i}} ; \vec{\alpha}\right)_{A V G}= & \frac{1}{N} \sum_{i=1}^{N}\left[\frac{d \Gamma_{K_{L}}}{d E_{\gamma}^{*} d \cos (\theta)}\left|a_{L}(t)^{(i)}\right|^{2}+\frac{d \Gamma_{K_{S}}}{d E_{\gamma}^{*} d \cos (\theta)}\left|a_{S}(t)^{(i)}\right|^{2}\right. \\
& \left.+2 \operatorname{Re}\left[\frac{d \gamma_{L S}}{d E_{\gamma}^{*} d \cos (\theta)} a_{L}(t)^{(i) \dagger} a_{S}(t)^{(i)}\right]\right] \\
= & \frac{1}{N} \sum_{i=1}^{N} \frac{d \Gamma_{L}}{d E_{\gamma}^{*} d \cos (\theta)}\left|a_{L}(t)^{(i)}\right|^{2}+\frac{1}{N} \sum_{i=1}^{N} \frac{d \Gamma_{K} K_{S}}{d E_{\gamma}^{*} d \cos (\theta)}\left|a_{S}(t)^{(i)}\right|^{2} \\
& +\frac{1}{N} \sum_{i=1}^{N} 2 \operatorname{Re}\left[\frac{d \gamma_{L S}}{d E_{\gamma}^{*} d \cos (\theta)} a_{L}(t)^{(i) \dagger} a_{S}(t)^{(i)}\right] \\
= & \frac{d \Gamma_{K_{L}}}{d E_{\gamma}^{*} d \cos (\theta)} \frac{1}{N} \sum_{i=1}^{N}\left|a_{L}(t)^{(i)}\right|^{2}+\frac{d \Gamma_{K_{S}}}{d E_{\gamma}^{*} d \cos (\theta)} \frac{1}{N} \sum_{i=1}^{N}\left|a_{S}(t)^{(i)}\right|^{2} \\
& +2 \operatorname{Re}\left[\frac{d \gamma_{L S}}{d E_{\gamma}^{*} d \cos (\theta)} \frac{1}{N} \sum_{i=1}^{N} a_{L}(t)^{(i) \dagger} a_{S}(t)^{(i)}\right] \\
= & \frac{d \Gamma_{K_{L}}}{d E_{\gamma}^{*} d \cos (\theta)}\left|a_{L}(t)\right|_{A V G}^{2}+\frac{d \Gamma_{K_{S}}}{d E_{\gamma}^{*} d \cos (\theta)}\left|a_{S}(t)\right|_{A V G}^{2} \\
& +2 \operatorname{Re}\left[\frac{d \gamma_{L S}}{d E_{\gamma}^{*} d \cos (\theta)}\left[a_{L}(t)^{\dagger} a_{S}(t)\right]_{A V G}\right]
\end{aligned}
$$

So it is possible to calculate $\mathcal{D}\left(\overrightarrow{x_{i}} ; \vec{\alpha}\right)$ using the average moduli of the wavefunctions, instead of calculating the average of all possible probability values. 


\section{Appendix G}

\section{Simulation of $K_{L, S} \rightarrow \pi^{+} \pi^{-} \gamma \gamma$}

One important physical process which must be taken into account is the presence of further radiative corrections, where one of the charged pions emits a second photon. This process can also be thought of as $K_{L, S} \rightarrow \pi^{+} \pi^{-} \gamma \gamma$. The emission of the second photon will modify the kinematics in such a way that accurate reconstruction of a $K_{L, S} \rightarrow \pi^{+} \pi^{-} \gamma$ decay is no longer possible, with the severity of the error scaling with the energy of the second photon. While the second photon will be emitted via bremsstrahlung, and will have a correspondingly low energy on average, it is still possible that the second photon will create a CsI cluster above threshold and be reconstructed. If both photons are reconstructed and satisfy all cuts, then the event will be rejected as we cut on ambiguous photon cluster solutions. Radiative corrections can then affect the acceptance three ways:

- Modification of event kinematics - can either increase or decrease acceptance depending on location of event in phase space

- Addition of a second photon cluster which can be used to reconstruct $\pi^{+} \pi^{-} \gamma$ when primary photon cluster is rejected or non-existent-increases acceptance

- Addition of a second photon cluster which can be used to reconstruct $\pi^{+} \pi^{-} \gamma$ and satisfy all analysis cuts, when the primary photon cluster already does so- decreases acceptance

As this process modifies the acceptance of events, it can also affect the maximum likelihood fit through the normalization factor. It thus becomes imperative to accurately 
model this effect in the KTeV Monte Carlo.

The presence of radiative corrections to $K_{L} \rightarrow \pi^{+} \pi^{-} \gamma$ first became clear in the analysis of the 1997 vacuum beam data[46] where the large statistics of that sample revealed a larger than expected tail in the $\pi^{+} \pi^{-} \gamma$ invariant mass plot at masses less than the kaon mass. In this analysis, the presence is again clear. To model these second order radiative corrections, we use version 2.0 of the PHOTOS software package [38] which has been integrated into the KTeV Monte Carlo.

While the use of PHOTOS results in much better data/MC agreement in the $\pi^{+} \pi^{-} \gamma$ mass plot, compared to the case of no radiative corrections, the agreement is not perfect. Notably, it over predicts the amount of $K_{L, S} \rightarrow \pi^{+} \pi^{-} \gamma \gamma$ events in the regenerator beam sample, resulting in a low-mass tail that is higher than data. We must then tune PHOTOS so that the Monte Carlo matches the data. PHOTOS is setup in such a way that it does not always produce a second photon when run. The probability for this secondary photon emission is assumed to be the source of the discrepancy with data, and must be modified somehow. We do this by defining an additional weighting factor $w_{\pi \pi \gamma \gamma}$ which is used to increase or decrease the frequency with which a second photon is generated. If $w_{\pi \pi \gamma \gamma}<1$ we desire fewer $K_{L, S} \rightarrow \pi^{+} \pi^{-} \gamma \gamma$ events to be generated, and this is accomplished by accepting only a fraction of events in which PHOTOS generates a photon. This accepted fraction is set to be equal to $w_{\pi \pi \gamma \gamma}$. If a particular event is rejected, PHOTOS is rerun once again and may or may not generate a photon on the second try. In this case, when PHOTOS does not generate a photon, the event is automatically accepted. On the other hand, if $w_{\pi \pi \gamma \gamma}>1$ a greater proportion of $K_{L, S} \rightarrow \pi^{+} \pi^{-} \gamma \gamma$ events should be generated. This is done by rejecting a fraction of events in which PHOTOS does not produce a photon, in which case PHOTOS is run again, and may or may not produce a photon. Here the fraction is set equal to $\frac{1}{w_{\pi \pi \gamma \gamma}}$. If PHOTOS does generate a photon, the event is accepted. Note that $w_{\pi \pi \gamma \gamma}=1$ corresponds to the PHOTOS default, where it is run once per event, and the result is always accepted.

Inspection of a large number of histograms of various distributions that are generated by the Monte Carlo reveals only subtle differences between the case with radiative corrections turned on and the case of them turned off. Only the plot of $\pi^{+} \pi^{-} \gamma$ invariant mass shows a clear signature of radiative corrections, as shown in FigureG.1. Attempted full reconstruction of $K_{L, S} \rightarrow \pi^{+} \pi^{-} \gamma \gamma$ also largely failed as a 
tool to see the radiative corrections, as only a few dozens of events pass all utilized analysis cuts. We then use the $\pi^{+} \pi^{-} \gamma$ mass plot to set the value of $w_{\pi \pi \gamma \gamma}$.

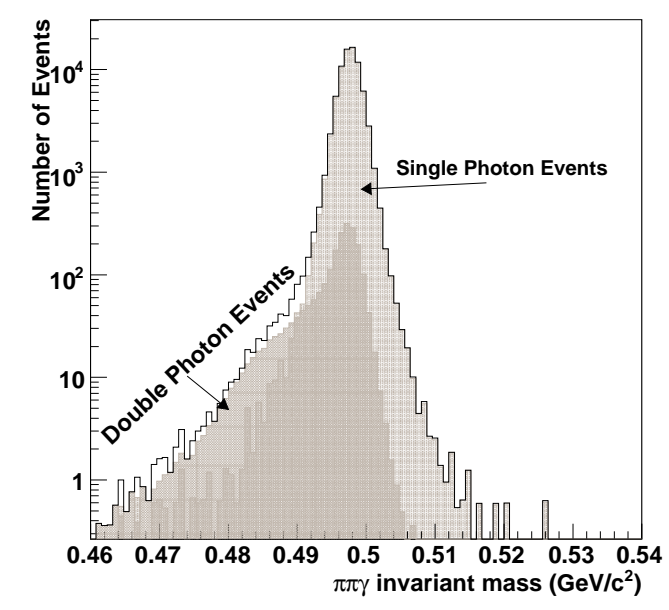

Figure G.1: A plot illustrating the shape and relative sizes of "pure" $K_{L, S} \rightarrow \pi^{+} \pi^{-} \gamma$ events and "pure" $K_{L, S} \rightarrow \pi^{+} \pi^{-} \gamma \gamma$ events. The sum is shown as the black histogram.

We first begin by measuring the relative contributions of $K_{L, S} \rightarrow \pi^{+} \pi^{-} \gamma$ and $K_{L, S} \rightarrow \pi^{+} \pi^{-} \gamma \gamma$ to the data after all analysis cuts except for the $\pi^{+} \pi^{-} \gamma$ mass cut. Two Monte Carlo samples are generated. The first sample is pure $K_{L, S} \rightarrow \pi^{+} \pi^{-} \gamma$ in which PHOTOS is turned off in this sample. The second is "pure" $K_{L, S} \rightarrow \pi^{+} \pi^{-} \gamma \gamma$ in which PHOTOS is turned on and the weight is set $w_{\pi \pi \gamma \gamma}=1 \times 10^{10}$ ensuring that every event contains a second photon. All selection criteria are applied to both samples. The result is three different plots of the $\pi^{+} \pi^{-} \gamma$ invariant mass - one of data, another of pure $K_{L, S} \rightarrow \pi^{+} \pi^{-} \gamma$ events and the last a plot of "pure" $K_{L, S} \rightarrow \pi^{+} \pi^{-} \gamma \gamma$ events. It is then possible to independently scale the normalization of the two Monte Carlo distributions until the sum of the two Monte Carlo plots best match the data. This is done using a $\chi^{2}$ minimization. One additional complication which must be taken into account is that of backgrounds which contribute to the wings of the $\pi^{+} \pi^{-} \gamma$ mass distribution and may affect the fit parameters that describe the relative size of the $K_{L, S} \rightarrow \pi^{+} \pi^{-} \gamma \gamma$ sample to $K_{L, S} \rightarrow \pi^{+} \pi^{-} \gamma$. In order to take background into account, we include a prediction of the shape of the background into the fit. A number of fits are done using different background shapes. These shapes are: 
- A constant background $A$, in which the normalization $A$ is floated

- An exponential background $B e^{\beta\left(m_{0}-m\right)}$, in which the normalization $B$, decay constant $\beta$ and the mass offset $m_{0}$ are floated.

- A constant background $A$ plus an exponential function $B e^{\beta\left(m_{0}-m\right)}$, in which the normalizations $A$ and $B$, decay constant $\beta$ and the mass offset $m_{0}$ are floated.

- A linear background function $A+C m$ plus an exponential function $B e^{\beta\left(m_{0}-m\right)}$ in which the normalizations $A$ and $B$, the slope $C$, decay constant $\beta$ and the mass offset $m_{0}$ are floated.

- No background

A fit is run using each different background prediction, and the weighted average of the all results is computed. The errors on the parameters are taken to be the statistical errors from the $\chi^{2}$ minimization, and are supplied by the program used in the fit-Minuit. This is done for both the vacuum and regenerator beams.

The end result of these fits are two scaling factors which fix the normalization between the $K_{L, S} \rightarrow \pi^{+} \pi^{-} \gamma$ and $K_{L, S} \rightarrow \pi^{+} \pi^{-} \gamma \gamma$ Monte Carlo samples. Applying these factors to each sample, we can then obtain the fraction $f$ of events out of the total for which a second photon was emitted after all cuts have been made except the $\pi^{+} \pi^{-} \gamma$ mass cut.

$$
f=\frac{N_{\pi^{+} \pi^{-} \gamma \gamma}}{N_{\pi^{+} \pi^{-} \gamma}+N_{\pi^{+} \pi^{-} \gamma \gamma}}
$$

For the 1999 regenerator sample, $f=0.023 \pm 0.002$, while for the 1999 vacuum beam sample, $f=0.0279 \pm .0025$ when a (constant+exponential) background is assumed. The fraction must then be corrected to reflect the fraction of events out of the total for which a second photon was emitted at the generation level, before any cuts. This can be done by dividing by the total acceptance factor for each sample:

$$
f_{G E N}=\frac{\frac{N_{\pi^{+}} \pi^{-} \gamma \gamma}{{ }^{\mathcal{A}} \pi^{+} \pi^{-} \gamma \gamma}}{\frac{\pi^{+} \pi^{-} \gamma}{\mathcal{A}^{+} \pi^{+} \pi^{-} \gamma}+\frac{{ }^{N} \pi^{+} \pi^{-} \gamma \gamma}{{ }^{\mathcal{A}} \pi^{+} \pi^{-} \gamma}}
$$


where $\mathcal{A}$ is the acceptance for a particular Monte Carlo sample after all cuts except for the $\pi^{+} \pi^{-} \gamma$ mass cut. For the 1999 regenerator sample, the acceptance ${ }^{1}$ is approximately 0.02535 when PHOTOS is set to always generate a photon, while the acceptance is 0.02507 for "pure" $K_{L, S} \rightarrow \pi^{+} \pi^{-} \gamma$ events without radiative corrections. For the 1999 vacuum sample, the numbers are 0.01901 and 0.02009 with and without the radiative correction from PHOTOS. This pattern holds across both years - the use of PHOTOS increases the acceptance for regenerator beam events, while also decreasing the acceptance for vacuum beam events. For the 1999 regenerator beam sample, $f_{G E N}^{F I T}=0.0228 \pm 0.0019$ while for the 1999 vacuum beam sample, $f_{G E N}^{F I T}=0.0294 \pm 0.0026$ when a (constant+exponential) background is assumed.

Correcting for acceptance will yield the fractions that the Monte Carlo should produce in order to best match the data. It can then be compared to the number that the Monte Carlo actually does produce when $w_{\pi \pi \gamma \gamma}=1$. The new value of the weight will then be given by the fraction of $K_{L, S} \rightarrow \pi^{+} \pi^{-} \gamma \gamma$ events that the Monte Carlo needs to produce in order to match data, divided by the fraction that the Monte Carlo produces by default.

$$
w_{\pi \pi \gamma \gamma}=\frac{f_{G E N}^{F I T}}{f_{G E N}^{D E F A U L T}}
$$

By default, PHOTOS generates a second photon for $4.56 \%$ of vacuum beam events, while it generates a second photon for $5.38 \%$ of the regenerator beam events. Comparing this with the results from the fit and acceptance correction, we find that the correction weight for PHOTOS is:

$$
\begin{array}{ll}
w_{\pi \pi \gamma \gamma}=0.515 \pm 0.034 & (R E G) \\
w_{\pi \pi \gamma \gamma}=0.870 \pm 0.056 & (V A C) \\
w_{\pi \pi \gamma \gamma}=0.638 \pm 0.051 & (A L L)
\end{array}
$$

which have been averaged over all possible background fits, and the error is dominated by the statistical errors of the fits.

The total average of $0.638 \pm 0.051$ is chosen as the new weighting factor for PHOTOS. It is this weight which is used in all Monte Carlo generated samples used in

\footnotetext{
${ }^{1}$ in this case, this acceptance is taken to be for decays for which $10 \mathrm{GeV} / \mathrm{c}<p_{K}<200 \mathrm{GeV} / \mathrm{c}$, $105.5 m<Z_{\text {vertex }}<159.9 m$ and $E_{\gamma}^{*}>4.0 \mathrm{MeV}$. As the analysis cut windows are tighter than this, these variables will contribute to the total loss
} 
this analysis.

Note that the correction factors are different between the regenerator and vacuum beams. In order to ensure that the result is not sensitive to this discrepancy, we shall vary the PHOTOS weighting factor between the vacuum and regenerator beam results and not just within one standard deviation of the average result when we estimate the systematic error due to uncertainty in the PHOTOS correction factor. 


\section{Appendix $\mathbf{H}$}

\section{Generation of Kinematic Variables}

One important aspect of the $K_{L, S} \rightarrow \pi^{+} \pi^{-} \gamma$ Monte Carlo was efficient generation of the phase space variables $E_{\gamma}^{*}$ and $\tau$. The desired distributions of these variables are both very sharply peaked for regenerator beam events, and are difficult to efficiently generate using the standard acceptance/rejection method. As a reminder, the standard acceptance/rejection method generates a deviate - a random number from some probability density function $p(x)$, by selecting a value of $\mathrm{x}$ between some minimum value $x_{\min }$ and some maximum value $x_{\max }$ according to a uniform distribution. This value of $\mathrm{x}$ is then accepted if:

$$
p(x)>w_{\max } \times \text { rand }
$$

where $w_{\max }$ is some number which is greater than $\mathrm{p}(\mathrm{x})$ for all $x_{\min }<x<x_{\max }$ and rand is a uniform random number between 0 and 1 . This method is illustrated in Figure H.1. In short, this method produces an uniform density of points in a $2 \mathrm{~d}$ plane between 0 and $w_{\max }$ and $x_{\min }<x<x_{\max }$, and will accept those points which fall under the curve $p(x)$. In Figure H.1, the gray area shows the inefficiency that results when this method is used for a strongly peaked function.

The use of this method will result in many trial values of $E_{\gamma}^{*}$ and $\tau$ being produced and rejected, especially at larger values of $E_{\gamma}^{*}$ and $\tau$. One solution which may be employed to increase the efficiency of this generation method is to draw values of $\mathrm{x}$ 
from something other than a uniform distribution, which resembles $p(x)$. This will bias the generated distribution of $\mathrm{x}$ after acceptance, so this bias must be removed using a reweighting factor.

The first step of this process is to choose a mapping between a uniform distribution and one of the phase space variables which will result in a distribution which somewhat approximates the decay rate $\mathcal{D}\left(\overrightarrow{x_{i}} ; \vec{\alpha}\right)$ that we wish to generate. For example, as shown in Section 7.2.1, the mapping

$$
\tau=\frac{-0.005}{c}+\frac{1.010}{c} t_{\text {rand }}^{3}
$$

was selected for the proper time selection, where $t_{\text {rand }}$ is a uniform deviate from 0 to 1. Note that the Transformation Method [41] is being used here. The use of this mapping will convert uniformly distributed values of $t_{\text {rand }}$ into non-uniformly distributed values of $\tau$. The pdf for $\tau$ that results will then be:

$$
\begin{aligned}
p(\tau) d \tau & =\left|\frac{d t_{\text {rand }}}{d \tau}\right| \\
& =\left|\frac{1}{3\left(\frac{1.010}{c}\right) t_{\text {rand }}^{2}}\right|
\end{aligned}
$$

where the fundamental transformation law of probabilities

$$
p(y)=p(x)\left|\frac{d x}{d y}\right|
$$

was used. This, combined with the mapping between $t_{\text {rand }}$ and $\tau$ yields:

$$
p(\tau) d \tau=\frac{1}{3}\left(\frac{1.010}{c}\right)^{-1 / 3}\left[\tau+\frac{0.005}{c}\right]^{-2 / 3} d \tau
$$

Note that this is the inverse of Equation 7.6, where a change of variable has been made from $t_{\text {rand }}$ to $\tau$.

Since the $\tau$ spectrum has been biased by $p(\tau)$, it is necessary to remove the bias by dividing the value of the decay rate $\mathcal{D}\left(\overrightarrow{x_{i}} ; \vec{\alpha}\right)$ by $p(\tau)$ before the acceptance/rejection decision ( as shown in Equation H.1 ) has been made. This is the reason Equation 7.11 takes the form it does. 


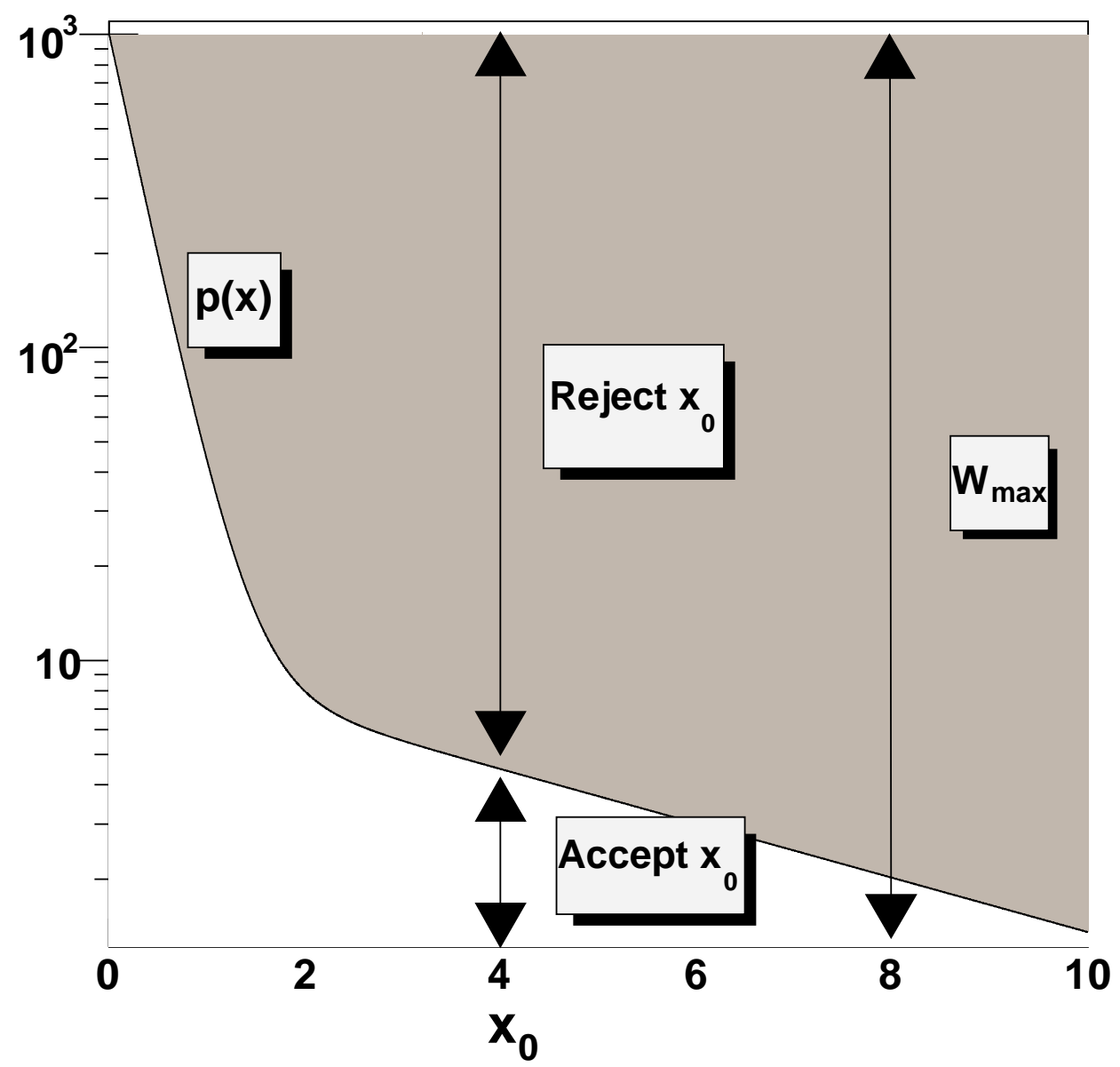

Figure H.1: A picture of the traditional acceptance rejection method. In this example, $x_{0}$ is chosen uniformly from 0 to $10, w_{\max }$ is multiplied by a random number between 0 to 1 , and if this product is smaller than $p\left(x_{0}\right)$, the value of $x_{0}$ is accepted. 

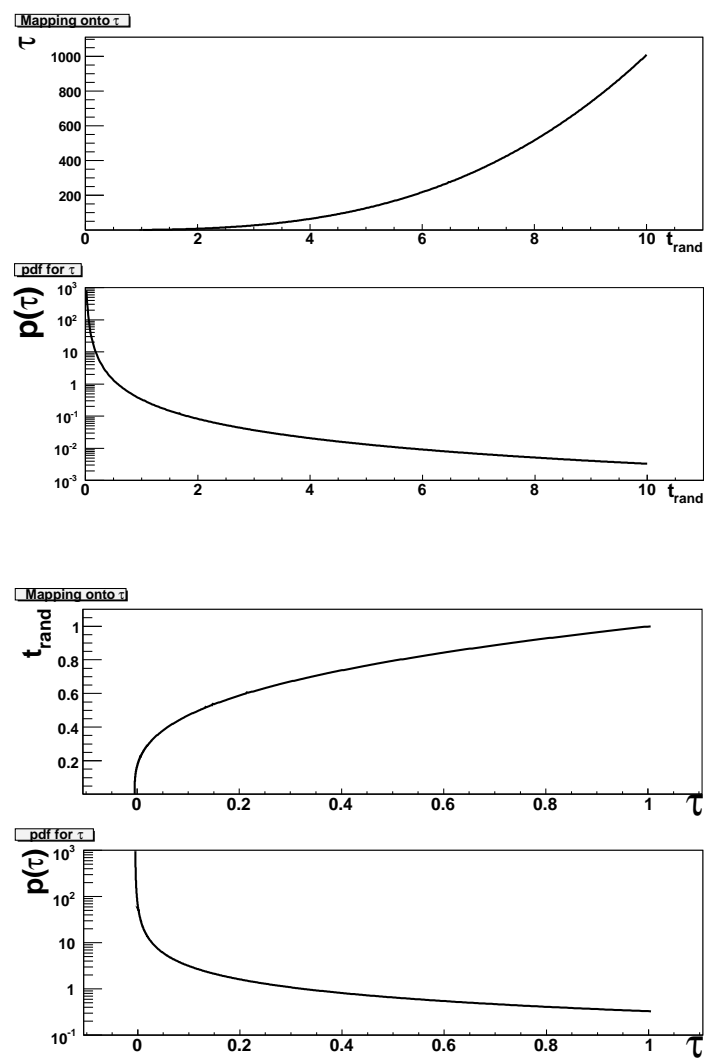

Figure H.2: a) Shows the mapping from the uniform random variable $t_{\text {rand }}$ to $\tau$, and the resulting pdf as a function of $t_{\text {rand }}$. b) Shows the inverse mapping from the uniform random variable $t_{\text {rand }}$ to $\tau$, and the resulting pdf as a function of $\tau$. Note that neither pdf is normalized. 
Appendix I

Plots for 1997 Vacuum Data 

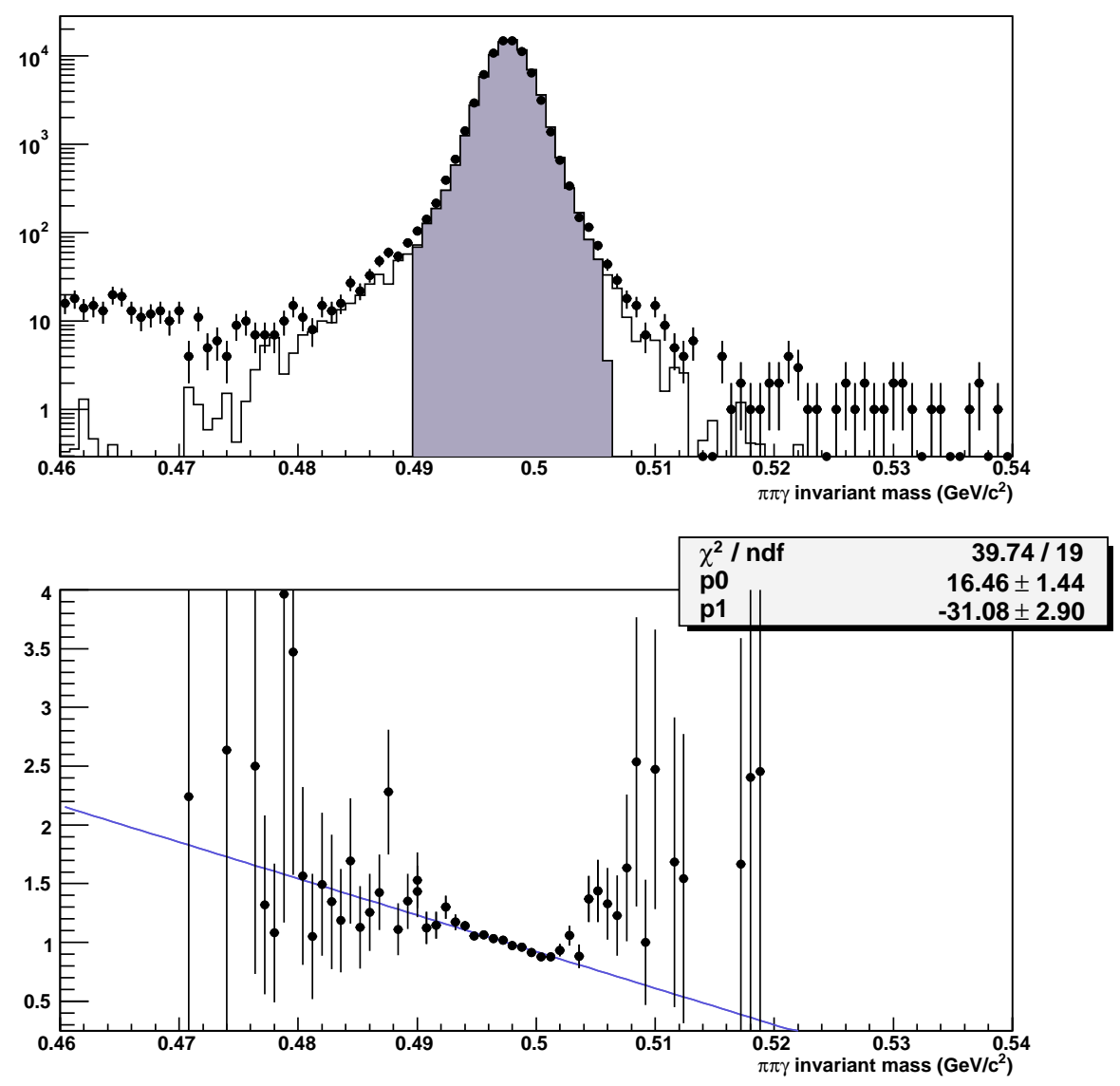

Figure I.1: A plot of the $\pi^{+} \pi^{-} \gamma$ invariant mass before the cut on $M_{\pi^{+} \pi^{-} \gamma}$ but after all other cuts. Data are points, while the histogram is of Monte Carlo simulated events. The solid part of the histogram denotes the areas that pass after this particular cut. A plot of the ratio of the number of data events to Monte Carlo events, before this cut, is shown on the bottom. The number of Monte Carlo events is normalized to be equal to the total number of data events in this plot. A fit of the ratio using a linear function is also shown. 

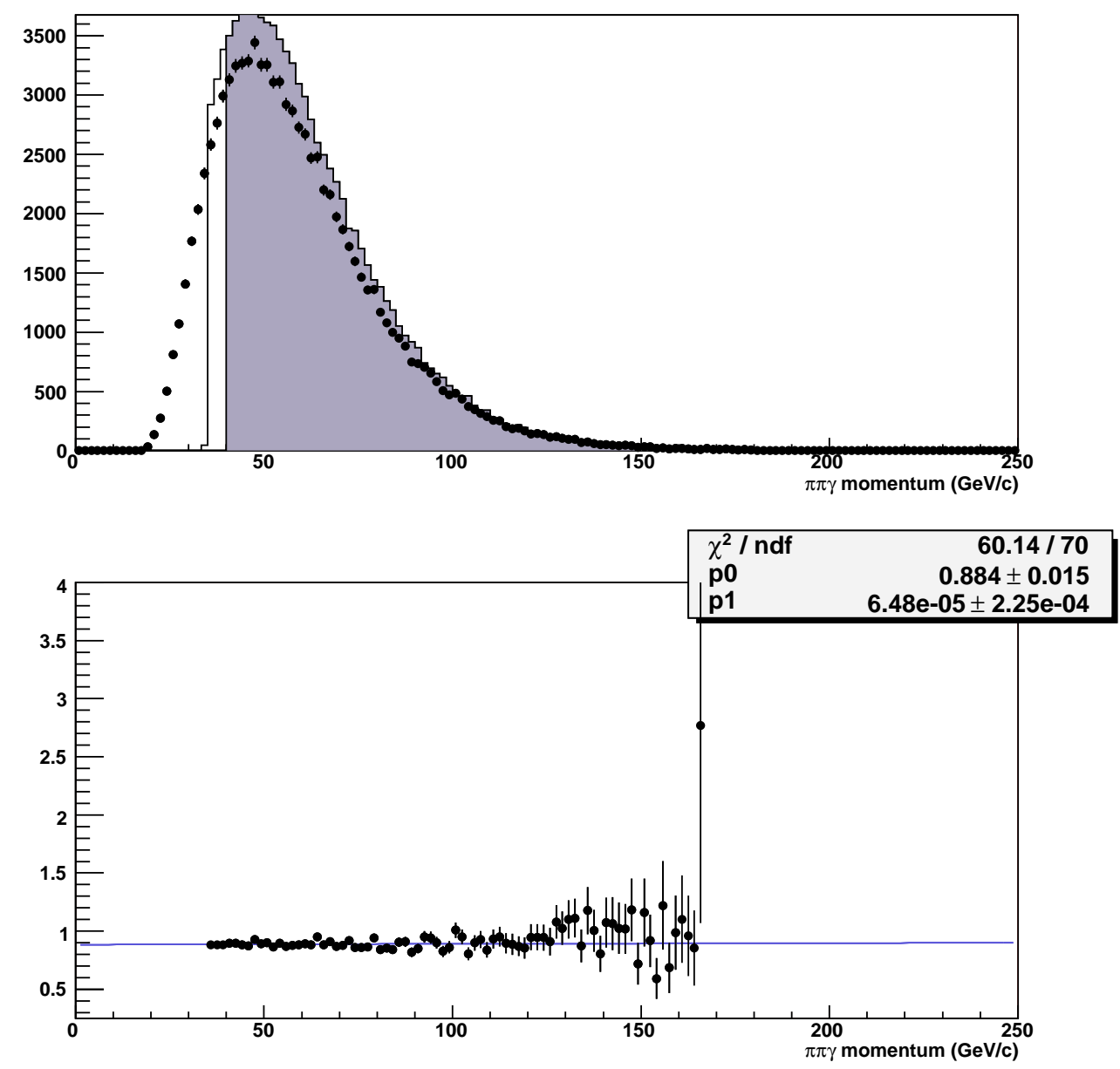

Figure I.2: The reconstructed kaon momentum before the cut. Data are points, while the histogram is of Monte Carlo simulated events. The solid part of the histogram denotes the areas that pass after this particular cut. A plot of the ratio of the number of data events to Monte Carlo events, before this cut, is shown on the bottom. The number of Monte Carlo events is normalized to be equal to the total number of data events in this plot. A fit of the ratio using a linear function is also shown. 

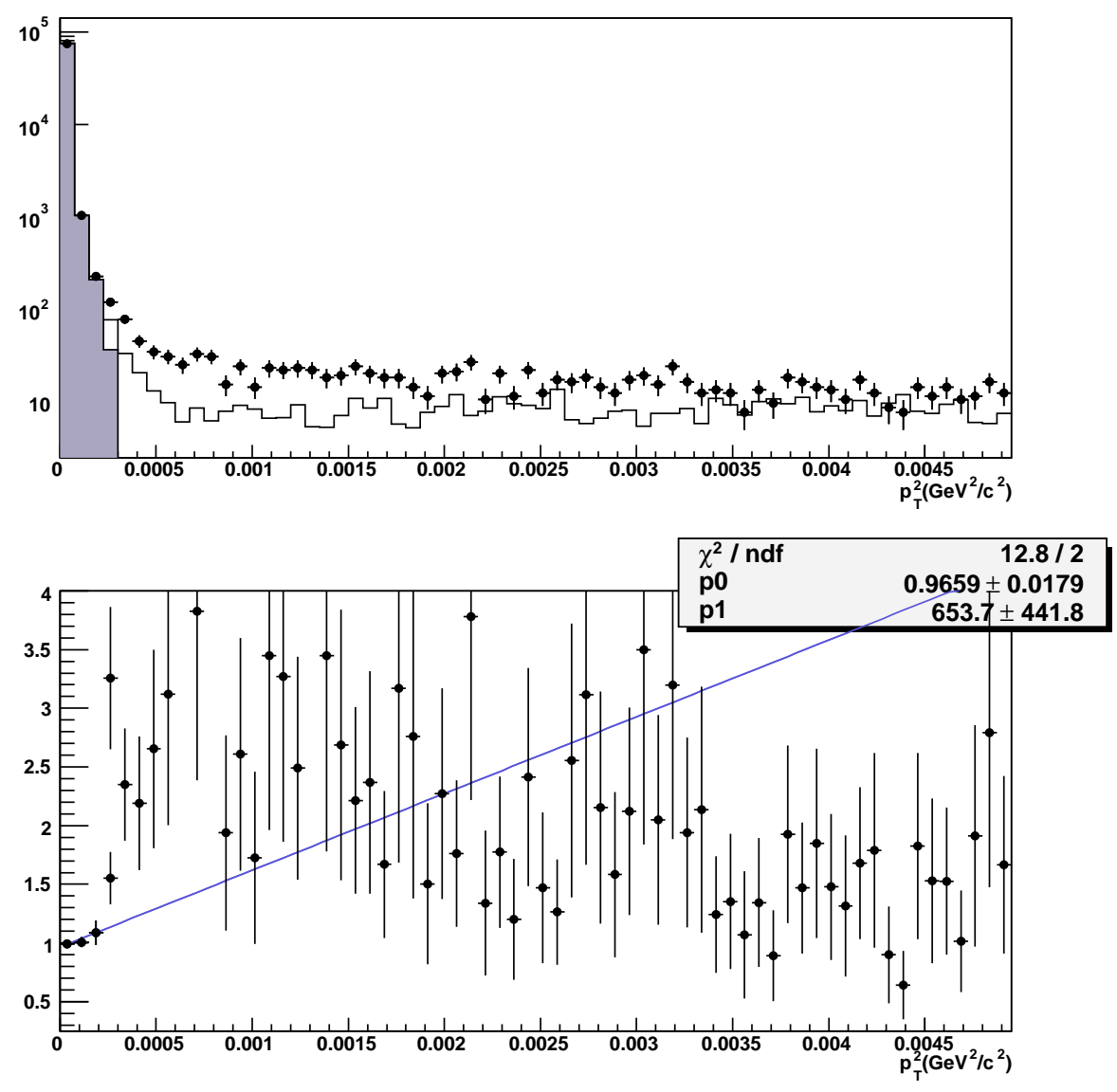

Figure I.3: A plot of $P_{T}^{2}$ with respect to the downstream face of the regenerator for the $\pi^{+} \pi^{-} \gamma$ system before the cut. Data are points, while the histogram is of Monte Carlo simulated events. The solid part of the histogram denotes the areas that pass after this particular cut. A plot of the ratio of the number of data events to Monte Carlo events, before this cut, is shown on the bottom. The number of Monte Carlo events is normalized to be equal to the total number of data events in this plot. A fit of the ratio using a linear function is also shown. 

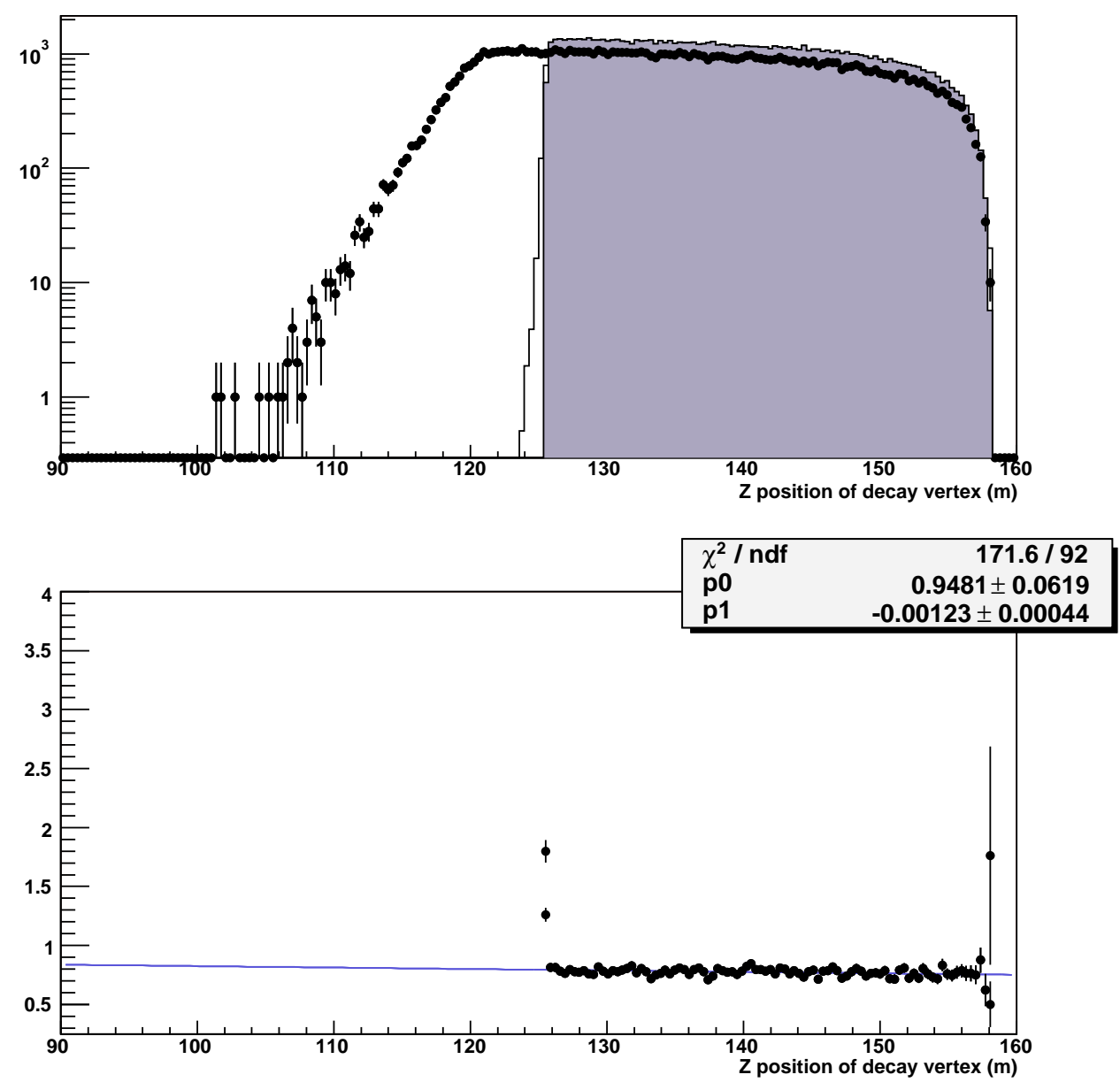

Figure I.4: A plot of the $\mathrm{z}$ location of the decay vertex, before the cut is applied. Data are points, while the histogram is of Monte Carlo simulated events. The solid part of the histogram denotes the areas that pass after this particular cut. A plot of the ratio of the number of data events to Monte Carlo events, before this cut, is shown on the bottom. The number of Monte Carlo events is normalized to be equal to the total number of data events in this plot. A fit of the ratio using a linear function is also shown. 

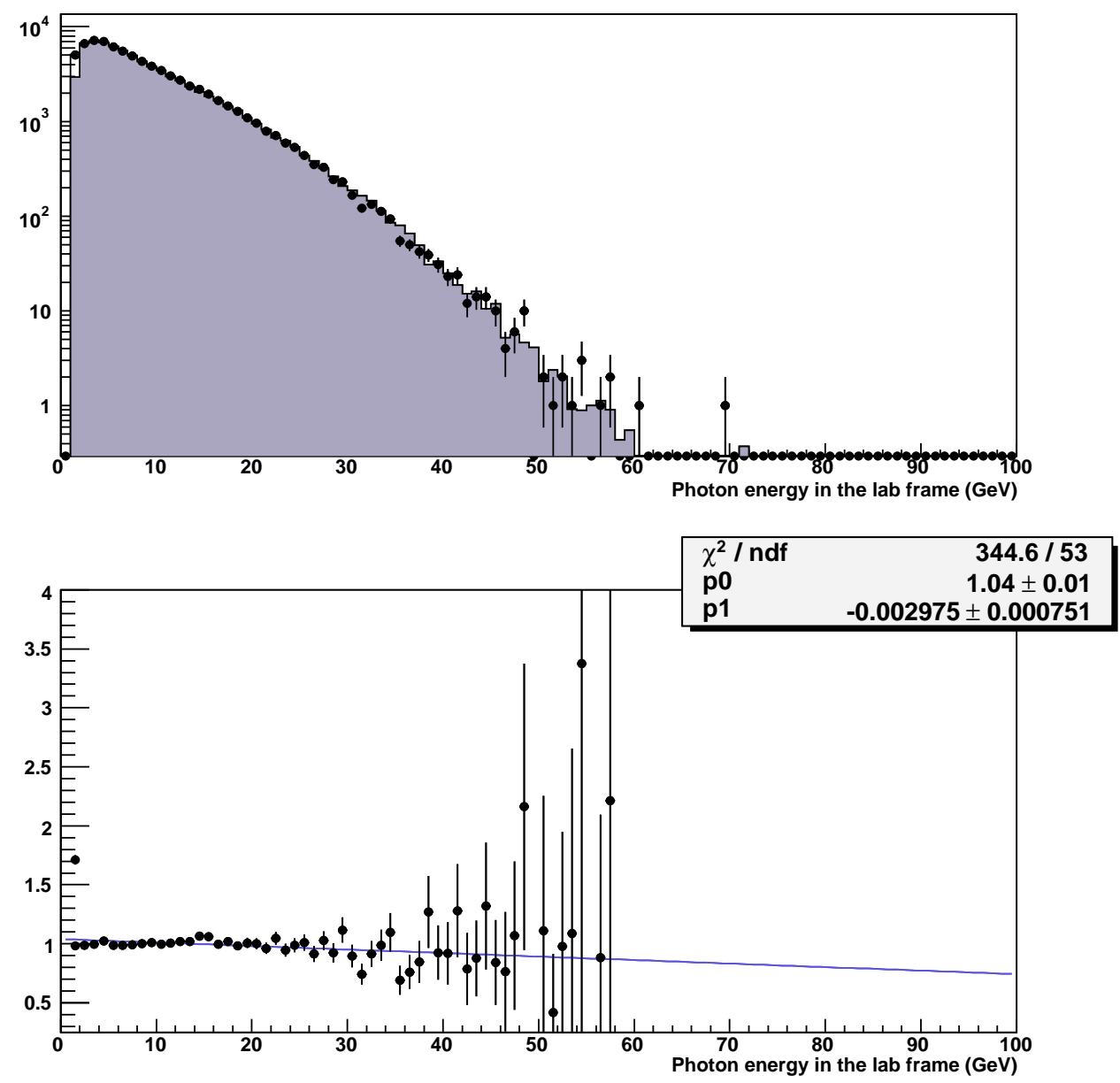

Figure I.5: The photon cluster energy in the lab frame, before the final analysis cut. Data are points, while the histogram is of Monte Carlo simulated events. The solid part of the histogram denotes the areas that pass after this particular cut. A plot of the ratio of the number of data events to Monte Carlo events, before this cut, is shown on the bottom. The number of Monte Carlo events is normalized to be equal to the total number of data events in this plot. A fit of the ratio using a linear function is also shown. 

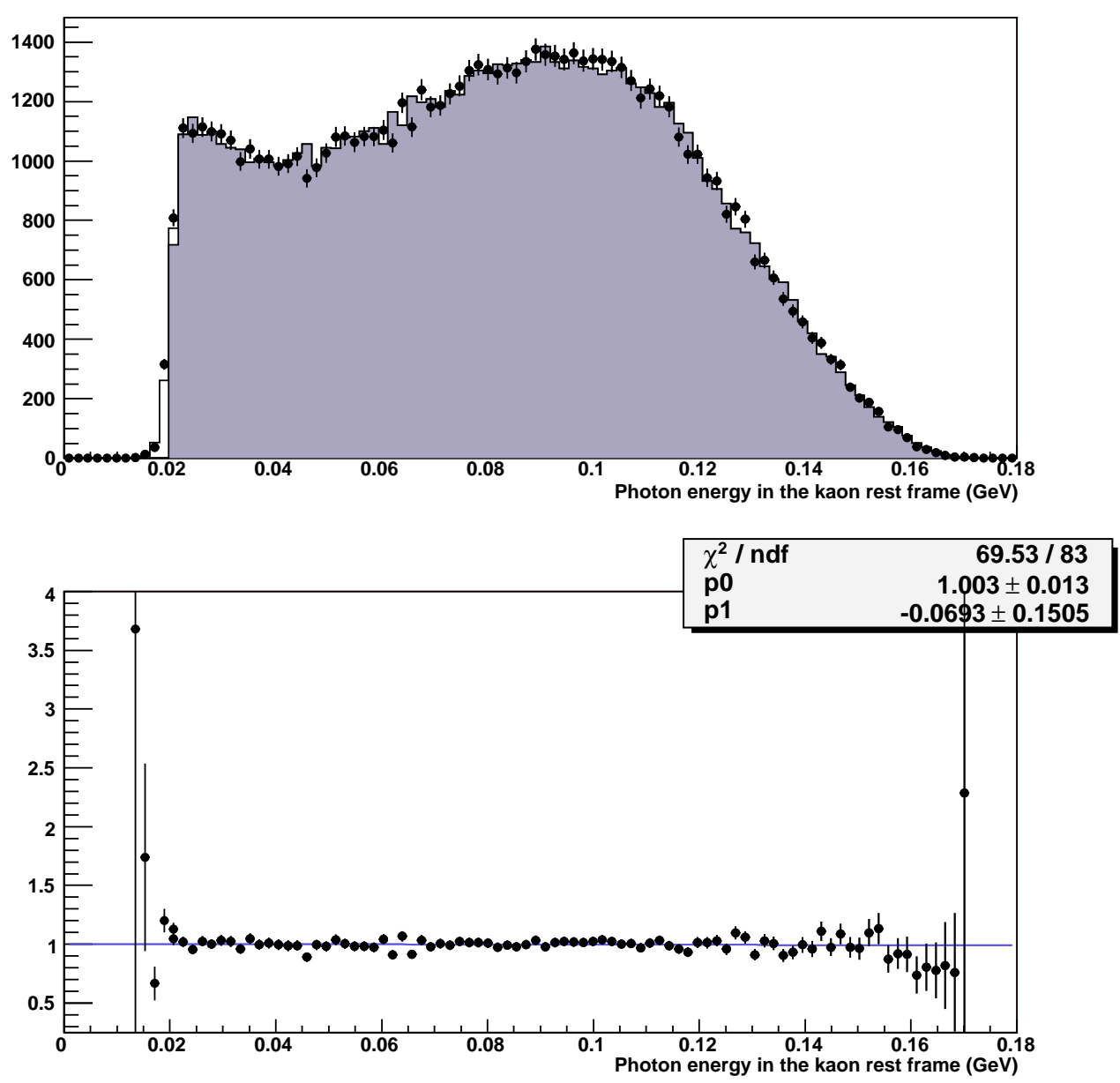

Figure I.6: A plot of the photon energy in the kaon rest frame before the cut. Data are points, while the histogram is of Monte Carlo simulated events. The solid part of the histogram denotes the areas that pass after this particular cut. A plot of the ratio of the number of data events to Monte Carlo events, before this cut, is shown on the bottom. The number of Monte Carlo events is normalized to be equal to the total number of data events in this plot. A fit of the ratio using a linear function is also shown. 

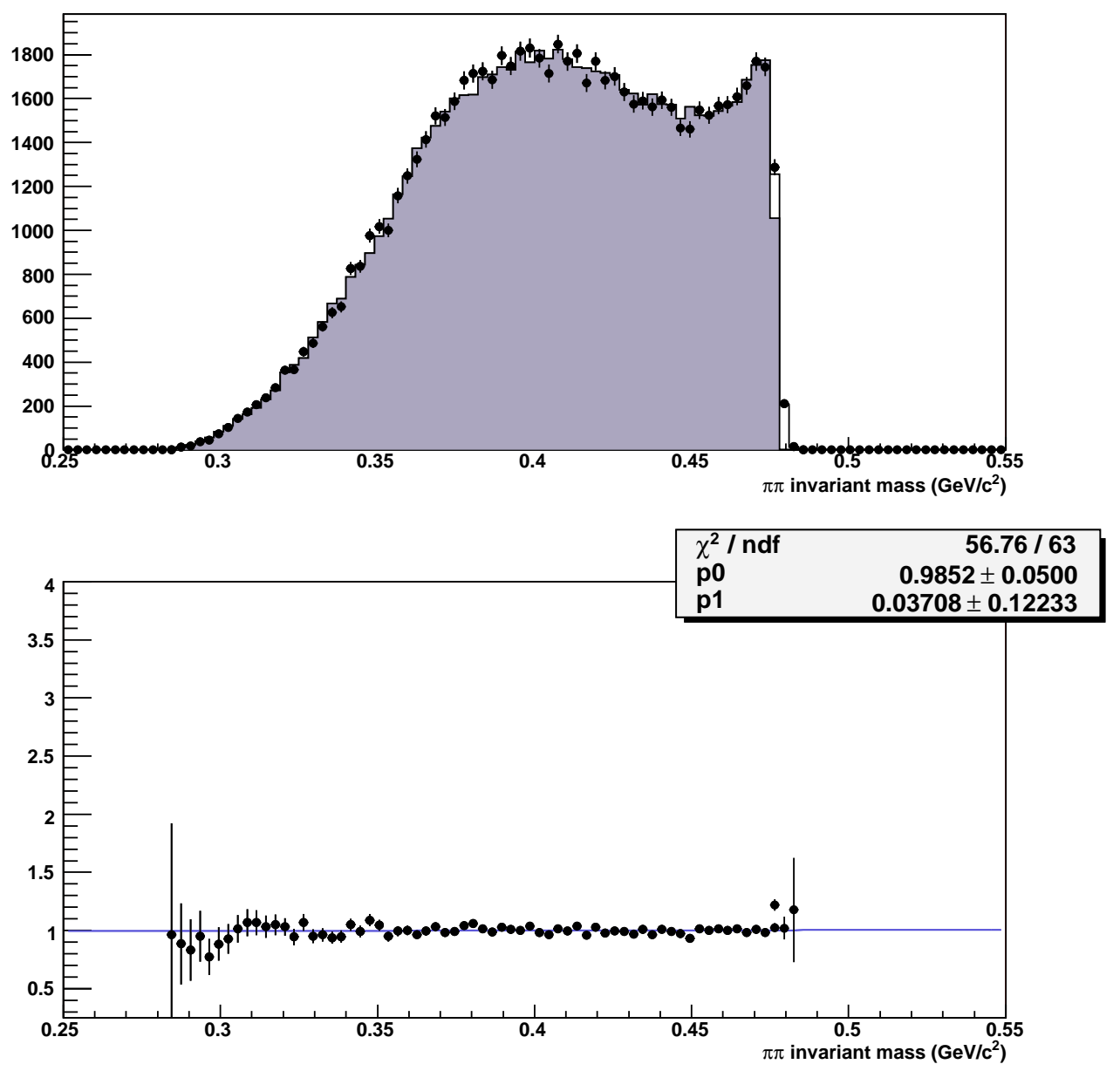

Figure I.7: A plot of the invariant mass of the pion pair before the $M_{\pi^{+} \pi^{-}}$cut. Note that any cut on $E_{\gamma}^{* K I N}$ will also be reflected here. Data are points, while the histogram is of Monte Carlo simulated events. The solid part of the histogram denotes the areas that pass after this particular cut. A plot of the ratio of the number of data events to Monte Carlo events, before this cut, is shown on the bottom. The number of Monte Carlo events is normalized to be equal to the total number of data events in this plot. A fit of the ratio using a linear function is also shown. 

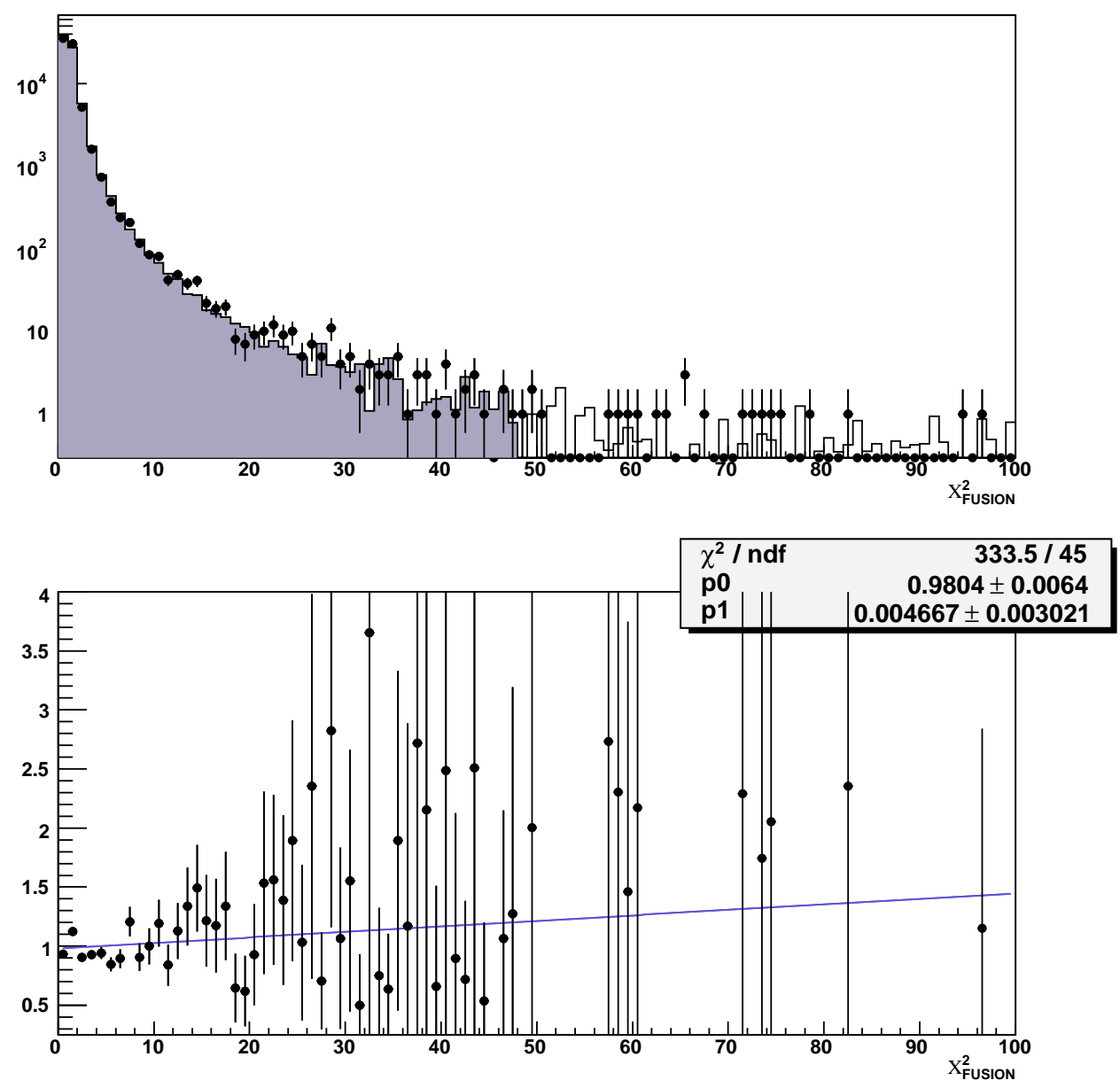

Figure I.8: A plot of fusion $\chi^{2}$ before the cut on this variable. Data are points, while the histogram is of Monte Carlo simulated events. The solid part of the histogram denotes the areas that pass after this particular cut. A plot of the ratio of the number of data events to Monte Carlo events, before this cut, is shown on the bottom. The number of Monte Carlo events is normalized to be equal to the total number of data events in this plot. A fit of the ratio using a linear function is also shown. 

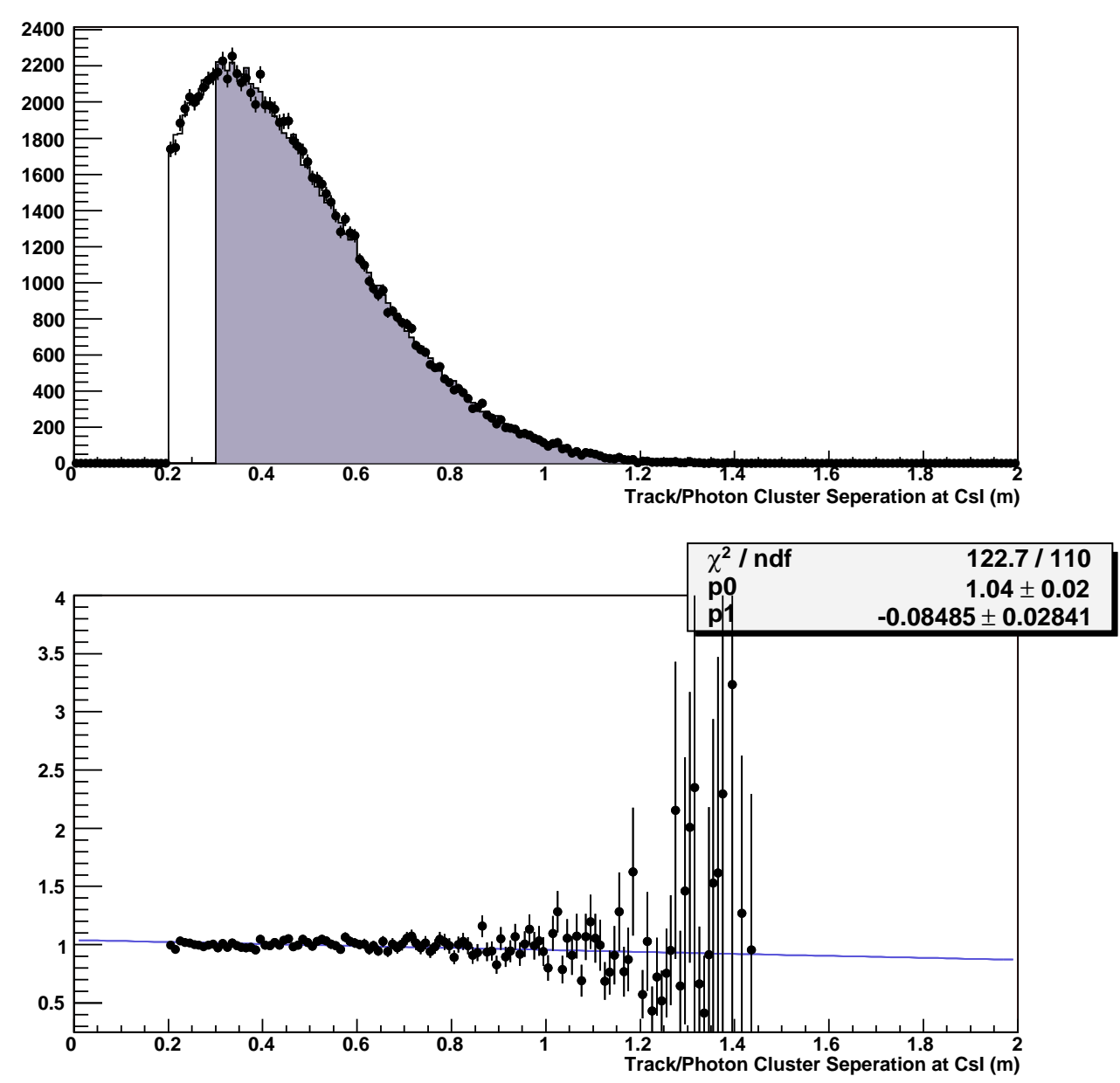

Figure I.9: Minimum of the two pion/gamma separations at the CsI for each event, before the cut. Data are points, while the histogram is of Monte Carlo simulated events. The solid part of the histogram denotes the areas that pass after this particular cut. A plot of the ratio of the number of data events to Monte Carlo events, before this cut, is shown on the bottom. The number of Monte Carlo events is normalized to be equal to the total number of data events in this plot. A fit of the ratio using a linear function is also shown. 

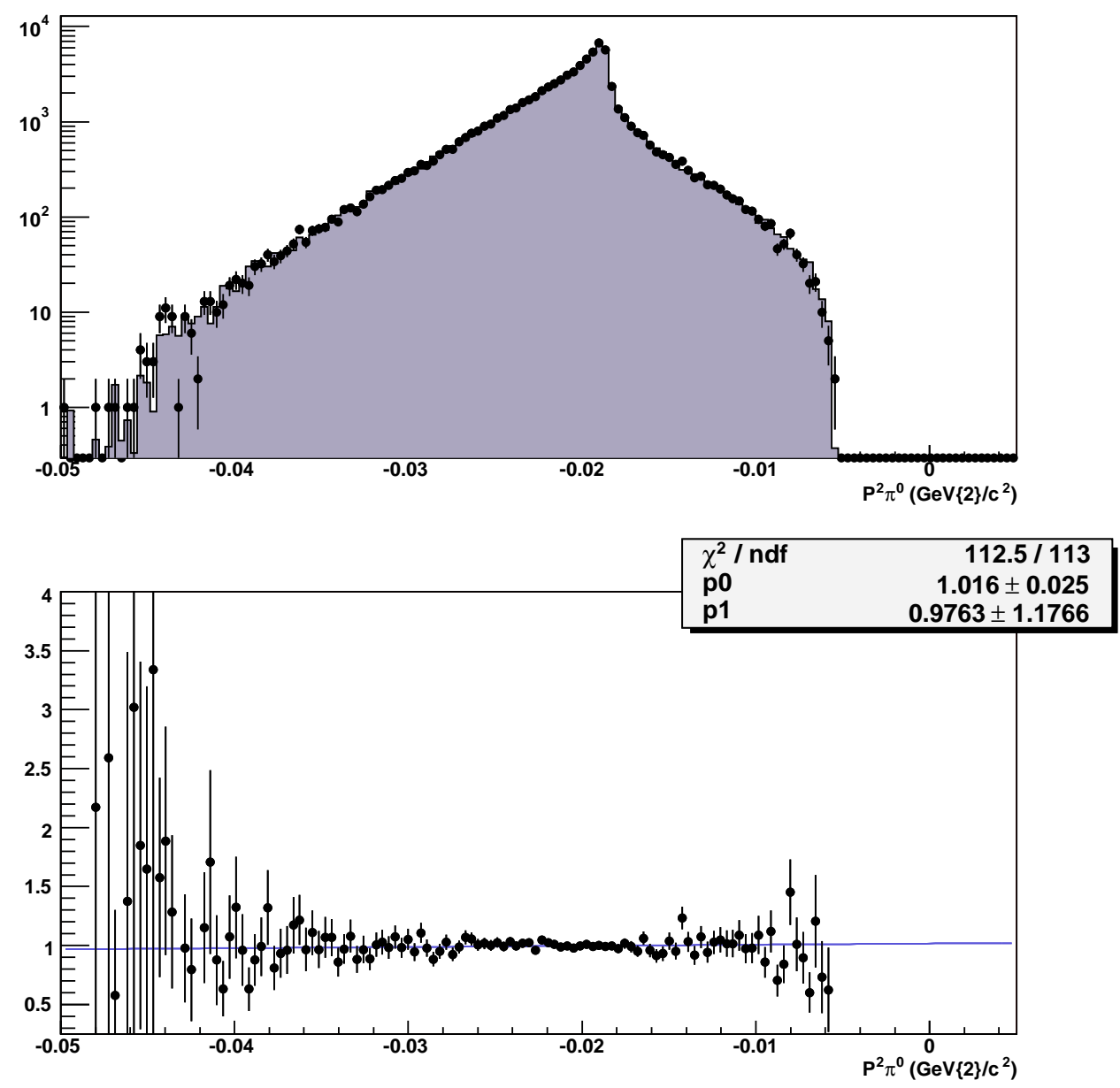

Figure I.10: A plot of $P_{\pi^{0}}^{2}$ with respect to the downstream face of the regenerator, before the final analysis cut. Data are points, while the histogram is of Monte Carlo simulated events. The solid part of the histogram denotes the areas that pass after this particular cut. A plot of the ratio of the number of data events to Monte Carlo events, before this cut, is shown on the bottom. The number of Monte Carlo events is normalized to be equal to the total number of data events in this plot. A fit of the ratio using a linear function is also shown. 

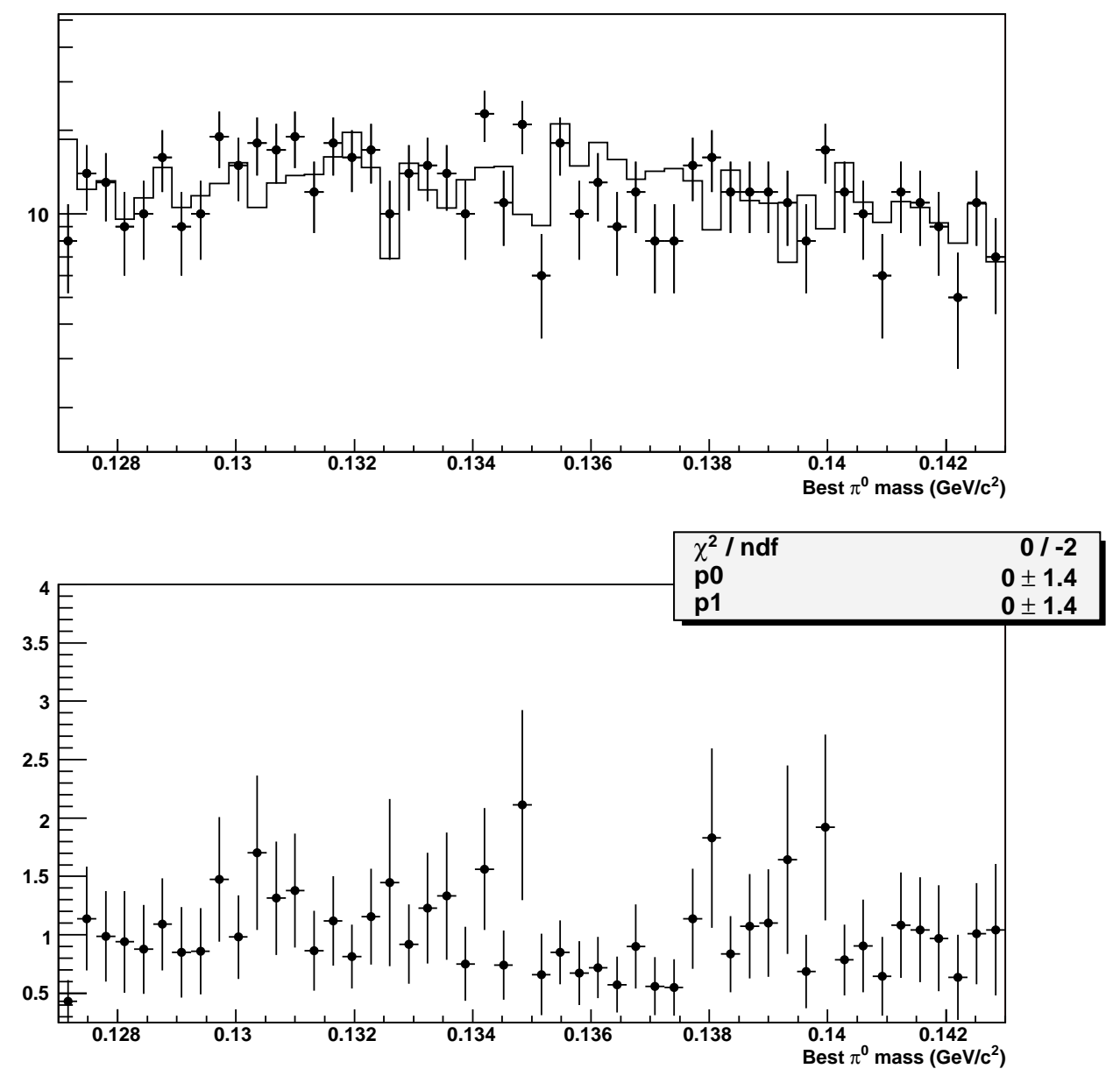

Figure I.11: A plot of the best $\pi^{0}$ mass found in each event using two clusters in the CsI calorimeter, before the cut on this variable. Data are points, while the histogram is of Monte Carlo simulated events. The solid part of the histogram denotes the areas that pass after this particular cut. A plot of the ratio of the number of data events to Monte Carlo events, before this cut, is shown on the bottom. The number of Monte Carlo events is normalized to be equal to the total number of data events in this plot. A fit of the ratio using a linear function is also shown. 

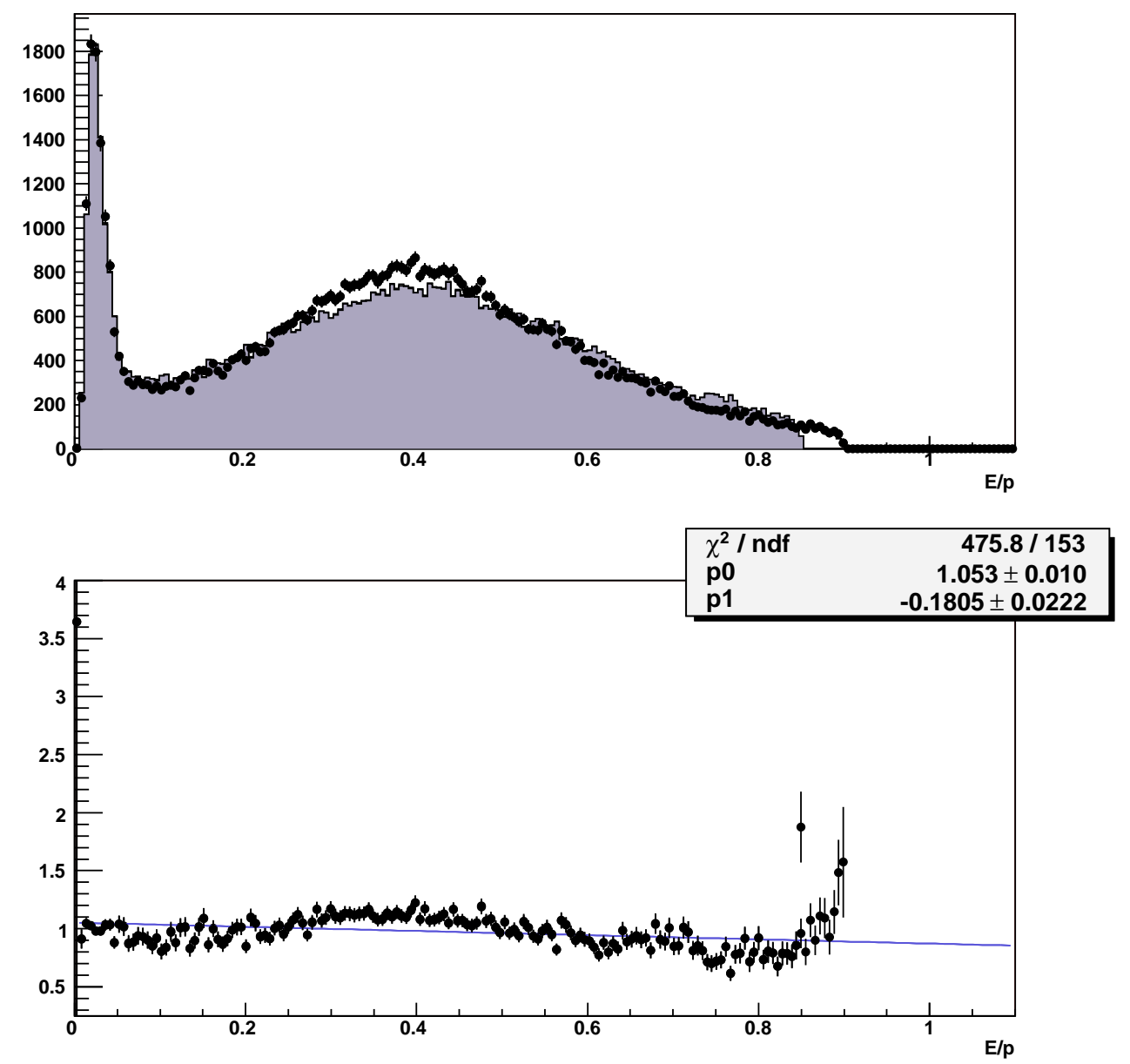

Figure I.12: A plot of the greater of the two values of $\mathrm{E} / \mathrm{p}$ for each event, before the final E/p cut. Data are points, while the histogram is of Monte Carlo simulated events. The solid part of the histogram denotes the areas that pass after this particular cut. A plot of the ratio of the number of data events to Monte Carlo events, before this cut, is shown on the bottom. The number of Monte Carlo events is normalized to be equal to the total number of data events in this plot. A fit of the ratio using a linear function is also shown. 

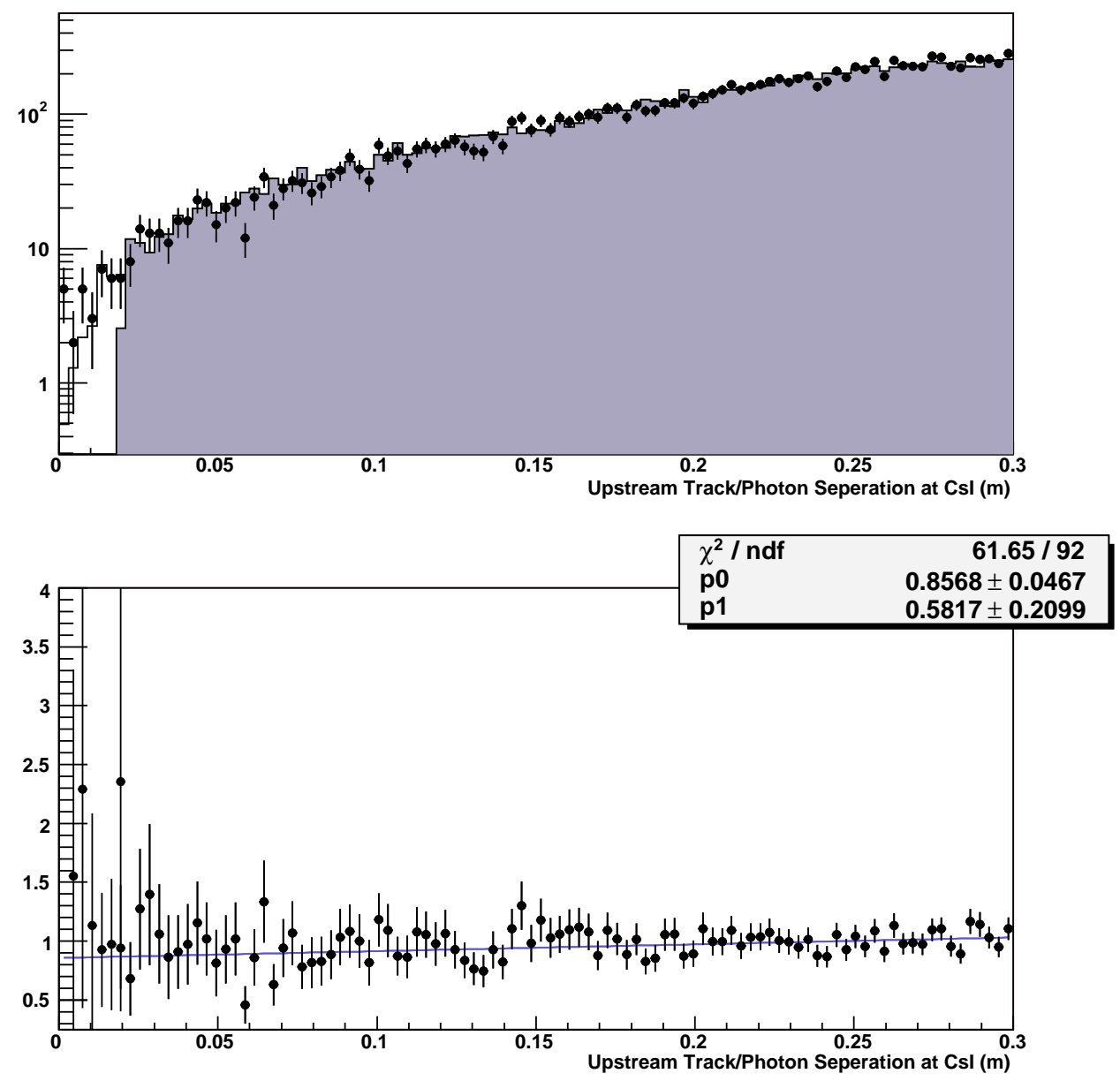

Figure I.13: A plot of the upstream track/photon separation distance at the CsI before the cut. Data are points, while the histogram is of Monte Carlo simulated events. The solid part of the histogram denotes the areas that pass after this particular cut. A plot of the ratio of the number of data events to Monte Carlo events, before this cut, is shown on the bottom. The number of Monte Carlo events is normalized to be equal to the total number of data events in this plot. A fit of the ratio using a linear function is also shown. 

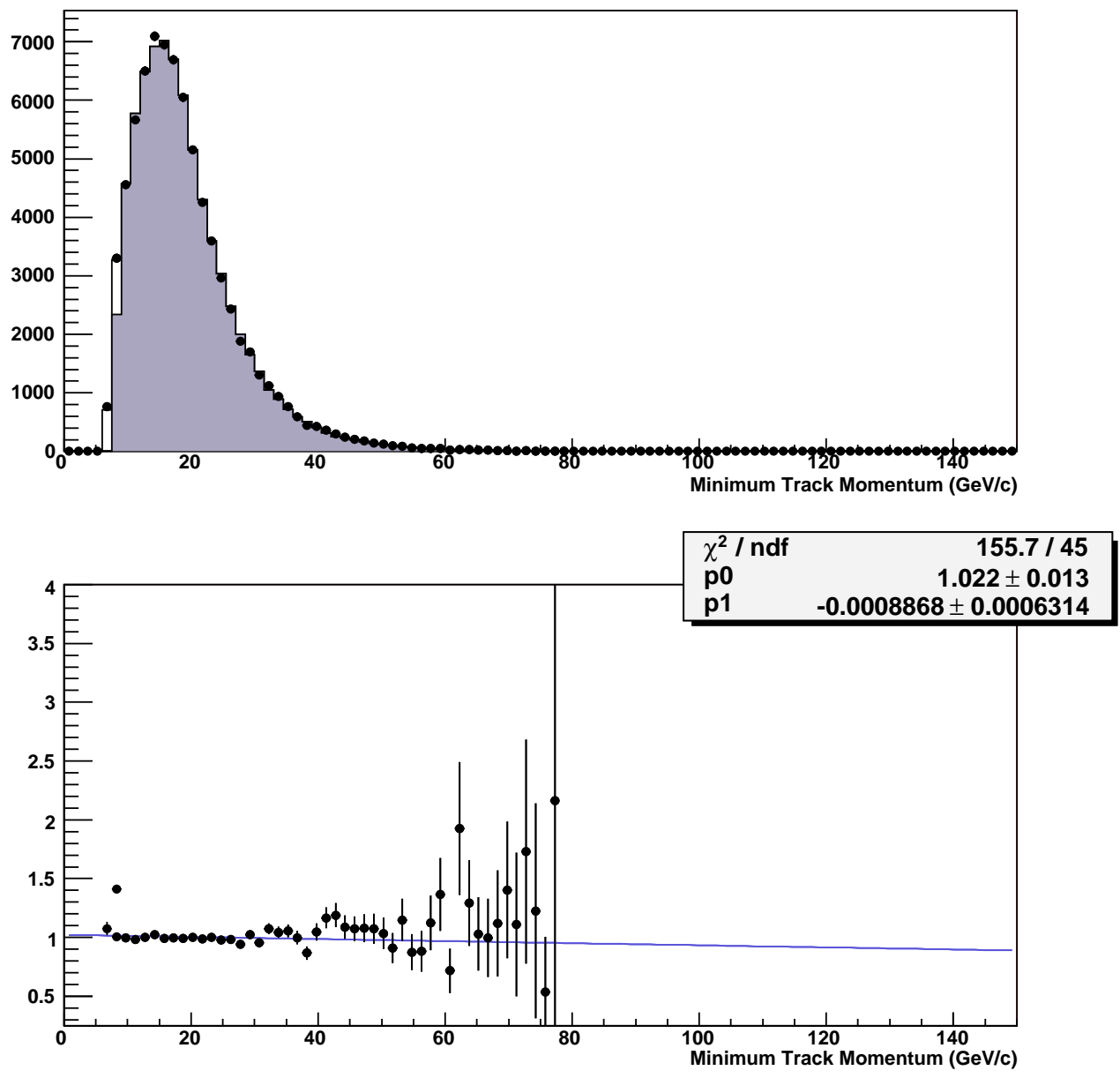

Figure I.14: A plot of the lesser of two track momenta per event before the cut. Data are points, while the histogram is of Monte Carlo simulated events. The solid part of the histogram denotes the areas that pass after this particular cut. A plot of the ratio of the number of data events to Monte Carlo events, before this cut, is shown on the bottom. The number of Monte Carlo events is normalized to be equal to the total number of data events in this plot. A fit of the ratio using a linear function is also shown. 

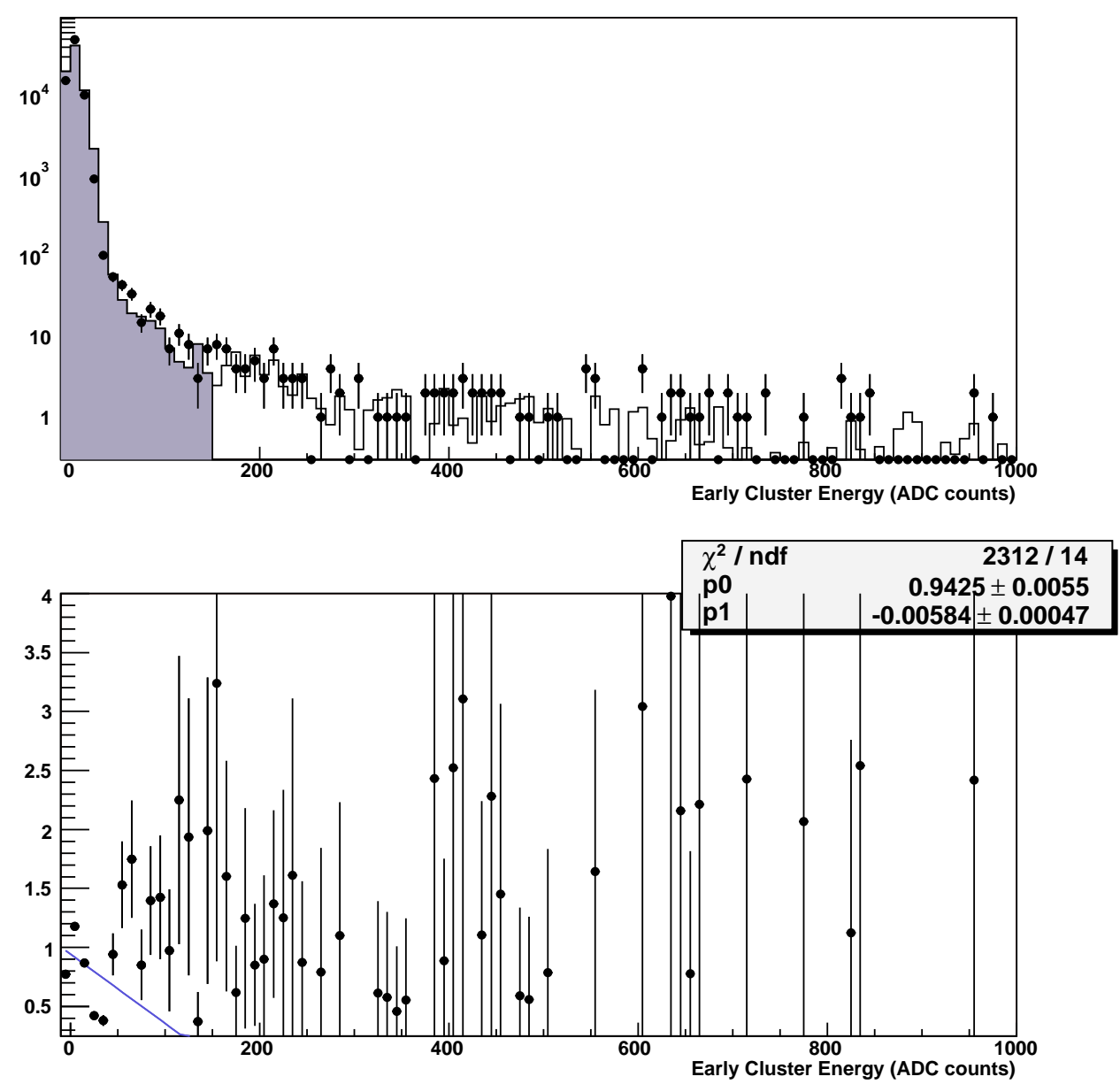

Figure I.15: A plot of the "early" energy of the photon candidate cluster before the cut. Data are points, while the histogram is of Monte Carlo simulated events. The solid part of the histogram denotes the areas that pass after this particular cut. A plot of the ratio of the number of data events to Monte Carlo events, before this cut, is shown on the bottom. The number of Monte Carlo events is normalized to be equal to the total number of data events in this plot. A fit of the ratio using a linear function is also shown. 

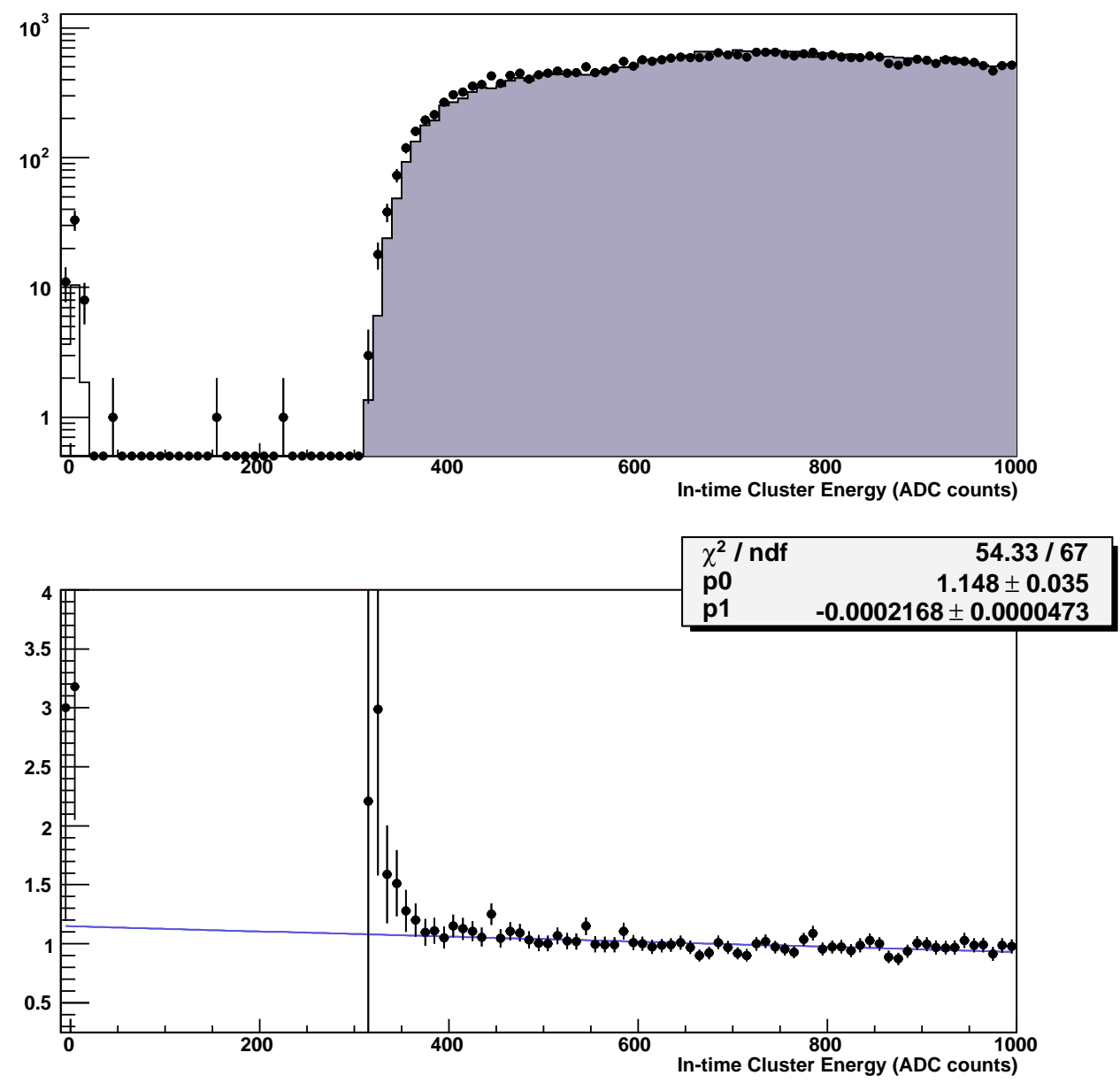

Figure I.16: A plot of the "in-time" energy of the photon candidate cluster before the cut. Data are points, while the histogram is of Monte Carlo simulated events. The solid part of the histogram denotes the areas that pass after this particular cut. A plot of the ratio of the number of data events to Monte Carlo events, before this cut, is shown on the bottom. The number of Monte Carlo events is normalized to be equal to the total number of data events in this plot. A fit of the ratio using a linear function is also shown. 

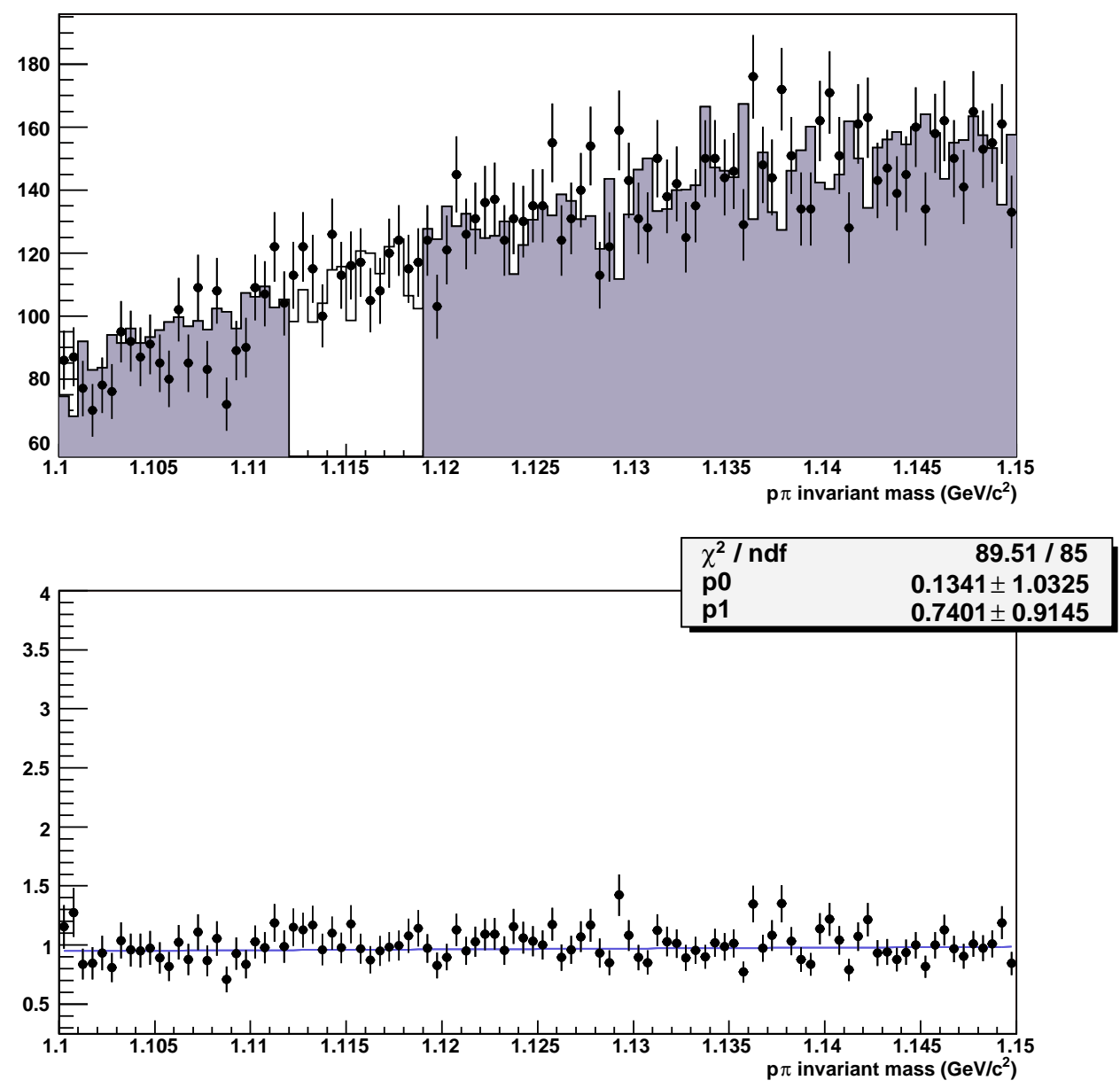

Figure I.17: A plot of the proton/pion invariant mass before the cut. Data are points, while the histogram is of Monte Carlo simulated events. The solid part of the histogram denotes the areas that pass after this particular cut. A plot of the ratio of the number of data events to Monte Carlo events, before this cut, is shown on the bottom. The number of Monte Carlo events is normalized to be equal to the total number of data events in this plot. A fit of the ratio using a linear function is also shown. 

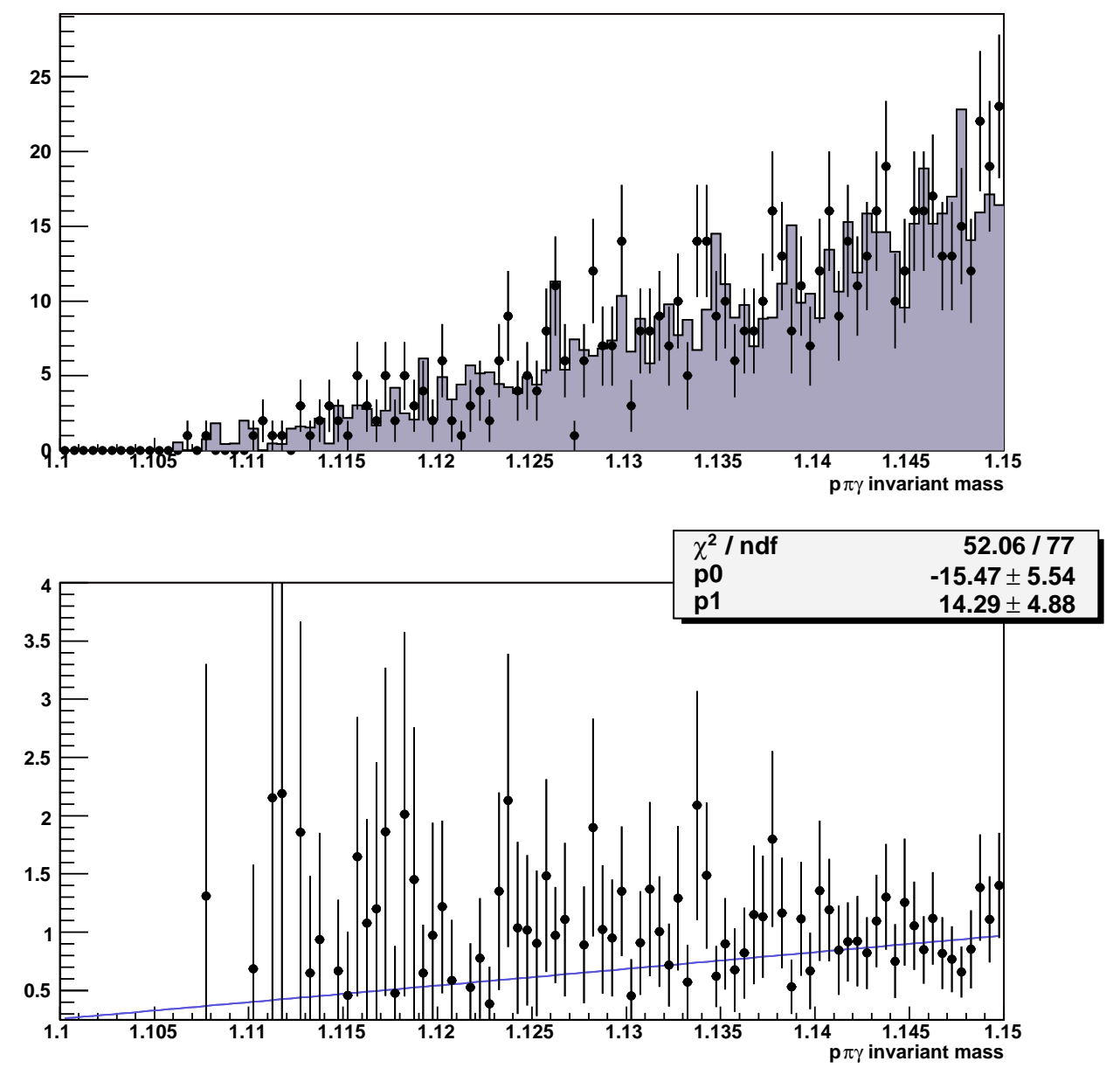

Figure I.18: A plot of the proton/pion/gamma invariant mass, before any cut on this variable. Data are points, while the histogram is of Monte Carlo simulated events. The solid part of the histogram denotes the areas that pass after this particular cut. A plot of the ratio of the number of data events to Monte Carlo events, before this cut, is shown on the bottom. The number of Monte Carlo events is normalized to be equal to the total number of data events in this plot. A fit of the ratio using a linear function is also shown. 

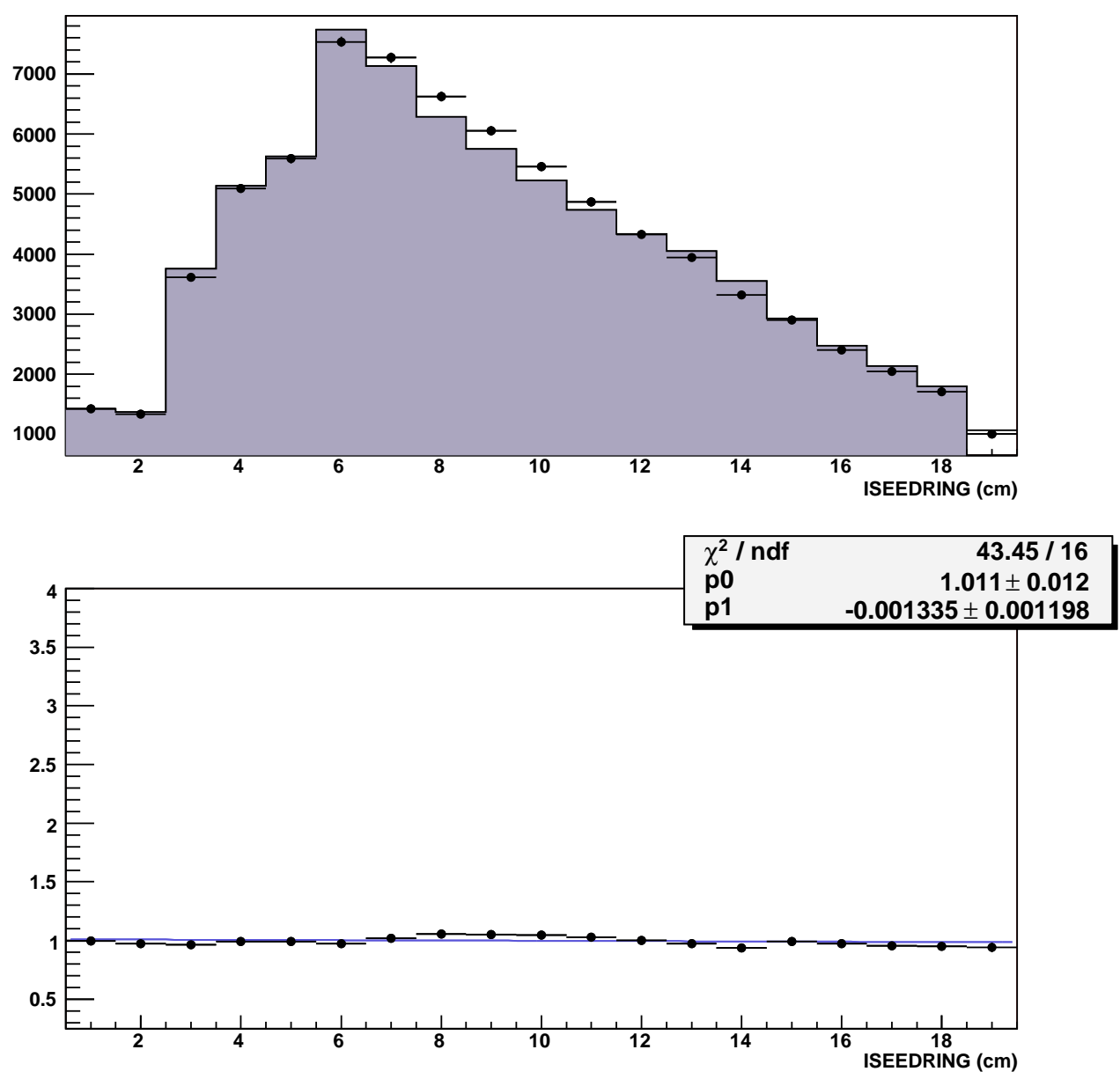

Figure I.19: A plot of the outer photon fiducial cut variable ISEEDRING, before the cut. Data are points, while the histogram is of Monte Carlo simulated events. The solid part of the histogram denotes the areas that pass after this particular cut. A plot of the ratio of the number of data events to Monte Carlo events, before this cut, is shown on the bottom. The number of Monte Carlo events is normalized to be equal to the total number of data events in this plot. A fit of the ratio using a linear function is also shown. 

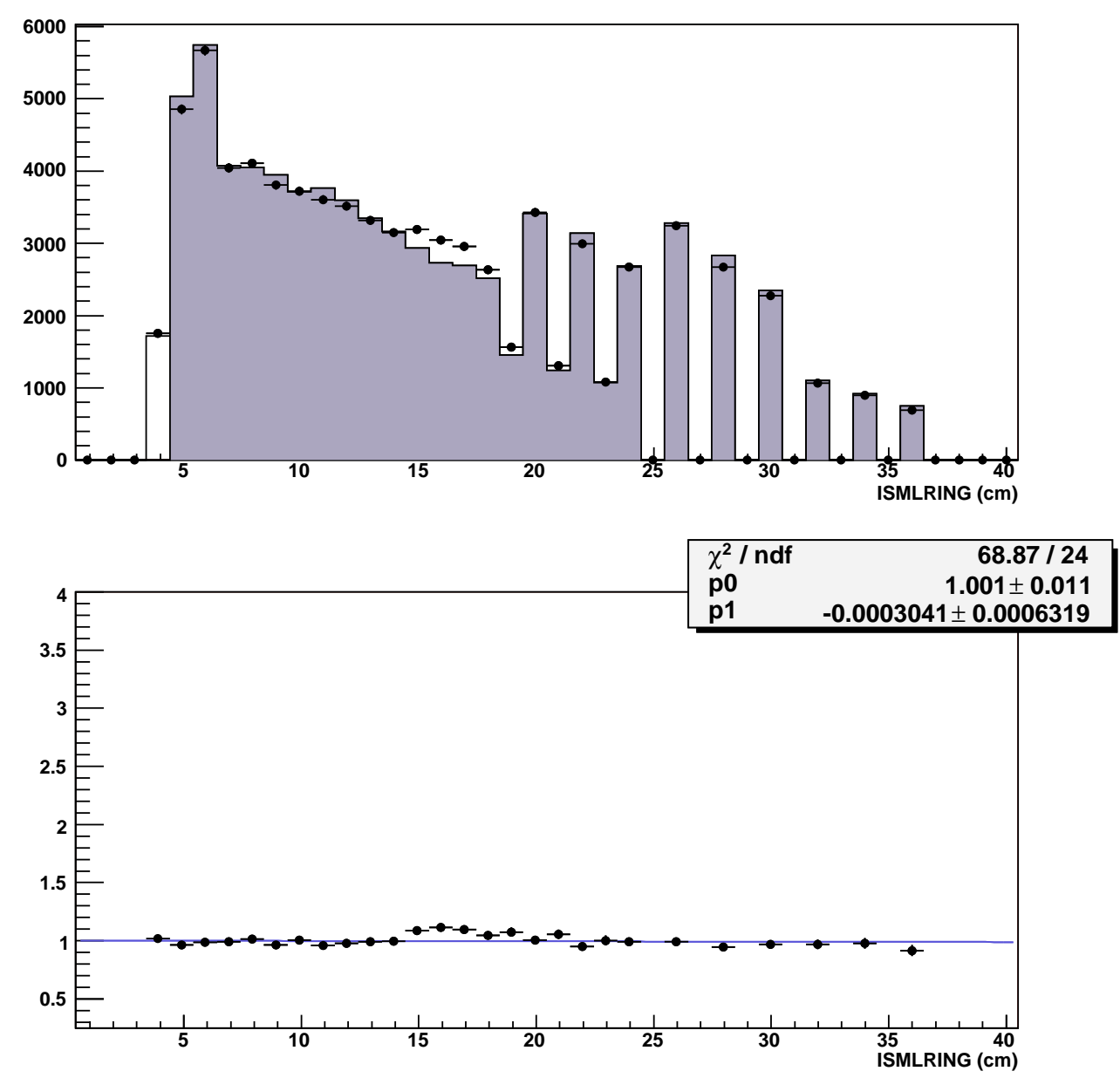

Figure I.20: A plot of the inner photon fiducial cut variable ISMLRNG before the cut. Data are points, while the histogram is of Monte Carlo simulated events. The solid part of the histogram denotes the areas that pass after this particular cut. A plot of the ratio of the number of data events to Monte Carlo events, before this cut, is shown on the bottom. The number of Monte Carlo events is normalized to be equal to the total number of data events in this plot. A fit of the ratio using a linear function is also shown. 

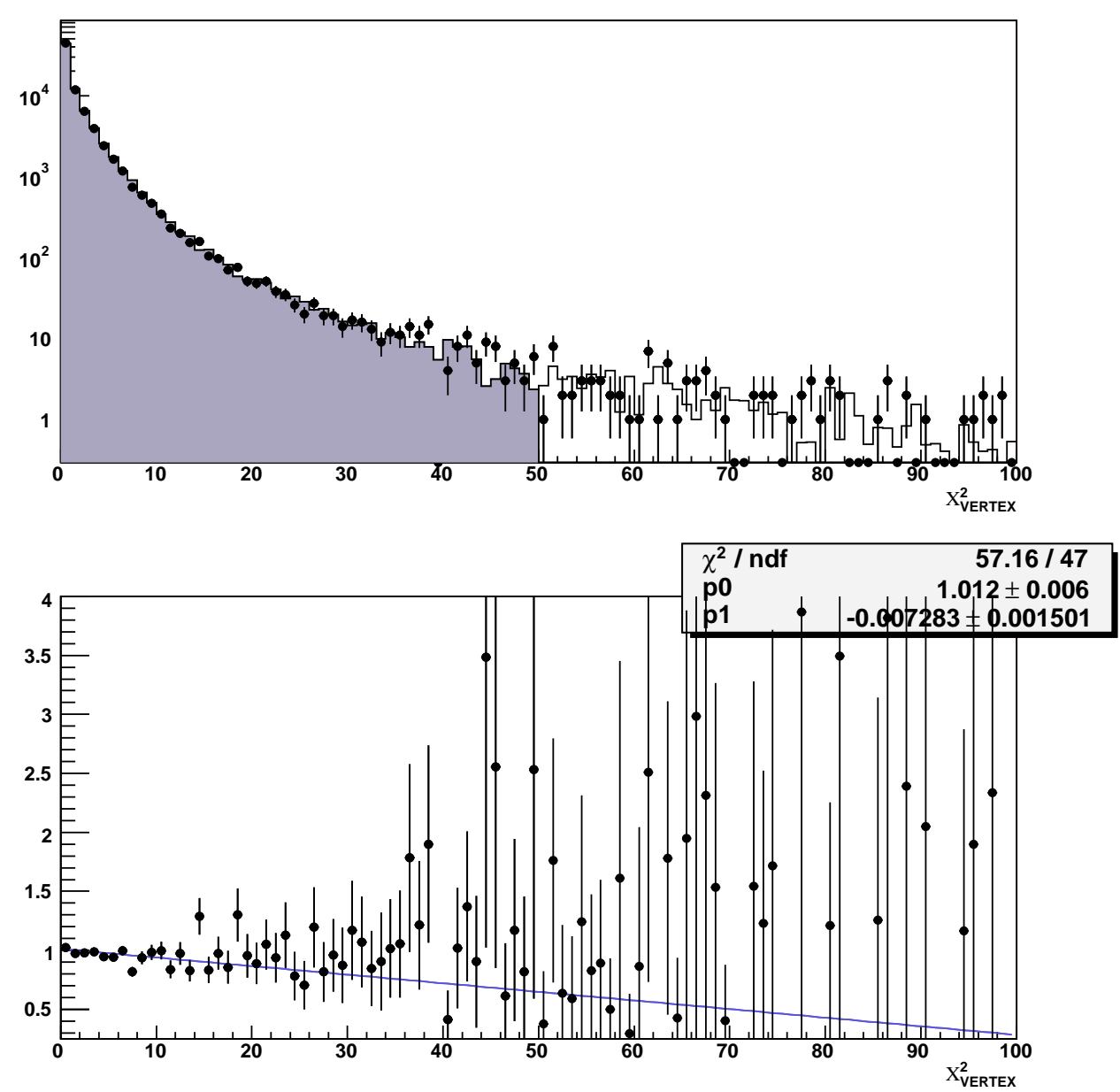

Figure I.21: A plot of vertex $\chi^{2}$ before the cut. Data are points, while the histogram is of Monte Carlo simulated events. The solid part of the histogram denotes the areas that pass after this particular cut. A plot of the ratio of the number of data events to Monte Carlo events, before this cut, is shown on the bottom. The number of Monte Carlo events is normalized to be equal to the total number of data events in this plot. A fit of the ratio using a linear function is also shown. 

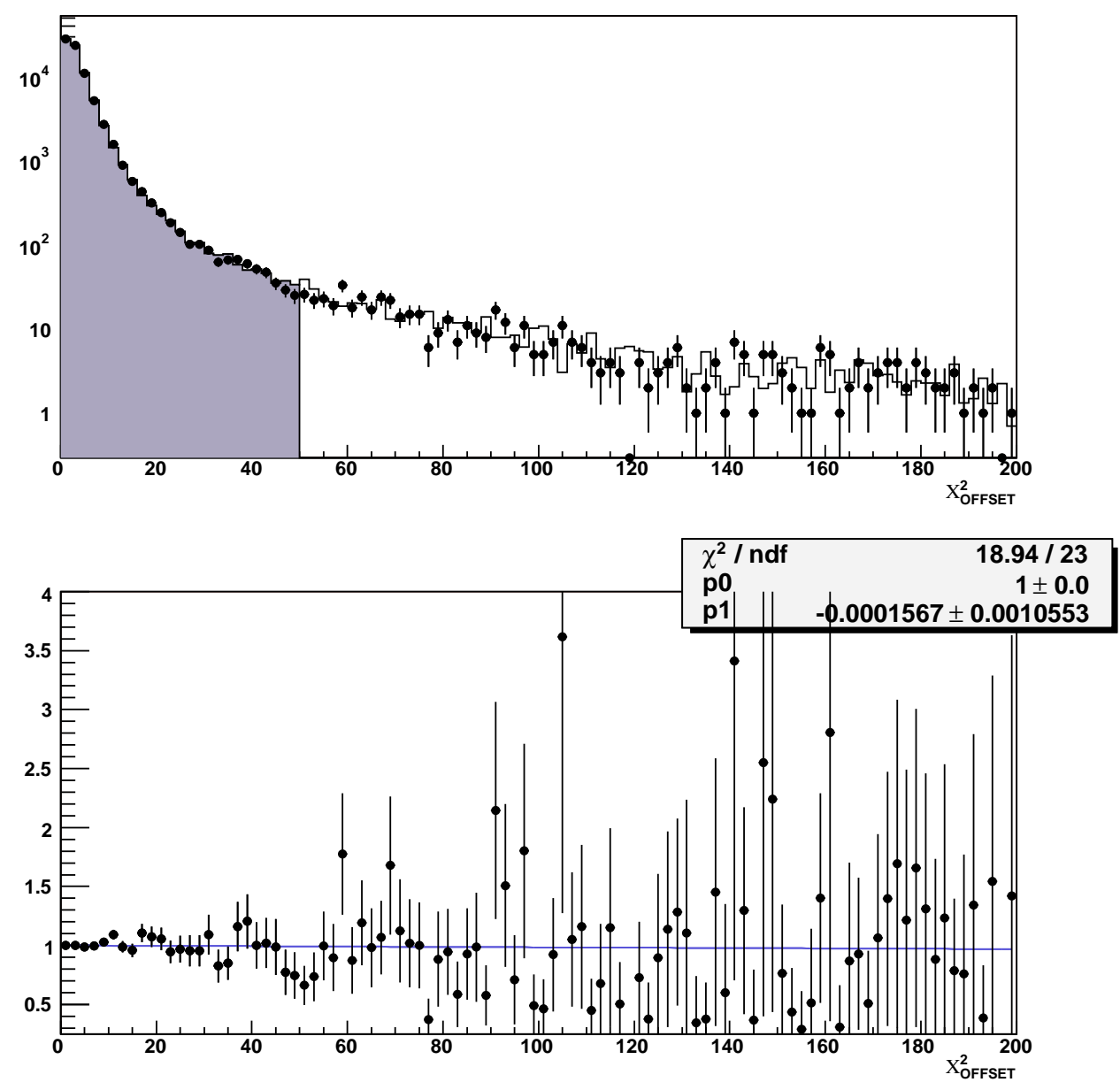

Figure I.22: A plot of the greater of the two track offset $\chi^{2}$ values in each event before the cut. Data are points, while the histogram is of Monte Carlo simulated events. The solid part of the histogram denotes the areas that pass after this particular cut. A plot of the ratio of the number of data events to Monte Carlo events, before this cut, is shown on the bottom. The number of Monte Carlo events is normalized to be equal to the total number of data events in this plot. A fit of the ratio using a linear function is also shown. 

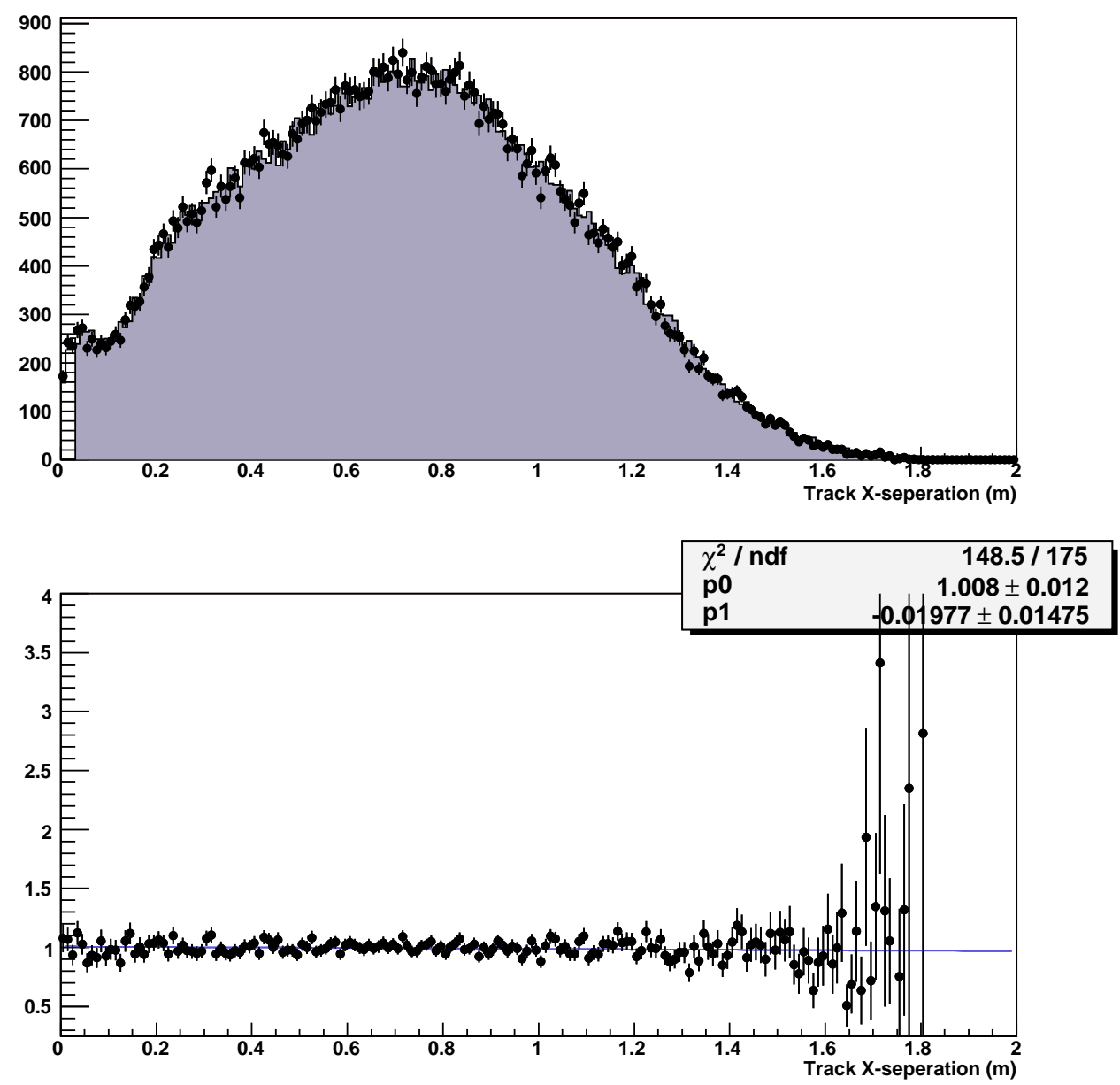

Figure I.23: A plot of the separation of the tracks in the $\mathrm{x}$ direction at the CsI, before the cut. Data are points, while the histogram is of Monte Carlo simulated events. The solid part of the histogram denotes the areas that pass after this particular cut. A plot of the ratio of the number of data events to Monte Carlo events, before this cut, is shown on the bottom. The number of Monte Carlo events is normalized to be equal to the total number of data events in this plot. A fit of the ratio using a linear function is also shown. 

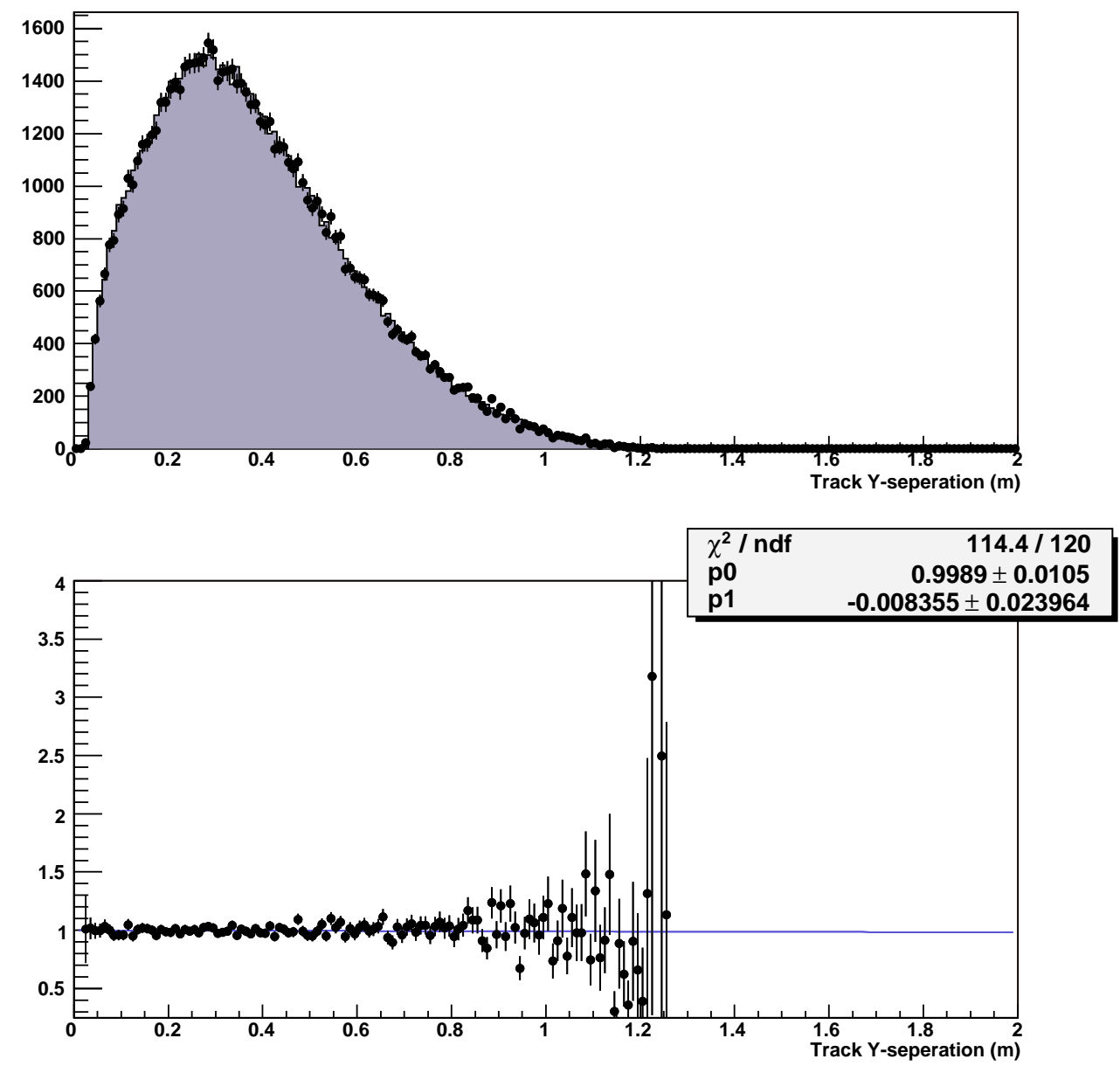

Figure I.24: A plot of the separation of the tracks in the y direction at the CsI, before the cut. Data are points, while the histogram is of Monte Carlo simulated events. The solid part of the histogram denotes the areas that pass after this particular cut. A plot of the ratio of the number of data events to Monte Carlo events, before this cut, is shown on the bottom. The number of Monte Carlo events is normalized to be equal to the total number of data events in this plot. A fit of the ratio using a linear function is also shown. 

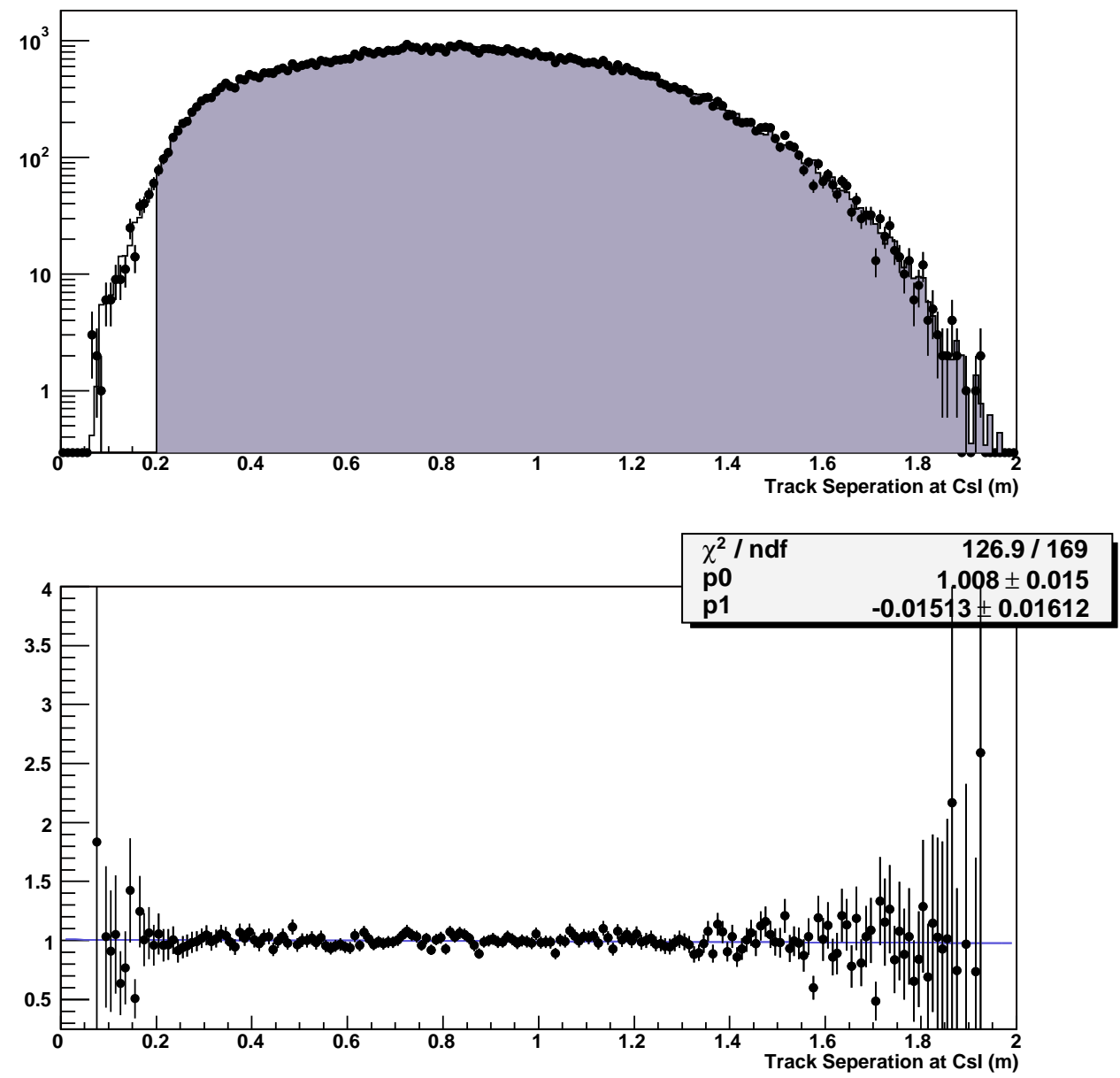

Figure I.25: A plot of the track separation at the CsI before the cut. Data are points, while the histogram is of Monte Carlo simulated events. The solid part of the histogram denotes the areas that pass after this particular cut. A plot of the ratio of the number of data events to Monte Carlo events, before this cut, is shown on the bottom. The number of Monte Carlo events is normalized to be equal to the total number of data events in this plot. A fit of the ratio using a linear function is also shown. 
Appendix $\mathbf{J}$

Plots for 1999 Regenerator Data 

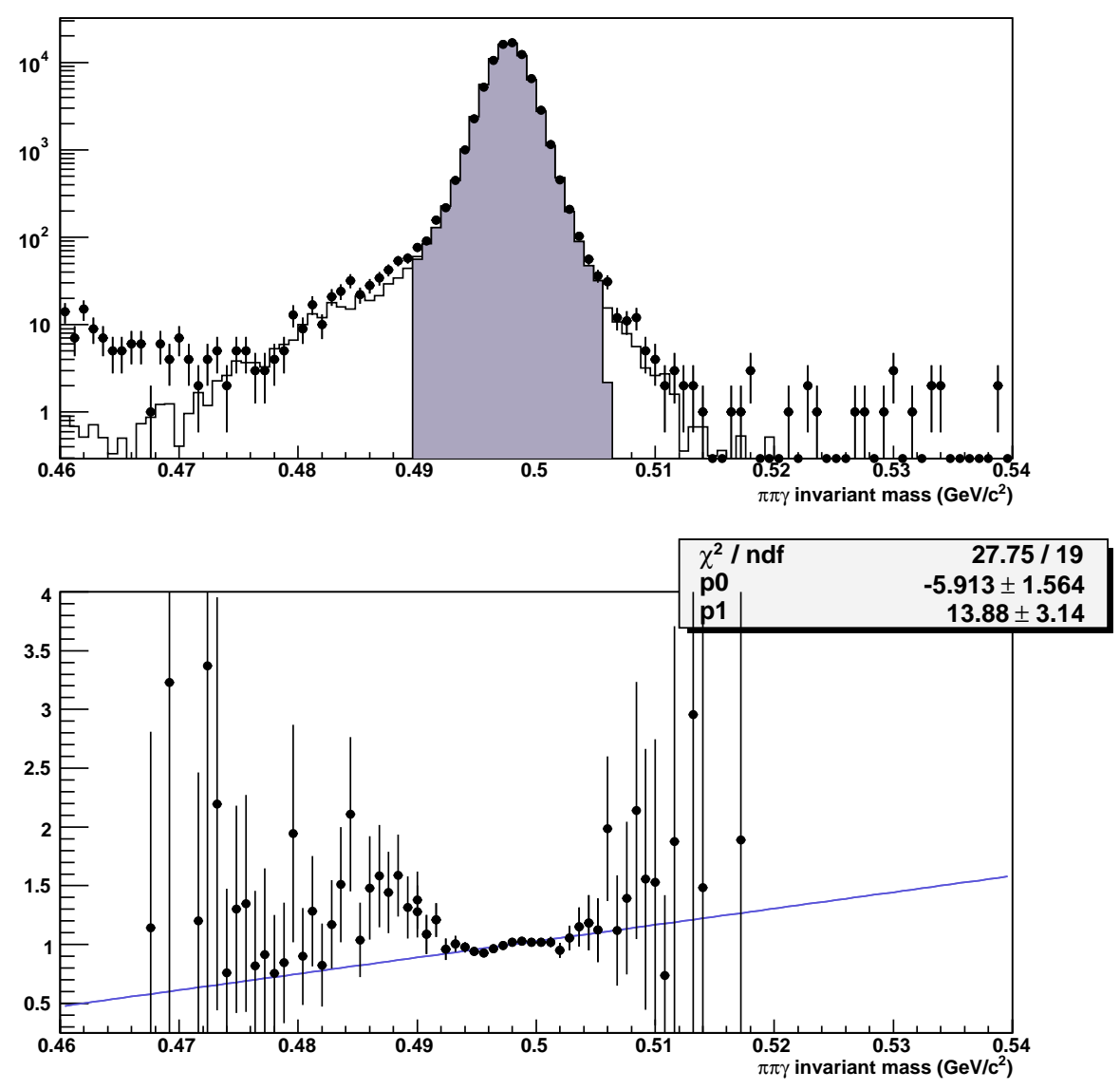

Figure J.1: A plot of the $\pi^{+} \pi^{-} \gamma$ invariant mass before the cut on $M_{\pi^{+} \pi^{-} \gamma}$ but after all other cuts. Data are points, while the histogram is of Monte Carlo simulated events. The solid part of the histogram denotes the areas that pass after this particular cut. A plot of the ratio of the number of data events to Monte Carlo events, before this cut, is shown on the bottom. The number of Monte Carlo events is normalized to be equal to the total number of data events in this plot. A fit of the ratio using a linear function is also shown. 

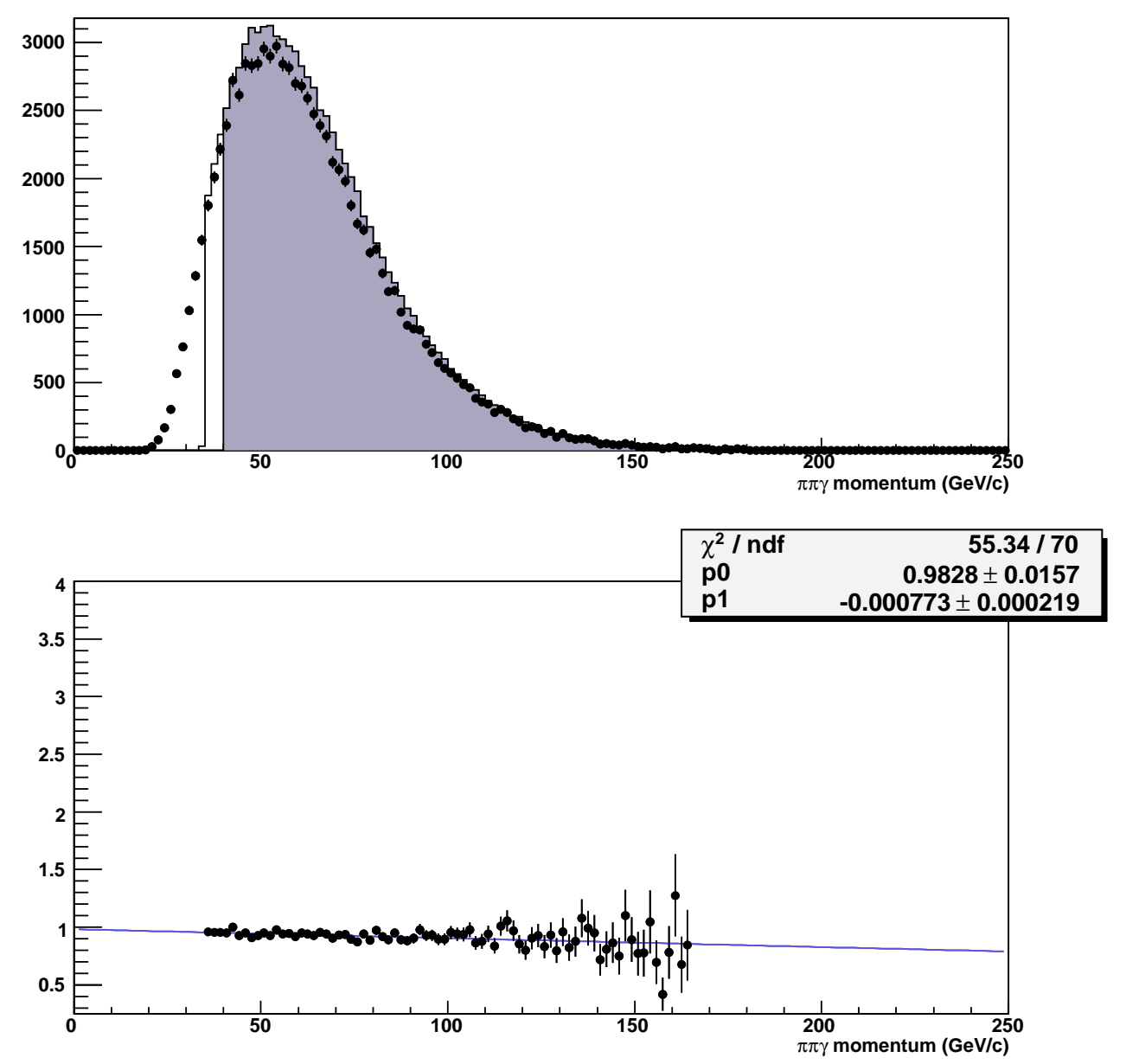

Figure J.2: The reconstructed kaon momentum before the cut. Data are points, while the histogram is of Monte Carlo simulated events. The solid part of the histogram denotes the areas that pass after this particular cut. A plot of the ratio of the number of data events to Monte Carlo events, before this cut, is shown on the bottom. The number of Monte Carlo events is normalized to be equal to the total number of data events in this plot. A fit of the ratio using a linear function is also shown. 

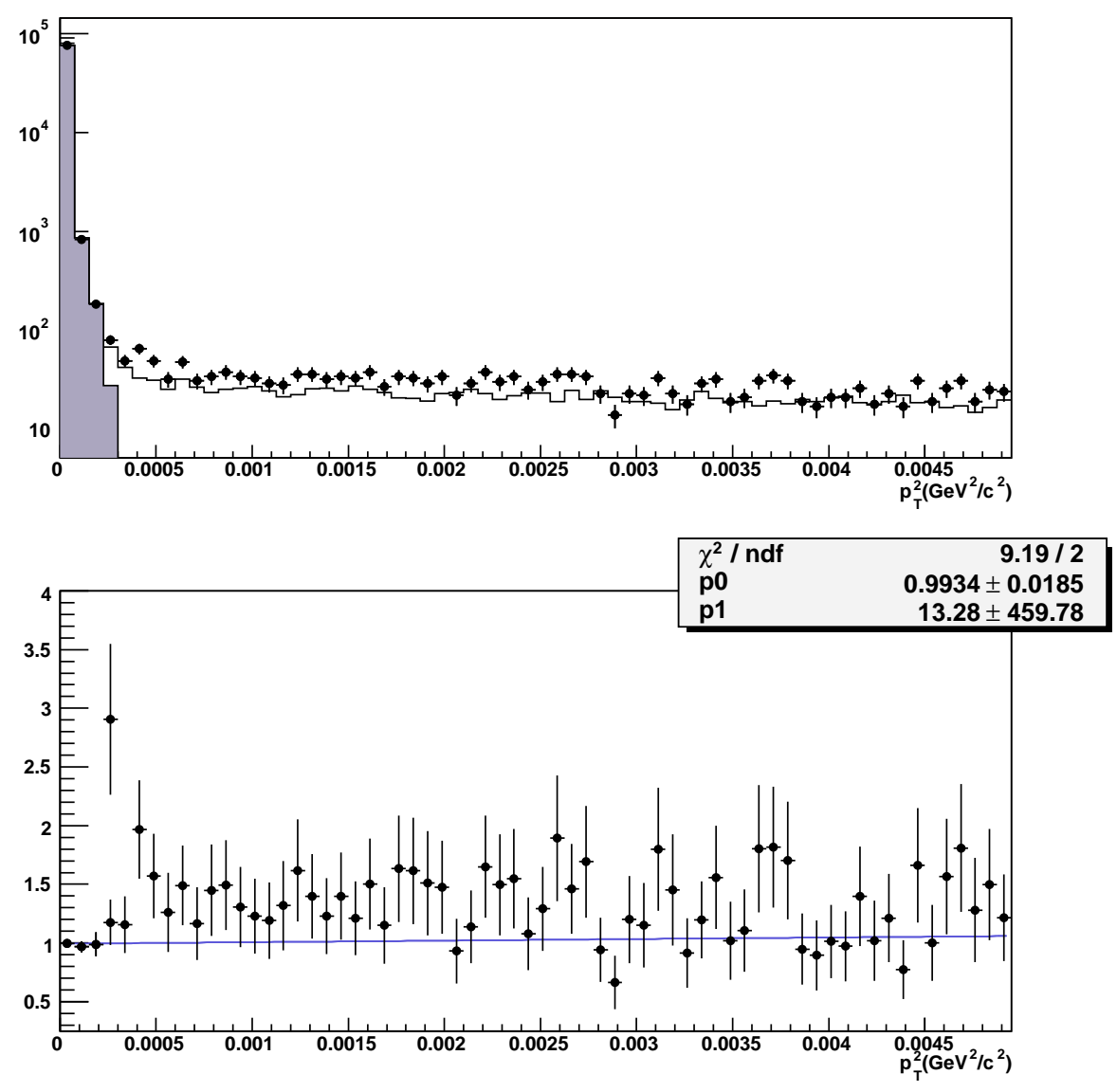

Figure J.3: A plot of $P_{T}^{2}$ with respect to the downstream face of the regenerator for the $\pi^{+} \pi^{-} \gamma$ system before the cut. Data are points, while the histogram is of Monte Carlo simulated events. The solid part of the histogram denotes the areas that pass after this particular cut. A plot of the ratio of the number of data events to Monte Carlo events, before this cut, is shown on the bottom. The number of Monte Carlo events is normalized to be equal to the total number of data events in this plot. A fit of the ratio using a linear function is also shown. 

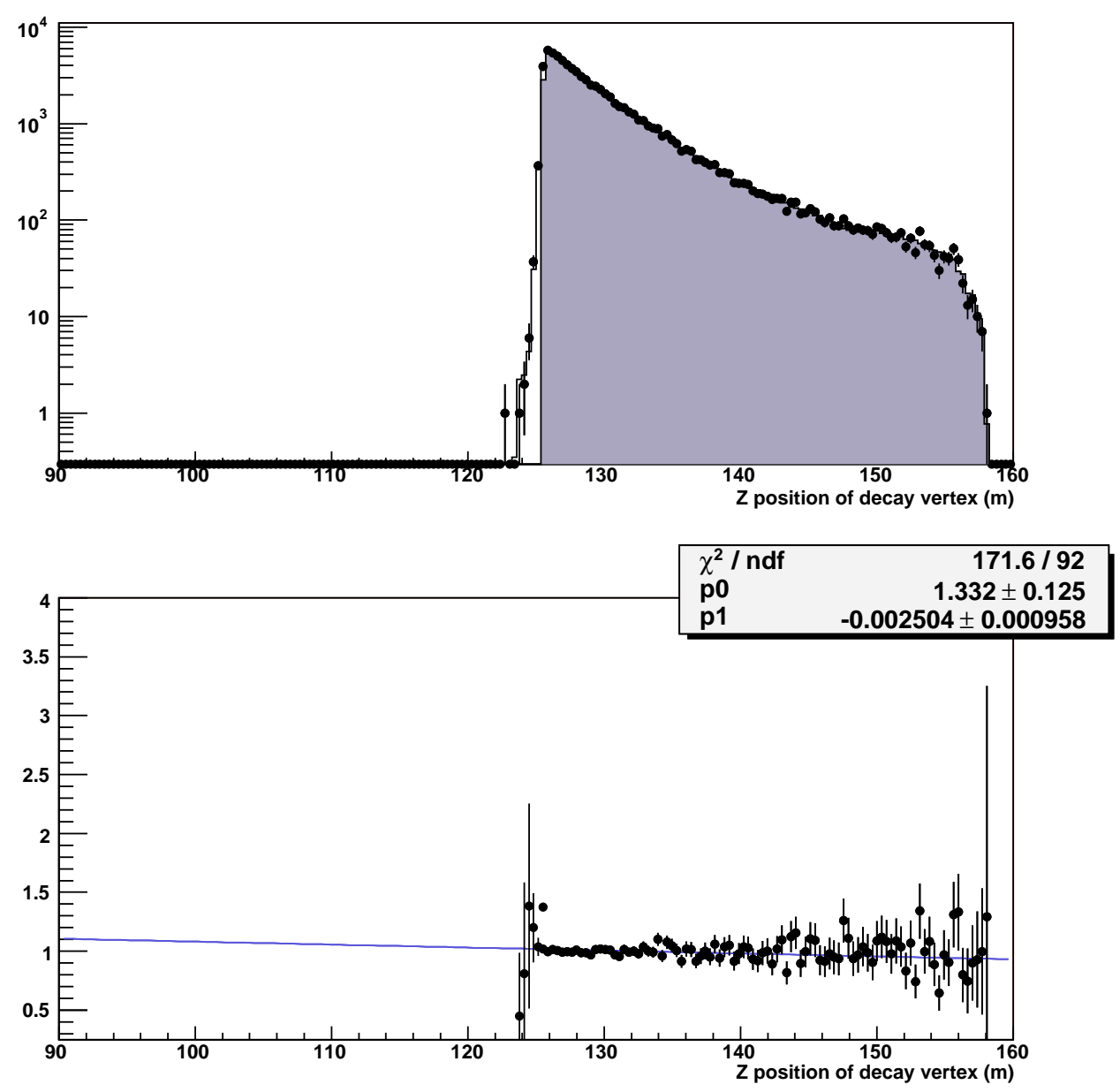

Figure J.4: A plot of the $\mathrm{z}$ location of the decay vertex, before the cut is applied. Data are points, while the histogram is of Monte Carlo simulated events. The solid part of the histogram denotes the areas that pass after this particular cut. A plot of the ratio of the number of data events to Monte Carlo events, before this cut, is shown on the bottom. The number of Monte Carlo events is normalized to be equal to the total number of data events in this plot. A fit of the ratio using a linear function is also shown. 

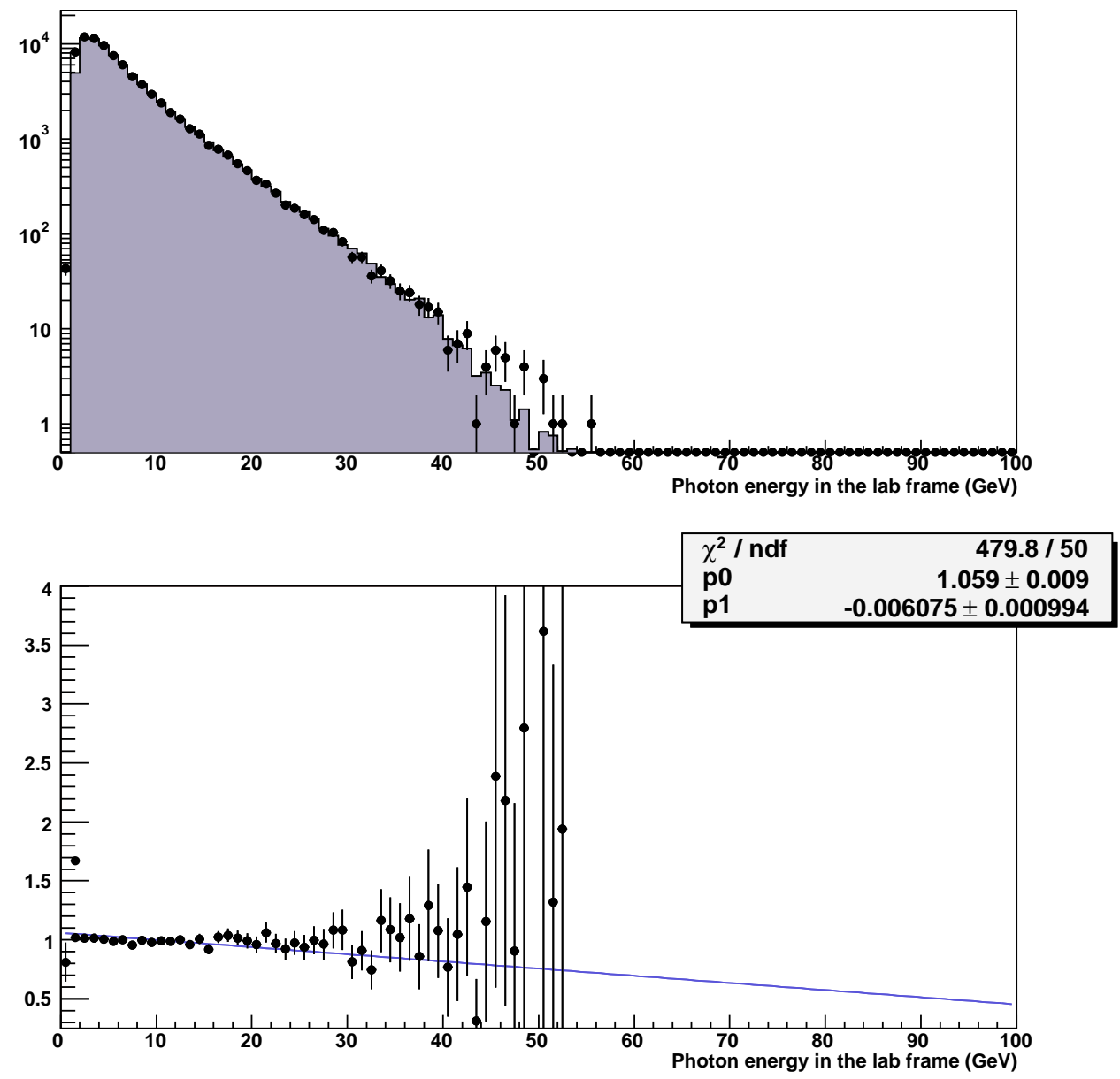

Figure J.5: The photon cluster energy in the lab frame, before the final analysis cut. Data are points, while the histogram is of Monte Carlo simulated events. The solid part of the histogram denotes the areas that pass after this particular cut. A plot of the ratio of the number of data events to Monte Carlo events, before this cut, is shown on the bottom. The number of Monte Carlo events is normalized to be equal to the total number of data events in this plot. A fit of the ratio using a linear function is also shown. 

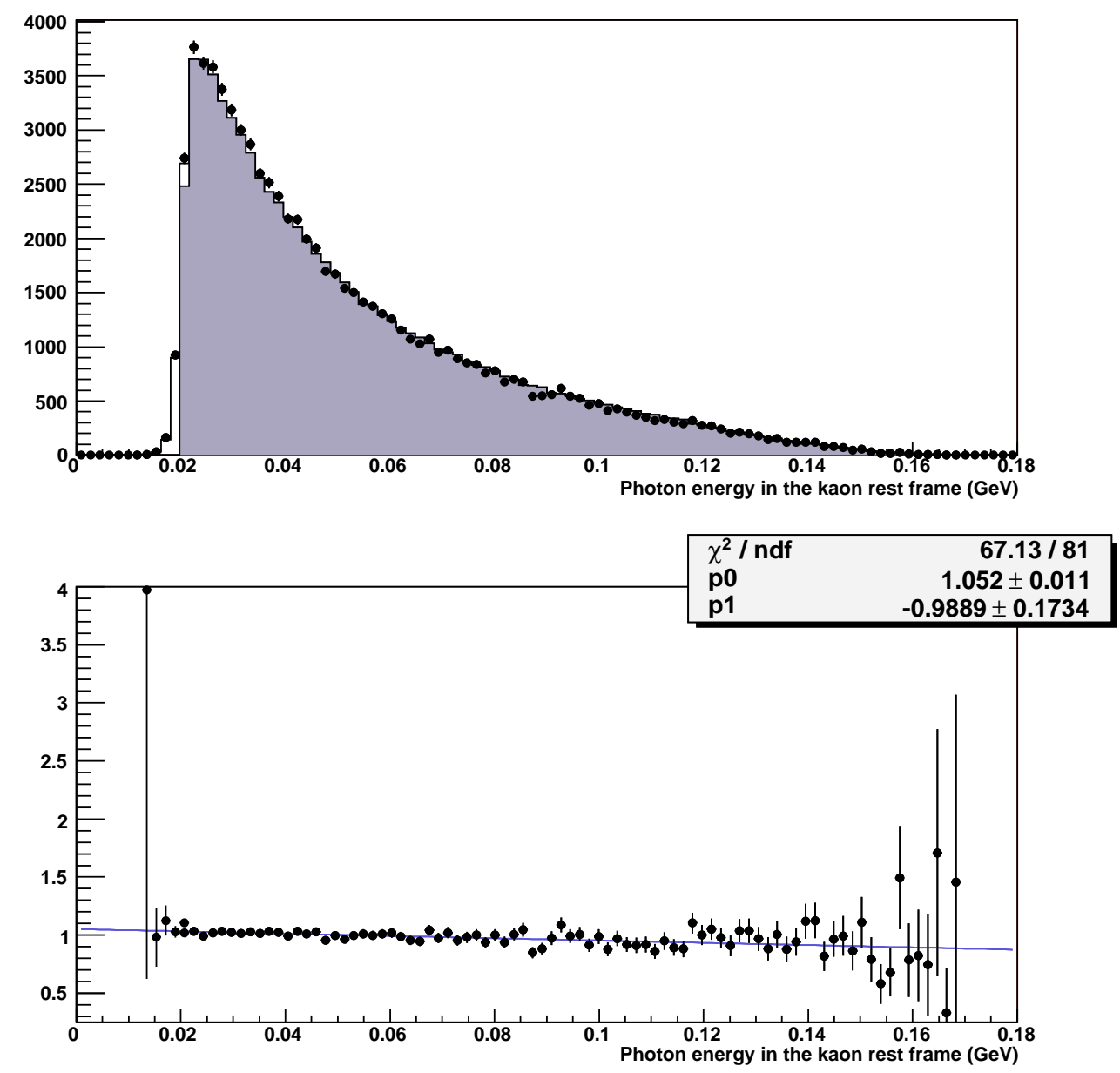

Figure J.6: A plot of the photon energy in the kaon rest frame before the cut. Data are points, while the histogram is of Monte Carlo simulated events. The solid part of the histogram denotes the areas that pass after this particular cut. A plot of the ratio of the number of data events to Monte Carlo events, before this cut, is shown on the bottom. The number of Monte Carlo events is normalized to be equal to the total number of data events in this plot. A fit of the ratio using a linear function is also shown. 

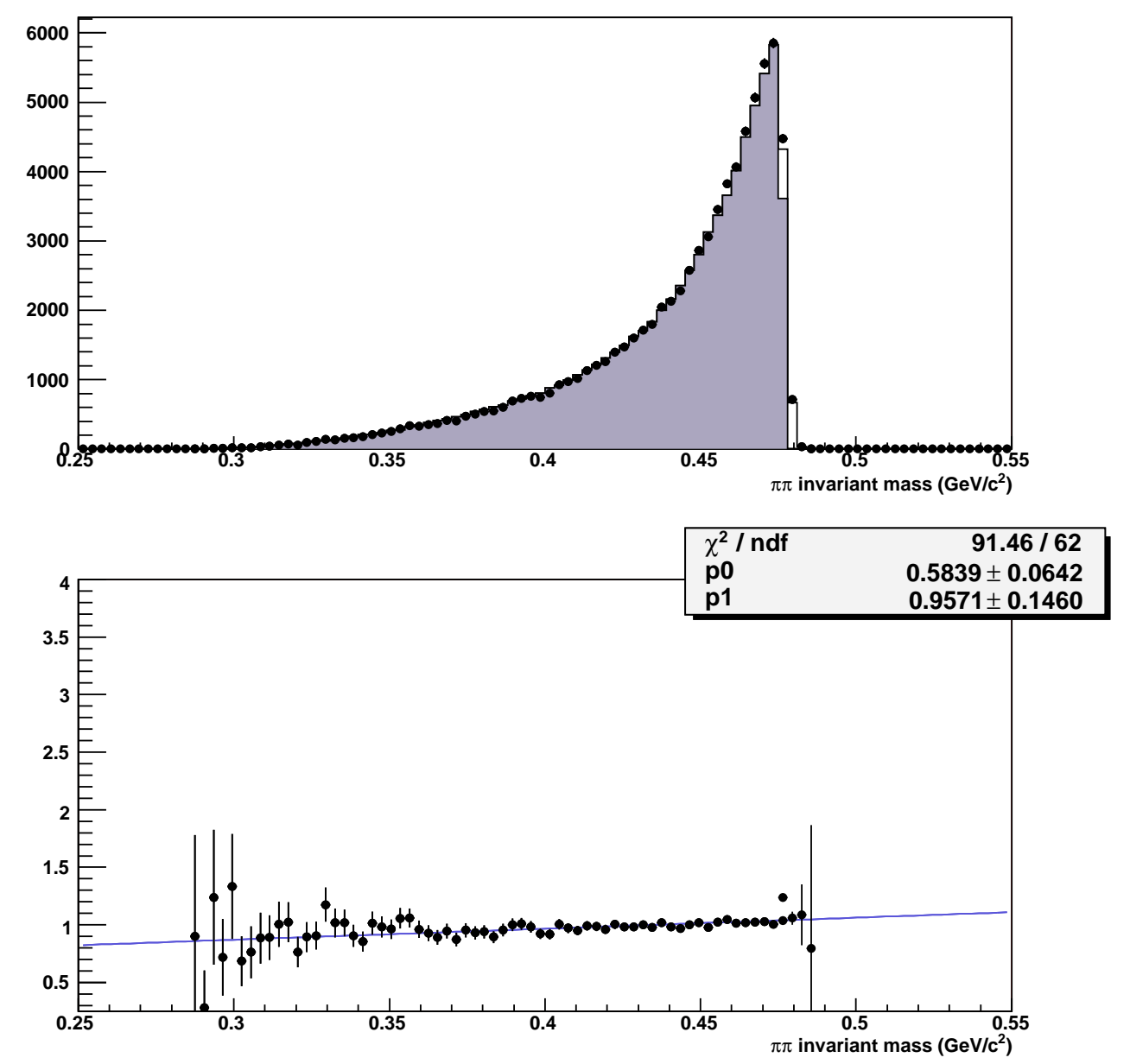

Figure J.7: A plot of the invariant mass of the pion pair before the $M_{\pi^{+} \pi^{-}}$cut. Note that any cut on $E_{\gamma}^{* K I N}$ will also be reflected here. Data are points, while the histogram is of Monte Carlo simulated events. The solid part of the histogram denotes the areas that pass after this particular cut. A plot of the ratio of the number of data events to Monte Carlo events, before this cut, is shown on the bottom. The number of Monte Carlo events is normalized to be equal to the total number of data events in this plot. A fit of the ratio using a linear function is also shown. 

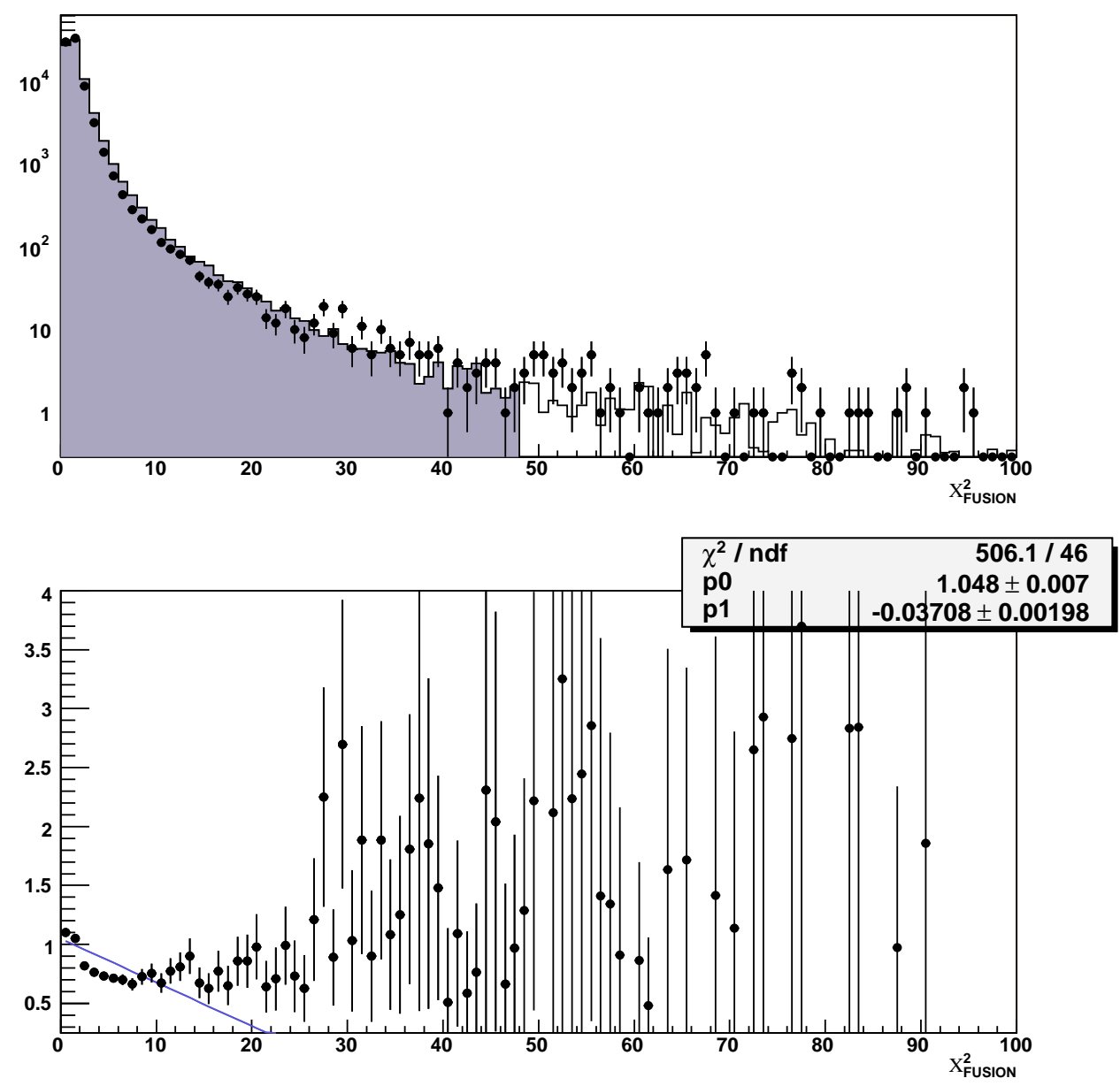

Figure J.8: A plot of fusion $\chi^{2}$ before the cut on this variable. Data are points, while the histogram is of Monte Carlo simulated events. The solid part of the histogram denotes the areas that pass after this particular cut. A plot of the ratio of the number of data events to Monte Carlo events, before this cut, is shown on the bottom. The number of Monte Carlo events is normalized to be equal to the total number of data events in this plot. A fit of the ratio using a linear function is also shown. 

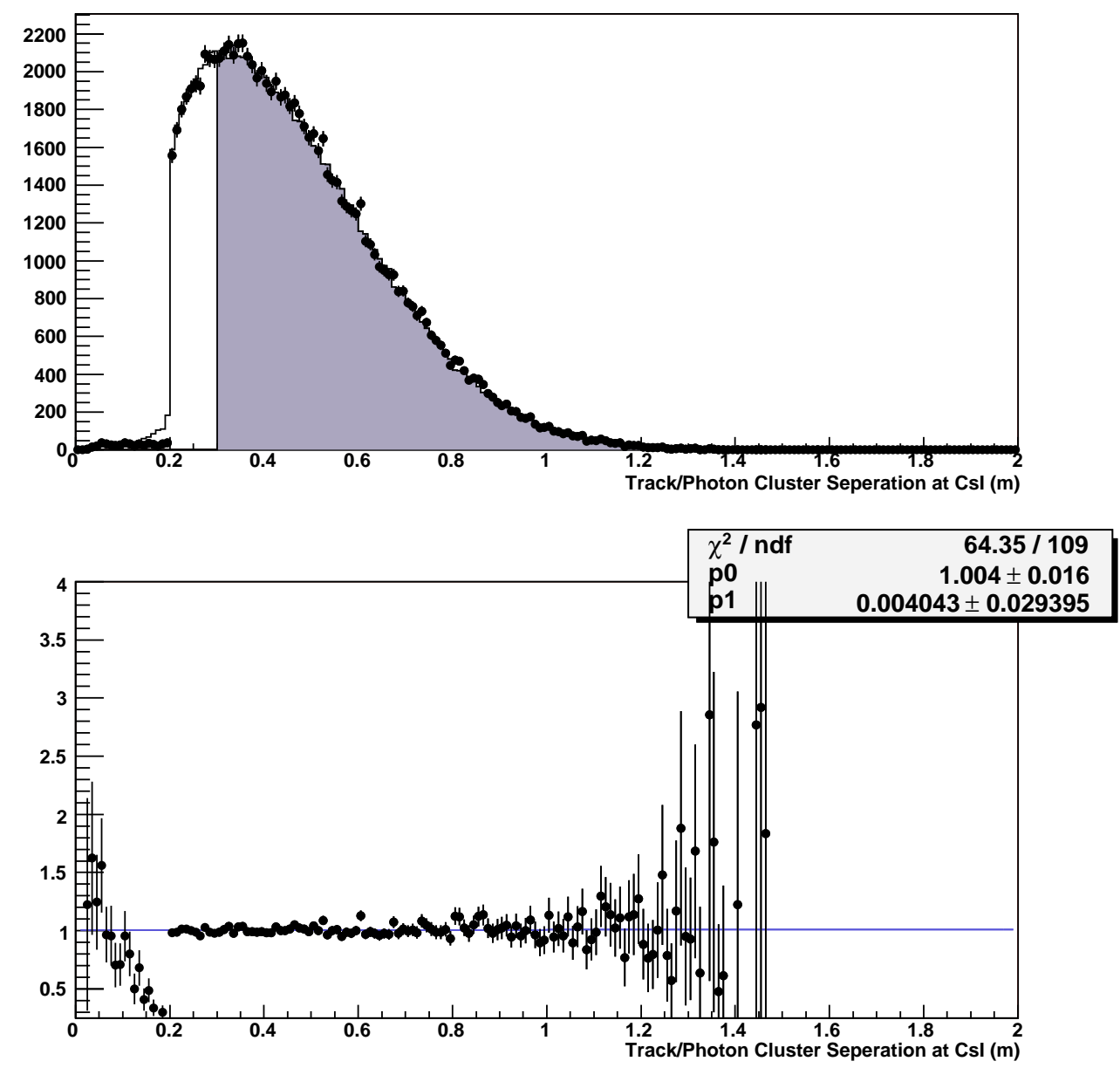

Figure J.9: Minimum of the two pion/gamma separations at the CsI for each event, before the cut. Data are points, while the histogram is of Monte Carlo simulated events. The solid part of the histogram denotes the areas that pass after this particular cut. A plot of the ratio of the number of data events to Monte Carlo events, before this cut, is shown on the bottom. The number of Monte Carlo events is normalized to be equal to the total number of data events in this plot. A fit of the ratio using a linear function is also shown. 

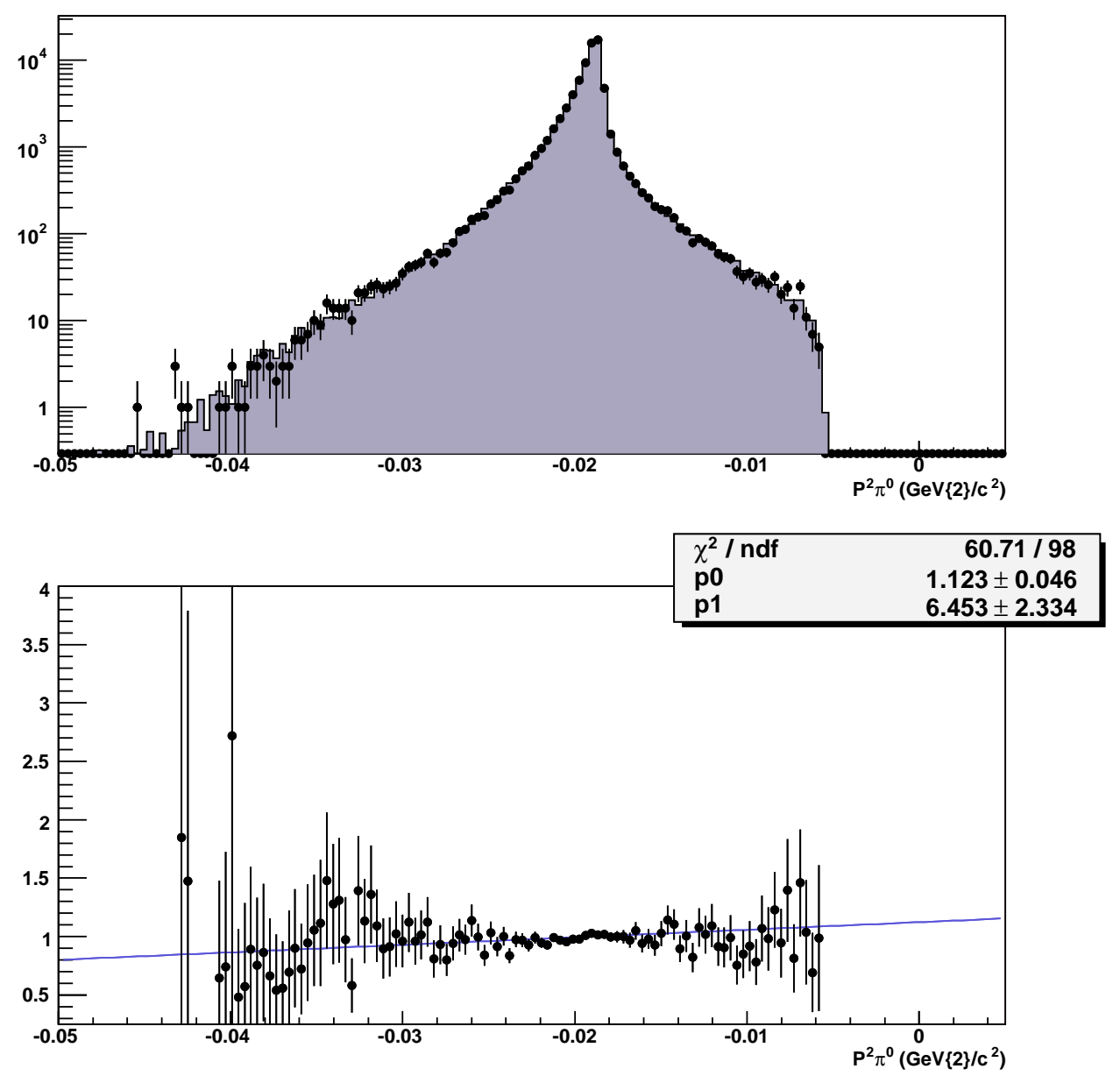

Figure J.10: A plot of $P_{\pi^{0}}^{2}$ with respect to the downstream face of the regenerator, before the final analysis cut. Data are points, while the histogram is of Monte Carlo simulated events. The solid part of the histogram denotes the areas that pass after this particular cut. A plot of the ratio of the number of data events to Monte Carlo events, before this cut, is shown on the bottom. The number of Monte Carlo events is normalized to be equal to the total number of data events in this plot. A fit of the ratio using a linear function is also shown. 

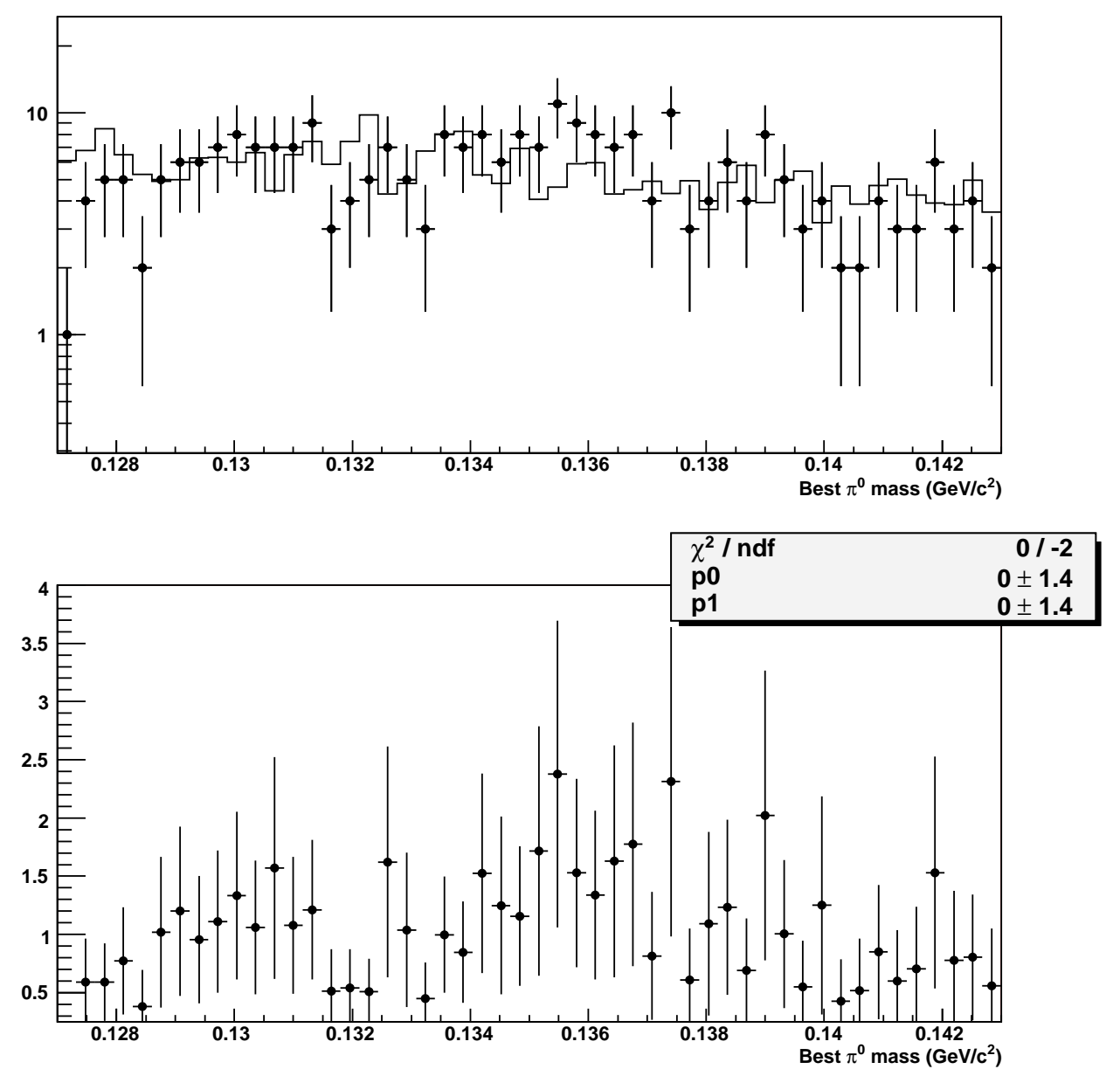

Figure J.11: A plot of the best $\pi^{0}$ mass found in each event using two clusters in the CsI calorimeter, before the cut on this variable. Data are points, while the histogram is of Monte Carlo simulated events. The solid part of the histogram denotes the areas that pass after this particular cut. A plot of the ratio of the number of data events to Monte Carlo events, before this cut, is shown on the bottom. The number of Monte Carlo events is normalized to be equal to the total number of data events in this plot. A fit of the ratio using a linear function is also shown. 

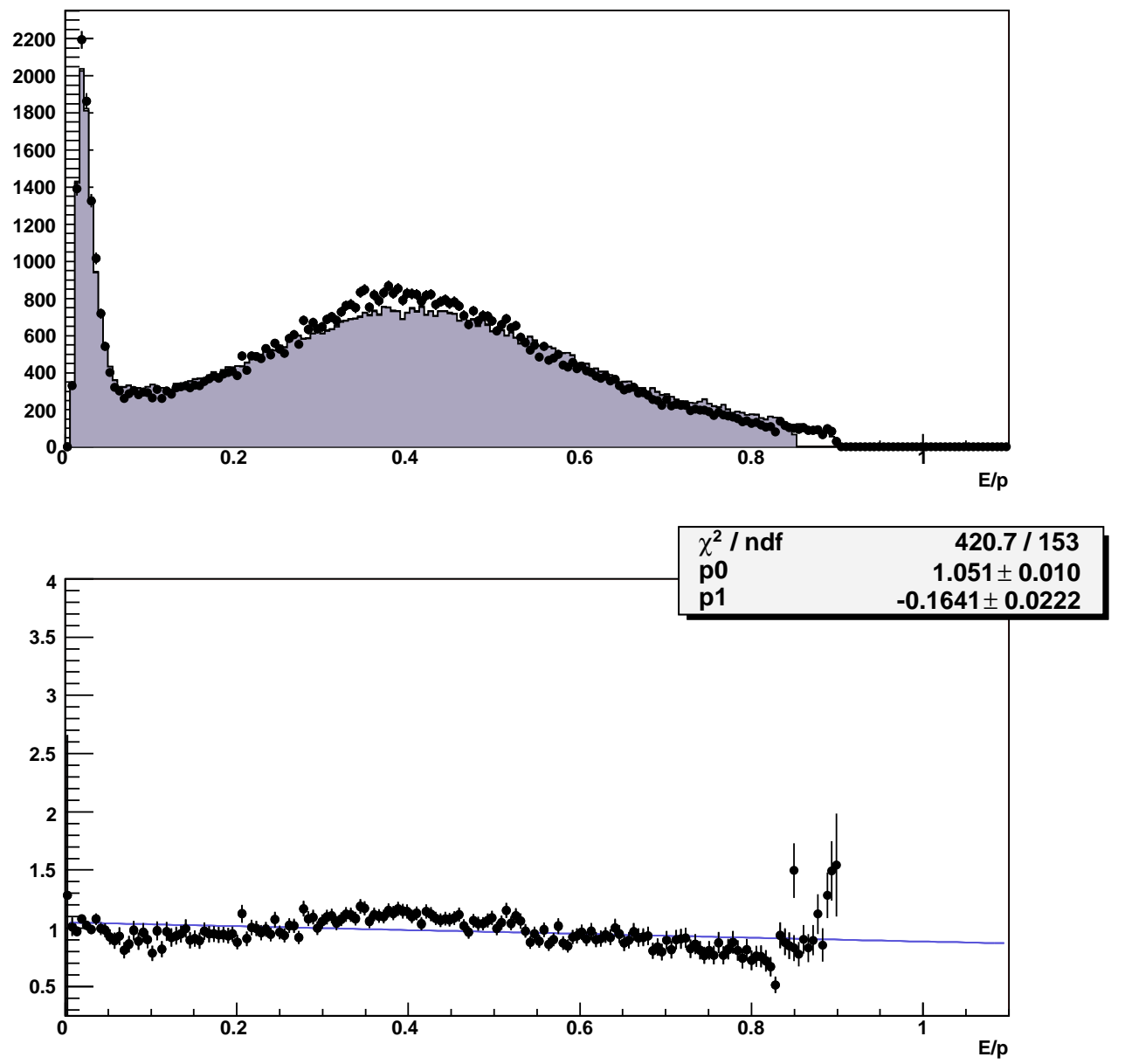

Figure J.12: A plot of the greater of the two values of $\mathrm{E} / \mathrm{p}$ for each event, before the final E/p cut. Data are points, while the histogram is of Monte Carlo simulated events. The solid part of the histogram denotes the areas that pass after this particular cut. A plot of the ratio of the number of data events to Monte Carlo events, before this cut, is shown on the bottom. The number of Monte Carlo events is normalized to be equal to the total number of data events in this plot. A fit of the ratio using a linear function is also shown. 

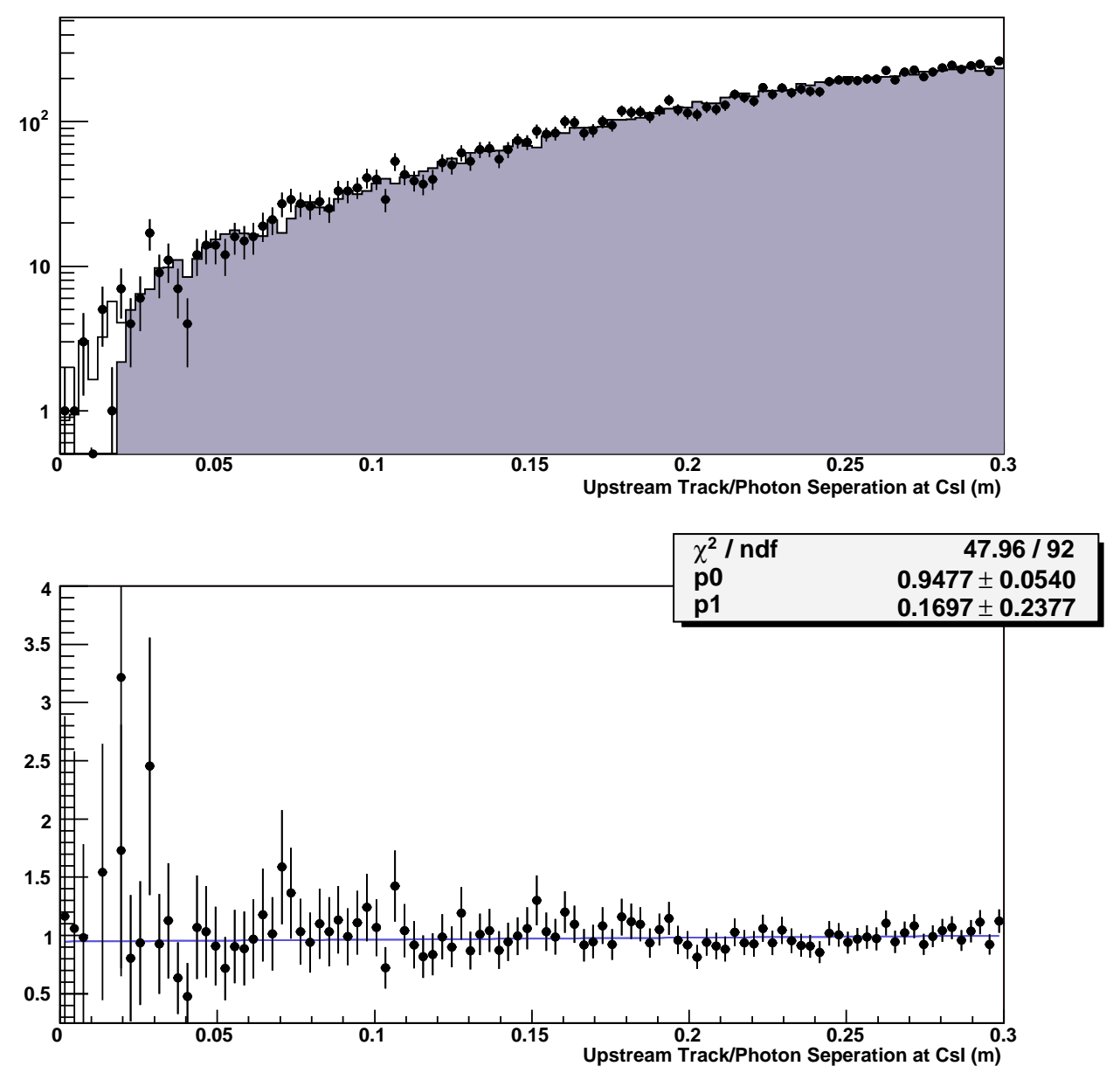

Figure J.13: A plot of the upstream track/photon separation distance at the CsI before the cut. Data are points, while the histogram is of Monte Carlo simulated events. The solid part of the histogram denotes the areas that pass after this particular cut. A plot of the ratio of the number of data events to Monte Carlo events, before this cut, is shown on the bottom. The number of Monte Carlo events is normalized to be equal to the total number of data events in this plot. A fit of the ratio using a linear function is also shown. 

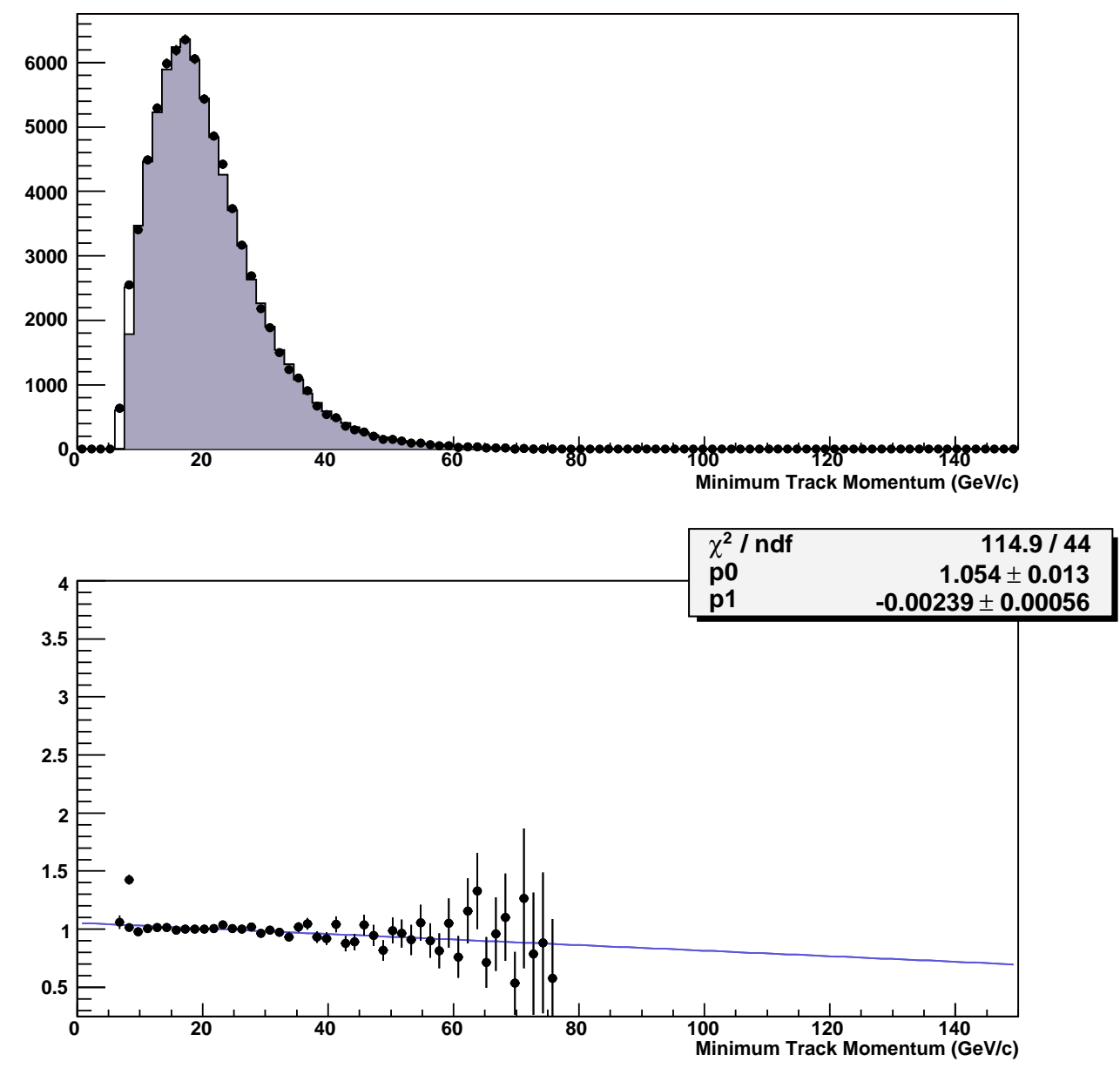

Figure J.14: A plot of the lesser of two track momenta per event before the cut. Data are points, while the histogram is of Monte Carlo simulated events. The solid part of the histogram denotes the areas that pass after this particular cut. A plot of the ratio of the number of data events to Monte Carlo events, before this cut, is shown on the bottom. The number of Monte Carlo events is normalized to be equal to the total number of data events in this plot. A fit of the ratio using a linear function is also shown. 

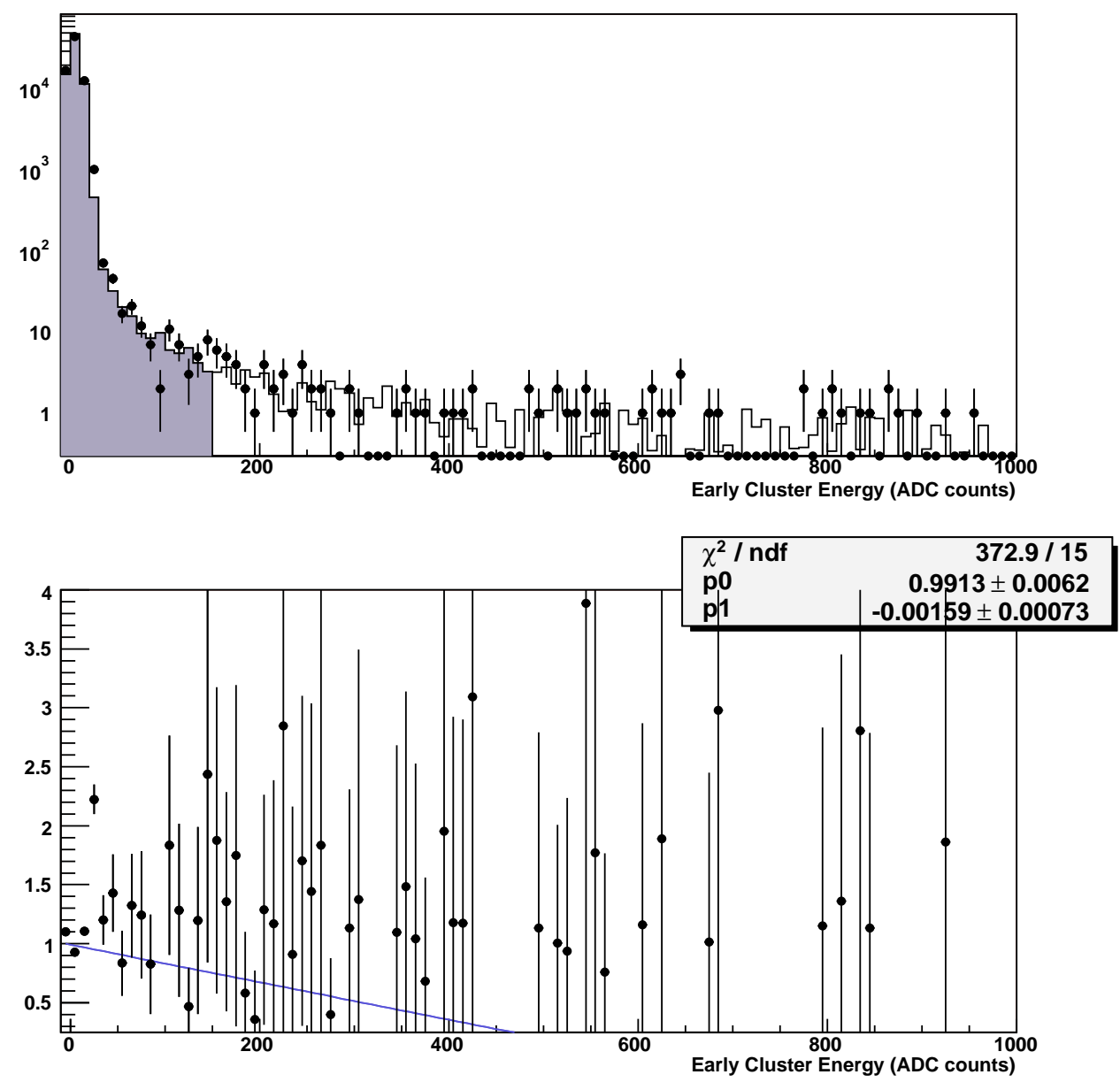

Figure J.15: A plot of the "early" energy of the photon candidate cluster before the cut. Data are points, while the histogram is of Monte Carlo simulated events. The solid part of the histogram denotes the areas that pass after this particular cut. A plot of the ratio of the number of data events to Monte Carlo events, before this cut, is shown on the bottom. The number of Monte Carlo events is normalized to be equal to the total number of data events in this plot. A fit of the ratio using a linear function is also shown. 

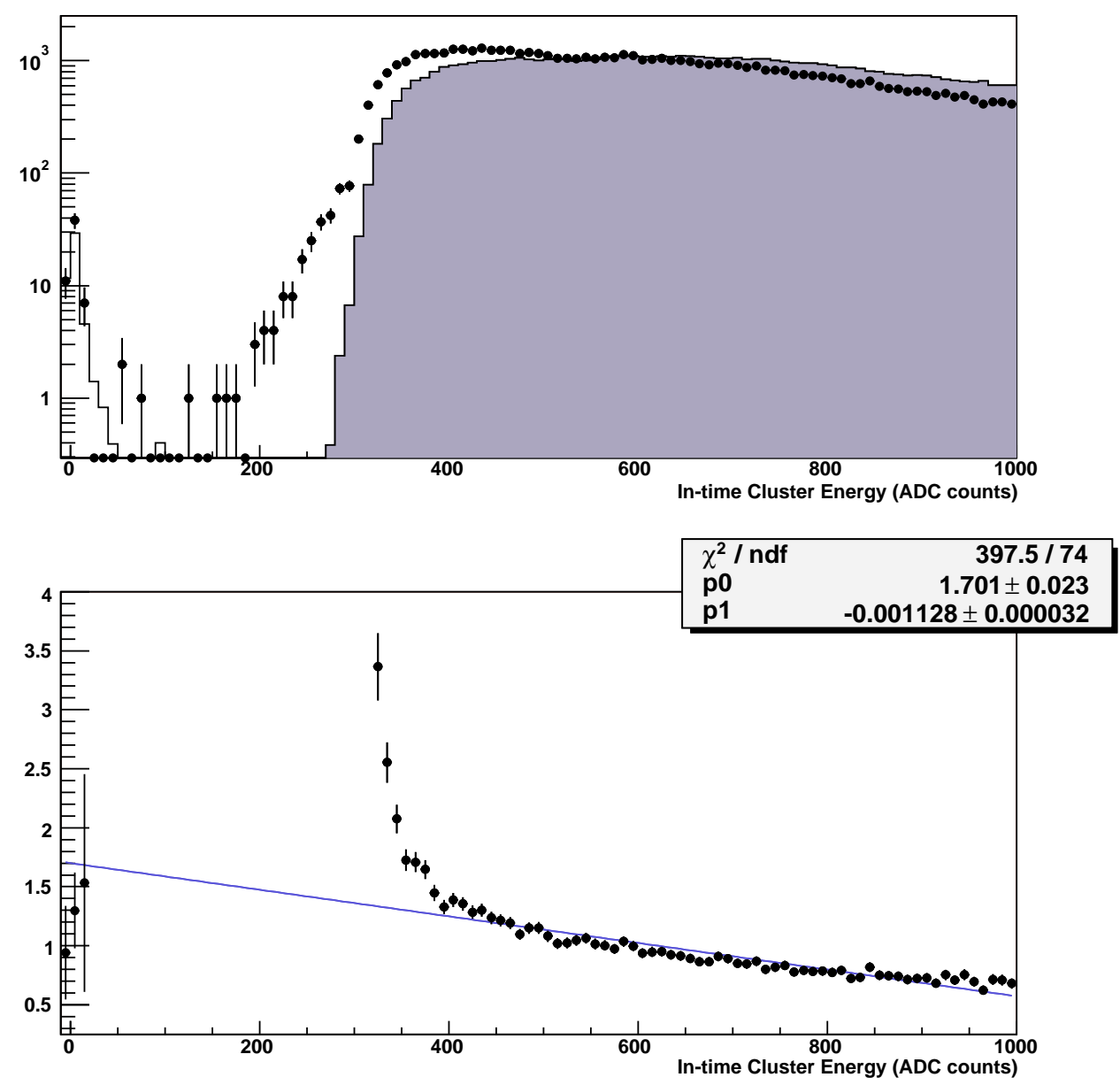

Figure J.16: A plot of the "in-time" energy of the photon candidate cluster before the cut. Data are points, while the histogram is of Monte Carlo simulated events. The solid part of the histogram denotes the areas that pass after this particular cut. A plot of the ratio of the number of data events to Monte Carlo events, before this cut, is shown on the bottom. The number of Monte Carlo events is normalized to be equal to the total number of data events in this plot. A fit of the ratio using a linear function is also shown. 

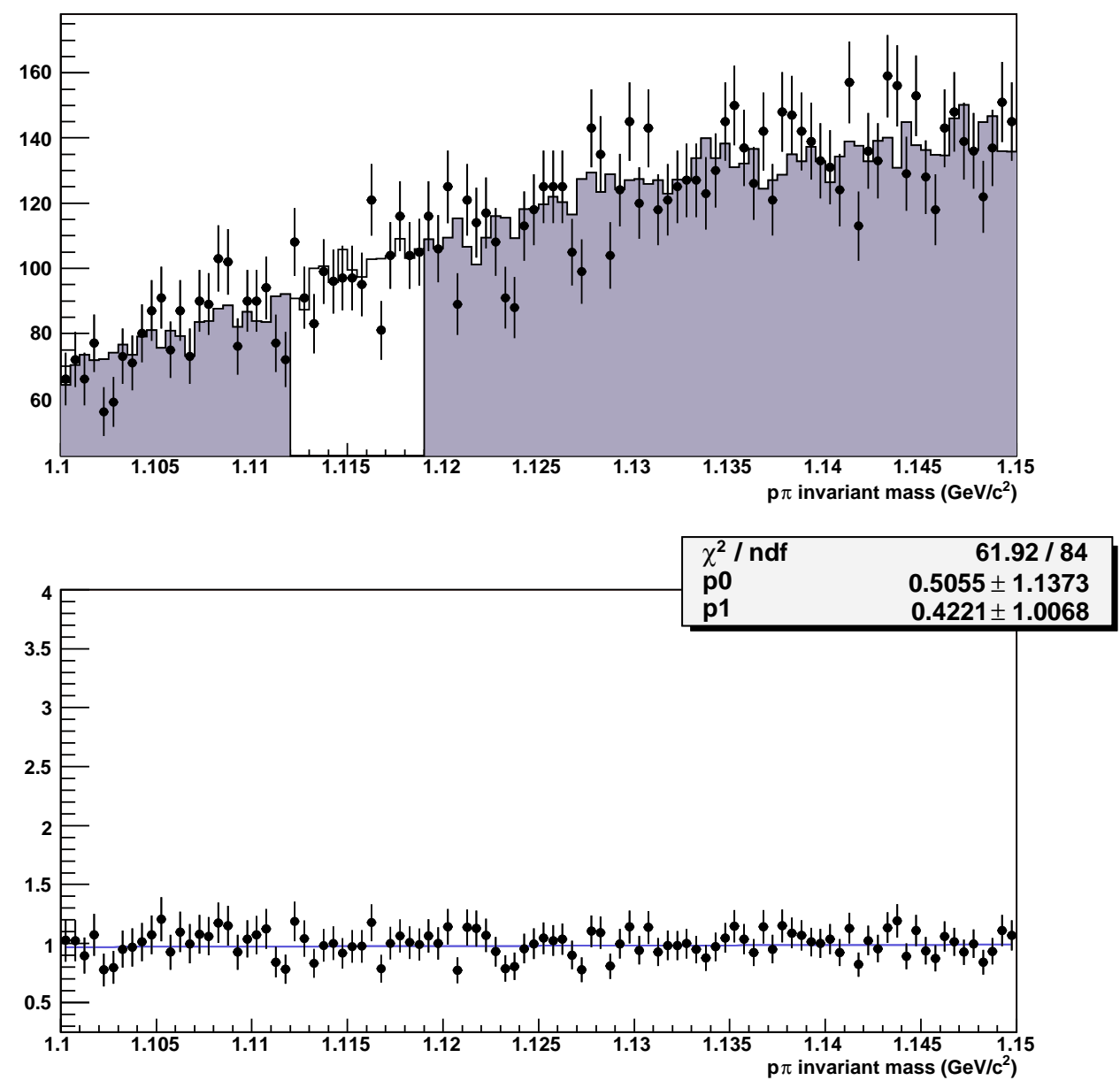

Figure J.17: A plot of the proton/pion invariant mass before the cut. Data are points, while the histogram is of Monte Carlo simulated events. The solid part of the histogram denotes the areas that pass after this particular cut. A plot of the ratio of the number of data events to Monte Carlo events, before this cut, is shown on the bottom. The number of Monte Carlo events is normalized to be equal to the total number of data events in this plot. A fit of the ratio using a linear function is also shown. 

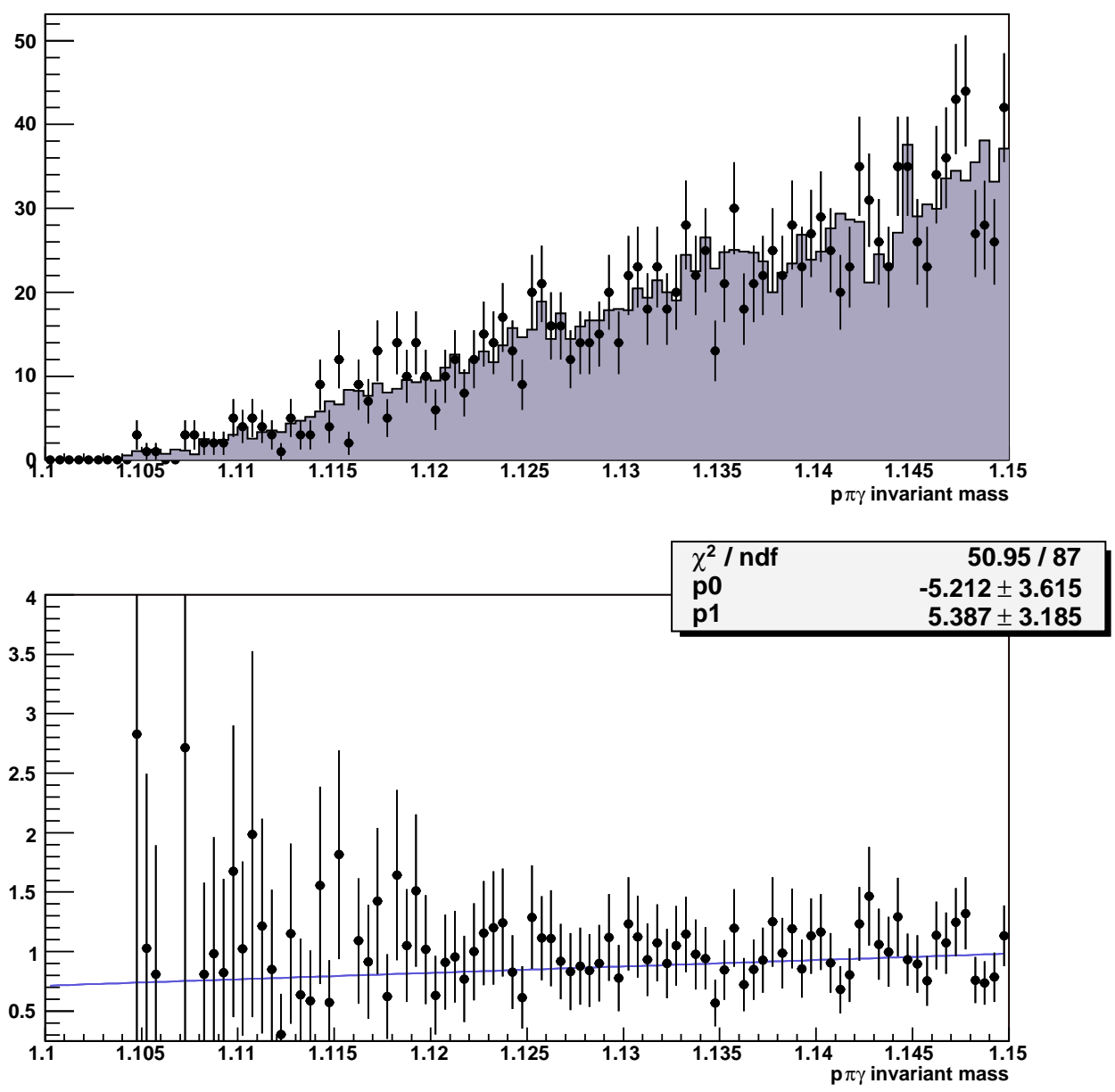

Figure J.18: A plot of the proton/pion/gamma invariant mass, before any cut on this variable. Data are points, while the histogram is of Monte Carlo simulated events. The solid part of the histogram denotes the areas that pass after this particular cut. A plot of the ratio of the number of data events to Monte Carlo events, before this cut, is shown on the bottom. The number of Monte Carlo events is normalized to be equal to the total number of data events in this plot. A fit of the ratio using a linear function is also shown. 

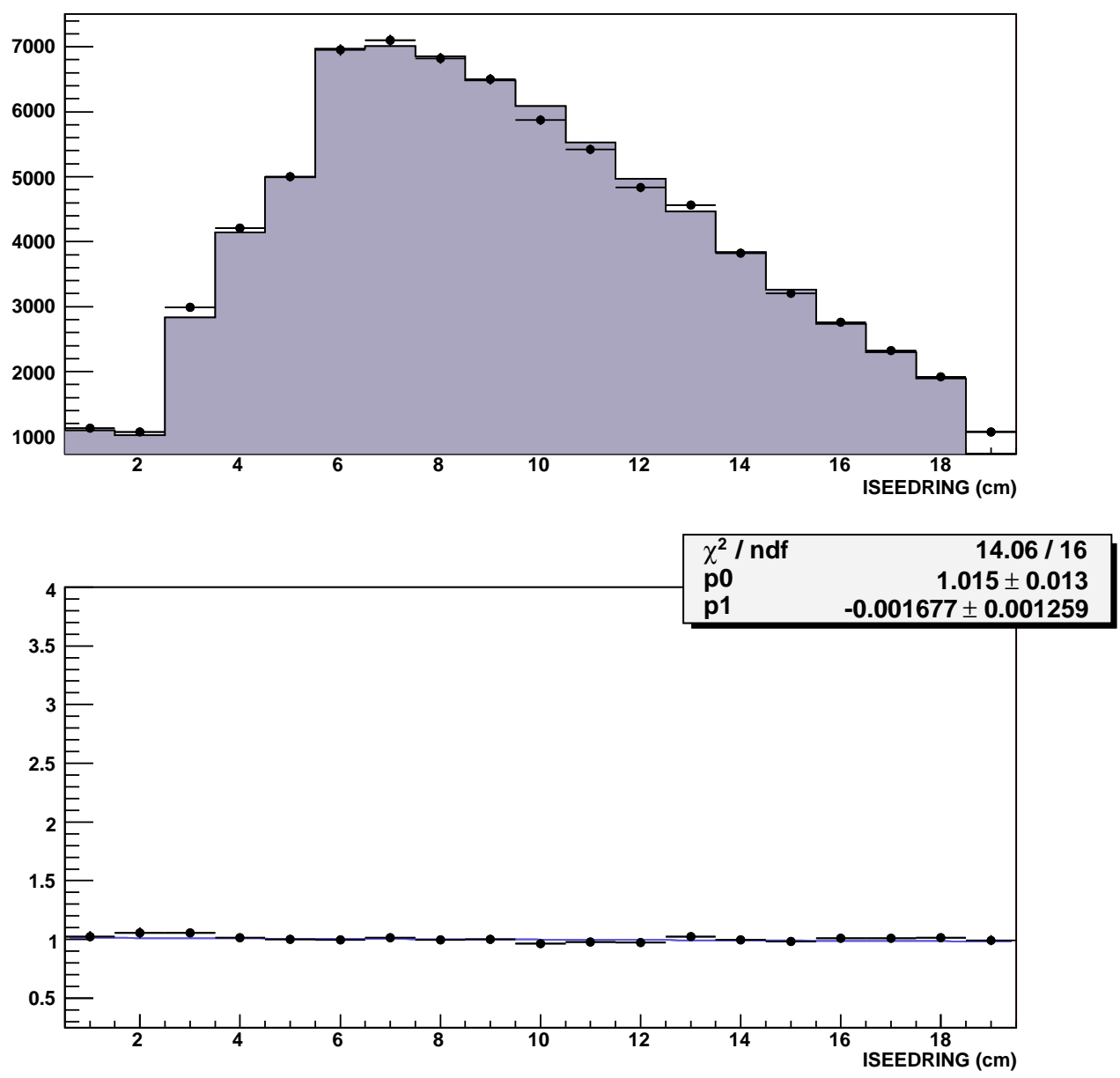

Figure J.19: A plot of the outer photon fiducial cut variable ISEEDRING, before the cut. Data are points, while the histogram is of Monte Carlo simulated events. The solid part of the histogram denotes the areas that pass after this particular cut. A plot of the ratio of the number of data events to Monte Carlo events, before this cut, is shown on the bottom. The number of Monte Carlo events is normalized to be equal to the total number of data events in this plot. A fit of the ratio using a linear function is also shown. 

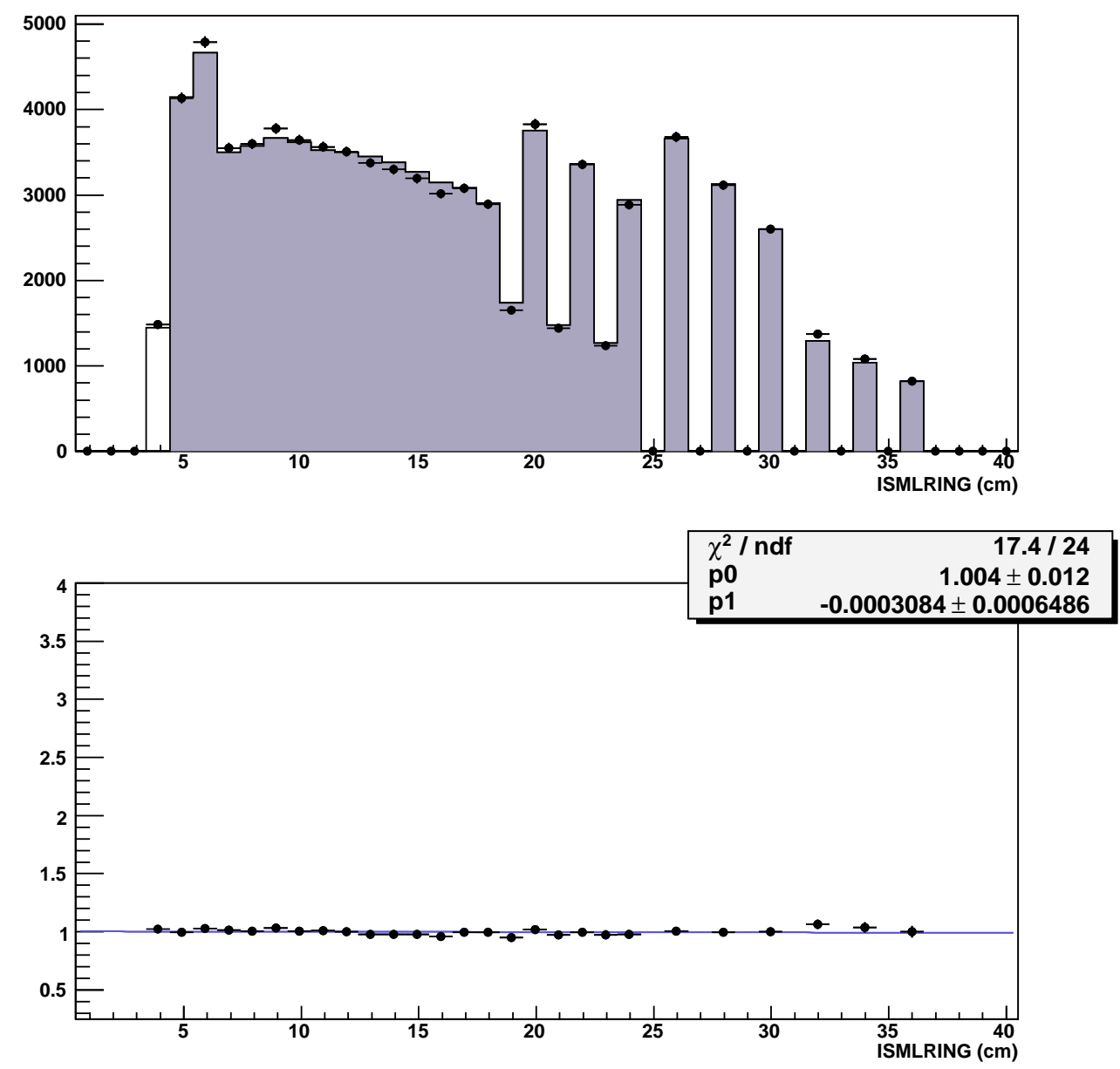

Figure J.20: A plot of the inner photon fiducial cut variable ISMLRNG before the cut. Data are points, while the histogram is of Monte Carlo simulated events. The solid part of the histogram denotes the areas that pass after this particular cut. A plot of the ratio of the number of data events to Monte Carlo events, before this cut, is shown on the bottom. The number of Monte Carlo events is normalized to be equal to the total number of data events in this plot. A fit of the ratio using a linear function is also shown. 

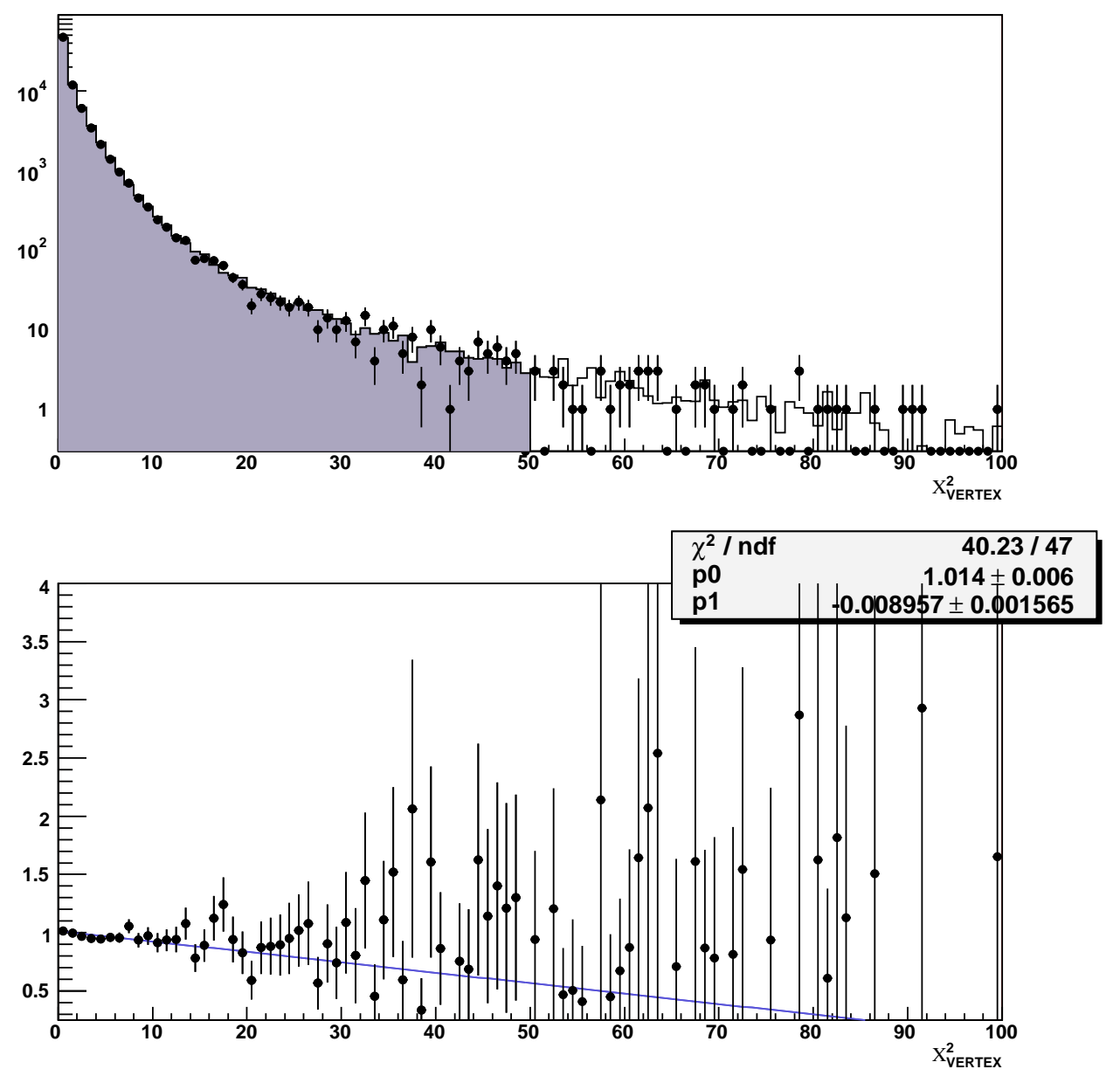

Figure J.21: A plot of vertex $\chi^{2}$ before the cut. Data are points, while the histogram is of Monte Carlo simulated events. The solid part of the histogram denotes the areas that pass after this particular cut. A plot of the ratio of the number of data events to Monte Carlo events, before this cut, is shown on the bottom. The number of Monte Carlo events is normalized to be equal to the total number of data events in this plot. A fit of the ratio using a linear function is also shown. 

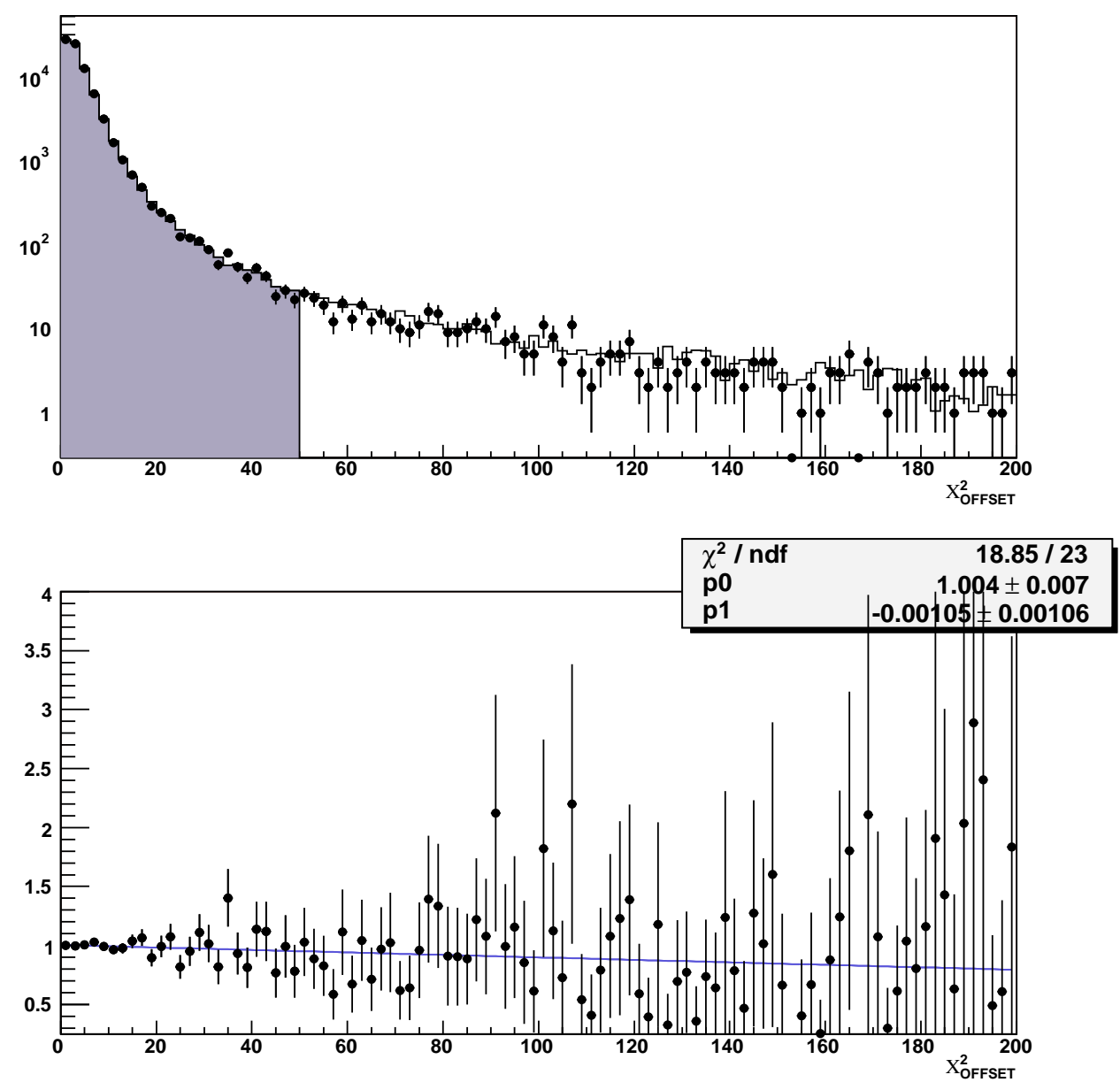

Figure J.22: A plot of the greater of the two track offset $\chi^{2}$ values in each event before the cut. Data are points, while the histogram is of Monte Carlo simulated events. The solid part of the histogram denotes the areas that pass after this particular cut. A plot of the ratio of the number of data events to Monte Carlo events, before this cut, is shown on the bottom. The number of Monte Carlo events is normalized to be equal to the total number of data events in this plot. A fit of the ratio using a linear function is also shown. 

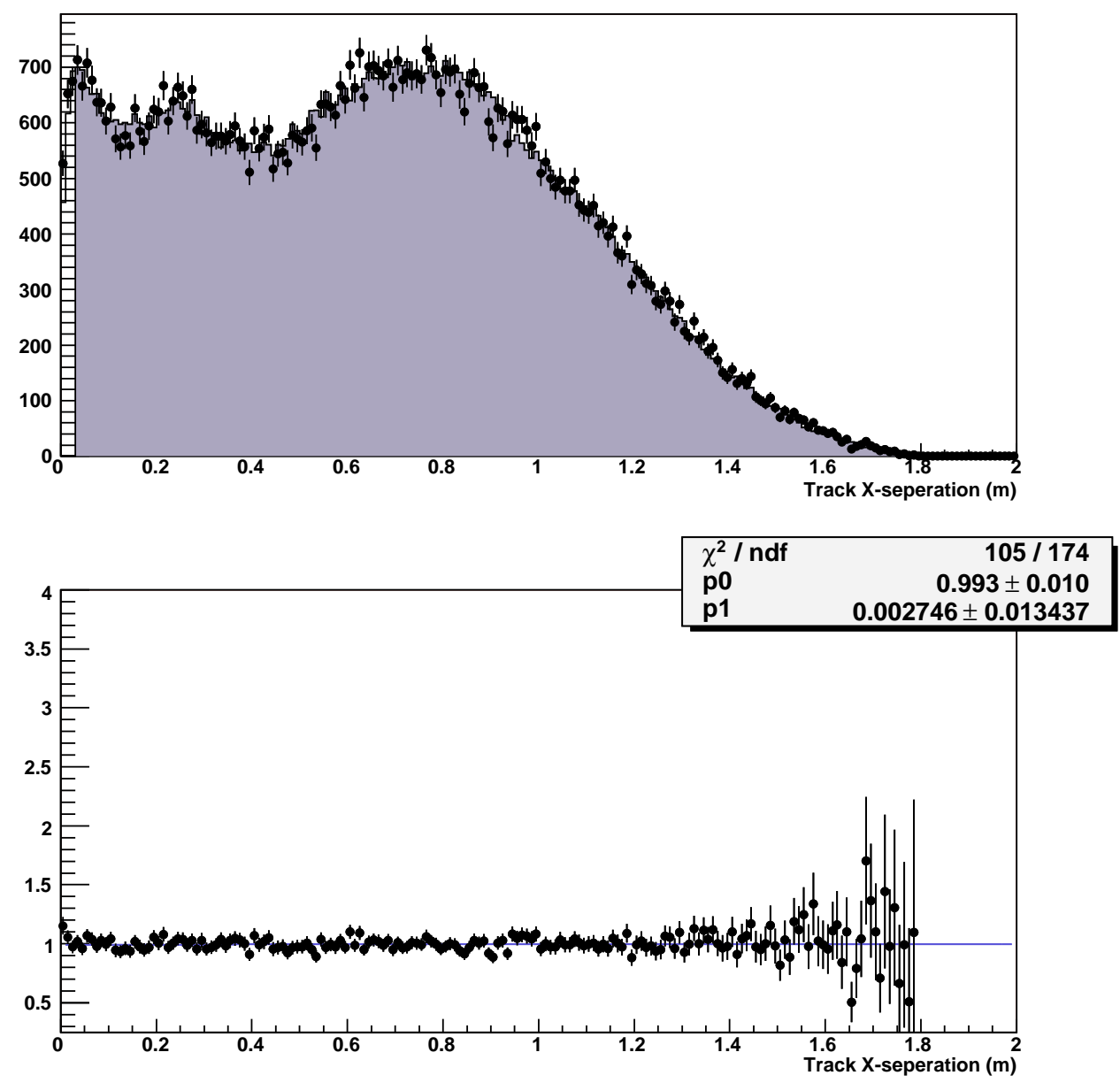

Figure J.23: A plot of the separation of the tracks in the $\mathrm{x}$ direction at the CsI, before the cut. Data are points, while the histogram is of Monte Carlo simulated events. The solid part of the histogram denotes the areas that pass after this particular cut. A plot of the ratio of the number of data events to Monte Carlo events, before this cut, is shown on the bottom. The number of Monte Carlo events is normalized to be equal to the total number of data events in this plot. A fit of the ratio using a linear function is also shown. 

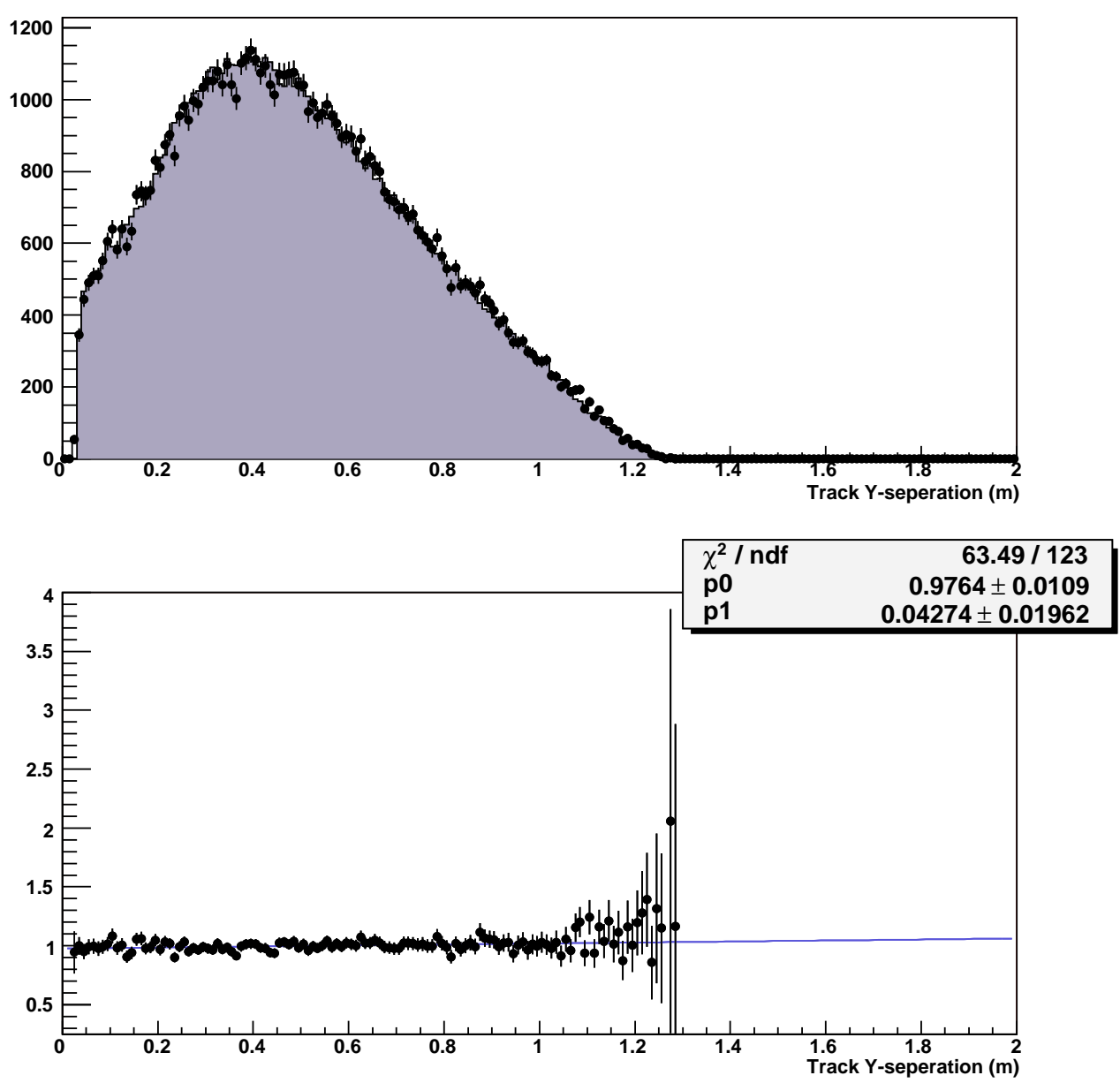

Figure J.24: A plot of the separation of the tracks in the y direction at the CsI, before the cut. Data are points, while the histogram is of Monte Carlo simulated events. The solid part of the histogram denotes the areas that pass after this particular cut. A plot of the ratio of the number of data events to Monte Carlo events, before this cut, is shown on the bottom. The number of Monte Carlo events is normalized to be equal to the total number of data events in this plot. A fit of the ratio using a linear function is also shown. 

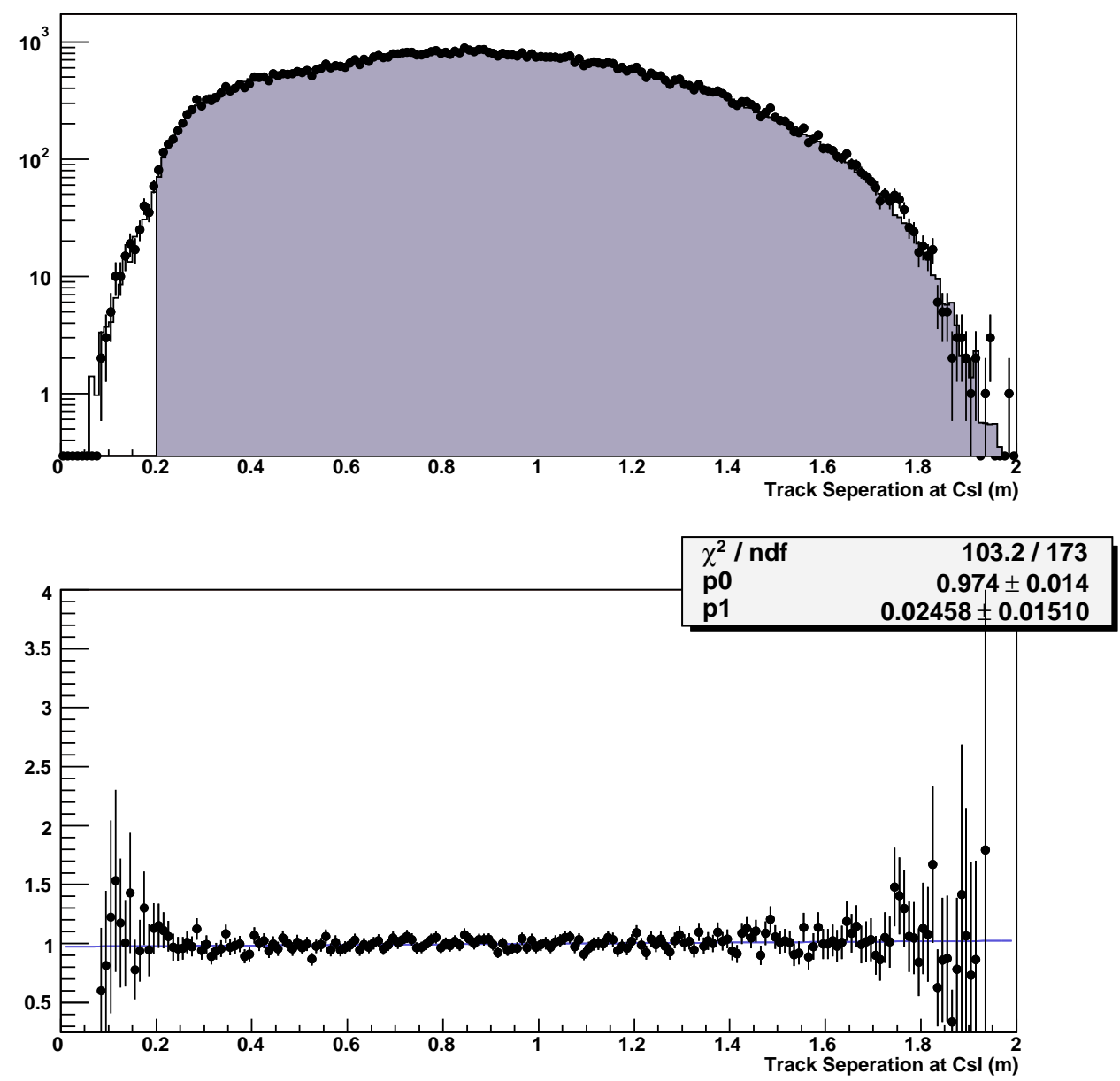

Figure J.25: A plot of the track separation at the CsI before the cut. Data are points, while the histogram is of Monte Carlo simulated events. The solid part of the histogram denotes the areas that pass after this particular cut. A plot of the ratio of the number of data events to Monte Carlo events, before this cut, is shown on the bottom. The number of Monte Carlo events is normalized to be equal to the total number of data events in this plot. A fit of the ratio using a linear function is also shown. 
Appendix K

Plots for 1999 Vacuum Data 

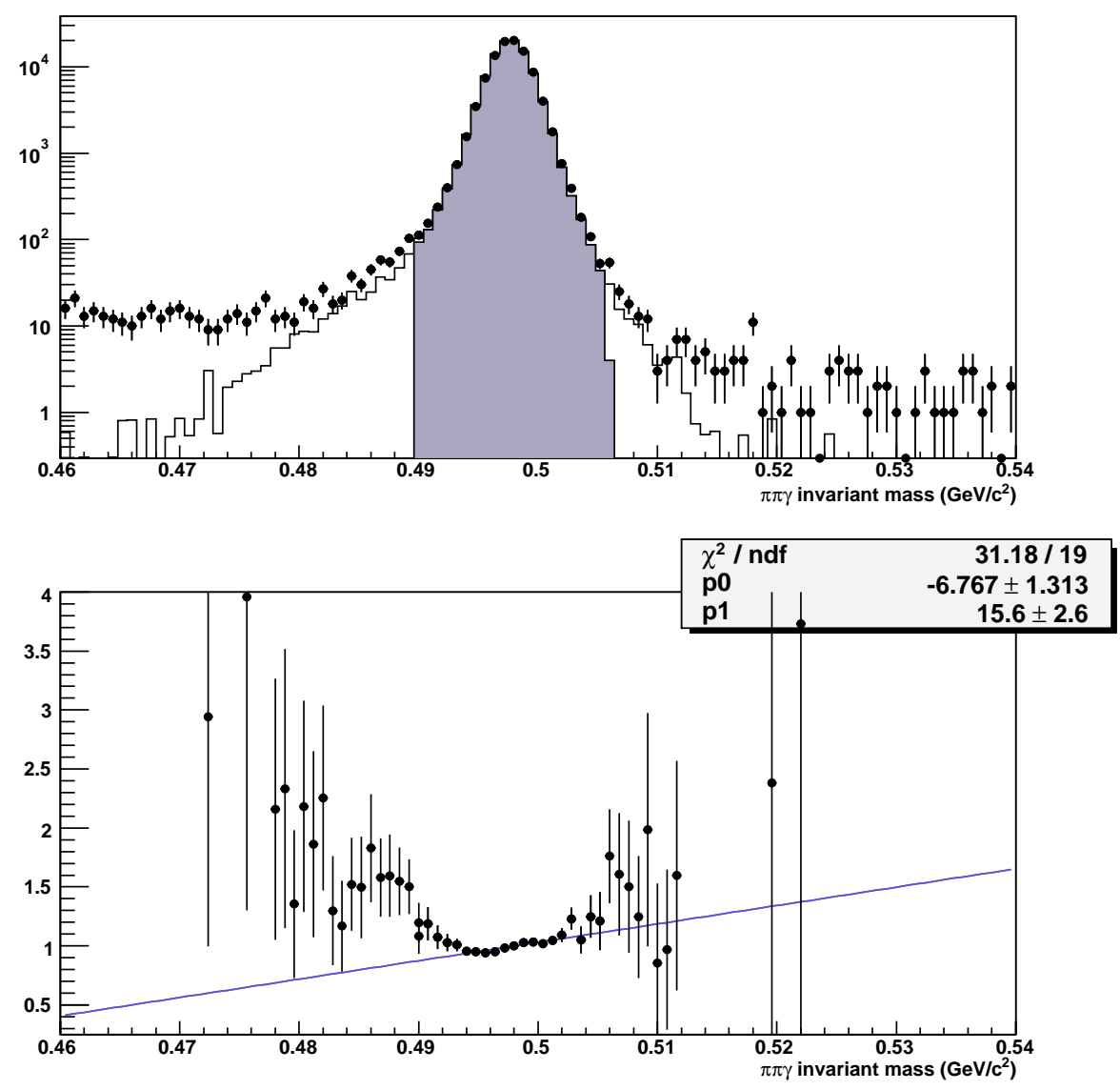

Figure K.1: A plot of the $\pi^{+} \pi^{-} \gamma$ invariant mass before the cut on $M_{\pi^{+} \pi^{-} \gamma}$ but after all other cuts. Data are points, while the histogram is of Monte Carlo simulated events. The solid part of the histogram denotes the areas that pass after this particular cut. A plot of the ratio of the number of data events to Monte Carlo events, before this cut, is shown on the bottom. The number of Monte Carlo events is normalized to be equal to the total number of data events in this plot. A fit of the ratio using a linear function is also shown. 

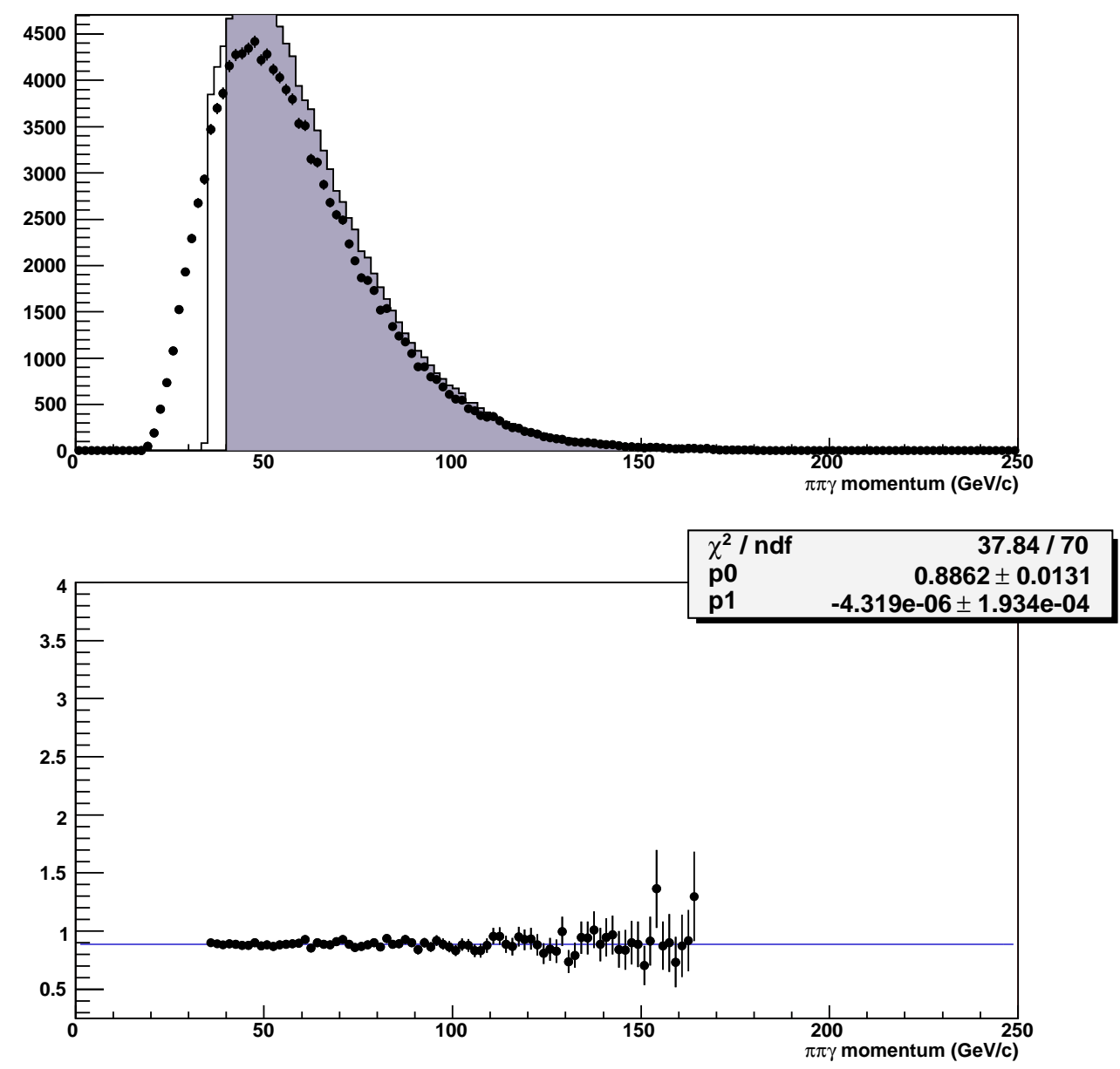

Figure K.2: The reconstructed kaon momentum before the cut. Data are points, while the histogram is of Monte Carlo simulated events. The solid part of the histogram denotes the areas that pass after this particular cut. A plot of the ratio of the number of data events to Monte Carlo events, before this cut, is shown on the bottom. The number of Monte Carlo events is normalized to be equal to the total number of data events in this plot. A fit of the ratio using a linear function is also shown. 

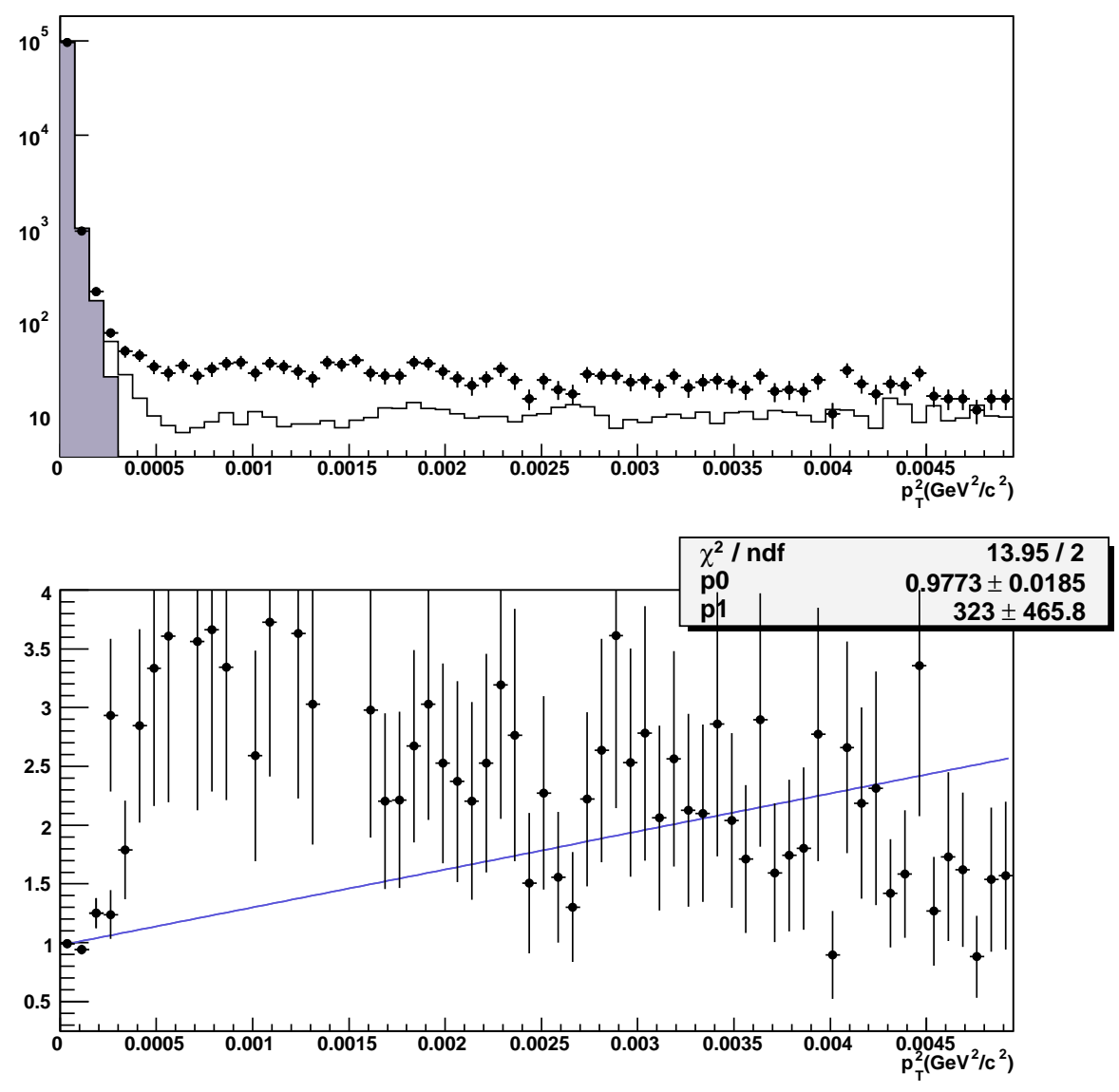

Figure K.3: A plot of $P_{T}^{2}$ with respect to the downstream face of the regenerator for the $\pi^{+} \pi^{-} \gamma$ system before the cut. Data are points, while the histogram is of Monte Carlo simulated events. The solid part of the histogram denotes the areas that pass after this particular cut. A plot of the ratio of the number of data events to Monte Carlo events, before this cut, is shown on the bottom. The number of Monte Carlo events is normalized to be equal to the total number of data events in this plot. A fit of the ratio using a linear function is also shown. 

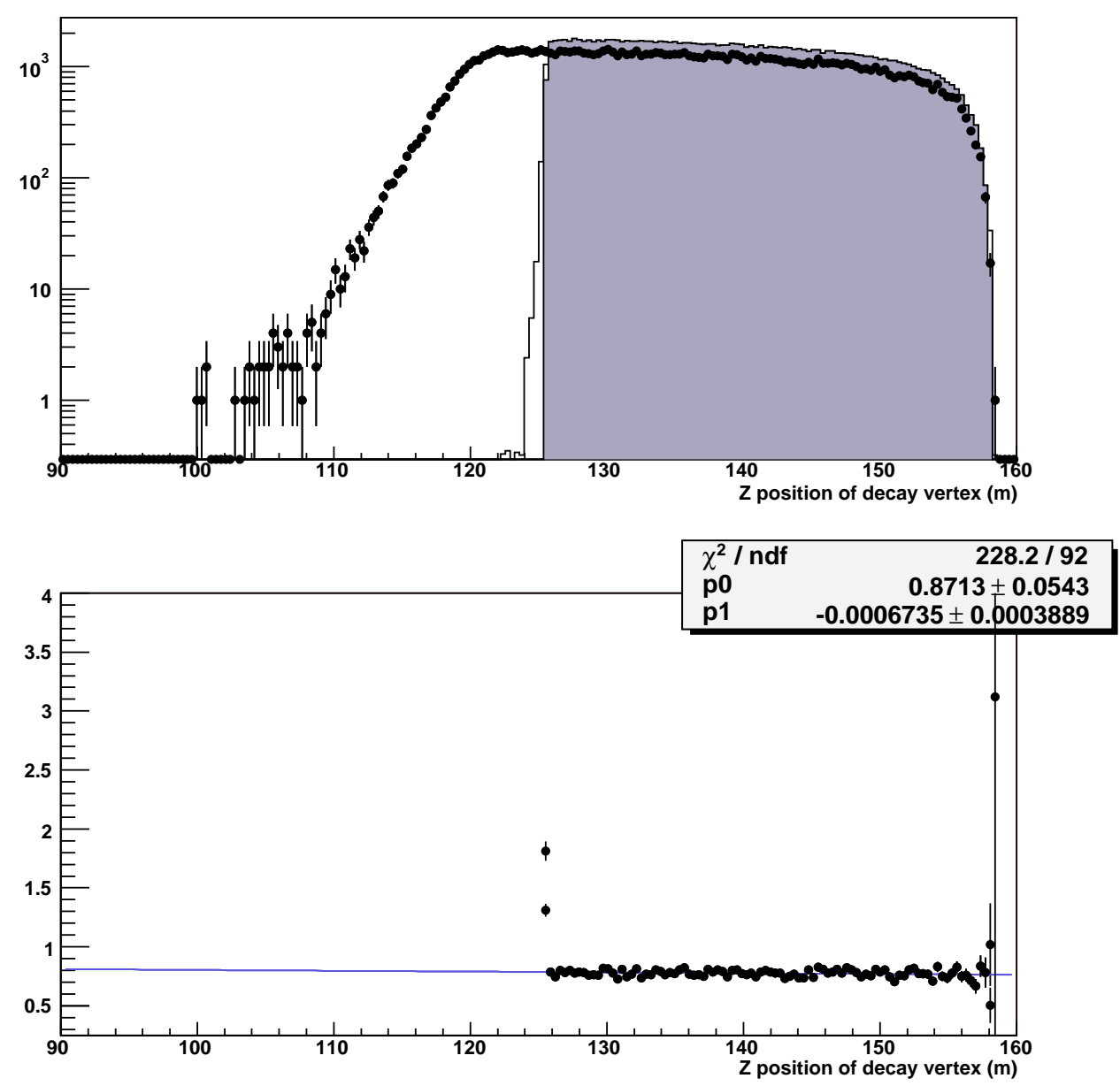

Figure K.4: A plot of the $\mathrm{z}$ location of the decay vertex, before the cut is applied. Data are points, while the histogram is of Monte Carlo simulated events. The solid part of the histogram denotes the areas that pass after this particular cut. A plot of the ratio of the number of data events to Monte Carlo events, before this cut, is shown on the bottom. The number of Monte Carlo events is normalized to be equal to the total number of data events in this plot. A fit of the ratio using a linear function is also shown. 

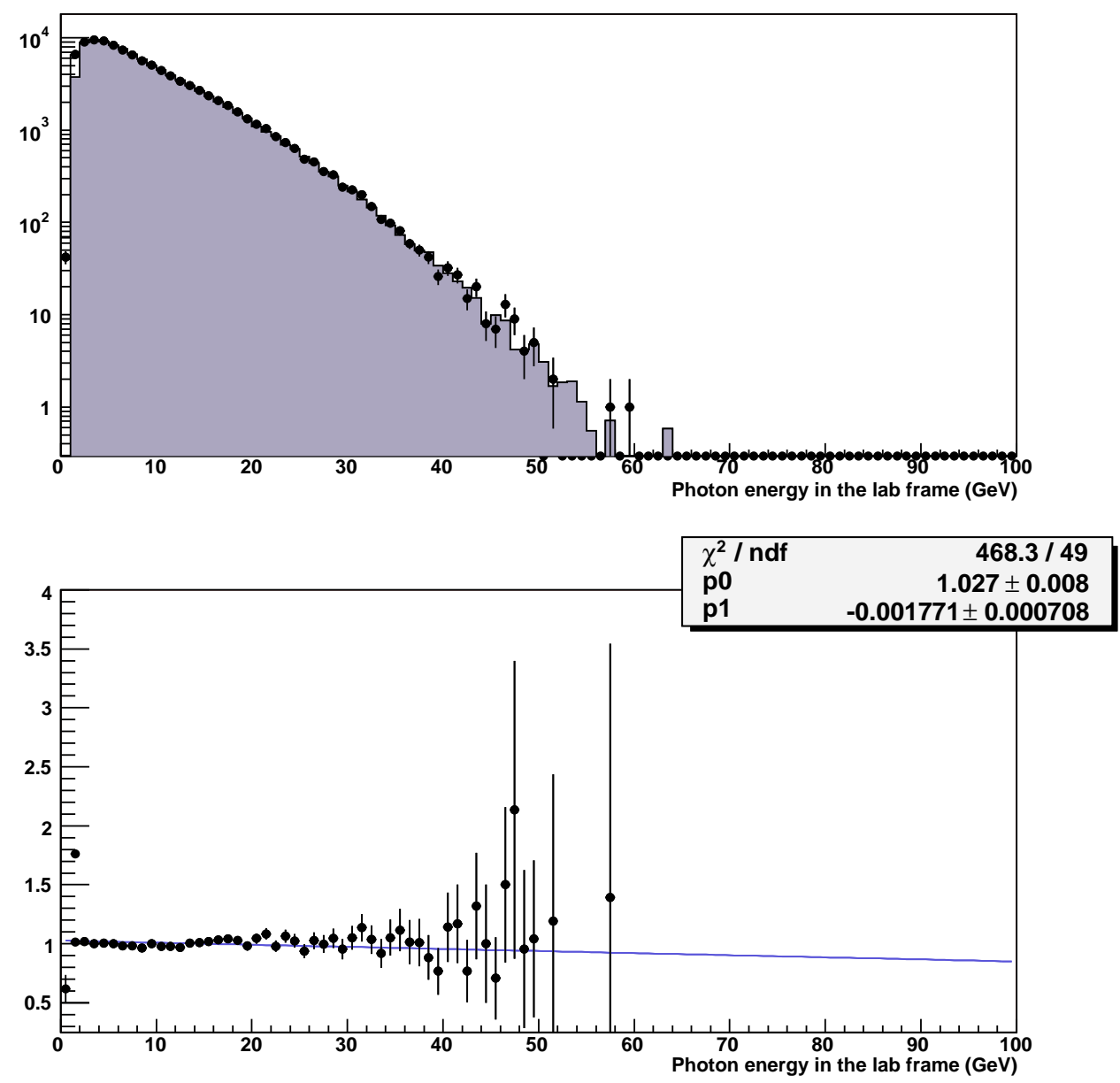

Figure K.5: The photon cluster energy in the lab frame, before the final analysis cut. Data are points, while the histogram is of Monte Carlo simulated events. The solid part of the histogram denotes the areas that pass after this particular cut. A plot of the ratio of the number of data events to Monte Carlo events, before this cut, is shown on the bottom. The number of Monte Carlo events is normalized to be equal to the total number of data events in this plot. A fit of the ratio using a linear function is also shown. 

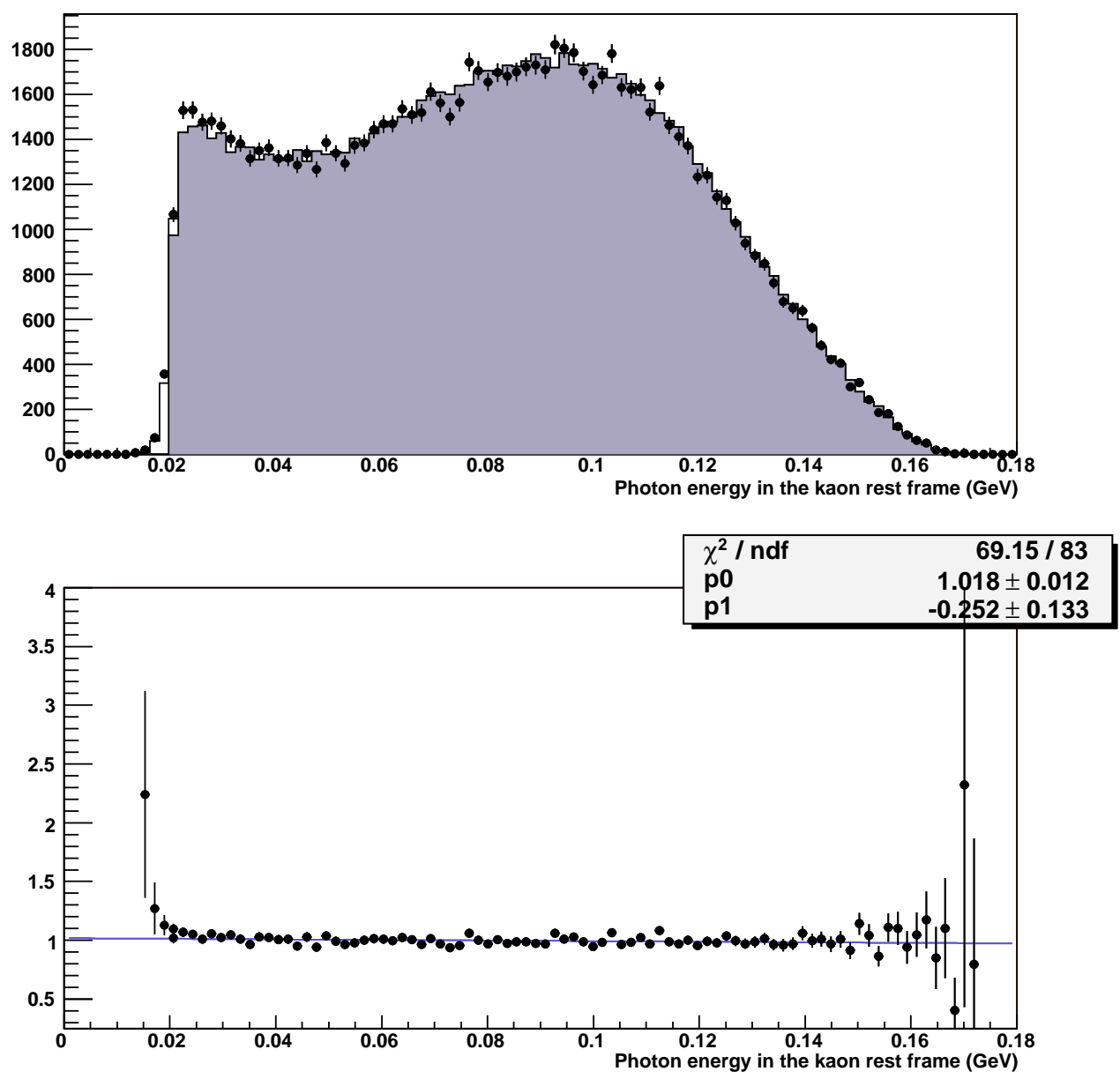

Figure K.6: A plot of the photon energy in the kaon rest frame before the cut. Data are points, while the histogram is of Monte Carlo simulated events. The solid part of the histogram denotes the areas that pass after this particular cut. A plot of the ratio of the number of data events to Monte Carlo events, before this cut, is shown on the bottom. The number of Monte Carlo events is normalized to be equal to the total number of data events in this plot. A fit of the ratio using a linear function is also shown. 

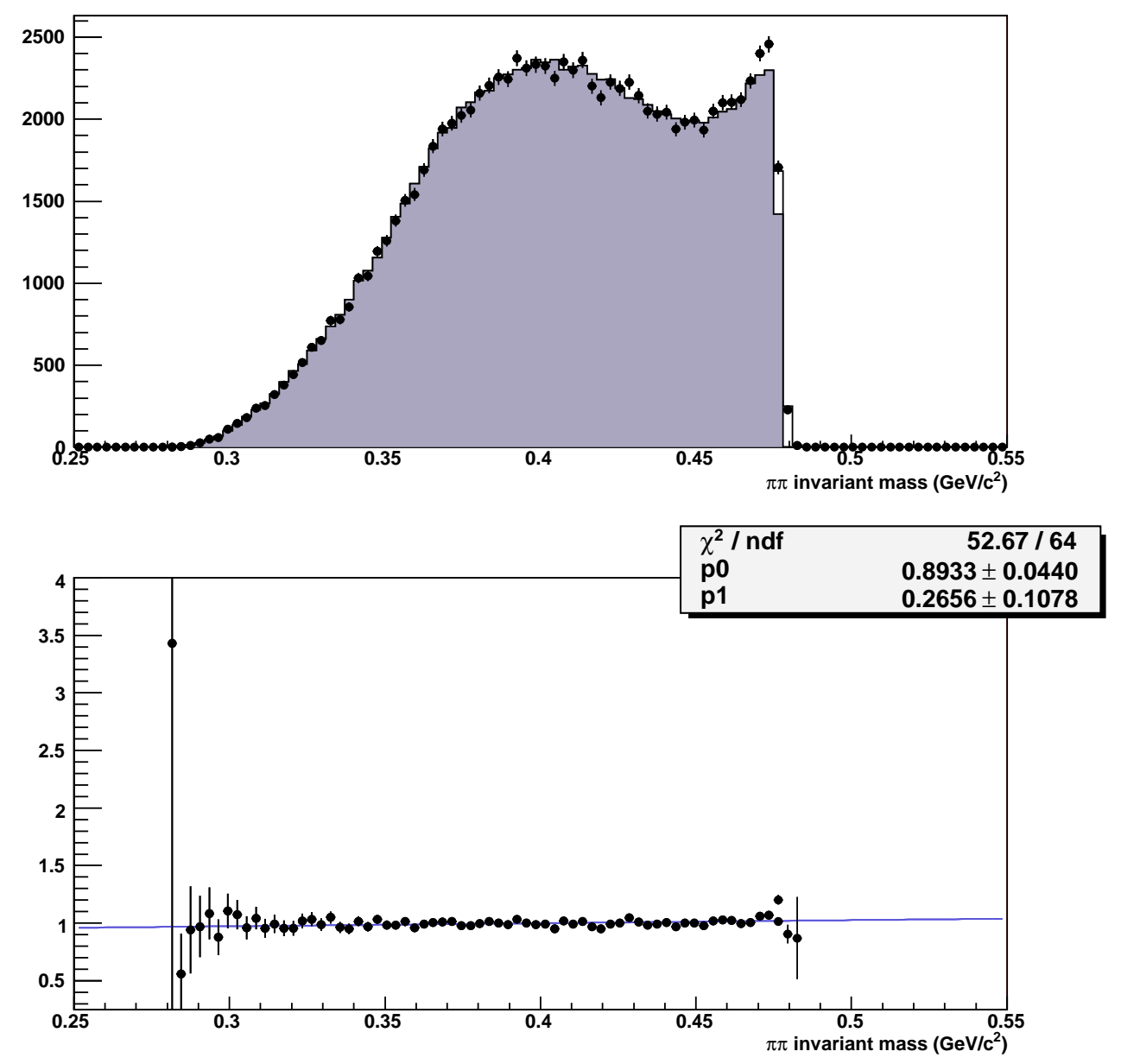

Figure K.7: A plot of the invariant mass of the pion pair before the $M_{\pi^{+} \pi^{-}}$cut. Note that any cut on $E_{\gamma}^{* K I N}$ will also be reflected here. Data are points, while the histogram is of Monte Carlo simulated events. The solid part of the histogram denotes the areas that pass after this particular cut. A plot of the ratio of the number of data events to Monte Carlo events, before this cut, is shown on the bottom. The number of Monte Carlo events is normalized to be equal to the total number of data events in this plot. A fit of the ratio using a linear function is also shown. 

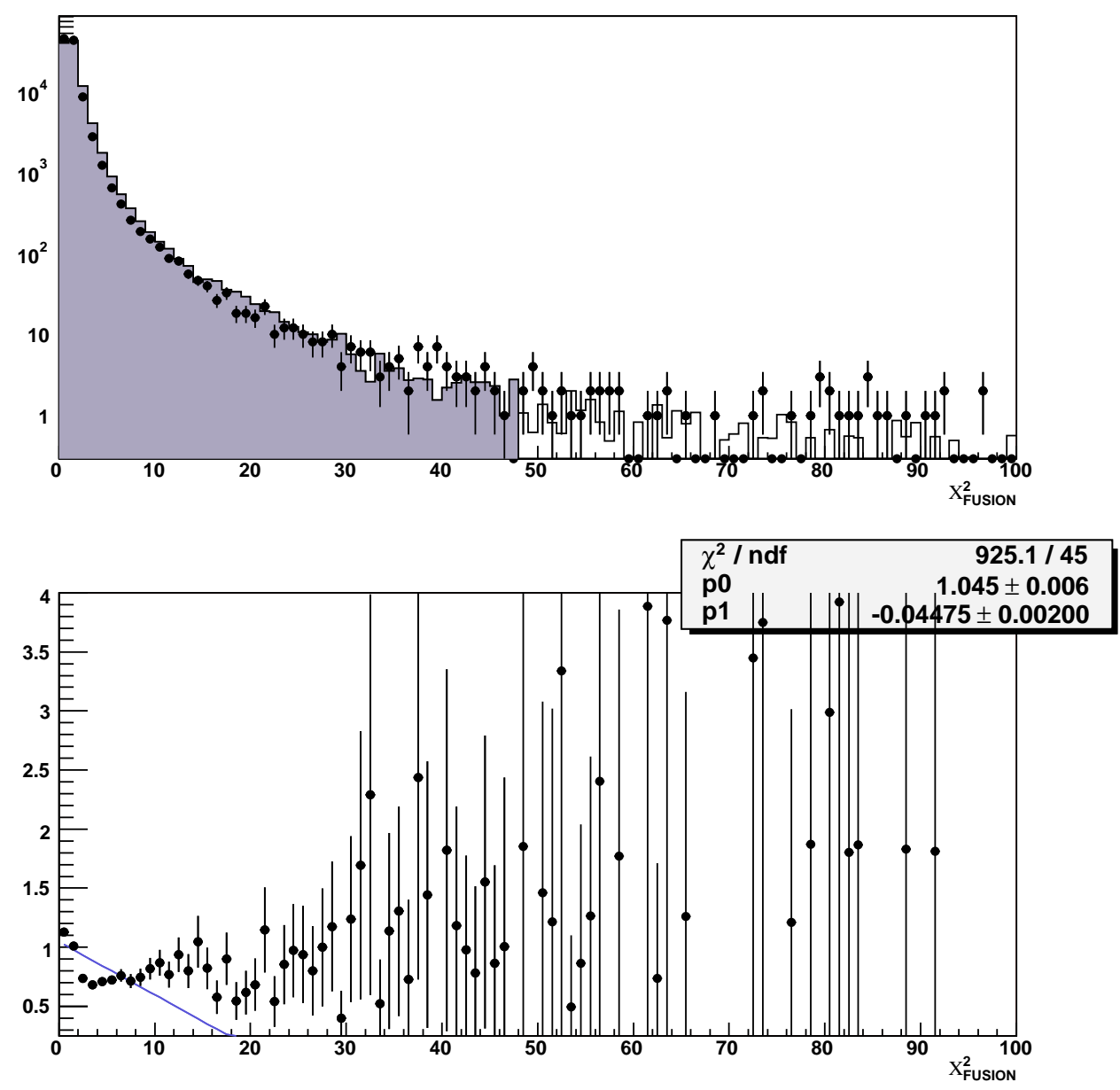

Figure K.8: A plot of fusion $\chi^{2}$ before the cut on this variable. Data are points, while the histogram is of Monte Carlo simulated events. The solid part of the histogram denotes the areas that pass after this particular cut. A plot of the ratio of the number of data events to Monte Carlo events, before this cut, is shown on the bottom. The number of Monte Carlo events is normalized to be equal to the total number of data events in this plot. A fit of the ratio using a linear function is also shown. 

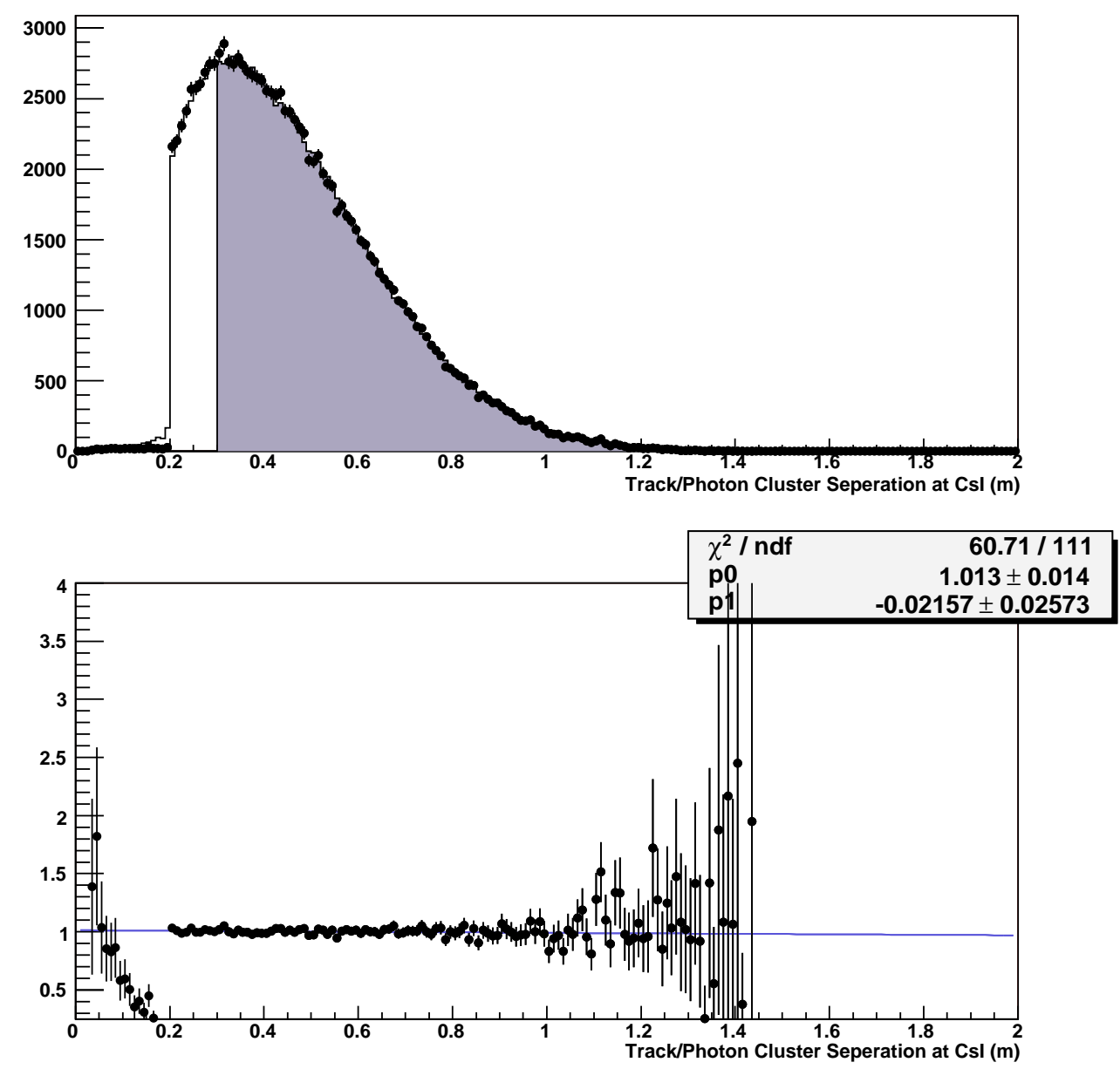

Figure K.9: Minimum of the two pion/gamma separations at the CsI for each event, before the cut. Data are points, while the histogram is of Monte Carlo simulated events. The solid part of the histogram denotes the areas that pass after this particular cut. A plot of the ratio of the number of data events to Monte Carlo events, before this cut, is shown on the bottom. The number of Monte Carlo events is normalized to be equal to the total number of data events in this plot. A fit of the ratio using a linear function is also shown. 

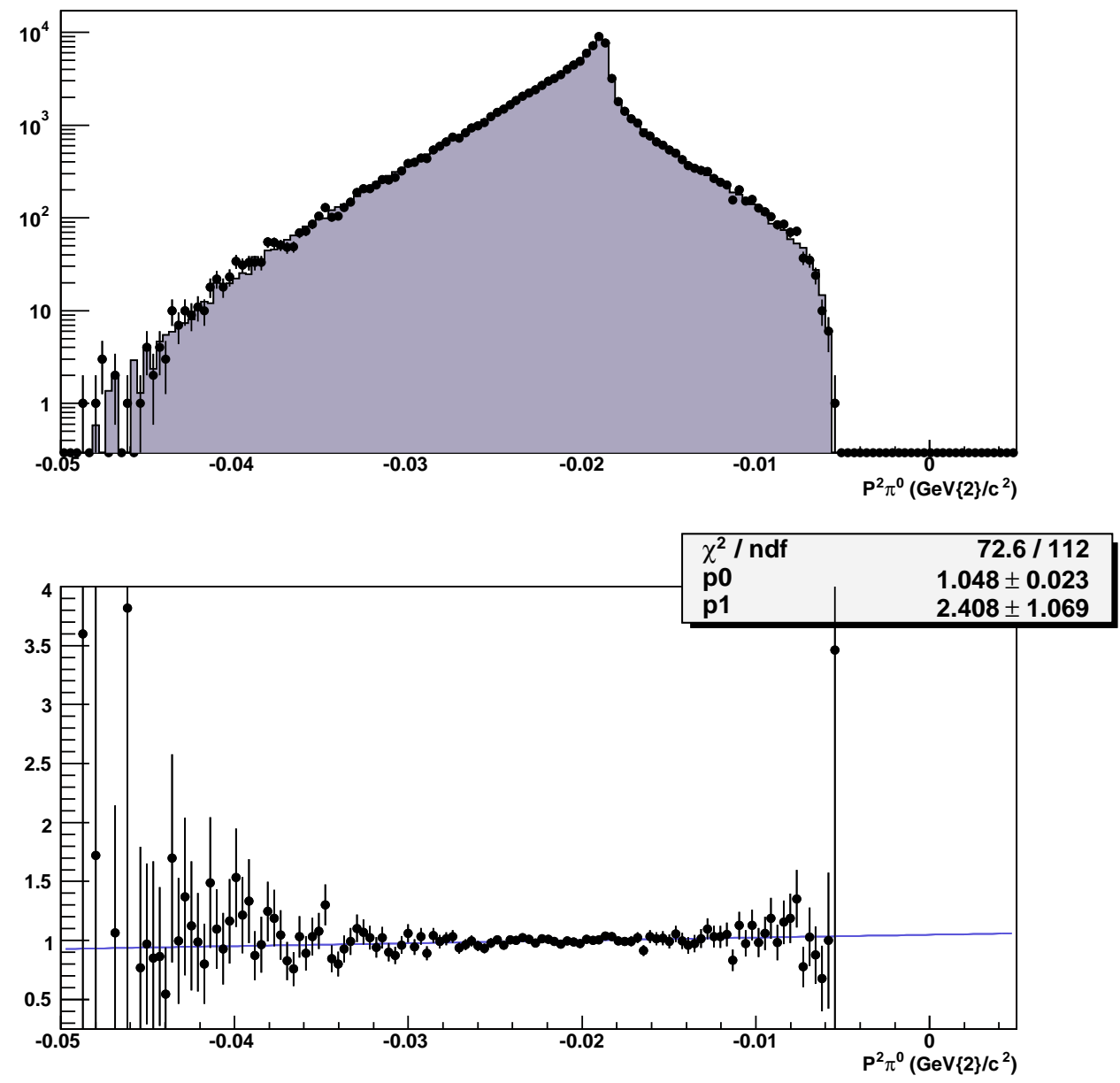

Figure K.10: A plot of $P_{\pi^{0}}^{2}$ with respect to the downstream face of the regenerator, before the final analysis cut. Data are points, while the histogram is of Monte Carlo simulated events. The solid part of the histogram denotes the areas that pass after this particular cut. A plot of the ratio of the number of data events to Monte Carlo events, before this cut, is shown on the bottom. The number of Monte Carlo events is normalized to be equal to the total number of data events in this plot. A fit of the ratio using a linear function is also shown. 

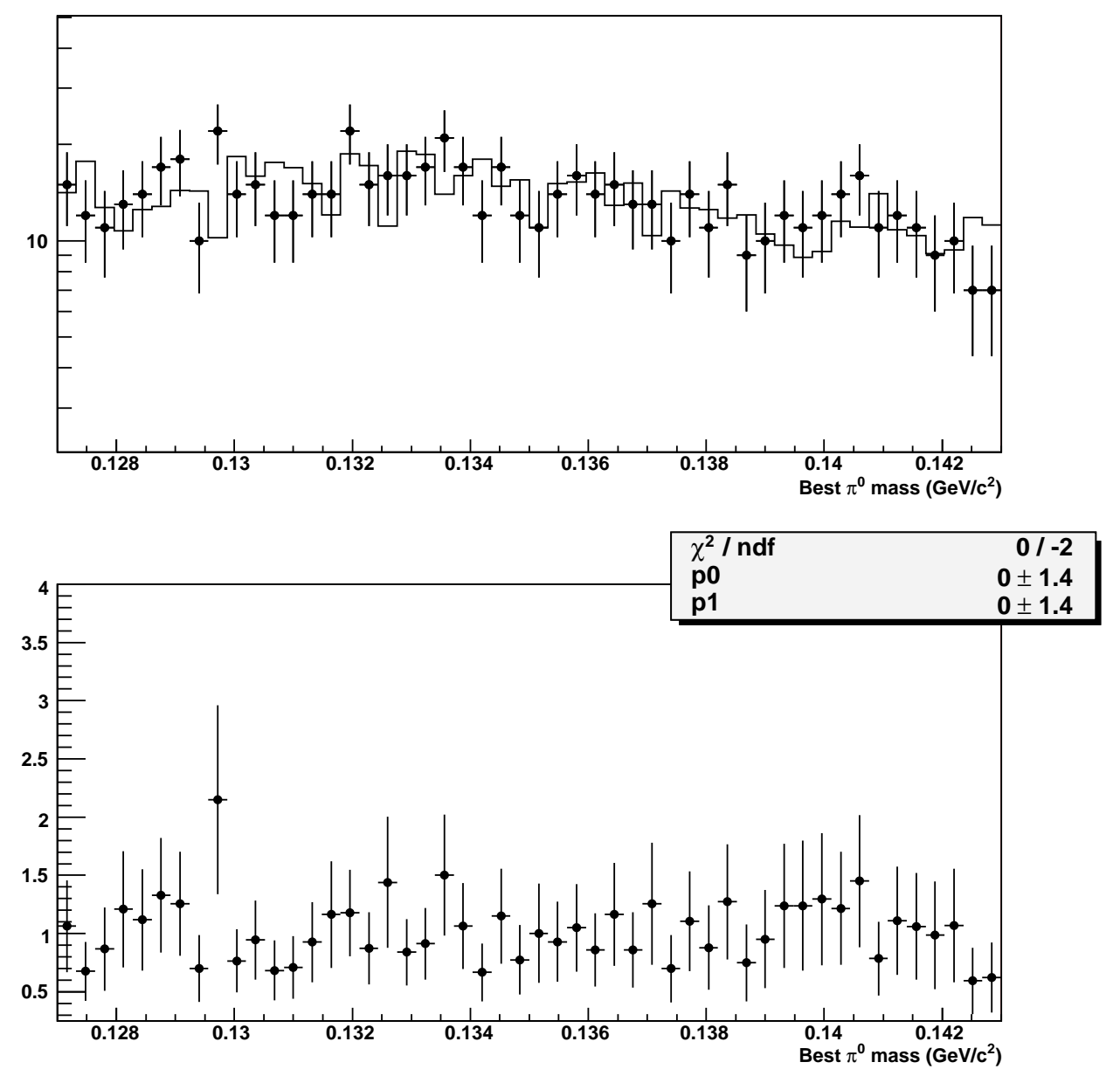

Figure K.11: A plot of the best $\pi^{0}$ mass found in each event using two clusters in the CsI calorimeter, before the cut on this variable. Data are points, while the histogram is of Monte Carlo simulated events. The solid part of the histogram denotes the areas that pass after this particular cut. A plot of the ratio of the number of data events to Monte Carlo events, before this cut, is shown on the bottom. The number of Monte Carlo events is normalized to be equal to the total number of data events in this plot. A fit of the ratio using a linear function is also shown. 

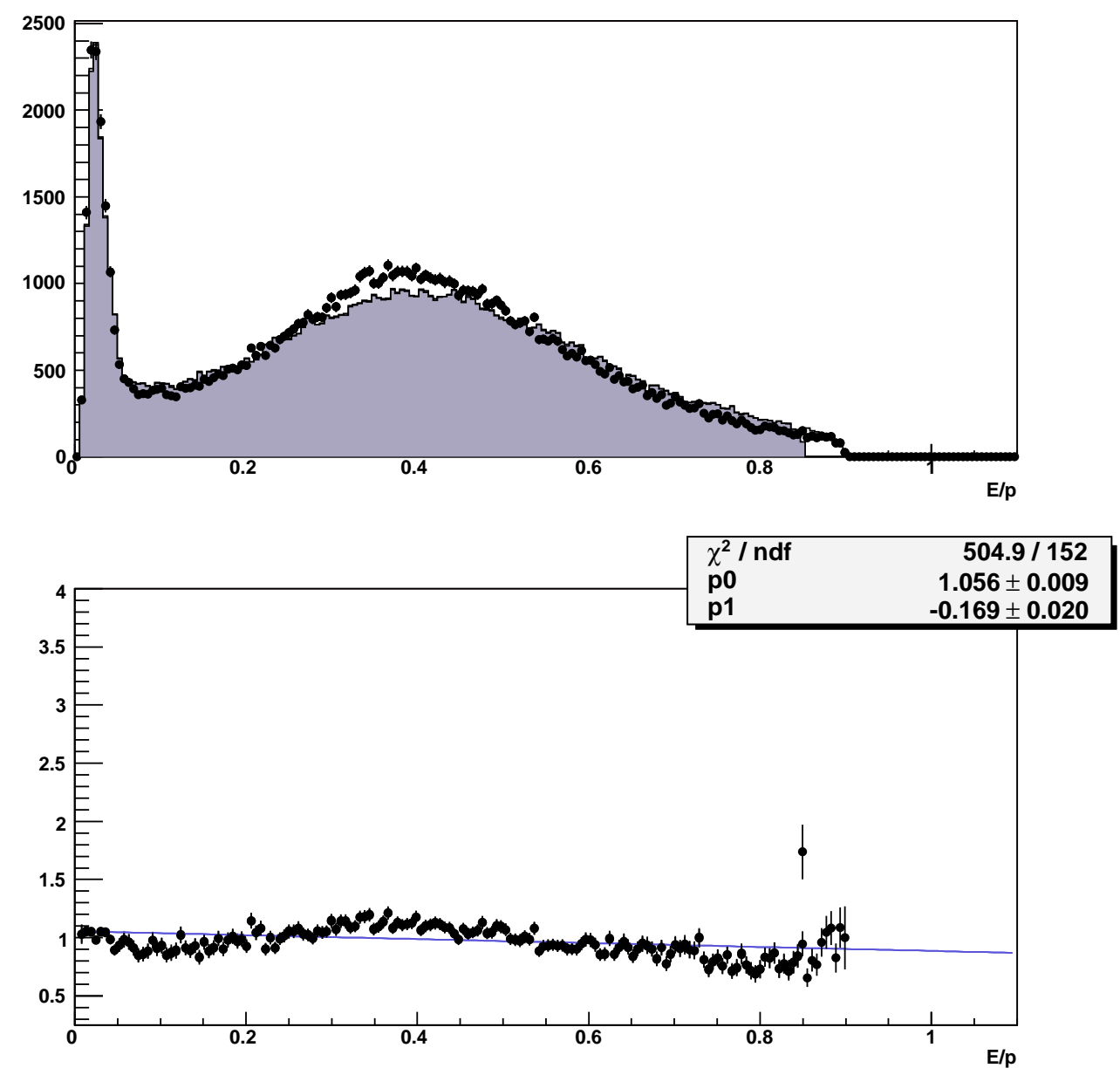

Figure K.12: A plot of the greater of the two values of $\mathrm{E} / \mathrm{p}$ for each event, before the final E/p cut. Data are points, while the histogram is of Monte Carlo simulated events. The solid part of the histogram denotes the areas that pass after this particular cut. A plot of the ratio of the number of data events to Monte Carlo events, before this cut, is shown on the bottom. The number of Monte Carlo events is normalized to be equal to the total number of data events in this plot. A fit of the ratio using a linear function is also shown. 

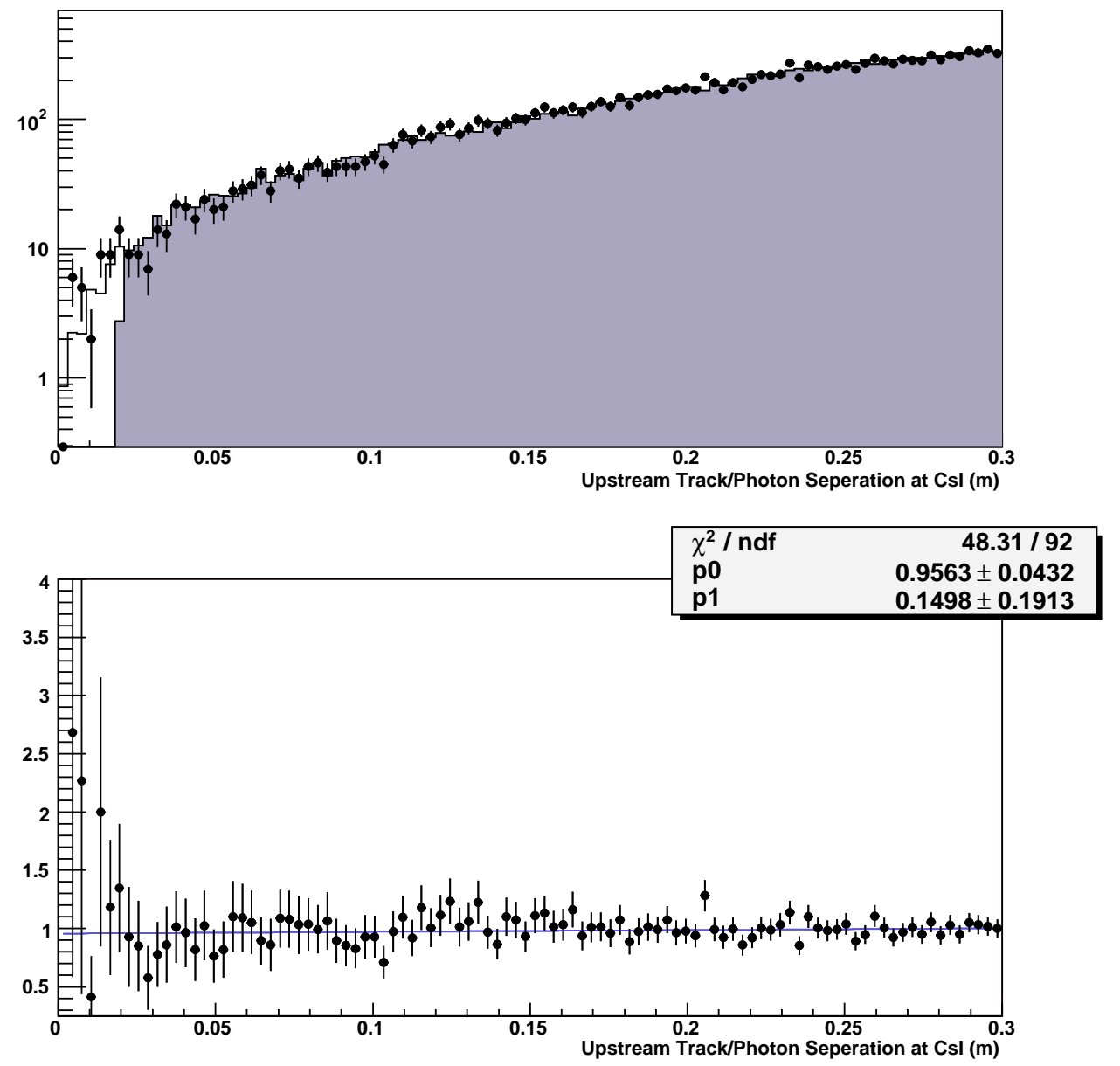

Figure K.13: A plot of the upstream track/photon separation distance at the CsI before the cut. Data are points, while the histogram is of Monte Carlo simulated events. The solid part of the histogram denotes the areas that pass after this particular cut. A plot of the ratio of the number of data events to Monte Carlo events, before this cut, is shown on the bottom. The number of Monte Carlo events is normalized to be equal to the total number of data events in this plot. A fit of the ratio using a linear function is also shown. 

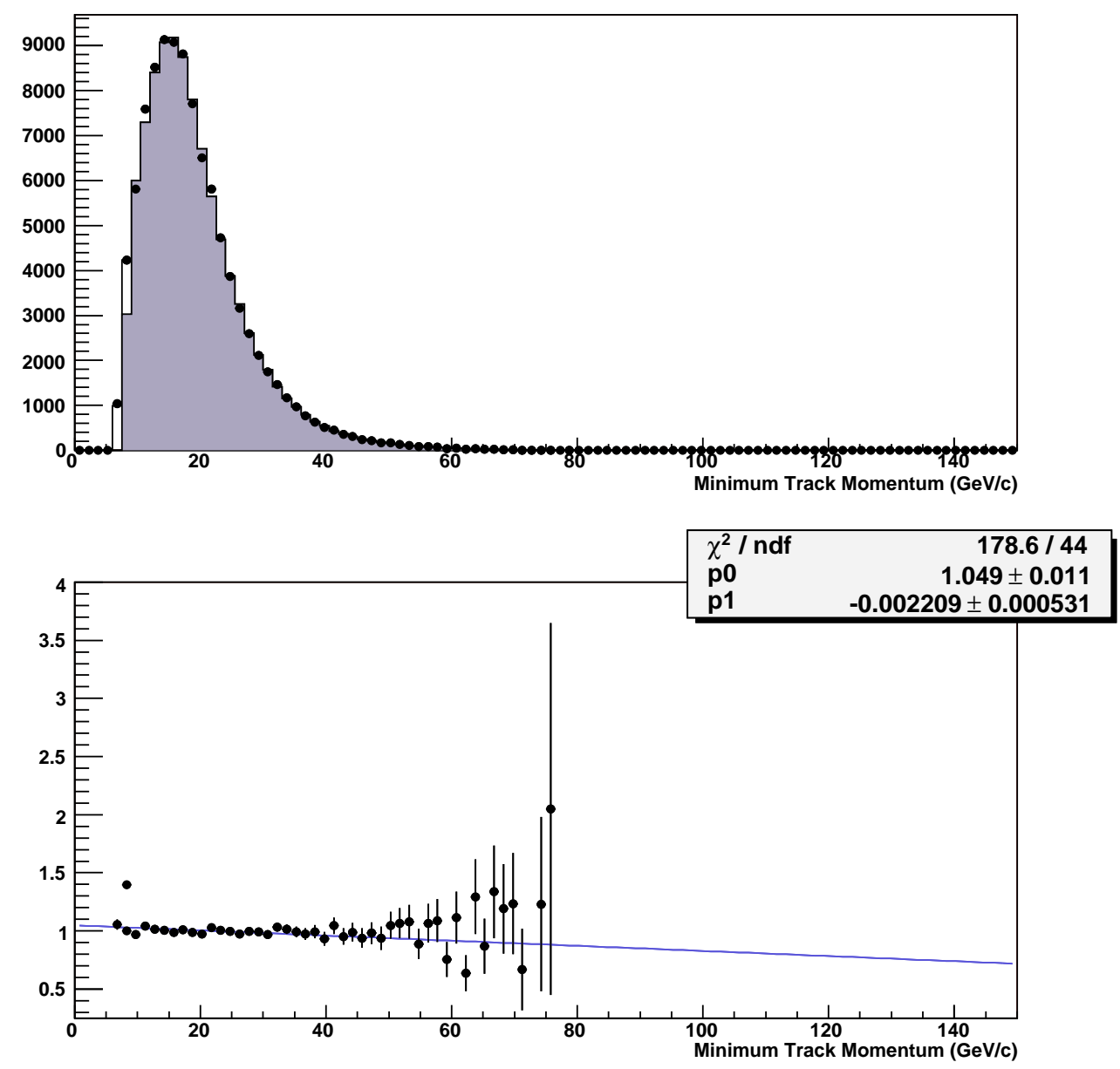

Figure K.14: A plot of the lesser of two track momenta per event before the cut. Data are points, while the histogram is of Monte Carlo simulated events. The solid part of the histogram denotes the areas that pass after this particular cut. A plot of the ratio of the number of data events to Monte Carlo events, before this cut, is shown on the bottom. The number of Monte Carlo events is normalized to be equal to the total number of data events in this plot. A fit of the ratio using a linear function is also shown. 

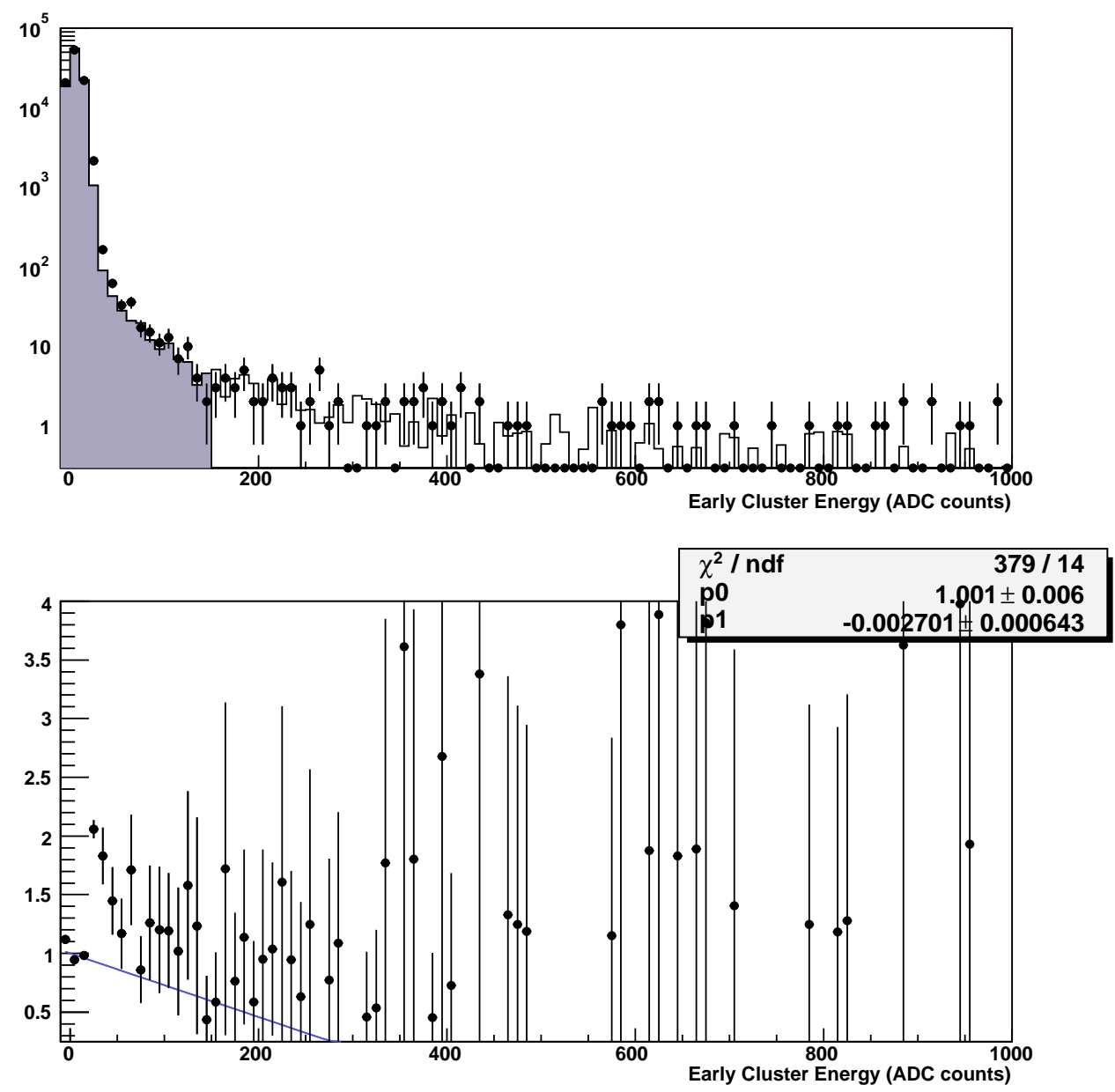

Figure K.15: A plot of the "early" energy of the photon candidate cluster before the cut. Data are points, while the histogram is of Monte Carlo simulated events. The solid part of the histogram denotes the areas that pass after this particular cut. A plot of the ratio of the number of data events to Monte Carlo events, before this cut, is shown on the bottom. The number of Monte Carlo events is normalized to be equal to the total number of data events in this plot. A fit of the ratio using a linear function is also shown. 

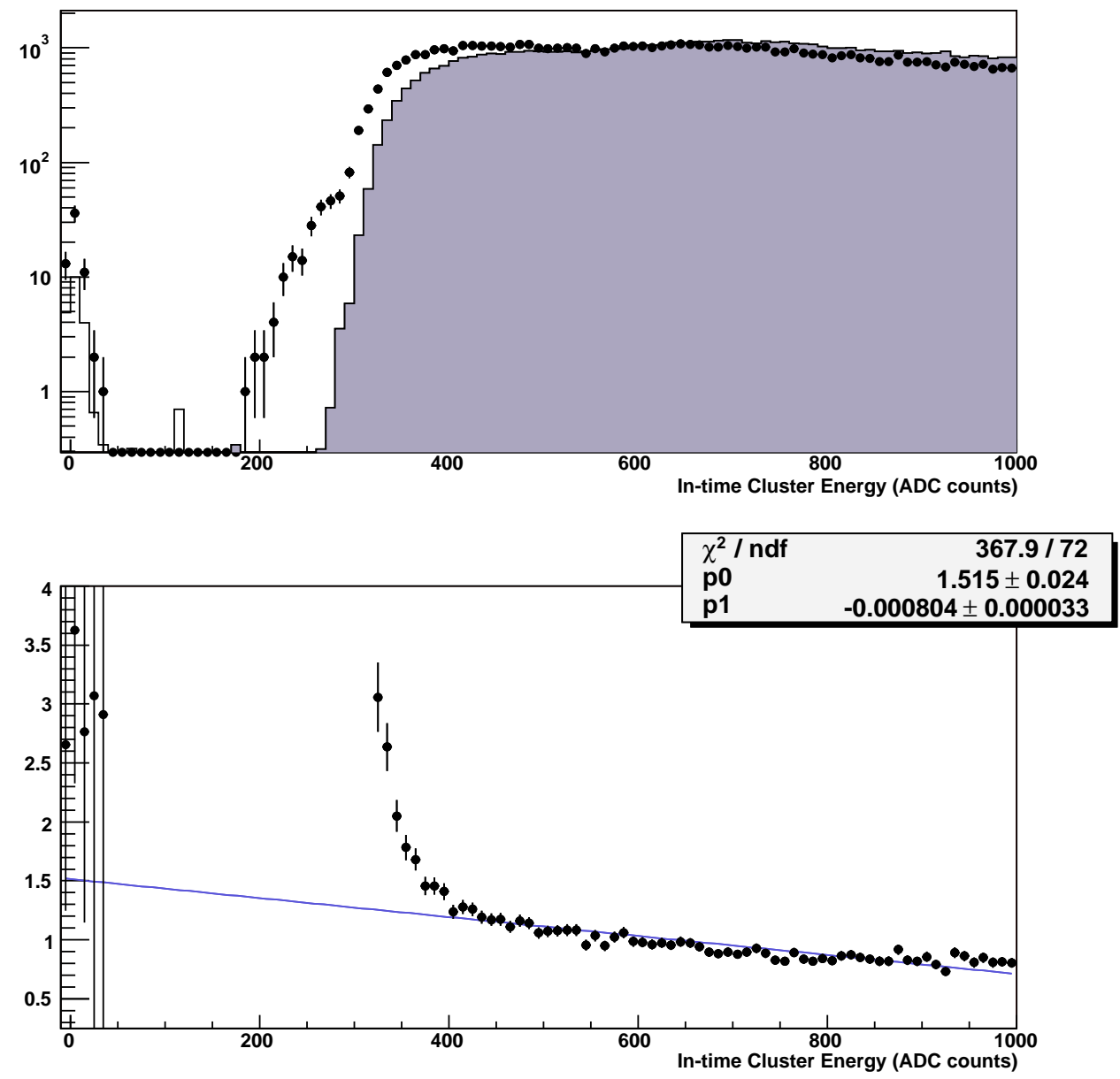

Figure K.16: A plot of the "in-time" energy of the photon candidate cluster before the cut. Data are points, while the histogram is of Monte Carlo simulated events. The solid part of the histogram denotes the areas that pass after this particular cut. A plot of the ratio of the number of data events to Monte Carlo events, before this cut, is shown on the bottom. The number of Monte Carlo events is normalized to be equal to the total number of data events in this plot. A fit of the ratio using a linear function is also shown. 

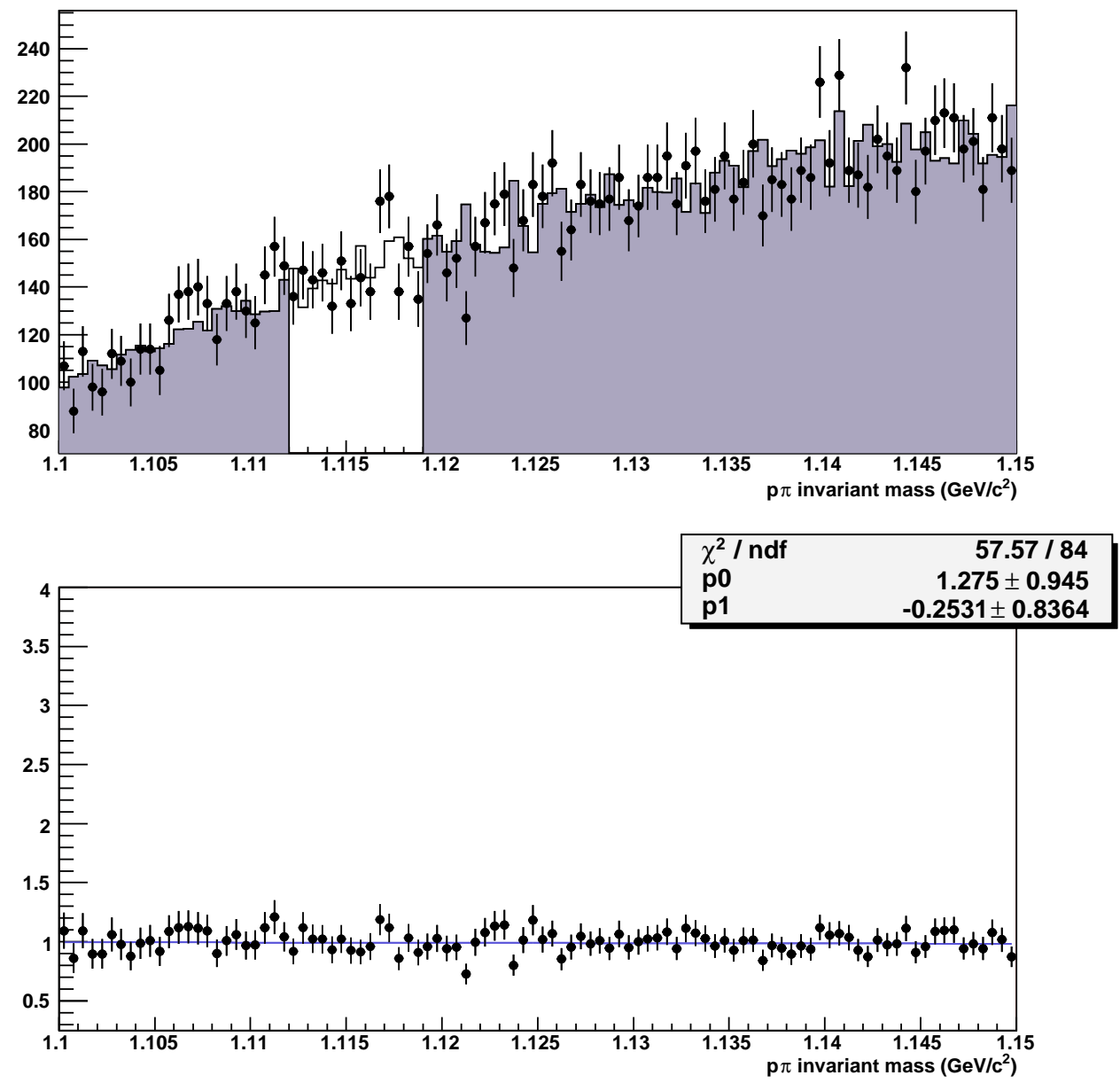

Figure K.17: A plot of the proton/pion invariant mass before the cut. Data are points, while the histogram is of Monte Carlo simulated events. The solid part of the histogram denotes the areas that pass after this particular cut. A plot of the ratio of the number of data events to Monte Carlo events, before this cut, is shown on the bottom. The number of Monte Carlo events is normalized to be equal to the total number of data events in this plot. A fit of the ratio using a linear function is also shown. 

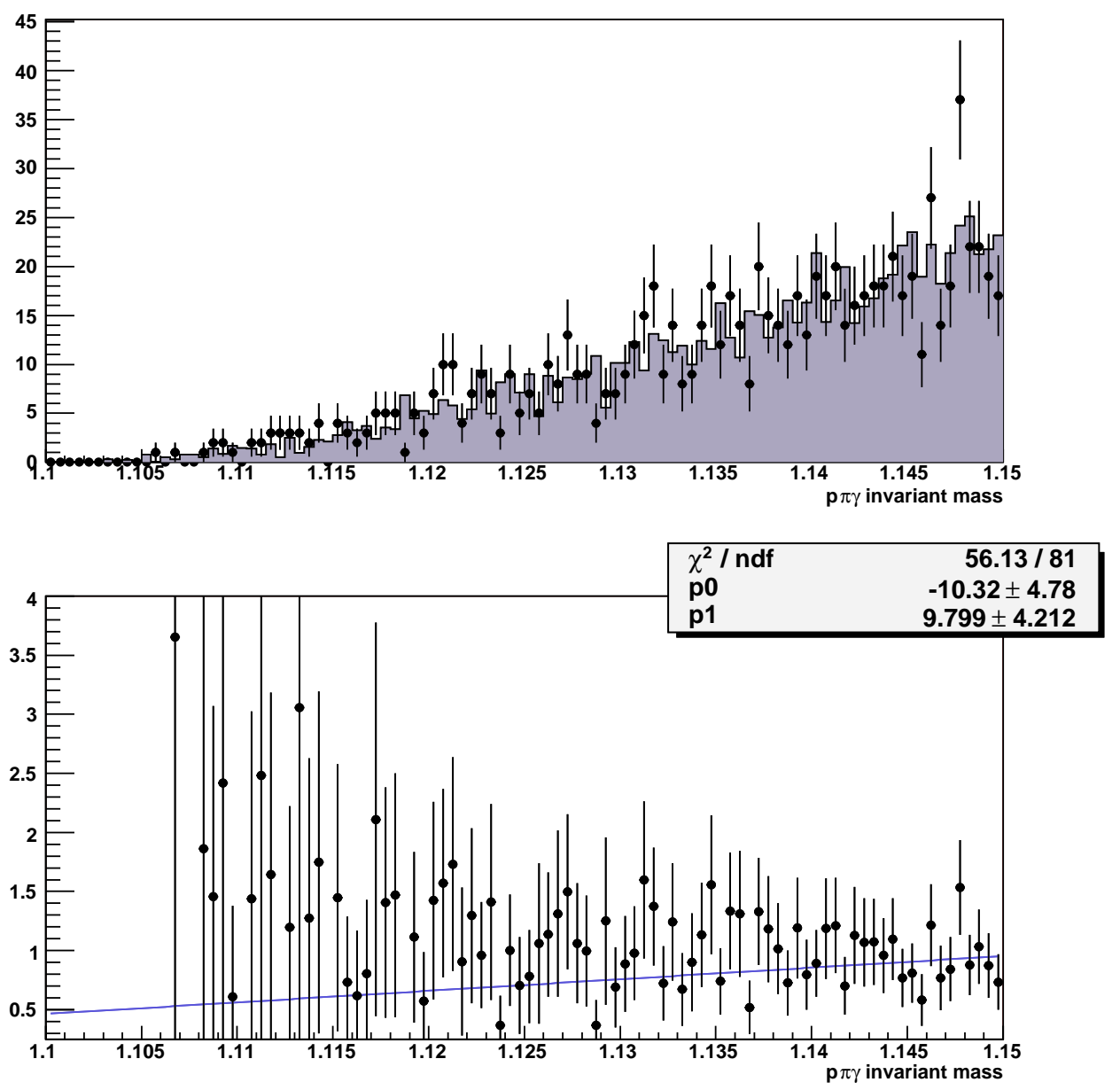

Figure K.18: A plot of the proton/pion/gamma invariant mass, before any cut on this variable. Data are points, while the histogram is of Monte Carlo simulated events. The solid part of the histogram denotes the areas that pass after this particular cut. A plot of the ratio of the number of data events to Monte Carlo events, before this cut, is shown on the bottom. The number of Monte Carlo events is normalized to be equal to the total number of data events in this plot. A fit of the ratio using a linear function is also shown. 

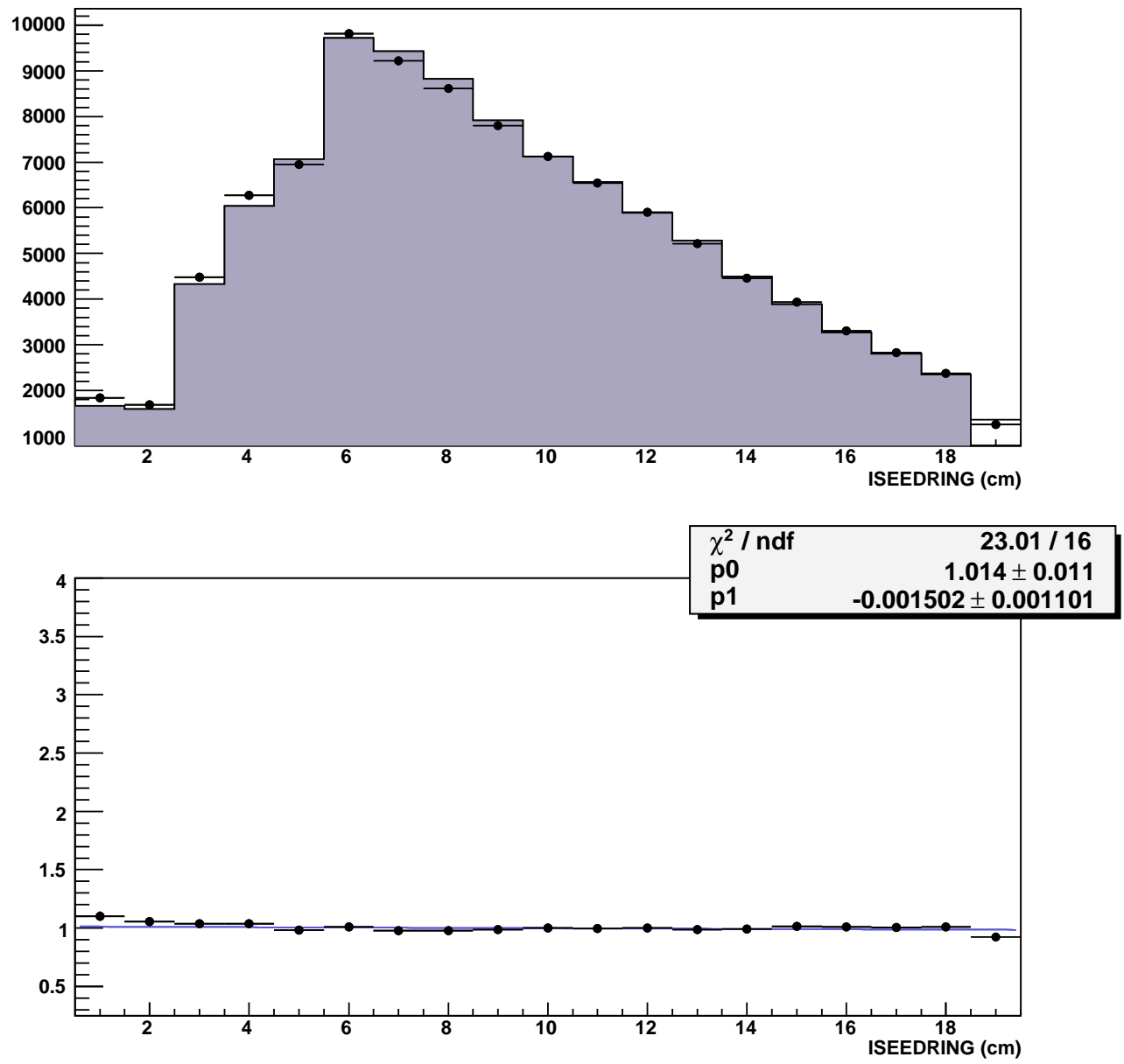

Figure K.19: A plot of the outer photon fiducial cut variable ISEEDRING, before the cut. Data are points, while the histogram is of Monte Carlo simulated events. The solid part of the histogram denotes the areas that pass after this particular cut. A plot of the ratio of the number of data events to Monte Carlo events, before this cut, is shown on the bottom. The number of Monte Carlo events is normalized to be equal to the total number of data events in this plot. A fit of the ratio using a linear function is also shown. 

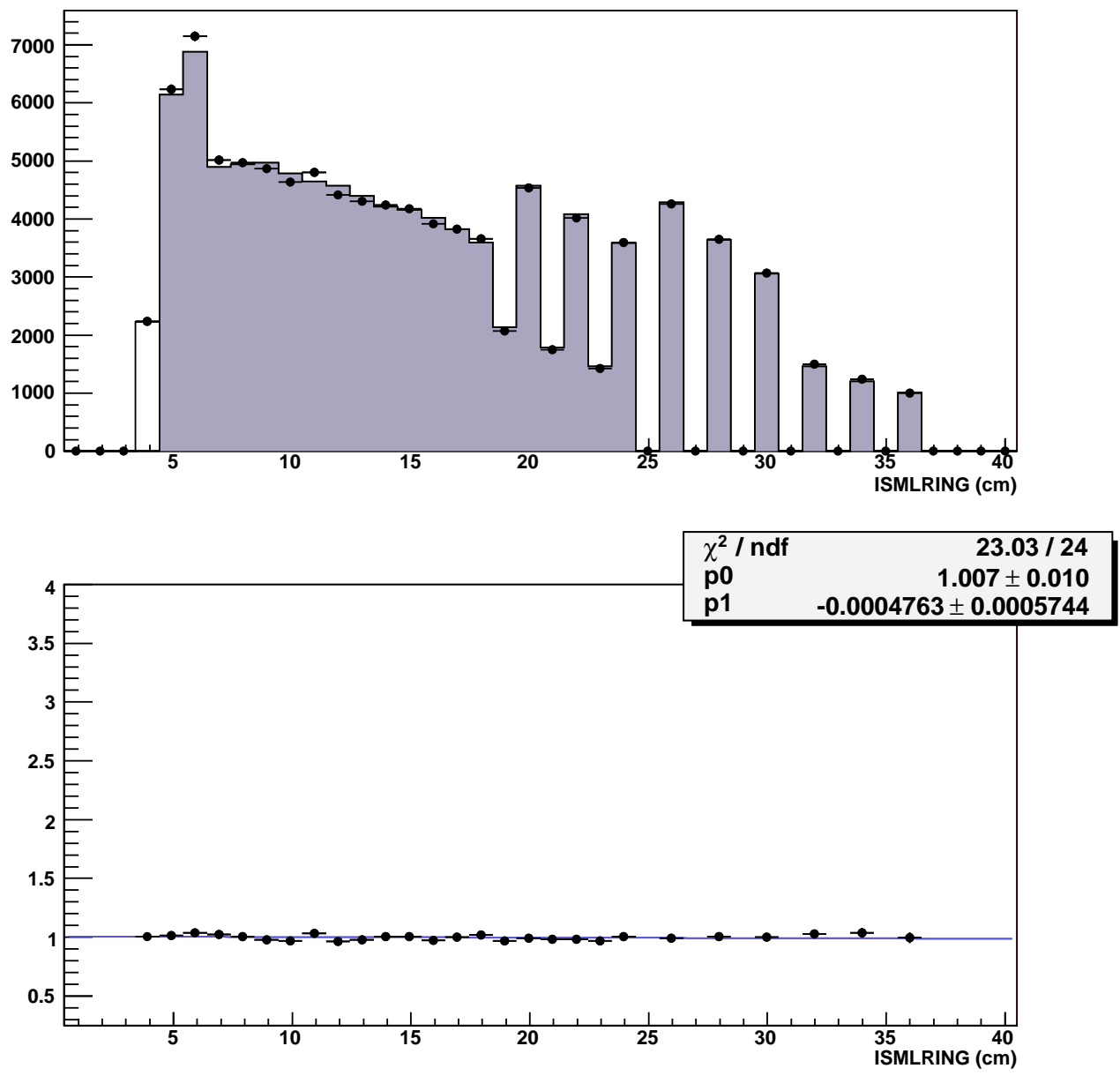

Figure K.20: A plot of the inner photon fiducial cut variable ISMLRNG before the cut. Data are points, while the histogram is of Monte Carlo simulated events. The solid part of the histogram denotes the areas that pass after this particular cut. A plot of the ratio of the number of data events to Monte Carlo events, before this cut, is shown on the bottom. The number of Monte Carlo events is normalized to be equal to the total number of data events in this plot. A fit of the ratio using a linear function is also shown. 

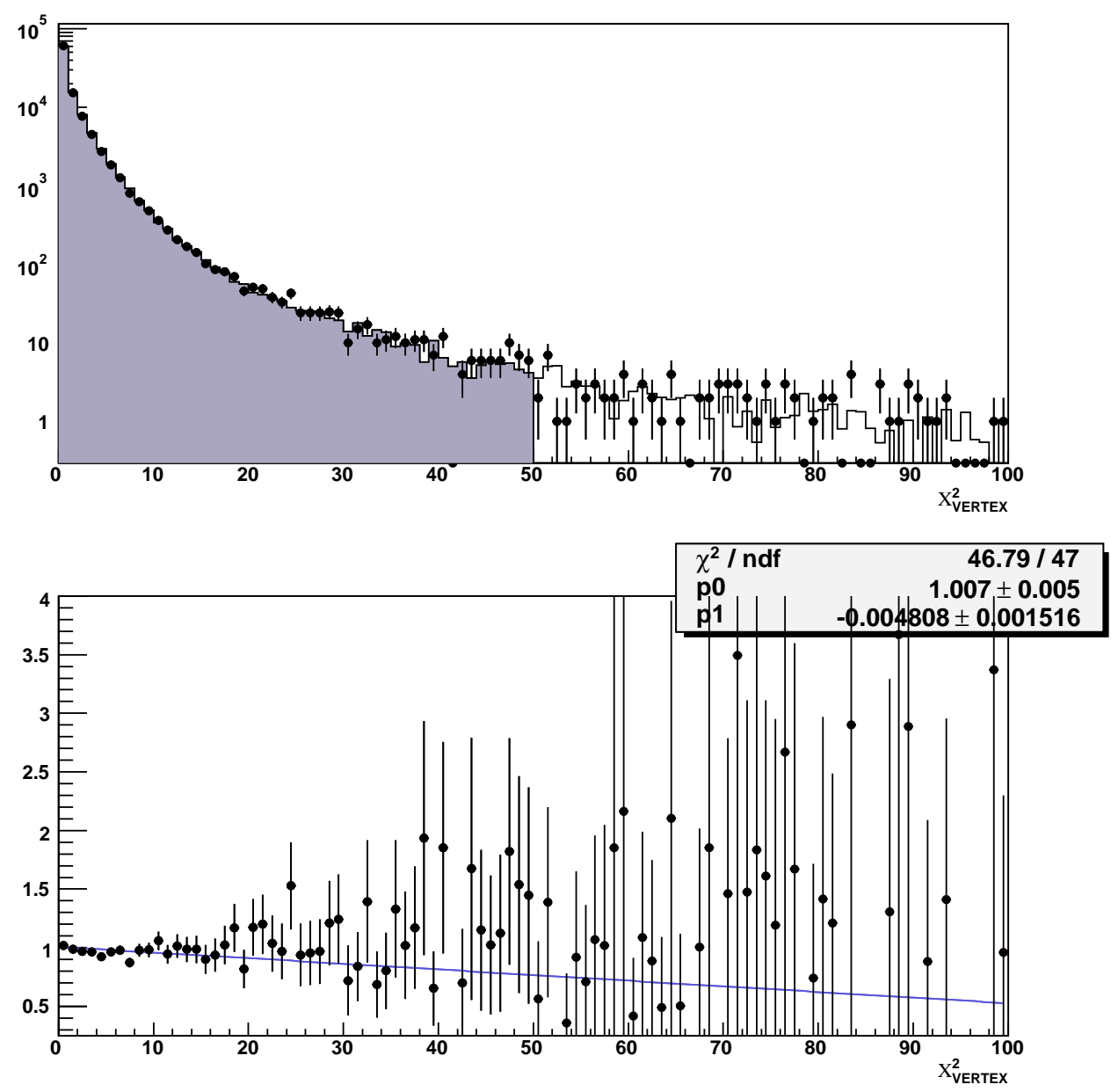

Figure K.21: A plot of vertex $\chi^{2}$ before the cut. Data are points, while the histogram is of Monte Carlo simulated events. The solid part of the histogram denotes the areas that pass after this particular cut. A plot of the ratio of the number of data events to Monte Carlo events, before this cut, is shown on the bottom. The number of Monte Carlo events is normalized to be equal to the total number of data events in this plot. A fit of the ratio using a linear function is also shown. 

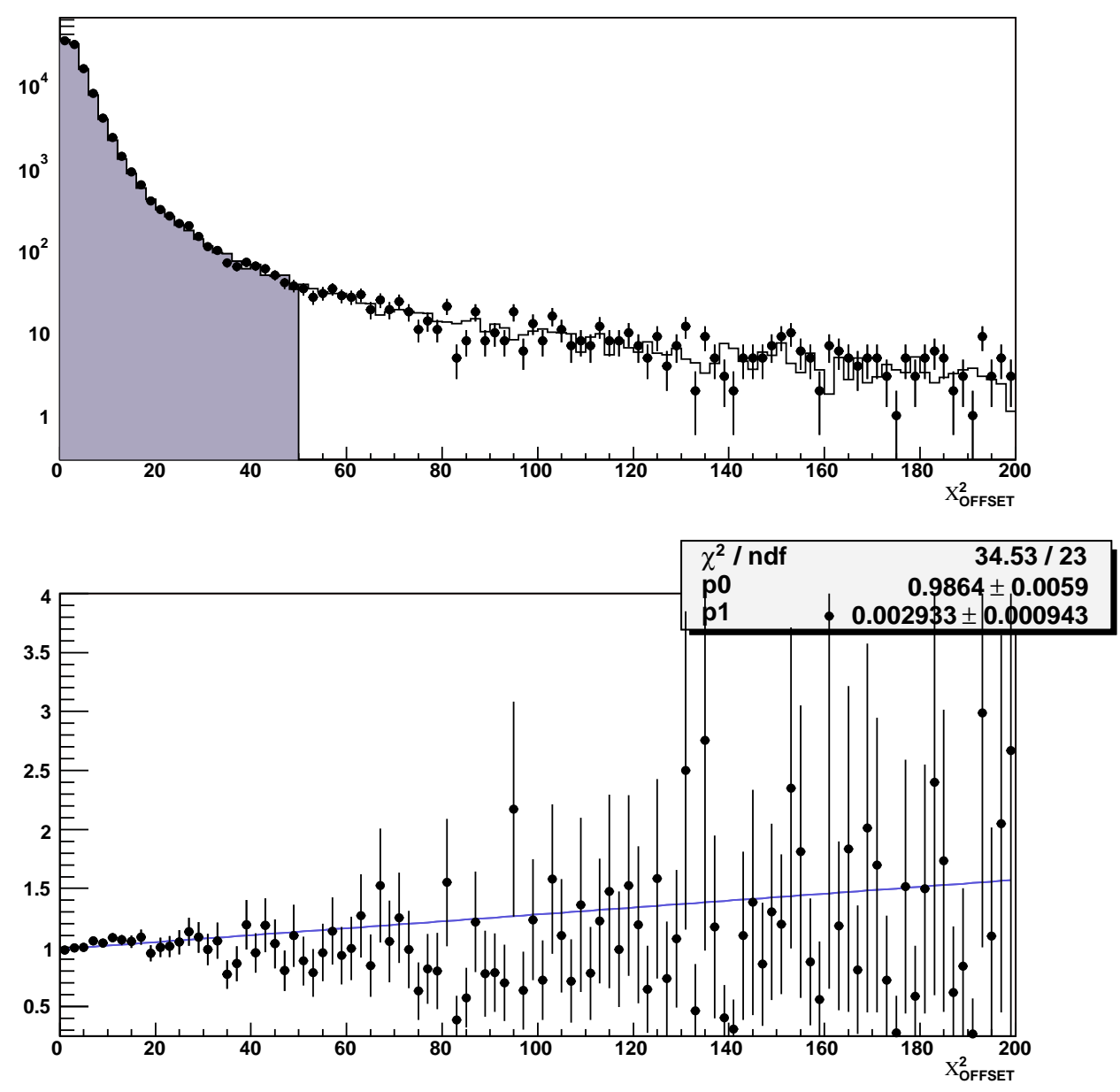

Figure K.22: A plot of the greater of the two track offset $\chi^{2}$ values in each event before the cut. Data are points, while the histogram is of Monte Carlo simulated events. The solid part of the histogram denotes the areas that pass after this particular cut. A plot of the ratio of the number of data events to Monte Carlo events, before this cut, is shown on the bottom. The number of Monte Carlo events is normalized to be equal to the total number of data events in this plot. A fit of the ratio using a linear function is also shown. 

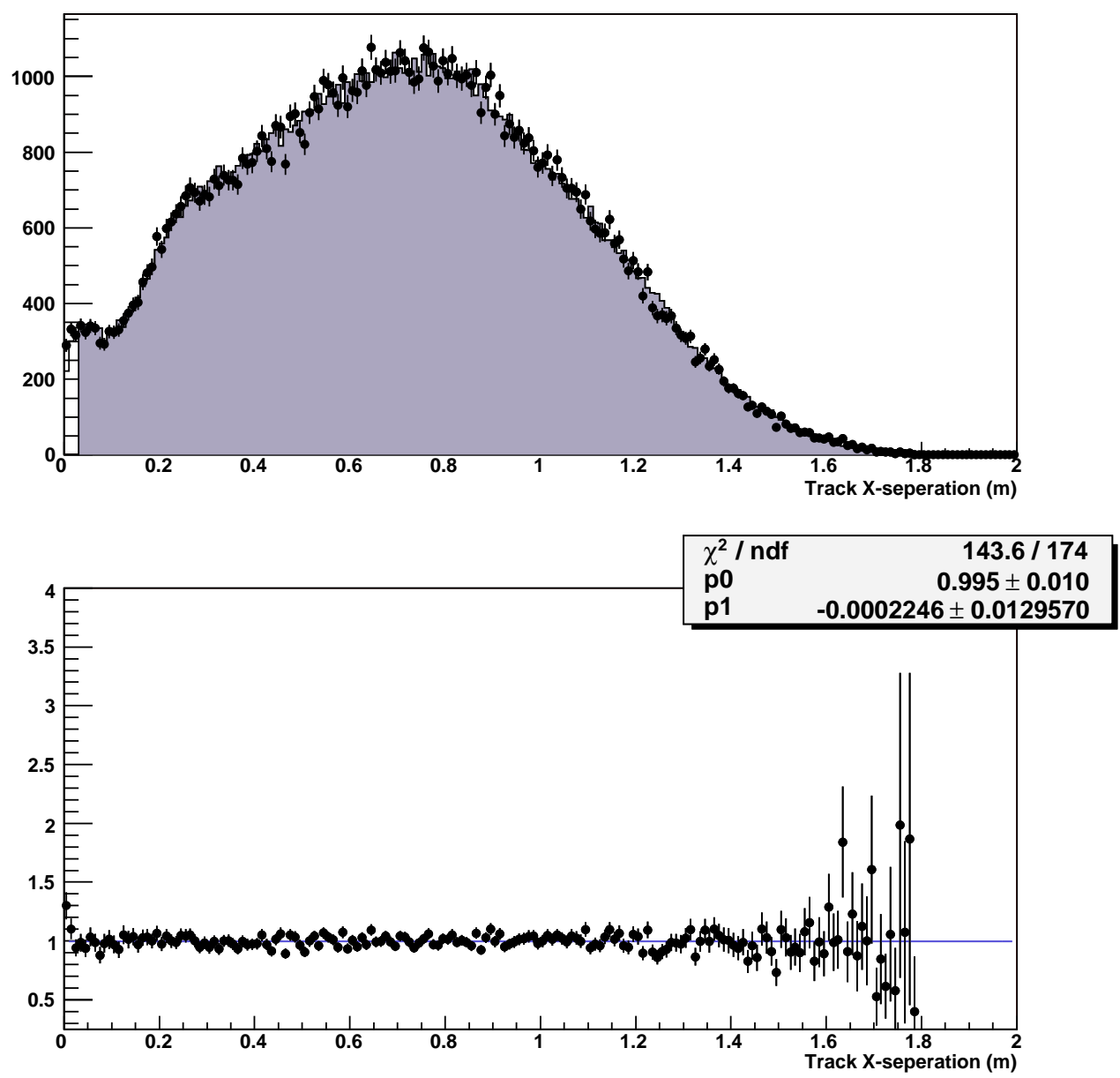

Figure K.23: A plot of the separation of the tracks in the $\mathrm{x}$ direction at the CsI, before the cut. Data are points, while the histogram is of Monte Carlo simulated events. The solid part of the histogram denotes the areas that pass after this particular cut. A plot of the ratio of the number of data events to Monte Carlo events, before this cut, is shown on the bottom. The number of Monte Carlo events is normalized to be equal to the total number of data events in this plot. A fit of the ratio using a linear function is also shown. 

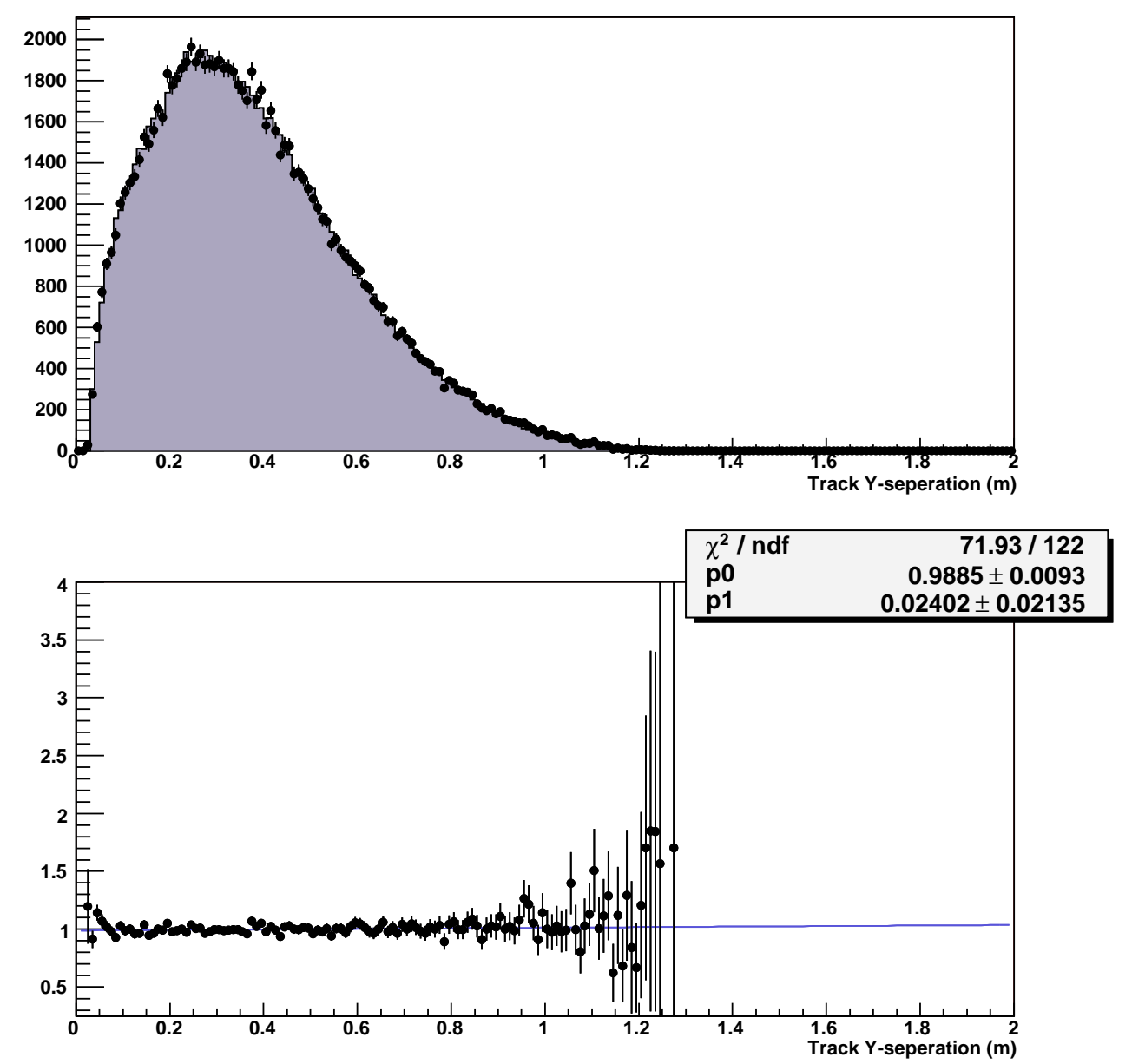

Figure K.24: A plot of the separation of the tracks in the y direction at the CsI, before the cut. Data are points, while the histogram is of Monte Carlo simulated events. The solid part of the histogram denotes the areas that pass after this particular cut. A plot of the ratio of the number of data events to Monte Carlo events, before this cut, is shown on the bottom. The number of Monte Carlo events is normalized to be equal to the total number of data events in this plot. A fit of the ratio using a linear function is also shown. 

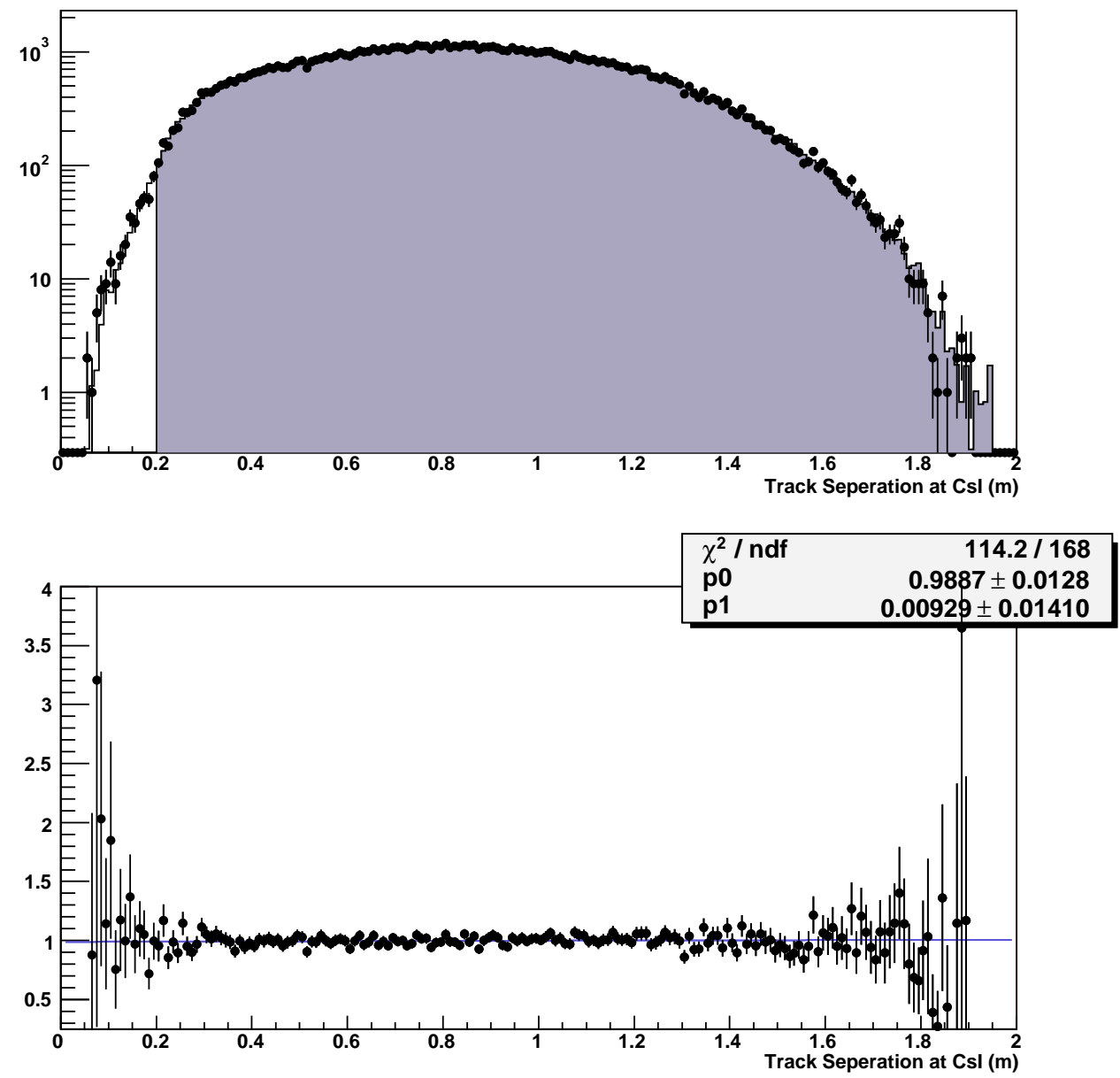

Figure K.25: A plot of the track separation at the CsI before the cut. Data are points, while the histogram is of Monte Carlo simulated events. The solid part of the histogram denotes the areas that pass after this particular cut. A plot of the ratio of the number of data events to Monte Carlo events, before this cut, is shown on the bottom. The number of Monte Carlo events is normalized to be equal to the total number of data events in this plot. A fit of the ratio using a linear function is also shown. 


\section{Bibliography}

[1] C. S. Wu, E. Ambler, R. W. Hayward, D. D. Hoppes, and R. P. Hudson. Experimental test of parity conservation in beta decay. Phys. Rev., 105(4):1413-1415, Feb 1957.

[2] J. H. Christenson, J. W. Cronin, V. L. Fitch, and R. Turlay. Evidence for the $2 \pi$ decay of the K(2)0 meson. Phys. Rev. Lett., 13:138-140, 1964.

[3] A. D. Sakharov. Violation of CP invariance, C asymmetry, and baryon asymmetry of the universe. Pisma Zh. Eksp. Teor. Fiz., 5:32-35, 1967.

[4] T. Alexopoulos et al. Measurements of $K_{L}$ branching fractions and the CP violation parameter $\left|\eta_{+-}\right|$. Phys. Rev., D70:092006, 2004.

[5] T. T. Wu and Chen-Ning Yang. Phenomenological analysis of violation of CP invariance in decay of K0 and anti-K0. Phys. Rev. Lett., 13:380-385, 1964.

[6] James Anthony Graham. Measurements of the direct CP-violating parameter $r e\left(\epsilon^{6} / \epsilon\right)$ and the kaon sector parameters $\delta_{m}, \tau_{S}$, and $\phi_{+-}$. UMI-30-29491.

[7] A. Alavi-Harati et al. Measurements of direct CP violation, CPT symmetry, and other parameters in the neutral kaon system. Phys. Rev., D67:012005, 2003.

[8] L. M. Sehgal and L. Wolfenstein. CP-violating interfrence effects in radiative $K^{0}$ decays. Phys. Rev., 162:1362-1368, 1967.

[9] G. Costa and P.K. Kabir. Possible CP-noninvariant effects in $\pi \pi \gamma$ decay of neutral kaons. Nuovo Cimento, A51:564, 1967. 
[10] G. Donaldson et al. Observation of the decay $K_{L} \rightarrow \pi^{+} \pi^{-} \gamma$. Phys. Rev. Lett., 33:554, 1974.

[11] A. S. Carroll et al. Study of the decay $K_{L} \rightarrow \pi^{+} \pi^{-} \gamma$. Phys. Rev. Lett., 44:529, 1980.

[12] E. J. Ramberg et al. Simultaneous measurement of $K_{S}$ and $K_{L}$ decays into $\pi^{+} \pi^{-} \gamma$. Phys. Rev. Lett., 70:2525-2528, 1993.

[13] A. Alavi-Harati et al. Study of the $K_{L} \rightarrow \pi^{+} \pi^{-} \gamma$ direct emission vertex. Phys. Rev. Lett., 86:761-764, 2001.

[14] E. Abouzaid et al. A measurement of the $\mathrm{K} 0$ charge radius and a $\mathrm{CP}$ violating asymmetry together with a search for CP violating E1 direct photon emission in the rare decay $K_{L} \rightarrow \pi^{+} \pi^{-} e^{+} e^{-}$. Phys. Rev. Lett., 96:101801, 2006.

[15] John Shields. The Search for the Emission of a CP-Violating E1 Photon in the $K_{L} \rightarrow \pi^{+} \pi^{-} \gamma$ Decay. PhD thesis, University of Virginia, 2004.

[16] Y. C. R. Lin and German Valencia. CP violation in $K_{L} \rightarrow \pi^{+} \pi^{-} \gamma$. Phys. Rev., D37:143, 1988.

[17] Pyung-won Ko and Tran N. Truong. $K_{L} \rightarrow 2 \gamma$ and $K_{L} \rightarrow \pi^{+} \pi^{-} \gamma$ decays. Phys. Rev., D43:4-8, 1991.

[18] Charles Picciotto. Analysis of $\eta, K_{L} \rightarrow \pi^{+} \pi^{-} \gamma$ using chiral models. Phys. Rev., D45:1569-1574, 1992.

[19] Giancarlo D'Ambrosio and Gino Isidori. CP violation in kaon decays. Int. J. Mod. Phys., A13:1-94, 1998.

[20] Jusak Tandean and G. Valencia. Reanalysis of CP violation in $K_{L} \rightarrow \pi^{+} \pi^{-} \gamma$. Phys. Rev., D62:116007, 2000.

[21] L. M. Sehgal and M. Wanninger. CP violation in the decay $K_{L} \rightarrow \pi^{+} \pi^{-} e^{+} e^{-}$. Phys. Rev., D46:1035-1041, 1992. 
[22] S. Eidelman, K.G. Hayes, K.A. Olive, M. Aguilar-Benitez, C. Amsler, D. Asner, K.S. Babu, R.M. Barnett, J. Beringer, P.R. Burchat, C.D. Carone, C. Caso, G. Conforto, O. Dahl, G. D’Ambrosio, M. Doser, J.L. Feng, T. Gherghetta, L. Gibbons, M. Goodman, C. Grab, D.E. Groom, A. Gurtu, K. Hagiwara, J.J. Hernández-Rey, K. Hikasa, K. Honscheid, H. Jawahery, C. Kolda, Kwon Y., M.L. Mangano, A.V. Manohar, J. March-Russell, A. Masoni, R. Miquel, K. Mönig, H. Murayama, K. Nakamura, S. Navas, L. Pape, C. Patrignani, A. Piepke, G. Raffelt, M. Roos, M. Tanabashi, J. Terning, N.A. Törnqvist, T.G. Trippe, P. Vogel, C.G. Wohl, R.L. Workman, W.-M. Yao, P.A. Zyla, B. Armstrong, P.S. Gee, G. Harper, K.S. Lugovsky, S.B. Lugovsky, V.S. Lugovsky, A. Rom, M. Artuso, E. Barberio, M. Battaglia, H. Bichsel, O. Biebel, P. Bloch, R.N. Cahn, D. Casper, A. Cattai, R.S. Chivukula, G. Cowan, T. Damour, K. Desler, M.A. Dobbs, M. Drees, A. Edwards, D.A. Edwards, V.D. Elvira, J. Erler, V.V. Ezhela, W. Fetscher, B.D. Fields, B. Foster, D. Froidevaux, M. Fukugita, T.K. Gaisser, L. Garren, H.-J. Gerber, G. Gerbier, F.J. Gilman, H.E. Haber, C. Hagmann, J. Hewett, I. Hinchliffe, C.J. Hogan, G. Höhler, P. Igo-Kemenes, J.D. Jackson, K.F. Johnson, D. Karlen, B. Kayser, D. Kirkby, S.R. Klein, K. Kleinknecht, I.G. Knowles, P. Kreitz, Yu.V. Kuyanov, O. Lahav, P. Langacker, A. Liddle, L. Littenberg, D.M. Manley, A.D. Martin, M. Narain, P. Nason, Y. Nir, J.A. Peacock, H.R. Quinn, S. Raby, B.N. Ratcliff, E.A. Razuvaev, B. Renk, G. Rolandi, M.T. Ronan, L.J. Rosenberg, C.T. Sachrajda, Y. Sakai, A.I. Sanda, S. Sarkar, M. Schmitt, O. Schneider, D. Scott, W.G. Seligman, M.H. Shaevitz, T. Sjöstrand, G.F. Smoot, S. Spanier, H. Spieler, N.J.C. Spooner, M. Srednicki, A. Stahl, T. Stanev, M. Suzuki, N.P. Tkachenko, G.H. Trilling, G. Valencia, K. van Bibber, M.G. Vincter, D. Ward, B.R. Webber, M. Whalley, L. Wolfenstein, J. Womersley, C.L. Woody, O.V. Zenin, and R.-Y. Zhu. Review of Particle Physics. Physics Letters B, 592:1+, 2004.

[23] B. De Wit and J. Smith. Field Theory in Particle Physics. North Holland, 1986.

[24] L. M. Sehgal and J. van Leusen. Violation of time reversal invariance in the decays $K_{L} \rightarrow \pi^{+} \pi^{-} \gamma$ and $K_{L} \rightarrow \pi^{+} \pi^{-} e^{+} e^{-}$. Phys. Rev. Lett., 83:4933-4936, 1999. 
[25] G. D'Ambrosio and N. Paver. Kaon decay interferometry as meson dynamics probes. Phys. Rev., D49:4560-4569, 1994.

[26] E. J. Ramberg et al. First measurement of the CP violation parameter $\eta_{+-\gamma}$ in neutral kaon decays. Phys. Rev. Lett., 70:2529-2532, 1993.

[27] John N. Matthews et al. New measurement of the CP violation parameter $\eta_{+-\gamma}$. Phys. Rev. Lett., 75:2803-2806, 1995.

[28] K. Arisaka et al. KTeV design report: Physics goals, technical components, and detector costs. FERMILAB-FN-0580.

[29] Peter Sven Shawhan. Observation of direct CP violation in $K_{S, L} \rightarrow \pi \pi$ decays. FERMILAB-THESIS-1999-21.

[30] Valmiki Prasad. Measurements of direct CP-violation and CPT-invariance in the neutral kaon system. FERMILAB-THESIS-2002-06.

[31] G. Colin Brown. A precision measurement of the charge asymmetry of semileptonic kaon decays behind a regenerator at KTeV. FERMILAB-THESIS-200404 .

[32] David G. Williams. A study of $\mathrm{NaBi}(\mathrm{WO} 4) 2$ as a cherenkov radiator. Defense Technical Information Center Accession Number : ADA403809.

[33] Peter L. Mikelsons. Search for the decay $K_{L} \rightarrow \pi^{0} e^{+} e^{-}$and study of the decay $K_{L} \rightarrow e^{+} e^{-} \gamma \gamma$. FERMILAB-THESIS-1999-40.

[34] Richard Kessler. $K \rightarrow \pi^{+} \pi^{-} \gamma$ level3, crunch and mc.

[35] T. Alexopoulos et al. Measurements of the branching fractions and decay distributions for $K_{L} \rightarrow \pi^{ \pm} \mu^{\mp} \nu \gamma$ and $K_{L} \rightarrow \pi^{ \pm} e^{\mp} \nu \gamma$. Phys. Rev., D71:012001, 2005.

[36] Michael Ronquest. Notes on the background estimation for the $1997 K_{L} \rightarrow$ $\pi^{+} \pi^{-} \gamma$ analysis.

[37] A.J. Malensek. 
[38] Elisabetta Barberio and Zbigniew Was. PHOTOS: A universal monte carlo for qed radiative corrections. version 2.0. Comput. Phys. Commun., 79:291-308, 1994.

[39] R. Brun, R. Hagelberg, M. Hansroul, and J. C. Lassalle. Geant: Simulation program for particle physics experiments. user guide and reference manual. CERNDD-78-2-REV.

[40] Harald Cramér. Mathematical Methods of Statistics. Princeton University Press, 1958.

[41] William H. Press, Saul A. Teukolsky, William T. Vetterling, and Brian P. Flannery. Numerical Recipes in Fortran, Second Edition. Cambridge University Press, 1992.

[42] R. Fletcher. A new approach to variable metric algorithms. Comp. J., 13:317$322,1970$.

[43] F. James and M. Roos. 'MINUIT' a system for function minimization and analysis of the parameter errors and correlations. Comput. Phys. Commun., 10:343367,1975 .

[44] Joel Heinrich. Pitfalls of goodness-of-fit from likelihood. 2003.

[45] Alexander Golossanov. Measurements of $\mathrm{CP}$ violation and $k^{0}$ charge radius using $K_{L} \rightarrow \pi^{+} \pi^{-} e^{+} e^{-}$decays. FERMILAB-THESIS-2005-47.

[46] E. Abouzaid et al. Measurement of direct photon emission in the $K_{L} \rightarrow \pi^{+} \pi^{-} \gamma$ decay mode. Phys. Rev., D74:032004, 2006.

[47] Silvia Goy Lopez. Study of the $K^{ \pm} \rightarrow \pi^{\mp} \pi^{0} \gamma$ decays at the NA48/2 experiment at the CERN SPS. PhD thesis, Torino University, 2006. Retrieved From http://goylopez.home.cern.ch/goylopez/thesis.pdf.

[48] Luigi Cappiello and Giancarlo D'Ambrosio. Form factor in $K^{+} \rightarrow \pi^{+} \pi^{0} \gamma$ : interference versus direct emission. Phys. Rev., D75:094014, 2007. 
[49] A. Lai et al. Investigation of $K_{L, S} \rightarrow \pi^{+} \pi^{-} e^{+} e^{-}$decays. Eur. Phys. J., C30:3349, 2003.

[50] M. Peskin and D. Schroeder. An Introduction to Quantum Field Theory. Westview Press, 1995.

[51] Kip S. Thorne, Charles W. Misner, and John Archibald Wheeler. Gravitation. W.H. Freeman, 1973. 